\title{
DIGITALCOMMONS
}

$5-1-2002$

\section{Vol. 1, No. 1 (Full Issue)}

JMASM Editors

Follow this and additional works at: http:// digitalcommons.wayne.edu/jmasm

\section{Recommended Citation}

Editors, JMASM (2002) "Vol. 1, No. 1 (Full Issue)," Journal of Modern Applied Statistical Methods: Vol. 1 : Iss. 1 , Article 29. DOI: $10.22237 /$ jmasm/1020254700

Available at: http://digitalcommons.wayne.edu/jmasm/vol1/iss1/29 


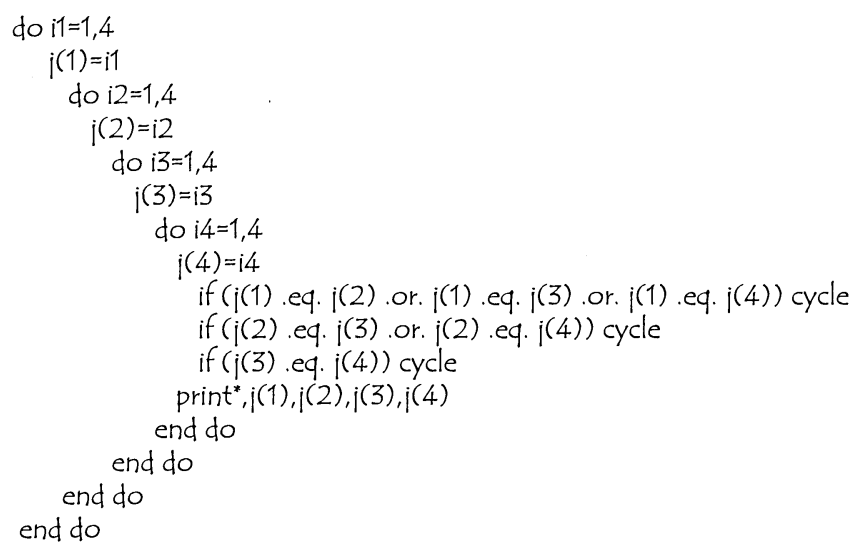

\section{Journal of Modern Applied Statistical Methods}

\begin{tabular}{|c|c|c|}
\hline \multicolumn{3}{|r|}{ Invited Articles } \\
\hline $2-12$ & Pranab K. Sen & $\begin{array}{l}\text { Shifting Goals And Mounting Challenges For Statistical } \\
\text { Methodology }\end{array}$ \\
\hline $13-18$ & R. Clifford Blair & $\begin{array}{l}\text { A Distribution-Free Maximum Test Of Location For Two } \\
\text { Independent Samples }\end{array}$ \\
\hline $19-23$ & W. Jay Conover & Some Locally Most Powerful Rank Tests For Correlation \\
\hline $24-31$ & $\begin{array}{l}\text { Rand R. Wilcox, } \\
\text { H. J. Keselman }\end{array}$ & Power Analysis When Comparing Trimmed Means \\
\hline
\end{tabular}

Shlomo S. Sawilowsky

Wayne State University

Editor

Bruno D. Zumbo

University of British Columbia

Associate Editor 


\section{Journal of Modern Applied Statistical Methods}

JMASM is an independent journal designed to provide an outlet for the scholarly works of applied nonparametric or parametric statisticians, data analysts, researchers, classical or modern psychometricians, quantitative or qualitative evaluators, and methodologists. Work appearing in Regular Articles and Brief Reports are peer reviewed; in Statistical Software Applications \& Review and JMASM Algorithms and Code are reviewed by the Editorial Board; and in Graduate Student Research are reviewed by graduate students.

Three areas are appropriate for JMASM: (1) development or study of new statistical tests or procedures, or the comparison of existing statistical tests or procedures, using computer-intensive Monte Carlo, bootstrap, jackknife, or resampling methods, (2) development or study of nonparametric, robust, permutation, exact, and approximate randomization methods, and (3) applications of computer programming, preferably in Fortran (all other programming environments are welcome), related to statistical algorithms, pseudo-random number generation, simulation techniques, and self-contained executable code to carry out new or interesting statistical methods. Elegant derivations, as well as articles with no take-home message to practitioners, have low priority. Articles based on Monte Carlo (and other computerintensive) methods designed to evaluate new or existing techniques or practices, particularly as they relate to novel applications of modern methods to everyday data analysis problems, have high priority.

Problems may arise from applied statistics and data analysis; experimental and nonexperimental research design; psychometry, testing, and measurement; and quantitative or qualitative evaluation. They should relate to the social and behavioral sciences, especially education and psychology. Applications from other traditions, such as actuarial statistics, biometrics or biostatistics, chemometrics, econometrics, environmetrics, jurimetrics, quality control, and sociometrics, are welcome. Applied methods from other disciplines (e.g., astronomy, business, engineering, genetics, logic, nursing, marketing, medicine, oceanography, pharmacy, physics, political science) are acceptable if the demonstration holds promise for the social and behavioral sciences.

\section{Editor}

Shlomo S. Sawilowsky, Wayne State University

Editorial Assistant

Jennifer Bunner

\section{Associate Editor}

Bruno D. Zumbo, University of British Columbia

\section{Editorial Board ${ }^{1}$}

Razia Azen, University of Wisconsin, Milwaukee Vance Berger ${ }^{2}$, National Cancer Institute

Gail Fahoome ${ }^{2}$, Wayne State University

Paolo Guidici, University of Pavia, Italy

Todd C. Headrick ${ }^{2}$, Southern Illinois University, Carbondale

Sin-Ho Jung, Indiana University

Chaeyoung Lee, Hallym University, Korea

Michael J. Nanna ${ }^{2}$, Arizona State University

Fadia Nasser, Tel Aviv University

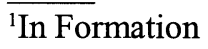

${ }^{2}$ Assistant Editor

Internet Sponsor

College of Education, Wayne State University
Professional Staff

Bruce Fay, Business Manager

Joe Musial, Marketing Director

Scott Compton, Internet Development

Ken Martin, Printing Consultant

WSU Graduate Student Staff

P. Monet Conner

Jacqueline Drouin

Mary Golinski

Jack Hill

Kalvin Holt

Irwin Jopps

Karen Lee

J. Sia Robinson

Lori Shingledecker

Boris Shulkin

Andrew Tierman

Keith Williams

Bonnie M. Motkyka, Undergraduate Student Intern, Grand Valley State University

Production Staff

Bulent Ozkan

Polavaram Dinakar Prasad
Printer: Entire Reproductions and Imaging Solutions Internet: www.entire-repro.com
248.299.8900 (Phone) e-mail:

248.299.8916 (Fax) sales@entire-repro.com 


\section{Journal Of Modern Applied Statistical Methods}

Invited Articles

2-12 Pranab K. Sen

$13-18$

R. Clifford Blair

$19-23$

W. Jay Conover

24-31 Rand R. Wilcox,

H. J. Keselman

Regular Articles

32-41

Suzanne R. Dubnika,

R. Clifford Blair, Thomas P. Hettmansperger

42-51 Christopher D. Corcoran, Cyrus R. Mehta

52-60 Shlomo S. Sawilowsky

$61-68$

Bruno D. Zumbo

69-73 Robert A. Malkin

74-82 Vance W. Berger, Cliff Lunneborg, Michael D. Ernst, Jonathan G. Levine

83-99 Michael J. Nanna

100-109 Kathleen Peterson

110-113 Michael Wolf-Branigin

114-125 Abraham Y. Nahm, Luis E. Solis-Galvan, S. Subba Rao, T. S. Ragu-Nathan
Shifting Goals And Mounting Challenges For Statistical Methodology

A Distribution-Free Maximum Test Of Location For Two Independent Samples

Some Locally Most Powerful Rank Tests For Correlation

Power Analysis When Comparing Trimmed Means

Rank-based Procedures For Mixed Paired And Two Sample Designs

Exact Level And Power Of Permutation, Bootstrap, And Asymptotic Tests For Trend

A Measure of Relative Efficiency For Location Of A Single Sample

An Adaptive Inference Strategy: The Case Of Auditory Data

An Unconditional Exact Test For Small Samples Matched Binary Pairs

Parametric Analyses In Randomized Clinical Trials

Hotelling's $\mathrm{T}^{2}$ vs The Rank Transform With Real Likert Data

Six Modifications Of The Aligned Rank Transform Test For Interaction

Applying Spatial Randomness To Community Inclusion

The Q-Sort Method: Assessing Reliability And Construct Validity Of Questionnaire Items At A Pre-Testing Stage 
126-130 Ernest P. Chiodo, Joseph L. Musial, J. Sia Robinson

131-138 Ricardo Ocaña-Riola,

Brief Reports

139-142 R. Clifford Blair, Stephen R. Cole

143-144 Shlomo S. Sawilowsky, The Trouble With Trivials $(p>.05)$ Jina Yoon

145-146 Shlomo S. Sawilowsky Barry S. Markman
An Error In Statistical Logic In the Application Of Genetic Paternity Testing

Two Methods To Estimate Homogeneous Markov Processes
Two-sided Equivalence Testing Of the Difference Between Two Means

Statistical Software Applications \& Review

147-156 Chao-Ying Joanne Peng, Modeling Strategies In Logistic Regression With SAS, SPSS, Systat, Tak-Shing Harry So

Using The t Test With Uncommon Sample Sizes $B M D P$, Minitab, And STATA

157-166 B. W. Frankland, Bruno D. Zumbo

Quantifying Bimodality Part I: An Easily Implemented Method Using SPSS

167-175 Margaret A. Posch

Asymptotic And Exact Tests in $2 \times \mathrm{C}$ Ordered Categorical Contingency Tables With StatXact 2.0 - 4 .0

\section{Graduate Student Research}

176-181 Jennifer Bunner, Shlomo S. Sawilowsky

Alternatives To $\mathrm{s}_{\mathrm{w}}$ In The Bracketed Interval For The Trimmed Mean

JMASM Algorithms and Code

182-190 Gail Fahoome

JMASM1: RANGEN 2.0 (Fortran 90/95)

191-194 Constantine Stamatopoulos JMASM2: Generation Of Combinations (Excel)

195-201 Todd C. Headrick

JMASM3: A Method For Simulating Systems Of Correlated Binary Data (Fortran 77)

End Material

Advertisements

Inside Back Cover

Instructions for Authors 


\title{
INVITED ARTICLES Shifting Goals And Mounting Challenges For Statistical Methodolgy
}

\author{
Pranab K. Sen \\ Departments of Biostatistics and Statistics, \\ University of North Carolina, Chapel Hill, NC
}

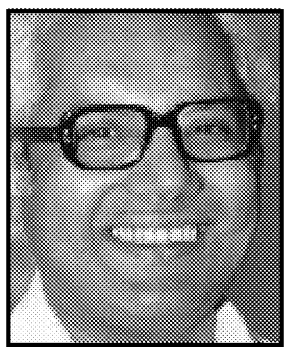

Modern interdisciplinary research in statistical science encompasses a wide field: agriculture, biology, biomedical sciences along with bioinformatics, clinical sciences, education, environmental and public health disciplines, genomic science, industry, molecular genetics, socio-behavior, socio-economics, toxicology, and a variety of other disciplines. Statistical science has historically had mathematical perspectives dominating theoretical and methodological developments. Yet, the advent of modern information technology has opened the doors for highly computation intensive statistical tools (i.e., software), wherein mathematical aspects are often de-emphasized. Knowledge discovery and data mining (KDDM) is now becoming a dominating force, with bioinformatics as a notable example. In view of this apparent discordance between mathematical (frequentist as well as Bayesian) and computational approaches to statistical resolutions, and a genuine need to formulate training as well as research curricula to meet growing demands, a critical appraisal of statistical innovations is made with due respect to its mathematical heritage, as well as scope of application. Some of the challenging statistical tasks are illustrated.

Keywords: Bioinformatics, Biostatistics, Clinical trials, Computational sequence analysis, Data mining, Dosimetry, KDDM, Pharmacogenomics, Quality of life

\section{Introduction}

Unlike most of the basic sciences, the evolution of statistical science has followed a somewhat different tract. Indeed, the question can be raised if statistics can be regarded as a science discipline. This might be partially due to its genesis and partially to the increased demand for statistical tools in almost all walks of life and science.

Statistics aimed to capture the power of observations mostly from empirical studies (as in clinical as well as other experimental sciences), and yet to formulate a general body of theory and methodology that provides rationality in a scientific and objective way. Striking a balance between mathematical exactness and natural

Pranab Kumar Sen is Cary C. Boshamer Professor, University of North Carolina, Chapel Hill. He is a Fellow of the American Statistical Association, and the Institute of Mathematical Statistics. He was awarded the 1998 Czech Union of Mathematicians and Physicists Commemoration Medal. His research has mostly been in nonparametrics, covering bioassays, psychometry, multivariate analysis, linear models, sequential analysis, survival analysis, toxicology, bioenvironmetrics, and stochastic processes. He has (co-)authored 10 texts and monographs and 540 publications, and (co-)edited 10 scientific volumes. He also cherishes the opportunity to indulge in literary works, and has published some of his poems. diversity (variation) has always been the basic goal in statistical reasoning. Nevertheless, combining empirical and methodological reasoning has never been an easy task; it has led to a lot of diversity in the current visualization of statistical science as an integrated and interdisciplinary field. At the same time, it has created some distinct division within the discipline (such as mathematical statistics, probability theory, applied probability theory, stochastic processes, applied statistics, experimental statistics, biometry, biostatistics, actuarial statistics, industrial statistics, public health statistics - notably, epidemiology -, environmetrics, nutrition, health administration and health care, and medical statistics, among others). These sectors are often demarcated with conventional frontiers of their own that do not overlap much.

As a result, the pedagogical, research, and practice portfolios in these sub-disciplines are often not so much compatible. Yet, it is undeniable that statistical reasoning is essential in all these disciplines, and hence, there must be a unifying factor in the statistical foundations. Moreover, the genesis of theoretical statistics (not to be mislabeled with the calculus of variations) is in a consortium of such as gambling, demography, genetics, anthropometry, agriculture, and biology as a whole. (Otherwise, statistics would have probably been a subversion of quantum physics or functional analysis!) It is also undeniable that in order to survive as an objective and scientific way of reasoning, statistics, as a discipline, needs the utmost 
patronage of mathematical logic as well as understanding. This remains pertinent to data analysis in a valid statistical way, and much of the discussion to follow will center on this point.

Is it natural to see (even) elementary school children playing with personal computers, and start data analysis (in the pretense of statistics) without having any idea of the subject matter? Perhaps, the advent of information technology might have changed the interface of statistical science in this way. If so, coping with data analysis may be the future goal of statistical science!

From a pedagogical perspective, (with the tendency of specialization in a of any broad discipline), it becomes imperative to examine undercurrents carefully, and to remodel programs accordingly. This relates to the necessity of examining the structure of high school level training in mathematical sciences with a view to orient the prospective college-bound students in statistical science (of course, with a good amount of planning so as not to create an imbalance in the training of people in this area with suitable job prospects and career development opportunities), to introduce the basics of statistical science at the high school to undergraduate levels, and to promote graduate level training for those who reach that level.

In this respect, the coverage of the subject material as well as students' mathematical levels needs to be planned and reviewed in a careful manner. In view of the enormously large number of college-bound students and the diversity of the subjects (compared to fifty years back), as well as, the differential job prospects (that evolutes quite dynamically), there are good reasons to appraise the conventional high school, undergraduate, and graduate curriculum in statistical science. The need for professional (licentiate) statisticians should be addressed as well, as discussed below. Perhaps, it would be more appropriate to depict the evolution of statistical sciences first, and then, in the light of that, to discuss the pertinent issues in other related items.

\section{The Evolution of Statistical Science}

A history of the evolution of statistical science could well be dependent on who wrote it and what was to be highlighted in the compilation. Evolution of probability theory and stochastic processes mostly took place in mathematical and physical sciences. It is clearly identifiable that the abstractions came generally from mathematics, but useful applications came generally from physical and engineering sciences. The classical Brownian motion and Brownian bridge are concrete examples in this respect.

Also, statistical mechanics reflects the interactive nature of classical probability theory and quantum mechanics in physics. The field of operations research and systems analysis has exhibited a high level of interaction between statistical reasoning with industrial engineering and operations management policies. Yet, a very systematic integration of probability theory and stochastic processes with mathematical statistics did not take place until about seventy-five years ago, and the channel is still quite open.

Of course, long before the evolution of modern mathematical statistics started, statistics arose as a study of the description of states, especially their economic and demographic aspects. A match of this origin of statistics can be traced to ancient China, Egypt, and India among other places. For example, chautha, one-quarter of a farmer's harvest, was to be given to the royal (or ruler's) depository, as a practice in India and other oriental places, and this could not have been done without some statistical sense.

Aside from such usages more than several thousand years ago, the more systematic tract of the evolution can be traced in the sixteenth century (CE) where several European countries (or kingdoms) developed some statistical reasoning for various administrative purposes. Common examples include actuarial and demographic usages. However, even astronomy was not outside this realm of statistics. For a nice source of this early historical perspective in statistics, refer to Kendall (1978) and Stigler (1986). They provide good historical accounts of the statistical heritage.

It is much less a problem to assess that developments in statistics in anthropometry, agriculture, genetics, education, psychology, and medicine sectors contributed no less generously to modern statistical theory (which is only just about 100 years old). In that way, mathematical statistics emerged as a discipline that incorporated probability theory and stochastic processes to institute mathematical treatment of data in a rigorous and scientific way, allowing variability to manifest as a natural part of the data diversity. In this way, it also inherited certain basic regularity conditions under which such objective mathematical treatises were adoptable for observational or experimental data models. Alas, it did not perceive the advent of modern information technology that could totally wipe out these methodological landmarks!

With the focus on the evolution of statistical methodology, it would be fair to acknowledge the developments in Great Britain along with contemporary as well as earlier developments in the Continental Europe. In the English sector, the scenario was dominated by the stalwarts: Karl Pearson, Ronald A. Fisher, Egon S. Pearson and J. Neyman, who actually moved to England and finally to USA. (Much earlier, the evolution of Bayes theorem initiated a novel line of attack, and even after a couple of centuries, statisticians are struggling to rationalize the Bayesian philosophy in diverse setups.) The K. Pearson Era could roughly be equated to prior to World War I, while the major thrust of Fisher's work was conducted post World War I period (1920 - 1939). 
The centenary of K. Pearson's classical goodness of fit test in 2001 prompted a special coverage of Biometrika to fathom the history of allied developments during the past 100 years. The (E. S.) Pearson - Neyman Era, overlapping with Fisher, captured the attention mostly during 1936-1950. Even though the Fisherian likelihoods and Neyman-Pearson hypothesis testing doctrines still reign in the statistical arena, there are threats for revolutionary changes coming from the knowledge discovery and data mining (KDDM) quarter, (and there is a need to watch out).

Although this evolution mostly in England had a clear biometric, anthropometric and genetics influence, in Russia (later Soviet Union, and now back to Russia) with genesis in gambling (St. Petersburg games), systematic developments took place in the area of probability theory and stochastic processes: A. A. Cuprov, A. A. Markov, V. I. Romanovsky, A. Khintchine, and A. N. Kolmogorov are specially noteworthy in this respect. Much of the foundations of modern probability theory and stochastic processes were laid down by the East European mathematicians, while in the second half of the 20th century, the evolution engulfed Western Europe as well the North American Continent.

There were also earlier developments in Western Europe, and in particular, R. von Mises's work on statistics and probability appears to be contemporary to R. A. Fisher's work; for the galaxy of other statisticians, refer to the historical accounts by Stigler (1986) and others. In the post World War II era, statistical science made its' most noticeable strides. In the USA, A. Wald opened the doors for sequential analysis and decision theory, H. Hotelling's earlier contributions to multivariate statistical analysis paved the way for more statistical methodology in psychometry, biometry and other fields. H. Cramer's treatise of mathematical methods of statistics captured the attention from all over, and the arena of statistical methodological research engulfed the USA with the Neyman school in California, and Hotelling, R. C. Bose, S. N. Roy, W. Hoeffding and $\mathrm{H}$. Robbins in North Carolina.

Mostly due to the pioneering leadership of P. C. Mahalanobis and P. V. Sukhatme, India was also on the global statistical map, with the emergence of R. C. Bose, A. Bhattacharya, S. N. Roy, C. R. Rao, and other contemporary stalwarts. In the Australian continent, E. J. G. Pitman emerged with superb statistical ideas that are still inspiring even after many decades. The next phase started in the mid 1960s when epidemiology, environmental science, medical and clinical sciences, and public health disciplines sought much more statistical analyses, leading to the emergence of modern biostatistics. After three more decades, this has become a completely different world: the Information Technology (IT) era: Where do statistical methodology go from here?

In retrospect, the growth and fall of abstract theoretical statistics could be visualized over the past 100 years. In this respect, the early phases are characterized by the foundation of statistical theory and methodology wherein mathematical concepts were developed and extensively appraised. In the later phases, driven by the natural forces of applications, data analysis concepts entered the arena, and in that respect, some fundamental changes in the basic statistical reasoning took place. This radical change is not unique in statistical sciences. In most of the basic sciences there also has been a significant drift from the theoretical to applied works.

Mathematics is the mother of all sciences, philosophy (logic), civilization, and cultural achievements too; mathematical reasoning is used in everyday life and decisions. The evolution of applied mathematics (including biomathematics) stems from the need of mathematics in other areas of scientific and socio-economic endeavors. Statistical science has its genesis partly in this mathematical universe and partly in the immense need for modeling (quantifying) and decision making in a variety of so-called inexact disciplines where uncertainty dominates the decision process, and thereby the exactness of mathematical logic stumbles into impasses. As such, statistical science has emerged as a discipline in its own stochastics clouds (over deterministics). It might not be out of the way to mention that this feature of uncertainty or unaccountable outcomes is also shared in economic decisions, although the nature in which that is handled could be quite different.

Mathematicians and theoretical physicists have bondage to mathematical abstractions and sophistications. Applied mathematicians and applied physicists like to use sophisticated mathematical tools to approximate real life problems. Statisticians are, however, somewhat divided by their diversity and professional outlook. Mathematical statisticians and probabilists have much greater affinity to mathematical concepts (e.g., analysis, abstractions, logic), while statisticians and applied probabilists are more akin to applied mathematicians, though committed to stochastics to a greater extent.

Applied (and bio-) statisticians are more data-oriented, and the information technology is changing the outlook across this broad spectrum. That is why there is the need to have an integral view of the statistical science, preserving its diversity and yet understanding its foundation and basic philosophy. Slicing a broader discipline into smaller chips may knowingly or unknowingly affect the integration of the discipline and create impasses for interactive research and training. No wonder, Professor P. C. Mahalanobis, while laying down the foundation of statistical science in India, 70 years ago, proclaimed the logo: Unity in Diversity.

Statistics does not aim to distort the prevailing diversity (variation) in a system; rather, it tries to appraise 
the picture in a unified way wherein the deterministic and stochastic aspects are appraised in a sound objective manner along with good indications of margins of possible incorrect decisions. The evolution of decision science to cope with uncertainties has its genesis in statistical science (although, the mode of statistical decisions in this respect is somewhat different than in the classical statistical decision theory). The theory of games, as has been developed with due respect to stochastics and importance of decision making, is a classical model for the incorporation of superb statistical reasoning in game theoretic as well as economic decision theory.

\section{Current Trends: Pedagogical Issues}

At this juncture of time, in statistical science, the traditional emphasis on agricultural sciences has been degraded in favor of biological, clinical, behavioral, biotechnological sciences, and public health. Though there is a renewed interest in financial statistics (incorporating sophisticated stochastics), the emphasis on traditional econometrics has been partly shifted to environmental, epidemiological and health management sciences (where there is a significant temporal component), and from classical genetics to microbiology and molecular biology, featured by excessively large dimensional data sets. As a result, in statistics curricula, abstract treatises of measure-theory based mathematical statistics, probability theory, and stochastic processes are giving way to more computationalintensive (informatics) and data-oriented (data analysis) teaching cum research programs all over the world. (Computational) Bayesian perspectives are mingling with numerical and simulation approaches to solve some harder theoretical problems. Faced with this complex, there is a profound need to appraise the following issues:

(1) What a teaching/training program in statistical science (at the high school/ undergraduate/graduate levels should we aim at? How should post-graduation job prospects be related to teaching/training of statisticians? How should statistics, biostatistics, and applied statistics teaching/training programs be synchronized in the light of job market as well as academic prospects?

(2) How do we attune the academic research program in statistical science so as to reflect the diversity and enormous scope of applicability (in an interdisciplinary setup as mentioned before)? There is a genuine need for blending mathematical sciences with dominating stochastics that crop up in these fields.

(3) Should statistical science be viewed as a subdivision of mathematical sciences, or computer sciences, or social sciences? As a matter of fact, can any of these broad disciplines engulf statistical science as a sub-discipline?
(4) How do we standardize teaching/training programs in statistical science so as to incorporate more interaction between theoretical and computational statistics? Information technology has opened up a broad avenue of new approaches to teaching of statistics - what are the pros and cons?

(5) Do we emphasize statistical science as an integral teaching/training program by itself? This needs recruitment of prospective students in statistics program, and synchronization of research with such an innovative program. How do we minimize the risk due to choosing unknowingly an inappropriate training program at the undergraduate college level, and how do we reallocate such mismatched entries?

An appraisal of these issues requires an assessment that might well be beyond the scope of this present article. Nevertheless, let me mention some salient points in this respect. What is the main objective of high school education in a country? Is it simply to produce a hallmark of literacy of citizens? With the change from agronomic to technological cum industrial setups in most of the countries, the high school programs are to be viewed from a much broader perspective, such as:

(1) Providing a basic education to growing citizens so that they feel comfortable in the society they live (culturally, socially as well as economically). The enlivenment of cultural and social aspects is generally brought up by the literature and social science subjects in the curriculum. The basic training in literature and mathematics may be the gateway to their passage to higher education.

(2) In the West, at the high school level, a reasonable amount of vocational training programs used to be the facilitating factors for high school graduates to stand on their own with respect to their daily needs in life. For example, typing skills, small mechanics to master household matters, automobile driving training to meet the daily need of commuting, etc.

(3) Sport activities to develop the team spirit and good sportsmanship.

(4) Religion classes to have socio-cultural understandings, and other related matters.

(5) Vocal as well as instrumental music classes, drama and journalism courses to develop arts faculties.

One essential thing in the whole curriculum is the development of social, community and ethical standards and obligations of high school students for the betterment 
of the society and community in which they live. Are we still sticking to this interface of our high school level training? In USA, in the melting pot, there are sometimes cracks that raise the question: what should be the primary goal of high school education, and how should be the curriculum developed to optimize this goal? I guess statistics has a lot to contribute towards this materialization.

In any setup, in the high schools, it would be quite evident to perceive the diversity of the students' family background, their level of motivation, diligence, drive, ability to comprehend academic material in diverse setups, as well as, their aims and ambitions in life. A good high-school program should aim to harness this diversity to the advantage of the society and country as a whole. No wonder that some level of specialization is instituted at the high school level. Students stronger in language skills are encouraged to have more advance placements in that direction, while better science and mathematics students are encouraged for higher education in science, engineering or medicine. Yet, in this diversion, it is apparent that not all high school students would be really ready for college level education; even if ideally all high school graduates plan to proceed to the traditional undergraduate education, will it be possible for colleges to absorb this heterogeneity and have a resolution without compromising their standards and main aims and objectives?

As such, alternative vocational training plans for those who choose not to go for academic college programs are also planned at the high school level. A number of years ago, in Japan, for example, only about fifteen percent of the high school graduates used to go for college education, while a majority for some other vocational training. In Europe, the college bound percentage used to be a bit higher, but not as much as in USA. Even there, many of the high school graduates used to get settled in life in their farming or other family occupations, leaving the luxury of college education to a smaller number of talented and academically oriented ones. The value system on which this was based might have changed drastically during the past three decades.

In addition, it remains to appraise the role of statistical science in this career decision-making task. Very few pupils at the high school level think of statistical science as a career objective, and also, in very few high schools, statistics curriculum may be strong enough to influence the pupil to think of such a possibility. Given this bleak picture, how could one expect a major turnover for statistics majors at the undergraduate level, and in turn, at the graduate level? To place proper emphasis on statistical science as a viable career alternative, it may be essential to sow the seeds at the high school level; that requires properly trained and dedicated teachers, and superb curriculum planning on the part of the school boards, state or nationwide. I am not too optimistic about the prospects.
The quality of undergraduate education is determined by the factors (i) quality of college-bound high school graduates, (ii) quality of faculty at the college level, (iii) curriculum of a program, and (iv) general economic conditions permitting the uninterrupted study environment for the students. It may be the impact of information technology that in the high schools, as well, there is a tremendous emphasis on the use of computing and programming skills in their study plan, so that in that process their drive for analytical thinking might be compromised to a certain extent. This feature has been observed to be persistent in USA, and my sense is that it is more or less the case in many other technologically advanced countries (the Far East Countries are different in this respect).

If such a change has taken place and is likely to continue in the foreseeable near future, it may have a profound impact on the training of mathematical sciences at the college level as well. In that way, there is bound to have some impact on statistical methodology as a part of this curriculum. In the high schools in USA, there is an enormous problem in recruiting adequate number of qualified and dedicated teachers who could assume the responsibility to accomplish the teaching and training task without compromising on the quality. This shortage is also linked to emphasis on nonacademic jobs (because of salary differentials and other factors).

The picture may not be too different in the undergraduate colleges, and there is a need to appraise how far existing faculty can promote the right environment for teaching and training in statistical science (that does not de-emphasize the basic role of statistical methodology). In the late 1980s it became quite apparent to college administrators that in many campuses, the undergraduate programs were very narrow or specialized in a sub-domain. There were general recommendations that liberal arts and science programs be brought back at the undergraduate level, and more specialized programs be relegated to the graduate level of training.

This recommendation certainly deserves a lot of commendation from educators from all over the world. This certainly makes the graduates to deal with real life in a better way, and also to choose their profession in a more thoughtful way (instead of being persuaded by the job prospects in specific areas, knowing that the fortunes do not stick to any specific area for a long time). Many campuses in USA are coping with this change in a subtle way, and in many places, statistics has been designated as a required part of the undergraduate training. Although this statistical-literacy is certainly boosting for people in statistical profession, it also raises the question: How much of statistical methodology should be prescribed for undergraduate non-mathematical sciences students? In what way, statistics acts as a binding power for diverse disciplines in this spectrum? How far statistics should be implemented in an 
undergraduate mathematical sciences curriculum? For students in experimental sciences major programs, how such statistics curriculum should be blended to suit both methodology and applications? All these issues merit careful appraisals.

It would not be out of the way to refer to interesting volume edited by Gal and Garfield (1997) dealing with the assessment challenge in statistics education, with varied contributions by a number of educators and researchers in statistics. Also, refer to a follow-up article by Garfield and Gel (1999). They outlined nicely some practical implications for college statistics teachers, and stressed on the following assessment challenges:

(1) Assessment of students in computer-assisted environments.

(2) Assessment of statistical literacy.

(3) Assessment of students' understanding 'big ideas'.

(4) Assessment of students' intuitions and reasoning involving probability concepts and processes.

(5) Assessment of outcomes of 'group work'.

(6) Developing models to use in evaluating and comparing curricula.

(7) Using assessment to determine what students understand after they interact with simulation software packages.

The main role of such an assessment is to enhance student learning, albeit in confrontation with the advances in computational challenges that crop up in college level statistics programs. Their assessment certainly serves a good purpose, though I would like it to take a step further in raising these issues not primarily for the college teachers but more for the educators who actually lay down the foundation of statistical curricula in high schools, undergraduate, graduate colleges, as well as, in professional and vocational schools. Teachers are more like the musicians while these planners are more the scripts that the teachers are to play. Although in their assessment, computational skills occupy a focal point, our motivation is to bring the compatibility of computational and analytic skills, when there are many extraneous factors which could have significant impact on the whole scenario.

As indicated earlier, not all high school students are undergraduate college bound, and many of them show up in community colleges and in other vocational training centers. Because of their intention or inability to pursue a more academic program (often, due to family or economic reasons, other than less impressive academic performance at the high school), any such training program runs on a somewhat different track (in terms of both time and level). Such vocational trainings serve a good purpose for the community and society as a whole (specially, when there is an acute shortage of such people in the profession), and hence, close attention should be paid to such programs.

For many of these vocational training, a curriculum, though specialized in a specific field (e.g., carpentry, electrical installation, builder's job, car mechanics), the growing need of using PCs and laptop PCs also calls for some basic statistical understanding, albeit with less methodological flavor. This might be more akin to data management and data analysis sectors. Therefore, the basic query arises: how much statistics should be in the instructional packages, and to what extent should the instructors be conversant with this material?

Statistics training at the (post-)graduate level is, in turn, dependent on the flow of interested, dedicated and qualified pupils from undergraduate classes as well as migration from other fields. The demand for traditional mathematical statistics post-graduate programs is likely to dwindle in the near future, and, more likely, biostatistics and other applied statistics programs would be expanded. Again, due to the tremendous influence of the current evolution in biotechnology and in information technology, there is a general tendency for incoming graduate school students to seek admission in some of the related programs than in mathematical or statistical sciences. At the same time, with due emphasis on the basic role of statistical data analysis in a broad spectrum of disciplines (including business management, finance, economics, and social sciences), there is the basic dilemma: how much to emphasize on statistical algorithms and packages without probably motivating them from methodologic justifications?

An answer to this query would depend on how we project the growth (or decline) of enrollment in graduate programs in different areas of statistical sciences (e.g., mathematical statistics, biostatistics, applied statistics, experimental statistics, etc.), and how to blend some of these programs to make this transition smoother. Fortunately, in many places, the mathematical statistics programs are undergoing such changes, and are incorporating more applied curriculum to facilitate the blending of methodology with good applications; the loser may be the abstract theory that might ultimately take shelter in hardcore mathematics curriculum. Over the past twenty years we have been observing a changing pattern of graduate students in USA in statistical sciences, most notably, in biostatistics.

As the number of US graduate students in various disciplines dwindled, even the more prestigious campuses have been flooded with international students, many of whom are from China. Most of these incoming students had good training in mathematics in a rather theoretical fashion, and in many cases, in their undergraduate 
programs, they had only mathematics, without much emphasis on other subjects, including statistics. In a changing environment in USA, not only they had to adapt to this new academic environment (which certainly have enriched the field in a way), but also they induce a lot of unanticipated changes wherein algorithms and computer skills dominate over statistical insights and interpretations. Although this model may explain how the interface of graduate studies in statistical science in USA is evolving in a different way than anticipated, it also raises the question: how to motivate immigrants from other disciplines to get tuned to the heartbeats of statistical methods while pursuing a graduate program in statistics?

There are a couple of other pedagogical issues that merit some appraisal. First, in most of the professional schools, namely, education, law, nursing, pharmacology, public health, dentistry, and medical and clinical sciences, more and more emphasis is placed on the use of statistical analysis in research as well as operational decision making. Traditionally, in many of these fields, both pupil and teachers were apprehensive of statistical mathematics, though some of that stuff became a part of their curriculum. To make it more effective, in many places, the instructors are chosen from affiliated statistics - biostatistics departments. This is certainly a very healthy sign. Teaching of basic statistics to such professional students require special care (to smooth out the somewhat different attitudes of students and statisticians at large), and this can be done by people having a sound depth in statistics (not just the packages that might be the 'handbook' for such professionals. At the same time, it needs the knowledge as well as genuine interest on the part of the statistics faculty to appreciate the specific field of application, so that things can be presented to the right audience in the right flavor, and with good interpretations and explanations.

Second, with the tremendous increase in demand of statistical analysis in every walk of life and science, a new class of statistical professionals has emerged; they are called licensed statisticians. A few years back, the American Statistical Association chartered this professional group, and provided broad guidelines for their ability to perform the task they would be asked for. Although it is expected that all these professional statisticians have some good statistical training at the graduate level, it is quite possible that many of them might have migrated from other fields. As such, to facilitate more interaction between good statistical packages and their underlying statistical methodology, it might be better to stress the need for adequate familiarity with statistical methodology so as to perform the licentiated job more adequately.

As pedagogical issues are intricately related to the needs of the society and mankind as a whole, the pedagogical value system continues to change progressively over time with the subtle changes in our social, cultural, political and economic factors. There are indications that such changes are not that subtle any more, and if so, we must prepare ourselves to possible radical changes in some of these aspects.

Not too long ago, among all disciplines, philosophy used to be the trump card, and in basic sciences, physics and chemistry were the royals. With the march of time, the emphasis on logic and philosophy in pedagogical setups has been downloaded in favor of applied sciences and computers. Basic training in mathematics has also gone through radical changes with more emphasis on computers: the PC's have made the slide-rules obsolete! Students in chemistry classes do not need to memorize the 'periodic charts' of atoms and molecules, nor they need to analyze a compound product by exclusive chemical analysis. High-level computers can simulate complex differential equations, and thereby predict anticipated reactions to a reasonable extent. Given these vast computational amenities, analytical thinking is being gradually replaced by simulation and computational skills.

Faced with this radical change, biological, clinical and other applied areas, once thought to the outside the domain of mathematical reasoning, are gaining momentum and catering for more attention from pedagogical as well as career decisions point of view. At the present time, there has been a sustained effort to promote more understanding of biological, clinical, public health, and environmental sciences through basic interdisciplinary research, and (bio)statistics is a binding force to incorporate quantitative reasoning in this broad interdisciplinary field. Biotechnology, in general, and bioinformatics in particular, are reshaping the sphere of human knowledge and endeavor. Yet, there are many challenging tasks ahead. Most imminent areas in this respect include the following questions and emerging arenas:

(1) Information technology: too much information to disseminate?

(2) Bioinformatics (and medical informatics): how large could a biological system be?

(3) Molecular biology and genomics; biotechnology.

(4) Chemometrics and environmetrics.

(5) Environmental health sciences, toxicology.

Most of these novel areas are characterized by very high-dimensional data sets (where there may be too much of information that can be disseminated in a manageable statistical way). Moreover, sometimes, it might be harder even to reformulate such problems in a way that would permit a convenient statistical way in looking for 
suitable resolutions. On top of that there could a lack of understanding on the part of a statistician of the biological intricacies, and on the part of scientists in other disciplines of the statistical intricacies. There is a pressing need for applicable statistical methodology for planning, modeling as well as analysis of such scientific endeavors.

Pedagogical aspects are very crucial in this respect. Without recruiting properly trained or knowledgeable persons for this needed statistical task, there could be genuine problems with drawing valid and efficient conclusions. On the other hand, attracting deserving trainees in this disciple needs proper nurturing at the high-school level, so that the prospective college-bound students do not all line-up with medical, law, engineering, or computer science prospects. Statistical methodologic orientation at the undergraduate training level is an essential ingredient in this venture, and I would like to stress it. Before this assessment, however, a few important points require comment.

Career placement prospects cannot be ruled out as a basic factor in teaching and training curriculums in statistical science. Yet this picture is progressively changing over time. After the World War II, in the British Commonwealth Countries, this curriculum used to be quite broad-based with a mixture of applications in various fields and methodology in the main stream, albeit, with some sacrifice of the abstractions that typically germinated from purely probabilistic and measure-theoretic approaches.

On the other hand, in the United States of America, as well as, in continental European countries, mostly statistics teaching programs were housed in mathematics (or mathematical sciences) curriculums, and as a result were substantially more measure-theoretic and mathematically more sophisticated. Applied statistics or experimental statistics programs were housed in engineering schools or in economics and business schools, and were tilted in the respective directions. Biostatistics programs were very few in number, and used to be in either the school of public health or sometimes in the medical schools.

This segregated picture changed gradually over time. More people in applied statistics programs tried to incorporate more statistical methodology in their applied work, and this led to the evolution of Technometrics, Biometrics and other journals. The positive aspect of this evolution could be seen from the increasing usage of statistical analysis in medical studies, and later on, many medical journals instituted a convention that without proper statistical support, empirical findings would not be considered adequate for publication.

A more radical change took place about thirty years ago when in the West, health regulatory agencies (e.g., the National Institutes of Health, and Food and Drug Administration in USA) advocated large scale clinical trials where statistical methodology seemed to have a visible role in the decision making process. The drug research groups and pharmaceutical industries were encouraged to have more statistical expertise for better prospects in their marketing and drug-approval ventures. At the present time, drug-research groups may still employ a significant number of statisticians (not all of whom might have the basic training in statistics).

\section{Whither Statistical Reasoning?}

In the preceding section, some of the areas where there is a pressing need for statistical reasoning were mentioned, and there are, in that way, some challenges too. I intend on elaborating on these points in more specific contexts, and then append a general discussion on the basic nature of the shifting goals along with these challenging problems.

(I) Genomics and bioinformatics. DNA and genomes have become household words, and there is a global effort to fathom out the mysteries of the intricate network of some 35,000 genes that constitute the human genome. Aided by biotechnological advancements, genomic science has captured the attention of researchers from all walks of life and science. The chemical words (A, C, G T) that constitute a DNA sequence exhibit qualitative variation, and on top of that there is very large number of sites. There are certain assumptions regarding the DNA activity, and it is not yet clear to what extent statistical methods (and probability theory) can be justified in this context.

The evolutionary interdisciplinary field of bioinformatics covers various aspects of mathematics, statistics, information technology, and of course molecular biology and genetics to a greater extent. However, at the present, it is not precisely known what constitutes the core of bioinformatics. Ewens and Grant (2001) have a nice way of stating the current status:

We take bioinformatics to mean the emerging field of science growing from the application of mathematics, statistics, and information technology, including computers and the theory surrounding them, to study and analysis of very large biological, and in particular, genetic data sets." The field has been fueled by the increase in DNA data generation leading to massive data sets already generated, and yet to be generated, in particular the data from the human genome project, as well as other genome projects. Bioinformatics does not aim to lay down fundamental mathematical laws that govern biological systems parallel to those laid down in 
physics. Such laws, if they exist, are a long way from being determined for biological systems. (Grant, 2001)

At this stage, the main utility of mathematics in this field is in the creation of tools that investigators can use to analyze data. Such tools involve statistical modeling of biological systems, and therefore, there is a genuine need for probability theory, stochastic processes, and statistics in the context of bioinformatics.

Gene scientists cannot scramble fast enough to keep up with the genomics, emerging at a furious pace and in astounding detail. As such, it would be improper to jump on conclusions based on data analysis alone (even under the ubiquitous KDDM umbrella). There is a genuine need to examine the stochastic evolutionary forces underlying large biological systems, in general, and genomics in particular. This venture turns us to look more intimately into the deeper stochastics aspects of macro biological systems, incorporate biological (and genetic) factors, as needed, and only then prescribe a statistical resolution.

This motivated Sen (2001) in the formulation of Biostochastics: Stochastic modeling and statistical analysis of very large biological (including genomic and polygenetic) data sets. Computational sequence analysis (CSA), large genetic data models, and computational biology, in general (Waterman 1995, Lange 1997), have a genuine need of biostochastics for proper methodologic justification. Let us elaborate this point with some specific models.

In the mode of an external analysis, Pinheiro et al. (2000) considered a MANOVA (multivariate analysis of variance) type statistical test for the homogeneity of several groups of people with respect to their DNA sequences, the primary motivation being the impact of HIV (human immunodeficiency virus) on the distribution of the nucleotides. These data models involve purely categorical responses and are immensely of high dimension.

They relate to extensions of the work of Light and Margolin $(1971,1974)$, because of high-dimensionality, where Hamming distance measure has been incorporated to eliminate some conceptual as well as computational difficulties. There are some genuine problems in pursuing a pure likelihood (or some of its variants) approach, and in that sense, the Hamming distance provides a meaningful resolution; more remains to study regarding its desirable or optimal properties.

Similarly, in the mode of an internal analysis, for testing the hypothesis of independence of mutations in a DNA strand, Karnoub et al. (1999) considered a conditional test, and more work is underway in this direction (Sen 2002a). There are many other interesting statistical problems in this area, and some of these are discussed in Ewens and Grant (2001), Waterman (1995) and others. Below, I discuss the salient features of such models in a general mold.

(II) The PBPK-PBTK Wanderworlds: Pharmaceutical and drug research activities have spurred up in an international phase. From a scientific approach this involves (1) Pharmacodynamics, (2) Pharmacokinetics, (3) Toxicokinetics, (4) Biomechanistics, and (5) PBPK (physiologically based phramacokinetic) models, to deal with the distribution, concentration, absorption, and ingestion of drugs and pharmaceutical elements in the human system (allowing the diversity of health and other conditions). At this time, from a statistical perspective, the vital components are (a) bioequivalence and relative potency studies, (b) dosageresponse regression modeling, and (c) assessing genetic undercurrents. There is a tremendous scope for (bio)mathematics and (bio)statistics to work together. Statistical theory and methodology have the key to the basic understanding, but more and more evolutionary work is needed to bring it down to the level of appropriate adoption.

(III) Environmental Pollution and Health Perspectives. Toxicity abounds in nature, environment and in the modern life style. Respirable suspended air particulate matters (RSAPM) constitute the main sources of air pollution, and are regarded as the carrier of toxicity, mostly inhaled through our respiratory system. There are other forms of environmental toxicity and pollutions (such as water and subsoil contamination), and together they work on human as well as other living organisms in a very complex manner.

Because of the latent nature of a large class of toxic substances that are in the environment, the extreme variability of human metabolism as well as their exposure to such toxic materials, yet unknown nature of many carcinogenic activities, and immense difficulties in effective assessment of prevailing toxicity, an exact mathematical dose-response formula is not appropriate here. Even the classical statistical regression analysis is not tenable here (as the basic regularity assumptions pertaining to modeling and analysis are hardly justifiable in such a case, and the sampling design may also be highly nonstandard).

There is a genuine need to incorporate the physiological (as well as molecular biological) mechanism and reactions of such toxic substances in human body (and mind, too), and in the midst of this highly stochastic and complex uptake-intake process, there is a three-phase statistical task for assessment of the aftermaths of environmental pollution, namely, (1) designing (planning) a scientific study that allows reliable measurement of the toxicity levels taking into spatial as well as temporal variation in a reasonable way, (2) formulating suitable models that are design-consistent and yet amenable to further statistical analysis with due biological and environmental 
understanding, and (3)analyzing the acquired experimental and observational outcome in a valid and yet simple way.

The sampling techniques for collection of environmental pollution data and associated auxiliary/explanatory variables are generally more complex, and the usual assumption of independence or homogeneity (i.e., spatiotemporal stationarity) may not generally hold, and as a result, much of the appeal of variograms and other standard statistical tools may therefore be lost in this context. Semiparametric formulations have greater appeal than parametric ones, and yet, the very basic semiparametric model may not fit the sampling design as well as the dose-response regressions.

In view of biological undercurrents, biomechanistic models (including the PBTK models) have greater appeal. Nonparametrics should have greater scope too, but may need a comparatively larger data set, a condition that may not always hold in practical applications. On top of that usually collected datasets by different regulatory agencies may conform to an exceedingly large dimension, so that extraction of statistically informative smaller subset of characteristics may often be a challenging task. Refer to Sen (2002b) for a detailed account of such related statistical problems.

The basic idea is to bring in the SARI (structureactivity relationship information) in the picture. The structure refers to the influx of toxicants (along with their bioconcentration factors) and their mode of uptake by human body. Activity refers to the biological, genetic and biochemical reactions that follow the intake of toxins in the body. The relationship is not directly observable and often is obscured by many physiological and behavioral complexities.

In a conventional sense, in a dose response regression, the dose is identifiable and the response is observable. In this context, the dose refers to the influx of toxicants, and there is a lot of variation in their intake by human body. The response refers to the occurrence of a disease or disorder which are more likely to be influenced by the toxicity influx. Nevertheless, the relationship is not so apparent, and the SARI can provide more biological information upon which a plausible regression model can be postulated.

For the SARI there is a profound need to seek biological and environmental impacts, and for statistical modeling and analysis, it is therefore necessary to take into account these factors as effectively as possible. The real challenge is to incorporate the SARI in statistical modeling and analysis. This needs more complex models, more biological and toxicologic feedbacks, imputation from animal studies (dosimetry), and delicate bioassays. Are we expecting statisticians trained in a conventional way to make breakthrough in this domain?

\section{Conclusion}

There is an annexation of a new frontier in statistical science from the ongoing evolution of biotechnology and information technology. This has posed some challenging tasks for statistical theory and methodology to cope with planning, modeling and analysis, genuinely needed for drawing scientific conclusions in a statistically sound manner. Most of these highly nonstandard problems may be characterized with the following features:

\section{(1) generally, (very) high-dimensional data models}

(2) non-continuous (i.e., binary/categorical or at best, discrete) response variables.

(3) usually, a complex, confounded network of extraneous (and often unobservable) factors

(4) insufficient justifications for adopting simpler theoretical models

(5) likely misspecification, identifiability, incompleteness, and validity problems

(6) scope for statistical reasoning not usually clear.

The wealth of statistical tools and concepts developed during the past six decades in dealing with statistical planning, modeling and analysis of observational and experimental data models, may be of very limited use in this new field. As such, we need to address our curricula, train our teachers, and motivate and organize our training programs to promote better understanding and interaction of statisticians (at large) with other scientists, and create a more congenial environment for the advance of our knowledge.

Although there has been a sustained growth of statistical packages to meet the increasing demand of data analysis, and also promising developments in statistical learning to provide more rationality to KDDM, it remains to see how these diverse tools can also be aged under statistical methods in a broadly interpretable way. I am not pessimistic in this venture, but we need to prepare ourselves better and do our needed homework in this multifaceted task in an adequate way. 
References

Anderson, R. J., \& Landis, J. R. (1980). CATANOVA for multidimensional contingency tables: nominal-scale response. Communications In Statistics: Theoretical Methods, 9, 1191-1206.

Anderson, R. J., \& Landis, J. R. (1982). CATANOVA for multidimensional contingency tables: ordinal scale response. Communications in Statistics: Theoretical Methods, 11, 257-270.

Chatfield, C. (1995). Model uncertainty, data mining and statistical inference (with discussion). Journal of the Royal Statistical Society, A158, 419-466.

Cox, D. R. (1995). Discussion of Chatfield's paper. Journal of the Royal Statistical Society, A158, 455456.

Durbin, R., Eddy, S., Krogh, A., \& Mitchison, G. (1998). Biological sequence analysis: Probabilistic models for proteins and nucleic acids. UK: Cambridge Univ. Press.

Ewens, W. J., \& Grant, G. R. (2001). Statistical methods for bioinformatics: An introduction. NY: Springer.

Fayyad, U. M. et al. (1996). Advances in knowledge discovery and data mining. Cambridge, MA: MIT Press.

Friedman, H. P., \& Goldberg, J. D. (2000). Knowledge discovery from data bases and data mining: New paradigms for statistics and data analysis. ASA Biopharma. Repr, 8(2), 1 -12.

Friedman, J., Hastie, T., \& Tibshirani, R. (2000). Additive logistic regression: A statistical view of boosting. Annals of Statistics, 28, 337-407.

Gal, I., \& Garfield, J. (Eds.) (1997). The assessment challenge in statistics education. Amsterdam: IOS Press.

Garfield, J., \& Gal, I. (1999). Assessment and statistics education: current challenges and directions. Inter. Statist. Rev., 67, 1-12.
Hastie, T., Tibshirani, R., \& Friedman, J. (2001). The elements of statistical learning: Data mining, inference and prediction. NY: Springer.

Hedges, L. V., \& Olkin, I. (1985). Statistical methods for meta analysis. London: Academic Press.

Karnoub, M., Seillier-Moiseiwitsch, F., \& Sen, P. K. (1999). A conditional approach to the detection of correlated mutations. Inst. Math. Statist. Lecture Notes, Monogr. No. 33, 221 - 235.

Kendall, M. G. (1978). The history of statistical method. International Encyclopedia of Statistics, 2, 10931101.

Pinheiro, H., Seillier-Moiseiwitsch, F., Sen, P. K., $\&$ Eron, J. (2000). Genomic sequence analysis and quasimultivariate CATANOVA. In Handbook of Statistics, 18, 713 - 746.

Stigler, S. M. (1986). The history of statistics: The measurement of uncertainty before 1900. Cambridge, MA: Harvard Univ. Press.

Sen, P. K. (2001). Excursions in biostochastics: Biometry to biostatistics to bioinformatics. Lect. Notes, Academia Sinica, Inst. Statist. Sci. Taipei, Taiwan.

Sen, P. K. (2002a). Computational sequence analysis and genomes: Statistical perspectives and controversies. Statistics Canada 2001 Conference. (Y. P. Chaubey, Ed.). UK: World Sc. Press.

Sen, P. K. (2002b, unpublished). Structure-activity relationship information incorporation in health related environmental risk assessment. Environmetrics.

Sen, P. K. (in press, 2000). Air pollution: Statistics and environmental health perspectives. Environmental Issues and Statistical Perspectives. Oxford University Press.

Waterman, M. S. (1995). Introduction to computational biology: Maps, sequences, and genomics. UK: Chapman-Hall. 


\title{
Combining Two Nonparametric Tests Of Location
}

\author{
R. Clifford Blair \\ Department of Epidemiology and Biostatistics \\ College of Public Health, \& \\ Jaeb Center For Health Research \\ University Of South Florida
}

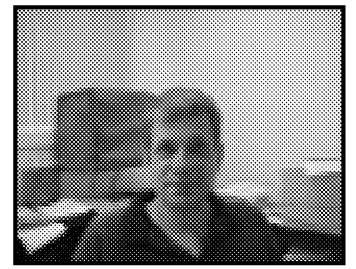

A distribution-free test is proposed whose power is similar to that of the Wilcoxon Rank-Sum or Terry-Hoeffding Normal Scores tests depending on which of these two tests is more powerful in a given data analysis situation, regardless of the population. This new statistic is distribution-free, and adds no new assumptions to those associated with the constituent tests. A table of critical values for the new statistic is given and some of its Type I error and power properties are examined.

Key words: Nonparametric tests, Shift in location, Wilcoxon rank-sum, Terry-Hoeffding, Normal scores

\section{Introduction}

Researchers are sometimes presented with situations in which two (or more) statistical tests appear to be equally appropriate for a given data analysis problem. In choosing between these tests the researcher may consider such factors as ease of computation, acceptability by peers, and availability of tables of critical values. Among the more important factors to influence such a choice would be the relative power of the statistics under consideration. Ceteris Paribus, one would desire to use the most powerful test available.

Unfortunately, it does not usually occur in such cases that one test is more powerful than its competitor among all plausible population models that may be appropriate for the data in the sample. Instead, one test or another may be more powerful than its primary competitor under a given set of circumstances. Thus, for example, one test might be preferred when the population has a lighttailed distribution, but may give way to its rival statistic when the distribution is heavy-tailed.

Factors that influence a test's power may be difficult to assess from available data. Moreover, these factors may interact in such complex patterns as to preclude any clear indication as to which test might be more powerful in a given situation. For certain inferential tests, the dilemma of test choice can be avoided through use of a "maximum" statistic (Cox, 1977). In essence, a maximum statistic is obtained by computing two or more statistics on a given data set, and choosing as the test statistic the one with the smallest associated $\mathrm{p}$-value.

R. Clifford Blair is Professor and Interim Chair, Department of Epidemiology and Biostatistics, College of Public Health, University of South Florida, Tampa, Florida. His areas of expertise are in computer-intensive statistical methods, multiple end point analysis, and control of family-wise error.
Consider that two independent samples layout. Two robust and powerful competitors are the Wilcoxon Rank-Sum test (W) and its normal scores counterpart, the Terry-Hoeffding (NS) (Terry, 1952) counterpart. Both procedures are used to test the null hypothesis that samples are from a common population. Asymptotic results suggest that these two tests may manifest substantial power differences, with the magnitudes and advantages of such differences depending on the shape of the population. The Asymptotic Relative Efficiencies (AREs) indicate that, in general, when alternatives are expressed as simple shifts in location, the normal scores test is more efficient than the rank test when sampling is from a light-tailed distribution. However, the normal scores test is at a disadvantage when the populations are heavy-tailed. (For details, see Chernoff and Savage, 1958; Hodges \& Lehmann, 1961; Lehmann, 1959; Mikulski, 1963; and Terry, 1952.)

Thus, the purpose of this paper is to present a simple maximum statistic that can be used in lieu of a choice between the Wilcoxon Rank-Sum and Terry-Hoeffding tests.

\section{Methodology}

The proposed statistic is obtained by computing both the rank-sum and the normal scores statistics, and choosing as the test statistic the one with the smaller p-value. In order to facilitate development of the sampling distribution of this maximum statistic, it is helpful to express W and NS in a common metric. In this case, both W and NS may be easily expressed in the form of a t statistic. (There are other possibilities, but existing software makes this choice computationally simpler.)

In the case of $\mathrm{W}$, this accomplished by replacing original observations with their respective ranks (with ranking being carried out without regard to group) and computing the usual independent samples $t$ statistic on those 
ranks. The resulting rank transformation statistic $\left(\mathrm{t}_{\mathrm{w}}\right)$ is a monotone function of W (Conover \& Iman, 1981). Its sampling distribution is well approximated by a t distribution with $n_{1}+n_{2}-2$ degrees of freedom (Iman, 1974).

Similarly, an expression for NS may be obtained by replacing observations with their respective normal scores, which are defined as the expected values of the order statistics under normality (Owen, 1962). The $t$ statistic is then computed on these normal scores. The resulting statistic $\left(\mathrm{t}_{\mathrm{NS}}\right)$ is a monotone function of NS, and it too may be referred to the $t$ distribution (Bradley, 1968).

Thus, the new test statistics $\left(\mathrm{t}_{\max }\right)$ is defined as

$$
\mathrm{t}_{\max }=\left\{\begin{array}{l}
t W ;|+W|>\mid+N S \\
t N S ;|+N S|>|+W|
\end{array}\right\}
$$

In the event $\left|t_{w}\right|=\left|t_{N S}\right|$, then either statistic may be used.

\section{Sampling Distribution}

The exact sampling distribution of tmax may be obtained by forming all possible permutations of the integers 1 to $n_{1}+n_{2}$ (where $n_{1}$ and $n_{2}$ represent the number of observations in each of two samples), computing $t_{\text {max }}$ on each set of integers, and forming the cumulative distribution of the values obtained. In this study, the cumulative distribution of $\mathrm{t}_{\max }$ was estimated by randomly permuting the integers $n$ to $2 n 500,000$ times with $t_{\max }$ being computed on each permutation.

Table 1 provides values for $\mathrm{n} 1=\mathrm{n} 2=\mathrm{n}=5(1) 40$, $40(5) 60,60(10) 120$. It should be noted that the sampling distribution of $t_{\text {max }}$ is discrete, and therefore, it was not always possible to find critical values (c) such that $\mathrm{p}\left(\mathrm{t}_{\max } \leq\right.$ $c)=\alpha$. As a result, values of $c$ were chosen so that $\mathrm{c}$ was as large as possible, while maintaining the inequality.

It can also be seen that in some instances, the magnitude of $\mathrm{c}$ increases when $\mathrm{n}$ is increased, contrary to what is usually expected. This occurs because $t_{w}\left(t_{v s}\right)$ may be the test statistic for one particular value of $n$, and $t_{\mathrm{NS}}$ $\left(t_{w}\right)$ for the situation where $n$ is increased. This does not lead to a violation of the above stated inequality, however, so that the test level is maintained.

\section{Results}

\section{Type I Errors}

The results of a Monte Carlo study are compiled in Table 2. The entries reflect the Type I error rates for $t_{w}$, $t_{\mathrm{NS}}$, and $t_{\max }$ when samples are of various sizes. Data were generated by randomly permuting the integers from 1 to $n_{1}$ $+\mathrm{n}_{2}$, with the three statistics being computed on each permutation. Entries in the table for $t_{w}, t_{\mathrm{NS}}$, and $t_{\max (a)}$ were obtained by referring the three statistics to the appropriate critical values in a $t$ table, using $n_{1}+n_{2}-2$ degrees of freedom. Entries for $t_{\max (b)}$ were obtained, in the case of $n_{1}$ $=\mathrm{n}_{2}$, by referencing $\mathrm{t}_{\max }$ to the critical values in Table 1 . In the case of $n_{1} \neq n_{2}$, entries for $t_{\max (b)}$ were obtained by referring $\mathrm{t}_{\max }$ to the critical value in Table 1 using $\mathrm{n}=.5\left(\mathrm{n}_{1}+\right.$ $\mathrm{n}_{2}$ ). (Recall that the critical values in Table 1 were obtained under the condition of $n_{1}=n_{2}=n$.) Twenty thousand repetitions of the experiment were carried out for each condition studied.

Several points should be made regarding the results of these simulations. (1) The $t$ distribution provides a good approximation for the distribution of $t_{w}$ and $t_{w s}$ is reaffirmed. (2) Critical values from Table 1 produce Type I error rates for $t_{\text {max }}$ near nominal levels both in the case of $\mathrm{n}_{1}=\mathrm{n}_{2}$ and in the case of $\mathrm{n}_{1} \neq \mathrm{n}_{2}$. (3) Referencing $\mathrm{t}_{\max }$ to $\mathrm{a}$ $t$ distribution with $n_{1}+n_{2}-2$ degrees of freedom results in only modest Type I error inflations. This result implies that the researcher who is willing to tolerate minor Type I inflations need not rely on the special table of critical values provided when conducting a test based on $t_{\text {max }}$.

\section{Power}

Let $\mathrm{P}_{\mathrm{tw}}(\alpha)$ and $\mathrm{P}_{\mathrm{tNS}}(\alpha)$ denote the power of the $t_{\mathrm{w}}$ and $\mathrm{t}_{\mathrm{NS}}$ tests, respectively, when carried out individually at the $\alpha$ level of significance. Let $\alpha^{*}>\alpha$ denote the effective level of significance of the maximum test when the critical value is chosen in this way. Because the maximum test rejects the null hypothesis when either $\mathrm{t}_{\mathrm{w}}$ or $\mathrm{t}_{\mathrm{Ns}}$ is significant, it follows that the power of the maximum test a level of significance $\alpha^{*}$ has a lower bound $\max \left(\mathrm{P}_{\mathrm{tw}}(\alpha)\right.$, $\left.\mathrm{P}_{\mathrm{tNS}}(\alpha)\right)$. As indicated by the simulation above, $\alpha^{*}$ can be expected to be only slightly larger than $\alpha$. Therefore, the power of the maximum test when conducted at level of significance $\alpha$ should never be much less than the power of the better of the two individual tests when each is conducted at level of significance $\alpha$.

Figures 1-3 depict the results of a Monte Carlo study designed to compare the power of $\mathrm{t}_{\mathrm{w}}, \mathrm{t}_{\mathrm{NS}}$, and $\mathrm{t}_{\max }$. These figures indicate, respectively, the results for the normal, uniform, and Cauchy distributions. In this study, $t_{w}$ and $t_{\mathrm{NS}}$ were referred to the appropriate $t$ distribution, while $t_{\max }$ was referred to the values found in Table 1 . Tests were conducted at the $\alpha=.05$ (two-tailed) level of significance. In the cases of the normal and uniform distributions, the alternative condition was constructed by adding a constant equal to $.5 \sigma$ to the scores in one group. In the case of the Cauchy distribution, which has infinite standard deviation, an arbitrary constant of 1.00 was used. Ten thousand repetitions of the experiment were carried out for each condition studied.

Figure 1 shows that there was generally little difference in the power of the three tests when sampling was from a normal population. Differences that did occur favored $t_{\mathrm{NS}}$ and $t_{\max }$. In the case of the uniform distribution, Figure 2 shows that $t_{\mathrm{NS}}$ was the most powerful test, with $t_{\max }$ showing power similar to, but slightly less than, that of 
Table 1. Two-tailed Critical Values For $\mathrm{t}_{\max }$.

\begin{tabular}{|c|c|c|c|c|c|c|}
\hline \multirow[b]{2}{*}{$\mathrm{n}$} & \multicolumn{4}{|c|}{ Level of Significance } & \multirow[b]{2}{*}{.020} & \multirow[b]{2}{*}{.010} \\
\hline & .400 & .200 & .100 & .050 & & \\
\hline 5 & .9727 & 1.4572 & 1.8312 & 2.4958 & 3.7812 & 5.0000 \\
\hline 6 & .9571 & 1.3978 & 1.8503 & 2.2804 & 3.3211 & 3.8468 \\
\hline 7 & .9525 & 1.3919 & 1.8688 & 2.2926 & 2.7464 & 3.6623 \\
\hline 8 & .9431 & 1.4124 & 1.8066 & 2.2630 & 2.7940 & 3.4503 \\
\hline 9 & .9238 & 1.4076 & 1.8122 & 2.2005 & 2.6706 & 3.0822 \\
\hline 10 & .9027 & 1.3942 & 1.8023 & 2.1603 & 2.6616 & 2.9771 \\
\hline 11 & .9232 & 1.3744 & 1.7861 & 2.1732 & 2.6429 & 2.9582 \\
\hline 12 & .9207 & 1.3830 & 1.7872 & 2.1517 & 2.5778 & 2.9292 \\
\hline 13 & .9180 & 1.3836 & 1.7925 & 2.1339 & 2.5852 & 2.8869 \\
\hline 14 & .9162 & 1.3744 & 1.7655 & 2.1349 & 2.5764 & 2.8852 \\
\hline 15 & .9210 & 1.3682 & 1.7688 & 2.1310 & 2.5580 & 2.8463 \\
\hline 16 & .9161 & 1.3762 & 1.7613 & 2.1052 & 2.5327 & 2.8400 \\
\hline 17 & .9104 & 1.3790 & 1.7582 & 2.1188 & 2.5340 & 2.8243 \\
\hline 18 & .9154 & 1.3778 & 1.7589 & 2.1025 & 2.5128 & 2.8039 \\
\hline 19 & .9177 & 1.3737 & 1.7533 & 2.0954 & 2.5135 & 2.8152 \\
\hline 20 & .9178 & 1.3675 & 1.7486 & 2.0909 & 2.5080 & 2.8043 \\
\hline 21 & .9164 & 1.3628 & 1.7426 & 2.0826 & 2.4981 & 2.7824 \\
\hline 22 & .9137 & 1.3755 & 1.7544 & 2.0942 & 2.4956 & 2.7761 \\
\hline 23 & .9168 & 1.3640 & 1.7497 & 2.0835 & 2.4950 & 2.7876 \\
\hline 24 & .9205 & 1.3729 & 1.7485 & 2.0911 & 2.5003 & 2.7775 \\
\hline 25 & .9202 & 1.3654 & 1.7506 & 2.0763 & 2.4780 & 2.7598 \\
\hline 26 & .9142 & 1.3657 & 1.7402 & 2.0776 & 2.4769 & 2.7582 \\
\hline 27 & .9219 & 1.3692 & 1.7479 & 2.0776 & 2.4821 & 2.7618 \\
\hline 28 & .9163 & 1.3709 & 1.7476 & 2.0790 & 2.4823 & 2.7575 \\
\hline 29 & .9220 & 1.3712 & 1.7492 & 2.0817 & 2.4730 & 2.7401 \\
\hline 30 & .9200 & 1.3702 & 1.7440 & 2.0709 & 2.4652 & 2.7421 \\
\hline 31 & .9210 & 1.3683 & 1.7387 & 2.0743 & 2.4694 & 2.7491 \\
\hline 32 & .9237 & 1.3654 & 1.7441 & 2.0742 & 2.4606 & 2.7357 \\
\hline 33 & .9189 & 1.3621 & 1.7385 & 2.0703 & 2.4573 & 2.7361 \\
\hline 34 & .9189 & 1.3633 & 1.7399 & 2.0710 & 2.4545 & 2.7302 \\
\hline 35 & .9211 & 1.3665 & 1.7419 & 2.0707 & 2.4645 & 2.7340 \\
\hline 36 & .9225 & 1.3628 & 1.7364 & 2.0697 & 2.4594 & 2.7300 \\
\hline 37 & .9183 & 1.3648 & 1.7370 & 2.0693 & 2.4532 & 2.7288 \\
\hline 38 & .9237 & 1.3688 & 1.3730 & 2.0691 & 2.4561 & 2.7286 \\
\hline 39 & .9203 & 1.3623 & 1.7361 & 2.0654 & 2.4495 & 2.7129 \\
\hline 40 & .9229 & 1.3642 & 1.7345 & 2.0619 & 2.4419 & 2.7104 \\
\hline 45 & .9221 & 1.3656 & 1.7341 & 2.0664 & 2.4419 & 2.7073 \\
\hline 50 & .9221 & 1.3640 & 1.7339 & 2.0588 & 2.4444 & 2.7018 \\
\hline 55 & .9170 & 1.3595 & 1.7309 & 2.0532 & 2.4338 & 2.7012 \\
\hline 60 & .9216 & 1.3643 & 1.7320 & 2.0587 & 2.4378 & 2.7061 \\
\hline 70 & .9205 & 1.3653 & 1.7293 & 2.0487 & 2.4245 & 2.6837 \\
\hline 80 & .9210 & 1.3619 & 1.7289 & 2.0473 & 2.4205 & 2.6747 \\
\hline 90 & .9237 & 1.3635 & 1.7280 & 2.0494 & 2.4269 & 2.6840 \\
\hline 100 & .9212 & 1.3627 & 1.7288 & 2.0492 & 2.4212 & 2.6847 \\
\hline 110 & .9195 & 1.3605 & 1.7257 & 2.0419 & 2.4136 & 2.6653 \\
\hline 120 & .9205 & 1.3602 & 1.7247 & 2.0421 & 2.4150 & 2.6691 \\
\hline
\end{tabular}


Table 2. Type I Error Rates Of $t_{w}, t_{N s}$, And $t_{\max }$ For Various Sample Sizes.

\begin{tabular}{|c|c|c|c|c|}
\hline \multirow[b]{2}{*}{$\mathrm{n} 1, \mathrm{n} 2$} & \multirow[b]{2}{*}{ Statistics } & \multicolumn{3}{|c|}{ Level Of Significance } \\
\hline & & .100 & .050 & .010 \\
\hline \multirow[t]{4}{*}{6,6} & $\mathrm{t}_{\mathrm{w}}$ & .095 & .067 & .015 \\
\hline & $\mathrm{t}_{\mathrm{Ns}}^{\mathrm{w}}$ & .107 & .058 & .015 \\
\hline & $t_{\max (2)}^{\text {Ns }}$ & .111 & .067 & .015 \\
\hline & $t_{\max (b)}$ & .097 & .049 & .008 \\
\hline \multirow[t]{4}{*}{$3,9 t_{w}$} & $\mathrm{t}_{\mathrm{w}}$ & .099 & .064 & .010 \\
\hline & $\mathrm{t}_{\mathrm{NS}}^{w}$ & .099 & .054 & .010 \\
\hline & $\mathrm{t}_{\max (a)}$ & .109 & .064 & .010 \\
\hline & $t_{\max (b)}$ & .109 & .064 & .010 \\
\hline \multirow[t]{4}{*}{$10,10 t_{\mathrm{w}}$} & $\mathrm{t}_{\mathrm{w}}$ & .105 & .052 & .012 \\
\hline & $\mathrm{t}_{\mathrm{Ns}}^{\mathrm{w}}$ & .099 & .050 & .012 \\
\hline & $t_{\max (a)}$ & .114 & .057 & .013 \\
\hline & $t_{\max (b)}$ & .099 & .049 & .011 \\
\hline \multirow[t]{4}{*}{$5,15 \mathrm{t}_{\mathrm{w}}$} & $\mathrm{t}_{\mathrm{w}}$ & .097 & .051 & .011 \\
\hline & $\mathrm{t}_{\mathrm{NS}}$ & .100 & .050 & .011 \\
\hline & $t_{\max (a)}$ & .112 & .058 & .013 \\
\hline & $\begin{array}{l}\max (a) \\
t_{\max (b)}\end{array}$ & .106 & .050 & .010 \\
\hline \multirow[t]{4}{*}{20,20} & $t_{w}$ & .104 & .050 & .010 \\
\hline & $\mathrm{t}_{\mathrm{NS}}$ & .102 & .052 & .011 \\
\hline & $t_{\max (a)}$ & .117 & .060 & .013 \\
\hline & $\begin{array}{l}\max (a) \\
t_{\max (b)}\end{array}$ & .102 & .052 & .010 \\
\hline \multirow[t]{4}{*}{10,30} & $t_{w}$ & .101 & .047 & .010 \\
\hline & $\mathrm{t}_{\mathrm{NS}}^{\mathrm{w}}$ & .098 & .047 & .009 \\
\hline & $\mathrm{t}_{\max (a)}$ & .116 & .055 & .011 \\
\hline & $\begin{array}{c}\mathfrak{t}_{\max (a)} \\
\text { tax (b) }\end{array}$ & .101 & .049 & .010 \\
\hline \multirow[t]{4}{*}{40,40} & $\mathrm{t}_{\mathrm{w}}$ & .100 & .049 & .010 \\
\hline & $\mathrm{t}_{\mathrm{NS}}^{\mathrm{w}}$ & .101 & .049 & .010 \\
\hline & $\mathrm{t}_{\max (\mathrm{a})}$ & .117 & .058 & .012 \\
\hline & $\begin{array}{l}t_{\max (a)} \\
x^{2}(b)\end{array}$ & .100 & .050 & .010 \\
\hline \multirow[t]{4}{*}{20,60} & $\mathrm{t}_{\mathrm{w}}$ & .101 & .052 & .009 \\
\hline & $\mathrm{t}_{\mathrm{NS}}^{\mathrm{w}}$ & .101 & .051 & .010 \\
\hline & $\mathrm{t}_{\max (\mathbf{a})}$ & .116 & .061 & .012 \\
\hline & $\begin{array}{l}t_{\max (a)} \\
t_{\max (b)}\end{array}$ & .101 & .052 & .009 \\
\hline \multirow[t]{4}{*}{60,60} & $\mathrm{t}_{\mathrm{w}}$ & .101 & .049 & .010 \\
\hline & $\mathrm{t}_{\mathrm{NS}}^{\mathrm{w}}$ & .101 & .048 & .010 \\
\hline & $\mathrm{t}_{\max (a)}$ & .117 & .058 & .012 \\
\hline & 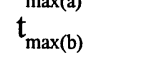 & .102 & .048 & .010 \\
\hline \multirow[t]{4}{*}{30,90} & $\mathrm{t}_{\mathrm{w}}$ & .100 & .049 & .011 \\
\hline & $\mathrm{t}_{\mathrm{NS}}^{\mathrm{w}}$ & .100 & .050 & .011 \\
\hline & $\mathrm{t}_{\max (\mathrm{a})}$ & .116 & .058 & .011 \\
\hline & $\begin{array}{l}\max (a) \\
t_{\max (b)} \\
\end{array}$ & .100 & .049 & .011 \\
\hline
\end{tabular}



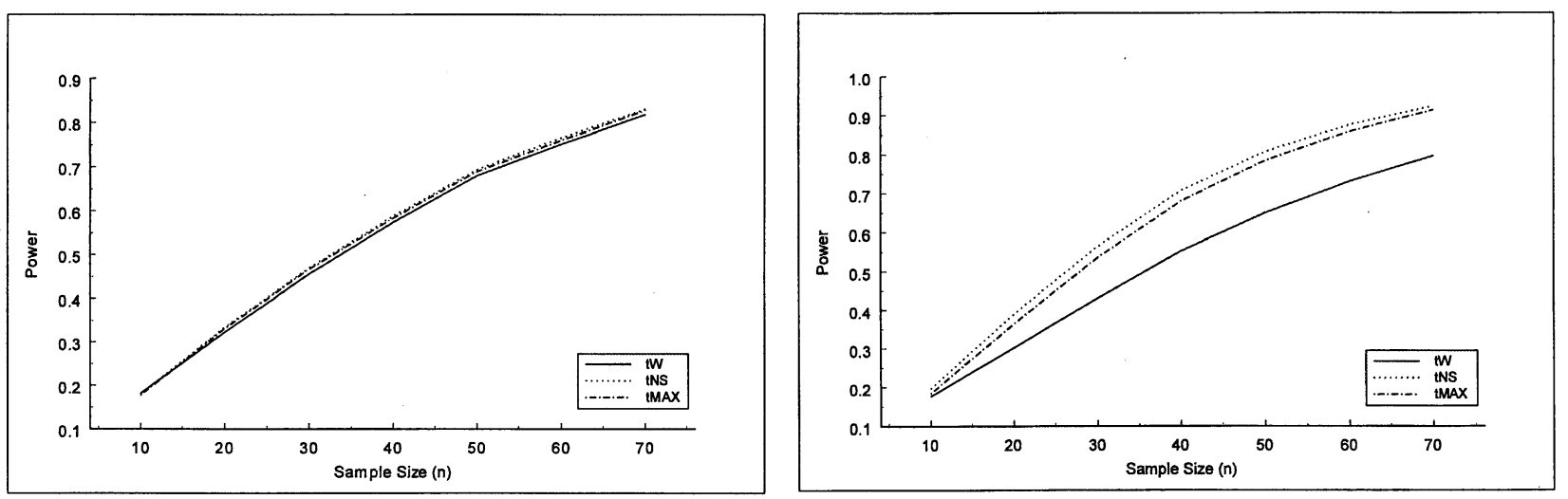

Figure 3. Power of $t W, t M S$ and $t M A X$ When Sampling is From a Cauchy Distriubtuon

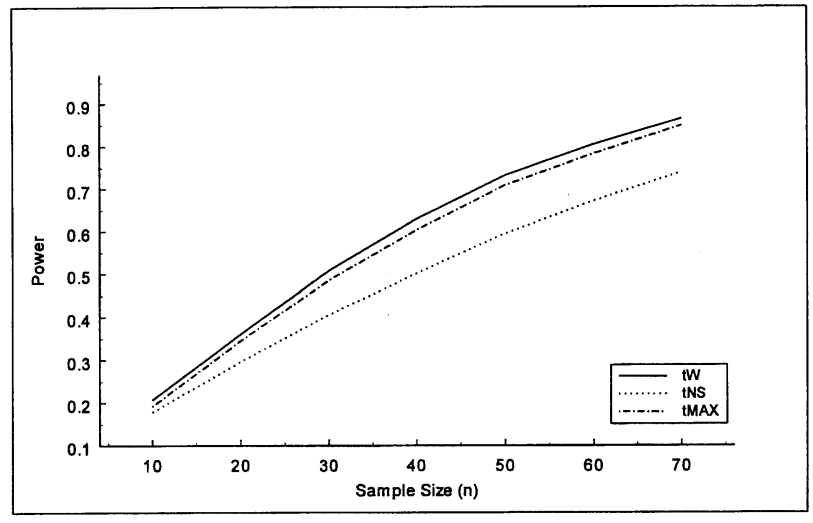

$\mathrm{t}_{\mathrm{Ns}}$. Under the heavy-tailed Cauchy distribution, $\mathrm{t}_{\mathrm{w}}$ was the most powerful statistic, with $\mathrm{t}_{\text {max }}$ once again demonstrating power similar to, but slightly less than, that of the most powerful test.

\section{Conclusion}

It is usually difficult for researchers to obtain sufficient information from a given data set so as to make reasonable choices between suitable statistical tests. It is important, therefore, that tests have power to detect broad classes of alternatives with high probability. The technique demonstrated here is a simple method for constructing such tests.

A major advantage of the test presented here lies in the fact that this test is automatically adaptive to the weight in the tail of the population from which the data were sampled. This is contrasted with various adaptive estimation procedures which require a preliminary estimate of tail weight.

It should also be noted that the maximum method may be extended to a wide variety of testing situations. For example, more than two statistics my be formed into a maximum test, with component tests being both parametric and non-parametric. A large number of other possibilities exist.

\section{References}

Bradley, J. V. (1968). Distribution-free statistical tests. Englewood Cliffs, NJ: Prentice-Hall.

Chernoff, H., \& Savage, I. R. (1958). Asymptotic normality and efficiency of certain nonparametric test statistics. Annals of Mathematical Statistics, 28, 972-994.

Conover, W. J., \& Iman, R. (1981). Rank transformations as a bridge between parametric and nonpara- 
metric statistics. The American Statistician, 35, 124-129. Cox, D. R. (1977). The role of significance tests. Scandinavian Journal of Statistics, 4, 49-70.

Hodges, J. L., \& Lehmann, E. L. (1961). Comparison of the normal scores and Wilcoxon tests. In (J. Neyman, ed.) Proceedings of the Fourth Berkeley Symposium on Mathematical Statistics and Probability, 1. Berkeley and Los Angeles, CA: University of California Press. Iman, R. L. (1974). An approximation to the exact distribution of the Wilcoxon-Mann-Whitney rank sum test statistic. Communications in Statistics, 5, 587-598.

Lehmann, E. L. (1959). Testing statistical hypotheses. NY: Wiley.

Mikulski, P. W. (1963). On the efficiency of optimal nonparametric procedures in the two sample case. Annals of Mathematical Statistics, 34, 22-32.

Owen, D. B. (1962). Handbook of statistical tables. Reading, MA: Addison-Wesley.

Terry, M. E. (1952). Some rank order tests which are most powerful against specific parametric alternatives. Annals of Mathematical Statistics, 23, 346-366. 


\title{
Some Locally Most Powerful Rank Tests For Correlation
}

\author{
W. J. Conover \\ College of Business Administration \\ Texas Tech University
}

Four examples are given to illustrate the ease and practicality of the procedure for finding locally most powerful rank tests for correlation. The first two examples deal with bivariate exponential models. The third example uses the bivariate normal distribution, and the fourth example analyzes the Morgenstern's general correlation model.

Keywords: Bivariate exponential, Bivariate normal, Morgenstern's model, Top-down correlation, Spearman's rho, Normal scores, Savage scores

Introduction

There are two primary difficulties in developing tests with good power properties for testing the null hypothesis of independence between two variables $\mathrm{X}$ and $\mathrm{Y}$, based on a bivariate random sample $\left(X_{i}, Y_{i}\right), i=1,2, \ldots, n$, against the alternative hypothesis of correlation. One difficulty is in finding a suitable model for the bivariate distribution, and the other is in developing a powerful test for correlation once the model is selected.

The bivariate normal distribution is a convenient model to use for many reasons. The parameter rho is the linear correlation coefficient, so correlation is convenient to address in this model. The most powerful test for correlation is well known, and the locally most powerful rank test (LMPRT) uses Fisher-Yates expected normal scores.

But the bivariate normal distribution does not fit some types of data very well. Therefore other classes of bivariate distributions have been developed in an attempt to find models more appropriate than the bivariate normal distribution, while retaining some of the nice analytical properties found in the bivariate normal distribution. In this paper the general bivariate density function $h(x, y ; \Theta)$ is considered, with some fairly general restrictions.

One popular family of bivariate distributions was proposed by Morgenstern (1956). Let $F(x)$ and $G(y)$ be the marginal distribution functions. A bivariate distribution function with those marginals is given by

$$
\mathrm{H}(\mathrm{x}, \mathrm{y} ; \theta)=\mathrm{F}(\mathrm{x}) \mathrm{G}(\mathrm{y})[1+\theta\{1-\mathrm{F}(\mathrm{x})\}[1-\mathrm{G}(\mathrm{y})\}]
$$

W. Jay Conover has been publishing papers in nonparametric statistic since1964. He is best known for his books, including Practical Nonparametric Statistics, which first appeared in 1971. The third edition was published in 1999. $\mathrm{He}$ is a Fellow of the American Statistical Association. where $\theta$ is the parameter that governs the degree of dependence between the random variables. Farlie (1961) found Spearman's rho to be the optimal correlation coefficient for testing the null hypothesis $\theta=0$ in Morgenstern's model (1.1), and studied the efficiency of less than optimal coefficients. Our derivation of the same result is much simpler and with fewer restrictions on the model.

Morgenstern's model (1.1) was generalized by Farlie (1960) to

$$
\mathrm{H}(\mathrm{x}, \mathrm{y} ; \theta)=\mathrm{F}(\mathrm{x}) \mathrm{G}(\mathrm{y})[1+\theta \mathrm{A}(\mathrm{x}) \mathrm{B}(\mathrm{y})]
$$

where $A(x)$ an $B(y)$ are bounded functions such that $A(\infty)$ $=\mathrm{B}(\infty)=0$. The model (1.2), and thus (1.1) also, is a special case of the general model studied in this paper.

Konijn (1956) studied correlation tests for the hypothesis $\theta_{2}=\theta_{3}=0$ in the model

$$
\begin{aligned}
& X=\theta_{1} W+\theta_{2} Z \\
& Y=\theta_{3} W+\theta_{4} Z
\end{aligned}
$$

where $\mathrm{W}$ and $\mathrm{Z}$ are independent random variables. Correlation tests for a similar class of alternatives

$$
\begin{aligned}
& X=(1-\theta) U+\theta Z \\
& Y=(1-\theta) V+\theta Z
\end{aligned}
$$

where $\mathrm{U}, \mathrm{V}$ and $\mathrm{Z}$ are independent random variables, and the null hypothesis is $\theta=0$, were investigated by Bhuchongkul (1964). Hájek and Sidák (1967, p.75, or see Hájek et al., 1999, p.77) discuss the nearly identical model

$$
\begin{aligned}
& \mathrm{X}=\mathrm{U}+\theta \mathrm{Z} \\
& \mathrm{Y}=\mathrm{V}+\theta \mathrm{Z}
\end{aligned}
$$

These models are more restrictive in their application than the more general model considered in this paper.

In this paper the general bivariate density function $\mathrm{h}(\mathrm{x}, \mathrm{y} ; \theta)$ is investigated. A theorem is presented that enables the locally most powerful rank test of $\theta=\theta_{0}$ to be derived under some fairly general conditions. Four examples are given to illustrate the usefulness of this result. 
Although the development stops short of finding the efficiencies of the obtained tests, in some cases the tests are well known, and their efficiencies have been studied.

\section{The Locally Most Powerful Rank Test For Correlation}

Let $(X, Y)$ have the joint density function $\mathrm{h}(\mathrm{x}, \mathrm{y} ; \theta)$ under $\mathrm{H}_{\mathrm{a}}$ and the density $\mathrm{h}\left(\mathrm{x}, \mathrm{y} ; \theta_{0}\right)=\mathrm{f}(\mathrm{x}) \mathrm{g}(\mathrm{y})$ under $\mathrm{H}_{0}$, the independence hypothesis, where $\mathrm{f}(\mathrm{x})$ and $\mathrm{g}(\mathrm{y})$ are the marginal density functions of $\mathrm{X}$ and $\mathrm{Y}$ respectively. Consider independent copies $\left(\mathrm{X}_{\mathrm{m}}, \mathrm{Y}_{\mathrm{m}}\right), \mathrm{m}=1, \ldots, \mathrm{n}$ of $(\mathrm{X}, \mathrm{Y})$, with ranks $R_{m}$ for $X_{m}$, and $Q_{m}$ for $Y_{m}$, where the $X$ 's and the $Y$ 's are ranked separately. Also define the scores

$\mathrm{a}(\mathrm{i}, \mathrm{j} ; \mathrm{h})=\mathrm{E}\left[\partial \ln \left\{\mathrm{h}\left(\mathrm{X}_{\mathrm{n}}^{(\mathrm{i})}, \mathrm{Y}_{\mathrm{n}}^{(\mathrm{j})} ; \theta\right)\right\} /\left.\partial \theta\right|_{\theta=\theta 0} \mid \mathrm{H}_{0}\right]$

where $X_{n}^{(i)}$ and $\left.Y_{n}{ }_{n}\right)$ are the $i^{\text {th }}$ and $j^{\text {th }}$ order statistics in a random sample of size $n$ from $f(x)$ and $g(y)$ respectively.

The following theorem was first proven by Shirahata (1974) in the p-variate case, for general regression alternatives. This is the simplified version for testing correlation in the bivariate case.

Theorem for locally most powerful rank correlation tests. Let $\mathrm{J}$ be some open interval around $\theta_{0}$. If

1. $\mathrm{h}(\mathrm{x}, \mathrm{y} ; \theta) / \mathrm{h}\left(\mathrm{x}, \mathrm{y} ; \theta_{0}\right)$ exists for $\theta \in \mathrm{J}$,

2. $\partial\{\mathrm{h}(\mathrm{x}, \mathrm{y} ; \theta)\} /\left.\partial \theta\right|_{\theta=\theta 0}$ exists for $\theta \in \mathrm{J}$,

3. $\mathrm{h}\left(\mathrm{x}, \mathrm{y} ; \theta_{0}\right)=\lim _{\theta} \rightarrow_{\theta 0} \mathrm{~h}(\mathrm{x}, \mathrm{y} ; \theta)$ exists for $\theta \in \mathrm{J}$, and

4. $\lim \theta \rightarrow \theta_{0} \iint|\partial\{\mathrm{h}(\mathrm{x}, \mathrm{y} ; \theta)\} / \partial \theta| \mathrm{dx} d \mathrm{y}$ $=\iint|\partial\{\mathrm{h}(\mathrm{x}, \mathrm{y} ; \theta)\} / \partial \theta|_{\theta=\theta 0} \mid \mathrm{dxdy}<\infty$,

then the locally $(\delta \rightarrow 0)$ most powerful rank test of $\mathrm{H}_{0}: \theta$ $=\theta_{0}$ against $\mathrm{H}_{\mathrm{a}}: \theta=\theta_{0}+\delta$ is given by the test with the critical region

$$
\begin{aligned}
& \mathrm{n} \\
& \Sigma \mathrm{a}\left(\mathrm{R}_{\mathrm{m}}, \mathrm{Q}_{\mathrm{m}} ; \mathrm{h}\right)>\mathrm{k}
\end{aligned}
$$

where $\mathrm{k}$ is chosen so the test will have an appropriate size $\alpha$.

Implementation of the previous theorem to find the scores $\mathrm{a}(\mathrm{i}, \mathrm{j} ; \mathrm{h})$ associated with the locally most powerful rank test for correlation involves the following steps.

1. Find the partial derivative of $\mathrm{h}(\mathrm{x}, \mathrm{y} ; \theta)$ with respect to $\theta$ and $\operatorname{set} \theta$ equal to $\theta_{0}$.

2. Divide the result in Step 1 by $h\left(x, y ; \theta_{0}\right)=f(x) g(y)$.

3. Substitute $X_{n}^{(i)}$ for $x$ and $Y_{n}^{(j)}$ for $y$ in the quotient in Step

2 , where $X_{n}^{(i)}$ and $Y_{n}^{(j)}$ are the $i \underline{\text { th }}$ and $j \underline{\text { th }}$ order statistics in random samples of size $\mathrm{n}$ from $\mathrm{f}(\mathrm{x})$ and $\mathrm{g}(\mathrm{y})$ respectively.

4. Find the expected value of the random variable in Step 3 under $\mathrm{H}_{0}$. That is, integrate the product of (a) the result of Step 2,

(b) the density function of the ith order statistic from $\mathrm{f}(\mathrm{x})$,

(c) and the density function of the jh $\mathrm{g}(\mathrm{y})$, over the entire range of values of $\mathrm{X}$ and $\mathrm{Y}$.

\section{Four Examples Of Locally Most Powerful Rank Tests}

These four examples show the ease with which the theorem of Section 2 can be applied to obtain locally most powerful rank tests for correlation. In all four examples the resulting test statistic is known, and the literature citations can be consulted to find tables for small sample sizes, and asymptotic approximations for large sample sizes.

The first two examples involve bivariate distributions, where both marginal distributions are exponential. The model in the first example allows only nonnegative correlations, and may be used when the alternative hypothesis is one of positive correlation. The model in the second example allows only nonpositive correlations, and may be used when the alternative hypothesis is one of negative correlation. In both examples, the locally most powerful rank test uses the top-down correlation coefficient of Iman and Conover (1987). The third example involves the bivariate normal distribution, and the fourth example looks at a very general bivariate distribution.

Example 1. Mardia (1970) presented a bivariate exponential distribution

$$
\mathrm{h}_{1}(\mathrm{x}, \mathrm{y} ; \theta)=\frac{1}{1-\theta} \mathrm{e}^{\frac{-\mathrm{x}+\mathrm{y}}{1-\theta}} \sum_{\mathrm{r}=0}^{\infty}\left[\frac{\theta \mathrm{xy}}{(1-\theta)^{2}}\right] \mathrm{r} / \mathrm{r} ! \mathrm{r} !
$$

$$
x>0, y>0,0 \leq \theta<1
$$

which has exponential marginal densities $\exp (-\mathrm{x})$ and $\exp (-\mathrm{y})$, and which degenerates to the product of those marginal densities $\exp \{-\mathrm{x}-\mathrm{y}\}$ for $\theta=0$, representing the case of independence. The correlation coefficient between $\mathrm{X}$ and $\mathrm{Y}$ is $\theta$. Although this model has standardized exponential distributions, the non-standardized exponential distributions, with a general scale variable, give the same result because the test derived is a function only of ranks within each variable, and the ranks are not changed by a change in the scale variable.

This model first appeared in Mardia (1962) as a special case of a bivariate gamma distribution that appeared in Kibble (1941). It has been attributed to various authors, such as to Downton (1970) by Hawkes (1972) and others, and to Nagao and Kadoya (1971) by Cordova and Rodreguez-Iturbe (1985), Johnson and Kotz (1972), and others. It is widely used as a model for the bivariate 
exponential distribution. A parametric test of the null hypothesis of independence is apparently unknown. The locally most powerful rank test is derived in the following.

$\mathrm{a}\left(\mathrm{i}, \mathrm{j} ; \mathrm{h}_{1}\right)=\mathrm{E}\left\{\left(\mathrm{X}_{\mathrm{n}}^{(\mathrm{i})-1}\right)\left(\mathrm{Y}_{\mathrm{n}}^{(\mathrm{j})}-1\right) \mid \mathrm{H}_{0}\right\}=\left(\mathrm{s}_{\mathrm{n}}(\mathrm{i})-1\right)\left(\mathrm{s}_{\mathrm{n}}(\mathrm{j})-1\right)$

where $s_{n}(i)$ and $s_{n}(j)$ are the expected values of order statistics from the exponential distribution. That is, step 1 in the previous section involves finding the derivative of $h_{1}(x, y ; \theta)$ with respect to $\theta$, and setting $\theta=0$. This gives

$$
\left.\frac{\partial}{\partial \theta} \mathrm{h}_{1}(\mathrm{x}, \mathrm{y} ; \theta)\right|_{\theta=0}=\mathrm{e}^{-\mathrm{x}-\mathrm{y}}(\mathrm{x}-1)(\mathrm{y}-1)
$$

The second step is to divide by $f(x) g(y)=e^{-x-y}$, which gives

$$
(\mathrm{x}-1)(\mathrm{y}-1)
$$

In the third step the ith and jth order statistics from the exponential distributions $\mathrm{f}(\mathrm{x})$ and $\mathrm{g}(\mathrm{y})$ replace $\mathrm{x}$ and $\mathrm{y}$ respectively. Thus the expected values, in step 4 , give the LMPRT scores in (3.2).

The scores in (3.2) are given by the formula

$$
s_{n}(i)=\sum_{j=0}^{i-1} \frac{1}{n-j}
$$

and are sometimes called Savage scores because they were introduced by Savage (1956). Their use in a rank correlation coefficient

$$
\mathrm{r}_{\mathrm{T}}=\frac{\sum \mathrm{s}_{\mathrm{n}}\left(\mathrm{R}_{\mathrm{m}}\right) \mathrm{s}_{\mathrm{n}}\left(\mathrm{Q}_{\mathrm{m}}\right)-\left(\sum \mathrm{s}_{\mathrm{n}}(\mathrm{i})\right)^{2} / \mathrm{n}}{\Sigma\left[\mathrm{s}_{\mathrm{n}}(\mathrm{i})\right]^{2}-\left(\sum \mathrm{s}_{\mathrm{n}}(\mathrm{i})\right)^{2} / \mathrm{n}}=\frac{\sum \mathrm{s}_{\mathrm{n}}\left(\mathrm{R}_{\mathrm{m}}\right) \mathrm{s}_{\mathrm{n}}\left(\mathrm{Q}_{\mathrm{m}}\right)-\mathrm{n}}{\mathrm{n}-\mathrm{s}_{\mathrm{n}}(\mathrm{n})}
$$

was studied by Iman and Conover (1987), and called the top-down correlation coefficient $r_{T}$ because of its tendency to emphasize the tail values. That is, items are ranked from least important or lowest, with rank 1, to most important or greatest, with rank $\mathrm{n}$. The top-down correlation coefficient gives more emphasis to the agreement among the most important items, and less emphasis to agreement among the least important items. Spearman's rho and Kendall's tau give equal importance to agreement at all ranks.

Exact quantiles for the null distribution of $\mathrm{r}_{\mathrm{T}}$ in a test of independence are given by $\operatorname{Iman}(1987)$ for $n \leq 14$. Therefore the locally most powerful rank test of $\mathrm{H}_{0}: \theta=0$ against $\mathrm{H}_{\mathrm{a}}: \theta>0$ in the bivariate exponential distribution given by (3.1) rejects $\mathrm{H}_{0}$ if and only if $\mathrm{r}_{\mathrm{T}}>\mathrm{k}$ for a suitably chosen value of $\mathrm{k}$.

Example 2. Gumbel (1960) introduced another bivariate exponential distribution $\mathrm{h}_{2}(\mathrm{x}, \mathrm{y} ; \theta)=\{(1+\theta \mathrm{x})(1+\theta \mathrm{y})-\theta\} \mathrm{e}^{-\mathrm{x}-\mathrm{y}-\theta \mathrm{xy}}$ $x>0, y>0,0 \leq \theta \leq 1$

with non-positive correlation coefficient:

$$
-1+\int_{0}^{\infty} \frac{e^{-y}}{1+\theta y} d y
$$

Note that the correlation coefficient is zero when $\theta=0$, and it decreases monotonically as $\theta$ increases. Therefore the LMPRT for correlation is also the LMPRT for $\theta$. This distribution degenerates to $\exp \{-\mathrm{x}-\mathrm{y}\}$ under $\mathrm{H}_{0}: \theta=0$. This widely known model was studied further by Gumbel (1961) and has been used more recently by Wei (1981) and Barnett (1983). As with the previous model, a parametric test of the null hypothesis of independence is apparently unknown. The locally most powerful rank test is derived in the following.

The optimal scores are again found to be functions of the Savage scores. Specifically the scores are: $\mathrm{a}\left(\mathrm{i}, \mathrm{j} ; \mathrm{h}_{2}\right)=\mathrm{E}\left\{-\left(\mathrm{X}_{\mathrm{n}}^{(\mathrm{i})}-1\right)\left(\mathrm{Y}_{\mathrm{n}}^{(\mathrm{j})}-1\right) \mid \mathrm{H}_{0}\right\}=-\left(\mathrm{s}_{\mathrm{n}}(\mathrm{i})-1\right)\left(\mathrm{s}_{\mathrm{n}}(\mathrm{j})-1\right)$

which leads to the locally most powerful rank test that rejects $\mathrm{H}_{0}$ when $\mathrm{r}_{\mathrm{T}}<\mathrm{k}$ for some suitably chosen negative number $\mathrm{k}$. Note that the negative value for $\mathrm{k}$ is due to the model, which allows only negative correlation in the restricted parameter range for $\theta$.

Example 3. The all-important bivariate normal distribution has density:

$\mathrm{h}_{3}(\mathrm{x}, \mathrm{y} ; \theta)=\left(2 \pi\left(1-\theta^{2}\right)\right)^{-1 / 2} \exp \left\{-\left(\mathrm{x}^{2}+\mathrm{y}^{2}-2 \theta \mathrm{xy}\right) / 2\right\}$

and correlation coefficient $\theta$. The scores for the locally most powerful rank test are given by:

$$
a\left(i, j ; h_{3}\right)=E\left(Z_{n}^{(i)}\right) E\left(Z_{n}^{(j)}\right)
$$

where $Z_{n}^{(i)}$ and $Z_{n}{ }^{(j)}$ are order statistics from the standard normal distribution. These scores are used in the wellknown normal scores statistic first given by Fisher and Yates (1957). This derivation of the locally most powerful rank test for the bivariate normal distribution, also given by Shirahata (1974), is much simpler than the previous ones, and uses a more general model than the rather restrictive models (1.3), (1.4) and (1.5).

Example 4. The class of bivariate distributions in introduced by Morgenstern (1956) has the bivariate distribution function:

$\mathrm{H}(\mathrm{x}, \mathrm{y} ; \theta)=\mathrm{F}(\mathrm{x}) \mathrm{G}(\mathrm{y})[1+\theta\{1-\mathrm{F}(\mathrm{x})\}\{1-\mathrm{G}(\mathrm{y})\}]$

for any marginal distribution functions $F(x)$ and $G(y)$. This model has been extended by Plackett (1965) and often appears in discussions of bivariate distributions (see for 
example Mardia, 1970, or Johnson and Kotz, 1972). Due to the unspecified nature of $F(x)$ and $G(y)$ no parametric test is possible. However, rank tests are possible. In fact the locally most powerful rank test is easily derived, as shown in the following.

When $H(x, y)$ is continuous then the density function is

$$
\mathrm{h}_{4}(\mathrm{x}, \mathrm{y} ; \theta)=\mathrm{f}(\mathrm{x}) \mathrm{g}(\mathrm{y})[1+\theta\{1-2 \mathrm{~F}(\mathrm{x})\}\{1-2 \mathrm{G}(\mathrm{y})\}]
$$

which reduces to the independence case $f(x) g(y)$ when $\theta=$ 0 . This example shows the full power of the method discussed in this paper for finding the locally most powerful rank test for independence. The scores $a\left(i, j ; h_{4}\right)$ in this case reduce to

$$
\begin{aligned}
\mathrm{a}\left(\mathrm{i}, \mathrm{j} ; \mathrm{h}_{4}\right) & =\mathrm{E}\left\{\left(2 \mathrm{~F}\left(\mathrm{X}_{\mathrm{n}}^{(i)}\right)-1\right)\left(2 \mathrm{G}\left(\mathrm{Y}_{\mathrm{n}}^{(j)}\right)-1\right)\right\} \\
& =\left(2 \mathrm{E}\left\{\mathrm{U}_{\mathrm{n}}^{(i)}\right\}-1\right)\left(2 \mathrm{E}\left\{\mathrm{U}_{\mathrm{n}}^{(j)}\right\}-1\right)
\end{aligned}
$$

where $U_{n}^{(i)}$ and $U_{n}^{(j)}$ represent order statistics from the uniform distribution on $(0,1)$. These are the scores used in the Spearman rank correlation coefficient, so Spearman's rho is the locally most powerful rank test for correlation for the entire class of Morgenstern distributions, assuming only that the bivariate distributions are continuous. This result was first obtained by Farlie (1961), but this method of proof is much simpler.

Note that $h_{4}(x, y ; \theta)$ is a density function with marginal densities $f(x)$ and $g(y)$ for all density functions $f$ and $g$. In particular if $f$ and $g$ are exponential density functions, $\mathrm{h}_{4}$ is another form of a bivariate exponential distribution. In this case the correlation coefficient is $\theta / 4$ (Gumbel, 1960) and it varies only within the narrow domain $[-.25, .25]$. Since the correlation coefficient is a monotonic function of $\theta$, the LMPRT for correlation in this bivariate exponential model uses Spearman's rho, instead of the top-down correlation coefficient of the previous two bivariate exponential models.

\section{Conclusion}

Asymptotic normality for the special cases of the test statistic given in the previous section is already known. In general, asymptotic normality under the null hypothesis results from Theorem 3 , and under contiguous alternatives from Theorem 4, of Shirahata (1974). Closely related results were also given by Behnan (1971), Ruymgaart et al. (1972), and Ruymgaart (1974).

\section{References}

Barnett, V. (1983). Reduced distance measures and transformations in processing multivariate outliers. Australian Journal of Statistics, 25(1), 64-75.

Behnan, K. (1971). Asymptotic optimality and ARE of certain rank-order tests under contiguity. The Annals of Mathematical Statistics, 42, 325-329.

Bhuchongkul, S. (1964). A class of nonparametric tests for independence in bivariate populations. The Annals of Mathematical Statistics, 35, 138-149.

Cordova, J. R., \& Rodriguez-Iturbe, I. (1985). On the probabilistic structure of storm surface runoff. Water Resources Research, 21, 755-763.

Downton, F. (1970). Bivariate exponential distributions in reliability theory. Royal Statistical Society Journal, 32, 408-417.

Farlie, D. J. G. (1960). The performance of some correlation coefficients for a general bivariate distribution. Biometrika, 47, 307-323.

Farlie, D. J. G. (1961). The asymptotic efficiency of Daniels' generalized correlation coefficients. Royal Statistical Society Journal, 23, 128-142.

Fisher, R. A., \& Yates, F. (1957). Statistical tables for biological, agricultural and medical research. (3rd ed.). Darien, Conn: Hafner.

Gumbel, E. J. (1960). Bivariate exponential distributions. Journal of the American Statistical Association, 55, 698-707.

Gumbel, E. J. (1961). Bivariate logistic distributions. Journal of the American Statistical Association, 56, 335-349.

Hájek, J., \& Sidák, Z. (1967). Theory of rank tests. New York: Academic Press.

Hájek, J., Sidák, Z., \& Sen, P.K. (1999). Theory of rank tests. (2nd ed.). New York: Academic Press.

Hawkes, A. G. (1972). A bivariate exponential distribution with applications to reliability. Royal Statistical Society Journal, 34, 129-133.

Iman, R. L. (1987). Tables of the exact quantiles of the top-down correlation coefficient for $\mathrm{n}=3(1) 14$. Communications in Statistics, Theory and Methods, 16(5), 1513-1540.

Iman, R. L., \& Conover, W. J. (1987). A measure of top-down correlation. Technometrics, 29(3), 351-357.

Johnson, N. L., \& Kotz, S. (1972). Distributions in statistics: Continuous multivariate distributions. New York: John Wiley.

Kibble, W. F. (1941). A two-variate gamma type distribution. Sankhya, 5, 137-150.

Konijn, H. S. (1956). On the power of certain tests for independence in bivariate populations. The Annals of Mathematical Statistics, 27, 300-323. 
Mardia, K. V. (1962). Multivariate pareto distributions. The Annals of Mathematical Statistics, 33, 10081015.

Mardia, K. V. (1970). Families of bivariate distributions. Darien, Conn: Hafner.

Morgenstern, D. (1956). Einfache beispiele zweidimensionaler Verteilungen. Mitteilungsblatt für Mathematische Statistik, 8, 234-235.

Nagao, M., \& Kadoya, M. (1971). Two-variate exponential distribution and its numerical table for engineering applications. Bull. Disaster Prev. Res. Inst., Kyoto Univ., 20, 183-215.

Plackett, R. L. (1965). A class of bivariate distributions. Journal of the American Statistical Association, $60,516-522$.
Ruymgaart, F. H. (1974). Asymptotic normality of nonparametric tests for independence. The Annals of Statistics, 2, 892-910.

Ruymgaart, F. H., Shorack, G. R., \& van Zwet, W. R. (1972). Asymptotic normality of nonparametric tests for independence. The Annals of Mathematical Statistics, 43, 1122-1135.

Savage, I. R. (1956). Contributions to the theory of rank order statistics - the two-sample case. The Annals of Mathematical Statistics, 27, 590-615.

Shiratata, S. (1974). Locally most powerful rank tests for independence. Bulletin of Mathematical Statistics, 16, 11-21.

Wei, L. J. (1981). Estimation of location difference for fragmentary samples. Biometrika, 68(2), 471-476. 


\section{Power Analyses When Comparing Trimmed Means}

\author{
Rand R. Wilcox \\ Department of Psychology \\ University of Southern California
}

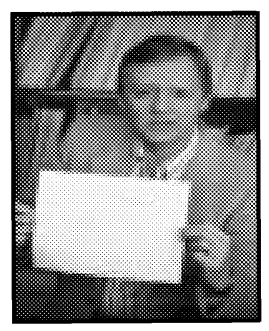

H. J. Keselman

Department of Psychology

University of Manitoba

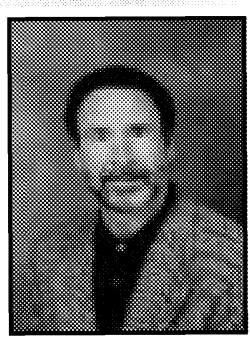

Given a random sample from each of two independent groups, this article takes up the problem of estimating power, as well as a power curve, when comparing $20 \%$ trimmed means with a percentile bootstrap method. Many methods were considered, but only one was found to be satisfactory in terms of obtaining both a point estimate of power as well as a (one-sided) confidence interval. The method is illustrated with data from a reading study where theory suggests two groups should differ but nonsignificant results were obtained.

Keywords: Bootstrap, Robust methods

Introduction

Power is a fundamental concern when comparing measures of location corresponding to two independent groups. Of course, when we fail to detect a difference, this might be because there is little or no difference between the measures of location, or perhaps the sample size was inadequate for detecting a difference that is substantively important. Surely the best-known and most commonly used method when addressing power is to assume both groups have normal distributions with a common variance, specify a (standardized) difference between means, choose $1-\beta$, the desired probability of rejecting the hypothesis of equal means for this specified difference, and then determine the required sample size to achieve this goal. Cohen (1977) provided an excellent summary of this strategy.

Another but less commonly used method for dealing with power is to use a post hoc analysis. That is, collect data, and based on the observed values estimate power or determine what sample size is required to achieve a

Rand R. Wilcox is a Professor of Psychology at the University of Southern California. He is a fellow of the Royal Statistical Society and the American Psychological Society. He has published over 170 journal articles, and has recently written his fifth book on statistics. Harvey Keselman is a fellow of the American Psychological Association and the American Psychological Society. He is a Professor of Psychology at The University of Manitoba, Winnipeg, Manitoba, Canada. His areas of interest include the analysis of repeated measurements, multiple comparison procedures, and robust estimation and testing. desired amount of power. As is evident, power is not an issue if the null hypothesis is rejected, but otherwise it is. A classic illustration of this approach is the two-stage strategy derived by Stein (1945). Extensions of the method to two or more groups have been proposed by various researchers during the ensuing years, a summary of which can be found in Wilcox (1996). Included are exact heterosecedastic methods when sampling from normal distributions. That is, under normality, both the probability of a Type I error and power can be simultaneously controlled. Other approaches to controlling power are reviewed by Hewett and Spurrier (1983).

Our goal in this paper is to consider how power analyses might be made when comparing $20 \%$ trimmed means rather than means. But unlike Stein-type procedures, our goal is to obtain both a point estimate of power plus a one-sided confidence interval. That is, if we fail to reject, we want to estimate power, based on the observed data. In particular, we want to estimate the power curve, the probability of rejecting as a function of the difference between the population trimmed means.

Our interest in $20 \%$ trimmed means stems from both its theoretical advantages summarized by Staudte and Sheather (1990) and Huber (1981), among others, plus its practical advantages when trying to deal with nonnormality. In particular, methods based on $20 \%$ trimmed means provide good control over Type I errors for a broader range of situations versus methods based on means, they maintain relatively high power under arbitrarily small departures from normality that destroy power when using means, and they provide accurate confidence intervals over a much 
broader range of situations versus conventional methods for means. Theory and simulations also indicate that trimmed means do a better job of reducing bias when testing hypotheses. Student's two-sample $t$, for example, is biased, meaning that the probability of rejecting is not minimized when the null hypothesis is true.

That is, power can decrease as the difference between the means increases. Comparing $20 \%$ trimmed means with a percentile bootstrap method virtually eliminates this problem among situations considered in extant publications. Moreover, the percentile bootstrap, used in conjunction with $20 \%$ trimmed means, performs remarkably well when the goal is to use a test that is reasonably equal-tailed. For a nontechnical summary of the many problems associated with means in particular and least squares in general, and how modern robust methods address these problems, see Wilcox (2001a). For a recent review of problems associated with conventional methods, see Keselman, Huberty, Lix, Olejnik, Cribbie, Donohue, Kowalchuk, Lowman,

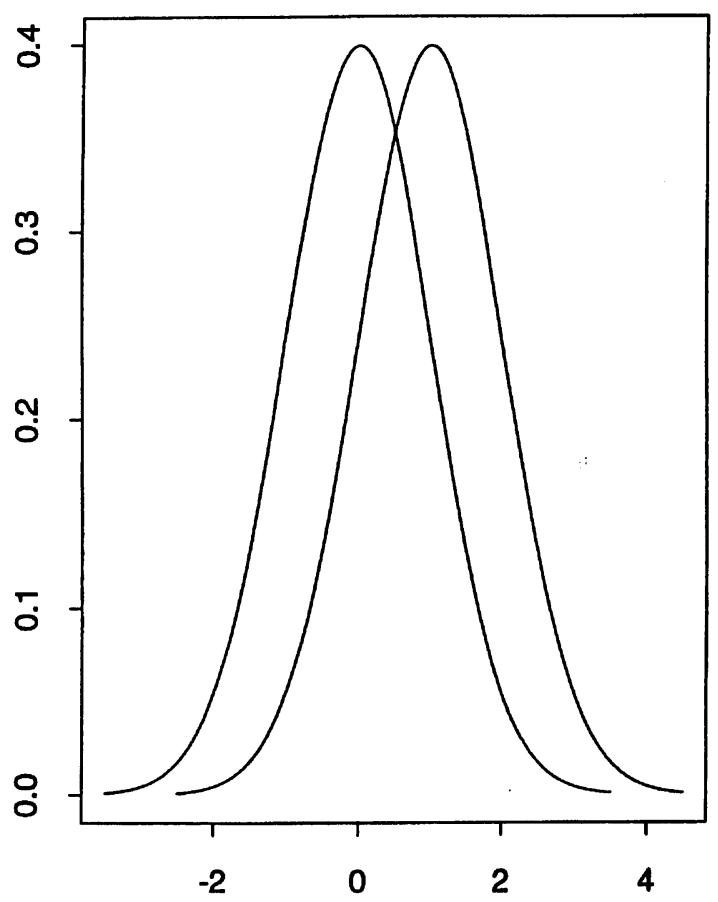

tosky, Keselman, and Levin (1998). Readers interested in the practical details of how to apply robust methods can refer to Wilcox (1997).

To help motivate this paper we begin with the usual planning strategy of determining an appropriate sample size under normality when using Student's t. The left panel of Figure 1 shows two normal curves having means 0 and 1 and a common variance of one. Let

$$
\Delta=\frac{\mu_{1}-\mu_{2}}{\sigma},
$$

where $\mu_{\mathrm{j}}$ and $\sigma_{j}^{2}$ are the mean and variance associated with the $j^{\text {th }}$ group $(j=1,2)$, and by assumption $\sigma_{1}^{2}=\sigma_{2}^{2}=\sigma^{2}$. Cohen argued that for normal distributions $\Delta=.8$ is a large effect, so from this view surely we want power to be reasonably high for the situation depicted in Figure 1. If we sample twenty-five observations from each group and test for equal means at the .05 level, power is .96. So based on this power analysis under normality, sample sizes of 25 would seem to suffice. But suppose we sample from the two distributions shown in the right panel instead. As is evident, they appear to be very similar to the

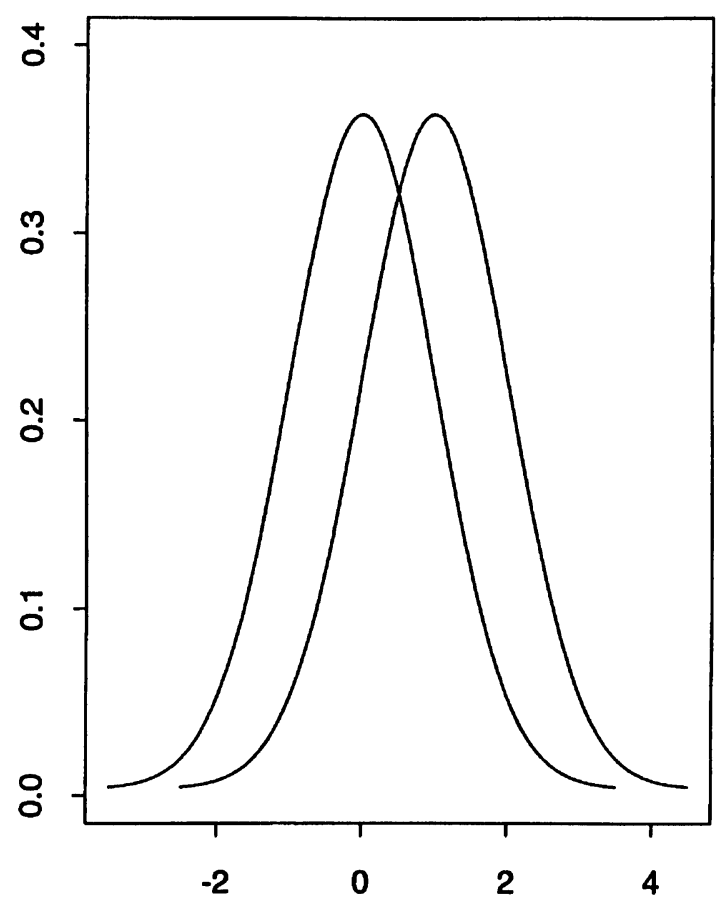

Figure 1

distributions shown in the left panel, only the curves in the right panel do not extend as far up the y-axis. Now Student's $\mathrm{t}$ has power .28 so the sample sizes are inadequate if a difference of $\mu_{1}-\mu_{2}=1$ is judged to be important. 
The distributions in the right panel of Figure 1 are from the family of mixed (or contaminated) normals. For the particular mixed normal considered here, sampling is from a standard normal distribution with probability .9, and with probability .1 an observation is sampled from a normal distribution with mean zero and standard deviation ten. The resulting distribution is very similar to a standard normal (in the Kolmogorov sense), but there is a crucial difference. The mixed normals in the right panel of Figure 1 have variances 10.9 and this is why power is relatively low. That is, a very slight departure from normality can result in very low power rendering our choice for sample sizes inadequate when conventional power analyses are made. This result follows immediately from Tukey (1960) as well as from general results on robustness summarized in Huber (1981), Staudte and Sheather (1990) and Wilcox (1997).

When attention is restricted to means, a Stein-type method of power analysis will tend to catch the error just described once observations are available. A member of this class of methods that can be used in the situation at hand was derived by Bishop and Dudewicz (1978). (Related methods derived by Hochberg, 1975, and Tamhane, 1977 , can be used to control the length of a confidence interval.) Once data are available, the Bishop-Dudewicz method indicates how many additional observations are required to achieve some specified level of power given a difference between the means that is deemed important. If few or no additional observations are required, this indicates that the original sample size was adequate. Briefly, the required sample size depends on the sample variances. Not surprisingly, the larger the sample variances, the larger the required number of observations in order to achieve high power. Because the sample variances tend to be large when sampling from the mixed normal distribution considered here, versus sampling from a normal, the BishopDudewicz method will tend to detect the fact that the original sample sizes were inadequate for the situation depicted in the right panel of Figure 1.

An obvious concern is that obtaining additional observations can be difficult. What would be nice is a method that achieves high power in both of the situations depicted in Figure 1. Methods based on a $20 \%$ trimmed mean accomplish this goal. For the normal distributions, if we apply Yuen's (1974) method for trimmed means, power is .89 (based on a simulation with 10,000 replications), and for the contaminated normals it is .78. That is, relatively little power is lost under normality versus using means, and power is not destroyed under a small departure from normality. This is one of several reasons $20 \%$ trimmed means have appeal. But if we fail to reject when comparing $20 \%$ trimmed means, again we have the issue of assessing why. That is, an estimate of power becomes important.
A natural strategy for assessing power, when using trimmed means, is to use some analog of the BishopDudewicz method. Theoretical results leading to Yuen's (1974) method (e.g., Staudte and Sheather, 1990; Wilcox, 1997) suggest an obvious analog, but we found that in simulations, control over power was unsatisfactory. Various modifications were tried, but all of them gave unsatisfactory results. There are some rather obvious bootstrap methods for estimating power (e.g., Efron \& Tibshirani, 1993). Unfortunately, the estimate can be rather inaccurate with small or even moderately large sample sizes. (Some of the many variations that were considered and found to be unsatisfactory are briefly described below.) Yet, another concern is that Yuen's method can be less satisfactory than two basic bootstrap methods for comparing trimmed means (e.g., Wilcox, 1997).

One of these is the percentile t bootstrap and another is the percentile method. It is known that when comparing means, the percentile $t$ bootstrap outperforms the percentile method (e.g., Westfall \& Young, 1993). However, for trimmed means, there is little separating the two methods when comparing two groups. But when there are more than two groups, the percentile method begins to perform better, in terms of probability coverage and Type I errors, than the percentile $t$ (Wilcox, 2001b). Moreover, results in Singh (1998) suggest how the power of both bootstrap methods might be improved. Wilcox (2001b) found that Singh's approach, when applied to the percentile method, gives reasonable control over the probability of a Type I error if the smallest sample size is at least 15. But when using the percentile $t$, Singh's method performed rather poorly. For these reasons we focus on the percentile bootstrap method.

Our goal, therefore, is to find a reasonable point estimate of power and to assess the accuracy of this estimate by computing a .95 one-sided confidence interval, the idea being that we want a conservative estimate of power. For example, if we estimate power to be .8 , and our one-sided confidence interval for the actual amount of power is $(.7,1)$, then we can be reasonably certain that power is at least 7 and this can be used to judge the sample sizes under consideration. (Of course we could compute a two-sided confidence interval for the estimated power, but the upper end of such a confidence interval seems less interesting than the lower end.)

\section{Methodology}

For two independent groups let $X_{i j}$ be a randomly sampled observation for the $j^{\text {th }}$ group, $\left(j=1,2 ; i=1, \ldots, n_{j}\right)$. The corresponding population $20 \%$ trimmed means are labeled $\mu_{t 1}$ and $\mu_{t 2}$ and the goal is to test

$$
H_{0}: \mu_{t l}=\mu_{t 2}
$$


We begin by describing the method for testing $H_{0}$ after which we turn to the problem of estimating power.

For the $j^{\text {th }}$ group let $X_{l j}^{*}, \ldots, X_{n_{j} j}^{*}$ be a bootstrap sample. That is, for each $j$, the values $X_{l j}^{*}, \ldots, X_{n_{j} j}^{*}$ are obtained by randomly sampling, with replacement, $n_{j}$ values from $X_{1 j}, \ldots, X_{n_{j} j}$. Let $p^{*}=P\left(\bar{X}_{t l}^{*}>\bar{X}_{t 2}^{*}\right)$, where for the $j^{\text {th }}$ group $\bar{X}_{t j}^{*}$ is the $20 \%$ trimmed mean based on the bootstrap sample. (See Wilcox $(1997$, p. 32) for details on how to compute a trimmed mean.) That is, $p^{*}$ is the probability that, when resampling from the empirical distribution associated with the first and second groups, a bootstrap sample trimmed mean from group 1 is greater than the bootstrap sample trimmed mean from group 2 . Notice that $p^{*}$ reflects the degree to which the empirical distributions differ. If the empirical distributions are identical, then $p^{*}$ $=.5$. Moreover, if the null hypothesis of equal trimmed means is true, then results in Hall (1986a), in combination with the influence function of the trimmed mean, imply that $p^{*}$ should have, approximately, a uniform distribution. The reason is that

$$
\bar{X}_{t}=\mu_{t}+\frac{1}{n} \sum I F\left(X_{i}\right)
$$

plus a remainder term that goes to zero as $n$ gets large, where $\operatorname{IF}\left(X_{i}\right)$ is the influence function (e.g., Staudte \& Sheather, 1990). That is, the sample trimmed mean can be written as an average of independent identically distributed random variables. That $p^{*}$ converges to a uniform distribution under the null hypothesis also follows from general results in Hall (1986b). Consequently, the closer $p^{*}$ is to 0 or 1 , the more evidence there is that the null hypothesis should be rejected. Reject $H_{0}: \mu_{t 1}=\mu_{t 2}$ at the $\alpha$ level if $p^{*} \leq \alpha / 2$ or if $p^{*} \geq 1-\alpha / 2$.

The value of $p^{*}$ can be estimated in a simple manner. For the $j^{\text {th }}$ group, obtain $B$ bootstrap trimmed means $\bar{X}_{b t j}^{*}, b=1, \ldots, B$. Let $I_{b}=1$ if $\bar{X}_{b t l}^{*}>\bar{X}_{b t 2}^{*}$, otherwise $I_{b}=0$, where $\bar{X}_{b t j}^{*}$ is the trimmed mean based on the $b^{\text {th }}$ bootstrap sample. Then an estimate of $p^{*}$ is

$$
\hat{p}^{*}=\sum_{b=1}^{B} I_{b} / B
$$

The hypothesis of equal trimmed means is rejected if $\hat{p}^{*} \leq \alpha / 2$ or $\hat{p}^{*} \leq 1-\alpha / 2$. It is readily verified that the hypothesis testing procedure just described is the percentile bootstrap method. Furthermore, the view of the percentile bootstrap just given provides a useful way of addressing power.

For convenience, let $\hat{p}_{m}^{*}=\min \left(\hat{p}^{*}, 1-\hat{p}^{*}\right)$ in which case $H_{0}$ is rejected if $\hat{p}_{m}^{*} \leq \alpha / 2$, where as usual $\alpha$ is the desired probability of a Type I error. Then power is $1-\beta=P\left(\hat{p}_{m}^{*} \leq \alpha / 2\right)$ when $H_{0}$ is false. One of our failed attempts at estimating power was to approximate the bootstrap sampling distribution of the bootstrap trimmed means with a normal distribution. The mean and variance of this distribution are easily estimated using well-known properties of the trimmed mean. But the resulting estimate of power was found to be unsatisfactory. Next we tried a Cornish-Fisher approximation of the bootstrap sampling distributions using results in Wilcox (1994). Again estimated power was unsatisfactory.

A method that was partially successful was a nested bootstrap estimate of power. This approach provided a reasonably unbiased estimate of the true power level under a shift model, but the standard error of the estimate was such that the assessed power might be inaccurate to the point of being misleading. That is, in many situations the actual amount of power is over-estimated, giving a false sense that the sample sizes used are adequate. What is needed is some way of computing confidence intervals for the actual power level, but a reasonable method for accomplishing this goal, when using the nested bootstrap, has not been found.

Now we describe the one method we have found so far that gives good results, in simulations, under a shift model. (Handling situations where distributions have unequal variances is discussed below.) As is evident, power is related to the standard errors of the trimmed means. Roughly, the strategy is to devise a function for estimating power when distributions are normal, where the estimate is based on some specified difference between the population trimmed means, say $\delta=\mu_{t l}-\mu_{t 2}$, and the standard error of $\bar{X}_{t 1}-\bar{X}_{t 2}$. Then, given data, an estimate of power is obtained simply by estimating the standard error and plugging it into the function just described. That is, we estimate $1-\beta=P\left(\hat{p}_{m}^{*} \leq \alpha / 2\right)$. To get a one-sided .95 confidence interval for the actual power level, we compute a one-sided .95 confidence interval using a percentile bootstrap in conjunction with our power function. To elaborate, temporarily consider a single random sample, $X_{1}, \ldots, X_{n}$. Let $X_{(1)} \leq X_{(2)}$ $\leq \ldots \leq X_{(n)}$ be the order statistics and let

$$
W_{i}=\left\{\begin{aligned}
X_{(g+l)}, & \text { if } X_{i} \leq X_{(g+l)} \\
X_{i}, & \text { if } X_{(g+l)}<X_{i}<X_{(n-g)} \\
X_{(n-g)}, & \text { if } X_{i} \geq X_{(n-g) .}
\end{aligned}\right.
$$

Set $g=[.2 n]$, where $[.2 n]$ is the greatest integer $\leq .2 n$. The $(20 \%)$ Winsorized sample mean is 


$$
\bar{X}_{w}=\frac{1}{n} \sum W_{i}
$$

and the sample Winsorized sum of the squared deviations is

$$
\begin{aligned}
\operatorname{SSD}_{w} & =(g+1)\left(X_{(g+l)}-\bar{X}_{w}\right)^{2}+\left(X_{(g+2)}-\bar{X}_{w}\right)^{2}+\ldots \\
& +\left(X_{(n-g-l)}-\bar{X}_{w}\right)^{2}+(g+1)\left(X_{(n-g)}-\bar{X}_{w}\right)^{2} .
\end{aligned}
$$

The (20\%) sample Winsorized variance is

$$
s_{w}^{2}=\frac{1}{n-1} \sum\left(w_{i}-\bar{X}_{w}\right)^{2} .
$$

Letting $\sigma_{\omega}^{2}$ be the population Winsorized variance, theoretical results, based on the influence function of the $20 \%$ trimmed mean, indicate that the squared standard error of the sample trimmed mean is

$$
\frac{\sigma_{w}^{2}}{.36 n}
$$

(e.g., Staudte \& Sheather, 1990). Following Yuen (1974), we estimate this squared standard error with

$$
d=\frac{S S D_{w}}{h(h-1)},
$$

where $h=n-2 g$ is the "effective" sample size. Of course one could use instead $s_{w}^{2} /(.36 n)$, but in small sample sizes $d$ has been found to perform better when testing hypotheses (Wilcox, 1997). There is no indication that the alternate estimate of the standard error provides added value for the problem at hand, so $d$ is used henceforth. Returning to the two-sample case, let

$$
\tau^{2}=\frac{\sigma_{w 1}^{2}}{.36 n_{1}}+\frac{\sigma_{w 2}^{2}}{.36 n_{2}}
$$

be the population squared standard error of $\bar{X}_{t 1}-\bar{X}_{t 2}$, where $\sigma_{w j}^{2}$ is the population Winsorized variance for the $j^{\text {th }}$ group. Our immediate goal is to find a function that determines power under normality given $\delta$ and $\tau$. We have been unsuccessful at finding an analytic function that has practical value, so we determined our required function by setting $n_{1}=n_{2}=100$ and via simulations based on 10,000 replications, we then determined the function $\gamma$ that approximates power for a wide range of $\gamma$ values corresponding to $\delta=0$ up to a $\delta$ for which power is close to one. Details about how to compute $\gamma$ are in an appendix. We then checked the accuracy of this function when sample sizes are small and equal $\left(n_{1}=n_{2}=20\right)$ and when sample sizes are unequal $\left(n_{1}=20\right.$ and $\left.n_{2}=40\right)$.

So given a $\gamma$, now we have a function for estimating power under normality and when the standard error is known. When the standard error $\tau$ is not known we simply estimate it with

$$
S=\sqrt{d_{1}+d_{2}},
$$

where $d_{j}$ is the value of $d$ for the $j^{\text {th }}$ group, and given a $\delta$ we now estimate power with $\gamma(\delta, S)$. But this estimate will not be exact, so we need a one-sided confidence interval to get a conservative estimate of power. To do this we use a percentile bootstrap method. We generate bootstrap samples from each group in the manner already described and for the $b^{\text {th }}$ bootstrap sample we let $S_{b}^{*}$ be the bootstrap estimate of the standard error which can be used to obtain a bootstrap estimate of power. This estimate is labeled $G_{b}^{*}=\gamma_{b}^{*}\left(\delta, S_{b}^{*}\right), b=1, \ldots, B$. Letting $G_{(l)}^{*} \leq \ldots \leq G_{(B)}^{*}$ be these $B$ values written in ascending order, and setting $L=[.05 n]$, we take $G_{(L)}^{*}$ as the lower end of our .95 confidence interval for the actual power, $\gamma$. We have tried both $B=800$ and $B=2,000$. Based on the simulations below, all indications are that $B=2,000$ offers no practical value over $B=$ 800 , so $B=800$ is assumed henceforth.

Results

A two-step simulation study was used to check our power estimation method for both normal and non-normal distributions. The first step was to use simulations, based on 10,000 replications, to estimate the actual power for four types of distributions: Normal, symmetric with heavy tails, asymmetric with relatively light tails, and asymmetric with relatively heavy tails. That is, we chose a set of $\delta$ values so that the true power, $\gamma$, would have a reasonable range of values between 0 and 1 . (The actual values for $\gamma$ will be described momentarily.)

Given $n_{1}$ and $n_{2}$, we generated observations for both groups and increased the values in the second group by $\delta$. Then for each replication we rejected the hypothesis of equal trimmed means if $\hat{p}_{m}^{*} \leq \alpha / 2$, and $\gamma$ was estimated with the proportion of times $H_{0}$ was rejected.

In the second step, we ran another simulation where power is estimated with our proposed method. That is, for each replication we computed $S$ which then is used to obtain a point estimate of $\gamma$, then we used our bootstrap method to compute a .95 confidence interval for $\gamma$, and the actual probability coverage was estimated with the proportion of confidence intervals containing the value of $\gamma$ determined in step 1 . The nominal probability coverage was set at .95 , so the intended probability of not containing the true power is $\alpha=.05$. That is, we estimate $\alpha$ with $\hat{\alpha}$, the proportion of intervals not containing the true power. 
In our simulations, observations were generated from $g$-and- $h$ distributions which includes normal distributions as a special case. If $Z$ is a standard normal random variable, then an observation, $X$, from the $g$-and- $h$ distribution is given by

$$
X=\left(\frac{e^{g z}-1}{g}\right) e^{h Z^{2} / 2}
$$

When $g=0$, this last expression is taken to be

$X=\mathrm{Ze}^{\mathrm{hZ}^{2} / 2}$. The case $g=h=0$ corresponds to a standard normal random variable. With $g=0, X$ has a symmetric distribution with increasingly heavier tails as $h$ gets large. As $g$ increases from 0 , the distribution becomes more skewed. Hoaglin (1985) gave a detailed description of the $\mathrm{g}$-and-h distribution. (For some additional properties, see Wilcox, 1997.) Table 1 lists the skewness $\left(k_{1}\right)$ and kurtosis $\left(k_{2}\right)$ for the four distributions considered here. When $h>1 /$ $k$ and $g>0, E(X-\mu)^{k}$ is not defined and the corresponding entry in Table 1 is left blank. It might be argued that $g=h=1$ is an unrealistic departure from normality, but one of our goals is to determine how our method performs under seemingly extreme conditions.

Table 1: Some properties of the g-and-h distribution.

\begin{tabular}{|c|c|c|c|}
\hline$g$ & $h$ & $\kappa_{1}$ & $\kappa_{2}$ \\
\hline 0.0 & 0.0 & 0.00 & 3.00 \\
\hline 0.0 & 1.0 & 0.00 & - \\
\hline 1.0 & 0.0 & 6.18 & 113.9 \\
\hline 1.0 & 1.0 & - & - \\
\hline
\end{tabular}

Table 2 contains $\hat{\alpha}$ values (estimated one-sided probability coverage for $\gamma$ ) for $n_{1}=n_{2}=20$. Simulations were conducted with $n_{1}=20$ and $n_{2}=40$; similar results were obtained with other sample sizes and are not reported. Next, we ran simulations with $n_{1}=40$ and $n_{2}=20$, but the second group has a standard deviation four times as large as the first group. For normal distributions the results were:

\begin{tabular}{|c|c|}
\hline$\delta:$ & $\begin{array}{lllll}1.0 & 1.4 & 2.0 & 2.4 & 3.0\end{array}$ \\
\hline$\hat{\alpha}:$ & 045.045.030.038. 045. \\
\hline$\gamma:$ & .189.314.539.685 .859 \\
\hline
\end{tabular}

Similar results were obtained when sampling from a symmetric heavy-tailed distribution, but unsatisfactory results were obtained when sampling from the two skewed distributions considered here. More precisely, the $\hat{\alpha}$ values now exceed .1. Setting $n_{1}=n_{2}=40$ does not correct this problem. That is, our proposed method now overestimates power. So in practical terms, if our technique indicates that power is low for given values of $\delta, n_{1}$ and $n_{2}$, all indications are that this is indeed the case. If the estimated power is judged to be sufficiently high, our simulations indicate that this will be the case for a shift model (where distributions differ in location only), or situations where distributions are symmetric. So we have some perspective on whether the sample sizes are sufficiently large. But for skewed distributions having unequal variances, the actual power might be less than what is indicated. So progress has been made for some important special cases, but more needs to be done.

Table 2: Estimates of $\alpha, n_{1}=n_{2}=20$.

\begin{tabular}{llllll}
\hline & \multicolumn{5}{c}{$g=h=0$} \\
$\delta:$ & 0.2 & 0.4 & 0.6 & 0.8 & 1.0 \\
$\hat{\alpha}:$ & .022 & .015 & .011 & .018 & .015 \\
$\gamma:$ & .101 & .257 & .506 & .741 & .904 \\
& & & & & \\
& & \multicolumn{2}{c}{$g=1, h=0$} & & \\
$\delta:$ & 0.4 & 0.6 & 0.8 & 1.0 & 1.2 \\
$\hat{\alpha}:$ & .022 & .027 & .039 & .051 & .071 \\
$\gamma:$ & .208 & .382 & .571 & .734 & .842 \\
& & & & & \\
& & \multicolumn{2}{c}{$g=0, h=1$} & & \\
$\delta:$ & 0.6 & 1.0 & 1.4 & 1.8 & 2.2 \\
$\hat{\alpha}:$ & .014 & .018 & .031 & .043 & .077 \\
$\gamma:$ & .199 & .433 & .652 & .796 & .884 \\
& & & & & \\
& & & & & \\
$\delta:$ & 0.8 & 1.2 & 1.6 & 2.0 & 2.8 \\
$\hat{\alpha}:$ & .014 & .025 & .039 & .065 & .120 \\
$\gamma:$ & .265 & .445 & .601 & .703 & .827 \\
& & & & &
\end{tabular}

Note: $\delta=\mu_{t 1}-\mu_{t 2}, \hat{\alpha}:$ estimate of $\alpha, \gamma:$ actual power being estimated.

An Illustration

We illustrate our method with data from a reading study. (The data were generously supplied by Frank Manis, Department of Psychology, University of Southern California.) For one of the measures studied, theoretical arguments suggest that two particular groups 
should differ, but no significant difference was found using the percentile bootstrap method described previously. (Non-significant results were obtained with Student's $t$ as well.) Figure 2 shows an estimate of the power curve for these data. (The $S$-PLUS functions used to create this plot are available from the first author upon request.) The upper solid line is the estimated power and the lower dashed line marks the one-sided confidence interval. The estimate is that a difference between the trimmed means of $\mu_{t 1}-\mu_{t 2}=600$ corresponds to power equal to .8, approximately, and the confidence interval indicates that power could be as low as .6. So in this particular case all indications are that power is inadequate except for a very large difference between the trimmed means. That is, the empirical results do not provide a compelling argument that the theory is wrong because if the groups differ by a substantial amount, there is a low probability of detecting this based on the sample sizes used.

\section{Conclusion}

The main result in this paper is a method for detecting situations where power is too low. Our method of estimating power provides perspective regarding a shift model regardless of whether the distributions differ in scale. Moreover, all indications are that a reasonably accurate estimate of power can be had when distributions differ in scale provided they are symmetric. But more needs to be done. In particular, an accurate confidence interval for power is needed when distributions are skewed and have unequal variances. Another goal of possible interest is a .95 confidence band for the estimated power curve. That is, rather than compute a .95 confidence interval for each $\delta$ of interest, compute a confidence band where for all $\delta$ values, the simultaneous probability coverage is .95 .

\section{References}

Bishop, T., \& Dudewicz, E. (1978). Exact analysis of variance with unequal variances. Test procedures and tables. Technometrics, 20, 419-420.

Cohen, J. (1977). Statistical power analysis for the behavioral sciences. New York: Academic Press.

Efron, B., \& Tibshirani, R. J. (1993). An Introduction to the Bootstrap. New York: Chapman \& Hall.

Hall, P. (1986a). On the number of bootstrap simulations required to construct a confidence interval. Annals of Statistics, 14, 1453-1462.

Hall, P. (1986b). On the bootstrap and confidence intervals. Annals of Statistics, 14, 1431-1452.

Hewett, J. E., \& Spurrier, J. D. (1983). A survey of two stage tests of hypotheses: Theory and application. Communications in Statistics-Theory and Methods, 12, 2307-2425.

Hoaglin, D. C. (1985) Summarizing shape numerically: The g-and-h distributions. In D. Hoaglin, F. Mosteller and J. Tukey (Eds.), Exploring data tables, trends, and shapes. 461-515. New York: Wiley.

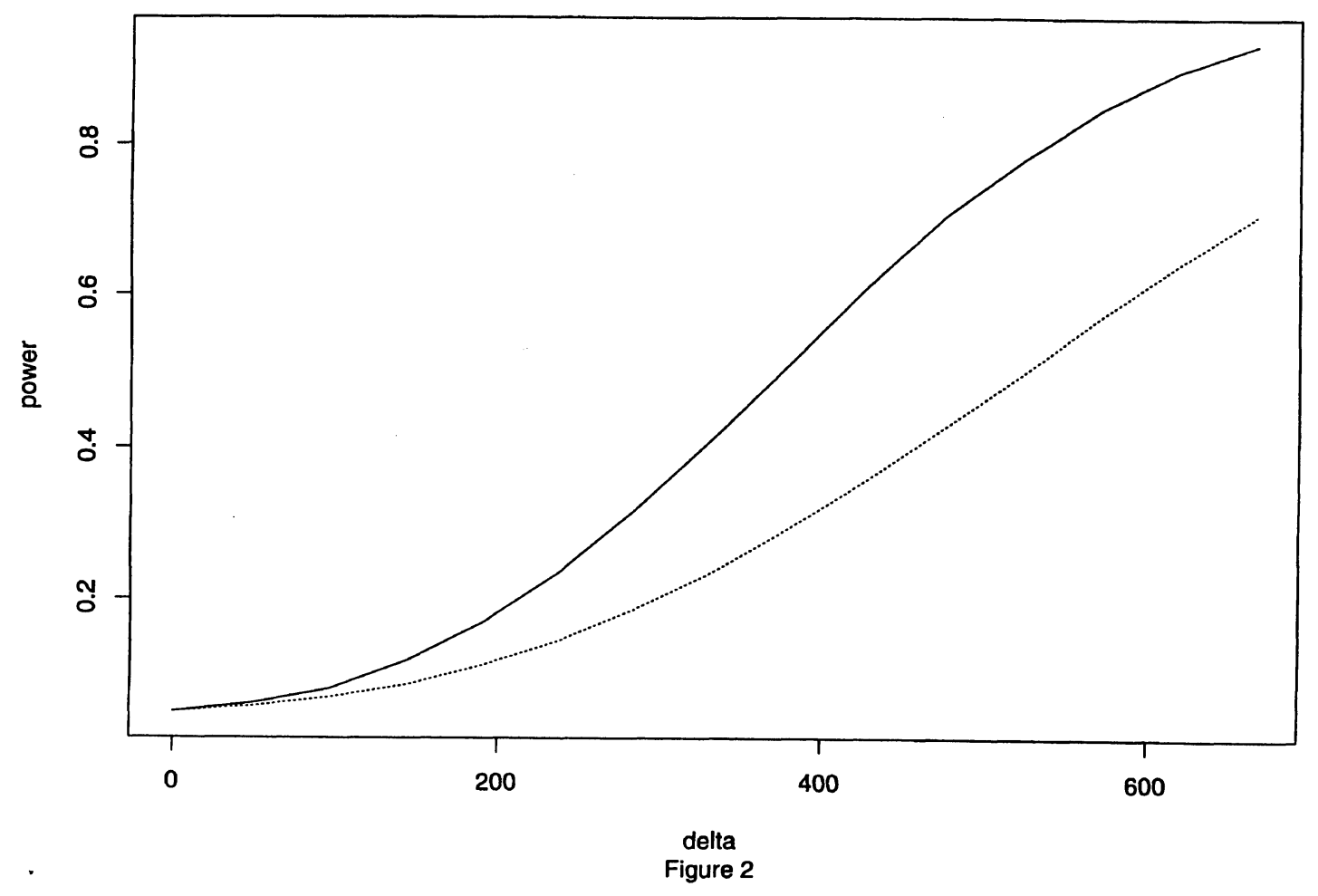


Hochberg, Y. (1975). Simultaneous inference under Behrens-Fisher conditions: A two sample approach. Communications in Statistics, 4, 1109-1119.

Huber, P. (1981). Robust statistics. New York: Wiley.

Hutson, A. D., \& Ernst, M. D. (2000). The exact bootstrap mean and variance of an L-estimator. Journal of the Royal Statistical Society, 62, 89-94.

Keselman, H. J., Huberty, C. J., Lix, L. M., Olejnik, S., Cribbie, R. A., Donohue, B., Kowalchuk, R. K., Lowman, L. L., Petosky, M. D., Keselman, J. C., \& Levin, J. R. (1998). Statistical practices of educational researchers: An analysis of their ANOVA, MANOVA, and ANCOVA analyses. Review of Educational Research, 68, 350-386.

Singh, K. (1998). Breakdown theory for bootstrap quantiles. Annals of Statistics, 26, 1719-1732.

Staudte, R. G., \& Sheather, S. J. (1990) Robust estimation and testing. New York: Wiley.

Stein, C. (1945). A two-sample test for a linear hypothesis whose power is independent of the variance. Annals of Statistics, 16, 243-258.

Tamhane, A. (1977). Multiple comparisons in model I one-way ANOVA with unequal variances. Communications in Statistics, A6, 15-32.
Tukey, J. W. (1960). A survey of sampling from contaminated normal distributions. In I. Olkin, et al. (Eds.), Contributions to probability and statistics. Stanford, CA: Stanford University Press.

Westfall, P. H., \& Young, S. S. (1993). Resampling based multiple testing. New York: Wiley.

Wilcox, R. R. (1994). Some results on the TukeyMcLaughlin and Yuen methods for trimmed means when distributions are skewed. Biometrical Journal, 36, 259273.

Wilcox, R. R. (1996). Statistics for the social sciences. San Diego: Academic Press.

Wilcox, R. R. (1997). Introduction to robust estimation and hypothesis testing. San Diego, CA: Academic Press.

Wilcox, R. R. (2001a). Fundamentals of modern statistical methods. New York: Springer.

Wilcox, R. R. (2001b). Pairwise comparisons of trimmed means for two or more groups. Psychometrika, $66,421-444$.

Yuen, K. K. (1974). The two-sample trimmed $t$ for unequal population variances. Biometrika, 61, 165170.

\section{Appendix}

The construction of our function for estimating power began by determining power corresponding to multiples of the standard error of the difference between the trimmed means. We considered 36 multiples of the standard error, beginning with zero (in which case $\mu_{t 1}-\mu_{t 2}=0$ ) and ending with 4.375 (meaning that the difference between the trimmed means is 4.375 standard errors). These 36 multiples of the standard error are given by $y_{\mathrm{i}}=(i-1) / 8, i=1$, ..., 36. So, for example, $y_{36}=4.375$ is the largest difference between the trimmed means we considered in standard errors. Let $h_{i}(i=1, \ldots, 36)$ be given by $500,540,607,706$, $804,981,1176,1402,1681,2008,2353,2769,3191,3646$, $4124,4617,5101,5630,6117,6602,7058,7459,7812$, $8150,8479,8743,8984,9168,9332,9490,9607,9700$, 9782, 9839, 9868 .

For example, suppose $h_{1}=500$ and $h_{3}=706$. The value $h_{\mathrm{i}}$, divided by 10,000 , is the power corresponding to $\mu_{t 1}-\mu_{t 2}=y_{i} \sqrt{2 * .01155}$ when $n_{1}=n_{2}=100$ and sampling is from a standard normal distribution. The constant .01155 is approximately equal to the squared standard error of the $20 \%$ trimmed mean. That is, $h_{i} / 10000$ gives power as a multiple of the standard error of $\bar{X}_{t 1}-\bar{X}_{t 2}$. Thus, for any two distributions, given $\delta$ and $S$, an estimate of the standard error of $\bar{X}_{t 1}-\bar{X}_{t 2}$ given by equation 2, power is estimated as follows: $v=[8 * \delta / S]+1$, where [.] is the greatest integer function, and let $d=8 *\left(\frac{\delta}{S}-\frac{v-1}{8}\right)$.Then, the estimated power is taken to be $\hat{\gamma}=\frac{h_{v}}{10000}+d *\left(\frac{h_{v+1}}{10000}-\frac{h_{v}}{10000}\right)$. In the event $v=36$, $h_{v+1}$ is taken to be 10000 in the previous equation. If $v>$ $36, \hat{\gamma}=1$.

Note: The research reported in this article was supported, in part, by a grant from the Natural Sciences and Engineering Research Council of Canada. 
Rank-Based Procedures for Mixed Paired and Two-Sample Designs

\author{
R. Clifford Blair \\ Department of Epidemiology and Biostatistics \\ College of Public Health, \& \\ Jaeb Center For Health Research \\ University Of South Florida
}

\author{
Thomas P. Hettmansperger \\ Department of Statistics \\ The Pennsylvania State University
}

This paper presents a rank-based procedure for parameter estimation and hypothesis testing when the data are a mixture of paired observations and independent samples. Such a situation may arise when comparing two treatments. When both treatments can be applied to a subject, paired data will be generated. When it is not possible to apply both treatments, the subject will be randomly assigned to one of the treatment groups. Our rank-based procedure allows us to use the data from the paired sample and the independent samples to make inferences about the difference in the mean responses. The rank-based procedure uses both types of data by combining the Wilcoxon signed-rank statistic and the Wilcoxon-Mann-Whitney statistic. The exact and asymptotic distributions of the test statistic under the null hypothesis are determined as well as the limiting distribution of the point estimate. We also consider the Pitman efficacy of our rank-based procedure and its efficiency with respect to mean-based procedures.

Keywords: Wilcoxon signed-rank statistic, Wilcoxon-Mann-Whitney statistic, Pitman efficacy.

\section{Introduction}

The purpose of this paper is to introduce a simple robust, nonparametric approach to testing and estimation in mixed paired and two sample designs. The new methods are more robust and have excellent efficiency when compared to more traditional methods based on the means. In addition, they are easy to compute and apply to data. Mixtures of paired and unpaired data may be realized in a variety of research contexts. We consider measurement data in this article. For a discussion of categorical data see Thompson (1995) and references therein.

For example, in a clinical trial designed to compare two methods of laser surgery that are used to correct a certain eye condition, patients with both eyes eligible for study may have one eye assigned to one surgical method, but the other treated with the competing method. Visual acuity or other measures taken on the treated eyes will likely

Suzanne R. Dubinicka is a Post-doctoral Fellow. This article was submitted while at the Department of Mathematical Sciences, Indiana University - Purdue University at Fort Wayne. Her research interests include nonparametric methods, survival analysis, measurement error, and permutation tests. R. Clifford is Professor, Department of Epidemiology and Biostatistics, University of South Florida. His research was partially supported by Jaeb Center for Health Research. Thomas P. Hettmansperger is Professor, Department of Statistics, The Pennsylvania State University. be correlated, thereby producing paired data. However, patients who have only one eye eligible for study will have the one eye randomly assigned to one of the competing treatments. This produces unpaired observations.

Other examples include designs in which subjects are to be observed at two points in time with some intervention between observations. Often in such situations, data may be missing at either the first or second observation times. Subjects with no missing data will thereby produce paired sets, while those with a missing data point will be unpaired. A special case of this example arises when pairwise comparisons are performed in repeated measures designs with missing observations.

Finally, in matched pair designs, some subjects in the pool may not have suitable matches. In such situations, the unmatched subjects can simply be randomly assigned to treatments, thereby taking advantage of all eligible study subjects.

As will be noted in one of the example analyses presented below, researchers are often uncertain as to how such data might be efficiently analyzed. Unfortunately, strategies wasteful of available information are often employed. When the underlying distributions are normal, there is a sufficient but not complete statistic. In this case, the maximum likelihood estimators may not behave very well and they are quite complex computationally. Consequently, we do not attempt to develop optimal methods. Instead, we suggest nonparametric methods that work well in a broad spectrum of models. Bhoj (1989) presented a 
thorough summary of tests based on means when the underlying distributions are normal. He proposed two new tests and compared various methods via Monte Carlo simulations. Perhaps the easiest version of such a test was proposed by Morrison (1973). See Ekbohm (1976, 1981); Hamdan, Khuri, and Crews (1978); and Woolson, Leeper, Cole and Clarke (1976) for additional early work based on means.

\section{Simple Rank Methods}

We consider the situation in which observations come from a bivariate distribution $f(x, y)$ with marginal densities $f_{I}(x)$ and $f_{2}(y)$; means $\theta_{I}$ and $\theta_{2}$; variances $\sigma_{I}^{2}$ and $\sigma_{2}^{2}$; and correlation $\rho$. We will assume that the marginal variances are equal and call this common variance $\sigma^{2}$. In addition, we will assume that the marginal densities of $X$ and $Y$ have the same shape and differ by at most a shift in location. We are concerned with making inferences about the difference in marginal means, $\Delta=\theta_{1}-\theta_{2}$. In particular, we will be interested in comparing a new treatment with an old treatment or with a control.

Paired data will be generated when the new and old treatments can be applied to each of the subjects. When complete pairs are available, we collect $\left(X_{1}, Y_{1}\right), \ldots,\left(X_{n}, Y_{n}\right)$. Let $D_{i}=X_{i}-Y_{i}, i=1, \ldots, n$. The $D_{i}$ have a probability density function $f_{d}$ with $E\left(D_{i}\right)=\Delta$ and $\operatorname{Var}\left(D_{i}\right)=2 \sigma^{2}(1-\rho)$.

Complete pairs may not always be available. In some situations, it will only be possible to apply one of the treatments to a subject. In this case, the treatments will be randomly assigned to the subjects, and two independent samples will be generated. We denote these samples of sizes $n_{1}$ and $n_{2}$ as $T_{1}, \ldots, T_{n_{1}}$ and $C_{1}, \ldots, C_{n_{2}}$, where the $T_{i}$ have density $f_{I}\left(t-\theta_{l}\right), E\left(T_{i}\right)=\theta_{l}, \operatorname{Var}\left(T_{i}\right)=\sigma^{2}$, and the $C_{j}$ have density $f_{2}\left(c-\theta_{2}\right), E\left(C_{j}\right)=\theta_{2}, \operatorname{Var}\left(C_{j}\right)=\sigma^{2}$.

Combining the paired data and the independent samples, we would like to make inferences about the parameter $\Delta$. In particular, we would like to determine if the new treatment is better than the old treatment, and to estimate the difference between the treatments. Thus, we construct a test $H_{o}: \Delta=0$ vs $H_{a}: \Delta>0$, an estimate of $\Delta$, and a confidence interval for $\Delta$.

An intuitively appealing statistic is the sum of the usual rank statistics for paired data, the Wilcoxon signedrank statistic, and for two independent samples, the Wilcoxon-Mann-Whitney statistic. Let

$$
\begin{aligned}
T^{+}(\Delta) & =S^{+}(\Delta)+U^{+}(\Delta) \\
& =\sum_{i \leq j} \sum I\left(\frac{D_{i}+D_{j}}{2}>\Delta\right)+\sum_{i=1}^{n_{1}} \sum_{j=1}^{n_{2}} I\left(T_{i}-C_{j}>\Delta\right)
\end{aligned}
$$

$$
=\sum_{i=1}^{n} R\left(\left|D_{i}\right|\right) I\left(D_{i}>0\right)+\sum_{j=1}^{n_{I}} R_{j}-\frac{n_{l}\left(n_{l}+1\right)}{2}
$$

where $I(A)=1$ if event $A$ occurs and $=0$ otherwise, $R\left(\left|D_{i}\right|\right)$ is the rank of $\left|D_{i}\right|$ among $\left|D_{I}\right|, \ldots,\left|D_{n},\right|$ and $R_{j}$ is the rank of $T_{j}$ in the combined independent samples.

\section{Distribution Theory}

From Hettmansperger and McKean (1998, Section $1.7 \& 2.4)$ it follows immediately that, under $H_{o}: \Delta=0$,

$$
\begin{aligned}
& E_{0} T^{+}(0)=\frac{n(n+1)}{4}+\frac{n_{1} n_{2}}{2} \\
& \operatorname{Var}_{0} T^{+}(0)=\frac{n(n+1)(2 n+1)}{24}+\frac{n_{1} n_{2}\left(n_{1}+n_{2}+1\right)}{12} .
\end{aligned}
$$

Theorem 1

Under $H_{0}: \Delta=0$, as $M \rightarrow \infty$,

$$
\frac{T^{+}(0)-E_{0} T^{+}(0)}{\sqrt{\operatorname{Var}_{0} T^{+}(0)}} \stackrel{D}{\rightarrow} Z \sim N(0,1)
$$

where

$$
\frac{n}{m} \rightarrow \gamma, \quad \frac{n_{1}+n_{2}}{m} \rightarrow 1-\gamma, \quad \frac{n_{1}}{n_{1}+n_{2}} \rightarrow \lambda, \quad \frac{n_{2}}{n_{1}+n_{2}} \rightarrow \lambda, \quad \text { and }
$$

$M=n+n_{1}+n_{2}, 0<\gamma<1$, and $0<\lambda<1$.

Thus, an asymptotic size $\alpha$ test will reject $H_{o}: \Delta=0$ in favor of $H_{a}: \Delta>0$ if

$$
\frac{T^{+}(0)-E_{0} T^{+}(0)-\frac{1}{2}}{\sqrt{\operatorname{Var}_{0} T^{+}(0)}} \geq z_{\alpha}
$$

where $Z_{\alpha}$ is the upper $\alpha$ quantile of the standard normal distribution.

A convolution can be used to compute the exact p-value. From the independence of $S^{+}(0)$ and $U^{+}(0)$,

$$
\begin{aligned}
P\left(T^{+} \geq t\right) & =\sum_{s} P\left(U^{+} \geq t-s\right) P\left(S^{+}=s\right) \\
& =\sum_{u} P\left(S^{+} \geq t-u\right) P\left(U^{+}=u\right)
\end{aligned}
$$

Hence, tables of probabilities and tail probabilities can be used to compute $P\left(T^{+} \geq t\right)$. The exact distribution of $T^{+}$ can be constructed recursively as well.

Define $\overline{\mathrm{P}}_{\mathrm{n}_{1}, \mathrm{n}_{2}}(\mathrm{k})$ as the number of data configurations such that $T^{+}=k$. Because there are $2^{n}\left(\frac{n_{1}+n_{2}}{n_{1}}\right)$ such configurations, 


$$
P\left(T^{+}(0)=k\right)=\frac{\bar{P}_{n, n_{1}, n_{2}}(k)}{2^{n}\left(\begin{array}{c}
n_{1}+n_{2} \\
n_{l}
\end{array}\right)}
$$

where $k=0,1, \ldots,[n(n+1)] / 2+n_{1} n_{2}$. Note that $T^{+}$is distribution-free. A recursive formula can be used to compute $\bar{P}_{n, n_{l}, n_{2}}(k)$.

Theorem 2

$$
\bar{P}_{n, n_{1}, n_{2}}(k)=\bar{P}_{n, n_{1}-1, n_{2}}\left(k-n_{2}\right)+\bar{P}_{n, n_{1}, n_{2}-1}(k)
$$

where $\bar{P}_{h, i, j}(k)=0$ or 1 as $k<0$ or $=0$, and

$$
\bar{P}_{h, i, 0}(k)=\bar{P}_{h, 0, j}(k)=\bar{P}_{h}(k)=\bar{P}_{h-I}(k-h)+\bar{P}_{h-1}(k)
$$

with $\bar{P}_{h}(k)=0$ if $k<0$ and $\bar{P}_{0}(k)=1$ or 0 as $k=0$ or $\neq 0$.

This recursive formula can be used to construct an exact test for our problem. The exact test will reject $H_{o}: \Delta=0$ in favor of $H_{a}: \Delta>0$ if $T^{+}(0) \geq c$, where $c$ is chosen as $P\left(T^{+}(0) \geq c\right) \doteq \alpha$. S-plus functions and Fortran programs have been written to compute the p-value for this test, as well as construct upper tail probabilities for the distribution of $T^{+}$for given $n, n_{1}$, and $n_{2}$. Table 1 was constructed using the $S$-plus functions. In this table, we have assumed that $n_{1}=n_{2}$ and computed selected upper quantiles for various values of $n$ and $n_{1}$. Because $T^{+}$is discrete, it is not usually possible to obtain the exact quantile desired. The values in the table give the values closest to the upper $5 \%, 2.5 \%$, and $1 \%$ quantiles.

We can also compute these upper quantiles using the normal approximations. For example, when $n=n_{1}=$ $n_{2}=5, P\left(T^{+} \geq 30\right) \doteq 0.058$ using the normal approximations in (4). Compare this to 0.059 in Table 1 using the exact distribution. For the same sample sizes, $P\left(T^{+} \geq 32\right) \doteq$ 0.029 and $P\left(T^{+} \geq 34\right) \doteq 0.013$. For larger sample sizes, the approximations are even better. For $n=n_{1}=n_{2}=10$, $P\left(T^{+} \geq 105\right) \doteq 0.051, P\left(T^{+} \geq 110\right) \doteq 0.026$, and $P\left(T^{+} \geq 116\right) \doteq 0.011$ using the normal approximation. Notice the first two agree with exact probabilities to three decimal places and the third differs from the exact probability by only 0.001 . We conclude that for most practical situations the normal approximation is adequate.

\section{Estimation}

To estimate $\Delta$, we consider the following form of our estimating function:

$$
T(\Delta)=\sum_{i \leq j} \sum \operatorname{sgn}\left(\frac{D_{i}+D_{j}}{2}-\Delta\right)+\sum_{i=1}^{n_{1}} \sum_{j=1}^{n_{2}} \operatorname{sgn}\left(T_{i}-C_{j}-\Delta\right) .
$$

where $\operatorname{sgn}(x)=1$ if $x>0,=0$ if $x=0$, and $=-1$ if $x<0$. Notice that $T(\Delta)=2 T^{+}(\Delta)-\left[n(n+1) / 2+n_{1} n_{2}\right]$ and $E_{\Delta} T(\Delta)=0$. We estimate $\Delta$ by $\hat{\Delta}_{\mathrm{R}}$ so that $T\left(\hat{\Delta}_{\mathrm{R}}\right)=0$. We find

$$
\hat{\Delta}_{\mathrm{R}}=\operatorname{med}\left\{\frac{D_{i}+D_{j}}{2}, T_{k}-C_{l} ; 1 \leq i \leq j \leq n, 1 \leq k \leq n_{l}, 1 \leq l \leq n_{2}\right\} \text {. }
$$

Theorem 3

Under $H_{o}: \Delta=0$, as $M \rightarrow \infty$ provided $0<\int f_{d}^{2}<\infty$

Table 1: Upper Quantiles for the Distribution of $T^{+}:\left(P_{t}, t\right)$ where $P_{t}=P\left(T^{+} \geq t\right)$

\begin{tabular}{c|cccccc} 
& \multicolumn{6}{c}{$n_{1}=n_{2}$} \\
$\mathrm{n}$ & 5 & 6 & 7 & 8 & 9 & 10 \\
\hline \multirow{5}{*}{5} & $(0.059,30)$ & $(0.050,38)$ & $(0.047,47)$ & $(0.048,57)$ & $(0.051,68)$ & $(0.047,81)$ \\
& $(0.028,32)$ & $(0.026,40)$ & $(0.028,49)$ & $(0.024,60)$ & $(0.023,72)$ & $(0.024,85)$ \\
& $(0.011,34)$ & $(0.012,42)$ & $(0.011,52)$ & $(0.011,63)$ & $(0.012,83)$ & $(0.011,89)$ \\
\hline \multirow{4}{*}{6} & $(0.044,35)$ & $(0.049,42)$ & $(0.046,51)$ & $(0.046,61)$ & $(0.048,72)$ & $(0.051,84)$ \\
& $(0.022,37)$ & $(0.027,44)$ & $(0.027,53)$ & $(0.023,64)$ & $(0.027,75)$ & $(0.027,88)$ \\
& $(0.014,38)$ & $(0.010,47)$ & $(0.011,56)$ & $(0.011,67)$ & $(0.009,80)$ & $(0.010,93)$ \\
\hline \multirow{4}{7}{7} & $(0.044,40)$ & $(0.046,47)$ & $(0.052,55)$ & $(0.050,65)$ & $(0.050,76)$ & $(0.053,88)$ \\
& $(0.023,42)$ & $(0.027,49)$ & $(0.026,58)$ & $(0.027,68)$ & $(0.025,80)$ & $(0.024,93)$ \\
& $(0.011,44)$ & $(0.010,52)$ & $(0.011,61)$ & $(0.010,72)$ & $(0.011,84)$ & $(0.009,98)$ \\
\hline \multirow{4}{*}{8} & $(0.052,45)$ & $(0.052,52)$ & $(0.055,60)$ & $(0.051,70)$ & $(0.050,81)$ & $(0.052,93)$ \\
& $(0.023,48)$ & $(0.025,55)$ & $(0.023,64)$ & $(0.023,74)$ & $(0.025,85)$ & $(0.024,98)$ \\
& $(0.012,50)$ & $(0.010,58)$ & $(0.011,67)$ & $(0.009,78)$ & $(0.009,90)$ & $(0.010,103)$ \\
\hline \multirow{4}{*}{9} & $(0.045,52)$ & $(0.053,58)$ & $(0.055,66)$ & $(0.050,76)$ & $(0.048,87)$ & $(0.049,99)$ \\
& $(0.027,54)$ & $(0.028,61)$ & $(0.025,70)$ & $(0.024,80)$ & $(0.025,91)$ & $(0.024,104)$ \\
& $(0.008,58)$ & $(0.010,65)$ & $(0.009,74)$ & $(0.010,84)$ & $(0.010,96)$ & $(0.010,109)$ \\
\hline \multirow{3}{*}{10} & $(0.045,59)$ & $(0.052,65)$ & $(0.052,73)$ & $(0.046,83)$ & $(0.051,93)$ & $(0.051,105)$ \\
& $(0.023,62)$ & $(0.023,69)$ & $(0.025,77)$ & $(0.023,87)$ & $(0.024,98)$ & $(0.026,110)$ \\
& $(0.011,65)$ & $(0.011,72)$ & $(0.010,81)$ & $(0.011,91)$ & $(0.010,103)$ & $(0.010,116)$ \\
\hline
\end{tabular}


and $0<\int f_{1}^{2}<\infty$, and the additional sample size limits in Theorem 1 are satisfied,

$$
\sqrt{M}\left(\hat{\Delta}_{R}-\Delta \stackrel{D}{\rightarrow} Z \sim N\left(0, \frac{1}{c_{T^{+}}^{2}}\right)\right.
$$

where

$$
c_{T^{+}}=\sqrt{12} \frac{\gamma^{2} \int f_{d}^{2}+\lambda(1-\lambda)(1-\gamma)^{2} \int f_{1}^{2}}{\sqrt{\gamma^{3}+\lambda(1-\lambda)(1-\gamma)^{3}}}
$$

is the Pitman efficiency of $T^{+}$. See (26) in the appendix.

A Confidence Interval and the Standard Error of $\hat{\Delta}$

To construct a confidence interval for $\Delta$, let $Y_{(1)} \leq \ldots \leq Y_{\left(N^{*}\right)}$ be the ordered values of the combined Walsh averages, $\left(D_{i}+D_{j}\right) / 2$, and pairwise differences, $T_{k}-C_{l}$, where $N^{*}=n(n+1) / 2+n_{1} n_{2}$. Then $\left[\mathrm{Y}_{(\mathrm{k}+1)}, \mathrm{Y}_{\left(\mathrm{N}^{*}-\mathrm{k}\right)}\right)$ is a $(1-\alpha) 100 \%$ confidence interval for $\Delta$ where $P\left(T^{+} \leq k\right)=P\left(T^{+} \geq N^{*}-k\right)=\alpha / 2$. Using a normal approximation with continuity correction

$k \doteq\left(\frac{n(n+1)}{4}+\frac{n_{1} n_{2}}{2}\right)-\frac{1}{2}-z_{\frac{\alpha}{2}} \sqrt{\frac{n(n+1)(2 n+1)}{24}+\frac{n_{1} n_{2}\left(n_{1}+n_{2}+1\right)}{12}}$.

This confidence interval can be used to estimate the asymptotic variance of $\hat{\Delta}_{R}$. Note that

$$
\frac{\sqrt{M}\left[Y_{\left(N^{*}-k\right)}-Y_{(k+1)}\right]}{2 z_{\alpha / 2}} P \frac{1}{\rightarrow} .
$$

See Hettmansperger and McKean (1998, Section 1.5 \& 2.4). Hence, we say $\hat{\Delta}_{R}$ is approximately normally distributed with mean $\Delta$ and variance $M^{-1} \hat{c}_{T^{+}}^{-1}$, where $\hat{c}_{T^{+}}^{-1}$ is defined by (9). In practice we would choose $\alpha$ around 0.10 .

Example 1

The Krypton Argon Regression of Neovascularization Study (KARNS) [The Krypton Argon Regression of Neovascularization Research Study Group (1993)] was designed to compare the efficacy of red krypton versus blue-green argon laser photocoagualation for the management of high-risk proliferative diabetic retinopathy. To this end a randomized prospective clinical trial was performed on patients with diabetes and neovasculariztion of the optic disc. Patients were randomized in the following manner: Patients with both eyes eligible for study had the right eye randomly assigned to one of the treatments, with the left eye then being assigned to the competing treatment. Patients who had only one eye eligible for study had that eye randomly assigned to one of the two treatments. As a result of this assignment scheme, part of the outcome data were paired and part were unpaired.

A variety of outcomes were measured 3 months after surgery. One outcome of interest was visual acuity as measured by the number of letters correctly read from the Early Treatment Diabetic Retinopathy Visual Acuity Chart. As noted by the authors, these measures had a substantial negative skew. Although the KARNS authors do not indicate exactly how these acuity scores were analyzed, they do note for other outcomes that paired and unpaired data were analyzed separately, thereby complicating the assessment of a possible treatment effect.

We have selected a subset of the data from this study to illustrate the rank-based methods of this paper; see Table 2. For this example, we have $n=20$ pairs of visual acuity measurements for those patients with both eyes eligible for study. In addition, we selected 20 patients with one eye eligible for study, with $n_{1}=10$ in the group receiving blue-green argon laser photocoagulation and $\mathrm{n}_{2}=10$ in the group receiving red krypton. The rank based estimate for the difference in mean visual acuities for the two groups is the median of the combined 310 Walsh averages and pairwise differences: $\hat{\Delta}_{R}=4.00$. In addition, to compute a $95 \%$ confidence interval for $\Delta_{R}$, we need to find $\mathrm{k}$ such that $P\left(T^{+} \leq k\right)=P\left(T^{+} \geq 310-k\right)=0.025$. Using the exact distribution for $T^{+}$, $P\left(T^{+} \leq 96\right)=P\left(T^{+} \geq 214\right)=0.0246$. Thus, a $95 \%$ confidence interval for $\Delta_{R}$ is $\left[\mathrm{Y}_{(97)}, \mathrm{Y}_{(214)}\right)=[-3,9.5)$. Also, suppose we are interested in testing that the mean visual acuity score is higher for the blue-green argon group. In this case, $S^{+}=135$ and $U^{+}=189$. Thus, our observed test statistic $T^{+}=189$ with an exact p-value of 0.126 . Using normal approximation, the approximate $\mathrm{p}$-value for this test is 0.124 . Note that there are 210 Walsh averages and 100 pairwise differences.

\section{Example 2}

Another situation in which these procedures may be used is a repeated measures design in which data are not available for all the time points. For example, the Optic Neuritis Treatment Trial (ONIT)/Longitudinal Optic Neuritis Study (LONS) [The Optic Neuritis Study Group (1991)] was conducted to assess the relative benefit to visual function of two corticosteroid treatments as compared with placebo for patients suffering from optic neuritis. This 
Table 2: Visual Acuity Measurements Paired Data

\begin{tabular}{ccccccccccc}
\hline \hline$X_{i}$ & 4 & 69 & 87 & 35 & 39 & 79 & 31 & 79 & 65 & 95 \\
$Y_{i}$ & 62 & 80 & 82 & 83 & 0 & 81 & 28 & 69 & 48 & 90 \\
\hline$D_{i}$ & -58 & -11 & 5 & -48 & 39 & -2 & 3 & 10 & 17 & 5 \\
\hline \hline$X_{i}$ & 68 & 62 & 70 & 80 & 84 & 79 & 66 & 75 & 59 & 77 \\
$Y_{i}$ & 63 & 77 & 0 & 55 & 83 & 85 & 54 & 72 & 58 & 68 \\
\hline$D_{i}$ & 5 & -15 & 70 & 25 & 1 & -6 & 12 & 3 & 1 & 9 \\
\hline \multicolumn{7}{c}{} \\
\hline \hline$T_{i}$ & 36 & 86 & 39 & 85 & 74 & 72 & 69 & 85 & 85 & 72 \\
$C_{j}$ & 88 & 83 & 78 & 30 & 58 & 45 & 78 & 64 & 87 & 65 \\
\hline
\end{tabular}

disease is characterized by the fact that patients usually experience a rapid loss of visual function over a period of about one week, with most of this function subsequently restored over a period of some months.

One of the important vision function measures used in this trial was the Farnsworth Munsell 100-Hue Test which assesses the patient's ability to make fine color discrimination. This test was administered to patients as baseline, six months, one year, and annually thereafter until the eighth year after disease diagnosis. A question arises as to when along this temporal course changes in color discrimination occur and when such changes no longer occur. It is of interest then to perform pairwise comparisons to make these assessments. In this case we use data from the placebo group, as interest lies in the natural course of the disease.

Problematic is the fact that, as expected, some patients miss their testing appointments for various reasons, thereby producing missing observations in the longitudinal data set. The testing procedures discussed in this paper can be used to test pairwise hypotheses in the face of missing data.

\section{Weighted Rank Methods}

Above, we based our inferences on simple sums of one and two sample methods. It should be possible with some added complexity to increase the efficiency by considering weighted sums. The weights may be constructed to depend on sample size proportions and correlation in the bivariate portion of the data. Of course, the efficiency depends on the underlying model distribution.
In this section, we explore various ways to construct weights, always trying to keep in mind the practical side of computing and applying the methods.

\section{Consider}

$$
T_{a}=a \frac{2}{n(n+1)} S^{+}+b \frac{1}{n_{1} n_{2}} U^{+}
$$

where $\mathrm{a}+\mathrm{b}=1$. Then, $T_{a}$ is an estimate of $\delta=a P\left(\left(D_{1}+D_{2}\right) / 2>0\right)+b P(T-C>0)$ and under $H_{0}: \Delta=0, \delta=1 / 2$.

Define, with $N=n_{1}+n_{2}$ and $a=n N /\left(n N+2 n_{1} n_{2}\right)$,

$$
T^{*}=\frac{2 N}{\left(n N+2 n_{1} n_{2}\right)(n+1)} S^{+}+\frac{2}{n N+2 n_{1} n_{2}} U^{+}
$$

for testing $H_{0}: \Delta=0$. Under $H_{0}, E_{0} T^{*}=1 / 2$ and

$$
\begin{aligned}
\operatorname{Var}_{0} T^{*}= & \left(\frac{n N}{n N+2 n_{1} n_{2}}\right)^{2} \frac{n(n+1)(2 n+1)}{24}+ \\
& \left(\frac{2 n_{1} n_{2}}{n N+2 n_{1} n_{2}}\right)^{2} \frac{n_{1} n_{2}(N+1)}{12} .
\end{aligned}
$$

Also, under $H_{0}, \mathrm{~T}^{*}$ has an asymptotic normal distribution. Thus, one can construct an asymptotic size $\alpha$ test based on $T^{*}$. This test is close to the optimal test derived in the appendix when the underlying distribution is bivariate normal.

We have, from (26) in the appendix,

$$
c_{T^{*}}=\sqrt{12} \frac{\gamma \int f_{d}^{2}+\lambda(1-\lambda)(1-\gamma) \int f_{1}^{2}}{\sqrt{\gamma+\lambda(1-\lambda)(1-\gamma)}} \text {. }
$$

For a bivariate normal with $\lambda=\frac{1}{2}$, Table 4 gives the efficiency of $\mathrm{T}^{*}$ relative to $T_{\text {opt,normal }}$ and relative to the simpler $T^{+}$from above. Note that $\mathrm{T}^{*}$ loses very little efficiency and is not below .95 for the range of $\gamma$ and $\rho$ values considered when compared to $T_{o p t, n o r m}$. On the other hand, it is quite a bit better than $T^{+}$for values of $\gamma$ below

Table 3: Efficiency of $T^{*}$ (a) with respect to $T_{\text {opt,norm }}$ and (b) with respect to $T^{+}$

\begin{tabular}{lllllll}
\hline \hline$\gamma$ & \multicolumn{2}{c}{$\rho=.2$} & \multicolumn{2}{c}{$\rho=.5$} & \multicolumn{2}{c}{$\rho=.8$} \\
\hline & $(\mathrm{a})$ & (b) & $(\mathrm{a})$ & $(\mathrm{b})$ & $(\mathrm{a})$ & $(\mathrm{b})$ \\
.2 & 0.987 & 1.187 & 1.000 & 1.360 & 0.952 & 1.818 \\
.5 & 0.990 & 1.000 & 1.000 & 1.000 & 0.975 & 1.000 \\
.8 & 0.996 & 1.058 & 1.000 & 1.034 & 0.992 & 1.001 \\
\hline
\end{tabular}


0.5 . It remains to show that $T^{*}$ has robust efficiency against its natural normal theory competition. This is shown later. We complete this section with discussion of the corresponding estimation and confidence interval.

Computing the rank estimate is not as straightforward as in the simple sum case. Again, we estimate $\Delta$ by $\hat{\Delta}_{R}^{*}$ such that $T^{*}\left(\hat{\Delta}_{R}^{*}\right)=1 / 2$. In this case, $T^{*}(\Delta)$ is a nondecreasing step function which steps down $2 N /\left[\left(n N+2 n_{1} n_{2}\right)(n+1)\right]$ units at each Walsh average and $2 /\left(n N+2 n_{1} n_{2}\right)$ units at each pairwise difference. Because the order of the Walsh averages and pairwise differences depends on the actual data, this estimate cannot be written in closed form. The estimate can be easily found in $S$-plus or Minitab. First, list the Walsh averages and pairwise differences along with the corresponding weights: $2 N /\left[\left(n N+2 n_{1} n_{2}\right)(n+1)\right]$ or $2 /\left(n N+2 n_{1} n_{2}\right)$. Then, sort the combined Walsh averages and pairwise differences with the associated weights in descending order. Starting with the largest item in the list, accumulate the weights until $1 / 2$ is reached. The Walsh average or pairwise difference corresponding to the weight required to reach $1 / 2$ is $\hat{\Delta}_{R}^{*}$. A similar problem exists in constructing a confidence interval for $\Delta$. We use the asymptotic normality of $\mathrm{T}^{*}$. Thus, an approximate $(1-\alpha) 100 \%$ confidence interval for $\Delta$ is $\left(\hat{\Delta}_{L}, \hat{\Delta}_{U}\right)$ where

$$
\begin{aligned}
& T^{*}\left(\hat{\Delta}_{L}\right) \doteq \frac{1}{2}+z_{\alpha / 2} \sqrt{\operatorname{Var}_{0} T^{*}} \\
& T^{*}\left(\hat{\Delta}_{U}\right) \doteq \frac{1}{2}-z_{\alpha / 2} \sqrt{\operatorname{Var}_{0} T^{*}}
\end{aligned}
$$

where $z_{\alpha / 2}$ is the upper $\alpha / 2$ quantile of the standard normal distribution. Then, use the sorting and accumulation for the estimate to determine the $\hat{\Delta}_{L}$ and $\hat{\Delta}_{U}$ values.

\section{Methodology}

\section{Comparison To Competitors}

In this section, we compare $\mathrm{T}^{*}\left(\right.$ or $\hat{\Delta}_{R}^{*}$ ) with various parametric and nonparametric competitors. We will use the Pitman efficiency as described in the appendix. Briefly, the Pitman efficiency of $\mathrm{T}^{*}$ (or $\hat{\Delta}_{R}^{*}$ ) with respect to $\mathrm{T}($ or $\hat{\Delta}$ ) is the ratio $e=\left(c^{*} / c\right)^{2}$, where $\mathrm{c}$ is called the efficacy. Efficiency values greater than one favor $\mathrm{T}^{*}\left(\right.$ or $\left.\hat{\Delta}_{R}^{*}\right)$. Pitman efficiency provides a single number comparison and has been shown to reflect small sample size as well as asymptotic behavior; see Hettmansperger (1984).

We discuss the comparisons in terms of estimates. The corresponding tests are often standardized versions of these estimates. In all cases, the estimation and test efficiency are identical and given by $e$. The competitors fall into two groups. One group is based on a linear models approach and includes a plug-in maximum likelihood estimator and an estimator derived from a test proposed by Bhoj (1989). The other group includes estimates for which the design is initially ignored and all the treatment observations are compared with all the control observations.

\section{Linear Model}

Consider the following linear model:

$$
Z=X \beta+\varepsilon
$$

where

$$
Z=\left[\begin{array}{l}
\mathbf{X} \\
\mathbf{Y} \\
\mathbf{T} \\
\mathbf{C}
\end{array}\right], X=\left[\begin{array}{cc}
1 & 1 \\
1 & -1 \\
1 & 1 \\
1 & -1
\end{array}\right], \boldsymbol{\beta}=\left[\begin{array}{l}
\beta_{1} \\
\beta_{2}
\end{array}\right]
$$

and

$$
\operatorname{Cov} \varepsilon=\Sigma=\sigma^{2}\left[\begin{array}{cccc}
\mathbf{I} & \rho \mathbf{I} & \mathbf{0} & 0 \\
\rho \mathbf{I} & \mathbf{I} & \mathbf{0} & \mathbf{0} \\
\mathbf{0} & \mathbf{0} & \mathbf{I} & \mathbf{0} \\
\mathbf{0} & \mathbf{0} & \mathbf{0} & \mathbf{I}
\end{array}\right]
$$

Note that $Z$ is a $\left(2 \mathrm{n}+\mathrm{n}_{1}+\mathrm{n}_{2}\right) \times 1$ matrix, $X$ is $\left(2 \mathrm{n}+\mathrm{n}_{1}+\mathrm{n}_{2}\right) \times 2, \beta$ is $2 \times 1, \Sigma$ is $\left(2 \mathrm{n}+\mathrm{n}_{1}+\mathrm{n}_{2}\right) \times$ $\left(2 \mathrm{n}+\mathrm{n}_{1}+\mathrm{n}_{2}\right)$. Then $\hat{\beta}=\left(X^{\prime} \Sigma^{-1} X\right)^{-1} X^{\prime} \underline{Z} \quad$ and $\operatorname{Var} \hat{\beta}=\left(X^{\prime} \Sigma^{-1} X\right)^{-1}$. Note that $\Delta=2 \beta_{2}$. To compute this estimate of $\Delta$, one must know the correlation $\rho$.

Theorem 4

$$
\text { If } \rho \text { is known and } M=n+n_{1}+n_{2} \text {, }
$$

$$
\begin{gathered}
\hat{\Delta}_{L M}(\rho)=\frac{1}{\left(n+n_{1}\right)\left(n+n_{2}\right)-n_{1} n_{2} \rho^{2}}\left\{n M(\bar{X}-\bar{Y})+n_{1} n_{2}\left(1-\rho^{2}\right)(\bar{T}-\bar{C})\right. \\
\left.-n(1-\rho)\left[\left(n_{1} \bar{X}-n_{2} \bar{Y}\right)-\left(n_{1} \bar{T}-n_{2} \bar{C}\right)\right]\right\}
\end{gathered}
$$

$$
\operatorname{Var} \hat{\Delta}_{L M}(\rho)=\frac{2 n(1-\rho)+\left(n_{1}+n_{2}\right)\left(1-\rho^{2}\right)}{\left(n+n_{1}\right)\left(n+n_{2}\right)-n_{1} n_{2} \rho^{2}} \sigma^{2}
$$

and as $M \rightarrow \infty$, provided $0<\sigma^{2}<\infty$ and the sample size limits of Theorem 1 are satisfied, 
$\sqrt{\mathrm{M}}\left(\hat{\Delta}_{\mathrm{LM}}(\rho)-\Delta\right) \stackrel{\mathrm{D}}{\longrightarrow} \mathrm{N}\left(0, \xi^{2}\right)$ where

$\xi^{2}=\frac{2 \gamma(1-\rho)+(1-\gamma)\left(1-\rho^{2}\right)}{[\gamma+\lambda(1-\gamma)][\gamma+(1-\lambda)(1-\gamma)]-\lambda(1-\lambda)(1-\gamma)^{2} \rho^{2}} \sigma^{2}$.

Note that $c_{L M}=\xi^{-1}$. In practice we would use $\hat{\Delta}(r)$ where $r$ is the correlation coefficient in the paired data. This estimate of $\Delta$ is also obtained using the plug-in method. For the assumed model, we derive the maximum likelihood estimator of $\Delta$ assuming that $\rho$ and $\sigma$ are known. Because $\rho$ and $\sigma$ are usually not known, we substitute, or plug-in, estimates of these unknown parameters into the expression for the estimate of $\Delta$. Using the plug-in method, we get $\hat{\Delta}_{p m l e}=\hat{\Delta}_{L M}(r)$ where $r$ is the sample correlation coefficient for the paired data. Ekbohm (1976) used this approach to construct test statistics and compared various early approaches in a Monte Carlo study. We do not recommend full maximum likelihood because it is computationally quite complex and may not be stable because there is no complete statistic for the model with all parameters treated as unknown.

Bhoj (1989) compared six different test statistics based on means in a Monte Carlo power study. The different approaches entail refinements that yield approximate $t$ distributions for the test statistics. In addition, some approaches consider the unequal variances case. See Bhoj's paper and references for a survey of parametric methods. We will concentrate on a new test proposed by Bhoj denoted $Z_{b}$ which emerged as generally superior to the other tests based on sample averages. The corresponding estimate was not explicitly developed by Bhoj, so we include a brief description here.

We follow the notation of Bhoj (1989). Let $\hat{\delta}=\omega \hat{\delta}_{2}+(1-\omega) \hat{\delta}_{3} \quad$ where $\quad \hat{\delta}_{2}=2 \bar{T}-\bar{X}-\bar{Y} \quad$ and $\hat{\delta}_{3}=\bar{X}+\bar{Y}-2 \bar{C}$. The value of $\omega$ depends on $\rho$ and was chosen to minimize $\operatorname{Var}(\hat{\delta})$; it is given by Formula (2.2) in Bhoj (1989). Next, note that under normality $\bar{D}=\bar{X}-\bar{Y}$ and $\hat{\delta}$ are independent. Hence, the linear combination of $\bar{D}$ and $\hat{\delta}$ with minimum variance is

$$
\hat{\Delta}_{b}=\frac{\sigma_{\hat{\delta}}^{2}}{\sigma_{\hat{\delta}}^{2}+\sigma_{\bar{D}}^{2}} \bar{D}+\frac{\sigma_{\bar{D}}^{2}}{\sigma_{\hat{\delta}}^{2}+\sigma_{\bar{D}}^{2}} \hat{\delta}
$$

where, in practice, unknown variances must be estimated. Finally, the quantity relevant for the efficiency calculations is $\sigma_{\hat{\Delta}_{b}}^{2}=\left(\sigma_{\hat{\delta}}^{-2}+\sigma_{\bar{D}}^{-2}\right)^{-1}$. A formula for $\sigma_{\hat{\delta}}^{2}$ is given by (2.3) in Bhoj (1989). Although this estimate appears to be quite different from the linear model estimate, in fact the variance can be shown to be identical with the variance of $\hat{\Delta}_{L M}(r)$ defined in Theorem 4 .

Hence, the linear model estimate includes the plug-in MLE and the Bhoj estimator. Our efficiency comparison then will compare $\hat{\Delta}_{R}^{*}$ to $\hat{\Delta}_{L M}(r)$.

The efficiency of $\hat{\Delta}_{R}^{*}$ with respect to the linear model estimate is

$$
\text { eff }\left(\hat{\Delta}_{R}^{*}, \hat{\Delta}_{L M}(\rho)\right)=\xi^{2} c_{T^{*}}^{2} .
$$

In the following discussion, we compare $\hat{\Delta}_{R}^{*}$ to $\hat{\Delta}_{L M}(\rho)$ via (22). However, this is also the Pitman efficiency of the rank test $T^{*}$ relative to a test based on $\hat{\Delta}_{L M}(\rho)$. Table 4 gives the efficiencies of $\hat{\Delta}_{R}^{*}$ with respect to the linear model estimate assuming an underlying bivariate normal distribution and $\lambda=1 / 2$.

\section{Results}

In Table 4, we see that when the underlying distribution is bivariate normal, the linear model estimate with $\rho$ known is more efficient than the rank-based estimate when $a^{*}$ is used regardless of the correlation, $\rho$, or the proportion of paired data, $\gamma$. The efficiencies are close to one, indicating that too much efficiency is not lost using the rank method.

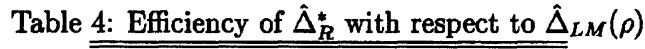

\begin{tabular}{llll}
$\gamma$ & $\rho=.2$ & $\rho=.5$ & $\rho=.8$ \\
\hline .2 & 0.942 & 0.955 & 0.909 \\
.5 & 0.945 & 0.955 & 0.931 \\
.8 & 0.951 & 0.955 & 0.948 \\
\hline
\end{tabular}

In practice, $\rho$ would be estimated from the data. When a Pearson correlation coefficient for the paired data is used, this introduces more variability into the linear model estimate of $\Delta$. Simulations show that the linear model estimate in which $\rho$ is estimated is still more efficient than the rank-based estimate using $a *$ under bivariate normality. In general, the gain in efficiency using this linear model estimate over the rank-based estimate is not great.

Instead of an underlying bivariate normal distribution, suppose the data are selected from a contaminated bivariate normal distribution. Let $\varepsilon$ represent the proportion of contamination. Then with probability $1-\varepsilon$, the data will be selected from our usual bivariate normal distribution with equal marginal variances $\sigma_{1}^{2}=\sigma_{2}^{2}=\sigma^{2}$ and 
correlation $\rho$. The rest of the data will be selected from a bivariate normal distribution with equal but larger marginal variances and possibly different correlation. It follows that the marginal distribution and distribution of differences are univariate contaminated normal distributions. Furthermore, if we mix a bivariate normal $\left(\mu_{1}=\mu_{2}=0, \sigma_{1}^{2}=\sigma_{2}^{2}=1, \rho\right)$ with a bivariate normal $\left(\mu_{1}=\mu_{2}=0, \sigma_{1}^{2}=\sigma_{2}^{2}=\sigma^{2}, \rho_{c}\right)$ then

$$
\begin{gathered}
\int f_{d, \varepsilon}^{2}=\frac{(1-\varepsilon)^{2}}{2 \sqrt{\pi} \sqrt{2(1-\rho)}}+\frac{\varepsilon^{2}}{2 \sqrt{\pi} \sqrt{2 \sigma^{2}\left(1-\rho_{c}\right)}} \\
+\frac{\sqrt{2} \varepsilon(1-\varepsilon)}{\sqrt{\pi} \sqrt{2(1-\rho)+2 \sigma^{2}\left(1-\rho_{c}\right)}} \\
\int f_{1, \varepsilon}^{2}=\frac{(1-\varepsilon)^{2}}{2 \sqrt{\pi}}+\frac{\varepsilon^{2}}{2 \sqrt{\pi} \sigma}+\frac{\sqrt{2} \varepsilon(1-\varepsilon)}{\sqrt{\pi} \sqrt{1+\sigma^{2}}} .
\end{gathered}
$$

Now $c_{T^{*}}$ is compared using $a^{*}$ and (12).

When the data are a sample from a contaminated bivariate normal distribution, the asymptotic variance of $\hat{\Delta}_{L M}$ is given by

$\xi_{\mathrm{c}}^{2}=\frac{u^{2}(u-w)\left[2 \gamma u^{2}+(1-\gamma)(u+w)\right]}{(u \gamma+\lambda(1-\gamma))(u \gamma+(1-\lambda)(1-\gamma)) u^{2}-\lambda(1-\lambda)(1-\gamma)^{2} w^{2}}$

where $u=(1-\varepsilon)+\varepsilon \sigma^{2}$ and $w=(1-\varepsilon) \rho+\varepsilon \rho_{c} \sigma^{2}$. Recall $\varepsilon$ is the proportion of contamination and $\rho_{c}$ and $\sigma^{2}$ are the correlation and the marginal variance, respectively, in the contaminated portion of the distribution. Thus, the efficiency of the $T^{*}$ methods with respect to the linear model methods under a contaminated bivariate normal distribution is given by $\xi_{c}^{2} c_{T^{*}}^{2}$.

Suppose the data are sampled from the contaminated bivariate normal distribution with $\rho_{c}=0$. This simulates the situation in which the components of the data are contaminated independently, as in the case of gross errors. Already for contamination of the order $5 \%, \hat{\Delta}_{R}^{*}$ is more efficient than $\hat{\Delta}_{L M}$. See Table 5 for efficiencies of $\hat{\Delta}_{R}^{*}$ with respect to $\hat{\Delta}_{L M}$ when $\varepsilon=0.05$ and $\sigma=2$. We find similar efficiencies for other levels of contamination and values of $\lambda \neq 1 / 2$. We conclude that $T^{*}$ (and $\hat{\Delta}_{R}^{*}$ ) are often more efficient than the linear based methods.
Table 5: Efficiency of $\hat{\Delta}_{R}^{*}$ with respect to $\hat{\Delta}_{\mathrm{LM}}$ with $\in=0.05, \sigma=2$, $\rho_{c=0, \lambda=1 / 2}$

\begin{tabular}{llll}
\hline$\gamma$ & $\rho=.2$ & $\rho=.5$ & $\rho=.8$ \\
\hline .2 & 1.235 & 1.232 & 1.138 \\
.5 & 1.039 & 1.182 & 1.137 \\
.8 & 1.161 & 1.160 & 1.146 \\
\hline
\end{tabular}

Without Regard To Design

In addition to the mean-based linear combination methods, one might consider a test statistic which treats the data as if it only consists of two samples. Let $T_{1}^{*}, \ldots, T_{n+n_{1}}^{*}$ and $C_{1}^{*}, \ldots, C_{n+n_{2}}^{*}$ represent observations from subjects receiving the treatment and the control, respectively. Then $T_{i}^{*}$ is either an observation from the paired data, $X_{j}$, or one from the independent samples, $T_{k}$. Similarly, for $C_{i}^{*}$. Let

$$
\bar{L}^{*}=\frac{1}{n+n_{1}} \sum_{i=1}^{n+n_{1}} T_{i}^{*}-\frac{1}{n+n_{2}} \sum_{j=1}^{n+n_{2}} C_{j}^{*}
$$

Thus, the computation of the statistic ignores the structure of the data. This was first investigated by Lin and Stivers (1974) and Ekbohm (1976). Bhoj (1989) included a version of this statistic in his simulation study. Usual computations reveal that the Pitman efficacy of $T_{M}^{*}$ is

$$
\mathrm{c}_{\overline{\mathrm{L}}^{*}}=\frac{1}{\sigma} \sqrt{\frac{[\gamma+\lambda(1-\gamma)][\gamma+(1-\lambda)(1-\gamma)]}{2(1-\rho) \gamma+1-\gamma}}
$$

The efficiency of $T^{*}$ with respect to $\bar{L} *$ is given under bivariate normality in Table 6 where it can be seen that for moderate to high correlation $T^{*}$ is superior even under normality.

Table 6: Efficiency of $T^{*}$ with respect to $\bar{L}^{*}, \lambda=1 / 2$

\begin{tabular}{llll}
\hline$\gamma$ & $\rho=.2$ & $\rho=.5$ & $\rho=.8$ \\
\hline .2 & 0.95 & 1.06 & 1.56 \\
.5 & 0.96 & 1.06 & 1.59 \\
.8 & 0.93 & 1.00 & 1.24 \\
\hline
\end{tabular}

The nonparametric counterpart to this approach is based on the Wilcoxon-Mann-Whitney statistic. The test consists of simply conducting the two sample rank test on the treatment versus the control observations irrespective of which part of the design they come from. The test will not have the usual Wilcoxon-Mann-Whitney permutation distribution. A normal approximation can be used to determine the p-value. However, certain quantities must be estimated. This approach was worked out in detail by Hollander, Pledger, and Lin (1974). The corresponding 
estimator $\hat{\Delta}_{H P L}$ is simply the median of all the pairwise differences between treatment and control observations. In the case of bivariate normality, formulas from the paper can be used to compute the efficiency of $\hat{\Delta}_{R}^{*}$ with respect to $\hat{\Delta}_{H P L}$. However, we wish to make the comparison for contaminated bivariate normal distributions as well. Hence, we report simulated efficiencies in Table 7 . They check with the formula values for the bivariate normal case. Note that $T^{*}\left(\right.$ and $\hat{\Delta}_{R}^{*}$ ) is generally superior to the rank test and $\hat{\Delta}_{H P L}$.

Table 7: Efficiency of $\hat{\Delta}_{R}^{*}$ with respect to $\hat{\Delta}_{H P L}, \lambda=1 / 2$

\begin{tabular}{lllllll}
\hline \hline$\gamma$ & \multicolumn{2}{c}{$\rho=.2$} & \multicolumn{2}{c}{$\rho=.5$} & \multicolumn{2}{c}{$\rho=.8$} \\
& Norm & CN & Norm & CN & Norm & CN \\
\hline .2 & 1.001 & 1.013 & 1.119 & 1.096 & 1.639 & 1.626 \\
.5 & 1.008 & 1.014 & 1.130 & 1.033 & 1.703 & 1.524 \\
.8 & 1.011 & 0.970 & 1.079 & 1.114 & 1.362 & 1.579 \\
\hline
\end{tabular}

Conclusion

A simple and efficient method for testing and estimation when the data collected is a mixture of paired and unpaired data is based on $T^{+}(\Delta),(1)$. The test statistic, point estimate, and confidence interval can be computed quickly and easily. The exact p-value is easily computed with an $S$ plus function for small sample sizes. The normal approximation works well for large sample sizes.

The $T^{*}(11)$ rank statistic is generally more efficient than $T^{+}$. The efficiency of $T^{*}$ compares very favorably with the optimal weighted rank statistic. The linear model mean based estimate of $\Delta$ is a bit more efficient than the R-estimate based on $T^{*}$ for a bivariate normal distribution, but we still estimate the correlation coefficient to use it. With a small amount of contamination the rank methods are superior. In addition, the rank methods, both test and estimate, are more robust in the sense that outliers will have less effect on them than on the mean based methods. In general, we recommend using $T^{*}$. However, if $n \geq n_{1}+n_{2}$, Table 3 suggests that $T^{+}$is almost as efficient as $T^{*}$. Hence, under those circumstances, we recommend using the simple methods based on $T^{+}$.

\section{References}

Bhoj, D. S (1989). On comparing correlated means in the presence of incomplete data. Biometrics Journal, 3, 279-288.

Dubnicka, S. R. (1998). Rank-based procedures for combined paired and unpaired data. Ph. D Thesis, The Pennsylvania State University.
Ekbohm, G. (1976). On comparing means in the paired case with incomplete data on both responses. Biometrika, 63, 299-304.

Ekbohm, G. (1981). On testing equality of means in the paired case with incomplete data on both responses. Biometrical Journal, 23, 251-259.

Hamdan, M., Khuri, A. \& Crews, S. (1978). A test for equality of means of two correlated normal variates with missing data on both responses. Biometrical Journal, 20, 667-674.

Hettmansperger, T. P. (1984). Statistical inference based on ranks. NY: John Wiley and Sons, Inc.

Hettmansperger, T. P., \& McKean, J. W. (1998). Robust nonparametric statistical methods. NY: Arnold.

Hollander, M., Pledger, G., \& Lin, P. -E. (1974). Robustness of the Wilcoxon test to a certain dependency between samples. The Annals of Statistics, 2(1), 177-181.

Lin, P., \& Stivers, L. (1974). On the difference of means with incomplete data. Biometrika, 61, 325-334.

Morrison, D. (1973). A test for equality of means of correlated variates with missing data on one response. Biometrika, 61, 101-105.

The Krypton Argon Regression Neovacularization Study Research Group (1993, November). Randomized comparison of krypton versus argon scatter photocoagulation for diabetic disc neovacularization. Opthalmology, 100(11), 1655-1664.

The Optic Neuritis Study Group (1991). The clinical profile of optic neuritis: Experience of the optic neuritis treatment trial. Arch. Opthal., 109, 1673-1678.

Thompson, P. (1995). A hybrid paired and unpaired analysis for the comparison of proportions. Statistics in Medicine, 14, 1463-1470.

Woolson, R., Leeper, J., Cole, J. \& .Clarke W. (1976). A Monte Carlo investigation of a statistic for a bivariate missing data problem. Communication in Statistics - Theory and Methods, A5, 681-658.

\section{Appendix}

Proof of Theorem 1: This follows immediately from the limiting normality of the stochastically independent signed rank and rank sum statistics. See Dubnicka (1998) for full details.

Proof of Theorem 2: Consider sequences that consists of an arrangement of $\mathrm{n}+$ and - signs followed by an ordering on $n_{1}$ T's and $n_{2}$ C's. A + in the $i^{\text {th }}$ position indicates that the difference $D_{j}$ is positive and $\left|D_{j}\right|$ is ranked $i^{\text {th }}$ among $\left|D_{l}\right|, \ldots,\left|D_{n}\right|$. A - in the $i^{\text {th }}$ positive means the $D_{j}$ is negative and $\left|D_{j}\right|$ is ranked $i^{\text {th }}$ among the absolute values. Also, a $\mathrm{T}(\mathrm{C})$ in the $j^{\text {th }}$ position indicates that the $j^{\text {th }}$ smallest response was obtained from a subject receiving the treatment (control). Then $T^{+}$is simply the sum of the ranks 
(positions) of the + signs plus the sum of the ranks of the T's minus $\left[n_{l}\left(n_{1}+1\right)\right] / 2$.

When either $i=0$ or $j=0$, the independent samples portion only contains observations from either the control group or the treatment group. In these cases, the independent samples do not contribute to the value of $T^{+}(0)$. Thus, the value of $T^{+}(0)$ depends only on the observations from the paired data. Hence, when either $i=0$ or $j=0$, we can ignore the subscripts $i$ and $j$. This completes the proof.

\section{Pitman Efficiency}

The Pitman efficacy of a test statistic $\mathrm{T}$ is defined as

$$
c_{T}=\lim _{\mathrm{M} \rightarrow \infty} \frac{\left.\frac{\mathrm{d}}{\mathrm{d} \Delta} \mathrm{E}_{\Delta} \mathrm{T}(0)\right|_{\Delta=0}}{\sqrt{\mathrm{M}} \sqrt{\operatorname{Var}_{0} \mathrm{~T}(0)}} .
$$

This quantity measures the standardized rate of change of the expected value of the statistic at the true value, 0 in this case. Relatively large values of $c_{T}$ indicate high sensitivity of the test and hence large $c_{T}$ is desirable. The computation of $c_{T}$ depends on knowing the mean of $T$ for general $\Delta$ and the variance under the true value.

Suppose further than $\hat{\Delta}$ is the estimate of $\Delta$ derived from $T$; then the asymptotic variance of $\sqrt{n}(\hat{\Delta}-\Delta)$ is $c_{T}^{-2}$. The Pitman testing and estimation efficiency of $T_{I}$ (and $\hat{\Delta}_{1}$ ) relative to $T_{2}$ (and $\hat{\Delta}_{2}$ ) is $e_{12}=\left(\mathrm{c}_{\mathrm{T} 1} / \mathrm{c}_{\mathrm{T} 2}\right)^{2}$. See Hettmansperger and McKean (1998) for details.

Proof of Theorem 3: The finiteness of the integrals is sufficient to guarantee the existence of $c_{T^{+}} \cdot \operatorname{Var}_{0} T^{+}(0)$ is given in (3). Also,

$$
\begin{aligned}
E_{\Delta} T^{+}(0)= & n\left[1-F_{d}(-\Delta)\right]+\frac{n(n-1)}{2} \int\left[1-F_{d}(-z-2 \Delta)\right] f_{d}(z) d z \\
& +n_{1} n_{2} \int\left[1-F_{l}(x-\Delta)\right] f_{l}(x) d z
\end{aligned}
$$

where $F_{d}$ represents the cumulative distribution function of the $D_{i}$ in the paired data and $F_{l}$ is the common cumulative distribution function of the $T_{i}$ and $C_{j}$ in the unpaired data. By symmetry,

$$
\left.\frac{d}{d \Delta} E_{\Delta} T^{+}(0)\right|_{\Delta=0}=n f_{d}(0)+n(n-1) \int f_{d}^{2}(z) d z+n_{1} n_{2} \int f_{l}^{2}(x) d x .
$$

Then

$$
c_{T^{+}}=\lim _{M \rightarrow \infty} \frac{n f_{d}(0)+n(n-1) \int f_{d}^{2}(z) d z+n_{1} n_{2} \int f_{1}^{2}(x) d x}{\sqrt{M} \sqrt{\frac{n(n+1)(2 n+1))}{24}+\frac{n_{1} n_{2}\left(n_{1}+n_{2}+1\right)}{12}}}
$$

and the result immediately follows.

The Pitman efficiency (10) is given by

$$
c_{T a}=\sqrt{12} \frac{a 2 \int f_{d}^{2}+b \int f_{I}^{2}}{\sqrt{a^{2} \frac{4}{\gamma}+b^{2} \frac{1}{\lambda(1-\lambda)(1-\gamma)}}} .
$$

We consider the weights that optimize the efficacy. The following lemma gives a formula for determining these weights and the maximum efficacy when the efficacy is of a particular form.

Lemma 1: Let $c^{*}=\left(a k_{1}+b k_{2}\right) /\left(a^{2} k_{3}+b^{2} k_{4}\right)^{1 / 2}, a+b=1$. Then $c^{*}$ is maximized by $a=k_{1} k_{4} /\left(k_{1} k_{4}+k_{2} k_{3}\right)$ and

$$
c_{\text {max }}^{*}=\left(\frac{k_{1}^{2}}{k_{3}}+\frac{k_{2}^{2}}{k_{4}}\right)^{1 / 2} \text {. }
$$

Applying Lemma 1 to $c_{T a}$, we find that $a_{o p t}=\gamma \int f_{d}^{2} /\left[\gamma \int f_{d}^{2}+2 \lambda(1-\lambda) \int f_{I}^{2}\right]$ maximizes the efficacy of $T_{a}$, and the maximum efficacy is

$$
c_{T a, \max }=\sqrt{12} \sqrt{\gamma\left(\int f_{d}^{2}\right)^{2}+\lambda(1-\lambda)(1-\gamma)\left(\int f_{l}^{2}\right)^{2}} .
$$

In order to develop an efficient and, at the same time, practical statistic, consider $a_{\text {opt }}$ when sampling from a bivariate normal distribution. We find, using $\int f_{d}^{2}=1 /\left(2 \sqrt{2 \pi \sigma^{2}(1-\rho)}\right)$ and $\int f_{1}^{2}=11\left(2 \sqrt{\pi \sigma^{2}}\right)$, that $a_{\text {opt,norm }}=\gamma /[\gamma+2 \lambda(1-\lambda)(1-\gamma) \sqrt{2(1-\rho)}] \quad$ where $\lambda \doteq n_{1} /\left(n_{1}+n_{2}\right)$ and $\gamma \doteq n /\left(n+n_{1}+n_{2}\right)$. We could estimate $\rho$ from the data; however, the efficiency is fairly flat around $\rho=1 / 2$, so we make that substitution to get

$$
a^{*}=\frac{\gamma}{\gamma+2 \lambda(1-\lambda)(1-\gamma)} \doteq \frac{n\left(n_{1}+n_{2}\right)}{n\left(n_{1}+n_{2}\right)+2 n_{1} n_{2}}
$$

This is the optimal weighted rank statistic when sampling from a bivariate normal distribution with $\rho=1 / 2 . \mathrm{T}^{*}$, (11), is based on $a^{*}$.

Proof of Theorem 4: The expressions for the estimator $\hat{\Delta}_{L M}(\rho)$ and its variance follow from routine but tedious calculations of $\left(X^{\prime} \Sigma^{-1} X\right)^{-1} X^{\prime} \Sigma^{-1} Z$ and $\left(X^{\prime} \Sigma^{-1} X\right)^{-1}$. The limiting normality also follows immediately. 


\section{Exact Level And Power Of Permutation, Bootstrap, And Asymptotic Tests Of Trend}

\author{
Christopher D. Corcoran \\ Department of Mathematics and Statistics \\ Utah State University
}

\author{
Cyrus R. Mehta \\ Cytel Software Corporation
}

We develop computational tools that can evaluate the exact size and power of three tests of trend (e.g., permutation, bootstrap and asymptotic) without resorting to large-sample theory or simulations. We then use these tools to compare the operating characteristics of the three tests. It is seen that the bootstrap test is ultra-conservative relative to the other two tests and as a result suffers from a severe deterioration in power. The power of the asymptotic test is uniformly larger than that of the other two tests, but it fails to preserve the Type I error for most of the range of the baseline response probability. The permutation test, being exact, is guaranteed to preserve the Type I error throughout the range of the baseline response probability. The price paid for this guarantee is a loss of power relative to the asymptotic test. The power loss is, however, small in most situations.

Keywords: Type I error, Power, Trend, Permutation tests, Bootstrapping

\section{Introduction}

Forty mice were divided into four equal groups. Each group was treated with a different dose of an animal carcinogen as a result of which some mice developed a tumor. The data are displayed in Table 1. The goal is to test for a doseresponse relationship. Specifically, let $\pi_{\mathrm{j}}$ be the Bernoulli probability that an animal treated at dose $d_{j}$ develops a tumor. We wish to test the null hypothesis

$\mathrm{H}_{0}: \pi_{1}=\pi_{2}=\pi_{3}=\pi_{4} \equiv \pi$

against the one-sided alternative hypothesis

$\mathrm{H}_{1}: \pi_{1} \leq \pi_{2} \leq \pi_{3} \leq \pi_{4}$

Table 1: Dose-Response Data for Animal Carcinogenicity Study

\begin{tabular}{||c||c|c|c|c||c||}
\hline \hline \multicolumn{1}{|c||}{ Response } & \multicolumn{4}{c||}{ Dose $d_{j}$ assigned to all mice in group $j$} & \multirow{2}{*}{ Total } \\
\cline { 2 - 6 } Status & $d_{1}=0$ & $d_{2}=1$ & $d_{3}=5$ & $d_{4}=50$ & \\
\hline Tumor & 1 & 0 & 1 & 3 & 5 \\
No Tumor & 9 & 10 & 9 & 7 & 35 \\
\hline Total & 10 & 10 & 10 & 10 & 40 \\
\hline \hline
\end{tabular}

with at least one inequality $n$ equation (1.2) being strict. The value of $\pi$, the common response probability under $\mathrm{H}_{0}$, is typically unknown.

An efficient test of the null hypothesis is the Cochran-Armitage test of trend (Cochran, 1954; Armitage,1955), in which the test statistic is

Christopher D. Corcoran is Assistant Professor, Department of Mathematics and Statistics, 202 LUND, Utah State University, Logan, UT, 84322-3900. Email: corcoran@math.usu.edu. Cyrus R. Mehta is President, Cytel Software Corporation, and Adjunct Professor of Biostatistics, Harvard School of Public Health.

$$
T(x)=\sum_{j=1}^{4} d_{j} x_{j}
$$

where $x_{\mathrm{j}}$ is the entry in row 1 and column $\mathrm{j}$ of a generic $2 \times 4$ contingency table, $x$, with column sums of 10 in each of the four columns. Substituting the Table 1 data into equation (1.3), the observed value of the test statistic is 155 . It is usual to test the null hypothesis by computing a p-value, defined as the probability under $\mathrm{H}_{0}$ of observing a table whose test statistic equals or exceeds 155. A major difficulty with performing this computation is that even under $\mathrm{H}_{0}$ the probability of observing any table depends on the unknown nuisance parameter $\pi$. We will evaluate three different methods for computing the p-value in the presence of this nuisance parameter. The three methods are bootstrap resampling, permutation resampling and normal approximation. We have two objectives in writing this paper. Our first objective is expository. We wish to clarify the distinction between the bootstrap and permutation resampling methods because these two terms are frequently confused. Our second objective is to compare the performance of all three methods with respect to Type I error and power.

\section{The Bootstrap P-Value}

The bootstrap p-value is obtained by resampling from the reference set, $\Gamma$, of all $2 \times 4$ tables with column sums equal to 10 . Under $H_{0}$ the probability of observing any $x \in \Gamma$ is

$$
f_{\pi}(x)=\prod_{j=1}^{4}\left(\begin{array}{l}
10 \\
x_{j}
\end{array}\right) \pi^{x_{j}}(1-\pi)^{10-x_{j}}
$$

a product of four binomial probabilities. It is not possible to resample tables from $\Gamma$ with probabilities given by (1.4) 
because $\pi$, the Bernoulli probability under $\mathrm{H}_{0}$, is unknown.

We can, however, replace $\pi$ with

$$
\hat{\pi}=\frac{5}{40}
$$

the maximum likelihood estimate (MLE) under the null hypothesis. The bootstrap p-value is then evaluated by resampling tables from $\Gamma$ with probabilities given by

$$
f_{\tilde{\pi}}\left(x_{j}\right)=\prod_{j=1}^{4}\left(\begin{array}{l}
10 \\
x_{j}
\end{array}\right) \hat{\pi}^{x_{j}}(1-\hat{\pi})^{10-x_{j}}
$$

Suppose we resample $M$ tables in this manner, denoted by $x_{1}, x_{2}, \ldots, x_{\mathrm{M}}$. The bootstrap p-value is evaluated as

$$
\tilde{p}_{b}(M)=\frac{\sum_{l=1}^{M} I\left\{T\left(x_{l}\right) \geq 155\right\}}{M}
$$

where $I\{$.$\} is the indicator function. In other words we$ resample tables from $\tilde{A}$ by treating the empirically observed value of $\pi$ as though it were the true value and estimate the bootstrap p-value as the fraction of resampled tables that are at least as extreme as the observed table with respect to the Cochran-Armitage test statistic. For the data in Table 1 the bootstrap p-value based on $M=100,000$ samples was found to be $\widetilde{\mathrm{p}}_{\mathrm{b}}(\mathrm{M})=0.0941$. In repeated samples this value would vary due to the sampling error associated with $\widetilde{\mathrm{p}}_{\mathrm{b}}(\mathrm{M})$. The sampling error decreases in proportion to the square root of $M$. In the limit as $M \rightarrow \infty$ the bootstrap p-value converges to the constant

$$
p_{b}=\sum_{\substack{x \in \Gamma \\ T(x) \geq 155}} f \hat{\pi}\left(x_{j}\right)
$$

For the data in Table 1, $p_{b}=0.0954$, which is almost the same as $\widetilde{\mathrm{p}}_{\mathrm{b}}(\mathrm{M})$ at $M=100,000$. Although increasing $M$ eliminates the sampling error associated with $\widetilde{\mathrm{p}}(M)$, it cannot eliminate the error associated with using $\hat{\pi}$ as an estimate for the unknown nuisance parameter $\pi$ in equation (1.7). Thus, the accuracy of bootstrap p-value depends on how well $\hat{\pi}$ approximates $\pi$ rather than on $M$, the number of times resampled from $\Gamma$.

The Permutation P-Value

The permutation p-value is obtained by conditioning on the sum of observed responses. Define the conditional reference set $\Gamma(5)$ to be all contingency tables for which the sum of entries in the first row is 5 . Then the conditional probability under $\mathrm{H}_{0}$ of observing any table $x$ $\in \Gamma(5)$ is given by

$$
h_{0}(x \mid 5)=\frac{f_{\pi}(x)}{\sum_{y \in \Gamma(5)} f_{\pi}(y)}
$$

which simplifies to

$$
h_{0}(x \mid 5)=\frac{\prod_{j=1}^{4}\left(\begin{array}{l}
10 \\
x_{j}
\end{array}\right)}{\left(\begin{array}{c}
40 \\
5
\end{array}\right)}
$$

Observe that equation (1.9) does not depend on $\pi$. The unknown nuisance parameter has been eliminated by conditioning on its sufficient statistic -the sum of entries in row 1 of Table 1.

The permutation p-value is obtained by resampling tables $x \in \Gamma(5)$ each with probability $h_{0}(x / 5)$. Suppose we resample $M$ tables in this manner, denoted by $x_{1}, x_{2}, \ldots, x_{\mathrm{M}}$. Then the permutation $\mathrm{p}$-value is evaluated as

$$
\tilde{p}_{e}(M)=\frac{\sum_{l=1}^{M} I\left\{T\left(x_{j}\right) \geq 155\right\}}{M}
$$

In other words, we resample tables from $\Gamma(5)$ with probability (1.9) and estimate the permutation p-value as the fraction of resampled tables that are at least as extreme as the observed table with respect to the Cochran-Armitage test statistic. For the data in Table 1 the permutation p-value based on $M=100,000$ samples was found to be 0.0553 . In repeated samples this value would vary due to the sampling error associated with $\widetilde{p}_{\mathrm{e}}(M)$. As before, the sampling error decreases in proportion to the square root of $M$.

For finite values of $M$ the permutation p-value specified in equation (1.10) is also referred to as the Monte Carlo p-value. We can eliminate the sampling error of the permutation or Monte Carlo p-value by letting $M \rightarrow \infty$ in which case (StatXact-4, p. 599) $\tilde{\mathrm{p}}_{\mathrm{e}}(M)$ converges to the constant quantity

$$
p_{e}=\sum_{\substack{\boldsymbol{x} \in \Gamma(5) \\ T(x) \geq 155}} h_{0}(\boldsymbol{x} \mid 5)
$$

Equation (1.11) does not contain any unknown nuisance parameters, nor is it subject to sampling error. Thus, this probability calculation is exact and $p_{\mathrm{e}}$ is referred to as the exact $\mathrm{p}$-value. For the data in Table $1, p_{\mathrm{e}}=0.0546$ which is almost the same as $\tilde{p}_{e}(M)$ at $M=100,000$. 
The Asymptotic P-Value

Because evaluation of equation (1.11) can be very computationally intensive, one frequently approximates this $\mathrm{p}$-value by appealing to the asymptotic normality of the distribution of $T(x)$. The asymptotic $p$-value is easily obtained as

$$
p_{a}=1-\Phi\left\{\frac{155-E(T \mid 5)}{\sqrt{\operatorname{Var}(T \mid 5)}}\right\}
$$

where $E(T \mid 5)$ and $\operatorname{Var}(T \mid 5)$ are the conditional mean and conditional variance, respectively, of $T(x)$ given $x \in \Gamma(5)$. Closed form expressions for these two conditional moments in terms of the margins of the observed contingency table are given by equations (2.30) and (2.31). Upon substituting into these equations we obtain $\mathrm{E}(T \mid 5)=70$ and $\operatorname{var}(T$ |5) $=1954.52$, where upon $p_{\mathrm{a}}=0.0273$.

\section{Methodology}

We have seen in above that the three p-values, $p_{b}, p_{e}$ and $p_{\mathrm{a}}$, are very different, ranging between 0.02 and 0.09 , and thereby leading to different conclusions about the null hypothesis. It is thus important to decide a priori which of the three methods, bootstrap, permutation or asymptotic, we intend to use for testing the null hypothesis. An objective way to compare the three methods is to determine, for a given nominal significance level, the actual significance level and power of each method. In this section we define these quantities and show how they may be computed. Below, we present the results of our comparisons.

We begin by generalizing the dose-response problem discussed in Section 1 to the comparison of $\mathrm{K}$ binomial populations with response probabilities $\pi_{1}, \pi_{2}, \ldots, \pi_{\mathrm{K}}$, respectively. We wish to test the null hypothesis

$$
\mathrm{H}_{0}: \pi_{1}=\pi_{2}=\ldots=\pi_{\mathrm{K}} \equiv \pi
$$

against the one-sided alternative hypothesis

$$
H_{1}: \pi_{1} \leq \pi_{2} \leq \ldots \leq \pi_{k}
$$

with at least one inequality $n$ equation (2.14) being strict. The value of $\pi$, the common response probability under $\mathrm{H}_{0}$, is unknown. Suppose we observe $x_{j}$ responses and $n_{j}$ $\mathrm{x}_{j}$ non-responses from population $j$. Table 2 displays the observed data in the form of a generic $2 \times \mathrm{K}$ contingency table, $x$.

Let $\Gamma$ denote the set of all $2 \times \mathrm{K}$ contingency tables

Table 2: Data from K Ordered Binomial Populations

\begin{tabular}{||c||c|c|l|c||c||}
\hline \hline \multirow{2}{*}{\multicolumn{1}{|c||}{$\begin{array}{c}\text { Response } \\
\text { Status }\end{array}$}} & \multicolumn{4}{c||}{ Binomial Populations } & \multirow{2}{*}{ Total } \\
\cline { 2 - 5 } & Pop_1 & Pop_2 & $\ldots$ & Pop_K & m \\
\hline Response & $x_{1}$ & $x_{2}$ & $\ldots$ & $x_{K}$ & m \\
Non-Response & $n_{1}-x_{1}$ & $n_{2}-x_{2}$ & $\ldots$ & $n_{K}-x_{K}$ & $N-m$ \\
\hline Total & $n_{1}$ & $n_{2}$ & $\ldots$ & $n_{K}$ & $N$ \\
\hline \hline
\end{tabular}

with column sums of $n_{j}, j=1,2, \ldots K$. For any $x \in \Gamma$ the Cochran-Armitage test statistic is defined as

$$
T(x)=\sum_{j=1}^{K} d_{j} x_{j}
$$

where the $d_{j}$ 's are pre-specified constants that correspond to doses in a dose-response setting. Our objective is to determine, for the bootstrap, permutation and asymptotic procedures, the true significance level and power of a onesided Cochran-Armitage test conducted at a nominal significance level of $\Gamma$. For the bootstrap and permutation procedures we will eliminate sampling error from the comparisons by assuming that the we sample an infinite number of times from the appropriate reference set. That is, we will let $M \rightarrow \infty$ and evaluate the performance of $\mathrm{p}_{\mathrm{b}}$ rather than $\widetilde{\mathrm{p}}_{\mathrm{b}}(M)$, and $p_{\mathrm{e}}$ rather than $\widetilde{\mathrm{p}}_{\mathrm{e}}(M)$. In order to make the size and power comparisons accurately, all the computations are based on exact distribution theory rather than relying on asymptotic approximations. Thus, the formulas presented above, for size and power are very difficult to compute. We use adaptations of the network algorithms described in Mehta, Patel and Senchaudhuri (1998) and Corcoran, Mehta, and Senchaudhuri (2000) to perform these computations.

Size and Power of the Bootstrap Procedure

Suppose we have observed the data displayed in Table 2, where the sum of entries in row 1 is $m$ and the total sample size is $\mathrm{N}$. After eliminating sampling error by letting the number of bootstrap samples $M$ be infinite, the bootstrap distribution of the Cochran-Armitage statistic is

$$
\operatorname{Pr}\{T(x)=t \mid m\}=\sum_{\substack{x \in \Gamma \\ T(x)=t}} \prod_{j=1}^{K}\left(\frac{m}{N}\right)^{x_{j}}\left(1-\frac{m}{N}\right)^{n_{j}-x_{j}}
$$

Suppose we wish to test the null hypothesis (2.13) at a nominal significance level $\alpha$. Let $t_{b}(m)$ be the level- $\alpha$ cut-off of the bootstrap distribution (2.16). That is,

$$
\sum_{\substack{\boldsymbol{X} \in \boldsymbol{\Gamma} \\ T(\boldsymbol{x}) \geq t_{b}(m)}} \prod_{j=1}^{K}\left(\frac{m}{N}\right)^{x_{j}}\left(1-\frac{m}{N}\right)^{n_{j}-x_{j}} \leq \alpha
$$

and for any $\mathrm{t}<\mathrm{t}_{\mathrm{b}}(m)$

$$
\sum_{\substack{\boldsymbol{X} \in \boldsymbol{\Gamma} \\ T(\boldsymbol{X}) \geq t}} \prod_{j=1}^{K}\left(\frac{m}{N}\right)^{x_{j}}\left(1-\frac{m}{N}\right)^{n_{j}-x_{j}}>\alpha
$$


Due to the discreteness of the distribution (2.16) the left hand side of (2.17) will usually be less than $\alpha$. For notational convenience we have suppressed the dependence of $\mathrm{t}_{\mathrm{b}}(m)$ on $\alpha$.

Conditional on $m$ the true size or Type I error of the bootstrap procedure is

$\mathcal{S}_{b}(m, \pi)=\sum_{\substack{x_{i \in r_{j}} \\ T\left(x^{\prime}\right) \geq t_{b}(m)}} \prod_{j=1}^{K}\left(\begin{array}{l}n_{j} \\ x_{j}\end{array}\right) \pi^{x_{j}}(1-\pi)^{n_{j}-x_{j}}$.

A priori, the unconditional Type I error of the bootstrap procedure is

$$
\mathcal{S}_{b}(\pi)=\sum_{m=0}^{N} \mathcal{S}_{b}(m, \pi) \operatorname{Pr}(m \mid \pi) .
$$

Let $\pi=\left(\pi_{1}, \pi_{2}, \ldots, \pi_{K}\right)$, where $\left\{\pi_{1}<\pi_{2}<\ldots<\pi_{k}\right\}$, denotes a specific alternative hypothesis. Conditional on $m$, the power of the bootstrap procedure to reject this alternative is

$$
\mathcal{P}_{b}(m, \pi)=\sum_{\substack{\boldsymbol{x} \in \Gamma \\
(\boldsymbol{x}) \geq t_{b}(m)}} \prod_{j=1}^{K}\left(\begin{array}{l}
n_{j} \\
x_{j}
\end{array}\right) \pi_{j}^{x_{j}}\left(1-\pi_{j}\right)^{n_{j}-x_{j}} .
$$

A priori, the unconditional power of the bootstrap procedure is

$$
\mathcal{P}_{b}(\pi)=\sum_{m=0}^{N} \mathcal{P}_{b}(m, \pi) \operatorname{Pr}(m \mid \pi)
$$

Size and Power of the Permutation Procedure

The permutation procedure differs from bootstrap procedure in the following way. In the bootstrap approach, the nuisance parameter $\pi$ under the null hypothesis was eliminated by substituting its MLE, $\hat{\pi}=m / \mathrm{N}$. In contrast, the permutation approach eliminates $\pi$ by conditioning on $m$, its sufficient statistic. Let $\Gamma(m)$ denote all tables $x$ $\in \Gamma$ for which the sum of entries in row lequals $m$. Then, after eliminating sampling error by letting the number of Monte Carlo samples $M$ be infinite, the permutation distribution of the Cochran-Armitage statistic is

$$
\operatorname{Pr}\{T(x)=t \mid m\}=\sum_{\substack{\boldsymbol{x} \in \boldsymbol{\Gamma}(\boldsymbol{m}) \\
\boldsymbol{T}(\boldsymbol{X})=t}} \frac{\prod_{j=1}^{K}\left(\begin{array}{l}
n_{j} \\
x_{j}
\end{array}\right)}{\left(\begin{array}{l}
N \\
m
\end{array}\right)}
$$

Let $\mathrm{t}_{\mathrm{e}}(m)$ be the level- $\alpha$ cut-off of the permutation distribution (2.23).That is,

$$
\sum_{\substack{x \in \Gamma(5) \\
T(\boldsymbol{x}) \geq t_{e}(m)}} \frac{\prod_{j=1}^{K}\left(\begin{array}{l}
n_{j} \\
x_{j}
\end{array}\right)}{\left(\begin{array}{l}
N \\
m
\end{array}\right)} \leq \alpha
$$

$$
\sum_{\substack{\boldsymbol{x} \in \Gamma(5) \\
T(\boldsymbol{X}) \geq t}} \frac{\prod_{j=1}^{K}\left(\begin{array}{l}
n_{j} \\
x_{j}
\end{array}\right)}{\left(\begin{array}{l}
N \\
m
\end{array}\right)}>\alpha
$$

Conditional on $m$ the true size or Type I error of the permutation procedure is

$$
\mathcal{S}_{e}(m, \pi)=\sum_{\substack{\boldsymbol{x} \in \Gamma(m) \\
T(\boldsymbol{x}) \geq t_{e}(m)}} \frac{\prod_{j=1}^{K}\left(\begin{array}{l}
n_{j} \\
x_{j}
\end{array}\right)}{\left(\begin{array}{l}
N \\
m
\end{array}\right)}
$$

A priori, the unconditional Type I error of the permutation procedure is

$$
\mathcal{S}_{e}(\pi)=\sum_{m=0}^{N} \mathcal{S}_{e}(m, \pi) \operatorname{Pr}(m \mid \pi)
$$

The conditional power of the permutation procedure to reject the alternative hypothesis $\left\{\pi_{1}<\pi_{2}<\ldots<\pi_{\mathrm{K}}\right\}$ given $m$ is

$$
\mathcal{P}_{e}(m, \pi)=\sum_{\substack{x \in \Gamma(m) \\
\tau(x) \geq t_{e}(m)}}\left[\frac{\prod_{j=1}^{K}\left(\begin{array}{l}
n_{j} \\
x_{j}
\end{array}\right) \pi_{j}^{x_{j}}\left(1-\pi_{j}\right)^{n_{j}-x_{j}}}{\sum_{y \in \Gamma(m)} \prod_{j=1}^{K}\left(\begin{array}{l}
n_{j} \\
y_{j}
\end{array} \pi_{j}^{y_{j}}\left(1-\pi_{j}\right)^{n_{j}-y_{j}}\right.}\right]
$$

A priori, the unconditional power of the permutation procedure is

$$
\mathcal{P}_{e}(\pi)=\sum_{m=0}^{N} \mathcal{P}_{e}(m, \pi) \operatorname{Pr}(m \mid \pi)
$$

Size and Power of the Asymptotic Procedure

The asymptotic procedure is very similar to the permutation procedure except that the numerically intensive computation of the level- $\alpha$ cut-off value is no longer required because the exact null permutation distribution (2.23) is replaced by its normal approximation. We have shown in Corcoran, et al. (2000), that the first two moments of this conditional distribution are

$$
E(T \mid m)=\left(\frac{m}{N}\right) \sum_{j=1}^{K} d_{j} n_{j}
$$

and

$$
\operatorname{Var}(T \mid m)=\left[\frac{m(N-m)}{N(N-1)}\right] \sum_{j=1}^{K}\left[d_{j}-\frac{E(T \mid m)}{m}\right]^{2} n_{j}
$$

and for any $\mathrm{t}<\mathrm{t}_{\mathrm{e}}(m)$ 
Assuming that the conditional distribution (2.23) is asymptotically normal with the above two moments, the level- $\alpha$ cut-off is evaluated as

$$
t_{\alpha}(m)=E(T \mid m)+z_{\alpha} \operatorname{Var}(T \mid m)
$$

where $Z_{\alpha}$ is the upper $\alpha$ percentile of the standard normal distribution.

Conditional on $m$ the true size or Type I error of the asymptotic procedure is

$$
\mathcal{S}_{a}(m, \pi)=\sum_{\substack{\boldsymbol{x} \in \Gamma(m) \\
T(\boldsymbol{X}) \geq t_{a}(m)}} \frac{\prod_{j=1}^{K}\left(\begin{array}{l}
n_{j} \\
x_{j}
\end{array}\right)}{\left(\begin{array}{l}
N \\
m
\end{array}\right)}
$$

A priori, the unconditional Type I error of the permutation procedure is

$$
\mathcal{S}_{a}(\pi)=\sum_{m=0}^{N} \mathcal{S}_{a}(m, \pi) \operatorname{Pr}(m \mid \pi)
$$

The conditional power of the asymptotic procedure to reject the alternative hypothesis $\left\{\pi_{1}<\pi_{2}<\ldots<\pi_{\mathrm{K}}\right\}$ given $m$ is

$$
\mathcal{P}_{a}(m, \pi)=\sum_{\substack{\boldsymbol{x} \in \Gamma(m) \\
T(\boldsymbol{x}) \geq t_{a}(m)}}\left[\frac{\prod_{j=1}^{K}\left(\begin{array}{c}
n_{j} \\
x_{j}
\end{array}\right) \pi_{j}^{x_{j}}\left(1-\pi_{j}\right)^{n_{j}-x_{j}}}{\sum y \in \Gamma(m) \prod_{j=1}^{K}\left(\begin{array}{c}
n_{j} \\
y_{j}
\end{array}\right) \pi_{j}^{y_{j}}\left(1-\pi_{j}\right)^{n_{j}-y_{j}}}\right]
$$

A priori, the unconditional power of the asymptotic procedure is

$$
\mathcal{P}_{a}(\pi)=\sum_{m=0}^{N} \mathcal{P}_{a}(m, \pi) \operatorname{Pr}(m \mid \pi)
$$

\section{Results}

Having formulated the unconditional power for the bootstrap, exact permutation, and asymptotic tests, we revisit the data of Table 1. Recall that the exact bootstrap p-value for these data is 0.0954 , the exact permutation $p$-value is 0.0546 , and the asymptotic $\mathrm{p}$-value is 0.0273 . At a 0.05 significance level, the three tests yield different substantive results. Observing the operating characteristics of each of the three tests may allow us to understand the apparent contradiction.

Computing the unconditional size and power of these three tests under any specific setting, however, is computationally challenging. Mehta, et al. (1998) first solved the problem of obtaining the quantities (2.27) and (2.29) by using a network algorithm to evaluate the distribution shown in (2.23), and hence to obtain $\mathrm{t}_{\mathrm{e}}(m)$. Corcoran, et al. (2000) extended this algorithm to enable computation of (2.34) and (2.36). These methods are currently available in the software package StatXact-5 (2001). Without an efficient tool such as a network algorithm, obtaining the critical value $t_{b}(m)$, as defined by equations (2.17) and (2.18), can likewise pose a difficult problem. In the Appendix we describe how one can use the network approach to find the exact conditional power of the bootstrap test. This algorithm provides a tool that-combined with the algorithms previously developed for the permutation and asymptotic tests - allows a researcher to assess the relative characteristics of these tests, under any set of conditions, without resorting to simulation or approximation.

We apply this approach to the design shown in Table 1. Figure 1 shows the actual Type I error as a function of the quantity $\pi$ under the null hypothesis (2.13). As the design is perfectly balanced, we need only plot size for $0<\pi=0.50$. Plot (a) of Figure 1 shows the actual Type I error of the three tests when the doses of (1.3) are $\left(d_{1}, d_{2}, d_{3}, d_{4}\right)=(0,1,2,3)$, plot (b) uses doses of $\left(d_{1}, d_{2}\right.$, $\left.d_{3}, d_{4}\right)=(0,1,2,4)$, and plot (c) uses dose scores of $\left(d_{1}, d_{2}\right.$, $\left.d_{3}, d_{4}\right)=(0,1,5,50)$. In all three plots, the asymptotic test violates the nominal significance level for most values of $\pi$. Under the dose scores used for plots (a) and (b), the asymptotic test violates the nominal significance level for $\pi$ greater than approximately 0.08 . For dose scores of $(0$, $1,5,50)$, the asymptotic test violates the nominal significance level for $\pi$ greater than approximately 0.05 . As expected, the exact test preserves the nominal significance level - never attaining the 0.05-level exactly due to the discreteness of the tail distribution. The bootstrap method, however,is comparatively very conservative.

Having examined the true significance levels of each test, we now compare the procedures with respect to power. Figures 2, 3, and 4 contain power plots for dose scores of $\left(d_{1}, d_{2}, d_{3}, d_{4}\right)=(0,1,2,3),\left(d_{1}, d_{2}, d_{3}, d_{4}\right)=(0$, $1,2,4)$, and $\left(d_{1}, d_{2}, d_{3}, d_{4}\right)=(0,1,5,50)$, respectively. Each of these three figures consists of four plots, corresponding to four values of $\pi_{1}: 0.01,0.05,0.10$, and 0.25 . For the sake of simplicity, each curve is plotted as a function of the parameter $\beta$ from the logistic dose-response model given by $\operatorname{logit}\left(\pi_{\mathrm{i}}\right)=\gamma+\beta \mathrm{d}_{\mathrm{i}}$, for $i=1,2,3,4$, where $\gamma=\operatorname{logit}\left(\pi_{1}\right)$ and $\operatorname{logit}(x)=\log [x /(1-x)]$.

From Figure 1 we observe - under any of the three sets of dose scores examined here - that the asymptotic test violates the nominal Type I error rate for $\pi_{1}=0.10$ and $\pi_{1}=0.25$, making this procedure viable only for $\pi_{1}=$ 0.01 and $\pi_{1}=0.05$. For these smaller choices of $\pi$, the asymptotic test indeed demonstrates a power advantage over the other two tests. However, with respect to the 
(a)

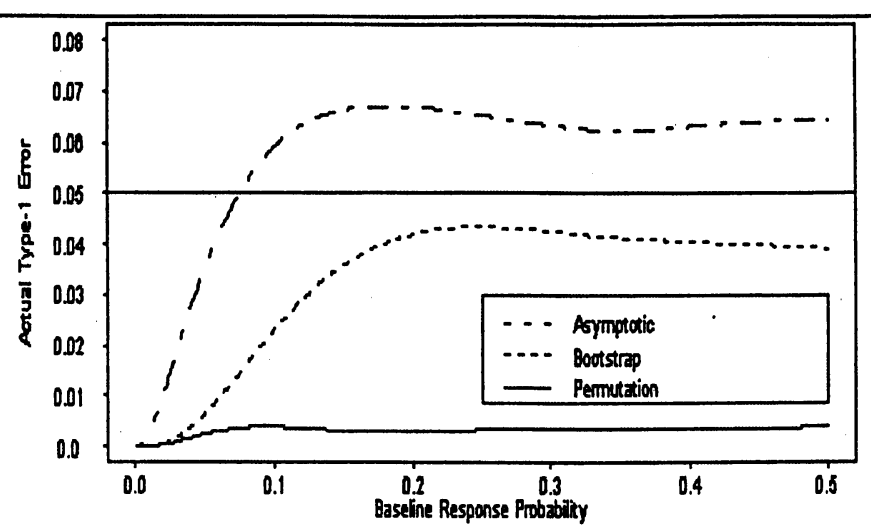

(b)

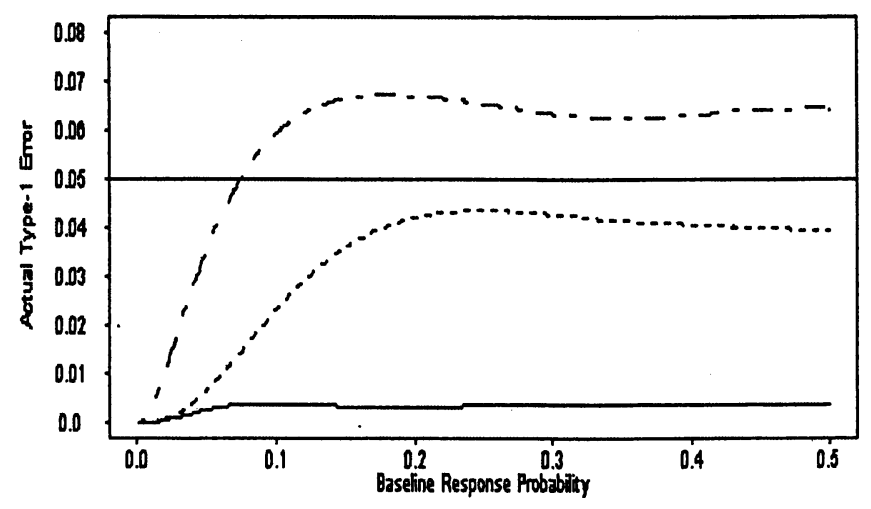

(c)

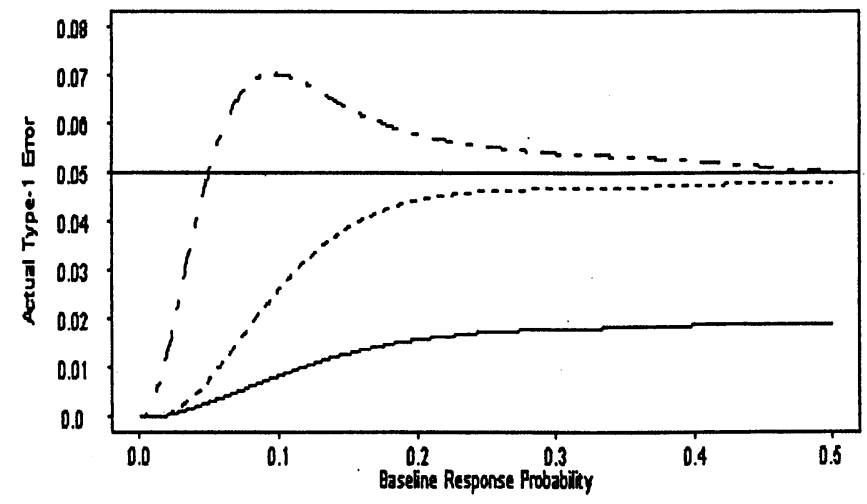

Figure 1: Actual type-1 error rate for asymptotic, permutation, and bootstrap trend tests when $K=4$, $n_{i}=10$ for $i=1,2,3,4$, and dose scores $\left(d_{1}, d_{2}, d_{3}, d_{4}\right)$ are (a) $(0,1,2,3),(\mathrm{b})(0,1,2,4)$, and (c) $(0,1,5,50)$.

experiment shown in Table 1, under the null hypothesis our best guess at the common response probability $\pi$ is 5 $/ 40=0.125$. As the asymptotic test exceeds the nominal significance level for probabilities in this range, one might have less faith in the accuracy of its associated $p$-value.

The exact power of the bootstrap procedure is clearly dominated by the permutation test under all conditions considered here, particularly when dose scores are equally-spaced (Figure 2) or almost equally-spaced (Figure 3).The conservatism and comparatively low power of the bootstrap procedure explain the relatively high $\mathrm{p}$ value obtained for the data of Table 1 . 


\section{Conclusion}

The primary purpose of a sample size calculation is to ensure that a study has sufficient power to detect a specific effect size. For example, when investigating a dose-response relationship of the form $\operatorname{logit}\left(\pi_{i}\right)=\gamma+\beta d_{i}$, one would typically have in mind a biologically or clinically meaningful slope, $\beta_{a}$ say, above which one could claim the existence of a trend in the data. The power of any test is the probability that the test will reject the null hypothesis that $\beta=0$ when in fact $\beta=\beta_{a}$. We have developed computational tools that, without resorting to approximations or simulations, can provide the exact power of three different tests of trend; permutation, bootstrap and asymptotic. It is seen that the test with the highest power is the asymptotic test, followed closely by the permutation test. The bootstrap test has considerably lower power than the others.

We have also developed computational tools for evaluating the exact Type I error of the three tests of trend. This is necessary because the power comparisons amongst the three tests are only meaningful if their Type I errors, or probabilities of falsely rejecting the null hypothesis, are bounded by the same value. We typically fix the Type I error bound at $\alpha=0.05$. The permutation test, being exact, is guaranteed to not exceed this error bound. To see this observe from equation (2.27) that the exact Type I error, $S_{\mathrm{e}}(\pi)$, for the permutation test is a weighted sum of terms of the form $S_{\mathrm{e}}(m, \pi)$, where, by equation (2.24) each such term cannot exceed $\alpha$, and the weights, $\operatorname{Pr}(m \mid \pi)$ are probabilities that sum to 1 . Therefore $S_{\mathrm{e}}$ cannot exceed $\alpha$ either, and the Type I error is guaranteed to be preserved. We cannot make the same argument for the bootstrap type1error, $S_{\mathrm{b}}(\pi)$, or for the asymptotic Type I error, $S_{\mathrm{a}}(\pi)$.

Figure 1 demonstrates that, for the entire range of the baseline response probability $\pi$, the Type I error of the permutation test is preserved. Figure 1 also reveals that the Type I error of bootstrap test remains below the stipulated 0.05 level throughout the range of the baseline response probability. This is an interesting finding because

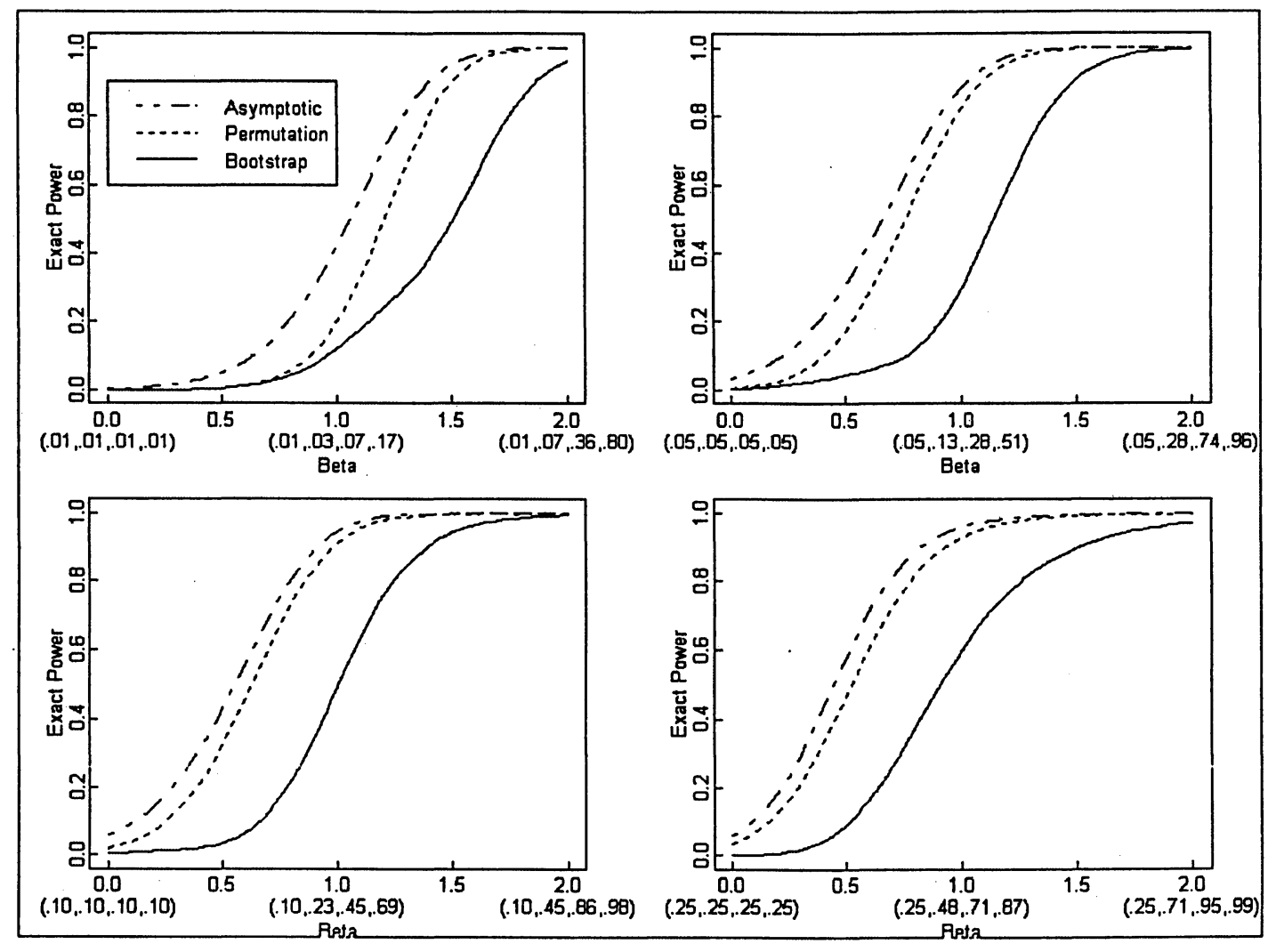

Figure 2: Exact power for asymptotic, permutation, and bootstrap trend tests when $K=4, n_{i}=10$ for $i=1,2,3,4$, and dose scores $\left(d_{1}, d_{2}, d_{3}, d_{4}\right)=(0,1,2,3)$, for (clockwise from upper left) $\pi_{1}=0.01$, $\pi_{1}=0.05, \pi_{1}=0.10$, and $\pi_{1}=0.25$. Power is computed as a function of $\beta$, based upon the logistic dose-response model $\operatorname{logit}\left(\pi_{i}\right)=\gamma+\beta d_{i}$, with $\gamma=\operatorname{logit}\left(\pi_{1}\right)$. 


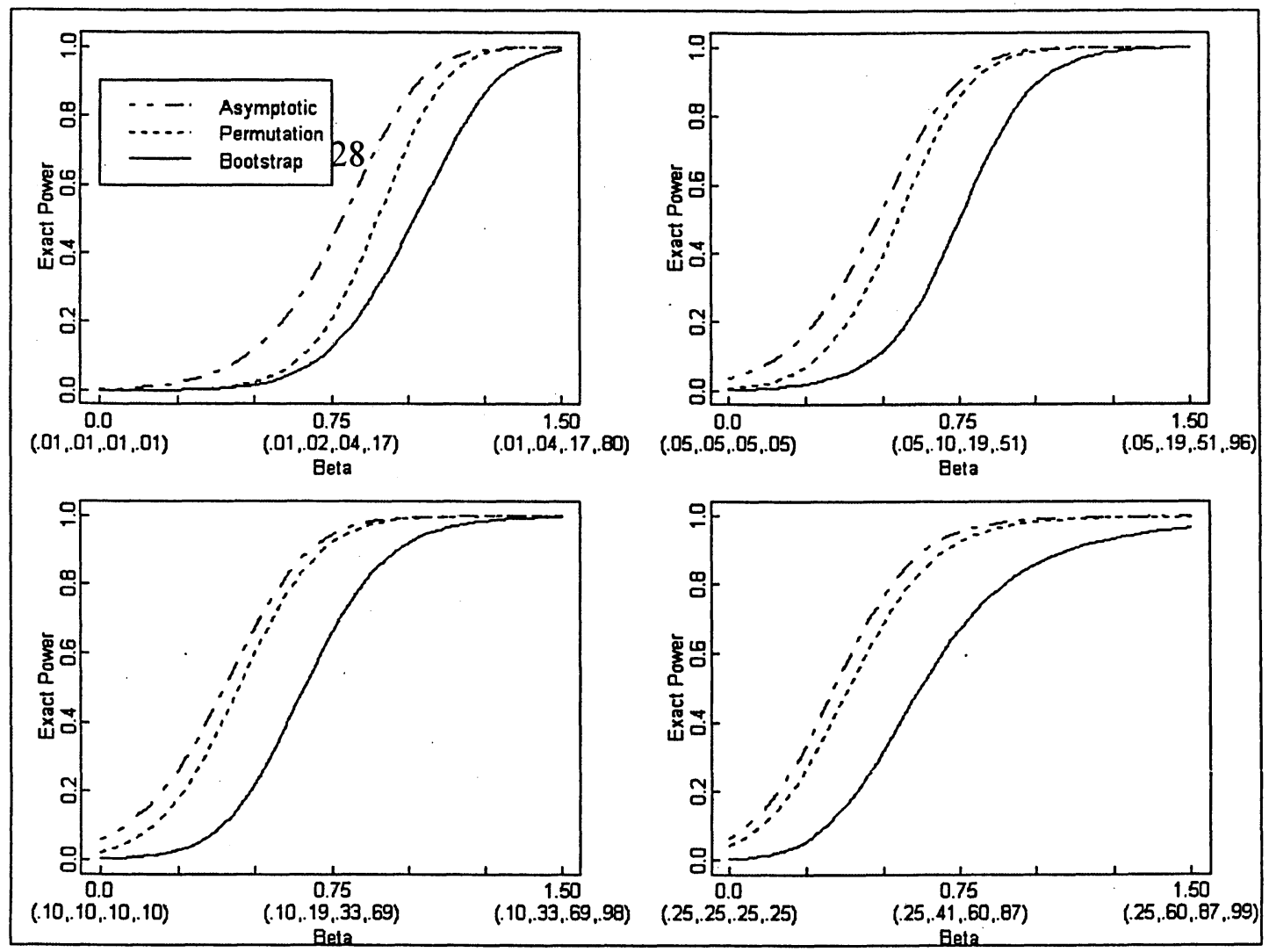

Figure 3: Exact power for asymptotic, permutation, and bootstrap trend tests when $K=4, n_{i}=10$ for $i=1,2,3,4$, and dose scores $\left(d_{1}, d_{2}, d_{3}, d_{4}\right)=(0,1,2,4)$, for (clockwise from upper left) $\pi_{1}=0.01$, $\pi_{1}=0.05, \pi_{1}=0.10$, and $\pi_{1}=0.25$. Power is computed as a function of $\beta$, based upon the logistic dose-response model $\operatorname{logit}\left(\pi_{i}\right)=\gamma+\beta d_{i}$, with $\gamma=\operatorname{logit}\left(\pi_{1}\right)$.

the bootstrap test is not exact and therefore not guaranteed to preserve the Type I error. Unfortunately, as shown in Figure 1, the exact Type I error of the bootstrap test is very much below 0.05 for the cases considered here. This extreme conservatism results in a substantial deterioration of power for the bootstrap test relative to the permutation test, as is evident from Figures 2 to 4 . Thus, one would never choose the bootstrap test in preference to the permutation test in a dose-response setting. This was not known previously and it was generally held that the two procedures have more or less the same operating characteristics.

The power comparisons between the permutation and asymptotic test are not as unambiguous. Although the asymptotic test is uniformly more powerful than the permutation test, Figure 1 shows that it does not preserve Type I errors. However, for very small values $\pi$, the Type I error is preserved and, if one could determine a priori that this nuisance parameter is suitably small, one might be justified in adopting the asymptotic test. This is an important finding that could only be discovered because of the availability of a computational tool for evaluating the exact power of an asymptotic test. The computational tool that we have developed can therefore be very helpful, not merely for evaluating the power of various exact and asymptotic tests, but also for determining conditions under which one might actually prefer to use an asymptotic test - because of its superior operating characteristics - rather than its exact permutational counterpart.

\section{References}

Armitage, P. (1955). Tests for linear trends in proportions and frequencies. Biometrics, 11, 375-386.

Cochran, W, G. (1954). Some methods for strengthening the common $\chi^{2}$ tests. Biometrics, 10, 417451.

Corcoran, C. D., Mehta, C. R., \& Senchaudhuri, P. (2000). Power comparisons for tests of trend in doseresponse studies. Statistics in Medicine, 19, 3037-3050.

Mehta, C. R., Patel, N. R., Senchaudhuri, P. (1998). Exact power and sample-size computations for the Cochran-Armitage trend test. Biometrics, 54, 1615-1621. 

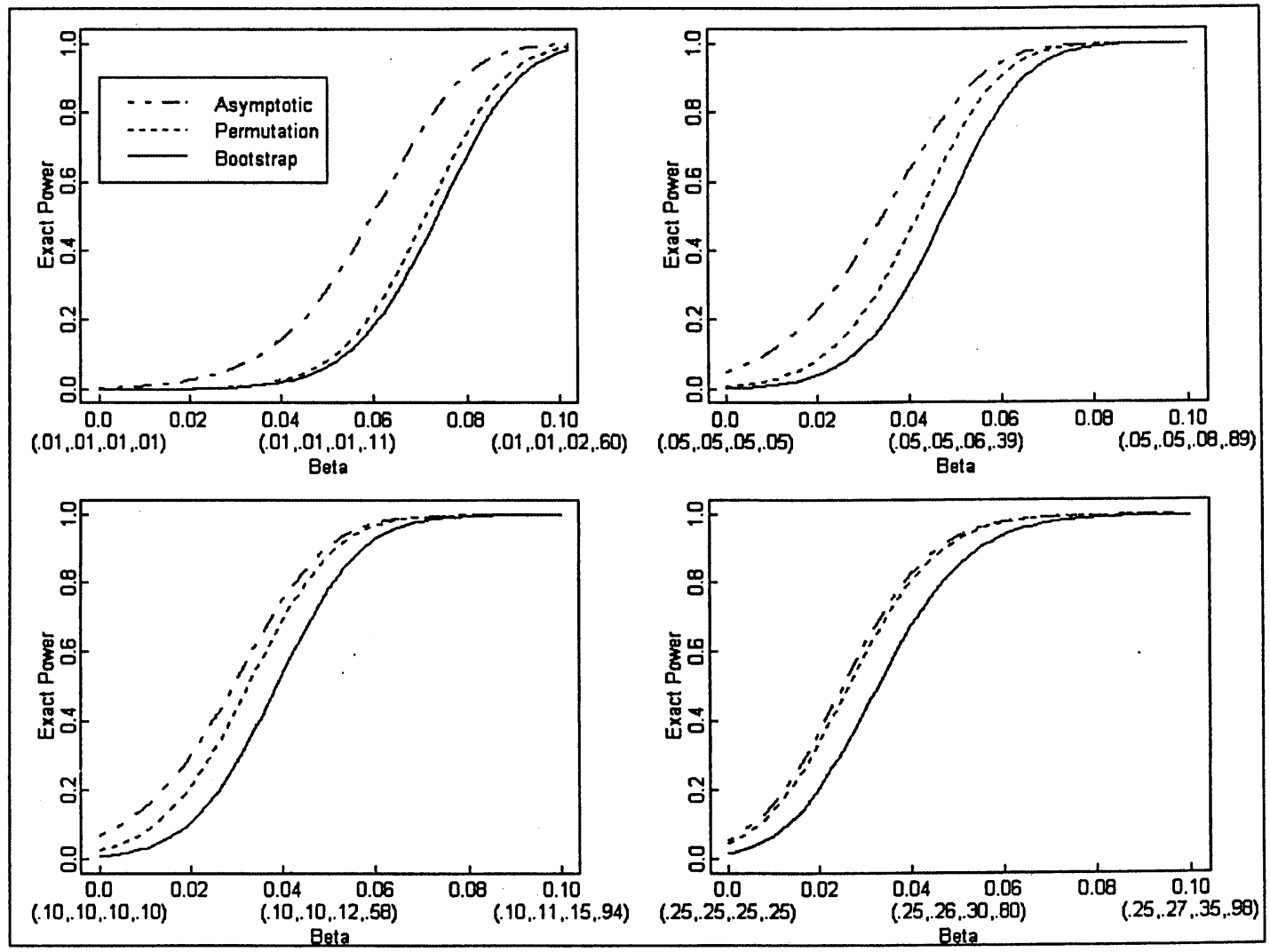

Figure 4: Exact power for asymptotic, permutation, and bootstrap trend tests when $K=4, n_{i}=10$ for $i=1,2,3,4$, and dose scores $\left(d_{1}, d_{2}, d_{3}, d_{4}\right)=(0,1,5,50)$, for (clockwise from upper left) $\pi_{1}=0.01$, $\pi_{1}=0.05, \pi_{1}=0.10$, and $\pi_{1}=0.25$. Power is computed as a function of $\beta$, based upon the logistic dose-response model $\operatorname{logit}\left(\pi_{i}\right)=\gamma+\beta d_{i}$, with $\gamma=\operatorname{logit}\left(\pi_{1}\right)$.

StatXact (2001). A software package for exact non-parametric inference. Versions 4 \& 5. Cytel Software Corporation: Cambridge, MA.

\section{Appendix}

Obtaining the Exact Power of the Bootstrap Test using the Network Algorithm

For a given $m$, it is sufficient to obtain the upper critical value $t_{b}(m)$ of the exact bootstrap tail distribution. We will follow the notation in the Appendix of Corcoran, et. al (2000), in toto:

1. For any $2 \times \mathrm{K}$ table with fixed $\mathrm{m}$, build a network as described with the following exceptions and additions:

(a) Augment the table with one column $-\mathrm{a}(\mathrm{K}+1)^{\mathrm{st}}$ column - such that the marginal total of the new column is $\mathrm{N}$, the total sample size of the new table is $2 \mathrm{~N}$, and the marginal total of the first row of the new table is $m+\mathrm{N}$, where $\mathrm{N}=\sum_{\mathrm{i}=1}^{\mathrm{k}} n_{\mathrm{i}}$.

(b)For the augmented table, $d_{K+1}=0$.

(c)For arcs connecting a node of the network to a successor node, define a new probability length where

$$
\hat{p}_{0, j+1}=\frac{n_{j+1}}{x_{j+1}}(\hat{\pi})^{x_{j+1}}(1-\hat{\pi})^{n_{j+1}-x_{j+1}},
$$

where $\hat{\pi}=\mathrm{m} / \mathrm{N}$.

2. Carry out the Backward induction pass as prescribed, with the following changes:

(a)In step 2 of the backward induction pass, define $\hat{\mathrm{TP}}_{0}\left(j, m_{j}\right)$ as the sum of the probability lengths (computed using the arc lengths $\hat{p}_{0, j+1}$ ) of all paths in $\Gamma_{m}\left(j, m_{j}\right)$.

(b)In step 3 of the backward induction pass, let $\hat{\mathrm{TP}}_{0}(K+1, m+N)=1$. 
(c)In lieu of steps 4(b) and 4(c) of the back ward induction pass, let

$$
\hat{\mathrm{TP}}_{0}\left(\mathrm{j}, \mathrm{m}_{\mathrm{j}}\right)=\sum \Gamma_{\mathrm{m}\left(\mathrm{j}, \mathrm{m}_{\mathrm{j}}\right)} \hat{\mathrm{p}}_{0, \mathrm{j}+1} \hat{\mathrm{TP}}_{0}\left(\mathrm{j}+1, \mathrm{~m}_{\mathrm{j}+1}\right)
$$

3. Carry out the Forward pass as prescribed, with the following alterations:

(a)In step 3 of the forward pass, define $\hat{p}_{0}(\tau)=\prod_{l=1}^{j} \hat{p}_{0, l}($ there is no need to compute $p_{0}(\tau)$ or $\left.p_{1}(\tau)\right)$.

(b)In step 4 of the forward pass, define

$$
\hat{c}_{i}(u)=\sum_{\substack{\tau \in \Upsilon_{m}\left(j, m_{j}\right), \tau(\tau)=u}} \hat{p}_{i}(\tau) .
$$

(There is no need to keep track of $c_{0}(u)$ or $c_{1}(\mathrm{u})$.
Use $\hat{C}_{i}(u)$ for steps 5, 6, and 7.)

(c)In step 8 of the forward pass, choose $t_{b}(m)$ to be the smallest $u^{*}$, given the nominal significance level $\alpha$, such that

$$
\sum_{\substack{u \in \Omega(K+1, m+N) \\ u \geq u^{*}}} \frac{\hat{c}_{0}(u)}{\hat{\operatorname{TP}}_{0}(0,0)} \leq \alpha
$$

4. Using the original $2 \times \mathrm{K}$ table, follow the prescribed backward and forward induction passes exactly, this time replacing $t(m)$ in step 1 of the forward pass with the critical value $t_{b}(m)$. 


\title{
A Measure Of Relative Efficiency For Location Of A Single Sample
}

\author{
Shlomo S. Sawilowsky \\ Evaluation \& Research \\ College of Education \\ Wayne State University
}

The question of how much to trim or which weighting constant to use are practical considerations in applying robust methods such as trimmed means (L-estimators) and Huber statistics (M-estimators). An index of location relative efficiency (LRE), which is a ratio of the narrowness of resulting confidence intervals, was applied to various trimmed means and Huber M-estimators calculated on seven representative data sets from applied education and psychology research. On the basis of LREs, lightly trimmed means were found to be more efficient than heavily trimmed means, but Huber M-estimators systematically produced narrower confidence intervals. The weighting constant of $\psi=1.28$ was found to be superior to various competitors suggested in the literature for $\mathrm{n}<50$.

Keywords: Huber M-estimator, $\psi$, Trimmed mean, Point estimator, Relative efficiency

\section{Introduction}

The development of robust methods in the past thirty-five years has produced a plethora of modern techniques for succinctly describing the most salient characteristics of a data set. With regard to measures of central tendency (location), the familiar mean, median, mode, and geometric mean have been augmented with L-estimators ( $L$ inear combination of order statistics), M-estimators (generalized Maximum likelihood), and R-estimators (inverted tests on $R$ anks of absolute values).These modern methods are examples of the "outright rejection" and the "accommodation" approaches to handling outliers (Barnett \& Lewis, 1994, p. 29). To understand the concepts of these robust methods, the mathematically inclined reader is referred to Hampel, et al. (1986), Hoaglin, Mosteller, and Tukey (1983), Huber (1981), or Staudte \& Sheather (1990). An excellent "first course" textbook introducing L- and Mestimators is Wilcox (1996).

Consider trimmed means, which are typical of Lestimators. The trimmed mean is calculated by sorting the data set, trimming a certain percentage of observations from the top and bottom of the scores, and calculating the average of the remaining scores. For example, the arithmetic mean of a data set containing the scores $75,71,70,76,72$, $73,73,70,30$, and 74 is 68.4 . A $2 \times 10 \%$ trim (symmetric trim of $10 \%$ of the smallest and largest observations) is calculated as follows:

Shlomo S. Sawilowsky, Wayne Statue University Distinguished Faculty Fellow, is Professor and Chair, Educational Evaluation and Research, 351 College of Education, Wayne State University, Detroit, MI, 48202, e-mail: shlomo@edstat.coe.wayne.edu. His interests are in nonparametric, robust, exact, permutation, and other computerintensive methods, especially via Fortran.
1. sort observations: $30,70,70,71,72,73,73,74,75,76$

2. trim $10 \% \times 10$ scores $=1$ score from both ends: 70,70 , $71,72,73,73,74,75$

3. calculate average:

$$
\frac{70+70+71+72+73+73+74+75}{8}=72.25
$$

In this example, the $2 \times 10 \%$ trimmed mean is shown to be resistant to the extreme score (30), resulting in a value of 72.25 which is more indicative of bulk of the scores. The arithmetic mean, in contradistinction, chased after the extreme low score, resulting in a measure of location that was lower than ninety percent of scores in the data set. Thus, the arithmetic mean is said to have a low breakdown point, because it is strongly influenced by even a single value, such as an outlier.

The data analyst might wonder if a different amount of trim would improve the estimate of location. The literature on this question is divided into two camps: the "heavily trim" (e.g., a $2 \times 25 \%$ trim was recommended by Rosenberger \& Gasko, 1983, p. 332-333; a 2×20\% trim was adopted by Wilcox, 1996, p. 16; 1998) and the "lightly trim" (either a $2 \times 10 \%$ or $2 \times 5 \%$ trim, considered by Hill \& Dixon, 1982; Huber, 1977, p. 1090; Stigler, 1977, p. 1063; Staudte \& Sheather, 1990, p. 133-134). Simulation evidence on a contaminated normal distribution indicated that the variances of trimmed means were minimized (and are thus one measure of the optimum trim or "optrim") for sample size $n=10$ when the trim was $16.1 \%$; for samples of size $\mathrm{n}=20$ it was almost half as much, as the optrim was $8.7 \%$ (Rosenberger \& Gasko, 1983, p. 319). The variance of estimators was minimized for data sampled from the double exponential and Cauchy distributions for samples of size $n=10$ with optrims of $34 \%$ and $40 \%$ (p. 330), respectively, and was $37 \%$ and $39 \%$ (p. 331), respectively, for samples of size $n=20$. Wilcox (1996) noted, "Currently there is no way of being certain how much trimming 
should be done in a given situation, but the important point is that some trimming often gives substantially better results, compared to no trimming" (p. 16).

The problem of selecting parameters in the robust measures literature, such as how much to trim, is not restricted to L-estimators. As an example with M-estimators, there are many choices pertaining to the weighting constant $\psi$ (also referred to as the bending constant or the tuning factor) used in the one-step Huber M-estimator. The M-estimator has a high breakdown point, determines empirically how much and from which side to trim, and has other desirable properties (Wilcox, 1996, p. 146, 204).

The formula for the one-step Huber M-estimator, with a weighting constant of $\psi=1.28$, is

Huber $_{\psi 1.28}$ M-Estimator $=$

$$
\frac{1.8977(M A D)\left(i_{2}-i_{1}\right)+\sum_{i=i_{1}+1}^{n-i_{2}} x_{i}}{n-i_{1}-i_{2}}
$$

It is calculated on the ten scores as follows:

1. sort observations: $30,70,70,71,72,73,73,74,75,76$

2. calculate median: 72.5

3. calculate MAD (Median Absolute Difference):

a. calculate $\mid x_{i}$ - median $|:| 30-72.5|=42.5|$,70 -

$72.5|=2.5| 70-,72.5|=2.5| 71-,72.5|=1.5| 72-$,

$72.5|=.5| 73-,72.5|=.5| 73-,72.5|=.5| 74-,72 . \mid=1.5$,

$|75-72.5|=2.5,|76-72.5|=3.5$

b. sort results: $.5, .5, .5,1.5,1.5,2.5,2.5,2.5$, $3.5,42.5$

c. $\mathrm{MAD}=$ median from step $\mathrm{b}: 2$

4. calculate $\frac{.6745\left(x_{i}-\text { median }\right)}{M A D}$

$\frac{.6745(30-72.5)}{2}=-14.333$

$\frac{.6745(70-72.5)}{2}=-.843$

$\frac{.6745(70-72.5)}{2}=-.843$

$\frac{.6745(71-72.5)}{2}=-.506$

$\frac{.6745(72-72.5)}{2}=-.169$

$\frac{.6745(73-72.5)}{2}=.169$

$$
\begin{aligned}
& \frac{.6745(73-72.5)}{2}=.169 \\
& \frac{.6745(74-72.5)}{2}=.506 \\
& \frac{.6745(75-72.5)}{2}=.843 \\
& \frac{.6745(76-72.5)}{2}=1.180
\end{aligned}
$$

5. count $i_{1}$ (number of values in step $4<-1.28$ ):

1 (observation a)

6 . count $i_{2}$ (number of values in step $4>1.28$ ):

0

7. calculate Huber ${ }_{\psi 1.28:}$

$=\frac{1.8977(2)(0-1)+(70+70+71+72+73+73+74+75+76)}{10-1-0}$
$=72.24$

The one-step M-estimator is nearly the same as the median in this illustration. Wilcox noted that typically the M-estimator is between the median and the mean (Wilcox, 1996, p. 147). (The constants .6745 and 1.8977 appearing in the calculation of the one-step Huber ${ }_{\psi 1.28} \mathrm{M}$ estimator refer to the inverse cumulative distribution function for $x=0.75$ for the standard normal curve $(\mu=0, \sigma=$ $1)$, and $\frac{\psi}{.6745}$, respectively. This formula, and its constants, are given by Wilcox, 1996, p. 147. It should be pointed out that the second constant, 1.8977 , pertains only to $\psi=$ 1.28. For example, use 2.2239 in calculating the one-step Huber $_{\psi 1.5}$ M-estimator.)

Some commonly used weights for the one-step Huber M-estimator include the following: (a) 1.28, the value used in the illustration, which corresponds to the .9 quantile of the standard normal distribution (Staudte \& Sheather, 1990, p. 117), (b) 1.339, used by a popular statistics software package (SPSS, 1996), (c) 1.345, because it represents the " 0.95 asymptotic efficiency on the standard normal distribution" (Rey, 1980, p. 108-109), (d) 1.4088 (Hampel, et al., 1983, p. 167), and (e) 1.5, (Andrews, et al., 1972, p. 13), which Stigler (1977) stated "does particularly well" (p. 1064). Other values are cited less frequently in the literature (e.g., Huber, 1964, p. 8485 , examined the upper bounds of the asymptotic variance for $\psi=0-3.0(.1)$ ), but in the absence of current usage they are not considered further.

The question remains as to which weighting constant should be used in practice. As noted by Lee (1995), the efficiency of M-estimation will "depend on the choice 
of $\psi$ " (p. 521). The casual approach in choosing $\psi$ was summarized by Hogg (1977), who stated that for optimum performance, "let the $\mathrm{k}[\psi]$ of the Huber statistic decrease as measures of skewness and kurtosis... increase; that is, for illustration, use Huber's P20 [ $\psi=2.0], \mathrm{P} 15$ [ $\psi=1.5]$, and $\mathrm{P} 12$ [ $\psi=1.2]$, respectively, as those measures increase. Users of the M-estimators actually seem to do this in practice anyway... to suit the problems at hand" (p. 1089, bracketed material added for clarification). The casual approach is obviously not satisfactory.

\section{Location Relative Efficiency (LRE)}

The issues raised above in terms of how much to trim and which weighting constant to use are important because they relate to the primary question of which procedure and which parameters of a procedure to use in estimating location in the single sample problem. Stigler's (1977) approach toward discovery of the best estimator was "to measure the absolute magnitude of an estimator's error relative to the size of errors achieved by other estimators" (p. 1062) for some famous physical science data sets. However, this technique is only applicable in the improbable situation where $\theta$, the population parameter for location, is exactly known. This is necessary in order to measure the variability of error, $\varepsilon$, which is taken to be $(\theta-\hat{\theta})$, the difference between the actual location parameter and the estimated location. (For other limitations, see Andrews, 1977, p. 1079; Hoaglin, 1977, p. 1087; Huber, 1977, p. 1091; and Pratt, 1977, p. 1092.)

Another method is to quantify the comparative efficiency of robust measures with their competitors. An example is the Cramér-Rao efficiency, which is the ratio of the lower bound of a competitor with that of the best estimator. Another method, proposed by Gastwirth and Cohen (1970), is the ratio of the variance of the best estimator divided by the variance of the competitor. Their statistic is called the relative efficiency. (See Rosenberger \& Gasko, 1983, p. 327, for further discussion, and Staudte, 1980 , p. 15, for a typical application.)

A problem with these two techniques is the need to know the best estimator, which if known, of course, would obviate the initial question. The best estimator is dependent on a variety of factors, including the distribution and sample size. In practice, unfortunately, the evidence cited in favor of the best estimator is typically just an assertion. Among other difficulties, the lower bound of the Cramér-Rao efficiency is frequently impossible to obtain. The Gastwirth and Cohen relative efficiency depends on the asymptotic variance, as opposed to the actual variance. (See Hampel, et al., 1986, p. 398, for a polemic favoring asymptotic variance over the actual variance, when taken together with asymptotic normality.)

A statistic was recently proposed (Sawilowsky,
1998) for determining the comparative efficiency of a location estimator for a single sample that precludes the necessity of knowing the best estimator. It avoids problems associated with assuming asymptotic normality in order to generalize asymptotic variances to actual variances. It is not hampered by limitations associated with the arithmetic mean and its impact on actual variances. Finally, it is simple to compute. This index is called the Location Relative Efficiency (LRE).

The impetus for the LRE was from Dixon and Tukey (1968). They calculated the $95 \%$ bracketed interval for the mean and for one through five units of Winsorized means. Then, they compared the length of the intervals of the Winsorized means with the length of the confidence interval associated with Student's $t$, characterizing this ratio as the "apparent efficiency" (p. 86). This is a Type III performance measure of a bracketed interval in the classification scheme discussed by Barnett and Lewis (1994; see their discussion for Types I and II), where they noted that, "a natural measure of its efficiency is the ratio of the lengths of the intervals" ( p. 75). Indeed, Huber (1972) noted that, "While for years one had been concerned mostly with what was latter called 'robustness of validity' (that the actual confidence levels should be close to, or at least on the safe side of the nominal levels), one realized that 'robustness of performance' (stability of power, or the length of confidence intervals) was at least as important" (p. 1045-1046, emphasis added).

The LRE for the $95 \%$ bracketed interval is defined as:

$$
L R E=\frac{\text { Huber }_{\Psi_{1.28(U 95 \% C . I .)}}-\text { Huber }_{\Psi_{1.28(L 95 \% C . I .)}}}{X_{(U 95 \% C . I .)}-X_{(L 95 \% C . I .)}}
$$

where the LRE is the range ( $U$ = upper bound, $L=$ lower bound) for the $95 \%$ bracketed interval for the one-step Huber M-estimator divided by the range for the $95 \%$ bracketed interval of the competitor. The Huber $_{\psi 1.28}$ is not asserted to be the best estimator. Rather, the resulting ratio may be greater than or less than one. LREs greater than one indicate the competitor yields confidence intervals that are narrower and thus more efficient than the Huber $_{\psi 1.28}$. LREs less than one indicate the Huber $_{\psi 1.28}$ is more efficient in that it produces narrower bracketed intervals.

The LRE was used to evaluate the performance of bracketed intervals produced by a variety of procedures (Sawilowsky, 1998). Four data sets given in Staudte and Sheather (1990, p. 133-137) were analyzed and the results were as follows: Huber ${ }_{\psi 1.28}(1.000), 2 \times 10 \%$ trim (.945), Sign (.895), Wilcoxon (.869), $2 \times 5 \%$ trim (.862), and Student's $\mathrm{t}(.625)$. However, note that the location parameter $\theta$ is not necessarily the same for these varied 
procedures (although Pratt argued that 'It doesn't matter what an estimator estimates, as long as it is a location parameter", 1977, p. 1092); and these results were based on four small data sets. The data sets pertained to empirical measures of the velocity of light $(n=66)$, the percentage of seafood in a product to determine if it complied with proposed labeling guidelines $(n=18)$, the proportion of DDT in kale $(\mathrm{n}=15)$, and Darwin's data on plant height ( $\mathrm{n}$ $=15$ ).

The physical science data sets explored by Stigler (1977) leave the same question "of whether these data adequately reflect anticipated applications, for example in the social sciences. I have little to add to my previous comments on this, other than to reiterate that I would welcome evidence on this point" (Stigler, 1977, p. 1098, emphasis added). The same is true of most published simulation work in the robustness literature, which was conducted on theo- retical distributions such as the deMoivreian (Gaussian), logistic, one-out (one score has scale three times the rest), one-wild (one score has scale ten times the rest), double exponential, and Cauchy (e.g., Rosenberger \& Gasko, 1983, p. 326-330). (The reliance on theoretical distributions has led to ridiculous statements, such as Hampel et al., 1986, citing Huber to say the following about the $t$ distribution with three degrees of freedom: " $t$ is a suitable example for what high-quality data can look like", p. 23!) Micceri $(1986,1989)$ canvassed the education and psychology literature and highlighted representative distributions as the most prevalent in social and behavioral science research. (These data sets were previously investigated, in terms of their impact on the t-test, by Sawilowsky \& Blair, 1992.) Descriptive statistics on the data sets, in the order that they were presented by Micceri (1986), are compiled in Table 1. The ordering does not, however,

Table 1. Descriptive Statistics For Seven Real Data Sets from Micceri (1986).

\begin{tabular}{|c|c|c|c|c|c|c|c|c|c|}
\hline Distribution & $\mathrm{N}$ & Median & $\mu$ & SE & $\sigma$ & $\gamma_{1}$ & $\gamma_{2}$ & $\mathrm{Z}_{\mathrm{U} 95 \% \mathrm{CI}}$ & $\mathrm{Z}_{\mathrm{L} 95 \% \mathrm{CI}}$ \\
\hline \multicolumn{10}{|l|}{ Achievement } \\
\hline Smooth Symmetric & 5,375 & 13 & 13.186 & .013 & 4.907 & .005 & -.340 & 13.211 & 13.160 \\
\hline \multicolumn{10}{|l|}{ Achievement } \\
\hline Discrete Mass At Zero & 2,429 & 13 & 12.919 & .019 & 4.415 & -.034 & .312 & 12.956 & 12.881 \\
\hline \multicolumn{10}{|l|}{ Achievement } \\
\hline Extreme Asymmetry & 2,768 & 27 & 24.497 & .018 & 5.788 & -1.330 & 1.106 & 24.553 & 24.462 \\
\hline \multicolumn{10}{|l|}{ Psychometric } \\
\hline Extreme Asymmetry & 2,047 & 11 & 13.667 & .021 & 5.754 & 1.638 & 1.522 & 13.709 & 13.626 \\
\hline \multicolumn{10}{|l|}{ Achievement } \\
\hline Digit Preference & 3,063 & 535 & 536.900 & .680 & 37.644 & -.065 & -.240 & 536.981 & 536.914 \\
\hline \multicolumn{10}{|l|}{ Psychometric } \\
\hline Extreme Bimodality & 665 & 4 & 2.971 & .037 & 1.687 & -.078 & -1.696 & 3.044 & 2.899 \\
\hline \multicolumn{10}{|l|}{ Achievement } \\
\hline Multimodality Lumpy & 467 & 18 & 21.148 & .044 & 11.917 & .194 & -1.199 & 21.234 & 21.062 \\
\hline
\end{tabular}

Notes: $\mu=$ population mean, $\sigma=$ population standard deviation, $\mathrm{SE}=$ standard error of the mean, $\gamma_{1}=$ skew, $\gamma_{2}=$ kurtosis, $\mathrm{Z}_{\mathrm{U} 95 \% \mathrm{CI}}, \mathrm{Z}_{\mathrm{L} 95 \% \mathrm{CI}}=$ Upper and Lower $95 \%$ Confidence Interval based on $\mathrm{Z}$ and the SE. Parameters are reported here in accordance with Micceri (1986), who took the position that the data sets were considered of sufficient size to proxy the population. 
reflect prevalence of occurrence. Clearly, the physical science data sets and convenient theoretical distributions mentioned above have little relevance for the applied social and behavioral science researcher.

Purpose of the Study

In the current study, the data sets provided by Micceri $(1986,1989)$ are used to assess the LRE of some robust methods of estimating location of a single sample.
The first question to be considered is whether the "heavily trim" or the "lightly trim" approach is more efficient in estimating location in real education and psychology data sets. Only symmetric trimming is considered because the applied researcher will most likely not have à priori knowledge of whether or not the parent population from which the data were sampled is asymmetric, and therefore will not know which side to trim. The second question is whether Huber's M-estimator is more efficient in estimating

Table 2. Median Location Relative Efficiency For Various Robust Measures Of Location and Sample Sizes For The Real Data Sets From Micceri (1986); Huber $_{\psi 1.28}=1.000 ; 1,000$ Repetitions.

n

\begin{tabular}{lllllllllll}
\hline & \multicolumn{10}{c}{$\mathrm{n}$} \\
\cline { 2 - 9 } Statistic & 10 & 20 & 30 & 40 & 50 & 60 & 70 & 80 & 90 & 100 \\
\hline
\end{tabular}


n

\begin{tabular}{|c|c|c|c|c|c|c|c|c|c|c|}
\hline Statistic & 10 & 20 & 30 & 40 & 50 & 60 & 70 & 80 & 90 & 100 \\
\hline & \multicolumn{10}{|c|}{ Extreme Asymmetry (Psychometric) } \\
\hline Huber $_{w 1339}$ & .967 & .966 & .965 & .966 & .965 & .966 & .966 & .966 & .966 & .966 \\
\hline Huber & .964 & .962 & .962 & .962 & .962 & .963 & .962 & .962 & .962 & .962 \\
\hline Huber ${ }_{11.4088}$ & .931 & .928 & .929 & .927 & .927 & .928 & .928 & .928 & .927 & .928 \\
\hline Huber & .904 & .901 & .897 & .892 & .892 & .891 & .889 & .887 & .887 & .887 \\
\hline Trim $_{2 \times 25 \%}$ & .510 & .503 & .449 & .457 & .443 & .444 & .424 & .443 & .418 & .432 \\
\hline $\operatorname{Trim}_{2 \times 20 \%}^{2 \times 2 \% \%}$ & .510 & .473 & .417 & .421 & .404 & .408 & .394 & .401 & .386 & .389 \\
\hline $\operatorname{Trim}_{2 \times 10 \%}$ & .431 & .408 & .375 & .375 & .365 & .354 & .355 & .354 & .359 & .356 \\
\hline $\operatorname{Trim}_{2 \times 5 \%}$ & $*$ & .382 & .361 & .361 & .352 & .341 & .342 & .343 & .346 & .341 \\
\hline
\end{tabular}

Digit Preference (Achievement)

$\begin{array}{lllllllllll}\text { Huber }_{\psi 1.339} & .980 & .984 & .986 & .996 & 1.000 & .999 & .997 & .996 & .998 & 1.002 \\ \text { Huber }_{\psi 1.345} & .978 & .983 & .988 & 1.003 & 1.000 & .998 & .997 & 1.002 & 1.001 & 1.002 \\ \text { Huber }_{\psi 1.4088} & .969 & 1.000 & .994 & .999 & 1.003 & 1.003 & 1.004 & 1.004 & 1.003 & 1.006 \\ \text { Huber }_{\psi 1.5} & 1.000 & 1.000 & 1.000 & 1.007 & 1.009 & 1.010 & 1.008 & 1.011 & 1.009 & 1.010 \\ & & & & & & & & & & \\ \operatorname{Trim}_{2 \times 25 \%} & .727 & .831 & .882 & .893 & .909 & .923 & .923 & .934 & .941 & .935 \\ \operatorname{Trim}_{2 \times 20 \%} & .727 & .841 & .892 & .904 & .917 & .929 & .932 & .933 & .945 & .936 \\ \operatorname{Trim}_{2 \times 10 \%} & .786 & .895 & .932 & .950 & .962 & .968 & .973 & .974 & .981 & .979 \\ \operatorname{Trim}_{2 \times 5 \%} & * & .913 & .952 & .966 & .983 & .984 & .990 & .991 & .999 & .994\end{array}$

Extreme Bimodality (Psychometric)

$\begin{array}{lllllllllll}\text { Huber }_{\psi 1.339} & .976 & .979 & .980 & .981 & .981 & .982 & .982 & .983 & 1.000 & 1.000 \\ \text { Huber }_{\psi 1.345} & .975 & .977 & .978 & .980 & .979 & .980 & .981 & .982 & 1.000 & 1.000 \\ \text { Huber }_{\psi 1.4088} & .955 & .961 & .970 & 1.000 & 1.000 & 1.000 & 1.000 & 1.000 & 1.000 & 1.000 \\ \text { Huber }_{\psi 1.5} & 1.000 & 1.000 & 1.000 & 1.000 & 1.000 & 1.000 & 1.000 & 1.000 & 1.000 & 1.000 \\ & & & & & & & & & & \\ \operatorname{Trim}_{2 \times 25 \%} & .545 & .523 & .594 & .588 & .603 & .598 & .620 & .607 & .619 & .617 \\ \operatorname{Trim}_{2 \times 20 \%} & .545 & .609 & .651 & .675 & .678 & .687 & .702 & .704 & .707 & .720 \\ \operatorname{Trim}_{2 \times 10 \%} & .629 & .763 & .826 & .849 & .884 & .897 & .910 & .937 & .946 & .925 \\ \operatorname{Trim}_{2 \times 5 \%} & * & .851 & .964 & .959 & 1.021 & 1.013 & 1.043 & 1.056 & 1.079 & 1.043\end{array}$

Multimodality \& Lumpy (Achievement)

$\begin{array}{lllllllllll}\text { Huber }_{\psi 1.339} & .987 & 1.000 & 1.000 & 1.004 & 1.006 & 1.009 & 1.013 & 1.012 & 1.014 & 1.015 \\ \text { Huber }_{\psi 1.345} & .986 & 1.000 & 1.000 & 1.006 & 1.008 & 1.013 & 1.014 & 1.013 & 1.016 & 1.017 \\ \text { Huber }_{\psi 1.4088} & 1.000 & 1.000 & 1.013 & 1.019 & 1.019 & 1.026 & 1.029 & 1.029 & 1.032 & 1.035 \\ \text { Huber }_{\psi 1.5} & 1.000 & 1.002 & 1.027 & 1.036 & 1.035 & 1.042 & 1.049 & 1.048 & 1.053 & 1.057 \\ & & & & & & & & & & \\ \operatorname{Trim}_{2 \times 25 \%} & .657 & .662 & .714 & .708 & .734 & .726 & .741 & .731 & .745 & .740 \\ \operatorname{Trim}_{2 \times 20 \%} & .657 & .732 & .757 & .775 & .788 & .798 & .805 & .805 & .811 & .815 \\ \operatorname{Trim}_{2 \times 10 \%} & .726 & .835 & .875 & .903 & .903 & .924 & .929 & .941 & .941 & .944 \\ \operatorname{Trim}_{2 \times 5 \%} & * & .897 & .951 & .963 & .963 & .983 & .995 & .995 & 1.003 & 1.002\end{array}$


location in real education and psychology data sets with a $1.28,1.339,1.345,1.4088$, or 1.5 weighting constant. Both of these questions will be answered on the basis of LREs. That is, evidence in support of a procedure will be in the form of a more efficient or narrower confidence interval.

\section{Methodology}

A Monte Carlo program was written for Minitab (1996) Release 11.1 using the data sets from Micceri (1986) and Minitab "macros" D.2.1 (one-sample trimmed mean, p. 318-319), and D.2.3-D.2.5 (one step Huber M-estimate, p. 321-323) from Staudte and Sheather (1990). Each data set was randomly sampled to produce sample sizes of $n=$ 10 (10) 100. (See Stigler, 1977, for a contrary view on "subsampling from large data sets", p. 1057.) These sample sizes were noted to be of interest by Goodall (1983, p. 395).

For the $2 \beta$-trimmed mean (i.e, two-sided trim of $\beta$ percent), degrees of freedom $(\mathrm{df})=\mathrm{n}-[2 \beta \mathrm{n}]-1$. The $\mathrm{df}$ are due to Tukey and McLaughlin (1963). The standard error for the one-step Huber M-estimator is the square root of the estimated asymptotic variance (Staudte \& Sheather, 1990, p. 132, formula 4.6.2).

As noted by Rosenberger and Gasko (1983), "Sometimes, in order to obtain a specified amount of trimming exactly, we need to trim a fraction of an observation; for example, a $5 \%$-trimmed mean from a sample of size 10 requires trimming half of each of the largest and smallest observations" (p. 309). They accomplished this feat by "giving fractional weight" (p. 310) to the remaining fraction. They noted that "Some authors trim only an integer number of observations from each extreme" (p. 310). The debate on this issue is amazingly involved; the reader is referred to their discussion on the matter (Rosenberger \& Gasko, 1983, p. 310-311). In the current study, trimming is rounded down to the whole number, which was the approach taken by Staudte and Sheather $(1990$, p. 134) and Wilcox (1998, in press). Therefore, the $2 \times 5 \%$ trim cannot be conducted for sample size $n=10$. Another anomaly is that the $2 \times 25 \%$ and $2 \times 20 \%$ trims yield identical samples (and therefore results) when $\mathrm{n}=10$.

\section{Results}

The study proceeded as follows. The LRE was calculated for each statistic. This process was repeated 1,000 times, sampling with replacement from the data set. Then, the median LRE for each statistic was computed from the 1,000 samples. The results are compiled in Table 2 .

The first question pertained to the amount of trimming that would yield the most efficient estimator. With the exception of the extreme asymmetric psychometric data set, lightly trimmed means produced narrower confidence intervals. In the best case (i.e., extreme bimodality psychometric with $n=20$ ), the $2 \times 5 \%$ trim produced confidence intervals about $63 \%$ narrower than the $2 \times 25 \%$ trimmed mean. In general, as the sample size increased, the confidence intervals produced by the various levels of trimming converged, although in half of the data sets the results were less than satisfactory even for $n=100$ in the sense that the LREs were substantially less than 1.0.

Indeed, the first question appears to be rather moot. On the basis of LREs, trimmed means systematically performed worse than the various Hubers. The trimmed means were only competitive for $\mathrm{n} \geq 90$ with the smooth symmetric achievement and the digit preference psychometric data sets. The latter data set is essentially a smooth symmetric data set with certain scores enjoying a propensity to protrude. In the worst case (i.e., extreme asymmetry psychometric with $\mathrm{n}=60$ ), trimmed means produced confidence intervals as much as $293 \%$ wider than the Huber ${ }_{\psi 1.28^{\circ}}$. Jackson (1986) noted that "A disadvantage of both trimming and Winsorizing is that they down-weight the highest and lowest order statistics whether or not all observations are sound. Thus, a proportion of the data values are always either omitted altogether or have their values changed towards the centre of the distribution" (p. 27). Perhaps this is the reason for the poor performance.

Again, some researchers express disdain in comparing statistics which estimate different quantities, and therefore, would not compare trimmed means directly with Huber statistics. Support for those researchers who find the comparisons useful is available from Pratt (1977), who argued that "It doesn't matter what an estimator estimates, as long as it is a location parameter" (p. 1092).

The second question pertained to the choice of $\psi$ in the one-step Huber M-estimator. The results in Table 2 suggest that $\psi=1.28$ is the best choice regardless of the nature of the data set for $\mathrm{n}<50$. For situations where $50 \leq$ $\mathrm{n} \leq 100, \psi=1.28$ remains an excellent choice, although $\psi$ $=1.5$ produced narrower confidence intervals more frequently.

\section{Conclusion}

The selection of robust methods requires more consumer input than clicking on a pull-down menu in a statistical package. This is because many robust procedures require making choices, such as the amount to trim or the value of a tuning parameter. Although there are many opinions to be found in the literature on which values to use, there has not been a systematic study of the impact of these choices. For example, this article considered the bracketed interval around the location for a single sample.

The results in this article pertain to the $95 \%$ bracketed interval. It was chosen because it was the level used by Dixon and Tukey (1968) (who provided the impetus for the creation of the LRE). Another reason is, "The 95\% confidence level appears to be used more frequently in 
practice than any other level" (Hahn \& Meeker, 1991, p. 38). Obviously, the results in this study should not be generalized to other levels (e.g., $90 \%$ or $99 \%$ ).

There were some assumptions made in this paper. First, it was assumed that bracketed intervals should have a fixed length. There have been attempts to improve on fixed-length bracketed intervals with those that "adjust so that their expected length depends ... on the data" (Low, 1997, p. 2548). Second, it was assumed that the bracketed confidenc intervals should be symmetric with respect to $\hat{\theta}$.

To restate the interpretation of the LRE, values less than one indicate the length of the bracketed interval is wider for a competitor than for the Huber ${ }_{\psi 1.28}$. It is desirable that the choice of $\psi$ for constructing the numerator of the LRE predominately result in a ratio less than one, and only occasionally should a competitor stand out in terms of its comparative performance. This study showed that the value of $\psi=1.28$ met this requirement. Specifically, for samples of size $\mathrm{n} \leq 30$, the LREs were greater than 1.0 for only 4 out of 184 (2.2\%) outcomes, and for only 10 out of $240(4.2 \%)$ outcomes for $n<50$.

The results were also generally less than 1.0 for all estimators for $50 \leq \mathrm{n} \leq 100$, save the Huber $_{w 1.5}$. An advantage in maintaining $\psi=1.28$ is that its bracketed intervals were never more than $6 \%$ wider than those for $\psi=1.5$ for $50 \leq \mathrm{n} \leq 100$, whereas results for $\psi=1.5$ were as much as $13 \%$ wider than $\psi=1.28$ for certain distributions and sample sizes. (Although it would complicate a simple statistic, and therefore is not recommended, the Monte Carlo results indicate that it would be beneficial to compute the LREs with $\psi=1.28$ for $10 \leq \mathrm{n}<50$, and $\psi=1.5$ for $50 \leq \mathrm{n}$ $\leq 100$.)

Huber (1981) defined robustness as "insensitivity to small deviations from the assumptions" (p. 1). Indeed, many previously conducted studies concentrated on robustness against contamination in the form of small deviations (e.g., one-out or one-wild). Barnett and Lewis (1994) noted that "Many such published procedures are robust against the possibility that the entire sample comes from some other distribution, possibly gamma or Cauchy, not too dissimilar to the normal but perhaps somewhat skew or fatter-tailed" (p. 56). The purpose of this paper was to examine the LREs of some robust measures where the sample comes from applied social and behavioral science data sets where the shape is quite dissimilar to the normal curve. The results indicate narrower $95 \%$ bracketed intervals for the one step Huber M-estimator when $\psi=1.28$ (as opposed to $\psi=1.339,1.345,1.4088$, and 1.5) for samples less than fifty. The results also indicate that, although lightly trimmed means of $2 \times 5 \%$ yield narrower $95 \%$ bracketed intervals than heavily trimmed means of $2 \times 25 \%$, trimmed means almost always result in significantly wider bracketed intervals than M-estimators for the real education and psychology data sets and sample sizes studied.

$$
\text { References }
$$

Andrews, D. F. (1977). Discussion. The Annals of Statistics, 5, 1078-1079.

Andrews, D. F., Bickel, P. J., Hampel, F. R., Huber, P. J., Rogers, W. H., \& Tukey, J. W. (1972). Robust estimates of location: survey and advances. Princeton, NJ: Princeton University Press.

Barnett, V., \& Lewis, T. (1994). Outliers in statistical data. ( $3^{\text {rd }}$ ed.) Chichester, UK: Wiley.

Dixon, W. J., \& Tukey, J. W. (1968). Approximate behavior of the distribution of Winsorized $t$ (Trimming/Winsorization 2). Technometrics, 10, 83-98.

Gastwirth, J. L, \& Cohen, M. L. (1970). Small sample behavior of some robust linear estimators of location. Journal of the American Statistical Association, 65, 946-973.

Goodall, C. (1983). M-estimators of location: An outline of the theory. In D. C. Hoaglin, F. Mosteller, and J. W. Tukey (Eds.), Understanding robust and exploratory data analysis. Chapter11. NY: Wiley.

Hahn, G. J., \& Meeker, W. Q. (1991). Statistical intervals: A guide for practitioners. NY: Wiley.

Hampel, F. R., Ronchetti, E. M., Rousseeuw, P. J., \& Stahel, W. A. (1986). Robust statistics: The approach based on influence functions. NY: Wiley.

Hill, M., \& Dixon, W. J. (1992). Robustness in real life: A study of clinical laboratory data. Biometrics, 38, 377-396.

Hoaglin, D. C. (1977). Discussion. The Annals of Statistics, 5, 1087-1088.

Hoaglin, D. C., Mosteller, F., \& Tukey, J. W. (1983). Understanding robust and exploratory data analysis. NY: Wiley.

Hogg (1977). Discussion. The Annals of Statistics, 5, 1088-1090.

Huber, P. J. (1981). Robust statistics. NY: Wiley.

Huber, P. J. (1977). Discussion. The Annals of Statistics, 5, 1090-1091.

Huber, P. J. (1972). The 1992 Wald Lecture. Robust statistics: A review. The Annals of Mathematical Statistics, 43, 1041-1067.

Huber, P. J. (1964). Robust estimation of a location parameter. The Annals of Mathematical Statistics, 35, 73-101.

Jackson, P. J. (1986). Robust methods in statistics. In (A. D. Lovie, Ed.), New developments in statistics for psychology and the social sciences, Chapter 2. London: The British Psychological Society and Methuen. 
Lee, H. (1995). Outliers in business surveys. In B. G. Cox, D. A. Binder, B. N. Chinnappa, M. J. Colledge, \& P. S. Kott (Eds.), Business survey methods. Chapter 26. NY: Wiley

Low, M. G. (1997). On nonparametric confidence intervals. The Annals of Statistics, 25, 2547-2554.

Micceri, T. (1986, November). A futile search for that statistical chimera of normality. Paper presented at the $31^{\text {st }}$ Annual Convention of the Florida Educational Research Association, Tampa, Fl.

Micceri, T. (1989). The unicorn, the normal curve, and other improbable creatures. Psychological Bulletin, 105, 156-166.

Minitab. (1996). Minitab, Release 11.1. State College, PA: Minitab, Inc.

Pratt, J. W. (1977). Discussion. The Annals of Statistics, 5, 1092-1094.

Rey, W. J. J. (1980). Introduction to robust and quasi-robust statistical methods. Berlin: Springer-Verlag.

Rosenberger, J. L., \& Gasko, M. (1983). Comparing location estimators: trimmed means, medians, and trimean. In D. C. Hoaglin, F. Mosteller, and J. W. Tukey (Eds.) Understanding robust and exploratory data analysis, Chapter 10. NY: Wiley.

Sawilowsky, S. S. (1998). Comment: Using robust statistics in social and behavioral science. British Journal of Mathematical and Statistical Psychology, 51, 4952.

Sawilowsky, S. S., \& Blair, R. C. (1992). A more realistic look at the robustness and Type II error properties of the $t$ test to departures from population normality. Psychological Bulletin, 111, 352-360.
Sawilowsky, S. S., \& Hillman, S. B. (1992). Power of the independent samples $t$ test under a prevalent psychometric measure distribution. Journal of Consulting and Clinical Psychology, 60, 240-243.

SPSS (1996). SPSS Base 7.0 Applications Guide. Chicago: SPSS.

Staudte, Jr., R. G. (1980). Robust estimation: Queen's papers in pure and applied mathematics No. 53. Kingston, Ontario, Canada: Queen's University.

Staudte, Jr., R. G., \& Sheather, S. J. (1990). Robust estimation and testing. NY: Wiley.

Stigler, S. M. (1977). Do robust estimators work with real data? The Annals of Statistics, 5, 1055-1098.

Tukey, J. W., \& McLaughlin, D. H. (1963). Less vulnerable confidence and significance procedures for location based on a single sample: trimming/Winsorization 1, Sankhya, 25, 331-352.

Wilcox, R. R. (1998). What are the goals and strategies of modern robust methods, why do they greatly increase chances of detecting differences between groups and associations among variables, and how do they enhance our understanding of data? British Journal of Mathematical and Statistical Psychology, 51, 1-40.

Wilcox, R. R. (1997). Some practical reasons for reconsidering the Kolmogorov-Smirnov test. British Journal of Mathematical and Statistical Psychology, 50, 9-20.

Wilcox, R. R. (1996). Statistics for the social sciences. San Diego, CA: Academic Press. 


\title{
An Adaptive Inference Strategy: The Case of Auditory Data
}

\author{
Bruno D. Zumbo \\ Department of Statistics \\ University of British Columbia
}

By way of an example some of the basic features in the derivation and use of adaptive inferential methods are demonstrated. The focus of this paper is dyadic (coupled) data in auditory and perceptual research. We present: (a) why one should not use the conventional methods, (b) a derivation of an adaptive method, and (c) how the new adaptive method works with the example data. In the concluding remarks we draw attention to the work of Professor George Barnard who provided the adaptive inference strategy in the context of the Behrens-Fisher problem - testing the equality of means when one doesn't want to assume that the variances are equal.

Keywords: Robustness, Inference, Statistical assumptions

Introduction

There are many uses of the expression "adaptive methods" in statistics and data analysis but, to my knowledge, all of them seek statistical procedures:

good for a broad class of possible underlying models, but which are not necessarily best for any one of them, where important parameters in the statistical procedure are specified after the sample is drawn, rather than fixed by prior considerations before the sample is observed, and

(iii) that let the sample data lead us toward plausible solutions to statistical problems.

Such adaptive methods are frequently characterized as being robust, that is, exhibiting strength in the face of real data situations where we know that most statistical models will seldom fit exactly the real situations; hence it does not seem productive to try to get the last ounce of mathematical efficiency out of some assumed situation. In my opinion, although he focused on estimation, the paper by Hogg (1974) is one of the clearest expositions of the basic tenants of adaptive methods.

The purpose of this article is to describe adaptive methods, in the context of an example, demonstrating both

Bruno D. Zumbo is Professor of Measurement, Evaluation, and Research Methodology as well as Associate Member of the Department of Statistics at the University of British Columbia. His areas of statistical research have included performance characteristics of standard procedures (both parametric and nonparametric) under non-standard conditions, as well as multivariate analysis and the statistical theories of measurement. the derivation and application of adaptive methods. Unlike Hogg (1974), the focus of the present paper is adaptive inference. The example discussed herein is of the commonly found scenario of testing the equality of means for two independent groups. In the example, we concern ourselves with within-group correlation, wherein the conventional methods of inference fix this within-group correlation, by prior considerations, to zero - i.e., independent observations within groups. This example treats the problem of pairwise within-groups correlations; that is, coupled data.

\section{Coupled Data}

Coupled data arise in the various fields of the social, behavioral, and health sciences. For example, relationship researchers regularly gather data from both members of the dyad (Kenny, 1995). The pairs can be heterosexual or homosexual couples, co-workers, family members or friendship pairs, to name a few examples. In perceptual research it is not unusual for researchers to report the number of organs (e.g., ears, eyes) tested, rather than the number of subjects. This latter situation, perceptual research, will be the focus of the present example.

In all of these cases, subjects or dyads are contributing two scores to the data pool. It can be reasonably argued that these two scores are not independent (i.e., uncorrelated) of one another. Data arising from such research should be referred to as coupled since each subject contributes a couplet of scores, and the correlation between these scores should be referred to as the intracouple correlation (Zumbo, 1996). This issue of coupled scores applies to audition, vision, and hemispheric laterality research, and any situation in which two lateral measures are made on one subject. Therefore, a defining characteristic of coupled data is that there are twice as many scores as there are subjects or dyads (i.e., there are $n$ scores and $n / 2$ subjects or dyads). Because the commonly used statistical inferential methods (not descriptive methods) assume that 
the $\mathrm{n}$ scores are independent, a potential problem may arise when a researcher bases their statistical analyses on the $n$ scores ignoring that they arise from $n / 2$ subjects or dyads. How, then, is one to perform inferential tests on data that are, potentially, highly interdependent - i.e., coupled data?

Before continuing with these new methods of analysis, I should perhaps take a closer look at the data structure for coupled data and discuss why we even need these new methods.

\section{Coupled Data Structure}

Coupled data arise in situations in which the observations in a study are not independent random variables, but rather are pairwise related. The researcher, however, is not interested in the differential effects of the elements of the pair. Coren and Hakstian (1990) initially brought this statistical problem to our attention in the area of auditory research. The statistical problem discussed by Coren and his associates has also been noted in vision research (Ederer, 1973; Rosner, 1982) and could conceivably occur in laterality studies, twin studies, or any experimental or quasi-experimental settings in which the assumption of independence within groups is violated by paired or, as I will refer to them, coupled data. Please note that what is being discussed here is obviously related to the units of analysis problem in survey or educational research wherein one deals with structured populations of respondents (e.g., clusters in sampling or classrooms in educational research). The methods presented herein could be extended to the classroom situation wherein one has more than two elements that are linked.

\section{An Example}

To illustrate the issues consider the data from a two-group completely randomized design given in Table 1. The data are from a hypothetical experiment reported in Zumbo and Zimmerman (1991) depicting auditory research. That is, assume an auditory researcher is interested in investigating whether there is a difference in hearing loss between two groups. The data is displayed in Table 1 .

It is important to note that the researchers are not interested in differences between the left and right ears but rather they gather data from both ears and they are interested in group differences. Therefore, the researcher has a total of 12 observations (i.e., 6 couplets or dyads) in group 1 and 12 observations (i.e., 6 couplets) in group 2. In Table 1, I have placed a box around a couplet, furthermore the top score within the box is the left ear. Traditionally, this design has been envisioned as a two-group completely randomized design and analyzed with a parametric statistical test (for example, in this case, the independent samples ttest with 22 degrees of freedom) treating the data arising from the two members of the dyad as if they were independent (see Coren and Hakstian, 1990, for examples).
Table 1. Coupled data example.

\begin{tabular}{|c|c|c|c|}
\hline \multicolumn{2}{|c|}{ Group 1} & \multicolumn{2}{|c|}{ Group 2} \\
\hline Dyad \# & $\mathbf{X}$ & Dyad \# & $\mathbf{X}$ \\
\hline 1 & 15.6 & 7 & 12.6 \\
\hline 1 & 15.9 & 7 & 12.4 \\
\hline 2 & 13.7 & 8 & 13.7 \\
\hline 2 & 13.9 & 8 & 14.2 \\
\hline 3 & 15.1 & 9 & 15.3 \\
\hline 3 & 15.5 & 9 & 14.5 \\
\hline 4 & 14.7 & 10 & 13.4 \\
\hline 4 & 15.2 & 10 & 12.3 \\
\hline 5 & 16.2 & 11 & 14.3 \\
\hline 5 & 15.7 & 11 & 14.7 \\
\hline 6 & 13.7 & 12 & 14.2 \\
\hline 6 & 14.0 & 12 & 13.8 \\
\hline $\begin{array}{l}\mathrm{n}=12 \\
\text { mean }= \\
\text { std. dev. }\end{array}$ & & $\begin{array}{l}\mathrm{n}=12 \\
\text { mean }= \\
\text { std. dev. }\end{array}$ & \\
\hline
\end{tabular}

What is wrong with treating this data with methods that fix the correlation to zero a priori?

The problem in dealing with these coupled data in this way is that for parametric tests a violation of within group independence can invalidate the statistical test (Zumbo, 1996; Zumbo \& Zimmerman, 1991). More precisely, it can be shown mathematically that for t-tests and ANOVA, a positive correlation within couples results in an inflation in Type I error rate while a negative correlation results in a reduction in Type I error rate. Therefore, if the data from the two ears are positively correlated the Type I error rate is inflated; however, if the data from the two ears are negatively correlated the Type I error rate is deflated.

More formally, a function can be derived showing how the Type I error rate is altered by coupled data. The appendix provides further technical detail. Denote $\alpha$ as the nominal Type I error rate of the t-test (usually .05), and $\varepsilon$ as the actual Type I error rate if we were to conduct the $\mathrm{t}$-test incorrectly ignoring the coupled data, $\mathrm{n}=\mathrm{n}_{1}=\mathrm{n}_{2}$ denotes the common sample size, and $\rho$ the intracouple correlation. The function is then written

$$
\mathrm{t}_{\varepsilon}=\mathrm{t}_{\alpha} \sqrt{\frac{\mathrm{n}-(1+\rho)}{(\mathrm{n}-1)(1+\rho)}} .
$$


Three points are noteworthy from the above equation. First, the amount that the Type I error rate is altered is a function of both the magnitude of the intracouple correlation and the sample size. Second, for a fixed sample size when $\rho=0 t_{\varepsilon}=t_{\alpha}$, while as $\rho$ approaches negative one in the limit $t_{\varepsilon}$ becomes larger than $t_{\alpha}$, and as $\rho$ approaches positive one in the limit $t_{\varepsilon}$ becomes smaller than $t_{\alpha}$. For example, for a nondirectional hypothesis test with 18 degrees of freedom $t_{\alpha}=2.10$, if $\rho=0$ then as expected $t_{\varepsilon}=2.10$; while for $\rho=-0.99999 t_{\varepsilon}=683.3$, and for $\rho=0.99999 t_{\varepsilon}=1.44$. Generally, then, if $\rho=0$ then $\varepsilon=\alpha$, a negative $\rho$ would result in $\varepsilon<\alpha$, while a positive $\rho$ would result in $\varepsilon>\alpha$. Finally, given that the distribution of $t$ scores and the distribution of $F$ scores are related by $t^{2}=F$, these results generalize to the fully randomized design ANOVA where,

$$
F_{\varepsilon}=F_{\alpha}\left(\frac{n-(1+\rho)}{(n-1)(1+\rho)}\right)
$$

Figure 1 is a graphical depiction of the relationship between Type I error rate and the correlation between the two observations that comprise the coupled data, $\rho$, for sample sizes of $4,6,8$, and 10 and values of $\rho$ ranging from -.90 to .90 . It should be noted that the Type I error rates reported in Figure 1 are the complement of the cumulative density function for the central $t$ with $\mathrm{v}$ degrees of freedom for the resulting $t_{\mathrm{v}:} \alpha$ from equation (A10) see the Appendix for details. The upper half of Figure 1 deals with a nondirectional model while the lower half deals with a directional model. First, it should be noted that the horizontal line traces the nominal Type I error rate and the vertical line traces $\rho$ equal to zero. The intersection of the horizontal and vertical lines is the Type I error rate for the i.i.d. case. Second, the general relationship is the same for directional and nondirectional hypotheses. That is, a positive correlation results in inflation in Type I error rate, whereas a negative correlation results in a decrease in Type I error rate. Finally, sample size appears to have very little impact except in the case of a correlation of 0.60 or larger wherein the smaller sample sizes result in a slightly more inflated Type I error rate (a difference of approximately .02 to .06). The minimal effect of sample size is demonstrated in Figure 2.

Thus, if one ignores the fact that one has dyadic or coupled data then there can be a serious inflation (or possible deflation if the correlation is negative) in the Type I error rate of the test. This implies that an alternative method of analysis is needed.

An Adaptive inferential method

An adaptive method for analyzing the example data can be found by re-deriving the independent samples $\mathrm{t}$-test allowing for a parameter in the t-test formula that measures the magnitude of the intracouple correlation, rather than apriori fix the correlation to zero. The

Figure 1. Type I error rates of the Student's t-test as a function of the correlation among the elements of the couple.

\section{A. Nondircelional Hypothesis}

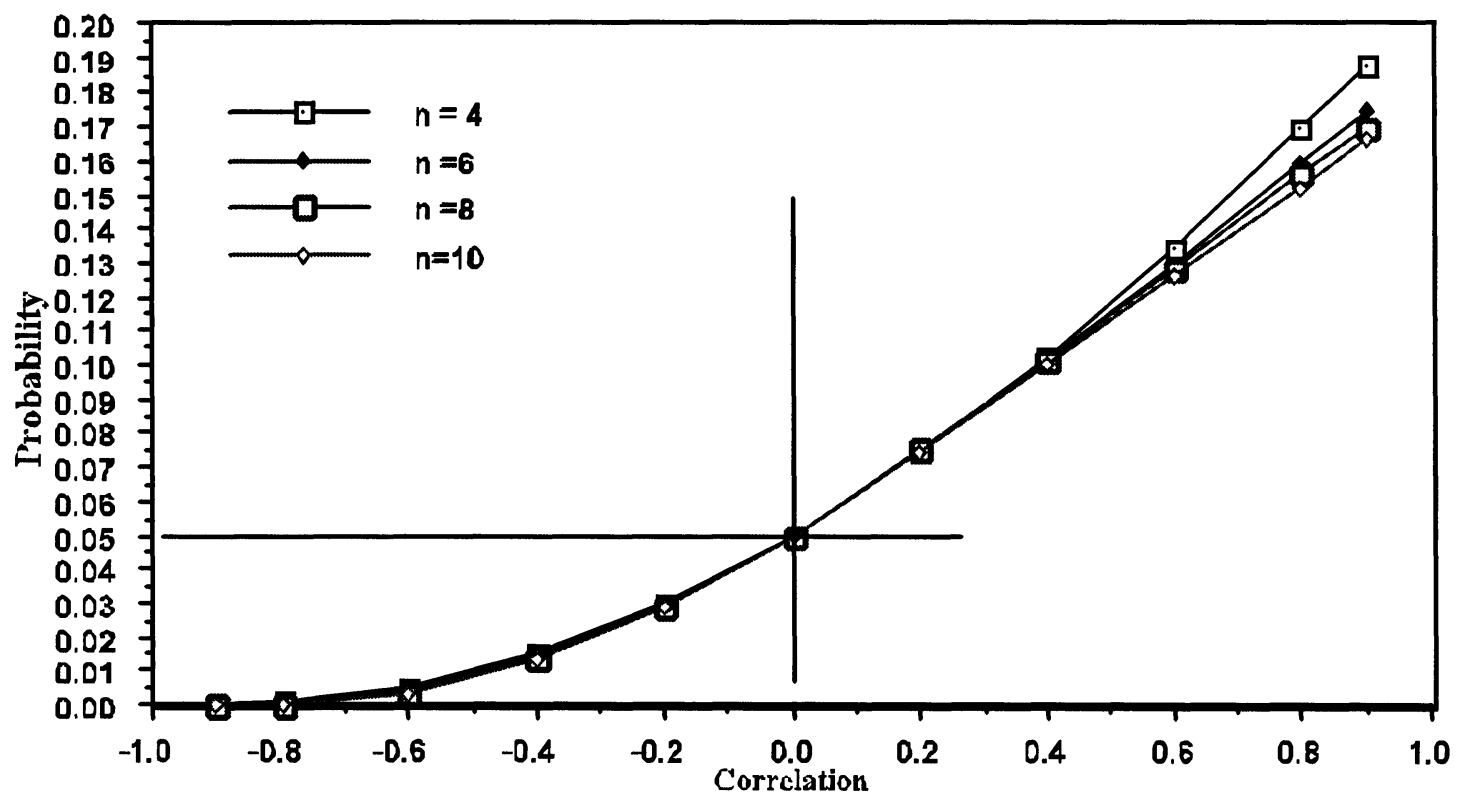


Figure 1 (Continued).

B. Dircctional Iypothesis

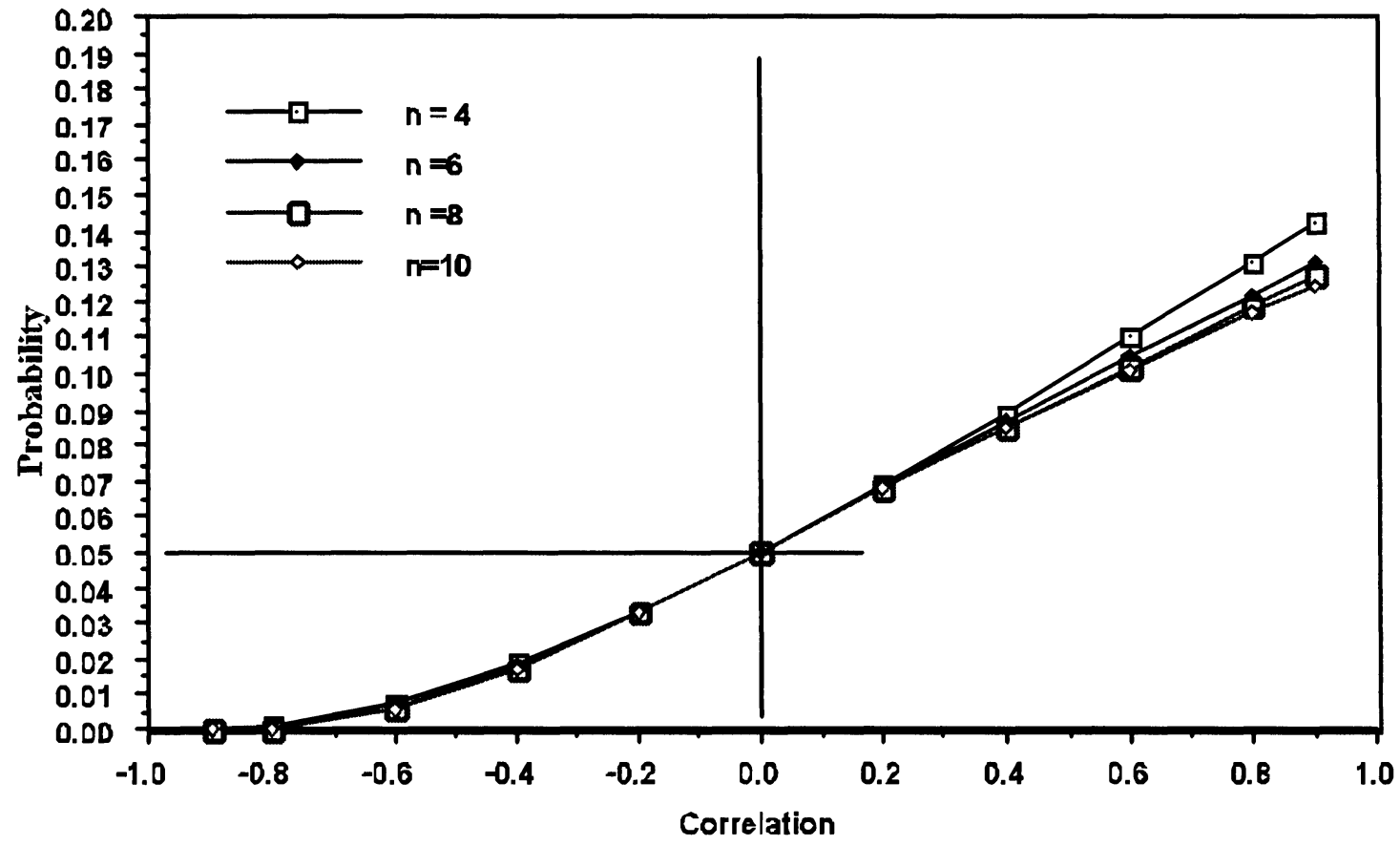

Figure 2. Type I error rates of the Student's t-test as a function of the correlation among the elements of the couple, $\mathrm{n}$ $=4$ and 10,000 (Directional Hypothesis).

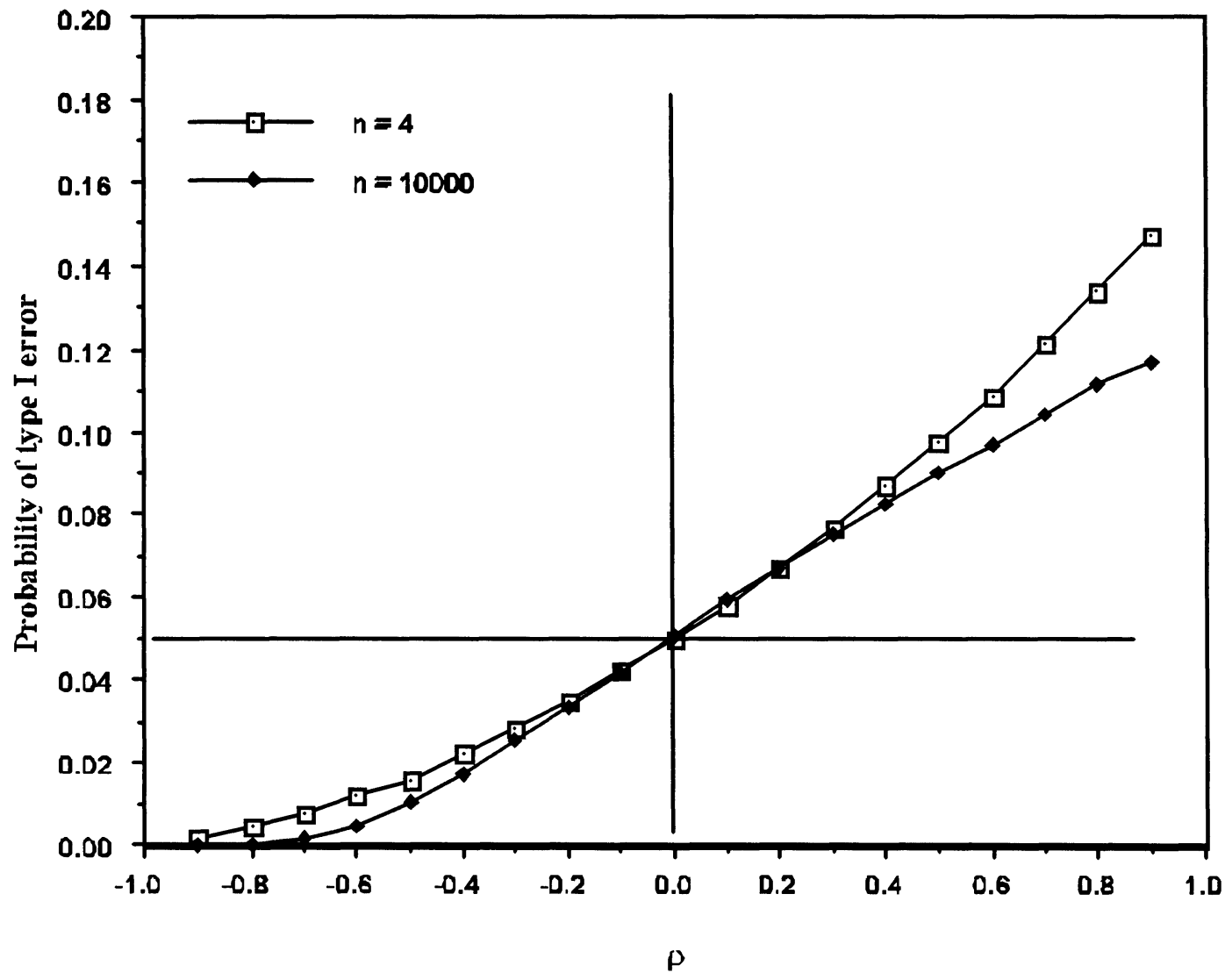


Appendix sketches such a derivation and leads to the replacement of the independent samples t-test by

$$
t(\rho)=\frac{\bar{X}_{1}-\bar{X}_{2}}{\sqrt{\frac{S S_{X_{1}}+S S_{X_{2}}}{n(n-1)} \times \frac{(n-1)(1+\rho)}{n-(1+\rho)}}}, \quad d f=2(n-1),
$$

wherein all of the symbols are described in the appendix and (2) applies for equal sample sizes and equal correlations for each group. Extending the strategy presented in the Appendix, one can derive the more general form allowing for unequal sample sizes and unequal correlations. The resulting more general $t$-test is

$$
\begin{gathered}
t\left(\rho_{1} \rho_{2}\right)=\frac{\bar{X}_{1}-\bar{X}_{2}}{\frac{S S_{X_{1}}}{n_{1}} \times\left(\frac{\left(1+\rho_{1}\right)}{n_{1}-\left(1+\rho_{1}\right)}\right)+\frac{S S_{X_{2}}}{n_{2}} \times\left(\frac{\left(1+\rho_{2}\right)}{n_{2}-\left(1+\rho_{2}\right)}\right)}, \\
\quad d f=n_{1+} n_{2}-2
\end{gathered}
$$

As an algebraic check, if the correlations for each group equal a common value, $\rho_{1}=\rho_{2}=\rho$, and the sample sizes for each group equal a common value, $\mathrm{n}_{1}=\mathrm{n}_{2}=\mathrm{n}$, then after some algebraic rearranging (2) equals (3). Furthermore, if $\rho_{1}=\rho_{2}=0$, then (3) simplifies to the standard unpooled version of Student's t-statistic for two independent samples.

For the purposes of our example we will use the $\mathrm{t}$-test in equation (2). First we compute the common correlation between the left and right ears, $\mathrm{r}=.883$, and then we compute a $90 \%$ interval for the correlation $(.686, .959)$ using the so-called Fisher's r-to-z transformation and applying the formula $z_{r} \pm 1.645 / \sqrt{N-3}$ where, in our case, $\mathrm{N}=12$. Equation (2) can now be applied for the point and interval estimates of the correlation. Table 2 contains these three t-test results and the (incorrect) result when the correlation is equal to zero, $t(0)$.

Clearly, it can be seen from Table 2 that there is no reason to suppose that the intracouple correlation is zero. Furthermore, it can be seen that the value of the test statistic is, as described earlier in this paper, sensitive to nonzero correlation. However, in presenting the results in the manner of Table 2, it can be assessed how sensitive the inference is to the assumption of zero correlation. If a nominal error rate of .05 is used, then the statistical decision is not effected by even a substantial non-zero correlation, whereas this would not be true for an error rate of 0.01 . Finally, it is important to note that this sort of sensitivity analysis needs to be conducted for each data set you have because in some cases the statistical decision may be affected by even a slight non-zero correlation.

It should be noted that this data is hypothetical and was generated with a standardized difference between the population means of 1.50 (Zumbo \& Zimmerman, 1991). That is, there is a substantial difference in the population means. (As a side note, a suggested method for analyzing this sort of data is to average across the two elements of the dyad and hence halving your sample size. This results in a statistically non-significant result, $\mathrm{t}(10)=2.13, p=0.06$.)

\section{Conclusion}

The purpose of this paper was to show how it might be more illuminating in day-to-day statistical applications to use an adaptive statistical strategy. For example, the adaptive $t$-test was computed for a plausible range of intracouple correlation values ranging from .686 to .959 . This, I believe, sheds more light on the problem than simply averaging over the two elements of the couple, which is a commonly recommended strategy (see Coren \& Hakstian, 1990) and resulted in a statistically non-significant finding that conceals the effect of intracouple correlation. The full range of correlations, including the point estimate, gives the ana-

\begin{tabular}{|c|c|c|c|c|}
\hline$\underline{\mathrm{t}(\rho)}$ & $\underline{\mathrm{t} \text {-value }}$ & degrees of freedom & p-value & magnitude of effect (point biserial correlation) \\
\hline $\mathrm{t}(0)$ & 3.03 & 22 & .006 & .295 \\
\hline$t(.686)$ & 2.26 & 22 & .034 & .189 \\
\hline $\mathrm{t}(.883)$ & 2.12 & 22 & .045 & .170 \\
\hline $\mathrm{t}(.959)$ & 2.07 & 22 & .050 & .163 \\
\hline
\end{tabular}
lyst a sense of the dependence of the result on the

Table 2. The resulting $\mathrm{t}$-test statistics at various values for $\rho$ in Equation (2). 
intracouple correlation. A similar approach could be used to study the units of analysis (wherein students are clustered within classrooms) in educational research. One could apply the same sort of analytic strategy as used in the Appendix and derive a t-test parameterized by an intraclass correlation. In doing their data analysis one could then investigate plausible values of the intraclass correlation and see how these values alter the statistical conclusion.

It should be noted that the coupled data problem is not the only problem that has been dealt with as adaptive inference. In fact, the approach presented herein is a strategy developed by Barnard $(1982,1984)$. He gave a similar treatment to the Behrens-Fisher problem by presenting a t-test that has as a parameter the ratio of the sample variances (see, e.g., Sprott \& Farewell, 1993).

Barnard showed that for the Behrens-Fisher case, the problem is to make inferences about the differences in means without fixing the ratio of the two variances, by prior considerations, to one. Barnard's method allows one to explore various values of the variance ratio (in fact, plausible values computed from the sample data, much like the intracouple correlation discussed above) and then one can see how constraining the value to one may, in fact, conceal the sensitivity that the t-test has to plausible values of the variance ratio. Although Barnard presented a method in the context of fiducial distributions, pivotals, robust pivotals, and pivotal likelihoods, the methods presented herein are an application of Barnard's analytic strategy of data-adaptive inference. In this data-adaptive inference, the data lead to sensible solutions.
References

Barnard, G. (1982). A new approach to the Behrens-Fisher problem. Utilitas Mathematica, 21, 261271.

Barnard, G. (1984). Comparing the means of two independent samples. Applied Statistics, 33, 266-271.

Coren, S., \& Hakstian, A. R. (1990). Methodological implications of interaural correlation: Count heads not ears. Perception \& Psychophysics, 48, 291-294.

Ederer, F. (1973). Shall we count number of eyes or number of subjects? Archives of Ophthalmology, 89, 12.

Hogg, R. V. (1974). Adaptive robust procedures: A partial review and some suggestions for future applications and theory. Journal of the American Statistical Association, 69, 909-923.

Kenny, D. A. (1995). The effect of nonindependence on significance testing in dyadic research. Personal Relationships, 2, 67-75.

Rosner, B. (1982). Statistical methods in ophthalmology: An adjustment for the intraclass correlation between eyes. Biometrics, 38, 105-114.

Sprott, D. A., \& Farewell, V. T. (1993). The difference between two normal means. The American Statistician, 47, 126-128.

Stevens, J. (1992). Applied multivariate statistics for the social sciences (2nd Ed.). Hillsdale, N.J.: Lawrence Erlbaum Associates.

Zumbo, B. D. (1996). Randomization test for coupled data. Perception \& Psychophysics, 58, 471-478.

Zumbo, B. D., \& Zimmerman, D. W. (1991). Further evidence for Coren and Hakstian's "Methodological implications of interaural correlation: Count heads not ears" and an alternative correction formula. Perception and Psychophysics, 50, 297-301.

Appendix

In the commonly used model-based general linear model, a random sample of size $\underline{\underline{n}}$ is a sequence of observations of independent identically distributed (i.i.d.) random variables, $\mathrm{X}_{1}, \mathrm{X}_{2}, \ldots, \mathrm{X}_{\mathrm{n}}$. Under this model

$$
\begin{gathered}
\sigma_{\bar{x}}^{2}=\frac{\sigma_{x}^{2}}{n}, \\
E\left(S^{2}\right)=\frac{n-1}{n} \sigma_{x}^{2}, \text { and } \\
\hat{\sigma}_{x}^{2}=\frac{S S_{x}}{n-1},
\end{gathered}
$$


where (A1) is the variance of a sample mean, (A2) is the mean of a sample variance, and (A3) is an unbiased estimate of the population variance. Here, I use the notation

$$
S S_{X}=\sum_{i=1}^{n}\left(X_{i}-\bar{X}\right)^{2}, \text { and }
$$

$\sigma_{x}^{2}$ denotes the population variance of the sample observations.

The derivation of equations (A1), (A2), and (A3) is simplified by the fact that the covariance terms in the general equation for the variance of a sum, $\mathrm{S}_{\mathrm{n}}$,

$$
\begin{aligned}
\sigma^{2}\left(S_{n}\right) & =\sigma^{2}\left(X_{1}+X_{2}+\ldots+X_{n}\right) \\
& =\sigma^{2}\left(X_{1}\right)+\sigma^{2}\left(X_{2}\right)+\ldots+\sigma^{2}\left(X_{n}\right)+\sum_{i \neq j} \operatorname{Cov}\left(X_{i}, X_{j}\right)
\end{aligned}
$$

are all zero; where $S_{n}=X_{1}+X_{2}+\ldots+X_{n}$.

This section derives expressions analogous to (A1), (A2), and (A3) that include nonzero covariance terms due to coupled data. If we let

$$
\rho=\frac{\operatorname{Cov}\left(X_{i}, X_{j}\right)}{\sigma_{X}^{2}}, i \neq j,
$$

and $\rho$ is the same for all $i$ and $j$, then for coupled data it turns out that

$$
\begin{aligned}
\sigma_{\bar{X}}^{2} & =\frac{\sigma_{x}^{2}}{n}[1+\rho] \\
E\left(S^{2}\right) & =\sigma_{x}^{2} \frac{[n-(1+\rho)]}{n}, \text { and } \\
\hat{\sigma}_{\bar{X}}^{2} & =\frac{S S_{X}}{n-(1+\rho)},
\end{aligned}
$$

As in expressions (A1) to (A3), $\mathrm{n}$ in (A4) to (A6) denotes the number of observations - except in this case they are not i.i.d. but rather are coupled data. As an algebraic check, if $\rho=0,(\mathrm{~A} 4),(\mathrm{A} 5)$, and (A6) reduce to (A1), (A2), and (A3), respectively.

One can now use (A4), (A5), and (A6) in lieu of their corresponding i.i.d. expressions to derive a Student's ttest for the balanced two-group completely randomized design assuming a common $\rho$ for both groups. That is, one can place a two-sided confidence interval around $\left(\mu_{1}-\mu_{2}\right)$ by using

$$
\left(\bar{X}_{1}-\bar{X}_{2}\right) \pm t_{v: \alpha / 2} \sqrt{\frac{S_{X_{1}}+S_{X_{2}}}{n(n-1)} \times \frac{(n-1)(1+\rho)}{n-(1+\rho)}}, v=2(n-1)
$$

where $v$ denotes the usual degrees of freedom, and $\mathbf{n}$ denotes the common sample size. Equation (A7) is re-expressed as equation (2) in the main body of the text, a t-test of the two independent groups balanced design.

Interestingly, applying Cochran's theorem (1934; Searle, 1971, Sections 2.5 and 2.6; 1982, p. 356) regarding the distribution of quadratic forms to (A7), it can be shown that (A7) is not distributed as $t$ and is therefore an approximate test. However, Zumbo and Zimmerman (1991) showed via Monte Carlo simulation that (A7) is an adequate 
approximation, maintaining its empirical Type I error rate very close to its nominal value. One can gain insight into how the approximation works by noting that the expected value of the variance, can be expressed as

$$
E\left(S^{2}\right)=\left(\sigma_{x}^{2} \times \frac{n-1}{n}\right) \times\left(1-\frac{\rho}{n-1}\right)
$$

and is clearly asymptotically unbiased.

Now, given (A7), I turn to the task of deriving a general expression indicating the severity of the alteration to the Type I error rate. Given that (A7) is an approximate test, the following results are not exact, but rather good approximations and should be indicative of the behavior of the Type I error rate.

Without loss of generality, let us consider the one-sided confidence interval computed for the population mean difference, $\left(\mu_{1}-\mu_{2}\right)$. Given the i.i.d. assumption, the one-sided confidence interval for small samples is denoted by

$$
\operatorname{Pr}\left[\left(\overline{\mathrm{X}}_{1}-\overline{\mathrm{X}}_{2}\right)+\mathrm{t}_{\mathrm{v}: \varepsilon} \sqrt{\frac{\mathrm{SS}_{\mathrm{X}_{1}}+\mathrm{SS}_{\mathrm{X}_{2}}}{\mathrm{n}(\mathrm{n}-1)}}<\left(\mu_{1}-\mu_{2}\right)\right]=\varepsilon
$$

where $t_{v: \varepsilon}$ equals the $100(\varepsilon)$ percentile of the $t$ distribution with $v=2(n-1)$. Now, given coupled data (A8) can be rewritten as

$$
\operatorname{Pr}\left[\left(\overline{\mathrm{X}}_{1}-\overline{\mathrm{X}}_{2}\right)+\mathrm{t}_{\mathrm{v}: \alpha} \sqrt{\frac{\mathrm{SS}_{\mathrm{X}_{1}}+\mathrm{SS}_{\mathrm{X}_{2}}}{\mathrm{n}(\mathrm{n}-1)} \times \frac{(\mathrm{n}-1)(1+\rho)}{\mathrm{n}-(1+\rho)}}<\left(\mu_{1}-\mu_{2}\right)\right]=\alpha
$$

where $t_{v}: \alpha$ denotes the $t$ value exceeded by probability $\alpha$. It should be noted that $\alpha$ is the nominal level of the test and $\varepsilon$ is the actual level achieved due to not accounting for the covariance due to coupled data.

Finally, setting equation (A8) equal to (A9) results in,

$$
t_{v: \alpha}=t_{v: \varepsilon} \sqrt{\frac{n-(1+\rho)}{(n-1)(1+\rho)}} .
$$

If the $\mathrm{n}$ observations are i.i.d., then $\rho=0$ and $\alpha=\varepsilon$. Therefore, if $\rho \neq 0$, then $\alpha$ can be quite different from $\varepsilon$. As noted above, (A10) can be used with directional or nondirectional hypotheses. Equation (A10) is listed as equation (1) in the main text of this paper. 


\title{
An Unconditional Exact Test For Small Samples Matched Binary Pairs
}

\author{
Robert A. Malkin \\ The Joint Department of Biomedical Engineering, \\ The University of Tennessee-Memphis and \\ The University of Memphis
}

When investigators have $\mathrm{N}$ pairs of binary data, a common test for an increased rate of response is McNemar's test. However, McNemar's is an approximate, conditional test. An exact, unconditional test exists, but requires restrictive assumptions. Critical values and power tables are presented for an exact, unconditional test free of these assumptions.

Keywords: Binary data, $2 \times 2$ tables, Matched pairs, Exact test

\section{Introduction}

For a $2 \times 2$ table of matched binary trials, a common large sample test is the McNemar's test [1]. For example, if blood is drawn from $\mathrm{N}$ patients and split into two tubes in which one is treated with a test drug and another with a control substance, then the results of each treatment would result in 1, a response, or 0, a non-response, for each tube. Each pair of tubes from a single patient would result in either a 10 (response to the test substance and a non-response to the control), 01,00 , or 11 . The results from all $\mathrm{N}$ patients, or matched-pairs, could be summarized by the number of pairs with each type of response, $m_{10}, m_{01}, m_{00}$ and $m_{11}$, respectively, where $N=m_{10}+m_{01}+m_{00}+m_{11}$. McNemar proposed to use the test statistic

$$
Z=\frac{m_{10}-m_{01}}{\sqrt{m_{01}+m_{10}}}
$$

to test the null hypothesis that $\operatorname{Pr}(10)=\operatorname{Pr}(01)$, or equivalently $\operatorname{Pr}(1 \bullet)=\operatorname{Pr}(\bullet 1)$ (the probability of a response in the test tube is equal to that in the control tube), against an appropriate alternative, such as a greater response to the test substance $\operatorname{Pr}(1 \bullet)>\operatorname{Pr}(\bullet 1) . \quad \mathrm{Z}$ is a non-exact, conditional statistic. That is, $Z$ is only approximately a normal deviate, and then only when $n=m_{10}+m_{01}$ is fixed before the experiment. However, the cost of a study typically depends on $N$, the total number of pairs, not $n$.

Robert A. Malkin is Herbert Herff Associate Professor at The Joint Department of Biomedical Engineering, The University of Tennessee-Memphis and The University of Memphis. Contact him at Department of Biomedical Engineering, ET330, Memphis, TN, 38152. E-mail at ramalkin@memphis.edu.
Planning for small clinical trials where budgets are limited, is difficult, if not impossible, when $N$ is unknown. Thus, the application of McNemar's test to small clinical trials is challenging.

Although there have been many proposed improvements to the McNemar's test, the first to propose an exact, unconditional test was Suissa and Shuster [2]. Their work was based on the exact formula for the power of the test, given by

$$
\pi(s, q, Z)=\sum \frac{m_{10} ! m_{01} ! m_{00} ! m_{11} !}{N !} s^{m_{10}} q^{m_{01}}(1-s-q)^{\left(m_{1}+m_{00}\right)}
$$

where $s=\operatorname{Pr}(10), q=\operatorname{Pr}(01)$, and the sum is taken over all $\left\{m_{10}, m_{01}, m_{00}, m_{11}\right\}$ such that $Z>z$, where $z$ is the critical value. Under the null hypothesis $s=q=$, say, $p / 2$, and therefore the $p$-value ( $\alpha$ or significance) is more easily expressed as $\pi(p, z)$. However, $p$ is unknown. To ensure that the actual p-value is less than the stated p-value, it is necessary to find the maximum of $\pi$ as a function of the unknown $p$. This maximum has not been found analytically to date. Therefore, Suissa and Shuster suggested numerically finding a supremum of $\pi(p, z)$ over $0 \leq p \leq 0.995$ for $N>10$. A supremum exists since the derivative is bounded [3]. The range $0.995 \leq p \leq 1.0$ was ignored by Suissa and Shuster because it required unusually long computing times and was said to represent an unlikely scenario [2]. Although unlikely, ignoring this region is somewhat arbitrary (why not 0.990 ?) and inevitably increases the possibility of a Type I error. There exists a more conservative test of the same type.

In this paper, I present new critical values for $N>5$. A simplified approach is presented to finding a supremum, appropriate for small values of $N$, which allows a supremum to be found over the entire interval $0 \leq p \leq 1.0$. 
Using the new critical values and calculation approach, new sample size (power) tables are presented. In short, I present the most conservative possible test for this problem.

\section{Methodology}

Under the null hypothesis, equation (1) can be simplified to [2]

$$
\pi(p, z)=\sum\left(\begin{array}{l}
N \\
n
\end{array}\right) p^{n}(1-p)^{N-n} F_{n}\left(n-i_{n}-1\right)
$$

where $F_{n}$ is the cumulative binomial distribution function. A supremum for this function with respect to the unknown $p$ exists, because the derivative is bounded. Specifically, the derivative is

$$
\frac{\partial \pi(p, z)}{p}=\sum\left(\begin{array}{l}
N \\
n
\end{array}\right) n p^{n-1}(1-p)^{N-n} F_{n}\left(n-i_{n}-1\right)-\sum\left(\begin{array}{l}
N \\
n
\end{array}\right) p^{n}(N-n)(1-p)^{N-n-1} F_{n}\left(n-i_{n}-1\right)
$$

A supremum for the magnitude of Formula (3) can be obtained by assuming that $F_{n}=1$ ( ince $F_{n} \leq 1$ ), and all the negative terms are zero. An equivalent magnitude is found by assuming that all the positive terms are zero. The maximum range for the sum is $0 \leq n \leq N$. Thus, equation (3) is bounded by

$$
\pm \sum_{n=0}^{N}\left(\begin{array}{l}
N \\
n
\end{array}\right) n p^{n-1}(1-p)^{N-n}
$$

From equation (2), when $p=0$ or $p=1, \pi=0$. So, a supremum need only be found over the open interval $0<p<1$. The maximum of $p^{r}(1-p)^{t-r}$, over the open interval $0<p<1$, is $\left(\frac{r}{s}\right)^{r}\left(1-\frac{r}{s}\right)^{s-r}$ [3]. Thus, for small $N$, the largest possible range of the slope of $\pi$ can be found by substituting the maximum value of $p^{n}(1-p)^{N-n}$ for each occurrence in the sum in (4). Using this substitution technique, it was discovered that the slope of $\pi$ is bounded by \pm 212.20 for $N \leq 29$.

A supremum for $\pi$ of any desired accuracy can be obtained with the knowledge of bounds for the slope of $\pi$ and the values of $\pi$ at appropriately selected points. For example, to find a supremum for $\pi(p, z)$ which is no more than 0.002 greater than the maximum, calculate $\pi(p, z)+0.001$ for every value of $p$ from $(0.001 / 212.20)$ up to $(1-0.001 / 212.20)$ in steps of $(0.001 / 212.20)$. The supremum is the maximum of the calculated values rounded up, in this case, to the nearest 0.001 .

\section{Critical Values and Sample Sizes}

The exact, unconditional critical values, $z$, for $N<30$ are given in Table 1 for one-sided tests with $p<0.05,0.025$, and 0.01. Suprema were calculated to be within 0.001 of the maxima. Symmetrically, this table can also be used for the two-sided tests with $p<0.10,0.05$ and 0.02 . Based on the critical values shown in Table 1 , the minimum number of matched pairs for the one-sided test was calculated to attain a power of at least $80 \%$ (Table 2) or $90 \%$ (Table 3) for a test of $\operatorname{Pr}(1 \bullet)>\operatorname{Pr}(\bullet 1)$. The parametric notation of Suissa and Shuster [2] has been retained: $\Psi=\operatorname{Pr}(10)+\operatorname{Pr}(01)$ and $\Delta=\operatorname{Pr}(10)-\operatorname{Pr}(01)$. Thus, a larger value of $\Psi$ for the same $\Delta$ indicates a smaller value of $\operatorname{Pr}(10)$, and therefore, requires a larger $N$.
Table 1 can be directly compared to Table 2 in [2]. Some values appear for the first time as Suissa and Shuster did not present the critical values for $N<10$. In addition, seven critical values which appear in both tables are different. Five of these differences, indicated by * in Table 1 , are attributable to the maximum lying outside the range $0 \leq p \leq 0.995$. Two additional differences are found as indicated by the $* *$ in Table 1 . In these two cases, the suprema for the significance value were approximately 0.000039 below the desired p-value, and, as with all suprema in their software, (kindly provided by Dr. Suissa) were rounded up at the fourth decimal place. For example, a suprema of 0.024961 was not considered to be sufficient to satisfy $p<0.025$. Such rounding is required for numerical calculations, but the software presented here rounded up at the 8 th decimal place. The differences in critical values, both due to rounding and ranging, are reflected in differences in the sample sizes shown in Tables 3 and 4.

\section{Illustration}

Assume that a new defibrillation waveform must be clinically tested to determine whether it is more efficient than a standard waveform at terminating ventricular fibrillation (VF), which is an acute condition and is fatal if untreated. For example, Chapman et al. [4] described a laboratory study of this type. Chapman used upwards of twenty VF inductions and terminations per laboratory 
Table 1

\begin{tabular}{llll}
\multicolumn{4}{c}{ Table 1 } \\
\hline $\mathrm{N}$ & $\mathrm{p}<0.05$ & $\mathrm{p}<0.025$ & $\mathrm{p}<0.01$ \\
\hline 5 & 1.74 & & \\
6 & 1.74 & 2.01 & \\
7 & 1.89 & 2.01 & 2.24 \\
8 & 1.74 & 2.13 & 2.24 \\
9 & 1.74 & 2.01 & 2.34 \\
10 & 1.90 & 2.01 & $2.53^{*}$ \\
11 & 1.74 & 2.12 & 2.34 \\
12 & 1.74 & 2.01 & 2.34 \\
13 & 1.74 & 2.01 & 2.50 \\
14 & 1.74 & 2.14 & 2.31 \\
15 & 1.81 & 2.01 & 2.33 \\
16 & 1.74 & 2.01 & $2.51^{*}$ \\
17 & 1.74 & 2.01 & 2.33 \\
18 & 1.74 & 2.01 & 2.36 \\
19 & 1.74 & 2.07 & 2.36 \\
20 & 1.79 & 2.01 & 2.36 \\
21 & 1.74 & 2.01 & 2.41 \\
22 & 1.74 & $2.14 *$ & $2.33^{* *}$ \\
23 & 1.74 & 1.97 & 2.33 \\
24 & 1.74 & 2.05 & 2.45 \\
25 & $1.80^{*}$ & 1.97 & 2.33 \\
26 & 1.74 & $1.97^{* *}$ & 2.36 \\
27 & 1.74 & $2.12^{*}$ & 2.36 \\
28 & 1.74 & 1.97 & 2.36 \\
29 & 1.74 & 2.05 & 2.42 \\
\hline
\end{tabular}

* Different from [2] due to supremum calculation; ** Different from [2] due to rounding differences

Table 2

\begin{tabular}{|c|c|c|c|c|c|c|c|c|c|}
\hline$\Delta$ & $\Psi$ & $\mathrm{p}<0.05$ & $\mathrm{p}<0.025$ & $\mathrm{p}<0.01$ & $\Delta$ & $\Psi$ & $\mathrm{p}<0.05$ & $\mathrm{p}<0.025$ & $\mathrm{p}<0.01$ \\
\hline \multirow[t]{3}{*}{0.30} & 0.35 & 21 & $25^{-}$ & $*$ & \multirow[t]{3}{*}{0.50} & 0.55 & 11 & 15 & 17 \\
\hline & 0.40 & 26 & $*$ & $*$ & & 0.60 & 13 & 16 & 21 \\
\hline & & & & & & 0.65 & 15 & 18 & 23 \\
\hline \multirow[t]{12}{*}{0.40} & 0.45 & 15 & 18 & 22 & & 0.70 & 17 & 20 & 25 \\
\hline & 0.50 & 17 & $23^{-}$ & 28 & & 0.75 & 18 & 23- & 28 \\
\hline & 0.55 & 20 & 24 & $*$ & & 0.80 & 19 & 23 & $*$ \\
\hline & 0.60 & 23 & 26 & $*$ & & 0.85 & 21 & $25-$ & $*$ \\
\hline & 0.65 & 25 & $*$ & $*$ & & 0.90 & 22 & $28-$ & $*$ \\
\hline & 0.70 & 27 & $*$ & $*$ & & 0.95 & 24 & $*$ & $*$ \\
\hline & 0.75 & 29 & $*$ & & 060 & 0.65 & $9+$ & 11 & 14 \\
\hline & & & & & & $\begin{array}{l}0.70 \\
0.75\end{array}$ & $\begin{array}{l}11 \\
11\end{array}$ & $\begin{array}{l}12 \\
15\end{array}$ & $\begin{array}{l}15 \\
18\end{array}$ \\
\hline & & & & & & 0.80 & 13 & 15 & 20 \\
\hline & & & & & & 0.85 & 14 & 17 & 22 \\
\hline & & & & & & 0.90 & 16 & 19 & $22-$ \\
\hline & & & & & & 0.95 & 16 & 20 & 25 \\
\hline
\end{tabular}

* $\mathrm{N}>30$, Calculate the sample size using approximation formula; - Different from [1] due to critical value difference; 
Table 3

\begin{tabular}{|l|l|c|c|c|}
\hline$\Delta$ & $\Psi$ & $\mathrm{p}<0.05$ & $\mathrm{p}<0.025$ & $\mathrm{p}<0.01$ \\
\hline 0.30 & 0.35 & 28 & $*$ & $*$ \\
& & & & 29 \\
0.40 & 0.45 & 19 & $23^{-}$ & $*$ \\
& 0.50 & 23 & 28 & $*$ \\
& 0.55 & 27 & $*$ & 22 \\
0.50 & 0.55 & 14 & 17 & 25 \\
& 0.60 & 17 & 20 & $*$ \\
& 0.65 & 20 & 23 & $*$ \\
& 0.70 & 22 & 25 & $*$ \\
& 0.75 & 24 & 28 & $*$ \\
& 0.80 & 26 & $*$ & \\
& 0.85 & 28 & $*$ & 17 \\
& & & & 20 \\
0.60 & 0.65 & 11 & 15 & 22 \\
& 0.70 & 13 & 16 & 25 \\
& 0.75 & 15 & 18 & 26 \\
& 0.80 & 17 & 20 & $*$ \\
& 0.85 & 18 & 23 & 23 \\
& 0.90 & 20 & 25 & \\
& 0.95 & 21 & & \\
\hline
\end{tabular}

$* \mathrm{~N}>30$, Calculate the sample size using approximation formula

- Different from [2] due to critical value difference

subject. Clinically, using more than one or two VF inductions for research purposes is uncommon because the danger to the patient increases rapidly as the number of inductions increases. Using the paired approach described here, the study could be designed to include two inductions and termination attempts per patient, one with the test stimulus waveform and one with a control stimulus waveform. When the same stimulus strength is used for both waveforms, the results are correlated matched-pairs of the type treated here, since some patients are easier to defibrillate than others, independent of waveform. If the test stimulus strength is selected to average about $40 \%$ effective in the population with the intent of finding waveforms that are dramatic improvements, say more than $80 \%$ effective in the population, such as those in [4], then $\Delta \approx 0.4$ and $\Psi \approx 0.56$. For $80 \%$ power, Table 2 indicates that a minimum of 20 patients should be planned for a one-sided test with $p<0.05$.

\section{Conclusion}

When the cost--in dollars or otherwise--of each sample pair is great, small samples are naturally preferred. Furthermore, some studies may be impossible when the total number of sample pairs cannot be predicted a priori. Under such conditions, the exact, unconditional approach presented here offers a conservative alternative to the McNemar's test.

\section{Acknowledgments}

This work was supported a faculty research grant from The University of Memphis. Editorial assistance was provided by Katara Herron.

\section{References}

1. McNemar Q. (1947). Note on the sampling error of the difference between correlated proportions or percentages. Psychometrika, 12, 153-157. 
2. Suissa S., \& Shuster J. J. (1991). The $2 \times 2$ matched-pairs trial: Exact unconditional design and analysis. Biometrics, 47(2), 361-369.

3. Suissa S., \& Shuster J. J. (1985). Exact unconditional sample sizes for the $2 \times 2$ binomial trial. J Royal Stat Soc, A148, 317-327.
4. Chapman P. D., Vetter J. W., Souza J. J., Wetherbee J. N., \& Troup P. J. (1989). Comparison of monophasic with single and dual capacitor biphasic waveforms for nonthoracotomy canine internal defibrillation. $\mathrm{J} \mathrm{Am} \mathrm{Coll}$ Cardiol, 14, 242-245. 


\section{Parametric Analyses In Randomized Clinical Trials}

\begin{abstract}
Vance W. Berger
Biometry Research Group

National Cancer Institute

Clifford E. Lunneborg

Statistics and Psychology

University of Washington

\author{
Jonathan G. Levine \\ Lincoln Technologies, Inc.
}

Department of Mathematical Sciences

Indiana University - Purdue University Indianapolis

One salient feature of randomized clinical trials is that patients are randomly allocated to treatment groups, but not randomly sampled from any target population. Without random sampling parametric analyses are inexact, yet they are still often used in clinical trials. Given the availability of an exact test, it would still be conceivable to argue convincingly that for technical reasons (upon which we elaborate) a parametric test might be preferable in some situations. Having acknowledged this possibility, we point out that such an argument cannot be convincing without supporting facts concerning the specifics of the problem at hand. Moreover, we have never seen these arguments made in practice. We conclude that the frequent preference for parametric analyses over exact analyses is without merit. In this article we briefly present the scientific basis for preferring exact tests, and refer the interested reader to the vast literature backing up these claims. We also refute the assertions offered in some recent publications promoting parametric analyses as being superior in some general sense to exact analyses. In asking the reader to keep an open mind to our arguments, we are suggesting the possibility that numerous researchers have published incorrect advice, which has then been taught extensively in schools. We ask the reader to consider the relative merits of the arguments, but not the frequency with which each argument is made.
\end{abstract}

Keywords: Exactness, Nonparametric, Permutation test, Reality-based analyses, Robustness, Validity

Introduction

Medical errors may be classified by the broken link in the chain connecting (a) study objectives to (b) medical data bases to (c) p-values to (d) study conclusions to (e) recommendations to $(\mathrm{f})$ accepted medical practice to $(\mathrm{g})$ actual medical practice. Medical errors attributable to physicians deviating from accepted practice, corresponding to the last link in the chain, (f) to (g), may attract the most malpractice suits and media attention. Yet the frequent insidious errors that occur at the second link, from (b) to (c), involving inappropriate statistical methodology, may result in even more damage (Bailar, 1976). In some cases, assumptions are required to calculate $p$-values, but when a platinum standard analysis is available so that "significance [may be] assessed in a way not involving unverifiable

Vance W. Berger is a Mathematical Statistician at the National Cancer Institute and an Adjunct Professor at the University of Maryland Baltimore County. Clifford E. Lunneborg is Emeritus Professor of Statistics and Psychology at the University of Washington, Seattle. His work focuses on the deployment of computer-intensive and design-based statistical methods. Michael D. Ernst is Assistant Professor in the Department of Mathematical Science at Indiana University - Purdue University Indianapolis. Jonathan G. Levine is at Lincoln Technologies, Inc. assumptions" (Tukey, 1993), it would be a (b) to (c) error not to use it.

In randomized clinical trials (RCTs), the random allocation of patients to treatment groups serves as the basis for valid between-group inference. In RCTs, then, neither random sampling from a target population nor unverifiable assumptions are required (Feinstein, 1993) to construct between-group tests that allow Type I errors (false positive findings) to occur at no greater than a specified rate $(\alpha)$. These platinum standard tests are design-based permutation tests that use as the reference distribution the set of actual potential allocation sequences (Berger, 2000a, Section 3.1). We will refer to design-based platinum standard permutation tests as exact in the remainder of the article, yet two caveats are needed to qualify the use of the word "exact" in this context.

First, design-based tests are exact for the strong null hypothesis, which specifies that each patient would respond identically to each treatment under study. This strong null hypothesis is not the complement of the superiority alternative hypothesis. There is an indifference region in which the weak null hypothesis (specifying common population response rates or means) is true but the strong null hypothesis is not. Design-based tests need not be exact on this region.

Second, exactness is not preserved, even for the strong null hypothesis, when the analysis is based on a 
randomization scheme other than the one that was actually used. Software may not always be available for constructing a test that mimics the actual randomization used. The size of the study may preclude the possibility of enumerating all possible permutations of treatment allocations consistent with the actual randomization used, and Monte Carlo approximations may be needed. So not every permutation test that is called exact is design-based, and it is not clear that permutation tests which are not design-based are more robust than parametric tests. Even design-based permutation tests, which are necessarily more robust (in the sense of keeping the power under $\alpha$ ) than parametric tests when the strong null hypothesis is true, may not be more robust than parametric tests when the weak null hypothesis is true and the strong null hypothesis is not. Although technically this opens the door to the possibility that in some cases the parametric test may be preferable to the best available permutation test, none of us can recall this argument being used in practice to justify a parametric analysis. Without a detailed investigation of the robustness of each test in the specific situation, we would consider the best way to decide between a parametric test and a permutation test to be the conditions for its exactness.

A parametric test requires both random sampling and proper specification of the distribution from which one is sampling randomly to be exact. In some sense, a different random allocation scheme, which is all that is needed for non-design-based permutation tests to be exact, comes closer to the actual random allocation scheme than random sampling from a known distribution does. In addition, inexactness caused by the use of Monte Carlo sampling can be bounded by selection of the number of points in the sample space.

For these reasons, we consider only cases in which the permutation test (even if not design-based) can safely be presumed to be more robust than the parametric test, and we note that this covers every case we have encountered in practice. The disturbing overuse of parametric analyses in these cases cannot be explained by the lag time required for new methods to gain acceptance in practice (Altman \& Goodman, 1994) - in fact permutation tests are not new (Ludbrook \& Dudley, 1998, Section 4.1). More likely, this trend is due to a combination of the reluctance of journal editors to accept correctness in place of precedent (Ludbrook \& Dudley, 1998) and some recent publications that endorse parametric analyses.

For example, Agresti and Coull (1998) cited the conservatism of exact methods as a reason to use approximate methods. Because their article was not especially focused on hypothesis testing or on RCTs, it does not strike us as entirely inconsistent with our views, although we do find it inappropriate to cite their article to justify the use of parametric tests in RCTs.

Other articles have specifically proposed that parametric tests be used in RCTs. For example, Barber and Thompson (2000) criticized the use of the exact Wilcoxon-Mann-Whitney (WMW) test instead of the parametric t-test in a RCT. Likewise, Shuster (1990) and Hewett et al. (2000) were both critical of Fisher's exact test for binary data.

We return to these articles after illustrating the discrepancy between the nominal $\alpha$ level and the actual $\alpha$ level. In arguing the obvious point that the actual level ought not exceed the nominal level (whether or not it is 0.05) we refute the application of Agresti and Coull's (1998) assertions to hypothesis testing in RCTs. We specialize this argument to the case of continuous data, using as an example a recent RCT to compare open access to routine appointments for inflammatory bowel disease (Williams et al., 2000a). We pay particular attention to the points made by Barber and Thompson (2000), and refute the key one about the relative merits of the t-test and the WMW test. We specialize to the comparison between the Chisquared test and Fisher's exact test for binary data, using as an example a recent study of the effect of neuromuscular training on knee injuries in female athletes (Hewett et al., 1999). Here we refute the points made by Shuster (1990) and Hewett et al. (2000). Then, we discuss and refute some of the reasons often cited for using a parametric test instead of an exact one. Finally, we provide recommendations.

\section{Strict Preservation of the Type I Error Rate $(\alpha)$}

As the probability of a false positive, the Type I error rate $(\alpha)$ has been called the regulator's risk in the drug evaluation context. This may suggest that only regulators need to concern themselves with the frequency, under null conditions (i.e., an ineffective medical intervention), with which analyses claim statistical significance (i.e., superior efficacy). This is a dangerous view, because even a medical error attributable to a break in the second link of the chain, (b) to (c), is still a medical error that can cause tremendous damage (Bailar, 1976). The "only assurance of the low likelihood of [the approval of ineffective compounds that have serious adverse effects] is the Type I error" which must "occur at tolerably low rates [for] the community [to] best be assured that the conclusions of the trial most likely reflect the anticipated experience of patients" (Moye, 1999, bracketed material added for clarity).

In this section, we take a careful look at the actual $\alpha$ level, and distinguish it from the tolerably low rate, which is (or should be) the nominal $\alpha$ level. In this discussion we must bear in mind the added importance of the Type I error rate due to the frequent unquestioning acceptance of positive between-group results (Berger, 2000a, Section 1; Voutilainen, 2001). We note that allowing the nominal $\alpha$ level to vary with the nature of the disease and the safety profile of the agent under study may be quite 
reasonable. In no way do we insist that 0.05 should always be used for $\alpha$. We do, however, insist that there be adherence to whatever $\alpha$ level is selected.

Berger (2000a, Section 3.1) demonstrated the potential for a parametric test to violate this basic tenet, and yet cover it up by calculating the $\alpha$ level incorrectly. This occurs because a parametric test is exact as an answer to one question, yet it is used for a different question. As an illustration, consider Table 14.2 in Section 14.2.6 of Berger and Ivanova (2001), based on the $2 \times 2$ contingency table $\{(12,10) ;(3,19)\}$ originally presented by Fox et al. (1993). Columns are response outcomes (no or yes) and rows are treatments, ondansetron (OND) vs. combination therapy (ODC). The chi-square p-values are exact as answers to the question "If one were to sample randomly from a chi-square distribution (with one degree of freedom), then what is the probability of finding results as extreme or more extreme than those we observed?".

Had the experiment actually employed random sampling from a distribution that actually had a chi-square distribution, then this question would be equivalent to "If one were to repeat the experiment performed, under null conditions (the equivalence of OND and ODC), then what is the probability of finding results as extreme or more extreme than those we observed?". In fact there was no random sampling, the population does not have a chi-square distribution, and the exact answer to the former question is not an exact answer to the latter question. To obtain an exact answer to the latter question, which is the one of interest, we hypothetically repeat the experiment. This means re-randomizing the allocation repeatedly, using the same randomness (probability structure) that was used to determine the actual allocation. Under the strong null hypothesis of no treatment effect the responses are independent of the allocation, so the responses do not change. This allows us to compute the test statistic for each of these hypothetical repeats of the experiment. This is how platinum standard permutation tests ensure exactness.

The one-sided actual $\alpha$ level is the probability, under the null hypothesis (OND and ODC being equally effective), of declaring that ODC is more effective than
OND. This declaration will be made if the p-value is as low as or lower than the nominal $\alpha$ level, so the nominal $\alpha$ level determines the number of ODC responses required by each test to claim significant superiority of ODC. If we pick a nominal $\alpha$ level of 0.0250 , then the rejection region consists of those outcomes for which the p-value is no greater than 0.0250 . It turns out that both tests would have the same rejection region, consisting of the outcomes for which ODC has 19 or more responses. But this event occurs with probability 0.0049 , and not 0.0250 . For this nominal $\alpha$ level both tests are conservative (and equally conservative). The outcome of ODC having 18 responses has a chi-square p-value of 0.0282 and a Fisher p-value of 0.0273 , so it does not qualify for inclusion in either 0.0250 rejection region (its p-value is too large). But if we change the nominal $\alpha$ level from 0.0250 to 0.0275 , then Fisher's exact test could fit the additional outcome into its rejection region, while the chi-square test could not. Because the chi-square rejection region still has null probability 0.0049 , it still has the same actual $\alpha$ level, 0.0049. But Fisher's exact test now has a larger rejection region, with null probability 0.0273 , which serves as its actual $\alpha$ level. In this case, Fisher's exact test is much less conservative than the chi-square test. This information appears in the table below.

With a nominal $\alpha$ level of 0.0500 , each test could include the 18 outcome but not the 17 outcome in its rejection region, because $\mathrm{p}(17)=0.1014>0.05$ and $p(17)=0.1017>0.05$, respectively, for Fisher's exact test and the chi-square test. Again, both tests are equally conservative. With a nominal $\alpha$ level of 0.2625 the tests again diverge, with Fisher's exact test rejecting for 17 but not 16 $(p=0.2628)$, while the chi-square test can reject for both $(p=0.2624$ for 16$)$, but not for $15(p=0.5000)$. With an actual $\alpha$ level of 0.1014 , Fisher's exact test is quite conservative; but the chi-square test, with its actual $\alpha$ level of 0.2628 , is anti-conservative. We have seen three distinct cases, but we did not see a case in which the chi-square test was simultaneously valid and less conservative than Fisher's exact test. Because the Fisher actual $\alpha$-level will

Table 1. Fisher's Exact Test Vs Chi-Square Test

\begin{tabular}{llllll}
\hline $\begin{array}{l}\text { Nominal } \alpha \\
\text { (one-sided) }\end{array}$ & \multicolumn{2}{l}{$\begin{array}{l}\text { Fisher's exact test } \\
\text { cut-off }\end{array}$ actual $\alpha$} & \multicolumn{2}{l}{$\begin{array}{l}\text { Chi-square test } \\
\text { cut-off }\end{array}$} & $\begin{array}{l}\text { Compared to Fisher's exact } \\
\text { test the chi-square test is }\end{array}$ \\
\hline & & & & & \\
0.0250 & 19 & 0.0049 & 19 & 0.0049 & equally conservative \\
0.0275 & 18 & 0.0273 & 19 & 0.0049 & more conservative \\
0.0500 & 18 & 0.0273 & 18 & 0.0273 & equally conservative \\
0.2625 & 17 & 0.1014 & 16 & 0.2628 & anti-conservative \\
\hline
\end{tabular}


be that attainable $p$-value closest to but not exceeding the nominal Type I error rate, no test can be simultaneously valid and less conservative than it. In fact, any exact test is minimally conservative in this sense. As such, even conservatism, which is often used as an argument against exact tests (Agresti \& Coull, 1998), favors the exact test unless the parametric test gains an unfair advantage by being anti-conservative.

For a more extreme example of a comparison between an anti-conservative parametric test and a conservative exact test, consider the $2 \times 2$ table $\{(8,2) ;(4,6)\}$. That is, there are $2 / 10$ successes in the control group, and $6 / 10$ successes in the active group. With a nominal $\alpha$ level of 0.05 one-sided, the actual $\alpha$ levels are 0.0099 for Fisher's exact test and 0.0849 for the chi-square test. Because 0.0849 is closer to 0.05 than 0.0099 is, some would argue that the chi-square test at the 0.0849 level is most appropriate. In fact, it may or may not be more appropriate than Fisher's exact test at the 0.0099 level, but these are not the only options. If the response variable is observed fairly soon after randomization, then one could consider an adaptive procedure in which recruitment to the study stops only when the conservatism is small enough. This might be judged to be the case if either the observed $p$-value interval (Berger, 2001) is entirely on one side of $\alpha$ or the pvalue interval that contains $\alpha$ is itself contained in a fairly tight pre-defined interval around $\alpha$. So a larger sample size might resolve this problem satisfactorily. But even without resorting to larger sample sizes, it is also clear that if the chi-square test can be run at an actual 0.0849 level, then 0.0849 is an attainable p-value, meaning that there is an outcome for which $8.49 \%$ of the outcomes are as or more extreme. This means that Fisher's exact test can also be conducted at the 0.0849 level.

So now there are two issues. First, is it acceptable to use a test with an actual $\alpha$-level larger than the planned 0.05? Second, if the answer to the first question is yes, then which test should be used at the 0.0849 level? Berger (2000b) noted the inappropriate willingness of some researchers to accept the general conservatism of exact tests, without considering the extent of conservatism of the exact test in question, as sufficient reason to answer yes to the first question. Yet the extent of conservatism of any exact test may be quantified by the p-value interval (Berger, 2001). Furthermore, conservatism is not a problem, because the lower the Type I error rate the better. The attendant loss of power may be a problem, so the power needs to be considered.

In any event, if the answer to the first question is no, then clearly Fisher's exact test must be used at the 0.0099 level. Regarding the second question, we note that with a nominal $\alpha$-level of 0.0849 both the Fisher and chi-square p-values would be significant exactly $8.49 \%$ of the time. There is still an important distinction, however, in that no more than $5 \%$ of the time would the Fisher pvalue be significant at the 0.05 level. This is not the case, however, for the chi-square test, for which the p-value would be significant at the 0.05 level $8.49 \%$ of the time. If events that should occur with probability one in a thousand "do not occur with this frequency, there is something seriously wrong with our understanding of probability" (Bailar, 1976). Likewise, if the $5^{\text {th }}$ percentile of a distribution is actually exceeded by $8.49 \%$ of the outcomes, then it is not really the $5^{\text {th }}$ percentile of the distribution. If the nominal $\alpha$ level is planned to be 0.05 , then it might be reasonable in some cases to use an actual $\alpha$ level that exceeds it, perhaps 0.0849 . To do so, and then after the fact report 0.05 as the $\alpha$ level used, is tantamount to planning a study with 200 patients, actually recruiting only 180 patients, yet still reporting the actual sample size as 200 . Because parametric tests are guilty of this type of deception, conservatism cannot justify their use in practice.

The Parametric t-test vs the Wilcoxon-Mann-Whitney (WMW) Test

In this section we consider the merits of the WMW test relative to the parametric $t$-test for unadjusted betweengroup comparisons on the basis of continuous data. We first point out that there are numerous versions of the WMW test (Bergmann, Ludbrook, \& Spooren, 2000), and this is likely what prompted Ludbrook (1996) to support exact tests in general yet specifically criticize the WMW test. In any event, it is the exact version of the WMW test that we consider. Williams et al. (2000a) used the WMW test (the exact version, we assume) to assess resource use and costs in a RCT comparing open access to routine appointments for inflammatory bowel disease.

Barber and Thompson (2000) commented that:

1) "resource use and cost data tend to have highly skewed distributions",

2) "t-test methods are only strictly valid for data that are normally distributed",

3) "the most appropriate simple method for comparing mean costs is the ordinary t-test",

4) "use of inappropriate methods for the analysis of cost data is all too common".

It is certainly true that many distributions are highly skewed, and even bell-shaped distributions need not be normally distributed. Furthermore, even if a variable has a normal distribution in the target population of interest, allowing for non-random sampling from this normal distribution allows for the possibility of accepting or rejecting an observation on the basis of the observation itself. Using the rejection method (Hoaglin, 1983) would then allow for the 
retained observations to have any distribution we want them to have. Hence, a lack of random sampling necessarily precludes the possibility of asserting normality of the sampling distribution of the data. If random allocation was used, then it is the only part of the study that was "experimental" (manipulated), and the sampling distribution is a permutation distribution. This permutation distribution may converge to normality as the sample size grows infinitely large, but we feel safe in agreeing with Geary (1947) and Hunter and May (1993) that no data (based on a finite sample size) have a normal distribution. So, we agree with Barber and Thompson's (2000) first point.

The second point has an ambiguity owing to the improper placement of the word "only" in the sentence. In light of the third point, it is conceivable that "only" was meant to start the sentence and limit the class of valid analyses to the t-test. However, given the context in which this sentence appears, it seems more likely that "only" was meant to follow "valid" so as to limit the situations in which the t-test is valid to those in which the data are normally distributed. If this latter interpretation is the correct one, then we agree with the second point. In fact, the perceived robustness to non-normality of parametric methods (as will be discussed) is somewhat of an illusion (Hunter \& May, 1993).

We develop our comments on the third point by first noting that Thompson and Barber (2000) claimed that "only the t-test on untransformed data can be appropriate for costs, since it is the only one that addresses a comparison of arithmetic means". Even conceding the point that it is reasonable to compare mean costs, we can still disagree with the third point, which may be interpreted broadly to include the exact t-test, although the use of the word "ordinary" makes it is more plausible that only the parametric ttest was intended. As articulated above, parametric tests (including the t-test) fail to preserve the Type I error rate in RCTs. As such, we cannot agree with the third point if it is interpreted the plausible way. In considering the more favorable interpretation of the third point, we note that our desire to maximize power to detect mean differences might suggest that the exact t-test would be ideal. However, mean differences may well be accompanied by differences in spread and/or shape (Hart, 2001), and the nature of these differences will affect which test is most powerful. In fact, one could construct an exact test using any test statistic, including the between-group mean difference in raw costs (the exact $t$-test), in ranks (the WMW test), or in Van der Waerden normal scores. Often the WMW test is more powerful and/or more robust than the t-test (Lachenbruch, 1992; Higgins \& Blair, 2000; Weinberg and Lagakos, 2001), so we cannot agree with the third point of Barber and Thompson (2000) even if it is interpreted to include the exact t-test.

Regarding the fourth point, we note that only an exact test can protect against a Type I error attributable to assuming normality. Using an exact test based on a test statistic that is broadly powerful to detect mean differences, and getting a low p-value, Williams et al. (2000a) convincingly demonstrated that "open access greatly reduces secondary care costs" (Williams et al., 2000b). Barber and Thompson (2000) demonstrated that for this data set, either the normality assumption was sufficiently flawed or the difference in means was sufficiently accompanied by shifts in shape and/or scale that the t-test failed to detect this difference. Apparently, Barber \& Thompson (2000) failed to recognize that their primary contribution is the demonstration of the truth of the fourth point, which they accomplished by illustrating that the frequently used parametric t-test can be quite misleading (Williams et al., $2000 \mathrm{~b}$ ), and is therefore inappropriate.

\section{The Chi-squared Test vs Fisher's Exact Test}

In a recent study of the effect of neuromuscular training (Hewett et al., 1999), the chi-square test was used to analyze knee injuries in female athletes. Clancy (2000) commented that "Because the observed and expected number of knee injuries was less than five in at least one cell, an approximate method is inappropriate. An appropriate method in this instance would have been a Fisher's exact test. Incidentally, use of this exact method demonstrated no statistical significance ..., suggesting that the extreme variability present in the small sample resulted in an incorrect finding when an approximate method was used. This provides all sports medicine researchers with a potent example of why appropriate statistical analysis is extremely important." We comment below on choosing tests based on expected cell counts. For now, note that Fisher's exact test is a misnomer, because as discussed above it is not exact unless there is random allocation that has as its only restriction that the treatment totals are fixed at their observed values (Berger, 2000a, Section 3.1). As the Hewett et al. (1999) study appears to have been nonrandomized, neither Fisher's exact test nor the chi-square test is exact in this context. Yet, in response Hewett et al. (2000) appeared to accept that Fisher's exact test was in fact exact, responding only that:

"the chi-square test is unconditional in that a significance probability produced by it refers to the long-term likelihood in repeated experiments of observing an outcome more extreme than ours, regardless of the marginal cell counts in these future experiments under the null hypothesis (more applicable and inclusive to future studies). Fisher's exact test, on the contrary, is conditional and is, technically, only applicable to future experiments like ours in which the 
marginal cell counts are fixed at the exact values that we obtained in our particular study. The significance probability of the Fisher's exact test is much more limited in scope than a chi-square probability, which is one of the reasons Fisher's exact test is rarely used by statisticians."

Hence, it is reasonable to assume that the same discussion would have ensued had the study actually been a RCT. A similar set of views was expressed over ten years ago by Shuster (1990, p. 26), who stated that Fisher's exact test "is not a true $\mathrm{p}$-value, since the additional proviso is made that in the replication of the experiment, you must match the total number of successes with that observed". The RCT design is well summarized by Kempthorne (1979) as Origin III sampling, for which valid probability statements about what might have happened with different samples are not supported (Berger, 2000a, Section 2.2). Applying a parametric test cannot extend the scope to which valid inferences apply, but producing the appearance of such extension can be dangerously seductive. Conditioning on the observed marginal totals, as Fisher's exact test does, is required for exactness and validity, and hence is not a weakness (Berger, 2000a, Section 4.3). In fact, by providing internal validity (exactness) through recognizing the limitations of the study design, Fisher's exact test can actually enhance, and not compromise, the possibility for external validity Berger (2000a, Section 5). As such, we find that while the chi-square test may have asymptotically good properties in the random sampling context, its use in RCTs reflects familiarity, and not appropriateness.

\section{So Why Do Researchers Use Parametric Analyses?}

In the Introduction we allowed for the possibility that one could argue convincingly that a given parametric test might be preferred to the best available permutation test provided that it were more robust in preserving the nominal Type I error rate on the indifference region and the strong null region. However, simply stating that parametric tests are robust, without comparing the robustness of a particular parametric test to that of a competing exact test, cannot be convincing. When such a comparison is not offered, and we have never seen one in practice, robustness cannot be offered as a reason to use a parametric test.

We demonstrated above that conservatism of exact tests is not a valid reason to select a parametric test either. Another reason that is often cited, especially if a preliminary test of the assumptions underlying the validity of the parametric test is conducted, is the frequent agreement of the exact and parametric tests. The lack of obvious problems resulting from all these years of using parametric tests has also been cited. One reason for using parametric tests that is not often cited, but may be deduced from the lack of attention dedicated to this issue, is that some may feel that this is a fourth decimal point issue that is not ready for prime time. We find no merit in any of these reasons. In the remainder of this section we provide journal editors and regulatory authorities with responses they can use if and when they encounter such arguments.

If credibility for the parametric test derives from assurances that its p-value will likely be close to the corresponding exact one, then this is tantamount to an admission that the exact test is the gold standard (or, perhaps, the platinum standard). Approximate tests cannot be any more exact than the exact tests they are trying to approximate and, as approximations to the exact tests, are correct only to the extent that they agree with the exact test. A "heads I win, tails you lose" situation then arises, because if the parametric and exact tests lead to essentially the same inference, then this is as much an argument in favor of the exact test as it is for the parametric test, and there is no benefit to using the parametric test. If they do not agree, then the exact test needs to be used.

Feinstein (1993) wrote that "a statistician defending the general use of $t$ and chi-square tests in modern research could point to their frequent accuracy. With the same argument, an old school clinician might point out that diabetes mellitus can usually be diagnosed by tasting the urine or applying Benedict's reagent. With the availability of better and equally easy ways to diagnose diabetes, however, these old procedures were gradually replaced by techniques that are more reliable. Similarly, when the aid of computers allows permutation or randomization tests to be performed easily, the t-test and chi-square test will probably begin to disappear as routine procedures. Even without computers, however, the permutation tests are the preferable and perhaps mandatory procedures to be used". Even in those cases in which the two tests agree perfectly, Altman (1982, p. 67) makes a compelling case that setting a precedent for poor methodology encourages other researchers to use the same poor methodology in the future. It is likely that in some of these future studies the results will be materially affected.

For these reasons, we believe that even if parametric tests tend to agree with exact tests, meaning that with "high" (left undefined) probability, the parametric pvalue will be "close" (also left undefined) to the exact pvalue, they still should not be used. Even with preliminary tests of the assumptions, the general similarity of the two tests does not exclude the possibility of discordant results between the tests for given data sets. This is because the preliminary test has as its null hypothesis the conditions that would allow for the use of the parametric test. The null hypothesis cannot be proven by a formal test of hypothesis, especially when the test suffers from poor power, as the preliminary tests to detect conditions that would render the parametric test unreliable (such as non-normality) 
often do.

For example, Little (1989) presented a $2 \times 2$ table, with cell counts $\{(170,2) ;(162,9)\}$. Because each expected cell count is at least 5, the chi-square test would be used, yet for one-sided testing (which is generally conducted at the 0.025 level) the chi-square test would find significance $(\mathrm{p}=0.0162)$ and Fisher's exact test would not $(\mathrm{p}=0.0299)$. Barber and Thompson (2000), Berger (2000a, Section 2.3), and Clancy (2000) presented other real data examples in which use of the parametric test matters more than in the fourth decimal. Given the danger in restricting the use of exact analyses to cases in which the need for such use is obvious, the prudent approach is to be suspicious of assumptions even when there is no apparent reason to be suspicious. The only way to validate a particular parametric $p$-value, and ensure that it differs from the corresponding exact $p$-value in only the fourth decimal place, is to compare it to the exact $p$-value. If the exact $p$-value needs to be computed to validate the approximate one, then why not simply use the exact one, the ready availability of which renders the extent to which an approximate test approximates it irrelevant (Berger, 2000a)?

As for the precedent for using parametric analyses without obvious damage, we note that $p$-values provided by inappropriate methodology are numbers between zero and one, and look just like p-values produced by appropriate methodology. Alarms do not go off when an inappropriate method is used. In fact, two forces conspire to conceal the damage caused by the use of inappropriate methodology by separating the manifestation of this damage from the antecedent usage of the inappropriate methodology. Specifically, when inappropriate methodology causes damage, there is both a diffusion of the damage to a set of patients who do not act or think as a unified individual and a lag time in the manifestation of this damage. Add to this that the patients may be sick anyway, and there is little hope of ever tracing the damage back to the cause. That damage actually does occur as the result of medical errors, and often goes unnoticed, has been well documented (Moore, 1995). How much easier would the life of an epidemiologist be if every risk were easily identified and linked to the damage it caused? In fact there have always been real risks that were not mitigated by our ignorance of their existence. The lack of an identifiable victim complaining about the use of parametric tests cannot be interpreted as the lack of a victim.

\section{Conclusion}

It has been said that for evil to prevail all it takes is a few good people to stand by and do nothing. The same could be said for the "scandal of poor medical research" (Altman, 1994). To avoid being part of the problem, all involved parties should insist on quality methodology. This applies especially to regulators and medical journal editors, who, given their "public duty to ensure that reports of research provide valid information" (WAME, 2001), might be seen as functioning as de facto regulators. Given that papers with poor methodology can cause harm (in numerous ways) and cannot be "unpublished" (Altman, 1982), consumers of medical publications (including practicing physicians and HMOs) should hold these publications to rigid standards before accepting and acting on the results (by altering reimbursement or prescribing patterns). That is, "because low p-values are not themselves persuasive but require solid methodology as a foundation, we must resist the pressure to view data positively that were produced from poor methodology" (Moye, 1999). Patients might want to ask their physicians about the evidence on which a decision is based. Given the importance of analyzing RCT data with methods that are applicable to and appropriate for RCTs, medical schools might consider offering degrees specifically in RCT design and analysis. Granting institutions might want to ensure that medical research is supported by a reality-based trialist who will build robustness into the analyses by making a minimum of unverifiable assumptions.

Because those who claim to be methodological experts often disagree among themselves, there is both conflicting information and misinformation being taught in schools and published in both the medical and the statistical literature. Two steps might put medical researchers in a better position to evaluate the analyses a statistician proposes. First, medical researchers could think hard about how best to analyze the data, possibly reading Feinstein (1993) carefully if the study is a RCT. Second, the medical researcher could require the statistician to justify the proposed analyses with logic and reason, instead of (or in addition to) references. It would help if the statistician would provide an informed consent document to spell out the assumptions and limitations of the proposed analyses. See the Appendix for an example dealing with parametric analyses. Although it is unlikely that statisticians who use parametric analyses would make themselves look bad by providing such a document, the medical researcher could bring some version of this document to the attention of the statistician to initiate the discussion about the analyses planned.

Developers of statistical software and authors of text books should offer analyses with a minimum of required assumptions and should make explicit the assumptions and limitations of all analyses. This presently is not the case (Bergmann, Ludbrook, \& Spooren, 2000). Reality-based trialists should, when confronting a researcher endorsing a parametric analysis, consider the advise of Bross (1990), who wrote that "if we politely call a method 'dubious', the criticism can be brushed off as a 'difference of opinion between experts'. However, if most 
statisticians call a method 'fraudulent', the criticism cannot be brushed off so easily.". Hopefully this article will prove useful to reality-based trialists in their efforts to argue effectively against parametric analyses, or at least using parametric analyses without carefully checking their situation-specific robustness.

As for the researcher who wants to resist realitybased analyses, and maintain the status quo of routinely using parametric tests, we agree with Bross (1990) that "the user of a statistical method has the responsibility for dealing with the scientific question: Are the assumptions valid? In particular, when human health and safety might be jeopardized ..., a statistician has a direct responsibility to protect the public health and safety by following failsafe principles in dealing with any assumptions". Given the logical basis for reality-based analyses, it is likely only a matter of time before the medical profession catches on that the normal theory that perplexed them in medical school actually has little or no place in RCTs. When this happens, and proper analyses become the rule instead of the exception, the emperor's new clothes will be seen for what they are, and some may well wonder why the naked emperor was allowed to rule for so long. We would not want to be in a position of having to explain why right up until the time that we were forced to cease and desist we continued to use inappropriate methods that resulted in medical errors leading to unnecessary morbidity and mortality. Although much work remains to fully elucidate the optimal methods for comparing medical interventions, and while there may never be a bias-proof system, there can be no excuse for not getting the easy ones right.

\section{References}

Agresti, A., \& Coull, B. A. (1998). Approximate is better than 'exact' for interval estimation of binomial proportions. The American Statistician, 52, 119-126.

Altman, D. G. (1982). Statistics in medical journals. Statistics in Medicine, 1, 59-71.

Altman, D. G. (1994). The scandal of poor medical research. British Medical Journal, 308, 283-284.

Altman, D. G., \& Goodman, S. N. (1994). Transfer of technology from statistical journals to the biomedical literature: Past trends and future predictions. JAMA, 272, 129-132.

Bailar, J. C. (1976). Bailar's laws of data analysis. Clin Pharmacol Ther, 20, 113-120.

Barber, J. A., \& Thompson, S. G. (2000). Would have been better to use t-test than Mann-Whitney U-test. British Medical Journal, 320, 7251, 1730.

Berger, V. W. (2000a). Pros and cons of permutation tests. Statistics in Medicine, 19, 1319-1328.
Berger, V. W. (2000b). Comment on Ludbrook and Dudley. The American Statistician, 54, 85-86.

Berger, V. W. (2001). The p-value interval as an inferential tool. Journal of the Royal Statistical Society $D$ (The Statistician), 50, 1, 79-85.

Berger, V. W., \& Ivanova, A. (2001). Permutation tests for phase III clinical trials. In (S. P. Millard and A. Krause (Eds.): Applied statistics in the pharmaceutical industry with case studies using S-PLUS. Springer-Verlag, New York, 349-374.

Bergmann, R., Ludbrook, J., \& Spooren, W. P. J. M. (2000). Different outcomes of the Wilcoxon-MannWhitney test from different statistics packages. The American Statistician, 54, 1, 72-77.

Bross, I. D. (1990). How to eradicate fraudulent statistical methods: Statisticians must do science. Biometrics, 46, 1213-1225.

Clancy, W. G. (2000). Letter to the editor. The American Journal of Sports Medicine, 28, 4, 615.

Feinstein, A. R. (1993). Permutation tests and 'statistical significance'. MD Computing, 10, 1, 28-41.

Fox, S. M., Einhorn, L. H., Cox, E., Powell, N., \& Abdy, A. (1993). Ondansetron versus ondansetron, dexamethasone, and chlorpromazine in the prevention of nausea and vomiting associated with multiple-day cisplatin chemotherapy. Journal of Clinical Oncology, 11, 23912395.

Geary R. C. (1947). Testing for normality. Biometrika, 34, 209-242.

Hart, A. (2001). Mann-Whitney test is not just a test of medians: Differences in spread can be important. British Medical Journal, 323, 391-393.

Hewett, T. E., Levy, M., Lindenfeld, T. N., \& Noyes, F. R. (2000). Letter to the editor. The American Journal of Sports Medicine, 28, 4, 615-616.

Hewett, T. E., Lindenfeld, T. N., Riccobene, J. V., and Noyes, F. R. (1999), "The Effect of Neuromuscular Training on the Incidence of Knee Injury in Female Athletes", The American Journal of Sports Medicine, 27, 6, 699-706.

Higgins, J. J., \& Blair, RC. (2000). Comment on Ludbrook and Dudley. The American Statistician, 54, 86.

Hoaglin, D. C. (1983). Generation of random variables. In N. L. Kotz and C. B. Read (Eds.): The Encyclopedia of statistical sciences, Volume 3. NY: John Wiley and Sons, 376-382.

Hunter, M. A., \& May, R. B. (1993). Some myths concerning parametric and nonparametric tests. Canadian Psychology, 34, 384-389.

Kempthorne, O. (1979). In dispraise of the exact test: Reactions. Journal of Statistical Planning and Inference, 3, 199-213. 
Lachenbruch, P. A. (1992). The performance of tests when observations have different variances. The Journal of Computations and Simulations, 40, 83-92.

Little, R. J. A. (1989). Testing the equality of two independent binomial proportions. The American Statistician, 43, 4, 283-288.

Ludbrook, J. (1996). The Wilcoxon-MannWhitney test condemned. British Journal of Surgery, 83, 136-137.

Ludbrook, J. L., \& Dudley, H. (1998). Why permutation tests are superior to $\mathrm{t}$ and $\mathrm{F}$ tests in biomedical research. The American Statistician, 52, 2, 127-132.

Moore, T. (1995). Deadly medicine. NY: HarperCollins.

Moye, L. A. (1999). End-point interpretation in clinical trials: The case for discipline. Controlled Clinical Trials, 20, 40-49.

Shuster, J. J. (1990). Handbook of sample size guidelines for clinical trials. Boca Raton: CRC Press.

Thompson, S. G., \& Barber, J. A. (2000). How should cost data in pragmatic randomized trials be analyzed?. British Medical Journal, 320, 1197-1200.

Tukey, J. W. (1993). Tightening the clinical Trial. Controlled Clinical Trials, 14, 266-285.

Voutilainen, P. E. (2001). Assessment of grouping variable should have been blind in trial of dementia. British Medical Journal, 322, 1491.

Weinberg, J. M., \& Lagakos, S. W. (2001). Linear rank tests under general alternatives, with application to summary statistics computed from repeated measures data. The Journal of Statistical Planning and Inference, 96, 109-127.

Williams, J. G., Cheung, W. Y., Russell, I. T., Cohen, D. R., Longo, M., \& Lervy, B. (2000a). Open access follow-up for inflammatory bowel disease: Pragmatic randomized trial and cost effectiveness study. British Medical Journal, 320, 544-548.
Williams, J. G., Cohen, D. R., \& Russell, I. T. (2000b). Authors' reply. British Medical Journal, 320, 7251,1730

World Association of Medical Editors (2001). Report of the world association of medical editors: Agenda for the future. Croatian Medical Journal, 42(2), 121-126.

Appendix

Sample Informed Consent Document for Statisticians to Provide to Medical Researchers (or Vice Versa)

By signing this form you agree that you have been informed of the following. In trusting me with your data, you recognize that I might perform analyses that are technically correct only if various conditions are true. The reality is that these conditions could not possibly be true. Yet in basing the analyses on the truth of these conditions we can follow the tradition of using such parametric analyses. It is unlikely that the results we obtain will differ very much from those we would have obtained had we used exact methods, which are readily available. In fact, it would not be difficult for me to compare the approximate results to the exact results, but I will not do so, because, as stated, it is unlikely that they will differ by very much. This means that there is the possibility that the parametric results will differ sufficiently from the exact results to lead to different conclusions. These conclusions may then be inappropriate, but this would not be discovered right away, because I will not compute the exact results. In the event that in the future it is revealed that damage resulted from the use of improper statistical methods, you agree to indemnify me. 


\title{
Hotelling's T² VS. The Rank Transform With Real Likert Data
}

\author{
Michael J. Nanna \\ Department of Educational Leadership and Policy Studies \\ Arizona State University
}

Monte Carlo research has demonstrated that there are many applications of the rank transformation that result in an invalid procedure. Examples include the two dependent samples, the factorial analysis of variance, and the factorial analysis of covariance layouts. However, the rank transformation has been shown to be a valid and powerful test in the two independent samples layout. This study demonstrates that the rank transformation is also a robust and powerful alternative to the Hotellings $\mathrm{T}^{2}$ test when the data are on a likert scale.

Keywords: Robustness, Statistical power, Parametric tests, Conditionally distribution-free tests, Multivariate tests

\section{Introduction}

Hotelling's $\mathrm{T}^{2}$ is a specific case of multivariate analysis of variance (MANOVA) having one independent variable with two levels and multiple dependent variables. It is the multivariate analogue of the independent samples $t$-test. Instead of examining differences in means between two samples on one dependent variable, Hotelling's $\mathrm{T}^{2}$ tests for the equality of centroids (mean vectors) between two groups. $\mathrm{T}^{2}$ is a parametric test having the usual assumptions that theoretically must be met for its valid application. For example, $\mathrm{T}^{2}$ assumes that the data of the underlying distributions is normally distributed, (i.e., assumes multivariate normality), that there is independence of observations, and that the variance/covariance matrices are equal.

The Rank Transform (RT) is a set of nonparametric-like (and sometimes referred to as conditionally distribution-free) procedures. The RT is performed by replacing original observations with their respective ranks, computing a parametric statistic on these ranks, and then referring the test statistic to the usual table of critical values (Conover \& Iman, 1981). It has been suggested that $\mathrm{RT}$ procedures have robustness and power properties similar to their parametric counterparts when normal theory assumptions are met, and have superior robustness and power properties when assumptions are not met (Conover \& Iman, 1981).

\section{Purpose of Current Study}

The purpose of this study is to examine the robustness and power properties of the two independent sample Hotelling's $\mathrm{T}^{2}$ test and the RT using Monte Carlo techniques with samples drawn from real data sets that are

Michael Nanna is a faculty associate in the division of Social and Philosophical Foundations within the Dept. of Educational Leadership and Policy Studies at Arizona State University. based on ordinal level likert scaled type data. An example of a common five-point likert scale is 1 "Strongly Disagree", 2 "Disagree", 3 "Neutral", 4 "Agree", and 5 "Strongly Agree". The discrete nature of the response set precludes the normality distribution assumption from being met.

Relevance to Social and Behavioral Science Research

Using Monte Carlo methods, it is possible to observe the operating characteristics of a statistic under real situations. This is important because statistics frequently support and/or drive decisions and policy in real applied settings such as education, psychology, medicine, and other social and behavioral science disciplines. Using inappropriate (or less efficient) statistics can lead to "analyses that are less powerful, and potentially to inferences that are invalid" (Hunter \& May, 1993, p. 386).

The implications are striking as examination of statistical power in clinical and applied research settings has consistently demonstrated low statistical power. The probability of committing a Type II error has been shown to reach .91 for detecting small effect sizes in applied data analysis (Cohen, 1962; Cohen, 1977; Sedlmeir \& Gigerenzer, 1989; Matyas \& Ottenbacher, 1993).

A discipline marked by studies with low statistical power means that researchers are unable to detect treatment effects that otherwise might provide the basis for developing more effective programs or interventions. Furthermore, strict reliance on statistical significance testing without consideration of robustness and power can lead to erroneous interpretation of research findings as researchers may decide to not follow up statistically insignificant results (Keppel, 1975). Lack of consensus and contradictory findings in the literature may also preclude a discipline from establishing a useful and dynamic body of knowledge (Ottenbacher, 1995). Issues of robustness and power are not theoretical problems, but rather pragmatic issues having important consequences in applied settings. 
Hotelling's $\mathrm{T}^{2}$

Many studies on Hotelling's $\mathrm{T}^{2}$ indicated it has acceptable Type I error rates under normal and various non-normal conditions (Jensen, 1982; Mardia, 1975; Kariya, 1981; Everitt, 1979; Harwell \& Serlin; 1995; Blair, Higgins, Karniski, \& Kromrey; 1994; Hopkins \& Clay; 1963; Algina \& Oshima; 1990; Ito \& Schull; 1964; Holloway \& Dunn, 1967). Other studies, however, have shown $\mathrm{T}^{2}$ displays inflated Type I error under asymmetry, even when sample sizes are relatively large (Chase \& Bulgren, 1971; Serlin \& Harwell, 1989; Utts \& Hettsmanperger, 1980; and Everitt, 1979).

Hakstian, Roed \& Lind (1979) offer the following summary concerning the robustness of $\mathrm{T}^{2}$ :

1. "The $\mathrm{HT}^{2}$ procedure is generally robust with respect to violation of the homogeneity of covariance matrix assumption for equal sample sizes, even when the ratio of sample size to number of dependent variables is small.

2. "For $n_{1} \neq n_{2}$, however, the test moves rapidly towards unacceptable Type I error rates as the degree of population covariance matrix heterogeneity is increased.

3. "The $\mathrm{T}^{2}$ procedure is not robust in the face of covariance matrix heterogeneity coupled with unequal n's, even for relatively mild departures from equality of the covariance matrices, sample sizes, or both." (p. $1,261)$

Rank Transformation

The RT is a set of procedures that substitutes the ranks of data for the raw data values and then calculates a usual parametric statistic on the ranked data. It has been suggested that rank tests provide a useful alternative method of analysis when the assumptions of parametric tests (i.e., $t$ and F) are not met. Initial simulation results indicated that the RT's robustness and power properties are similar to its normal theory counterpart when assumptions are met, and are often superior when assumptions are not met (Conover \& Iman, 1981).

Conover and Iman (1981) suggested the use of the $\mathrm{RT}$ as a bridge between parametric and nonparametric statistics for many different data analysis situations. Subsequently, however, considerable journal space has been given to the RT's lack of robustness and power for the two dependent samples, factorial analysis of variance, and factorial analysis of covariance layouts, so there is no need to review those results here. The RT has been shown, however, to be a robust and powerful alternative to its parametric counterpart in the context of both the two independent samples and the one-way analysis of variance layouts.

In terms of the multivariate two independent samples layout, Bhattacharyya, Johnson and Neave (1971), Tiku and Singh (1982), and Nath (1982) examined the robustness and comparative power properties of $\mathrm{T}^{2}$ and the rank transformed $\mathrm{T}^{2}\left(\mathrm{~F}_{\mathrm{R}}\right)$ and found both statistics to be robust for data samples from a uniform distribution. However, Type I error rates for both statistics exceeded that of nominal alpha under the exponential and lognormal distributions (Everitt, 1979). Similar results were also found by Nath and Duran (1983). Zwick (1986) demonstrated that the RT is robust and often more powerful than the F test, but did not recommend its routine application due to its highly specific and volatile behavior. These studies, however, were limited to artificial distributions.

\section{Scaling: Ordinal Measurement}

The robustness and power of a statistic using data which is scaled at the ordinal level of measurement is important given the number of measures that exist in rehabilitation medicine, psychology, and education use ordinal data - particularly Likert scales. There are numerous studies that address the issue of scales of measurement and its impact on a statistics performance but are beyond the scope of this paper. For a review on this issue, consult Anderson (1961); Boneau (1961); Senders (1958); Siegal (1956); Stevens (1946, 1951); Hsu and Feldt (1969); Heeren and D’Agostino (1987); Nanna \& Sawilowsky, (1998); Siegal, (1956); Stevens, (1946); Gaito, (1986); Lord, (1953); and Zumbo \& Zimmerman, (1991).

Indeed, measurement issues have been debated in the statistics and measurement literature for decades in the context of the "weak measurement vs. strong statistics" controversy. On the basis of considerable simulation evidence (see, e.g., Sawilowsky, 1990; Hunter \& May, 1993; Zumbo \& Zimmermen, 1993; and Sawilowsky, 1993), additional discussion on this issue will be dismissed from consideration in choosing between parametric and nonparametric tests.

There is a paucity of research on the properties of statistics applied to likert scaled data. The independent samples t-test was shown to be robust with respect to Type I errors in simulation studies conducted by Heeren \& D'Agostino (1987) and Hsu \& Feldt (1969). These results were replicated, and extended in terms of statistical power, for both the t-test and its rank transformation counterpart by Nanna \& Sawilowsky (1998) for likert scaled data.

The likert scaled data used for this study was obtained from the Functional Independence Measure (FIM), which is one of the most widely used assessment instruments in medical rehabilitation. In fact, "about $60 \%$ of rehabilitation facilities nationwide use the FIM" (Stineman, et al., 1996, p. 1101; see also Granger, et al., 1986). It was developed to provide uniform assessment of severity of 
disability and to specify medical rehabilitation outcomes. (e.g., physical, cognitive and social variables associated with disability.) It is an 18 item assessment tool comprised of multiple, 7-point likert scales which consist of scores which range from complete dependence (1) to complete independence (7). The scale was originally designed so that ratings on all 18 items were summed into a single score that was then used to estimate overall burden of care (Stineman, et al. 1996). Total FIM scores range from 18 (complete dependence) to 126 (complete independence).

FIM scores are based on the observation of a patient meeting specific objective physical and behavioral criteria and are usually rated by clinical observation at the time of admission, and again prior to discharge from rehabilitation services. It is intended to measure levels of disability regardless of the underlying pathological condition and is considered independent of the rater's clinical background (Brynes \& Powers, 1989; Keith, et al., 1987; Granger, et al., 1990). Previous studies have indicated high levels of instrument reliability $(\mathrm{r}=.95$, Byrnes \& Powers, $1989)$ and interrater agreement $(.93 \& .97$, Hamilton, et al., 1991). Ottenbacher, et al., (1996), concluded that the FIM "provided good interrater reliability across a wide variety of raters with different professional backgrounds and levels of training. The median interrater reliability value was .95 and was based on a large cumulative sample of patients representing a wide variety of disability levels and medical conditions" (p. 1230).

\section{Methodology}

Monte Carlo techniques were used to independently sample from both admit and discharge data sets of several FIM score distributions. As suggested by Micceri (1989), real data sets were used to model admit and discharge populations (as opposed to using mathematically convenient distributions) associated with each FIM score.

The FIM scores used in this study were obtained by evaluating 903 geriatric patients admitted to a large MidWestern rehabilitation facility from 1991 to 1995 . Patients were evaluated using the FIM at the time of admission, and again at the time of discharge. Seven $(1,3,4,5,6,7$, 13) of the eighteen individual admit and discharge FIM score distributions were selected for further study and are depicted in Figures 1-7. The histograms of the remaining FIM score distributions were similar to these seven, and for parsimony, are not addressed in the current study.

Differences obtained from independently sampling with replacement from the pre-test (admit) and then post-test (discharge) scores were used to represent gain due to treatment interventions (i.e., real treatment effects) as opposed to artificially modeled treatments (i.e., adding a constant to the initial distribution of scores to model a shift in location parameter) as has been done in previous studies. Although no formal effect sizes are calculated for this study, differences between the admit and discharge populations may serve as evidence of the presence of effect sizes. Because the distributions used in this study are meant to represent the actual populations, effect sizes are implicit in the difference between the means of the admit and discharge populations (see Table 1.). Therefore, sampling directly from the population distributions obviated the need to model synthetic treatment effects in this Monte Carlo study. Upon admission to the hospital, patients participated in multiple treatment regimens during their stay at the hospital before being discharged. The treatment interventions are presumed to account for the difference in means between admit and discharge distributions.

A subscale score was constructed for a separate study and, for this study, was used to model data scaled at a more continuous level of measurement. This subscale distribution, named OT, is a composite score comprised of seven FIM items that measure domains commonly assessed by occupational therapists. A list of descriptive statistics (e.g., mean, standard deviation, skew, and kurtosis) for each admit and discharge distribution used in this study can be found in Table 1.

A Fortran program was written for the IBM compatible Pentium PC accessing IMSL (1987) subroutines to sample with replacement for sample sizes $n_{1}=n_{2}=(5,15)$, $(10,10),(10,20),(15,15),(15,45),(30,30),(25,75)$, and $(50,50)$ for the number of dependent variable combinations of 2 and 5 . In order to insure the validity of the Monte Carlo simulation, samples were initially taken from a multivariate normal distribution to examine actual Type I error rates produced under normality.

The first portion of this study consisted of examining the robustness of both $\mathrm{T}^{2}$ and the RT in both twodependent and five-dependent variable combinations. The robustness portion of the study was performed by independently sampling with replacement from each FIM admit distribution using different sample size and dependent variable combinations, calculating the appropriate statistic, and comparing obtained alpha with nominal alpha levels. A similar technique was employed for the five dependent variable layout. For five dependent variables, additional runs were performed when sampling from each individual distribution five times (e.g., FIM 7 distribution serving as each dependent variable). A subset of the results are presented in this paper and are representative of the remaining results. A copy of the remaining results for dependent variable combinations are available from the author.

The second portion of this study consisted of examining the comparative power of $\mathrm{T}^{2}$ vs. the RT. This was accomplished by independently sampling, with replacement, from FIM admit distributions, and then comparing that with a sample taken from the related discharge 
Table 1. Descriptive Statistics for Admit and Discharge Fim Distributions.

\begin{tabular}{|c|c|c|c|c|}
\hline Distribution & $\underline{\text { Mean }}$ & Std. Dev. & $\underline{\text { Kurtosis }}$ & $\underline{\text { Skew }}$ \\
\hline Fim 1 Admit & 5.37 & 1.50 & .69 & -1.00 \\
\hline Fim 1 Disch. & 5.93 & 1.30 & 3.63 & -1.79 \\
\hline Fim 3 Admit & 3.64 & 1.33 & -.22 & -.05 \\
\hline Fim 3 Disch. & 4.81 & 1.47 & -.02 & -.84 \\
\hline Fim 4 Admit & 4.40 & 1.47 & .09 & -.49 \\
\hline Fim 4 Disch. & 5.25 & 1.48 & .61 & -.96 \\
\hline Fim 5 Admit & 3.19 & 1.44 & -.51 & .29 \\
\hline Fim 5 Disch. & 4.45 & 1.66 & -.73 & -.58 \\
\hline Fim 6 Admit & 3.87 & 1.61 & -1.01 & -.17 \\
\hline Fim 6 Disch. & 4.96 & 1.71 & -.22 & -.92 \\
\hline Fim 7 Admit & 4.32 & 1.88 & -1.07 & -.41 \\
\hline Fim 7 Disch. & 5.29 & 1.88 & -.14 & -1.05 \\
\hline Fim 13 Admit & 1.15 & .65 & 22.38 & 4.73 \\
\hline Fim 13 Disch. & 2.25 & 1.62 & -.37 & 1.02 \\
\hline OTFim Admit & 28.34 & 8.53 & .67 & -.05 \\
\hline OTFim Disch. & 35.54 & 9.02 & .59 & -.80 \\
\hline
\end{tabular}

distributions. The $\mathrm{T}^{2}$ test and the RT were calculated for the sample size and FIM admit/discharge distribution combinations outlined previously in the robustness portion of the simulation study. Each experiment was repeated at the $.10, .05$, and .01 alpha levels.

For the power portion of the study, group (admit vs. discharge) served as the primary independent variable (i.e., group one consisted of scores obtained from the admit distributions, and group two consisted of scores obtained from the associated discharge distributions). FIM scores served as the dependent variables for each group (i.e., combinations of either 2 or 5 FIM scores which served as the dependent variables). Differences in randomly selected FIM scores from the admit distributions were tested against randomly selected scores from their related discharge distributions. Random and independent samples with replacement were selected for each sample size and number of dependent variable combinations.

It is emphasized that independent samples tests are appropriate for this study as individual scores were randomly selected with replacement from each distribution and not pairs of scores. In a separate study using the same data distributions, Nanna \& Sawilowsky (1998) took independent random samples from several of the admit distributions used in this study and using Monte Carlo methods correlated them with independent samples drawn from their corresponding discharge distributions. They repeated this experiment 1,000,000 times, and found the long run correlation to be $r=-.0083$. 
Figure 1. Distribution of FIM 1 Scores

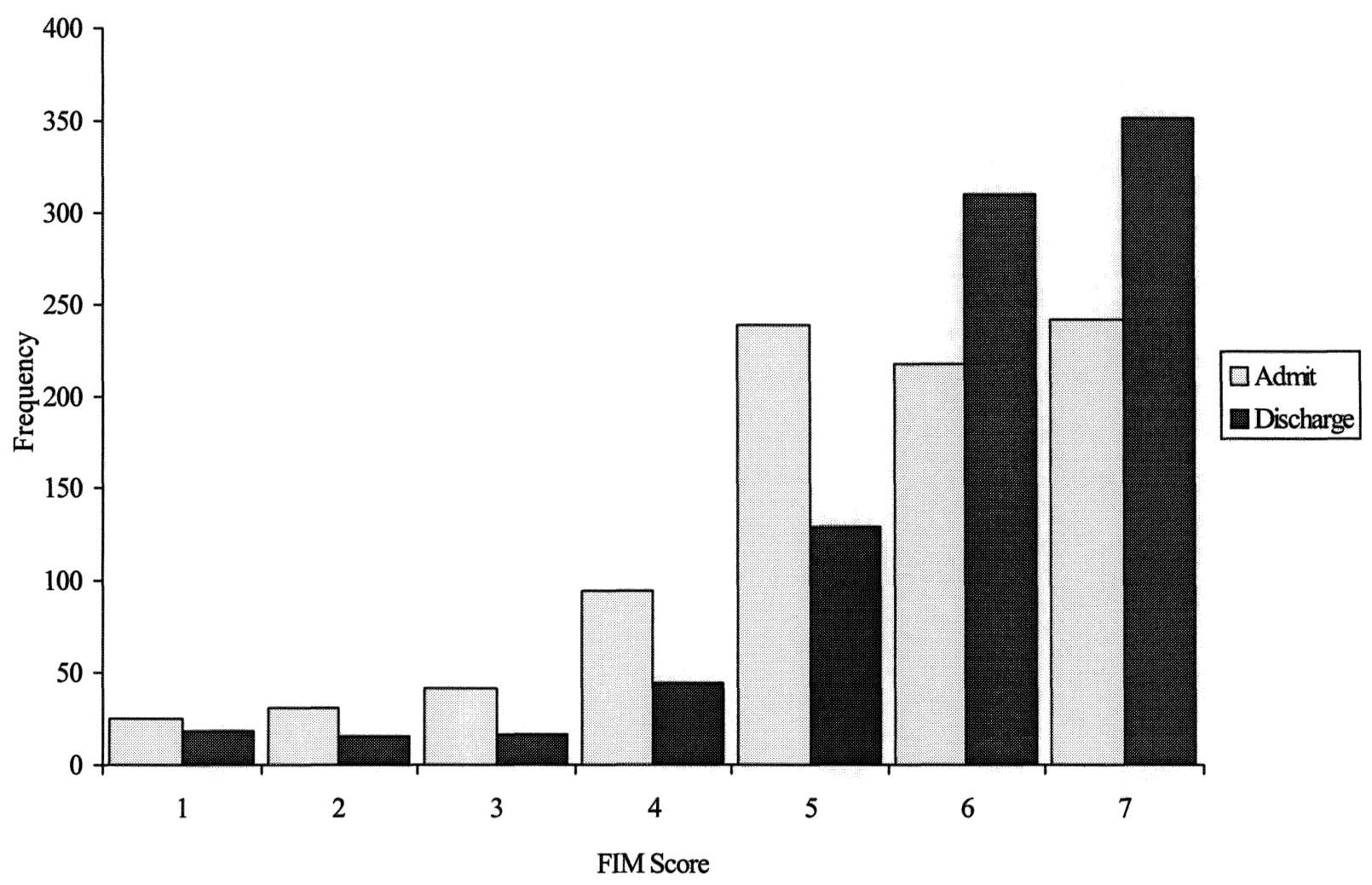

Figure 2. Distribution of FIM 3 Scores

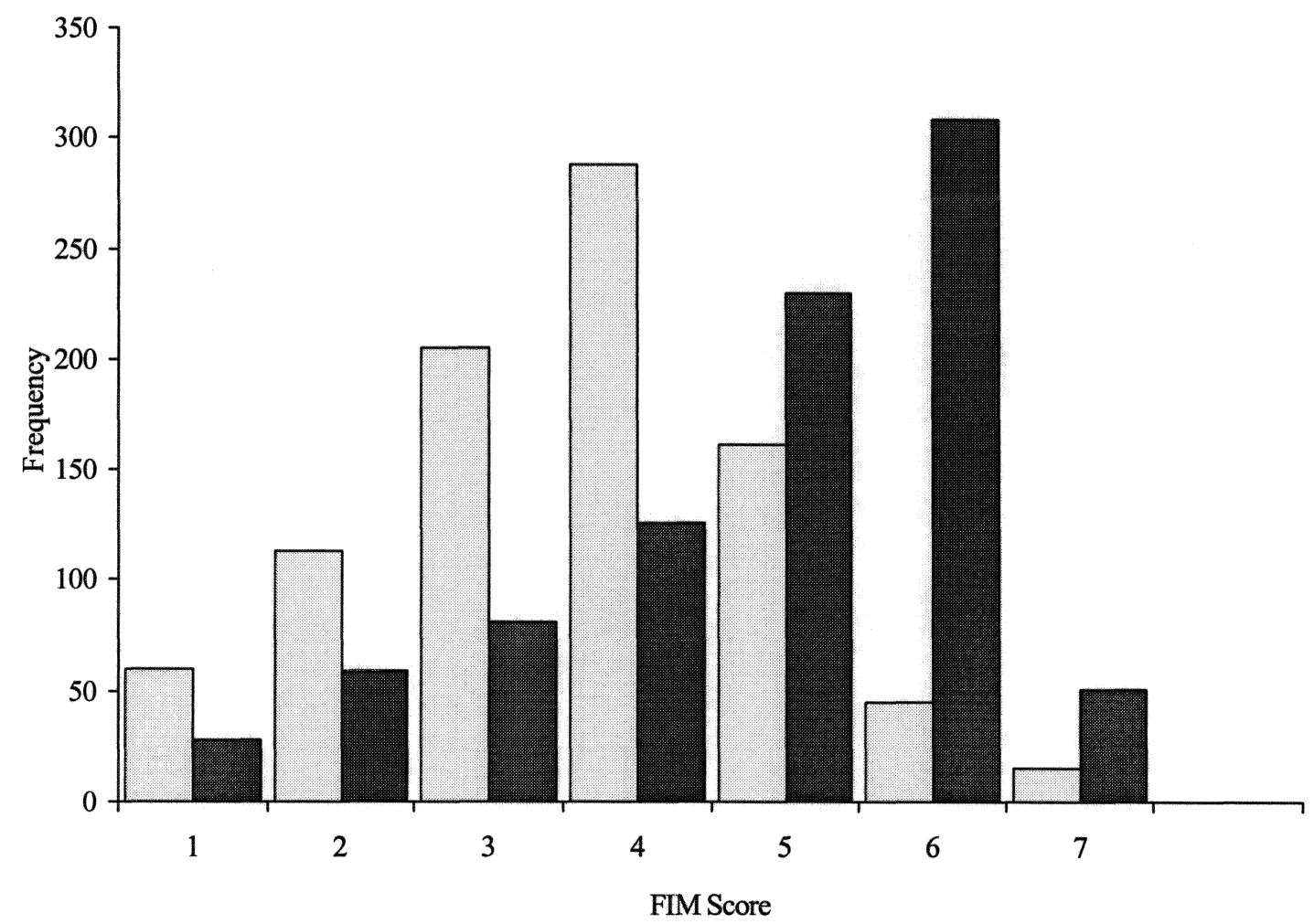


Results

Robustness - Two Dependent Variables

Results of each test were recorded for both statistics. The number of replications per experiment was 10,000 and the proportion of rejections served as an indication of robustness or power for each statistic. The one-tailed power of $\mathrm{T}^{2}$ and the RT test were compared at the $0.10,0.05$, and 0.01 alpha levels. Results for the .05 alpha level are presented in this paper with the remaining results $(.10$ and .01 alpha levels) available from the first author.

The ability to detect differences between the admit and discharge data distributions (i.e., differences in the centroids or mean vectors), served as an estimate of power for each statistic. Power results in this study are estimates only as effect sizes may be different between the $\mathrm{T}^{2}$ and RT tests. However, the results do reflect realistic conditions.
It is important to note that the discharge distributions are comprised of scores from the same individuals as in the admit distributions after receiving treatment. The null hypothesis being tested is the independent hypothesis and not the matched-pairs hypothesis as samples are drawn independently and separately from both the admit and discharge distributions. An example of the null hypothesis being tested in the power portion of this study using three dependent variables (e.g., FIM 1, FIM 3, and FIM 5) is as follows:

$$
H_{o}:\left(\begin{array}{l}
\mu_{F I M 1 a} \\
\mu_{F I M 3 a} \\
\mu_{F I M 5 a}
\end{array}\right)=\left(\begin{array}{l}
\mu_{F I M 1 d} \\
\mu_{F I M 3 d} \\
\mu_{F I M 5 d}
\end{array}\right)
$$

Figure 3. Distribution of FIM4 Scores

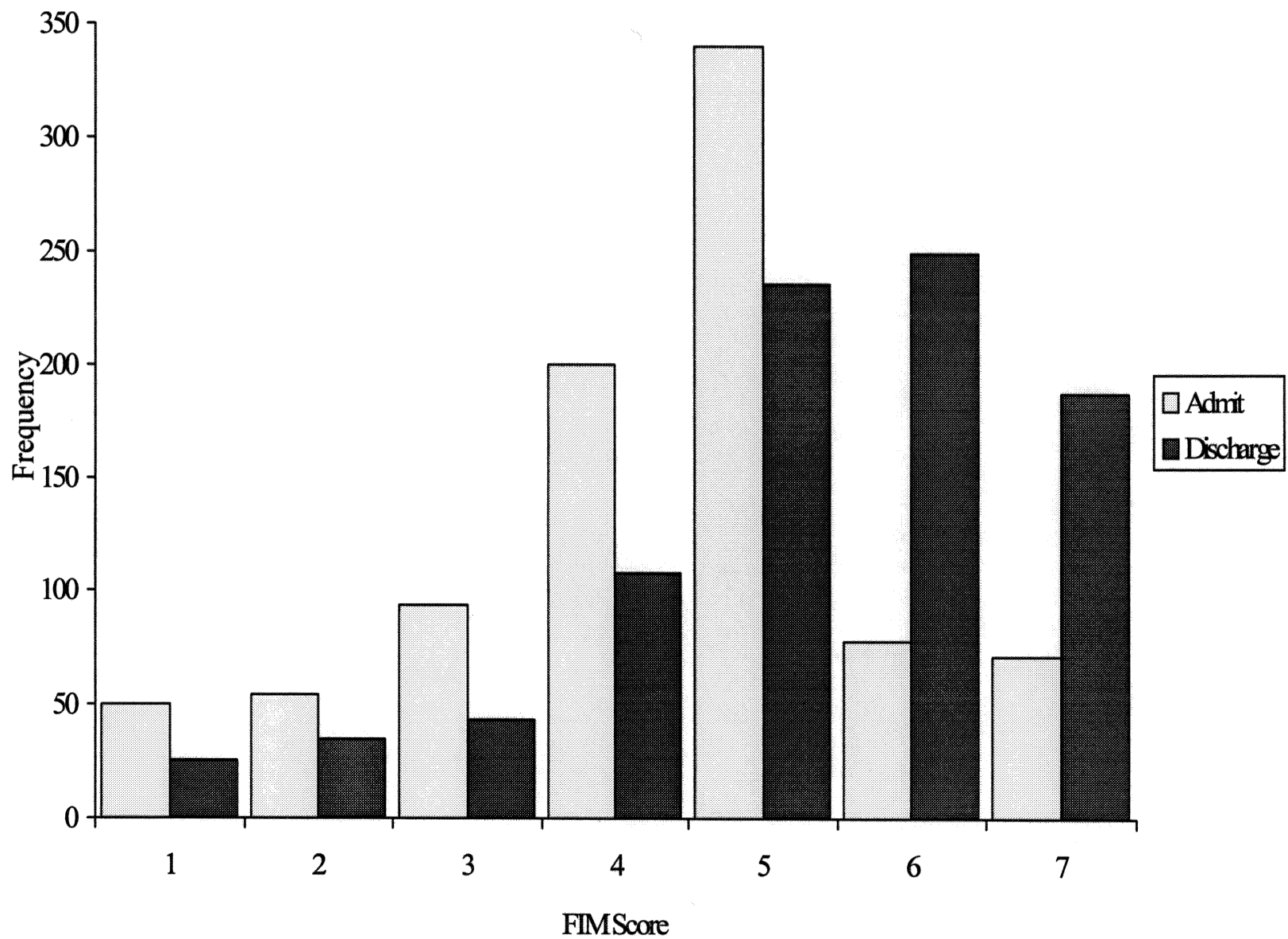


Figure 4. Distribution of FIM 5 Scores

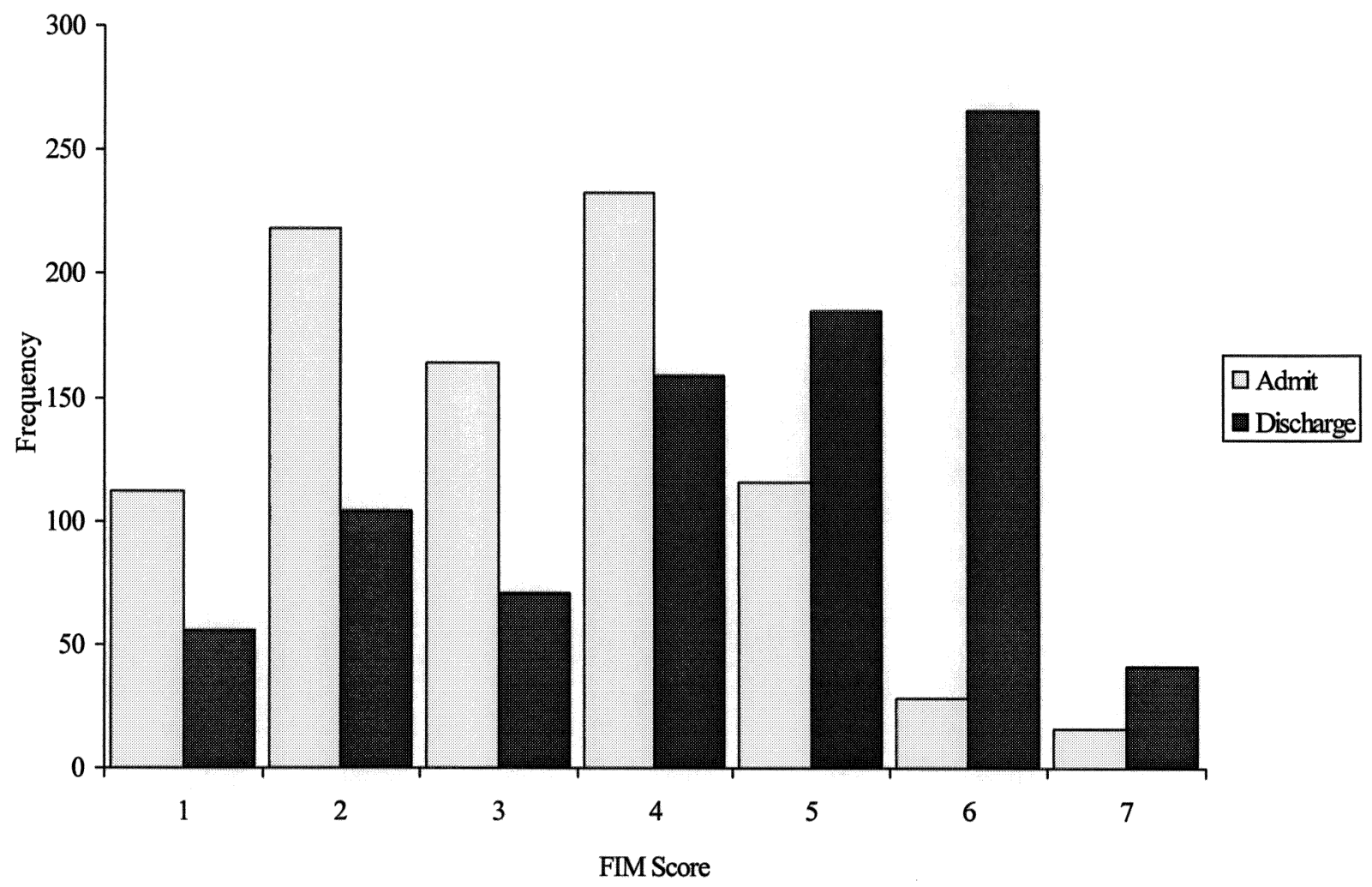

Figure 5. Distribution of FIM 6 Scores

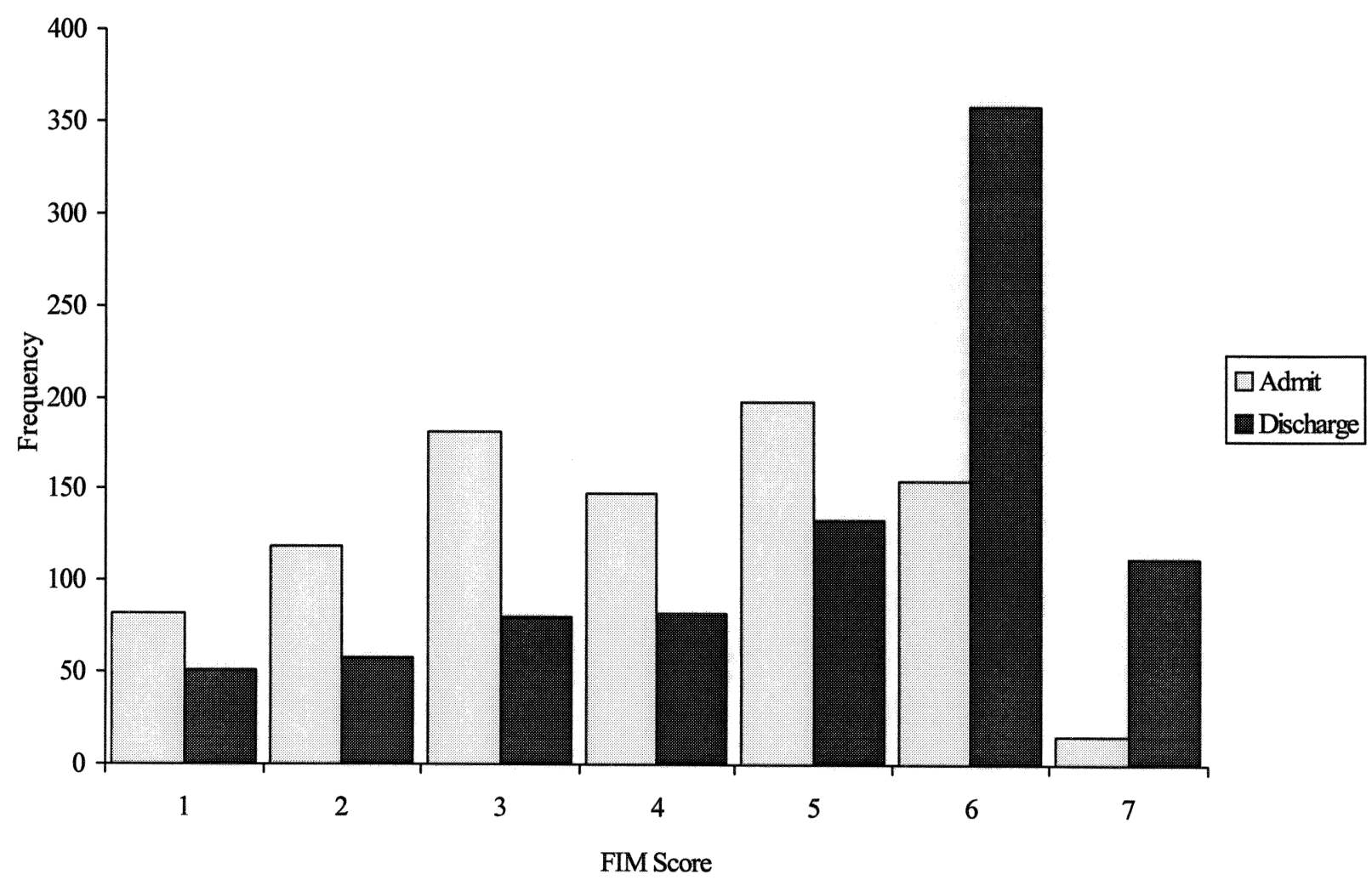


Figure 6. Distribution of FIM 7 Scores

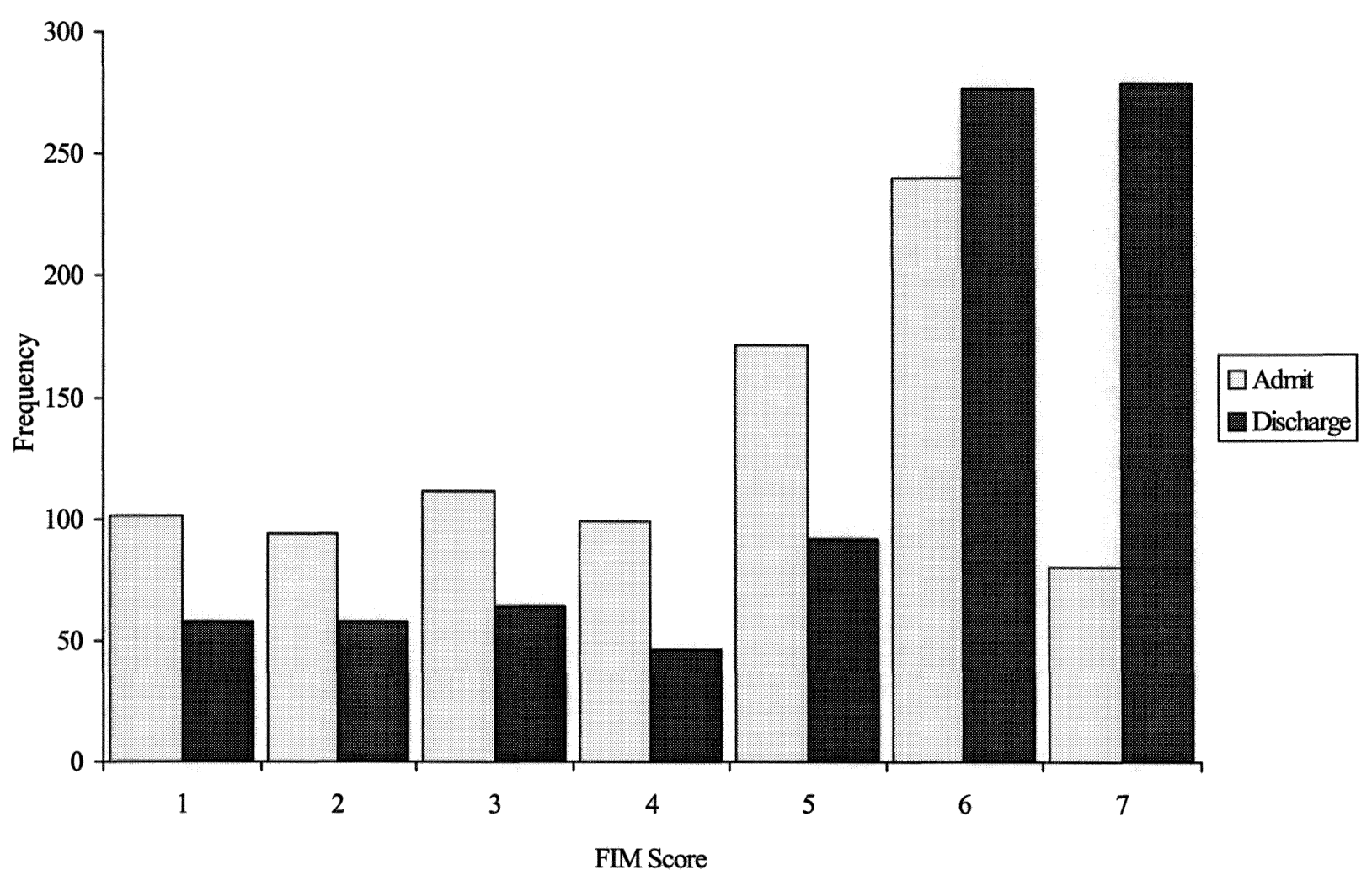

Figure 7. Distribution of FIM 13 Scores

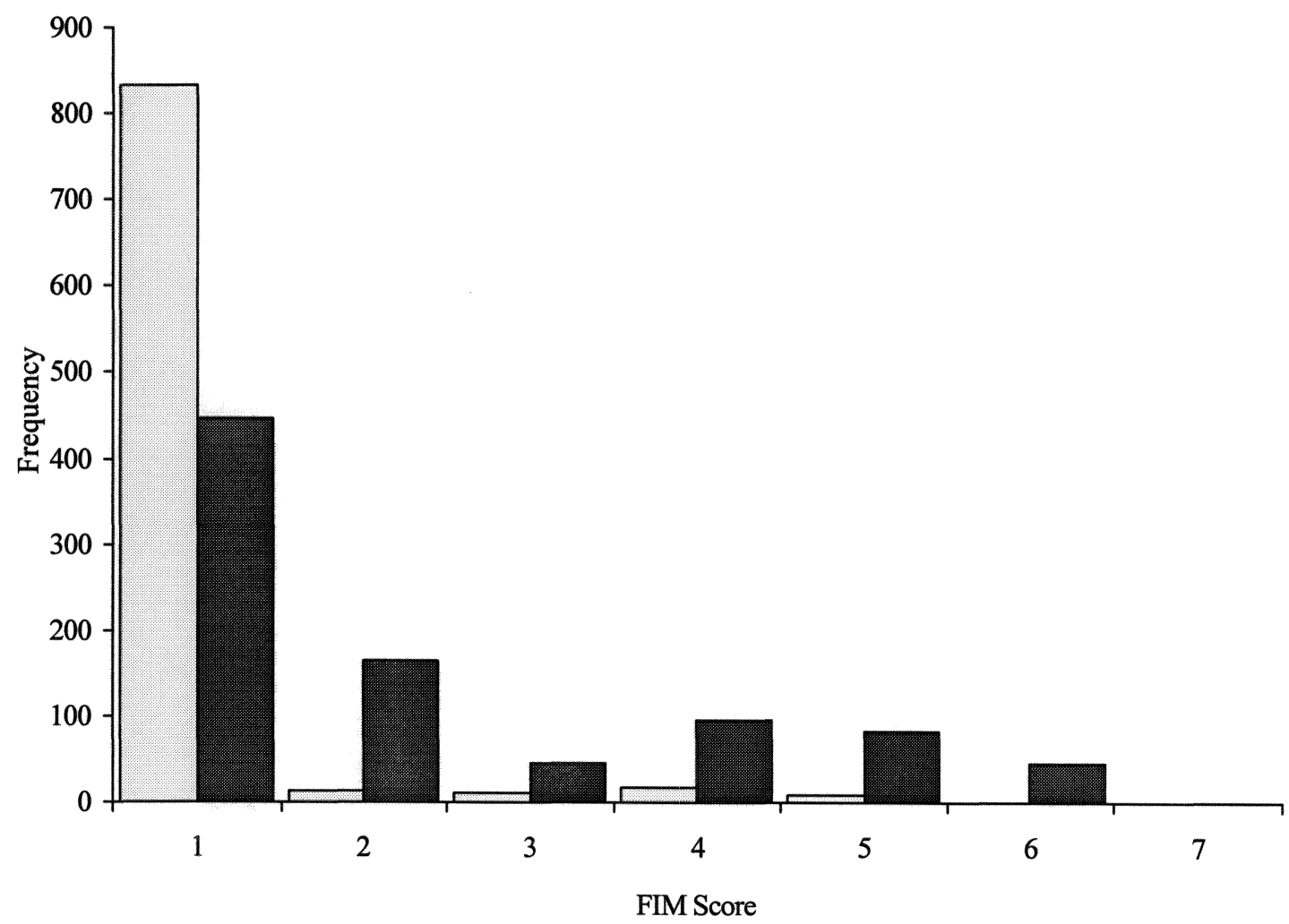


In general, both $\mathrm{T}^{2}$ and $\mathrm{RT}$ were robust with respect to Type I error using Bradley's (1978) "liberal" (p.146) criteria $(|\rho-\alpha| \leq \alpha / 2)$ but often non-robust using more stringent criteria in other reasonable conditions. This is particularly true the presence of a highly skewed distribution such as FIM 13. The presence of the highly skewed FIM 13 distribution caused both tests to become very conservative. This was true for each alpha level examined (i.e., $.10, .05$, and .01$)$.

This distribution is roughly analogous to the $\mathrm{L}$ shaped distribution investigated by Bradley $(1977 ; 1980$; 1982) which led to similar occurrences in the univariate case. Indeed, in situations where Fim 13 was sampled, the degree of non-robustness was quite pronounced with Type I error rates for both $\mathrm{T}^{2}$ and RT becoming extremely conservative, reaching a low of .0177 (normal alpha $=.05$ ) at sample size $(5,15)$ using the combination of FIM 1 and FIM 13.

Levels of non-robustness, as expected, were dependent on the interplay of several factors including sample size and alpha level. It is evident that, as in the univariate case, blanket statements concerning robustness of $\mathrm{T}^{2}$ and RT cannot be made. Moreover, where non-robustness was present, the RT was frequently more conservative than $\mathrm{T}^{2}$ and, in general, levels of robustness did not improve as sample size increased.
Robustness - Five Dependent Variables

Results for the five-dependent variable condition were similar to the two-dependent variable and are located in Table 4 . Both $\mathrm{T}^{2}$ and RT were relatively robust with respect to Type I error under most combinations but relatively non-robust at others. As in the two-dependent variable condition, when levels of non-robustness were found they tended to be conservative in the presence of extreme asymmetry such as that displayed in FIM 13. What is remarkable is that each test became conservative when FIM 13 was present, regardless of the combinations of other shapes. That is, the effect of a skewed distribution was not offset by the presence of more "tame" distributions. Unlike the two-dependent variable condition, however, both tests tended to improve as sample size increased. There were, of course, several noted exceptions.

The levels of robustness had little deviation from nominal alpha except when the distribution sampled was highly skewed (e.g., FIM 13). See Table 5.

\section{Power - Two Dependent Variables}

Results for this portion of the study are located in Table 3. In general, across all data sets, alpha levels, and sample size combinations, the RT was consistently more powerful than $\mathrm{T}^{2}$. Although, the power of both tests, as expected, increased with increased sample size, the RT, on

Figure 8. Distribution of OT Composite Score

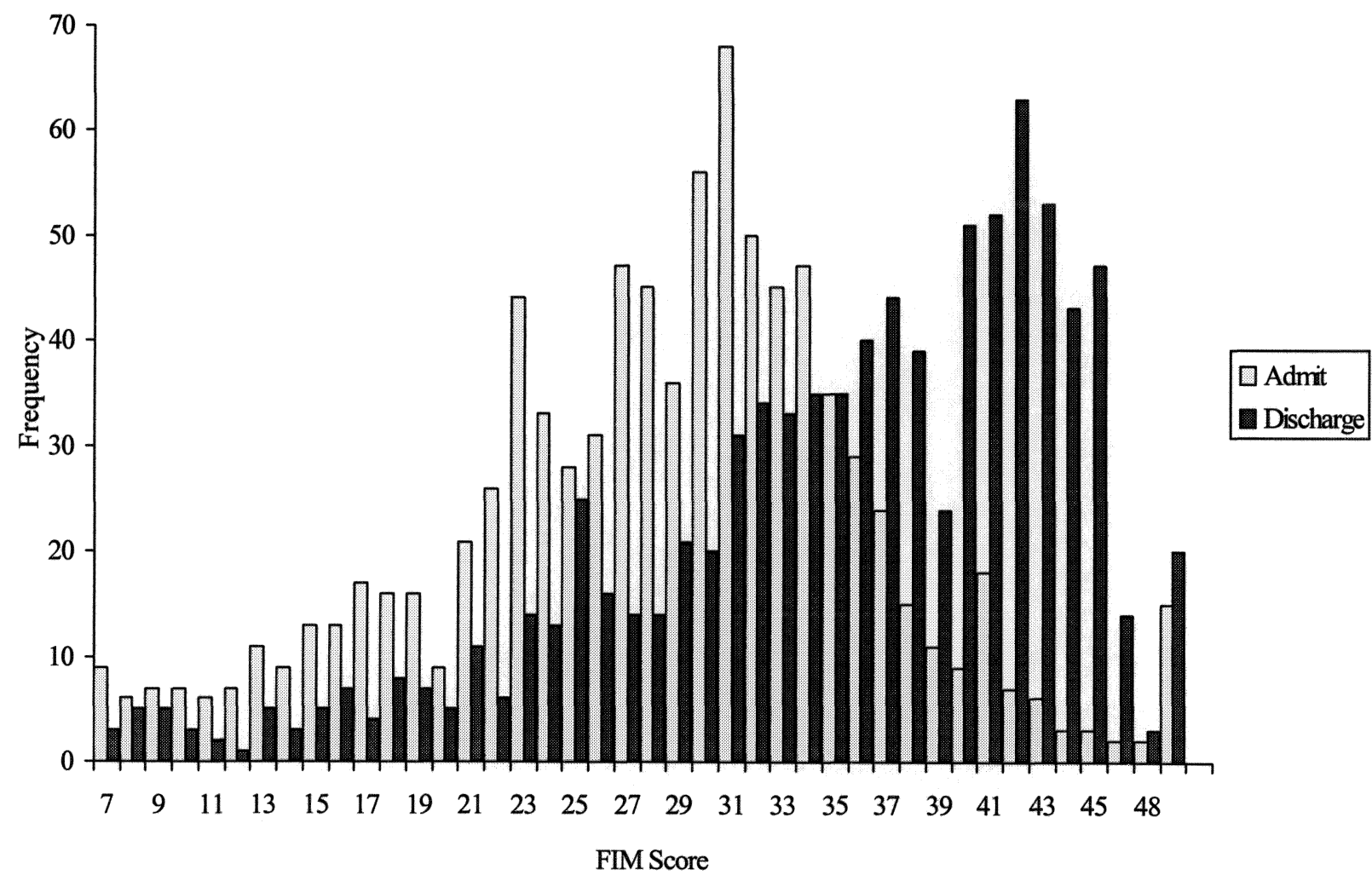


Table 2: Type I Error Rates for Two-dependent Variable Combinations at Alpha $=.05$.

\begin{tabular}{|c|c|c|c|c|c|c|c|c|c|}
\hline \multirow{2}{*}{$\begin{array}{l}\text { Fim } \\
\text { Distributions }\end{array}$} & \multirow[b]{2}{*}{$\underline{\text { Statistic }}$} & \multicolumn{8}{|c|}{ Sample Size } \\
\hline & & $\underline{5,15}$ & $\underline{10,10}$ & $\underline{10,20}$ & $\underline{15,15}$ & $\underline{15,45}$ & $\underline{30,30}$ & $\underline{25,75}$ & $\underline{50,50}$ \\
\hline \multirow[t]{2}{*}{1,3} & $\mathbf{H T}^{2}$ & .0468 & .0468 & .0371 & .0513 & .0487 & .0469 & .0507 & .0496 \\
\hline & $\mathrm{RT}$ & .0486 & .0510 & .0382 & .0527 & .0491 & .0499 & .0534 & .0513 \\
\hline \multirow[t]{2}{*}{1,7} & $\mathbf{H T}^{2}$ & .0456 & .0529 & .0503 & .0493 & .0427 & .0520 & .0501 & .0468 \\
\hline & $\mathrm{RT}$ & .0497 & .0570 & .0522 & .0515 & .0458 & .0503 & .0503 & .0501 \\
\hline \multirow[t]{2}{*}{1,13} & $\mathbf{H T}^{\mathbf{2}}$ & .0365 & .0199 & .0265 & .0177 & .0463 & .0246 & .0470 & .0308 \\
\hline & $\mathrm{RT}$ & .0329 & .0211 & .0253 & .0199 & .0426 & .0233 & .0467 & .0309 \\
\hline \multirow[t]{2}{*}{$1, \mathrm{Ot}$} & $\mathbf{H T}^{2}$ & .0496 & .0469 & .0484 & .0477 & .0464 & .0467 & .0532 & .0449 \\
\hline & $\mathrm{RT}$ & .0532 & .0527 & .0525 & .0514 & .0472 & .0486 & .0527 & .0485 \\
\hline \multirow[t]{2}{*}{3,7} & $\mathbf{H T}^{2}$ & .0486 & .0494 & .0487 & .0523 & .0500 & .0463 & .0530 & .0472 \\
\hline & $\mathrm{RT}$ & .0485 & .0506 & .0511 & .0522 & .0479 & .0457 & .0511 & .0484 \\
\hline \multirow[t]{2}{*}{3,13} & $\mathbf{H T}^{2}$ & .0368 & .0200 & .0279 & .0204 & .0413 & .0244 & .0425 & .0312 \\
\hline & $\mathrm{RT}$ & .0316 & .0186 & .0252 & .0210 & .0386 & .0247 & .0413 & .0296 \\
\hline \multirow[t]{2}{*}{4,7} & $\mathbf{H T}^{\mathbf{2}}$ & .0486 & .0482 & .0454 & .0487 & .0514 & .0520 & .0514 & .0501 \\
\hline & $\mathrm{RT}$ & .0518 & .0491 & .0489 & .0512 & .0498 & .0532 & .0507 & .0508 \\
\hline \multirow[t]{2}{*}{4,13} & $\mathbf{H T}^{2}$ & .0392 & .0192 & .0249 & .0201 & .0396 & .0233 & .0477 & .0315 \\
\hline & $\mathrm{RT}$ & .0342 & .0187 & .0239 & .0209 & .0367 & .0233 & .0490 & .0302 \\
\hline \multirow[t]{2}{*}{$4, \mathrm{Ot}$} & $\mathbf{H T}^{2}$ & .0512 & .0521 & .0487 & .0516 & .0478 & .0493 & .0487 & .0488 \\
\hline & $\mathrm{RT}$ & .0513 & .0564 & .0503 & .0541 & .0486 & .0511 & .0504 & .0500 \\
\hline \multirow[t]{2}{*}{6,7} & $\mathbf{H T}^{\mathbf{2}}$ & .0496 & .0537 & .0517 & .0510 & .0502 & .0537 & .0500 & .0532 \\
\hline & $\mathrm{RT}$ & .0506 & .0536 & .0512 & .0523 & .0503 & .0540 & .0508 & .0531 \\
\hline \multirow[t]{2}{*}{6,13} & $\mathbf{H T}^{\mathbf{2}}$ & .0399 & .0223 & .0255 & .0192 & .0451 & .0259 & .0500 & .0327 \\
\hline & $\mathrm{RT}$ & .0319 & .0203 & .0225 & .0192 & .0412 & .0246 & .0444 & .0307 \\
\hline \multirow[t]{2}{*}{$6, \mathrm{Ot}$} & $\mathbf{H T}^{2}$ & .0512 & .0509 & .0482 & .0513 & .0456 & .0496 & .0510 & .0525 \\
\hline & $\mathrm{RT}$ & .0502 & .0547 & .0511 & .0544 & .0460 & .0511 & .0501 & .0506 \\
\hline \multirow[t]{2}{*}{$13, \mathrm{Ot}$} & HT2 & .0375 & .0208 & .0256 & .0203 & .0489 & .0215 & .0447 & .0290 \\
\hline & $\mathrm{RT}$ & .0314 & .0218 & .0243 & .0208 & .0412 & .0226 & .0422 & .0296 \\
\hline
\end{tabular}

average, tended to reject the null hypothesis anywhere from $1 \%$ to $17 \%$ more often than $\mathrm{T}^{2}$. And although differences were moderate at times, the power of RT was rarely less than $\mathrm{T}^{2}$. The distribution of FIM 13 scores had a negative impact on power at small sample sizes compared with other distribution combinations, but each test generally rehabilitated itself as sample size increased. However, power in the presence of FIM 13 scores must be interpreted in the 
Table 3: Type I Error Rates for Five-dependent Variable Combinations at Alpha $=.05$.

\begin{tabular}{|c|c|c|c|c|c|c|c|c|c|}
\hline Fim & \multirow[b]{2}{*}{ Statistic } & \multicolumn{6}{|c|}{ Sample Size } & \multirow[b]{2}{*}{25,75} & \multirow[b]{2}{*}{50,50} \\
\hline Distributions & & 5,15 & 10,10 & 10,20 & 15,15 & 15,45 & 30,30 & & \\
\hline \multirow[t]{2}{*}{$1,4,5,6,7$} & $\mathbf{H T}^{2}$ & .0499 & .0501 & .0529 & .0480 & .0515 & .0508 & .0501 & .0494 \\
\hline & RT & .0515 & .0544 & .0524 & .0499 & .0509 & .0529 & .0516 & .0497 \\
\hline \multirow[t]{2}{*}{$1,5,6,7,13$} & $\mathbf{H T}^{\mathbf{2}}$ & .0439 & .0350 & .0359 & .0322 & .0445 & .0343 & .0478 & .0408 \\
\hline & RT & .0410 & .0363 & .0356 & .0342 & .0422 & .0346 & .0444 & .0410 \\
\hline \multirow[t]{2}{*}{$1,6,7,13, \mathrm{Ot}$} & $\mathbf{H T}^{\mathbf{2}}$ & .0424 & .0349 & .0381 & .0368 & .0464 & .0374 & .0472 & .0453 \\
\hline & $\mathrm{RT}$ & .0371 & .0362 & .0362 & .0370 & .0451 & .0361 & .0491 & .0427 \\
\hline \multirow[t]{2}{*}{$3,4,5,6,7$} & $\mathbf{H T}^{2}$ & .0487 & .0535 & .0508 & .0509 & .0456 & .0527 & .0511 & .0465 \\
\hline & RT & .0499 & .0547 & .0520 & .0515 & .0489 & .0524 & .0519 & .0479 \\
\hline \multirow[t]{2}{*}{$3,5,6,7,13$} & $\mathbf{H T}^{2}$ & .0470 & .0354 & .0365 & .0327 & .0490 & .0373 & .0496 & .0411 \\
\hline & $\mathrm{RT}$ & .0435 & .0359 & .0352 & .0330 & .0463 & .0365 & .0466 & .0401 \\
\hline \multirow[t]{2}{*}{$3,6,7,13, \mathrm{Ot}$} & $\mathbf{H T}^{2}$ & .0443 & .0374 & .0380 & .0362 & .0445 & .0351 & .0505 & .0439 \\
\hline & $\mathrm{RT}$ & .0371 & .0399 & .0354 & .0378 & .0422 & .0366 & .0492 & .0418 \\
\hline \multirow[t]{2}{*}{$4,5,6,7,13$} & $\mathbf{H T}^{2}$ & .0431 & .0372 & .0395 & .0347 & .0467 & .0397 & .0508 & .0386 \\
\hline & $\mathrm{RT}$ & .0418 & .0361 & .0385 & .0343 & .0461 & .0376 & .0463 & .0394 \\
\hline \multirow[t]{2}{*}{$4,6,7,13, \mathrm{Ot}$} & $\mathbf{H T}^{\mathbf{2}}$ & .0485 & .0346 & .0362 & .0365 & .0470 & .0373 & .0463 & .0446 \\
\hline & $\mathrm{RT}$ & .0444 & .0363 & .0342 & .0372 & .0428 & .0367 & .0452 & .0412 \\
\hline \multirow[t]{2}{*}{$5,6,7,13, \mathrm{Ot}$} & $\mathbf{H T}^{2}$ & .0418 & .0341 & .0378 & .0335 & .0453 & .0355 & .0506 & .0397 \\
\hline & RT & .0392 & .0333 & .0379 & .0355 & .0438 & .0368 & .0474 & .0375 \\
\hline
\end{tabular}

context of its associated Type I error.

Power - Five Dependent Variables.

Results for this portion of the study are located in Table 6. Although the RT maintained power over $\mathrm{T}^{2}$ in most situations, the difference in magnitude tended to be less than in the two-dependent variable condition. There are instances, however, where the RT is substantially more powerful than $\mathrm{T}^{2}$. However, large differences in power were less frequent and both tests tended to perform equally well with greater frequency as sample size increased. An additional result seemed to be that the impact of FIM 13 scores was less profound. Under the conditions where each distribution was sampled multiple times (see Table 7), the only remarkable results were, again, when Fim 13 served as the sole dependent variable distribution (i.e., FIM 13 sampled five times). In general, RT worked better at small sample sizes, but $\mathrm{T}^{2}$ recovered as sample size increased.

Summary of Results

Results suggest that both $\mathrm{T}^{2}$ and the RT are robust under most non-normal situations in the independent samples case when data are scaled at the Ordinal level. These results hold for both the two-dependent variable and five-dependent variable conditions. The most noted exception for both conditions was in the presence of a highly skewed distribution such as the FIM 13 used in this study. With respect to power, $\mathrm{T}^{2}$ recovers somewhat as sample size reaches $(25,75)$ and $(50,50)$, however, RT maintains a modest power advantage at all sample sizes and 
Table 4: Type I Error Rates for Five-dependent Variables Sampling Using One Distribution for Each Variable at Alpha $=.05$.

\begin{tabular}{|c|c|c|c|c|c|c|c|c|c|}
\hline \multirow{2}{*}{$\begin{array}{l}\text { Fim } \\
\text { Distributions }\end{array}$} & \multirow[b]{2}{*}{ Statistic } & \multicolumn{8}{|c|}{ Sample Size } \\
\hline & & 5,15 & 10,10 & 10,20 & 15,15 & 15,45 & 30,30 & 25,75 & 50,50 \\
\hline \multirow[t]{2}{*}{1} & $\mathbf{H T}^{2}$ & .0485 & .0476 & .0474 & .0491 & .0520 & .0515 & .0462 & .0529 \\
\hline & $\mathrm{RT}$ & .0460 & .0546 & .0512 & .0564 & .0524 & .0527 & .0461 & .0522 \\
\hline \multirow[t]{2}{*}{4} & $\mathbf{H T}^{2}$ & .0495 & .0487 & .0469 & .0515 & .0488 & .0481 & .0531 & .0509 \\
\hline & $\mathrm{RT}$ & .0493 & .0580 & .0467 & .0496 & .0492 & .0516 & .0505 & .0521 \\
\hline \multirow[t]{2}{*}{7} & $\mathbf{H T}^{2}$ & .0505 & .0513 & .0531 & .0540 & .0516 & .0523 & .0531 & .0475 \\
\hline & $\mathrm{RT}$ & .0506 & .0560 & .0526 & .0546 & .0509 & .0545 & .0529 & .0489 \\
\hline \multirow[t]{2}{*}{13} & $\mathbf{H T}^{2}$ & - & - & - & - & .0382 & .0015 & .0422 & .0079 \\
\hline & $\mathrm{RT}$ & - & - & - & - & .0296 & .0008 & .0351 & .0069 \\
\hline \multirow[t]{2}{*}{ Ot } & $\mathbf{H T}^{2}$ & .0453 & .0475 & .0535 & .0507 & .0467 & .0484 & .0508 & .0475 \\
\hline & $\mathrm{RT}$ & .0478 & .0529 & .0562 & .0559 & .0507 & .0505 & .0483 & .0493 \\
\hline
\end{tabular}

* Results were not available for sample sizes less than $(15,45)$ for FIM 13 . Due to the highly skewed and discrete nature of this distribution and given the restrictions on the number of repetitions in this simulation study, no variance could be calculated for small sample sizes.

distribution combinations. There were noted exceptions. For instance, $\mathrm{T}^{2}$ displayed almost negligible power advantages over RT when distribution combinations $(1,3),(1,7)$, and $(1,13)$ were sampled for reasons unknown.

Although $\mathrm{T}^{2}$ is more powerful under normality, the RT has been shown to be only slightly less powerful (Bhattacharyya, Johnson, \& Neave, 1971; Tiku \& Singh, 1982; Nath \& Duran, 1983) and in non-normal situations is accepted as being more powerful in many cases (Nath, 1982; Nath \& Duran, 1983; Zwick, 1986). In this study, the RT consistently outperformed $\mathrm{T}^{2}$ at nearly every sample size, alpha level, and dependent variable combination. Unlike previous studies examining robustness and power which often employ the use of artificial distributions and/ or treatment effects, this study examined the operating characteristics of $\mathrm{T}^{2}$ and the RT under real conditions. In fact, the results of this study have direct implications to the substantive field from which these distributions came, as well as other fields with similar types of data.

\section{Conclusion}

With respect to ordinal scaled data in the form of likert scaled data commonly obtained in applied data analysis situations and its inherent violation of normality when testing for equality of centroids, the Rank Transformation procedure provides an increase in power over Hotelling's $\mathrm{T}^{2}$ while maintaining acceptable Type I error rates. This is particularly true with two-dependent variables and smaller sample sizes. The results must not be extrapolated beyond the context of this study to other data analysis layouts, such as the multivariate factorial analysis of variance. The robustness results of this study on the multivariate two independent samples layout with likert scaled data were in agreement with, and extend the results found by Heeren \& D'Agostino (1987) and Hsu \& Feldt (1969) on the univariate independent samples t-test; and the robustness and power results found by Nanna \& Sawilowsky (1998) for the rank transformation analog to the univariate two independent samples layout with likert scaled data.

\section{References}

Akritas, M.G. (1990). The rank transform method in some two-factor designs. Journal of the American Statistical Association, 85, 73-78.

Akritas, M.G. (1991). Limitations of the rank transform procedure: A study of repeated measures designs, part 1. Journal of the American Statistical Association, $86,457-460$. 
Table 5: Power for Two-dependent Variable Combinations at Alpha $=.05$.

\begin{tabular}{|c|c|c|c|c|c|c|c|c|c|}
\hline \multirow{2}{*}{$\begin{array}{l}\text { Fim } \\
\text { Distributions }\end{array}$} & \multirow[b]{2}{*}{$\underline{\text { Statistic }}$} & \multicolumn{8}{|c|}{ Sample Size } \\
\hline & & $\underline{5,15}$ & $\underline{10,10}$ & 10,20 & $\underline{15,15}$ & $\underline{15,45}$ & $\underline{30,30}$ & $\underline{25,75}$ & $\underline{50,50}$ \\
\hline \multirow[t]{2}{*}{1,3} & $\mathbf{H T}^{2}$ & .3225 & .3953 & .5205 & .5693 & .7683 & .8755 & .9429 & .9837 \\
\hline & $\mathrm{RT}$ & .3255 & .4357 & .5733 & .6245 & .8357 & .9174 & .9740 & .9919 \\
\hline \multirow[t]{2}{*}{1,7} & $\mathbf{H T}^{2}$ & .2155 & .2211 & .3096 & .3323 & .4857 & .5924 & .7095 & .8315 \\
\hline & $\mathrm{RT}$ & .2075 & .2686 & .3570 & .4024 & .5823 & .7094 & .8214 & .9179 \\
\hline \multirow[t]{2}{*}{1,13} & $\mathbf{H T}^{2}$ & .1150 & .2404 & .3042 & .5067 & .6717 & .9256 & .9598 & .9967 \\
\hline & $\mathrm{RT}$ & .1058 & .3371 & .4203 & .6584 & .8473 & .9795 & .9922 & .9997 \\
\hline \multirow[t]{2}{*}{$1, \mathrm{Ot}$} & $\mathbf{H T}^{2}$ & .3176 & .3967 & .5224 & .5754 & .7614 & .8698 & .9408 & .9817 \\
\hline & $\mathrm{RT}$ & .3290 & .4434 & .5867 & .6454 & .8470 & .9287 & .9800 & .9949 \\
\hline \multirow[t]{2}{*}{3,7} & $\mathbf{H T}^{2}$ & .3300 & .4429 & .5587 & .6216 & .8050 & .9099 & .9657 & .9942 \\
\hline & $\mathrm{RT}$ & .3599 & .5018 & .6445 & .7009 & .8916 & .9525 & .9898 & .9984 \\
\hline \multirow[t]{2}{*}{3,13} & $\mathbf{H T}^{2}$ & .2411 & .4723 & .5792 & .7417 & .9115 & .9897 & .9981 & .9997 \\
\hline & $\mathrm{RT}$ & .2723 & .5661 & .7187 & .8491 & .9738 & .9980 & 9999 & .9999 \\
\hline \multirow[t]{2}{*}{4,7} & $\mathbf{H T}^{2}$ & .2381 & .2959 & .3948 & .4396 & .6089 & .7518 & .8365 & .9346 \\
\hline & $\mathrm{RT}$ & .2549 & .3635 & .4781 & .5396 & .7363 & .8555 & .9317 & .9787 \\
\hline \multirow[t]{2}{*}{4,13} & $\mathbf{H T}^{2}$ & .1432 & .3221 & .3914 & .6033 & .7843 & .9633 & .9849 & .9988 \\
\hline & $\mathrm{RT}$ & .1617 & .4274 & .5493 & .7497 & .9246 & .9903 & .9989 & .9999 \\
\hline \multirow[t]{2}{*}{$4, \mathrm{Ot}$} & $\mathbf{H T}^{2}$ & .3626 & .4719 & .5836 & .6541 & .8351 & .9243 & .9752 & .9954 \\
\hline & $\mathrm{RT}$ & .3872 & .5349 & .6670 & .7334 & .9156 & .9688 & .9938 & .9992 \\
\hline \multirow[t]{2}{*}{6,7} & $\mathbf{H T}^{2}$ & .2636 & .3325 & .4326 & .4832 & .6637 & .8083 & .8878 & .9635 \\
\hline & $\mathrm{RT}$ & .2823 & .3965 & .5200 & .5743 & .7854 & .8905 & .9556 & .9893 \\
\hline \multirow[t]{2}{*}{6,13} & $\mathbf{H T}^{2}$ & .1604 & .3638 & .4498 & .6422 & .8290 & .9739 & .9903 & .9998 \\
\hline & $\mathrm{RT}$ & .1803 & .4690 & .6042 & .7822 & .9476 & .9946 & .9986 & 1.000 \\
\hline \multirow[t]{2}{*}{$6, \mathrm{Ot}$} & $\mathbf{H T}^{2}$ & .3818 & .5024 & .6238 & .6875 & .8624 & .9416 & .9804 & .9967 \\
\hline & $\mathrm{RT}$ & .4135 & .5622 & .7058 & .7643 & .9345 & .9763 & .9960 & .9994 \\
\hline \multirow[t]{2}{*}{$13, \mathrm{Ot}$} & $\mathrm{HT}^{2}$ & .2392 & .4762 & .5869 & .7454 & .9070 & .9883 & .9966 & .9998 \\
\hline & RT & .2686 & .5872 & .7294 & .8612 & .9778 & .9987 & 1.000 & 1.000 \\
\hline
\end{tabular}

Algina, J. \& Oshima, T. (1990). Robustness of the independent samples Hotelling's $\mathrm{T}^{2}$ to variance-covariance heteroscedasticity when sample sizes are unequal and in small ratios. Psychological Bulletin, 108(2), 308-
Anderson, N. H. (1961). Scales and statistics: Parametric and nonparametric. Psychological Bulletin, 58, 305-316. 313. 
Table 6: Power for Five-dependent Variable Combinations at Alpha $=.05$.

\begin{tabular}{|c|c|c|c|c|c|c|c|c|c|}
\hline \multirow[b]{2}{*}{$\begin{array}{l}\text { Fim } \\
\text { Distributions }\end{array}$} & \multirow[b]{2}{*}{$\underline{\text { Statistic }}$} & \multicolumn{8}{|c|}{ Sample Size } \\
\hline & & $\underline{5,15}$ & $\underline{10,10}$ & $\underline{10,20}$ & $\underline{15,15}$ & $\underline{15,45}$ & $\underline{30,30}$ & $\underline{25,75}$ & $\underline{50,50}$ \\
\hline \multirow[t]{2}{*}{$1,4,5,6,7$} & $\mathbf{H T}^{2}$ & .3811 & .4998 & .6751 & .7382 & .9282 & .9831 & .9960 & .9998 \\
\hline & $\mathrm{RT}$ & .3904 & .5705 & .7524 & .8066 & .9694 & .9942 & .9996 & 1.000 \\
\hline \multirow[t]{2}{*}{$1,5,6,7,13$} & $\mathbf{H T}^{2}$ & .3202 & .4950 & .6665 & .7930 & .9544 & .9953 & .9999 & 1.000 \\
\hline & $\mathrm{RT}$ & .3302 & .5882 & .7694 & .8798 & .9881 & .9995 & 1.000 & 1.000 \\
\hline \multirow[t]{2}{*}{$1,6,7,13, \mathrm{Ot}$} & $\mathbf{H T}^{2}$ & .3312 & .5105 & .6891 & .8065 & .9600 & .9964 & .9999 & .9999 \\
\hline & $\mathrm{RT}$ & .3528 & .6197 & .8080 & .9003 & .9932 & 9999 & 1.000 & 1.000 \\
\hline \multirow[t]{2}{*}{$3,4,5,6,7$} & $\mathbf{H T}^{2}$ & .4549 & .6134 & .7919 & .8506 & .9758 & .9970 & .9997 & 1.000 \\
\hline & $\mathrm{RT}$ & .4853 & .6824 & .8626 & .9020 & .9937 & .9992 & 1.000 & 1.000 \\
\hline \multirow[t]{2}{*}{$3,5,6,7,13$} & $\mathbf{H T}^{2}$ & .3920 & .6135 & .7923 & .8892 & .9873 & .9991 & .9999 & 1.000 \\
\hline & $\mathrm{RT}$ & .4251 & .7005 & .8781 & .9440 & .9977 & .9999 & 1.000 & 1.000 \\
\hline \multirow[t]{2}{*}{$3,6,7,13, \mathrm{Ot}$} & $\mathbf{H T}^{2}$ & .4116 & .6232 & .7979 & .8926 & .9869 & .9997 & 1.000 & 1.000 \\
\hline & $\mathrm{RT}$ & .4474 & .7194 & .8929 & .9547 & .9989 & .9999 & 1.000 & 1.000 \\
\hline \multirow[t]{2}{*}{$4,5,6,7,13$} & $\mathbf{H T}^{2}$ & .3416 & .5370 & .7097 & .8235 & .9720 & .9995 & .9998 & 1.000 \\
\hline & $\mathrm{RT}$ & .3636 & .6275 & .8222 & .9061 & .9945 & .9999 & 1.000 & 1.000 \\
\hline \multirow[t]{2}{*}{$4,6,7,13, \mathrm{Ot}$} & $\mathbf{H T}^{2}$ & .3574 & .5447 & .7290 & .8378 & .9744 & .9984 & .9996 & 1.000 \\
\hline & $\mathrm{RT}$ & .3886 & .6595 & .8460 & .9254 & .9963 & .9997 & 1.000 & 1.000 \\
\hline \multirow[t]{2}{*}{$5,6,7,13, \mathrm{Ot}$} & $\mathbf{H T}^{2}$ & .3949 & .6108 & .7912 & .8870 & .9869 & .9996 & 1.000 & 1.000 \\
\hline & $\mathrm{RT}$ & .4204 & .7065 & .8814 & .9472 & .9982 & 1.000 & 1.000 & 1.000 \\
\hline
\end{tabular}

Bhattacharyya, G. K., Johnson, R.A., \& Neave, H. R. (1971). A comparative power study of the bivariate rank sum test and $\mathrm{T}^{2}$. Technometrics, 13(1), 191-198.

Blair, R. C., Sawilowsky, S. S., \& Higgins, J. J. (1987). Limitations of the rank transform in tests for interaction. Communications in Statistics: Computation and Simulation, B16, 1133-1145.

Blair, R. C., Higgins, J. J., Karniski, W., \& Kromrey, J. D. (1994). A study of multivariate permutation tests which may replace Hotelling's $\mathrm{T}^{2}$ in prescribed circumstances. Multivariate Behavioral Research, 29(2), 141-163.

Boneau, C. A. (1961). A note on measurement scales and statistical tests. American Psychologist, 16, 260261.

Bradley, J. V. (1968). Distribution-free statistical tests. Englewood Cliffs, NJ: Prentice Hall.
Bradley, J. V. (1977). A common situation conducive to bizarre distribution shapes. The American Statistician, 31(4), 147-150

Bradley, J. V. (1980b). Nonrobustness in classical tests on means and variances: a large scale sampling study. Bulletin of the Psychonomic Society, 15(4), 275298.

Bradley, J. V. (1982). The insidious L-shaped distribution. Bulletin of the Psychonomic Society, 20(2), 8588.

Byrnes, M. B. \& Powers, F. F. (1989). FIM: Its use in identifying rehabilitation needs in the head injured patient. Journal of Neuroscience Nursing, 21, 61-63.

Chase, G. R. \& Bulgren, W. G. (1971). A monte carlo investigation of the robustness of $\mathrm{T}^{2}$. Journal of the American Statistical Association, 66, 499-502. 
Table 7: Power for Five-dependent Variables Using the Same Distribution for All Sampled Variables at Alpha $=.05$.

\begin{tabular}{|c|c|c|c|c|c|c|c|c|c|}
\hline \multirow[b]{2}{*}{$\begin{array}{l}\text { Fim } \\
\text { Distributions }\end{array}$} & \multirow[b]{2}{*}{ Statistic } & \multicolumn{8}{|c|}{ Sample Size } \\
\hline & & $\underline{\mathbf{5 , 1 5}}$ & $\underline{10,10}$ & $\underline{10,20}$ & $\underline{15,15}$ & $\underline{15,45}$ & $\underline{\mathbf{3 0 , 3 0}}$ & $\underline{25,75}$ & $\underline{\mathbf{5 0 , 5 0}}$ \\
\hline \multirow[t]{2}{*}{1} & $\mathbf{H T}^{2}$ & .2908 & .2329 & .4023 & .3739 & .6404 & .7232 & .8603 & .9336 \\
\hline & $\mathrm{RT}$ & .2383 & .2654 & .4047 & .4254 & .6699 & .7970 & .9036 & .9685 \\
\hline \multirow[t]{2}{*}{4} & $\mathbf{H T}^{2}$ & .3633 & .4633 & .6475 & .7083 & .9123 & .9732 & .9951 & .9997 \\
\hline & $\mathrm{RT}$ & .3812 & .5509 & .7362 & .8092 & .9638 & .9919 & .9994 & 1.000 \\
\hline \multirow[t]{2}{*}{7} & $\mathbf{H T}^{2}$ & .3015 & .3699 & .5235 & .5883 & .8254 & .9293 & .9784 & .9962 \\
\hline & $\mathrm{RT}$ & .3240 & .4899 & .6610 & .7296 & .9372 & .9834 & .9979 & .9999 \\
\hline \multirow[t]{2}{*}{13} & $\mathbf{H T}^{2}$ & .0250 & .4770 & .6342 & .9263 & .9967 & 1.000 & 1.000 & 1.000 \\
\hline & $\mathrm{RT}$ & .0466 & .6910 & .8767 & .9878 & 1.000 & 1.000 & 1.000 & 1.000 \\
\hline \multirow[t]{2}{*}{ Ot } & $\mathbf{H T}^{2}$ & .6409 & .7925 & .9227 & .9541 & .9983 & 1.000 & 1.000 & 1.000 \\
\hline & $\mathrm{RT}$ & .6865 & .8520 & .9649 & .9792 & .9998 & 1.000 & 1.000 & 1.000 \\
\hline
\end{tabular}

Cohen, J. (1962). The statistical power of abnormal-social psychological research: A review. Journal of Abnormal and Social Psychology, 65, 145-153.

Cohen, J. (1977). Statistical power analysis for the behavioral sciences. (Rev. Ed). New York. Academic Press.

Conover, W. J. \& Iman, R. L. (1976). On some alternative procedures using ranks for the analysis of experimental designs. Communications in Statistics - Theoretical Methods, A5(14), 1349-1368.

Conover, W. J. (1980). Practical nonparametric statistics. $2^{\text {nd }}$ Ed., NY: John Wiley.

Conover, W. J., \& Iman, R. L. (1981). Rank transformations as a bridge between parametric and nonparametric statistics. American Statistician, 35 124-129.

Eaton, M. L. \& Efron, B. (1970). Hotelling's T ${ }^{2}$ test under symmetry conditions. Journal of the American Statistical Association, 65, 702-711.

Everitt, B. S. (1979). A monte carlo investigation of the robustness of Hotelling's one- and two-sample $\mathrm{T}^{2}$ test._Journal of the American Statistical Association. 74(365), 48-51.

Gaito, J. (1986). Some issues in the measurementstatistics controversy. Canadian Psychology, 27, 63-68.

Granger, C. V., Hamilton, B.B., Keith, R.A., Zielezny, M., \& Sherwin, F.S. (1986). Advances in functional assessment for medical rehabilitation. Topics in $\mathrm{Ge}$ riatric Rehabilitation, 1, 59-74.
Granger, C. V., Cotter, A. C., Hamilton, B. B., Fiedler, R. C., \& Hens, M. M. (1990). Functional assessment scales: A study of persons with multiple sclerosis. Archives of Physical Medicine \& Rehabilitation, 71, 870875.

Hakstian, A. R., Roed, J. C., \& Lind, J. C. (1979). Two-sample $\mathrm{T}^{2}$ procedure and the assumption of homogeneous covariance matrices. Psychological Bulletin, 86, 1255-1263.

Hamilton, B. B., Laughlin, J. A., Granger, C.V., \& Kayton, R.M. (1991). Interrater agreement of the seven level Functional Independence Measure (FIM). Archives of Physical Medicine \& Rehabilitation, 72, 790.

Harwell, M. R. \& Serlin, R. (1995). An empirical study of the Type I error rates of five multivariate tests for the single-factor repeated measures model. Paper presented at the annual meeting of the American Educational Research Association. April, San Francisco.

Heeren, T. \& D'Agostino, R. (1987). Robustness of the two independent samples t-test when applied to ordinal scaled data. Statistics in Medicine, 6, 79-90.

Holloway, L. N. \& Dunn, O. J. (1967). The robustness of Hotelling's $\mathrm{T}^{2}$. Journal of the American Statistical Association, 62, 124-136.

Hopkins, J. W. \& Clay, P. P. F. (1963). Some empirical distributions of bivariate $\mathrm{T}^{2}$ and homoscedasticity criterion $M$ under unequal variance and leptokurtosis. Journal of the American Statistical Association, December. 1049-1053. 
Hora, S. C., \& Conover, W. J. (1984). The F statistic in the two-way layout with rank-score transformed data. Journal of the American Statistical Association, 79, 668-673.

Hora, S. C. \& Iman, R. L. (1988). Asymptotic relative efficiencies of the rank-transformation procedure in randomized complete block design. Journal of the American Statistical Association, 83, 462-470.

Hsu, T. C. \& Feldt, L. S. (1969). The effect of limitations on the number of criterion score values on the significance level of the F-test. American Educational Research Journal, 6(4), 515-527.

Hunter, M. A., \& May, R. B. (1993). Some myths concerning parametric and nonparametric tests. Canadian Psychology, 34(4), 384-389.

Iman, R. L. (1974). A power study of a rank transform for the two-way classification model when interaction may be present. The Canadian Journal of Statistics, 2(2), 227-239.

Iman, R. L., Hora, S. C. \& Conover, W. J. (1984). Comparison of asymptotically distribution-free procedures for the analysis of complete blocks. Journal of the American Statistical Association, 79(387), 674-685.

Ito, K. \& Schull, W. J. (1964). On the robustness of the $\mathrm{T}^{2}$ test in multivariate analysis of variance when variance-covariance matrices are not equal. Biometrika, 51(1), $71-82$.

Jensen, D. R. (1982). Efficiency and robustness in the use of repeated measurements. Biometrics, 38, 813825 .

Johnson, R. A. \& Wichern, D. W. (1982). Applied multivariate statistics. Englewood Cliffs, NJ: Prentice Hall, Inc.

Kariya, T. (1981). A robustness property of Hotelling's $\mathrm{T}^{2}$ test. The Annals of Statistics, 9(1), 211-214.

Katz, S., Ford, A. B., Moskowitz, R. W., Jackson, B. A., \& Jaffe, M. W. (1963). Studies of illness in the aged: The index of ADL. A standardized measure of the biological and psychosocial function. Journal of the American Medical Association, 185, 914-919.

Keith, R. A., Granger, C. V., Hamilton, B. B., \& Sherwin, F. S. (1987). The Functional Independence Measure: A new tool for rehabilitation. In M. G. Eisenberg \& R. C. Grzesiak (Eds.), Advances in clinical rehabilitation, 1, 6-18. New York: Springer.

Kelley, L. D., \& Sawilowsky, S. S. (1997). Nonparametric alternatives to the $\mathrm{F}$ statistic in analysis of variance. Journal of Statistical Computing and Simulation, 58343-359.

Keppel, G. (1975). Design and analysis: A researchers handbook. Englewood Cliffs, NJ: Prentice Hall.

Lord, F.M. (1953). On the statistical treatment of football numbers. American Psychologist, 8, 750-751.
Mardia, K.V. (1975). Assessment of multinormality and the robustness of Hotelling's $\mathrm{T}^{2}$ test. Applied Statistics, 24(2), 163-171.

Matyas, T.A., \& Ottenbacher, K.J. (1993). Confounds of insensitivity and blind luck: Statistical conclusion validity in stroke rehabilitation clinical trials. Archives of Physical Medicine \& Rehabilitation, 74, 559-565.

Micceri, T. (1989). The unicorn, the normal curve, and other improbable creatures. Psychological Bulletin, 105(1), 156-166.

Nanna, M. J., \& Sawilowsky, S.S. (1998). Analysis of Likert scale data in disability and medical rehabilitation research. Psychological Methods, 3, 55-67.

Nath, R. (1982). An investigation of the rank transform in the multivariate one-sample location problem. Journal of Statistical Computing and Simulation, 16, 139-155.

Nath, R., \& Duran, B.S. (1983). A robust test in the multivariate two-sample location problem. American Journal of Mathematical and Management Sciences, 3(3), 225-249.

Ottenbacher, K. J., \& Barrett, K. A. (1989). Measures of effect size in the reporting of rehabilitation research. American Journal of Physical Medicine \& Rehabilitation, 68(2), 52-58.

Ottenbacher, K. J. \& Barrett, K. A. (1990). Statistical conclusion validity of rehabilitation research: A quantitative analysis. American Journal of Physical Medicine \& Rehabilitation, 69(2), 102-107.

Ottenbacher, K. J. (1991). Statistical conclusion validity: Multiple inferences in rehabilitation research. American Journal of Physical Medicine \& Rehabilitation, 70(6), 317-322.

Ottenbacher, K. J. (1992). Statistical conclusion validity and Type IV errors in rehabilitation research. $A r$ chives of Physical Medicine \& Rehabilitation, 73, 121125.

Ottenbacher, K. J. (1995). Why rehabilitation research does not work (as well as we think it should). $A r$ chives of Physical Medicine and Rehabilitation, 76, 123129.

Ottenbacher, K.J., Hsu, Y., Granger, C.V., \& Fiedler, R.C. (1996). The reliability of the Functional Independence Measure: A quantitative review. Archives of Physical Medicine \& Rehabilitation, 77, 1226-1232.

Sawilowsky, S.S., Blair, R.C., \& Higgins, J.J. (1989). An investigation of the type I error and power properties of the rank transform procedure in factorial ANOVA. Journal of Educational Statistics, 1(3), 255-267.

Sawilowsky, S. S. (1990). Nonparametric tests of interaction in experimental design. Review of Educational Research, 60(1), 91-126.

Sawilowsky, S. S. (1993). Comments on Using Alternatives to Normal Theory Statistics in Social and Behavioural Science. Canadian Psychology, 34, 432-439. 
Sawilowsky, S. S. \& Blair, R. C. (1992). A more realistic look at the robustness and Type II error properties of the t-test to departures from population normality. Psychological Bulletin, 111(2), 352-360.

Sawilowsky, S. S. \& Hillman, S. B. (1992). Power of the independent samples t-test under a prevalent psychometric measure distribution. Journal of Consulting \& Clinical Psychology, 60(2), 240-243.

Sedlmeir, P., \& Gigerenzer, G. (1989). Do studies of statistical power have an effect on the power of studies? Psychological Bulletin, 105, 309-316.

Serlin, R. C., \& Harwell, M. R. (1989). A comparison of Hotelling's $\mathrm{T}^{2}$ and Puri and Sen's rank test for the single-factor, repeated measures design. Paper presented at the annual meeting of the American Educational Research Association, San Francisco.

Siegal, S. (1956). Nonparametric statistics. New York: McGraw-Hill.

Stevens, S. S. (1946). On the theory of scales and measurement. Science, 103, 677-680.

Stineman, M. G., Shea, J. A., Jette, A., Tassoni, C. J., Ottenbacher, K. J., Fiedler, R., \& Granger, C. V. (1996). The Functional Independence Measure: Tests of scaling assumptions, structure, and reliability across 20 diverse impairment categories. Archives of Physical Medicine \& Rehabilitation, 77, 1101-1108.
Tiku, M. L. \& Singh, M. Robust statistics for testing mean vectors of multivariate distributions. Communications in Statistics - Theoretical Methods, 11(9), 9851001.

Utts, J. M., \& Hettmansperger, T. P. (1980). A robust class of tests and estimates for multivariate location. Journal of the American Statistical Association, 75, 939-946.

Zimmerman, D. W. (1991). Failure of the MannWhitney test: A note on the simulation study of Gibbons and Chakraborti. Journal of Experimental Education, 60, 359-364.

Zumbo, B. D., \& Zimmerman, D. W. (1991). Levels of measurement and the relation between parametric and nonparametric statistical tests: a review of recent findings. A handbook for data analysis in the behavioural sciences, 1, 481-517.

Zumbo, B. D. \& Zimmerman, D. W. (1993a). Is the selection of statistical methods governed by level of measurement? Canadian Psychology, 34, 390-400.

Zwick, R. (1986). Rank and normal scores alternatives to Hotelling's $\mathrm{T}^{2}$. Multivariate Behavioral Research, 21, 169-186. 


\title{
Six Modifications Of The Aligned Rank Transform Test For Interaction
}

\author{
Kathleen Peterson \\ Macomb Intermediate School District, Michigan, \\ \& Oakland University
}

Testing for interactions in multivariate experiments is an important function. Studies indicate that much data from social studies research is not normally distributed, thus violating that assumption of the ANOVA procedure. The aligned rank transformation test (ART), aligning using the means of columns and rows, has been found, in limited situations, to be robust to Type I error rates and to have greater power than the ANOVA. This study explored a variety of alignments, including the median, Winsorized trimmed means (10\%) and (20\%), the Huber 1.28 M-estimator, and the Harrell-Davis estimator of the median. Results are reported for Type I errors and power.

Keywords: ANOVA, Interactions, Aligned rank transform, Nuisance parameter

\section{Introduction}

Conover and Iman (1981) suggested a rank transform test (RT) that ranks the data before doing an ANOVA as a bridge between parametric and non-parametric statistics. However, the RT was found to be erratic with respect to both Type I and Type II errors as a test of interaction in the context of a 3 x 4 design (Blair, Sawilowsky, \& Higgins, 1987) and a $2 \times 2 \times 2$ design (Sawilowsky, Blair \& Higgins, 1989). Sawilowsky and Blair (1987) commented: "Not only was the test dramatically non-robust at times, but it also demonstrated very poor power properties in many situations. This was particularly true under those conditions in which interactions were present." (p. 13)

In a review of existing non-parametric tests for interactions, Sawilowsky (1990) narrowed the search for the best test down to five: Bradley's Collapsed and Reduced technique (1979), adjusted (or aligned) rank transform, (Blair \& Sawilowsky, 1990), Puri and Sen L (1985), Shoemaker's extended median test (1985), and the Hettmansperger test (1984). Sawilowsky commented on the computational difficulty of the Hettmansperger test, and pointed out that of the other four, the adjusted [aligned] rank transform appears to reach desirable power properties with the smallest sample size.

Kelley and Sawilowsky (1997) found good results for the adjusted rank test. Their study indicated that this test aligned by means had superior power properties when compared to the ANOVA if the distribution is heavytailed or skewed, and the F test has only a slight power advantage when testing for interactions if the populations are symmetric with light tails.

It has been noted that there were some minor

Kathleen Peterson is a consultant for the Macomb Intermediate School District. Her duties include working with Macomb County secondary teachers who are integrating statistics into their math/science programs. She is also an adjunct faculty member at Oakland University. inflations with regard to Type I errors in layouts higher than the $2 \times 2$. For example, with nominal alpha set to .05 , null interactions in the presence of non-null main effects resulting in Type I error rates as high as .065 . The question arises whether some other estimate of the nuisance parameter, other than the arithmetic mean, might better preserve the Type I error rate. The study described here followed suggestions by Toothaker and Newman(1994) and Sawilowsky (1990) for further study of the aligned rank transform test for interaction using alignments other than the mean.

\section{Methodology}

This Monte Carlo study of a $3 \times 4$ design was designed to examine the Type I error rate and power of six alignment statistics and the $\mathrm{F}$ statistic, when sampling from a variety of normal and non-normal distributions.

The six statistics used for alignment purposes were: the sample mean ( $\left.\mathrm{ART}_{\mathrm{m}}\right)$, the sample median $\left(\mathrm{ART}_{\mathrm{md}}\right)$, the lightly trimmed $(2 \times 10 \%)$ Winsorized mean $\left(\mathrm{ART}_{\mathrm{tm}}\right)$, a heavily trimmed $(2 \times 20 \%)$ Winsorized mean $\left(\mathrm{ART}_{\text {tmh }}\right)$, the Huber $\psi_{1.28}\left(\mathrm{ART}_{\mathrm{H}}\right)$ (Hoaglin, Mosteller, \& Tukey, 1983), and the Harrell-Davis (1982) estimator of the median (ART ${ }_{\mathrm{HD}}$ ).

For the $\mathrm{ART}_{\mathrm{m}}$, estimates of the main effects were removed by calculating the means for each row and column of data. Then the mean for each row was subtracted from the observations in that row. After that, the mean of each column was subtracted from the remaining values in that column. After alignment the remaining values were ranked; then an ANOVA was done on the ranks to test for an interaction. Other alignments were done in a similar manner. Alpha levels of .05 and .01 were used.

In a search for a statistic to be used in situations where normality is not assured an important issue is: "What distributions should be studied?" There have been arguments for using real data sets (e.g., Stigler, 1977; Micceri, 1989; Sawilowsky \& Hillman, 1992). Wilcox (1995) 
argued for using theoretical distributions with salient features (such as kurtosis or skewness) motivated by theoretical considerations, and considering what happens when these features are altered. But Micceri (1986) pointed out in a study of 440 large data sets from social science research that in some cases, (although he used a variety of quantitative techniques to assess tail weights, asymmetry, and modality), classification could only be done by visual inspection of the pseudo-population (large sample) or a combination of visual inspection and quantitative assessments.

Micceri (1989) also pointed out that the data sets which exhibited extremely light tails (similar to the uniform distribution) tended to be asymmetric, suggesting that simulated studies based on such symmetric mathematical functions such as the uniform, logistic, double exponential, Cauchy, and $t$ with few degrees of freedom may not represent real-world data to any reasonable extent.

Although there are an infinite number of non-normal distributions, having knowledge that a statistic is appropriate for many situations encountered in social studies research is more reassuring than knowing that a statistic works with some theoretical distributions, especially when sample sizes may not be large enough to determine if the population studied has those characteristics. For this reason, this study was done using, besides the Normal distribution, large, real data sets typical of those commonly found in social studies research.

A data set from Micceri's 1986 study (referred to as the Extremely Asymmetric Data Set) with $n=2,768$, was used for the simulation. It was assumed that this data set and the subsequent ones listed were large enough to proxy a population.

Another data set from this study, with $\mathrm{n}=5,375$ and referred to as the Smooth Symmetric Data Set, is typical of gain scores, which usually showed some degree of symmetry but often had heavy tails.

Micceri found that $81.2 \%$ of the 440 data sets showed considerable or extreme lumpiness or digit preference. A data set from this group, with $n=467$, referred to as the Multi-modal and Lumpy Data Set was also used.

Another Micceri achievement test data set used is the Discrete Mass at Zero with Gaps set, with $n=2,429$. This data set is typical of data where there is a pretest in which one subgroup has not been exposed to the material tested and the other group has some familiarity with the subject.

A data set with $\mathbf{n}=887$, referred to as the Likert Scale data set, is data from a medical rehabilitation setting (Nanna \& Sawilowsky, 1998). This set used a seven-point Likert scale.

Because previous studies (Sawilowsky, Blair, \& Higgins, 1989) indicated that some rank transformation tests for interaction break down in the presence of main effects, the following effect conditions were studied:

a. Condition 1: all effects null.

b. Condition 2: main effects with no interaction with $b_{3}=a_{1}=c \sigma$ and $b_{1}=b_{2}=b_{4}=a_{3}=$ $-c \sigma$, where $\mathrm{c}=.25-2.5(.25)$ and represents the shift.

c. Condition 3: no main effects and a disordinal interaction with $(\mathrm{ab})_{11}=(\mathrm{ab})_{12}=(\mathrm{ab})_{33}=$ $(\mathrm{ab})_{34}=\mathrm{c} \sigma$, and $(\mathrm{ab})_{13}=(\mathrm{ab})_{14}=(\mathrm{ab})_{31}=$ $(\mathrm{ab})_{32}=-\mathrm{c} \sigma$.

d. Condition 4: ordinal interaction with two main effects, with $(a b)_{11}=.5 c \sigma$ and $a_{1}=$ $(\mathrm{ab})_{14}=-\mathrm{c} \sigma$.

A Monte Carlo program was written as a Minitab (1998) Release 12.1 "macro", to take advantage of some existing Minitab routines. Minitab macros trims 1.mtb, os.mtb, and hd.mtb from Wilcox (1996) were used. A problem arose relative to the os.mtb macro, used for the Huber statistic. When a data set has a large number of ties, especially near the center of the data set, it is possible for the MAD (the Median Absolute Deviation from the median) to be zero. The program was modified so that in these cases the median was used as the one-step estimator of the Huber $\psi_{1.28}$, because the median is the starting point for the iterative process determining the Huber $\psi_{1.28}$ (Hoaglin, Mosteller, \& Tukey, 1983).

Samples sizes of 5, 10, 15 and 20 per cell were used. There were 5,000 repetitions for each experimental combination.

\section{Results}

The results of the Monte Carlo study are reported by effect condition. Condition 1 has all effects null, and Condition 2 has main effects with no interaction. Therefore the concern with these two conditions is the Type I error rates for the interaction.

\section{Condition 1}

Figure 1 displays an over-all view of the Type I error rates, by the aligning statistics, for all distributions and all sample sizes for alpha $=.05$. It shows a slight overall tendency for the alignment statistics studied to inflate alpha. The F statistic, the $\mathrm{ART}_{\mathrm{m}}$ and $\mathrm{ART}_{\mathrm{md}}$ are the best, in that order, with the $\mathrm{ART}_{\mathrm{m}}$ having only one value violating the stringent definition for robustness, $\alpha \pm .1 \alpha$, based on a sample size of 5000. For the $\underline{F}$ statistic, all values meet the stringent definition of robustness. All the other statistics except the $\mathrm{ART}_{\text {md }}$ have some values violating the moderate $(\alpha \pm .25 \alpha)$ criterion for robustness, with the $\mathrm{ART}_{\mathrm{HD}}$ being the worst.

For the $\mathrm{ART}_{\mathrm{m}}, \mathrm{ART}_{\mathrm{md}}, \mathrm{ART}_{\mathrm{trl}}$ and $\mathrm{ART}_{\text {trh }}$ any 
values beyond the stringent boundary were for the Extreme Asymmetry Data. The $\mathrm{ART}_{\mathrm{H}}$ and the $\mathrm{ART}_{\mathrm{HD}}$ had liberal rates for almost all the distributions studied. The $\mathrm{F}$ statistic was robust in all cases. Exact figures for each combination of sample size, statistic, distribution and alpha level are available at kpeterson@misd.net.

The results for Condition 1, Alpha $=.01$ were similar, although slightly more liberal. In addition to the elevated rates for the Extreme Asymmetry data, there were violations of the stringent goal for the $\mathrm{ART}_{\mathrm{md}}$ and $\mathrm{ART}_{\text {trr }}$, and the moderate goal for the $\mathrm{ART}_{\text {trh }}$ with the Likert distribution. There were liberal figures for most distributions for the $\mathrm{ART}_{\mathrm{H}}$ and the $\mathrm{ART}_{\mathrm{HD}}$, although it should be pointed out that the worst violation was a rate of .0358 , for the $\mathrm{ART}_{\mathrm{HD}}$ with the Extreme Asymmetry Data Set.

\section{Condition 2}

For Condition 2 (no interaction but main effects) all the statistics, including the F statistic, displayed a slight tendency to inflate alpha, but all the $\mathrm{F}$ rates were within the limits for a stringent definition of robustness. The $\mathrm{ART}_{\mathrm{md}}$ had only one out of 240 rates extreme enough to fall in the moderate interval (at $\mathrm{n}=20$ for the Likert distribution). Only the $\mathrm{ART}_{\text {trl }}$ (for the Extreme Asymmetry distribution, at $n=5$ ) had a value beyond the limit for moderate robustness.

For Condition 2 (no interaction with two main effects), alpha .01, all seven statistics had problems with the Extreme Asymmetry data for $\mathrm{n}=5$. The $\mathrm{F}$ statistic had one rate (out of 240) beyond the liberal level (with the Extreme Asymmetry data); the $\mathrm{ART}_{\mathrm{md}}$ had three rates which didn't meet stringent criteria (one of them was with the Likert data); the $\mathrm{ART}_{\text {trl }}, \mathrm{ART}_{\text {th }}$ and $\mathrm{ART}_{\mathrm{H}}$ had almost all rates for the Extreme Asymmetry data too large for moderate robustness. Each statistic except the F statistic had at least one (but no more than three) violations with the Likert data set. The violations tended to lessen in number and severity as sample size increased. Again, to keep perspective, the largest value was .0198 , for the $\mathrm{ART}_{\text {trl }}$ with the Extreme Asymmetry data set.

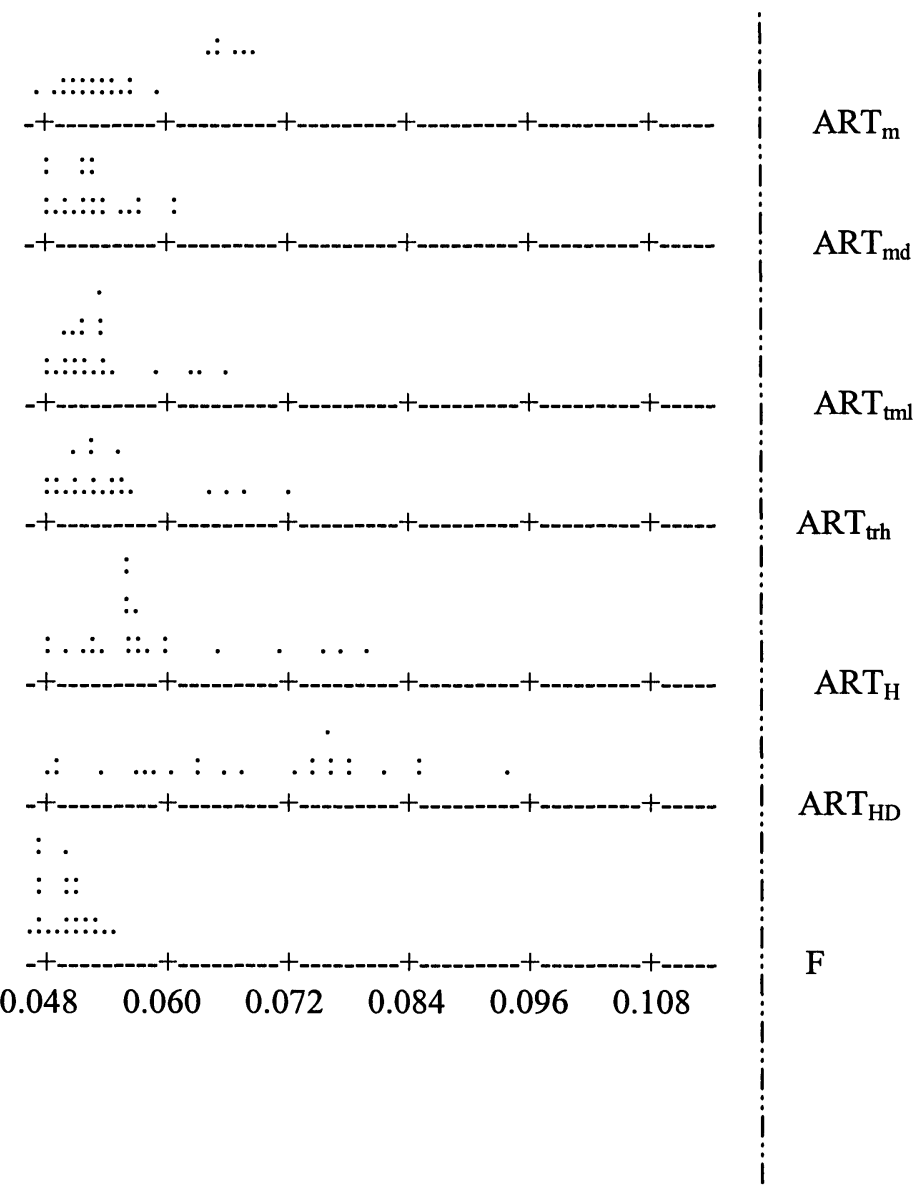

Figure 1. Type I error rates by statistic for Condition 1 (all-effects-null) with nominal alpha $=.05$. All four sample sizes are grouped together. 
Condition 3

Condition 3 is a disordinal interaction with no main effects. Figure 2 displays histograms which show the differences in power for each statistic in comparison to the F statistic for each of the 240 sample size/ distribution/ shift level combinations for alpha $=.05$.
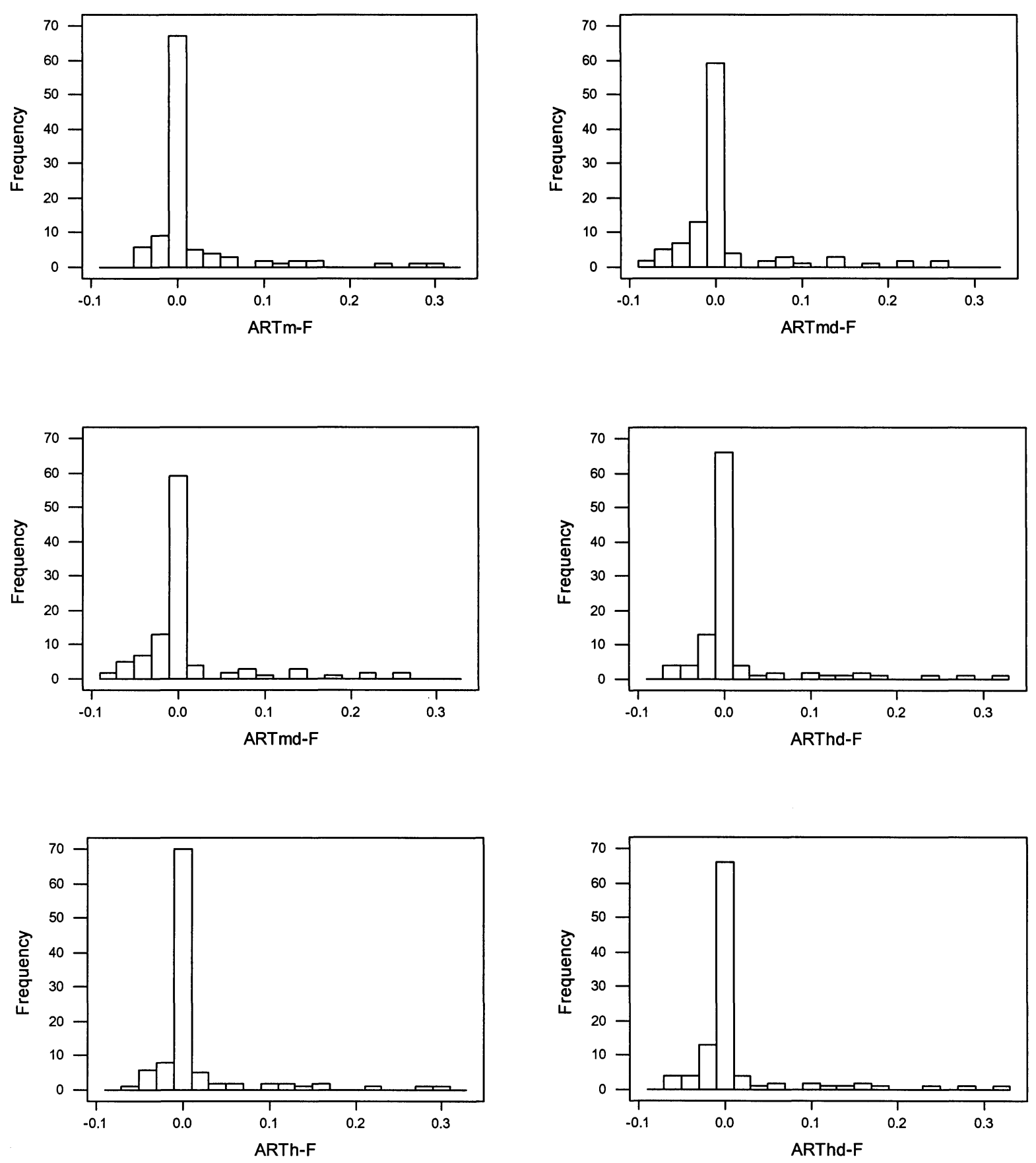

Figure 2. Histograms for Differences: Alignment Statistic Power Minus F Statistic Power (Condition 3, Alpha .05). 
Examination of the histograms reveals that:

1. In most cases, differences between a given statistic and the F statistic are minimal, very close to zero.

2. For all six statistics, the data is skewed to the right, indicating that there are some cases where the statistic in question is much more powerful than the $\mathrm{F}$ statistic.

3. The three statistics with the heaviest and longest left tails (indicating less power than the F statistic) are the ART ${ }_{\text {md }}$, $\mathrm{ART}_{\mathrm{H}}$, and the $\mathrm{ART}_{\mathrm{HD}}$.

Table 1. Descriptive Statistics for Power Differences: Alignment Statistic Minus F Statistic for Condition 3, Alpha $=.05$.

\begin{tabular}{lcccccccc}
\hline Variable & $\mathrm{N}$ & Mean & Median & StDev & Minimum & Maximum & Q1 & Q3 \\
\hline $\mathrm{ART}_{\mathrm{m}} \mathrm{F}$ & 104 & 0.01664 & 0.0000 & 0.05826 & -0.0408 & 0.3066 & -0.00155 & 0.00600 \\
$\mathrm{ART}_{\text {md }}-\mathrm{F}$ & 104 & 0.01047 & 0.0000 & 0.06065 & -0.0840 & 0.2556 & -0.01235 & 0.00015 \\
$\mathrm{ART}_{\text {tr }}-\mathrm{F}$ & 104 & 0.01608 & 0.0000 & 0.05731 & -0.0442 & 0.2974 & -0.00120 & 0.00455 \\
$\mathrm{ART}_{\text {trh }}-\mathrm{F}$ & 104 & 0.01415 & 0.0000 & 0.05313 & -0.0464 & 0.2900 & -0.00115 & 0.00435 \\
$\mathrm{ART}_{\mathrm{H}}-\mathrm{F}$ & 104 & 0.01466 & 0.0000 & 0.05773 & -0.0536 & 0.2960 & -0.00235 & 0.00350 \\
$\mathrm{ART}_{\text {HD }}-\mathrm{F}$ & 104 & 0.01321 & 0.0000 & 0.06163 & -0.0654 & 0.3114 & -0.00490 & 0.00040 \\
\hline
\end{tabular}

There were 48 power graphs generated for Condition 3 ( 6 distributions, 4 sample sizes, 2 alpha levels). Figures 3 and 4 show several of the situations where the alignment statistics show considerably more power than the $F$ statistic. The ART ${ }_{\text {md }}$, which had shown good type I error rates, showed a lack of power in some cases. This became more pronounced for Condition 4.

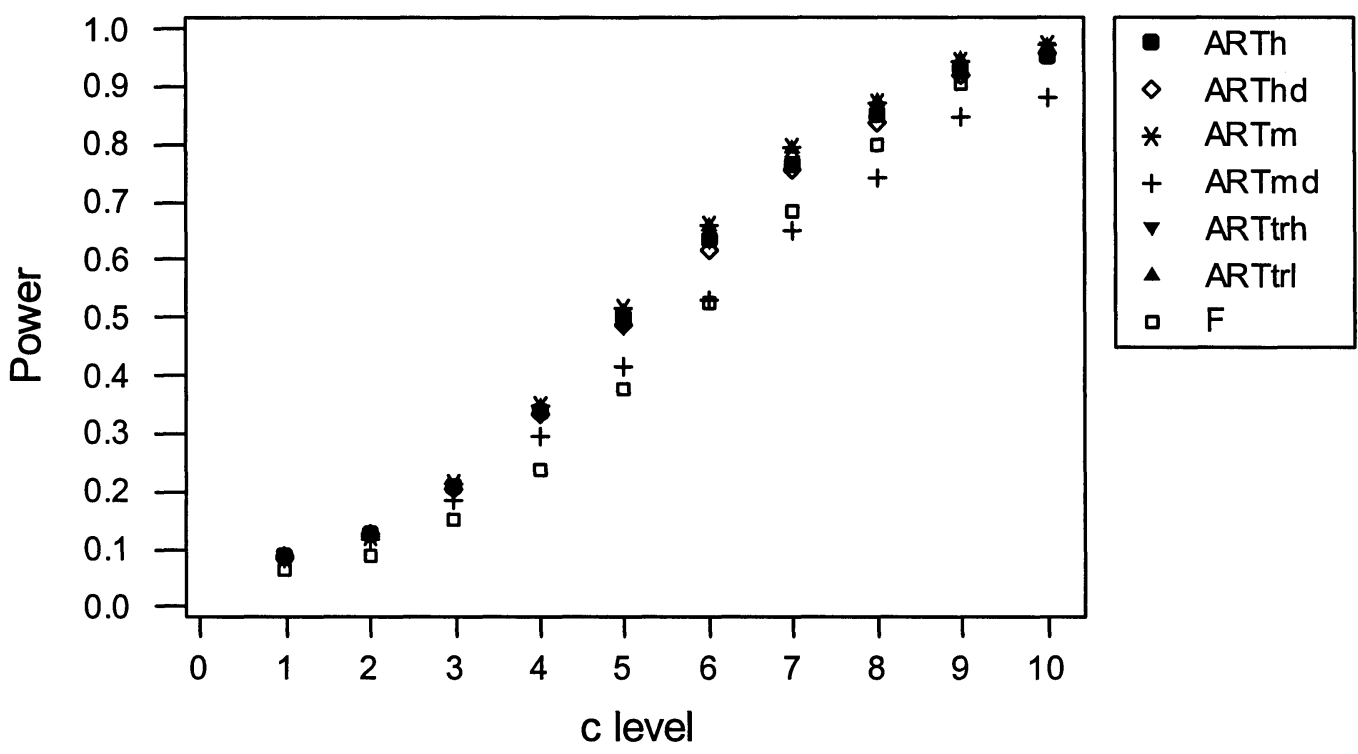

Figure 3. Power graph for Condition 3 (no main effects with a disordinal interaction) for the Extreme Asymmetry data set with nominal alpha $=.05$ and $\mathrm{n}=5$. The $\mathrm{C}$ level is the multiple of $.25 \sigma$ used for shift. 


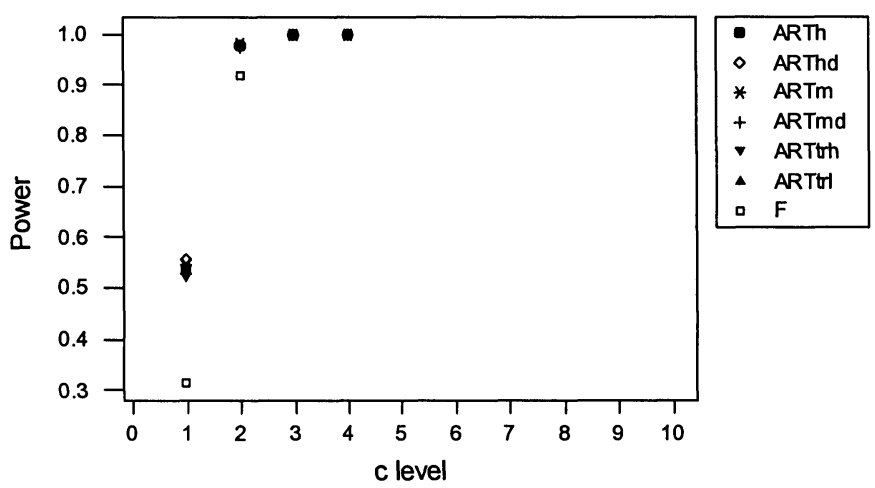

Figure 4. Power for Condition 3 (no main effects, disordinal interaction, Extreme Asymmetry), $\alpha=.05, \mathrm{n}=10$.

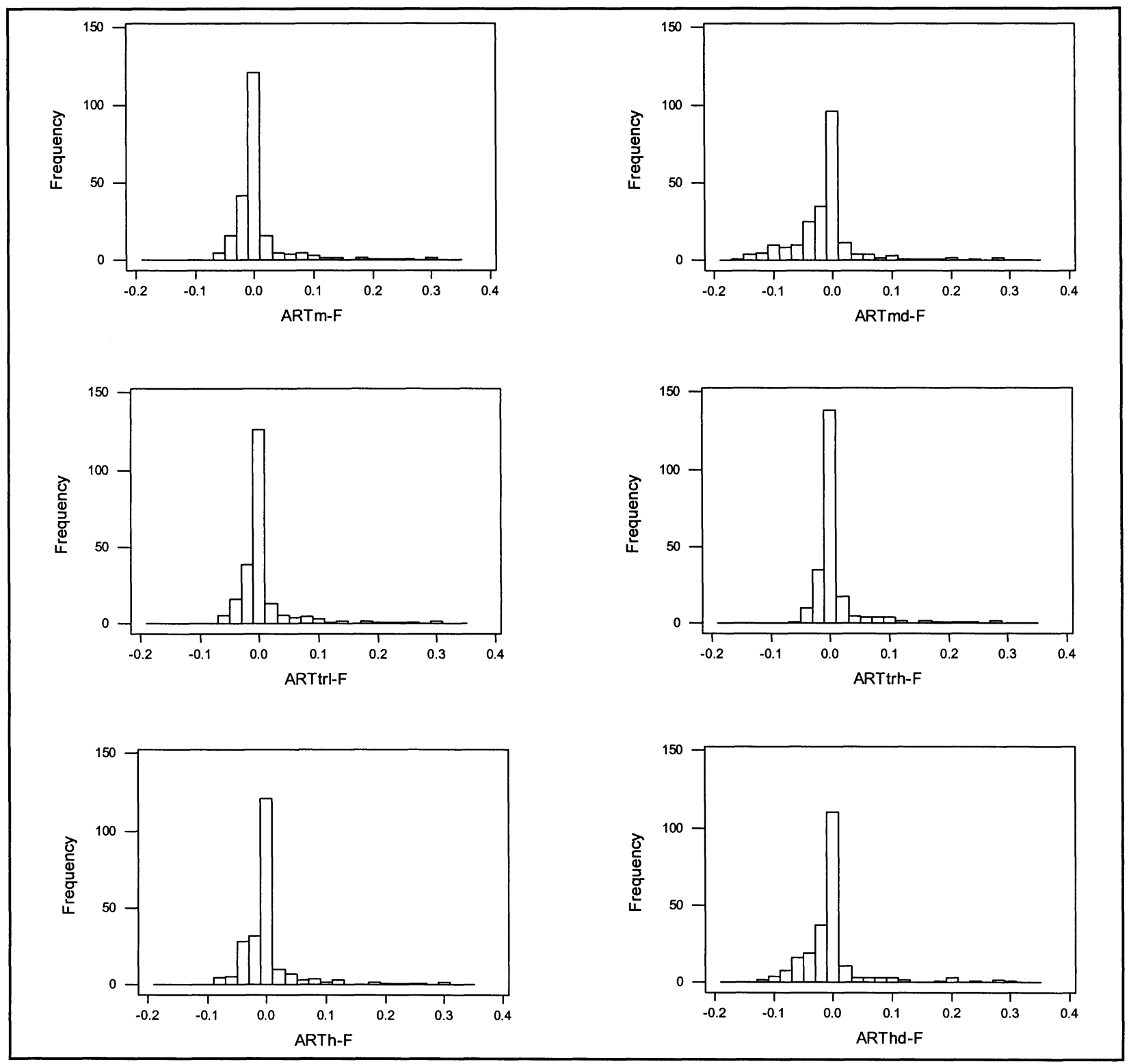

Figure 5. Histograms for Differences: (Alignment Statistic Power - F Statistic Power) for Condition 4, $\alpha=.05$. 
Condition 4

Condition 4 modeled an ordinal interaction with two main effects. Although it took longer (more shift) to reach full power under this condition, many of the patterns observed with Condition 3 were observed here, too.

Figure 5, with histograms showing the power differences between the six aligning statistics and the F statistic, shows that, similar to Condition 3 results, the vast majority of differences are very close to zero. The data is skewed to the right, indicating cases where the alignment statistic is considerably more powerful than the $\underline{F}$ statistic, and the left tails (indicating a lack of power relative to the $\mathrm{F}$ ) are the longest and heaviest for the $\mathrm{ART}_{\mathrm{md}}, \mathrm{ART}_{\mathrm{H}}$ and $\mathrm{ART}_{\mathrm{HD}}$

Table 2, which summarizes the differences between each of the alignment statistics and the $F$ statistic used to construct the histograms in Figure 5, shows that the most extreme case of lack of power relative to the $F$ test is with the $\mathrm{ART}_{\mathrm{HD}}(-.1510)$, and the most extreme case of superior power relative to the $F$ statistic is with the $A_{R T}$
(.29880), with the $\mathrm{ART}_{\mathrm{m}}$ next with 29700 . A comparison of the differences by mean, median, minimum, maximum, $\mathrm{Q} 1$ and Q2 shows that the $\mathrm{ART}_{\mathrm{m}}, \mathrm{ART}_{\mathrm{trr}}$, and $\mathrm{ART}_{\mathrm{trh}}$, have a very slight advantage over the other alignment statistics.

Figures 6-8 show several situations where the lack of power of the $\mathrm{F}$ and $\mathrm{ART}_{\mathrm{md}}$ are apparent. The $\underline{\mathrm{F}}$ statistic showed a large deficiency in power for the Extreme Asymmetry data. The $\mathrm{ART}_{\mathrm{md}}$ showed a deficiency for most of the distributions.

\section{Conclusion}

If Type I error is the major concern, the $\mathrm{F}$ statistic, $\mathrm{ART}_{\mathrm{md}}$ and $\mathrm{ART}_{\mathrm{m}}$, in that order, were the most promising in this study. These statistics had no violations of a moderate definition of robustness, $\alpha \pm .25 \alpha$, adjusted for sample size, for condition 1 and no violations of a stringent criterion, $\alpha$ $\pm .1 \alpha$, for Condition 2. The $\mathrm{ART}_{\mathrm{H}}$ and $\mathrm{ART}_{\mathrm{HD}}$ were the least satisfactory, with rates as high as $3.5 \alpha$ with the Extreme Asymmetry data when no main effects were present,

Table 2. Descriptive Statistics for Power Differences: (Alignment Statistic - F Statistic) for Condition 4, $\alpha=.05$.

\begin{tabular}{lcrrrrrrr}
\hline Variable & $\mathrm{N}$ & Mean & Median & StDev & Minimum & Maximum & Q1 & Q3 \\
\hline $\mathrm{ART}_{\mathrm{m}}-\mathrm{F}$ & 229 & 0.00925 & -0.0004 & 0.05363 & -0.0638 & 0.2970 & -0.0119 & 0.0045 \\
$\mathrm{ART}_{\mathrm{md}}-\mathrm{F}$ & 229 & -0.01081 & -0.0050 & 0.06102 & -0.1510 & 0.2804 & -0.0332 & 0.0000 \\
$\mathrm{ART}_{\text {trl }} \mathrm{F}$ & 229 & 0.00855 & -0.0004 & 0.05293 & -0.0648 & 0.2952 & -0.0119 & 0.0035 \\
$\mathrm{ART}_{\text {th }} \mathrm{F}$ & 229 & 0.01001 & -0.0002 & 0.04803 & -0.0530 & 0.2798 & -0.0072 & 0.0053 \\
$\mathrm{ART}_{\mathrm{H}}-\mathrm{F}$ & 229 & 0.00425 & -0.0012 & 0.05455 & -0.0816 & 0.2988 & -0.0160 & 0.0027 \\
$\mathrm{ART}_{\mathrm{HD}}-\mathrm{F}$ & 229 & -0.00266 & -0.0022 & 0.05773 & -0.1192 & 0.2956 & -0.0244 & 0.0004 \\
\hline
\end{tabular}

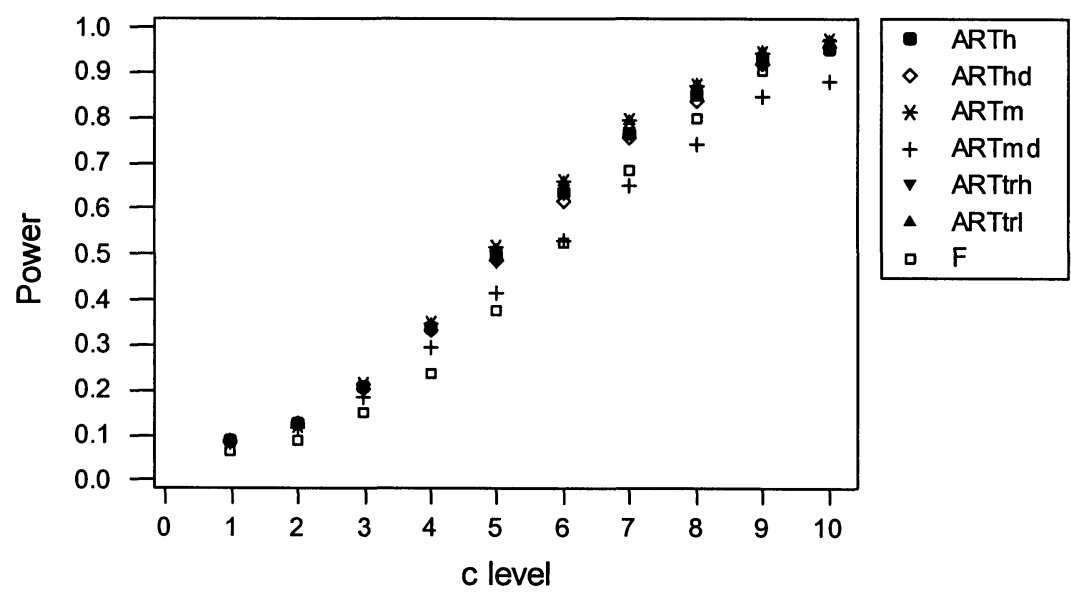

Figure 6. Power graph for Condition 4 (main effects with an ordinal interaction) for the Extreme Asymmetry data set with nominal alpha $=.05$ and $n=5$. The c level is the multiple of $.25 \sigma$ used for shift. 


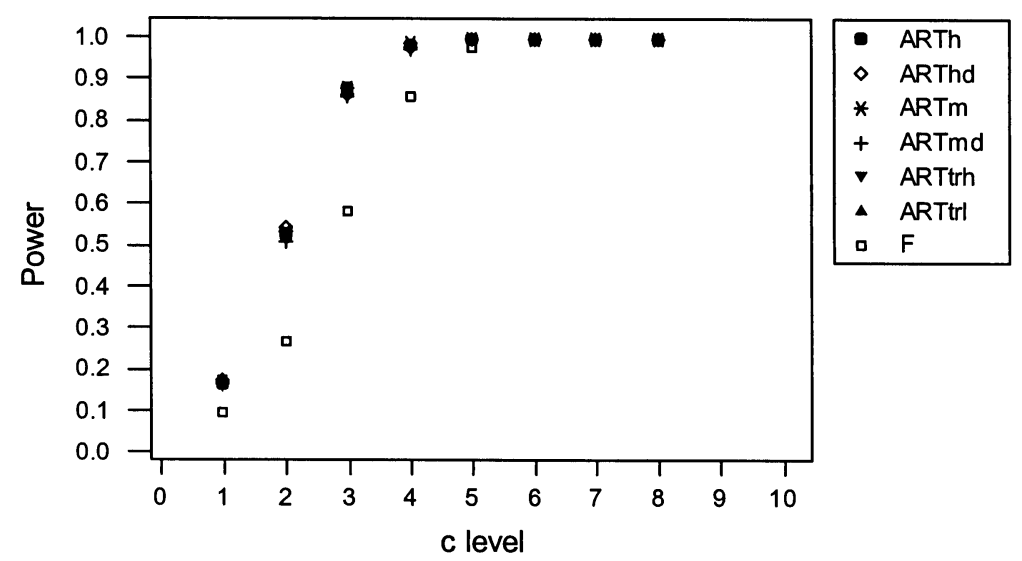

Figure 7. Power graph for Condition 4 (main effects with an ordinal interaction) for the Extreme Asymmetry data set with nominal alpha $=.05$ and $\mathrm{n}=20$. The $\mathrm{c}$ level is the multiple of $.25 \sigma$ used for shift.

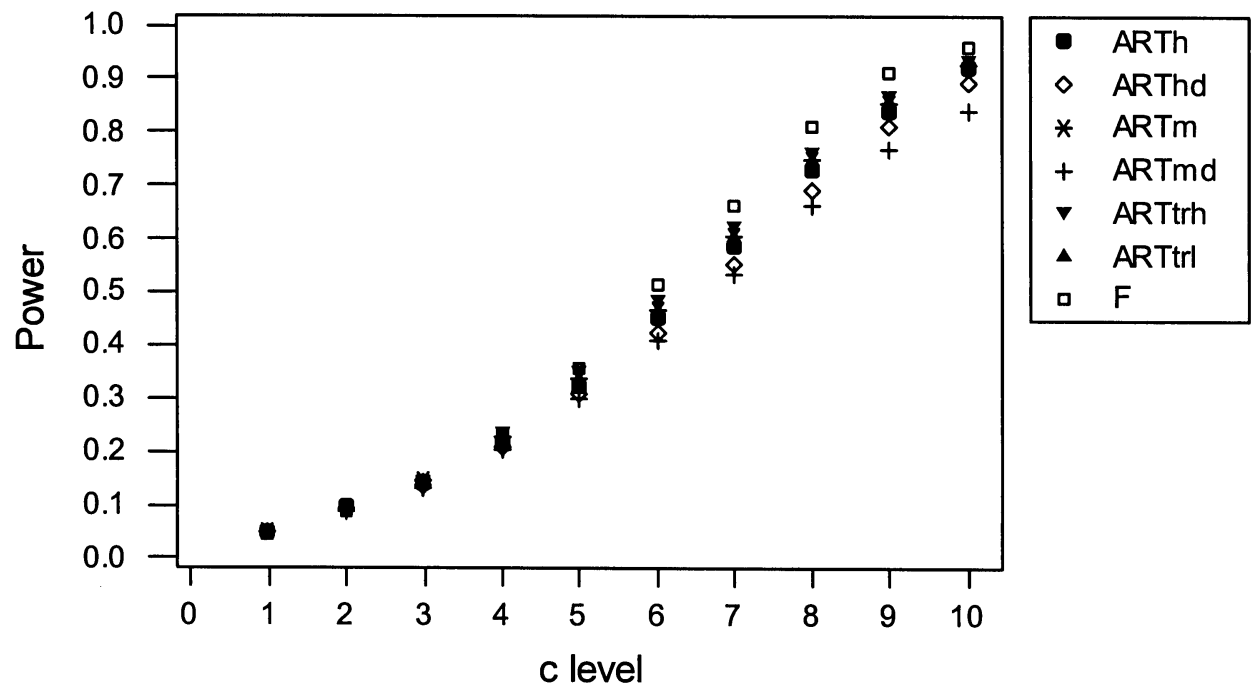

Figure 8. Power graph for Condition 4 (main effects with an ordinal interaction) for the Multi-modal and Lumpy data set with nominal alpha $=.05$ and $n=5$. The $\mathrm{c}$ level is the multiple of $.25 \sigma$ used for shift.

and poor results with other distributions.

This study affirms the weak power of the $\mathrm{F}$ statistic, in comparison to the $\mathrm{ART}_{\mathrm{m}}$, as reported by Kelley and Sawilowsky (1997), with Extreme Asymmetry data, in a $2 \times 2 \times 2$ design. The $\mathrm{F}$ statistic also performed poorly with the Likert data.

In addition, the median alignment showed lower power levels for most of the distributions studied, especially with Condition 4 , an ordinal interaction with main effects. The good Type I error rates for the F statistic and the $\mathrm{ART}_{\mathrm{md}}$ do not compensate for the much larger power deficiencies.

The $\mathrm{ART}_{\mathrm{H}}$ and the $\mathrm{ART}_{\mathrm{HD}}$ showed problems with both Type I error and power. The best statistics in terms of power for Conditions 3 and 4 were $\mathrm{ART}_{\text {trt }}, \mathrm{ART}_{\text {trr }}$, and the $\mathrm{ART}_{\mathrm{m}}$. The $\mathrm{ART}_{\mathrm{m}}$ had a slight advantage in terms of Type I error rates; the two trims a slight power advantage.

Kelley and Sawilowsky (1997), in their study of the Blair-Sawilowsky test (which has been referred to in this study as $\mathrm{ART}_{\mathrm{m}}$ ) and other nonparametric tests for 
interaction in a $2 \times 2 \times 2$ layout came to this conclusion:

It is recommended that when testing for interactions in a $2 \times 2 \times 2$ layout, Analysis of Variance [F statistic] be used with data known to be symmetric with light tails, such as the normal and uniform distributions, and the Blair-Sawilowsky [ART ${ }_{m}$ ] test be used with heavy-tailed or skewed data. If the shape of the distribution is unknown, the Blair and Sawilowsky test is recommended because it frequently exhibited considerably more power than the ANOVA [F]. In the apparently rare circumstances where data are obtained from a normal curve, this test will only be slightly less powerful than the ANOVA F test. (p. 357)

This study supports the value of the BlairSawilowsky $\left(\mathrm{ART}_{\mathrm{m}}\right)$ and extending its application to a $3 \mathrm{x}$ 4 layout. It also raises the possibility of other alignments $\left(\mathrm{ART}_{\mathrm{trl}}\right.$ and $\left.\mathrm{ART}_{\mathrm{trh}}\right)$ being as useful or even more so in other situations as mentioned above.

The F statistic has been considered an all-purpose statistic, used without consideration of the population. As has been indicated, this can lead to major errors. Although there is a natural tendency to want to find a substitute all-purpose statistic, there are many issues that would have to be addressed before any of these three could assume that role. Among them are: the nature of the interaction and number of non-null effects, other designs, the issue of unequal variances, and additional distribution issues.

Tukey (1984) described the practical power of a test as being the statistical power of a test multiplied by the probability that someone would actually use the test. This study has indicated three statistics as being somewhat equal for power and Type I error rates. Unless future studies indicate a big difference in the usefulness of the ART and $\mathrm{ART}_{\text {th }}$, Tukey's criterion would favor the $\mathrm{ART}_{\mathrm{m}}$ because it can be done quite easily on most statistical software packages. However, a macro for the Winsorized trimmed mean is available (Wilcox, 1996).

\section{References}

Blair, R. C., \& Sawilowsky, S. S. (1990, April). A test for interaction based on the rank transform. Paper presented at the annual meeting of the American Educational Research Association, Boston.
Blair, R. C., Sawilowsky, S. S., \& Higgins, J. J. (1987). Limitations of the rank transform statistic in tests for interactions. Communications in Statistics-Simulation and Computation, 16(4), 1133-1145.

Bradley, J. V. (1979). A nonparametric test for interactions of any order. Journal of Quality Technology, 11(4), 321-327.

Conover, W. J. \& Iman, R. I. (1981). Rank transformations as a bridge between parametric and nonparametric statistics. American Statistician, 35(3), 124-129.

Glass, G. V., Peckham, P. D., \& Sanders, J. R. (1972). Consequences of failure to meet assumptions underlying the fixed effects analysis of variance and covariance. Review of Educational Research, 42(30), 237-288.

Harrell, R. E., \& Davis, C. E. (1982). A new distribution-free quantile estimator. Biometrika, 69, 635-640.

Hettmansperger, T. F. (1984). Statistical inference based on ranks. New York: John Wiley.

Hoaglin, D. C., Mosteller, F. \& Tukey, J. W. (1983). Understanding Robust and Exploratory Data Analysis. New York: John Wiley.

Kelley, D. L., \& Sawilowsky, S. S. (1997). Nonparametric alternatives to the F statistic in analysis of variance. Journal of Computer Simulations, 58, 343-359.

Micceri, T. (1989). The unicorn, the normal curve, and other improbable creatures. Psychological Bulletin, 105(1), 156-166.

Micceri, T. (1986, Nov.). A futile search for that statistical chimera of normality. Paper presented at Florida Educational Research Association Annual Conference, Tampa, Florida. Minitab, Inc.

Minitab, Release 12.1 (1998). State College, PA:

Nanna, M. J., \& Sawilowsky, S. S.(1998) Analysis of Likert scale data in disability and medical rehabilitation research. Psychological Methods, 3(1), 55-67.

Puri, M. L., \& Sen, P. K. (1985). Nonparametric methods in general linear models. New York: Wiley.

Sawilowsky, S. S. (1990). Nonparametric tests of interaction in experimental design. Review of Educational Research, 60(1), 91-126.

Sawilowsky, S. S., \& Blair, R. C. (1987, April). An investigation of the Type I error and power properties of the rank transform procedure in factorial ANOVA. Paper presented at the annual meeting of the American Educational Research Association. Washington, DC.

Sawilowsky, S. S., Blair, R. C., \& Higgins, J. J. (1989), An investigation of the Type I error and power properties of the rank transform procedure in factorial anova, Journal of Educational Statistics, 14(3), 255-267.

Sawilowsky, S. S., \& Hillman, S. B. (1992). Power of the independent samples $t$ test under a prevalent psychometric measure distribution. Journal of Consulting and Clinical Psychology, 60, 240-243. 
Shoemaker, L. H. (1986). A nonparametric method for analysis of variance. Communications in Statistics, 15(3), 609-632.

Stigler, S. M. (1977). Do robust estimators work with real data? The Annals of Statistics, 5(6), 1055-1098.

Toothaker, L. E., \& Newman, D. (1994). Nonparametric competitors to the two-way ANOVA. Journal of Educational and Behavioral Statistics, 19(3), 237-273.

Tukey, J. (1984). Untitled lecture at Woodrow Wilson Summer Program for Teachers, Princeton University, Princeton, NJ.
Wilcox, R. R. (1995). ANOVA: The practical important of heteroscedastic methods, using trimmed means versus means, and designing simulation studies. British Journal of Mathematical and Statistical Psychology, 48, 99-114.

Wilcox, R. R. (1996). Statistics for the social sciences. San Diego, CA: Academic Press. 


\title{
Applying Spatial Randomness To Community Inclusion
}

\author{
Michael Wolf-Branigin \\ Caliber Associates \\ Fairfax, VA
}

\begin{abstract}
A spatial analytic methodology incorporating true locations is demonstrated using Monte Carlo simulations as a complement to current psychometric and quality of life indices for measuring community inclusion. Moran's $I$, a measure of spatial autocorrelation, is used to determine spatial dependencies in housing patterns for multiple variables, including family/friends involvement in future planning, home size, and earned income. Simulations revealed no significant spatial autocorrelation, which is a socially desirable result for housing locations for people with disabilities. Assessing the absence of clustering provides a promising methodology for measuring community inclusion.
\end{abstract}

Keywords: Spatial analysis, Monte Carlo methods, Community inclusion, Spatial randomness

\section{Introduction}

During the past decades employment, housing, educational policies, and other services for people with developmental disabilities have shifted to community settings in the United States. Self-determination, individualized budgets, and consumer-focused planning techniques are rapidly gaining influence within the disability field as people with disabilities are encouraged to explore and connect with their community, seek new experiences, learn how to live, work, recreate, and go to school with non-disabled peers (Mount, 1987; Rhodes, McFarland, \& Knight, 1995). This has resulted in more people with disabilities who work and live in increasingly more independent employment and housing settings (Braddock, Hemp, Parish, Westrich, \& Park, 1997). The objective has been to provide improved and relevant services in a fully integrated environment.

Spatial analysis, a methodology for assessing patterns, networks, and interactions, is frequently used to measure access to services for the general public by urban planners and geographers. However, the potential benefits of spatial analysis have yet to be considered by human service professionals to assess accessibility and inclusion for people with developmental disabilities. The use of spatial data as a component of social program evaluation practice is easily suggested by the fact that approximately $80 \%$ of human service organization databases have some locational element included (Hutchinson \& Daniel, 1995).

Spatial analyses might lead to increased understanding of relationships, and the degree to which people with disabilities interact with their environment. An example would be a study of community inclusion for people

Michael Wolf-Branigin is Senior Associate, Caliber Associates, 10530 Rosehaven Street, Suite 400, Fairfax, VA, 22030. His research activities are in substance abuse treatment, education policy for children with special needs, consumer-choice models, and disability and health. $\mathrm{He}$ is an accreditation surveyor with CARF, The Rehabilitation Accreditation Commission. with developmental disabilities who have moved from institutional or segregated type housing to community settings. These community settings include small group homes, semi-independent living, or independent living apartments.

Purpose of the Study

The purpose of this article is to introduce spatial analytic methods that compare true housing locations against randomly generated patterns using Monte Carlo simulations. The results of the simulations will be used to complement quality of life, psychometric, and attitudinal scales for examining levels of community inclusion and social interactions. This leads to the primary research question: Does spatial analysis, specifically a random pattern of location (i.e., lack of spatial autocorrelation) as identified through Moran's I, provide a useful method for measuring physical community inclusion for people with developmental disabilities?

Several explanatory variables (percentage of earned income to total income, size of the individual's support system, level of disability, and number of people with whom the individual lives who also have a disability) will be used to identify spatial dependencies. For example, do people with disabilities who have some earned income have a greater level of community inclusion than people who only receive income from governmental sources? This quantification of location allowed spatial features to be geographically referenced and mapped. Concurrently quantification of attributes facilitates the identification and analysis of spatial relationships.

With the greater adoption of community inclusion and normalization principles in the human service delivery sector, people with developmental disabilities are increasingly finding housing options in their communities. This and similar shifts of human services from a centralized service providers to more individualized approaches has created the need for evaluative strategies focusing on 
environmental interactions. Decentralized services, such as housing patterns of people with developmental disabilities, are a goal of community inclusion.

\section{Moran's I}

Within this community inclusion context, the absence of spatial autocorrelation (SAC) will be desirable. That means there should be no interaction or dependence between points (Diggle, 1983). The ideal pattern has been referred to as complete spatial randomness (CSR).

The test statistic, Moran's I, measures the degree of spatial autocorrelation (Cressie, 1993). It can be decomposed from an overall value to individual observations for each case (Anselin, 1995a). These decomposed values are referred to as local indicators of spatial association (LISAs). Point pattern analysis was used because of the additional advantage of requiring only location and attribute data for analysis.

Moran's I requires interval-scale observations and is useful in describing the spatial patterns of distributions. It is essentially a Pearson product-moment correlation coefficient modified to account for a spatial weight matrix (Getis, 1991). Values range from -1.0 to 1.0. A high value (approaching 1.0) indicates a clustered pattern, while a low value (approaching -1.0 ) indicates a scattered pattern. A value near zero indicates randomness. As individuals become more included in their communities the desire is for the patterns to approximate zero $(0.0)$.

\section{Method}

Data were obtained for individuals who currently reside in a variety of community-based settings. The types of homes include supported living, shared lives, foster and adoptive families, and small group homes. Data were also collected on the number of non-related people (with disabilities) who live in the same home.

Data were collected for eighty-six randomly selected individuals. The desired sample size was derived from Monte Carlo studies by Anselin \& Keleijian (1997), who suggested that samples of at least $\mathrm{N}=81$ with normally distributed error terms achieves the asymptotic form of Moran's I test, based on independent variable residuals. The same study indicated that the test possesses good overall power, but may under-reject the null hypothesis when it is in fact true with a smaller sample $(\mathrm{N}=48)$. This sample size for the autocorrelation analysis is also consistent with Cohen (1992) for medium effect sizes of .30 and .15, respectively.

The physical inclusion of people in their communities was assessed using a variety of spatial analytic approaches centering on point pattern analysis. Independent variables included the degree to which people with disabilities had involvement of family, friends and allies in planning their futures; number of people with disabilities residing in their home; and the level of individual earned income. included:

Following random selection, research procedures

1. collecting data at the individual consumer level,

2. mapping of this data in a two dimensional space,

3. performing autocorrelation analysis of housing locations using Moran's I,

4. creating local indicators of spatial association (LISA) for use as dependent variable, and

5. generating 999 random permutations for assessing pseudo-significance.

Data were collected from agency records and a review of annual planning meeting records. Data concerning the income types and levels for the income records was obtained from the current records of the consumer located in the medical records office of the organization.

Monte Carlo simulations were performed with SpaceStat software (Anselin, 1995b). Developing local indicators of spatial association (LISA) as the dependent variable required the creation of a spatial weights matrix using SpaceStat software. Statistics calculated included Moran's I for the distance bands indexes. Simulations for assessing the robustness of the Moran's I were conducted. This method followed Yung and Chan's (1999) three-step procedure, including defining a pseudo-population, resampling, and evaluation. Runs of 999 drawn from the sample were performed for the exploratory variables that were believed to be potentially spatially autocorrelated.

\section{Descriptive Statistics}

\section{Results}

Descriptive statistics are summarized below. The most common size of household was six persons (36.9\%). Twelve (14.3\%) individuals resided alone in independent or semi-independent housing. The proportion of earned income was highly variable as demonstrated by the standard deviation (.1591) being approximately twice the mean (.07639). Having one non-professional person at the planning meeting was the most frequent $(45.2 \%)$ followed by having two people present $(28.6 \%)$. Males and females comprised $56 \%(n=47)$, and $44 \%(n=37)$, respectively of the sample. Many people needed assistance with mobility. This was defined as the person not being able to drive, transport self to daily activities or use public transportation. Forty-eight (57.14\%) did not need assistance while $36(42.86 \%)$ did. 
Table 1. Autocorrelation for Distance Band Measures

\begin{tabular}{|l|c|c|c|c|}
\hline Explanatory Variable & Moran's I & Mean & Standard Deviation & Probability \\
\hline & & & & \\
Proportion of earned income & -.019 & -.010 & .0331 & .436 \\
Size of Home & .032 & -.012 & .0368 & .330 \\
People at Planning Meeting & -.014 & -.014 & .0364 & .250 \\
Level of Disability & -.013 & -.013 & .0353 & .222 \\
Mobility & -.013 & -.013 & .0344 & .123 \\
\hline
\end{tabular}

Note: Empirical significance based on 999 random permutations.

\section{Spatial Analyses}

Monte Carlo procedures using Moran's I spatial autocorrelation statistics for distance band measures did not reveal significant clustering for any of the exploratory variables. See Table 1. The distance band figures for proportion of earned income were $(I=-.019 ; \mathrm{p}=.436)$, home size $(I=-.032 ; p=.330)$, number of people at the person's annual planning meeting $(\mathrm{I}=-.014 ; \mathrm{p}=.250)$, level of disability $(\mathrm{I}=-.013 ; \mathrm{p}=.222)$, and level of mobility $(\mathrm{I}=$ $-.013 ; \mathrm{p}=.123$ ) respectively. The values for Moran's I were small (between -.019 and .032), thus indicating the absence of significant spatial autocorrelation. This suggests that the housing pattern as measured through spatial autocorrelation as a construct of complete spatial randomness was random.

\section{Conclusion}

A goal of this research was to demonstrate that spatial analysis (and more specifically point pattern analysis) methods incorporating true observations with Monte Carlo simulations provides a potentially useful tool for organizations providing housing services to people with developmental disabilities. Although measuring autocorrelation through complete spatial randomness (CSR) may be a goal of inclusion in an ideal situation, certain restraints such as access to public transportation may make this goal unrealistic. Therefore, evaluations at the local organizational level must be aware and sensitive to these exigencies. Conversely, some level of clustering may be a reasonable response to meet these demands.

Spatial analysis appears promising for measuring the degree that physical inclusion of people with developmental disabilities occurs in their communities. The results address the degree to which people were dispersed geographically throughout their community. Further application of these spatial methods could focus on issues prevalent in the current body of literature, including: the modifiable areal (or geographic) unit problem (MAUP), sensitivity issues when measuring households versus individuals, integration of spatial statistics with geographical information systems, and improved identification of inclusion through implementing a network analysis approach. Investigating autocorrelation through Monte Carlo simulations could be simplified by using all the addresses of housing locations maintained by an organization. Having a sample with multiple people residing at one location lead to statistical coding problems. Simulations with the current data set using two spatial software packages (SpaceStat and Systat 9.0 Spatial Statistics) were problematic due to co-located data. Using the addresses only once in the data collection process and analysis would eliminate this problem. Weights for the number of people at each location could be calculated into the analysis.

Paramount to understanding the logic of spatial analysis is to realize that it is an inductive process. Exploratory and interactive spatial analysis often relies on induction as opposed to a deductive hypothesis testing approach. This study provided initial support for the use of Monte Carlo simulations in spatial point pattern.

\section{References}

Anselin, L. (1995a). Local indicators of spatial association - LISA. Geographical Analysis 27, 2:93-115.

Anselin, L. (1995b). SpaceStat Version 1.80 User's Guide. Regional Research Institute, West Virginia University.

Anselin, L., \& Kelejian, H. (1997). Testing for spatial error autocorrelation in the presence of endogenous regressors. International Regional Science Review, 20, $1 \& 2$. Regional Research Institute. University of West Virginia.

Braddock, D., Hemp, R., Parish, S., Westrich, J., \& Park, H. (1997). The State of the States in Developmental Disabilities (5th ed.). Washington, D.C. AAMR. 
Chou, Y-H. (1997). Exploring spatial analysis in geographic information systems. Santa Fe: Onward Press.

Cohen, J. (1992). A power primer. Psychological Bulletin, 112, 155-159.

Cressie, N. (1993). Statistics for spatial data. New York: Wiley.

Diggle, P. (1983). Statistical analysis of spatial point patterns. New York, Academic Press.

Gettis, A. (1991). Spatial interaction and spatial autocorrelation: A cross-product approach. Environment and Planning, $A$ 23, 1269-1277.

Hutchinson, S., \& Daniel, L. (1995). Inside Arcliew. Santa Fe: Onward Press.

Mount, B. (1987). Personal futures planning: Finding directions for change (Doctoral dissertation, University of Georgia, 1987). University Microfilms International, Order No. 8714642.

Rhodes, D., McFarland, K., \& Knight, P. (1995). Evolution of consumerism in Rehabilitation counseling: A theoretical perspective. Journal of Rehabilitation, 61 (3).

Systat 9.0 Statistics II (1999). Chicago: SPSS, Inc.

Yung, Y-F., \& Chan, W. (1999). Statistical analyses using bootstrapping: Concepts and implementation. In (R. H. Hoyle, ed.) Statistical strategies for small sample research. Thousand Oaks, CA: Sage.

$$
\begin{gathered}
\text { Appendix } \\
\text { Moran's I Formula } \\
I=\frac{n \Sigma_{i} \Sigma_{j} \delta_{i j}\left(x_{i}-\bar{x}\right)\left(x_{j}-\bar{x}\right)}{S_{0} \Sigma_{i}\left(x_{i}-\mathrm{x}\right)^{2}}
\end{gathered}
$$

where $S_{o}=\Sigma_{i} \Sigma_{j} \delta_{1 j}$.

The expected value for the statistic is

$$
E(I)=-(n-1)^{-1}
$$

with the variance being

$$
\operatorname{Var}(I)=\frac{n^{2} S_{1}-n S_{2}+3 S_{0}{ }^{2}}{S_{0}{ }^{2}\left(n^{2}-1\right)}
$$

where

$$
S_{1}=(1 / 2) \Sigma_{i} \Sigma_{j} \quad\left(\delta_{i j}+\delta_{j i}\right)^{2}
$$

and

$$
S_{2}=\Sigma_{i}\left(\Sigma_{i} \delta_{i j}+\Sigma_{j} \delta_{j i}\right)^{2}
$$

The number of geographic units is represented by $n$, The spatial relationship between the $i$-th and $j$-th units is $\delta_{i j}$, the frequency of the spatial phenomenon is $x_{i}$, and $\mathrm{S}$ ${ }_{0}$ being the number of pairs in the spatial relationship (Chou, 1995). 


\section{The Q-Sort Method: Assessing Reliability And Construct Validity Of Questionnaire Items At A Pre-Testing Stage}

\author{
Abraham Y. Nahm \\ Management and Marketing Department \\ University of Wisconsin \\ S. Subba Rao \\ Department of Management \\ The University of Toledo
}

\author{
Luis E. Solis-Galvan \\ Operations and Technology Management Department \\ Instituto de Empresa \\ T. S. Ragu-Nathan \\ Department of Information Systems, Marketing, ECommerce \& Sales \\ The University of Toledo
}

This paper describes the Q-sort, which is a method of assessing reliability and construct validity of questionnaire items at a pre-testing stage. The method uses Cohen's Kappa and Moore and Benbasat's Hit Ratio in assessing the questionnaire. Two examples are provided on how the method was applied in survey research.

Keywords: Q-sort, Surveys, Reliability, Construct validity, Kappa, Hit ratio

Introduction

This paper describes the Q-sort method, which is a method of assessing reliability and construct validity of questionnaire items that are being prepared for survey research. The method is applied at the pre-test stage, which comes after the item generation through literature search and before the administering of questionnaire items as a survey. The method is cost efficient and simple, yet provides ample insight into potential problem areas in the questionnaire items that are being tested.

\section{Theoretical Basis}

The Q-sort method is an iterative process in which the degree of agreement between judges forms the basis of assessing construct validity and improving the reliability of the constructs. The method consists of two stages. In the first stage, two judges are requested to sort the questionnaire items according to different constructs, based on which the inter-judge agreement is measured. In the second stage, questionnaire items that were identified as being too ambiguous, as a result of the first stage, are reworded or deleted, in an effort to improve the agreement between the judges. The process is carried out repeatedly until a satisfactory level of agreement is reached.

Abraham Y. Nahm, Management and Marketing Department, University of Wisconsin - Eau Claire, P.O. Box 4004, Eau Claire, Wisconsin, 54702-4004.E-mail: nahmay@uwec.edu. Luis E. Solis-Galvan, Operations and Technology Management Department, Instituto de Empresa, Madrid, Spain. S. Subba Rao, Department of Management, The University of Toledo, Toledo, Ohio. T. S. Ragu-Nathan, Department of Information Systems, Marketing, ECommerce and Sales, The University of Toledo, Toledo, Ohio.
The following example describes the theoretical basis for the Q-sort method and the two evaluation indices to measure inter-judge agreement level: Cohen's Kappa (Cohen, 1960) and Moore and Benbasat's "Hit Ratio" (Moore and Benbasat, 1991).

Assume two judges independently classified a set of $\mathrm{N}$ components as either acceptable or rejectable. After the work was finished the following table was constructed:

\begin{tabular}{ccccc}
\hline & \multicolumn{3}{c}{ Judge 1 } & \\
\cline { 3 - 4 } & & Acceptable & Rejectable & Totals \\
\hline Judge 2 & Acceptable & $\mathrm{X}_{11}$ & $\mathrm{X}_{12}$ & $\mathrm{X}_{1+}$ \\
& Rejectable & $\mathrm{X}_{21}$ & $\mathrm{X}_{22}$ & $\mathrm{X}_{2+}$ \\
\hline Totals & $\mathrm{X}_{+1}$ & $\mathrm{X}_{+2}$ & $\mathrm{~N}$ \\
\hline
\end{tabular}

Note: $X_{i j}=$ the number of components in the $\mathrm{i}^{\text {th }}$ row and $\mathrm{j}^{\text {th }}$ column, for $i, j=1,2$.

The above table can also be constructed using percentages by dividing each numerical entry by N. For the population of components, the table will look like:

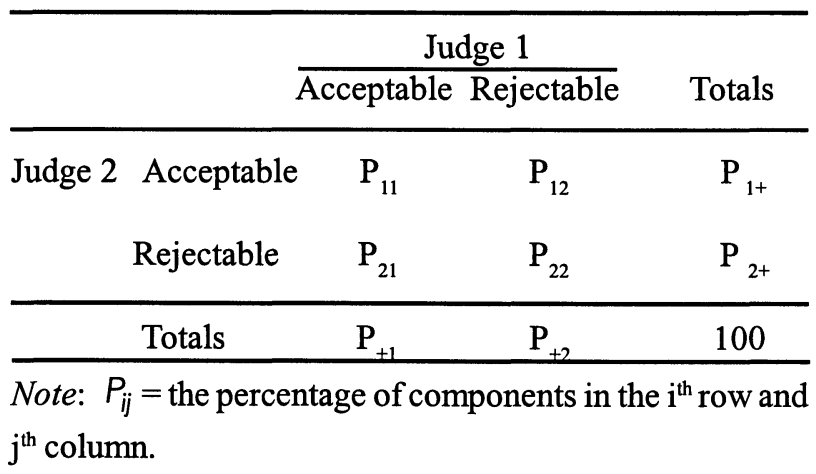


This table of percentages is used to describe the Cohen's Kappa coefficient of agreement. The simplest measure of agreement is the proportion of components that were classified the same by both judges, i.e., $\Sigma_{\mathrm{i}} \mathrm{P}_{\mathrm{ii}}=\mathrm{P}_{11}+$ $\mathrm{P}_{22}$. However, Cohen suggested comparing the actual agreement, $\Sigma_{\mathrm{i}} \mathrm{P}_{\mathrm{ii}}$, with the chance of agreement that would occur if the row and columns are independent, i.e., $\Sigma_{i} P_{i+} P_{+i}$ The difference between the actual and chance agreements, $\Sigma_{i} P_{i i}-\Sigma_{i} P_{i+} P_{+i}$ is the percent agreement above which is due to chance. This difference can be standardized by dividing it by its maximum possible value, i.e., $100 \%-\Sigma_{i}$ $\mathrm{P}_{\mathrm{i}+} \mathrm{P}_{+\mathrm{i}}$. The ratio of these is denoted by the Greek letter Kappa and is referred to as Cohen's Kappa.

Thus, Cohen's Kappa as a measure of agreement can be interpreted as the proportion of joint judgement in which there is agreement after chance agreement is excluded. The three basic assumptions for this agreement coefficient are: 1) the units are independent, 2) the categories of the nominal scale are independent and mutually exclusive, and 3) the judges operate independently.

For Kappa, no general agreement exists with respect to required scores. However, several studies have considered scores greater than 0.65 to be acceptable (e.g. Vessey, 1984; Jarvenpaa 1989). Landis and Koch (1977) have provided a more detailed guideline to interpret Kappa by associating different values of this index to the degree of agreement beyond chance. They suggest the following guideline:

1. Excellent Agreement: Kappa $=.76-1.00$

2. Fair To Moderate Agreement: Kappa $=.40-.75$

3. Poor Agreement: Kappa $=.39$ or less

A second overall measure of both the reliability of the classification scheme and the validity of the items was developed by Moore and Benbasat (1991). The method required analysis of how many items were placed by the panel of judges for each round within the target construct. In other words, because each item was included in the pool explicitly to measure a particular underlying construct, a measurement was taken of the overall frequency with which the judges placed items within the intended theoretical construct. The higher the percentage of items placed in the target construct, the higher the degree of inter-judge agreement across the panel which must have occurred.

Scales based on categories which have a high degree of correct placement of items within them can be considered to have a high degree of construct validity, with a high potential for good reliability scores. This procedure is more a qualitative analysis than a rigorous quantitative procedure. There are no established guidelines for determining good levels of placement, but the matrix can be used to highlight any potential problem areas. The following exemplifies how this measure works.
Consider the simple case of four theoretical constructs with ten items developed for each construct. With a panel of three judges, a theoretical total of 30 placements could be made within each construct. Thereby, a theoretical versus actual matrix of item placements could be created as shown in the table below (including an ACTUAL "N/A: Not Applicable" column where judges could place items which they felt fit none of the categories). (See Table A on next page.)

The item placement ratio (the "Hit Ratio") is an indicator of how many items were placed in the intended, or target, category by the judges. Examination of the diagonal of the matrix shows that with a theoretical maximum of 120 target placements (four constructs at 30 placements per construct), a total of 102 "hits" were achieved, for an overall "hit ratio" of $85 \%$. More important, an examination of each row shows how the items created to tap the particular constructs are actually being classified. For example, row $\mathrm{C}$ shows that all 30 -item placements were within the target construct, but that in row B, only $60 \%$ $(18 / 30)$ were within the target. In the latter case, 8 of the placements were made in construct $A$, which might indicate the items underlying these placements are not differentiated enough from the items created for construct A. This finding would lead one to have confidence in a scale based on row $C$, but be hesitant about accepting any scale based on row B. An examination of off-diagonal entries indicates how complex any construct might be. Actual constructs based on columns with a high number of entries in the off-diagonal might be considered too ambiguous, so any consistent pattern of item misclassification should be examined.

\section{Examples}

Research on Post-industrial Manufacturing

Nahm (2000) studied the relationship between external environment, internal environment ("managerial beliefs and attitudes" and "organizational structure"), and manufacturing practices through a large-scale survey. As part of this research, questionnaire items were developed to measure the constructs "external environment," "managerial beliefs and attitudes," and "organizational structure" (items for "manufacturing practices" were adopted from Koufteros, Vonderembse, \& Doll, 1998). The development of the instrument was carried out in two steps. The first step was item generation. The purpose of this step was to create pools of items for each of the constructs that fit the construct definitions.

Once the item pools were created, items for the various constructs were re-evaluated to eliminate redundant or ambiguous items. This was done through structured interviews with practitioners from five different manufacturing firms. The focus was to check the relevance of 
Table A

\begin{tabular}{|c|c|c|c|c|c|c|c|c|}
\hline & & A & B & $\begin{array}{l}\text { Act } \\
\text { C }\end{array}$ & D & N/A & Total & $\%$ Hits \\
\hline \multirow[t]{4}{*}{ Theoretical } & $\mathrm{A}$ & 26 & 2 & 1 & 0 & 1 & 30 & 87 \\
\hline & $\mathrm{B}$ & 8 & 18 & 4 & 0 & 0 & 30 & 60 \\
\hline & $\mathrm{C}$ & 0 & 0 & 30 & 0 & 0 & 30 & 100 \\
\hline & $\mathrm{D}$ & 0 & 1 & 0 & 28 & 1 & 30 & 93 \\
\hline
\end{tabular}

Note: Item placements, 120 ; hits, 102 ; overall hit ratio, $85 \%$.

each construct's definitions and clarity of wordings of sample questionnaire items. The result was the following number of items in each pool:

External Environment

Level of Market Heterogeneity

Degree of Market Segmentation

Length of Product Life Cycle

Driving Force for Manufacturing Technology

Number of Customer Requirements

Sub-Total

Managerial Beliefs and Attitudes

Goals for Investing in Facilities and Equipment

Organization of Work

Scope in Decision Making

Management Mechanism

Focus of Managerial Tasks

Focus of Supplier Relationships Management

Sub-Total

Organizational Structure

Locus of Decision Making

Nature of Formalization

Number of Layers in Hierarchy

Level of Horizontal Integration

Level of Communication

Sub-Total

Total

Items placed in a common pool were subjected to two sorting rounds by two independent judges per round. The procedure had practitioners from the industry act as judges and sort the items from the first stage into separate constructs, based on similarities and differences among items. Based on the placements made by the judges the items could then be examined and inappropriately worded or ambiguous item could be eliminated or reworded. Two goals for this stage were: to attempt to identify any ambiguous items, and to pre-assess the construct validity of the various scales being developed.

First, judges sorted the questionnaire items into construct categories. Each item was printed on a $3 \times 5$ inch index card. The cards were shuffled into random order for presentation to the judges. Each judge sorted the cards into categories. A "not available" category definition was included to ensure that the judges did not force any item into a particular category. During the two sorting rounds, two different pairs of judges were utilized. Each 48 set of judges included a manufacturing engineer/manager or a top management executive to ensure that the perceptions of the target population would be included in the analysis. Prior to sorting the cards, the judges were briefed with a standard set of instructions that were previously tested with a separate judge to ensure comprehensiveness and comprehensibility. Judges were allowed to ask as many questions as necessary to ensure they understood the procedure.

To assess the reliability of the sorting conducted 61 by the judges, two different measures were made. First, for each pair of judges in each sorting step, their level of agreement in categorizing items was measured using Cohen's Kappa.

First sorting round.

In the first round, the inter-judge raw agreement scores averaged 0.74 (Table 1), the initial overall placement ratio of items within the target constructs was $84 \%$ (Table 2), and the Kappa scores averaged 0.73. A summary of the first round inter-judge agreement indices is shown in Table 3. Following the guidelines of Landis and Koch (1977) for interpreting the Kappa coefficient, the value of 0.73 indicates a moderate, but almost excellent level of agreement beyond chance for the judges in the first round. This value is slightly lower than the value for raw agreement, which is 0.74 (Table 1). The level of item placement ratios averaged $84 \%$. For instance, the lowest item placement ratio value was $67 \%$ for the "level of communication" construct, indicating a low degree of construct validity. On the other hand, several constructs ("driving 
force for manufacturing technology" and "number of layers in hierarchy") obtained a $100 \%$ item placement ratio, indicating a high degree of construct validity.

In order to improve the Cohen's Kappa measure of agreement, an examination of the off-diagonal entries in the placement matrix (Table 2) was conducted. Any ambiguous items (fitting in more than one category) or too indeterminate items (fitting in no category) were either deleted or reworded. Overall, 29 items were deleted, and 25 items were reworded.

\section{Second sorting round.}

Again, two judges were involved in the second sorting round, which included the reworded items developed after the first sorting round. In the second round the inter-judge raw agreement scores averaged 0.80 (Table 4), the initial overall placement ratio of items within the targets constructs was $88 \%$ (Table 5), and the Kappa scores averaged 0.78 . A summary of the second round inter-judge agreement indices is shown in the second column of Table 3. The value for Kappa coefficient of 0.78 is higher than the value obtained in the first round, and indicates an excellent fit, based on the guidelines of Landis and Koch (1977) for interpreting the Kappa coefficient. The level of item placement ratios averaged $88 \%$. The lowest item placement ratio value was that of $69 \%$ for the "scope in decision making" construct, indicating a low degree of construct validity. Again several constructs ("goals for investing in facilities and equipment," "focus of supplier relationships management," and "nature of formalization") obtained a $100 \%$ item placement ratio, indicating a high degree of construct validity.

In order to further improve potential reliability and construct validity, an examination of the off-diagonal entries in the placement matrix (Table 5) was conducted. Again, any ambiguous items (fitting in more than one category) or too indeterminate items (fitting in no category) were either deleted or reworded. Overall, 29 items were further deleted, and 15 items were reworded.

At this point, we stopped the Q-sort method at round two, for the raw agreement score of 0.80 , Cohen's Kappa of 0.78 , and the average placement ratio of $88 \%$ were considered as an excellent level of inter-judge agreement, indicating high level of reliability and construct validity.

\section{Research on Quality Management}

The second example is from Solis-Galvan (1998) who studied the relationship between quality management and manufacturing competitive capabilities. The basic steps for the instrument development were the same as with $\mathrm{Nahm}$ (2000). The first step was item generation. The following number of items were generated in each pool:
Management Based Quality Practices

Top Management Support $\quad 11$

Strategic Quality Planning 5

$\begin{array}{ll}\text { Sub-Total } & 16\end{array}$

Employee Based Quality Practices

Employee Training 5

Employee Involvement 5

Employee Empowerment 5

Employee Recognition 4

Sub-Total 19

Information Based Quality Practices

Quality Information Availability 5

Quality Information Usage 4

Benchmarking 4

$\begin{array}{ll}\text { Sub-Total } & 13\end{array}$

Customer Based Quality Practices

Customer Orientation 9

Customer Closeness 6

Sub-Total 15

Product/Process Based Quality Practices

Product Design $\quad 8$

SPC Usage 3

Process Design 3

Sub-Total 14

Supplier Based Quality Practices

Supplier Quality 7

Total $\quad 84$

The second step was item testing. The basic procedure was to have quality managers, plant managers, and top management executives judge and sort the items from the first stage into separate quality management categories, based on similarities and differences among items. A group of potential judges were identified from the local Chapter of the American Society for Quality. All of the potential participants were representatives of the population targeted for this study, and considered as knowledgeable in the quality field, and with the required experience and position to assess the impact of TQM practices in their organization. From this group, a random sample of six judges was selected to participate during this stage.

First sorting round.

Two judges were involved in the first round, which included items developed for the quality management constructs (84 items). In the first round, the inter-judge raw agreement scores averaged 0.71 (60 agreements among 84 
items), the initial overall placement ratio of items within the target constructs was $84 \%$, and the Kappa scores averaged 0.64 (Table 6).

Following the guidelines of Landis and Koch for interpreting the Kappa coefficient, the value of 0.64 indicates a moderate level of agreement beyond chance for the judges in the first round. This value is slightly lower than the value for raw agreement, which is 0.71 . The level of item placement ratios averaged $84 \%$. For instance, the lowest item placement ratio value was $55 \%$ for the "customer orientation" construct, indicating a low degree of construct validity. On the other hand, several constructs ("employee recognition," "quality information availability," "quality information usage," "usage of statistical quality control," and "benchmarking") obtained a $100 \%$ item placement ratio, indicating a high degree of construct validity.

In order to improve the Cohen's Kappa measure of agreement, an examination of the items that had a mismatch between the theoretical category and the actual placements by the judges was conducted. The analysis led to the rewording of ambiguous or indeterminate items. One additional item was included in the "product design" construct after reviewing its definition. Feedback obtained from both judges lead to the creation of two additional items for the "customer closeness" construct and one additional item for the "supplier quality" construct.

\section{Second sorting round.}

Two judges were involved in the second sorting round, which included the reworded and new items developed after the first sort round. In the second round the inter-judge raw agreement scores averaged 0.70 (62 agreements among 88 items).

A summary of the second round inter-judge agreements indices is shown in the second column of Table 6. The value for Kappa coefficient of 0.68 was higher than the value obtained in the first round, but still indicated a moderate level of agreement beyond chance for the judges in the second round. The level of item placement ratios averaged $82 \%$. The lowest item placement ratio was $50 \%$ for the "employee involvement" construct, indicating a low degree of construct validity. Several constructs ("employee recognition," "quality information availability," "quality information usage," "usage of statistical quality control," and "process design") obtained a $100 \%$ item placement ratio, indicating a high degree of construct validity.

Examination of the items that had a mismatch between the theoretical and actual placements resulted in rewording four items belonging to the constructs "customer orientation," "product design," and "supplier quality." One additional question suggested by two judges was added to the "SPC usage" construct.
Third sorting round.

Two judges participated in the third sorting round, which included the reworded items and the new item added to the "SPC usage" construct. In the third round the interjudge raw agreement scores averaged 0.81 ( 72 agreements among 89 items), the overall placement ratio of items within the targets constructs was $89 \%$, and the Kappa scores averaged 0.81 (Table 6).

The value for Kappa coefficient of 0.81 is significantly higher than the value obtained in the second round, and indicates an excellent level of agreement beyond chance. The level of item placement ratios averaged $89 \%$. The lowest item placement ratio value was that of $75 \%$ for the "quality information usage" construct, indicating a moderate to good degree of construct validity. The constructs "employee training," "quality information availability," "usage of statistical quality control," "process design," "supplier quality," and "benchmarking" obtained a $100 \%$ item placement ratio. This placement of items within the target construct shows that a high degree of construct validity and potential reliability were achieved.

\section{Conclusion}

Until now, researchers engaged in survey research had no simple way of assessing the reliability and construct validity of their questionnaire items before the large scale survey except for conducting a pilot survey on a reduced scale. By applying the Q-sort method, one can now assess them in a timely and cost-efficient manner. The method is a qualitative rather than a quantitative method, and thus may not be compared to the usefulness and the statistical power of a pilot survey. Nevertheless, in light of the importance of a quality scale development in survey research, a method like the one described here, which is a cost-efficient and simple yet insightful method of assessing reliability and construct validity of measurement items, should be of great help to researchers.

\section{References}

Cohen, J. (1960). A coefficient of agreement for nominal scales. Educational and Psychological Measurement, Spring, 37-46.

Jarvenpaa, S. (1989). The effect of task demands and graphical format on information processing strategies. Management Science, 35, 285-303.

Koufteros, X. A., Vonderembse, M. A., \& Doll, W. J. (1998). Developing measures of time-based manufacturing. Journal of Operations Management, 16, 21-41.

Landis, J. R., \& Koch, C. G. (1977). The measurement of observer agreement for categorical data. Biometrics, 33, 159-174. 
Moore, G. C., \& Benbasat, I. (1991). Development of an instrument to measure the perceptions of adopting an information technology innovation. Information Systems Research, 2, 192-222.

Nahm, A. Y. (2000). Exploring the relationship between external environment, internal environment, and manufacturing practices: An industrial/post-industrial perspective. Unpublished doctoral dissertation, The University of Toledo, Ohio.
Solis-Galvan, L. E. (1998). An exploratory study of the relationship between quality management and manufacturing competitive capabilities. Unpublished doctoral dissertation, The University of Toledo, Ohio.

Vessey, I. (1984). An investigation of the psychological processes underlying the debugging of computer programs. Unpublished doctoral dissertation, The University of Queensland, Queensland, Australia. 
Appendix

Table 1

Inter-Judge Raw Agreement Scores: First Sorting Round

\begin{tabular}{|c|c|c|c|c|c|c|c|c|c|c|c|c|c|c|c|c|c|c|}
\hline \multirow{2}{*}{\multicolumn{2}{|c|}{ Constructs $^{\mathrm{a}}$}} & \multicolumn{17}{|c|}{ Judge 1} \\
\hline & & 1 & 2 & 3 & 4 & 5 & 6 & 7 & 8 & 9 & 10 & 11 & 12 & 13 & 14 & 15 & 16 & NA \\
\hline \multirow{10}{*}{$\begin{array}{l}\mathrm{J} \\
\mathrm{u} \\
\mathrm{d} \\
\mathrm{g} \\
\mathrm{e}\end{array}$} & 1 & 6 & 2 & & & 1 & & & & & & & & & & & & \\
\hline & 2 & & 6 & & & 1 & & & & & & & & & & & & \\
\hline & 3 & & & 8 & 1 & & & & & & & & & & & & & \\
\hline & 4 & & & & 11 & & & & & & & & & & & & & \\
\hline & 5 & 2 & & & 1 & 9 & & & & & & & & & & & & \\
\hline & 6 & & & & & & 6 & & & & & & & & & & & \\
\hline & 7 & & & & & & 1 & 5 & 3 & 1 & 1 & & & & & & & \\
\hline & 8 & & & & & & & 4 & 6 & 3 & 1 & & & & & & & \\
\hline & 9 & & & & & & & 1 & & 7 & & & & & & & & \\
\hline & 10 & & & & & & 1 & & & & 8 & 1 & & & & & & \\
\hline \multirow{7}{*}{2} & 11 & & & & & & & & 1 & & & 9 & & & & & & \\
\hline & 12 & & & & & & & & & & & & 8 & & 2 & & & \\
\hline & 13 & & & & & & & & & & & & 2 & 9 & & 2 & & \\
\hline & 14 & & & & & & & & & & & & & & 8 & & & \\
\hline & 15 & & & & & & & & & & & & 1 & 1 & & 10 & & \\
\hline & 16 & & & & & & & & & & & & & & 3 & 2 & 5 & 1 \\
\hline & NA & & & & & & & 1 & 1 & & & & & & & & & \\
\hline
\end{tabular}

Note. Total items placement, 163; number of agreements, 121; agreement ratio, 0.74.

${ }^{a}$ Constructs: 1 . Market heterogeneity

2. Degree of market segmentation

3. Length of product life cycle

4. Driving force for manufacturing technology

5. Number of customer requirements

6. Goals for investing in facilities and equipment

7. Organization of work

8. Scope in decision-making

9. Management mechanism

10. Focus of managerial tasks

11. Focus of supplier relationships management

12. Locus of decision-making

13. Nature of formalization

14. Number of layers in hierarchy

15. Level of horizontal integration

16. Level of communication 
Table 2

Items Placement Ratios: First Sorting Round

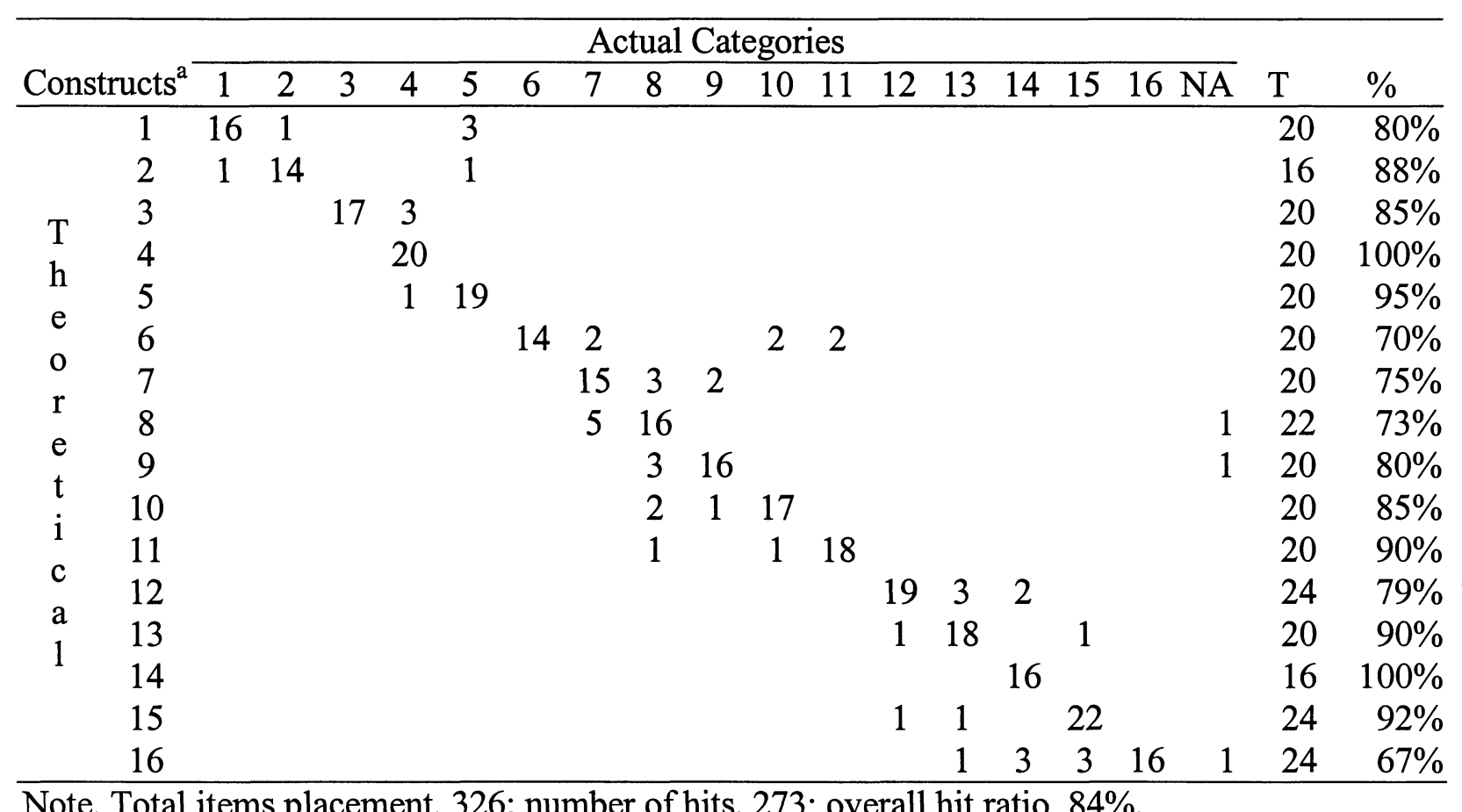

Note. Total items placement, 326; number of hits, 273 ; overall hit ratio, $84 \%$.

${ }^{\mathrm{a}}$ The names of contructs are as listed in Table 1. 
Q-SORT METHOD: RELIABILITY AND CONSTRUCT VALIDITY

Table 3

Inter-Judge Agreements

\begin{tabular}{|c|c|c|}
\hline Agreement Measure & Round 1 & Round 2 \\
\hline Raw Agreement & 0.74 & 0.80 \\
\hline Cohen's Kappa & 0.73 & 0.78 \\
\hline \multicolumn{3}{|l|}{ Placement Ratio Summary } \\
\hline External environment & $80 \%$ & $86 \%$ \\
\hline Degree of market segmentation & $88 \%$ & $93 \%$ \\
\hline Length of product life cycle & $85 \%$ & $81 \%$ \\
\hline Driving force for manufacturing technology & $100 \%$ & $95 \%$ \\
\hline Number of customer requirements & $95 \%$ & $85 \%$ \\
\hline Goals for investing in facilities and equipment & $70 \%$ & $100 \%$ \\
\hline Organization of work & $75 \%$ & $93 \%$ \\
\hline Scope in decision making & $73 \%$ & $69 \%$ \\
\hline Management mechanism & $80 \%$ & $86 \%$ \\
\hline Focus of managerial tasks & $85 \%$ & $94 \%$ \\
\hline Focus of supplier relationships management & $90 \%$ & $100 \%$ \\
\hline Locus of decision making & $79 \%$ & $83 \%$ \\
\hline Nature of formalization & $90 \%$ & $100 \%$ \\
\hline Number of layers in hierarchy & $100 \%$ & $81 \%$ \\
\hline Level of horizontal integration & $92 \%$ & $91 \%$ \\
\hline Level of communication & $67 \%$ & $75 \%$ \\
\hline Average & $84 \%$ & $88 \%$ \\
\hline
\end{tabular}


123

NAHM, SOLIS-GALVIN, RAO, \& RAGU-NATHAN

Table 4

Inter-Judge Raw Agreement Scores: Second Sorting Round

\begin{tabular}{|c|c|c|c|c|c|c|c|c|c|c|c|c|c|c|c|c|c|c|}
\hline \multirow{2}{*}{\multicolumn{2}{|c|}{ Constructs $^{\mathrm{a}}$}} & \multicolumn{17}{|c|}{ Judge 3} \\
\hline & & 1 & 2 & 3 & 4 & 5 & 6 & 7 & 8 & 9 & 10 & 11 & 12 & 13 & 14 & 15 & 16 & $\mathrm{NA}$ \\
\hline & 1 & 5 & 1 & & & 1 & & & & & & & & & & & & \\
\hline & 2 & 1 & 6 & & & & & & & & & & & & & & & \\
\hline & 3 & & 1 & 6 & 1 & & & & & & & & & & & & & \\
\hline & 4 & & & & 10 & & & & & & & & & & & & & \\
\hline & 5 & & & & 1 & 7 & & & & & & & & & & & & 2 \\
\hline $\mathrm{J}$ & 6 & & & & & & 7 & & & & 1 & & & & & & & \\
\hline $\mathrm{u}$ & 7 & & & & & & & 6 & 3 & 1 & & & & & & & & \\
\hline$d$ & 8 & & & & & & & & 3 & & 1 & 1 & & & & & & \\
\hline $\mathrm{g}$ & 9 & & & & & & & & 2 & 5 & & & & & & & & \\
\hline $\mathrm{e}$ & 10 & & & & & & & & & & 7 & & & & & & & \\
\hline & 11 & & & & & & & & & & & 10 & & & & & & \\
\hline 4 & 12 & & & & & & & & & & & & 7 & & & 1 & & \\
\hline & 13 & & & & & & & & & & & & 2 & 9 & & 1 & & \\
\hline & 14 & & & & & & & & & & & & 1 & & 5 & & & \\
\hline & 15 & & & & & & & & & & & & & 1 & & 9 & & \\
\hline & 16 & & & & & & & & & & & & & 1 & & 1 & 5 & \\
\hline & NA & & & & & & & & & & & & & & 2 & & & \\
\hline
\end{tabular}

Note. Total items placement, 134; number of agreements, 107; agreement ratio, 0.80 .

${ }^{\mathrm{a}}$ The names of contructs are as listed in Table 1. 
Table 5

Items Placement Ratios: Second Sorting Round

\begin{tabular}{|c|c|c|c|c|c|c|c|c|c|c|c|c|c|c|c|c|c|c|c|c|}
\hline \multirow{2}{*}{\multicolumn{2}{|c|}{ Constructs $^{\mathrm{a}}$}} & \multicolumn{17}{|c|}{ Actual Categories } & \multirow[b]{2}{*}{$\mathrm{T}$} & \multirow[b]{2}{*}{$\%$} \\
\hline & & 1 & 2 & 3 & 4 & 5 & 6 & 7 & 8 & 9 & 10 & 11 & 12 & 13 & 14 & 15 & $16 I$ & $\overline{\mathrm{NA}}$ & & \\
\hline & 1 & 12 & 1 & & & 1 & & & & & & & & & & & & & 14 & $86 \%$ \\
\hline & 2 & 1 & 13 & & & & & & & & & & & & & & & & 14 & $93 \%$ \\
\hline & 3 & & 1 & 13 & 2 & & & & & & & & & & & & & & 16 & $81 \%$ \\
\hline $\mathrm{T}$ & 4 & & & 1 & 19 & & & & & & & & & & & & & & 20 & $95 \%$ \\
\hline $\mathrm{h}$ & 5 & & & & 1 & 17 & & & & & & & & & & & & 2 & 20 & $85 \%$ \\
\hline $\mathrm{e}$ & 6 & & & & & & 14 & & & & & & & & & & & & 14 & $100 \%$ \\
\hline o & 7 & & & & & & & 13 & & 1 & & & & & & & & & 14 & $93 \%$ \\
\hline $\mathrm{r}$ & 8 & & & & & & & 3 & 11 & & 1 & 1 & & & & & & & 16 & $69 \%$ \\
\hline e & 9 & & & & & & & & 2 & 12 & & & & & & & & & 4 & 86 \\
\hline $\mathrm{t}$ & 10 & & & & & & 1 & & & & 15 & & & & & & & & 16 & 94 \\
\hline i & 11 & & & & & & & & & & & 20 & & & & & & & 20 & $100 \%$ \\
\hline c & 12 & & & & & & & & & & & & 15 & 2 & & 1 & & & 18 & $83 \%$ \\
\hline a & 13 & & & & & & & & & & & & & 18 & & & & & 18 & $100 \%$ \\
\hline 1 & 14 & & & & & & & & & & & & 1 & & 13 & & & 2 & 16 & $81 \%$ \\
\hline & 15 & & & & & & & & & & & & & 2 & & 20 & & & 22 & $91 \%$ \\
\hline & 16 & & & & & & & & & & & & 2 & 1 & & 1 & 12 & & 16 & $75 \%$ \\
\hline
\end{tabular}

Note. Total items placement, 268; number of hits, 237; overall hit ratio, $88 \%$.

${ }^{a}$ The names of contructs are as listed in Table 1. 
Table 6

Inter-Judge Agreements

\begin{tabular}{|c|c|c|c|}
\hline Agreement Measure & Round 1 & Round 2 & Round 3 \\
\hline Raw Agreement & 0.71 & 0.70 & 0.81 \\
\hline Cohen's Kappa & 0.64 & 0.68 & 0.81 \\
\hline \multicolumn{4}{|l|}{ Placement Ratio Summary } \\
\hline Top Management Involvement & $86 \%$ & $91 \%$ & $91 \%$ \\
\hline Strategic Quality Planning & $80 \%$ & $80 \%$ & $90 \%$ \\
\hline Employee Training & $80 \%$ & $100 \%$ & $100 \%$ \\
\hline Employee Involvement & $80 \%$ & $50 \%$ & $80 \%$ \\
\hline Employee Empowerment & $80 \%$ & $70 \%$ & $80 \%$ \\
\hline Employee Recognition & $100 \%$ & $80 \%$ & $88 \%$ \\
\hline Availability of Quality Information & $100 \%$ & $100 \%$ & $100 \%$ \\
\hline Usage of Quality Information & $100 \%$ & $100 \%$ & $75 \%$ \\
\hline Customer Orientation & $55 \%$ & $67 \%$ & $83 \%$ \\
\hline Closeness to Customers & $91 \%$ & $63 \%$ & $75 \%$ \\
\hline Product Design & $75 \%$ & $78 \%$ & $83 \%$ \\
\hline Statistical Process Control Usage & $100 \%$ & $100 \%$ & $100 \%$ \\
\hline Process Management & $66 \%$ & $100 \%$ & $100 \%$ \\
\hline Supplier Relationships & $93 \%$ & $88 \%$ & $100 \%$ \\
\hline Benchmarking & $100 \%$ & $88 \%$ & $100 \%$ \\
\hline Average & $84 \%$ & $82 \%$ & $89 \%$ \\
\hline
\end{tabular}




\title{
An Error In Statistical Logic In The Application Of Genetic Paternity Testing
}

\author{
Ernest P. Chiodo \\ Internal Medicine, School of Medicine \\ Wayne State University
}

\author{
Joseph L. Musial \\ Department of Internal Medicine \\ Henry Ford Health System
}

\author{
J. Sia Robinson \\ East Side Academy, Detroit, Michigan
}

\begin{abstract}
A Bayes probability computer program was written in Fortran to examine issues related to genetic paternity testing. An application was given to demonstrate the effects improper assumptions of prior probability of culpability. The seriousness of such errors include the potential of assigning paternity to wrongly accused men, or wrongly refuting paternity.
\end{abstract}

Key words: Bayes, Paternity testing.

\section{Introduction}

Genetic testing has been widely used in criminal cases as well as in cases involving establishment of paternity. In the United States, the incidence of paternity cases appears to be very large especially when compared to live birth rates. For example, the Centers for Disease Control reports that there were 3,959,417 live births nationally during calendar year 1999 (Ventura, Martin, Curtin, Menacker, \& Hamilton, 2001). Among these births, a total of $1,308,560(33 \%)$ were delivered by unmarried women. Unpublished data from The Wayne County Circuit Court, located in Southeastern Michigan, notes that there were 11,104 case filings during the year 2000 (Wayne County Circuit Court, 2001). Case filings are generated when legal paternity has not been established.

Every legal practitioner dealing with genetic testing in either a criminal or paternity context should know how this testing can lead to incorrect conclusions. The statistical assumptions made during paternity testing can cause the results of testing to be misleading and unreliable. Although the focus of this article is on a serious error in statistical methodology frequently occurring in paternity testing, the same error may also occur in criminal DNA

Ernest P. Chiodois a physician and an attorney. He is board certified in internal medicine, occupational medicine, public health, and general preventive medicine. Contact him at 25656 Schoenherr, Suite B, Warren, MI 48089, E-mail: echiodo@pol.net. Joseph L. Musial, Ph. D., is an Education Specialist for the Department of Internal Medicine at Henry Ford Health System, Detroit, MI. J. Sia Robinson is an instructor at East Side Academy, Detroit Public Schools, and adjunct lecturer, College of Education, Wayne State University. The authors acknowledge Jerome J. Fekin, Jr., for providing the case filing data, and Nicol R. Shamey, Plymouth-Canton, MI, School District. testing with dire consequences. An attorney practicing criminal or family law needs to understand the statistical assumptions that may cause the results of genetic testing to be misleading and unreliable. This understanding allows the knowledgeable advocate an opportunity to dispute testing results that are commonly and wrongly assumed to be infallible.

The mathematics in this article is limited in scope to the application of a simple formula. In addition, there is incentive to engage in the minimal mental effort needed to understand this article, because it provides the thoughtful attorney with a powerful advocacy tool. The central issue is the common error of assuming equal prior (pre-test) probabilities for an event in the face of ignorance concerning the actual probabilities. This common error called the "principle of indifference" may cause genetic testing using Bayes formula to be misleading (Issac, 1995).

The probability of the event $A$ is written as $P(A)$ and operates under the condition: $0 \leq \mathrm{P}(\mathrm{A}) \leq 1.0$. An impossible event has a probability of zero and a certain event has a probability of 1.0. Probabilities are mutually exclusive, which means there is no overlap. Tossing a coin illustrates this concept. There can be only one possible outcome: heads or tails. However, rarely are events dichotomous, straightforward probabilities. Instead, there are frequently a significant number of previous research findings with different probability levels.

This provides the entry point of Bayesian statistics. Thomas Bayes was an $18^{\text {th }}$ century English clergyman who devised a formula to generate a conditional probability (Borowski \& Borwein, 1991; Freund, 1973). The basic tenet of Bayesian statistics is the inclusion of conditional or prior probabilities. Often, the prior probabilities are not mechanically or deterministically generated, but rather, are based on expert judgment. Motulsky (1995) noted "usually the prior probability is not a real probability but is rather a subjective feeling. Some statisticians (Bayesians) think it is okay to convert feelings to numbers (" $99 \%$ sure" 
or " $70 \%$ sure"), which they define as the prior probability. Other statisticians (frequentists) think that you should never equate subjective feelings with probabilities" (p. 145).

The field of inferential statistics maintains a certain element of inaccuracy. Specifically, there are two types of errors associated with hypothesis testing: (1) a Type I error, or "false-positive", which occurs when a researcher asserts that there was a significant finding when in fact none existed; and, (2) a Type II error, or "false negative", which occurs when a researcher fails to observe a significant difference.

The following medical example illustrates the inherent problems associated with conditional probabilities. Assume that a 40 year-old female, who resides in an upper middle class suburb, presents with a newly diagnosed case of lung cancer to her primary care physician. The physician may use conditional probabilities generated by the Centers for Disease Control in order to determine the national incidence of this type of lung cancer among 40 year-old females. This approach appears reasonable, but caution is in order. During the history and physical, the patient may have failed to report that her former residence of many years was located near a toxic waste dump. This means that the history of environmental exposure may have contributed to the presenting cancer growth. By not including this in the construction of the prior probability, the physician may fail to consider other comorbid diseases associated with environmental exposure.

The problems associated with Bayesian statistics are also relevant to paternity cases. Suppose a man is accused of being the father of a child. He is found to have a genetic marker that only occurs in $1 \%$ of the male population. The child is tested and is also found to have the same genetic marker. The mother does not have the genetic marker. It is known that whenever a father has the marker it is always passed to the child. In this case the man contests paternity. Let:

$$
\begin{aligned}
& \mathrm{A}=\text { The man is the father of the child } \\
& \mathrm{B}=\text { The child has the same genetic marker as the } \\
& \text { man } \\
& A^{\prime}=\text { The man is not the father of the child } \\
& \mathrm{P}(\mathrm{A} / \mathrm{B})=\text { The probability that the man is the father of }
\end{aligned}
$$

$$
\begin{gathered}
\mathrm{P}\left(\mathrm{A}^{\prime}\right)=\text { The assumed prior probability before testing } \\
\text { that the man is not the father. }
\end{gathered}
$$

Bayes formula is as follows:

$$
P(A / B)=\frac{[P(B / A) P(A)]}{\left[P(B / A) P(A)+P\left(B / A^{\prime}\right) P\left(A^{\prime}\right)\right]}
$$

In this case $\mathrm{P}(\mathrm{B} / \mathrm{A})$ is 1 because there is a $100 \%$ probability that the child will get the genetic marker if the man is the father*. $\mathrm{P}\left(\mathrm{B} / \mathrm{A}^{\prime}\right)$ is 0.01 because the child has the same probability of having the genetic marker as the general population (one percent) if the man is not the father.

Recognize that only $\mathrm{P}(\mathrm{A})$ and $\mathrm{P}\left(\mathrm{A}^{\prime}\right)$ need to be identified before plugging the values into Bayes formula. $\mathrm{P}\left(\mathrm{A}^{\prime}\right)$ is simply $1-\mathrm{P}(\mathrm{A})^{* *}$. Therefore, all that remains is to identify $\mathrm{P}(\mathrm{A}) \mathrm{P}(\mathrm{A})$ is the assumed probability prior to testing that the man is the father of the child. In paternity testing this is often assumed to be $50 \%(.50)$. This assumption is made because there is a controversy concerning paternity. The mother of the child claims that the man is the father. The man claims that he is not the father. A prior (pre-test) probability of $50 \%$ is assumed as a default value for $\mathrm{P}(\mathrm{A})$.

If the above values are entered into Bayes formula the following result occurs:

$\mathrm{P}(\mathrm{A} / \mathrm{B})=[(1)(0.5)] /[(1)(0.5)+(0.01)(0.5)]=0.9901$

Therefore, there is a greater than $99 \%$ probability of paternity when using a prior (pre-test) probability of $50 \%(\mathrm{P}(\mathrm{A})=0.5)$.

The Joint AMA-ABA Guidelines (Hummel, 1976; Kilmer, 1993) for likelihood of paternity are in Table 1.

Table 1. Paternity Guidelines.

\begin{tabular}{ll}
\hline Test Probability & Interpretation \\
\cline { 2 - 2 }$<80 \%$ & not useful \\
$80 \%-90 \%$ & undecided \\
$90 \%-95 \%$ & likely \\
$95 \%-99 \%$ & very likely \\
$99.1 \%-99.75 \%$ & extremely likely \\
$99.8 \%-99.9 \%$ & practically proven \\
\hline
\end{tabular}


In Michigan, paternity is presumed when the DNA profile determination determines a probability of paternity of $99 \%$ or higher (Hummel, 1976; Kilmer, 1993). In Michigan, blood tests for paternity are generally admissible in evidence at trial (Kilmer, 1993). Consequently, the man in the above example would be presumed under Michigan law to be the father of the child.

However, the results will change drastically if a lower prior (pre-test) probability of paternity is used. Instead of a $50 \%$ prior (pre-test) probability of paternity assume that $\mathrm{P}(\mathrm{A})$ is 0.001 . This change to a low prior probability changes the results of Bayes formula.

$$
\mathrm{P}(\mathrm{A} / \mathrm{B})=[(1)(0.001)] /[(1)(0.001)+(0.01)(0.999)]=0.091
$$

The change in the prior probability results in only a slightly greater than $9 \%$ probability of paternity. This would not result in a presumption of paternity and would in most cases be viewed as strong evidence against paternity.

The drastic change in probabilities that occur with a change in prior (pre-test) probability highlights a serious error in statistical methodology known as the "principle of indifference" (Isaac, 1995). The principle of indifference is the error of assuming equality when the actual probabilities are unknown. In paternity testing the prior (pretest) probability is often assumed to be $50 \%$. This assumption is made because the true probability of paternity is not known. The mother claims that the man is the father. The man denies paternity. Because it is not known who is telling the truth a fifty-fifty split on the prior (pre-test) probability is made. However, this assumption about the prior (pre-test) probability $\mathrm{P}(\mathrm{A})$ may cause a highly misleading result as the above example illustrates.

It is well known by statisticians that the principle of indifference is a serious methodological error. If there is no knowledge concerning the prior (pre-test) probability it is better to make no assumptions rather than to assume a 50-50 chance based on ignorance. Such an error leads to an assumption of a high probability that is transformed by the mathematics to an even higher probability (Isaac, 1995). A man who is able to present credible evidence that he never previously met a woman should not be assigned a prior (pre-test) probability of $50 \%$ of being the father of her child. A $50 \%$ prior (pre-test) probability is an arbitrarily value set at an unreasonably high level. Conversely, if a woman is able to produce credible evidence that she was alone with a man in an isolated location during the time period of conception, she is entitled to a prior (pre-test) probability of greater than $50 \%$. This is needed because an inappropriately low prior (pre-test) probability can result in a misleadingly low test result. In both of the above cases the application of the principle of indifference lead to misleading results with tragic consequences.
Methodology

A Bayes probability computer program was written using Fortran 90. A total of 27 prior probabilities ranging from 0.001 to 0.90 were loaded into the program. A prior probability curve was plotted using the resulting probabilities. The Fortran 90 computer program appears below:

program one

implicit none

real:: $p(27)$

real::ba,ba1,pp,ppp

integer::i

open(1,file='probs',status='new')

$! p(27)$ is array of prior probabilities

!pp is prior probability

!ppp is resulting probability

!ba is $P(B / A)$ value of 1

!ba1 is $P\left(B / A^{\prime}\right)$ value of .01

$\mathrm{ba}=1.0$

ba $1=.01$

$\mathrm{p}(1)=.001$

$\mathrm{p}(2)=.002$

$\mathrm{p}(3)=.003$

$p(4)=.004$

$\mathrm{p}(5)=.005$

$\mathrm{p}(6)=.006$

$p(7)=.007$

$\mathrm{p}(8)=.008$

$p(9)=.009$

$\mathrm{p}(10)=.01$

$\mathrm{p}(11)=.02$

$\mathrm{p}(12)=.03$

$\mathrm{p}(13)=.04$

$\mathrm{p}(14)=.05$

$\mathrm{p}(15)=.06$

$p(16)=.07$

$\mathrm{p}(17)=.08$

$\mathrm{p}(18)=.09$

$\mathrm{p}(19)=.1$

$\mathrm{p}(20)=.2$

$\mathrm{p}(21)=.3$

$\mathrm{p}(22)=.4$

$\mathrm{p}(23)=.5$

$\mathrm{p}(24)=.6$

$\mathrm{p}(25)=.7$

$\mathrm{p}(26)=.8$

$\mathrm{p}(27)=.9$

!Call prior probabilities from array.

do $\mathrm{i}=1,27$

$\mathrm{pp}=\mathrm{p}(\mathrm{i})$

!Calculate resulting probability 
$\mathrm{ppp}=((\mathrm{ba}) *(\mathrm{pp})) /\left((\mathrm{ba})^{*}(\mathrm{pp})+(\mathrm{ba} 1)^{*}(1-\mathrm{pp})\right)$

write $(*, *)$ 'For a prior probability of:',pp

write $(*, *)$ 'the resulting probability is:',ppp

write $(*, *)$

write $(*, *)$

!Write results to external file.

write $(1,10)$ pp,ppp

10 format $(2 \mathrm{f} 8.4)$

end do

stop

end program one

The probabilities ranged from 0.091 to 0.9989 and were negatively skewed. (See Figure 1 on next page.) The distribution begins to rapidly grow when a 0.2 prior probability was loaded. The probability of 0.091 suggests that the male defendant had a 9\% chance that he was the father. Michigan's prior probability of 0.5 resulted in a $99 \%$ probability of paternity. However, this simulation was not based upon real world prior probabilities. Instead, all prior probabilities were arbitrarily loaded. The resulting probabilities, or establishing paternity, ranged from 9 to $99 \%$.

\section{Conclusion}

Genetic testing is seriously flawed when improper assumptions of prior probability of culpability are made. In the arena of paternity testing this has the great potential of assigning paternity to wrongly accused men. It also has the equally tragic potential of wrongly refuting paternity. In the arena of criminal law the same errors concerning assumptions about prior probability present the great risk of loss of life and liberty. The skillful legal advocate must know the potential of abuse of genetic testing and be prepared to expose the abuse when it occurs. The conditional probabilities employed by courts of law should be based upon objectivity rather than subjectivity.

The reader is invited to try various probabilities into the following Fortran 90 Bayes Probability program:

program two

implicit none

real::p

real::pp

write $(* *)$ 'Enter prior probability ( $\leq 3$ decimals):'

$\operatorname{read}(* *) \mathrm{p}$

$\mathrm{pp}=\left((1)^{*}(\mathrm{p}) /((1) *(\mathrm{p})+(.01) *(1-\mathrm{p}))\right.$

write $(*, *)$ 'The resulting probability is:',pp

stop

end program two

Endnotes

* In probability mathematics a 100 percent probability is 1. A 50 percent probability is 0.5 .

** $P\left(A^{\prime}\right)$ is the opposite of $P(A) . P\left(A^{\prime}\right)$ is equal to one minus $P(A)$ since in probability mathematics the sum of all the possibilities is one.

\section{References}

Borowski, E. J., \& Borwein, J. M. (1991). The Harper Collins dictionary of mathematics. New York: Harper Collins.

Freund, J. E. (1973). Introduction to probability. New York: Dover Publications.

Hummel. (1976). Joint AMA-ABA guidelines: Present status of serologic testing in problems of disputed parentage, 10 Fam LQ 247.

Isaac, R. (1995). The pleasures of probability. New York: Springer-Verlag.

Kilmer, J. B. (1993). Paternity and surrogate parenting agreements. In J. A. Curtis, S. Bassett, \& L. M. Collins (Eds.) Michigan family law (4 ${ }^{\text {th }}$ ed.). Ann Arbor, MI: The Institute of Continuing Legal Education. §17-14 and $\S 17.15$.

Motulsky, H. (1995). Intuitive biostatistics. New York: Oxford University Press.

Ventura, S. J., Martin, J. A., Curtin, S. C., Menacker, F., \& Hamilton, B. E. (2001, April 17). National vital statistics reports. Births: Final data for 1999, 49(1). Centers for Disease Control.

Wayne County Circuit Court (2001). Unpublished Data. Detroit, Michigan. 


\section{BAYES PRIOR PROBABILITY CURVE}

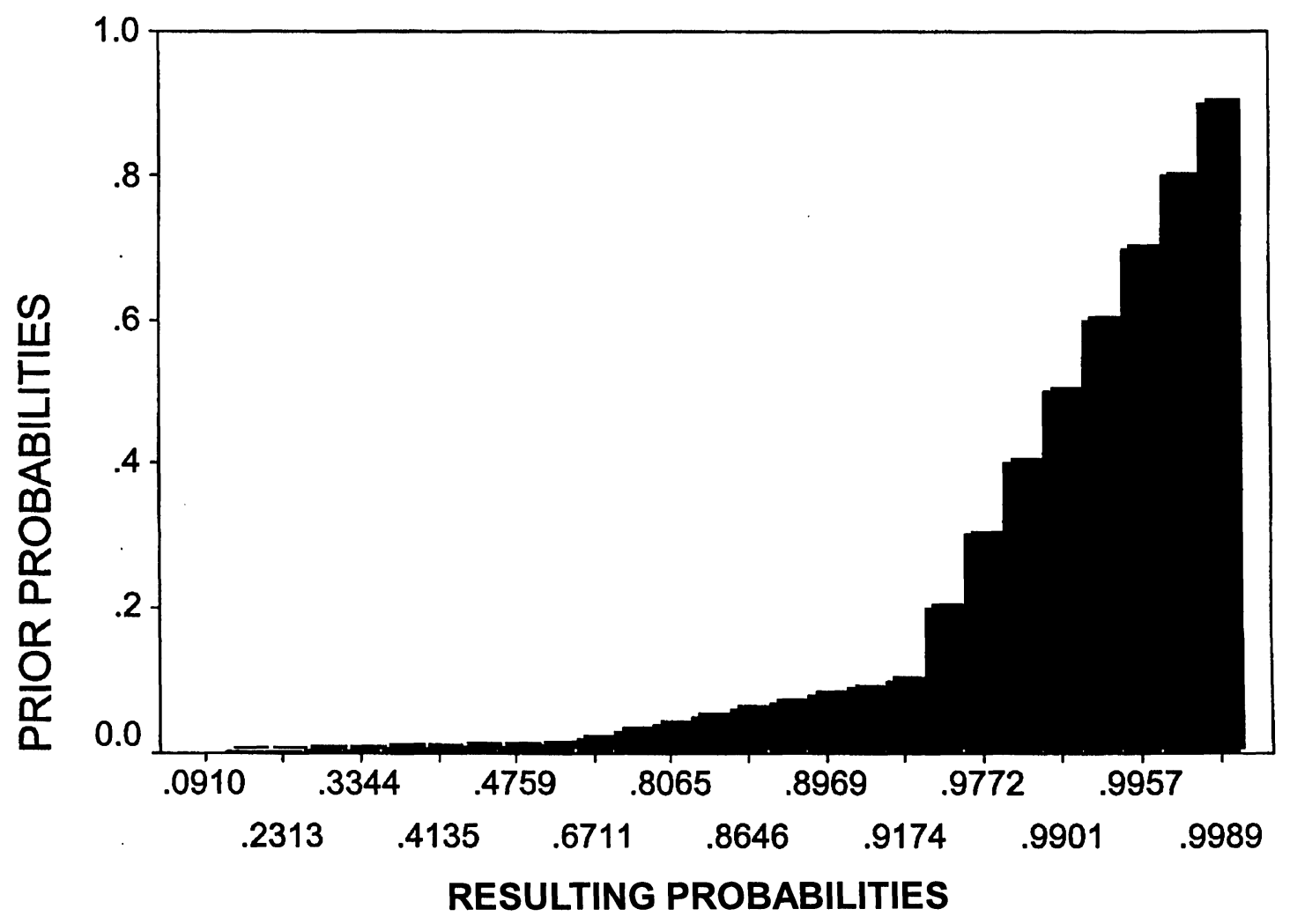

Figure 1. 


\title{
Two Methods To Estimate Homogenous Markov Processes
}

\author{
Ricardo Ocaña-Rilola \\ Escuela Andaluza de Salud Pública \\ Granada (Spain)
}

\begin{abstract}
Multi-state Markov processes have been introduced recently in Health Sciences in order to study disease history events. This sort of model have some advantages respect to traditional survival analysis, therefore they are an important line of research into stochastic processes applied to Epidemiology. However these types of models increase the complexity of analysis, even for simpler processes, and standard software is limited. In this paper, two methods for fitting homogeneous Markov models are proposed and compared.
\end{abstract}

Keywords: Stochastic processes, Markov processes, Breast cancer data

Introduction

Statistical models such Kaplan-Meier ${ }^{1}$, proportional haz$\operatorname{ards}^{2}$, or competing risks ${ }^{3}$ are used in survival analysis to study the distribution of the time elapsed between two events. An example would be the diagnosis date of a disease and the date of death of the patient. More recently, stochastic processes, and in particular Markov processes, ${ }^{4}$ have been introduced to analyze problems such as this. ${ }^{5}$ With this sort of model it is possible to analyze jointly the evolution of the patients through different states of their disease, obtaining a complete and detailed study on disease history. They are designated multistate models or multistate stochastic processes.

Aalen ${ }^{5}$ and Andersen and Borgan ${ }^{6} 7$ showed that survival analysis can be treated as a particular type of twostate Markov process where the transition intensity from the transient state live to the absorbent dead is the hazard function of the variable survival time. Similarly, competing risks models can be described as a stochastic process with a transient state live and several absorbing states, corresponding to the different causes of death. The advantage of including traditional survival analysis under the framework of Markov processes resides in the possibility of studying the detailed evolution of the patients through different states or stages before death, even when the exact transition time occurs is not known. ${ }^{8,9}$

Markov processes have been used in to study AIDS,,$^{10,11,12}$ use of contraceptives ${ }^{13}$ and cancer. ${ }^{9}$ These studies employed different methodologies depending on the

Ricardo Ocaña-Riola. Escuela Andaluza de Salud Pública, Campus Universitario de Cartuja, Apdo de Correos 2070, 18080 Granada, SPAIN, E-mail: ricardo@easp.es. This research was developed at the Escuela Andaluza de Salud Pública and financed by grant number IN92-D24255738 from the Programa Nacional de Formación de Personal Investigador en España of the Ministerio de Educación y Ciencia. The author thanks Jacques Estève for comments and suggestions. particular conditions of each case. In practice, it is often useful to use a homogeneous Markov process in order to model disease history data because generally they are easier to interpret. Moreover, the assumption that the process is homogeneous simplifies the methods used to fit the model.

In this context, the main objective of this paper is to propose and to compare two methods, both of which are computationally tractable, to estimate homogeneous Markov models in continuous time. To illustrate the methods described in the following sections, breast cancer data from Granada (South of Spain), are considered. These data are continuous with one absorbing state.

\section{Breast Cancer Data}

Breast cancer in Granada is the most frequent cancer in woman. ${ }^{14}$ It represents $19 \%$ of diagnosed cases of cancer, and $17 \%$ of the cancer deaths in women in the Granada province. The data originated from the Granada Cancer Registry population base, developed by the Andalusian School of Public Health and integrated in the European Network of Cancer Registries.

The study was carried out with 241 women with breast cancer diagnosed in $1985 / 86$, who received radical treatment and had a period free of symptoms. The followup ended on 31 of December 1990. Approximately 5\% of the cases were lost in follow-up. The 5-years Kaplan-Meier survival rate was $58 \%$ for these patients, with the $95 \%$ confidence interval between $52 \%$ and $65 \%$.

The data are represented by a three-state Markov model with two transient states and one absorbing. These states are state $1=$ "With Symptoms", state 2 = "Without Symptoms", and state $3=$ "Death". The transitions are represented in Figure 1.

Each patient was observed on the date of the diagnosis, the date of the last treatment, the date of the recurrence, where the date of the recurrence was defined as "the first attendance with evidence of relapse"15, and date of death. Thus, it is considered that at the start of followup all the patients are with symptoms (state 1), when the last treatment has been completed the patient is without 


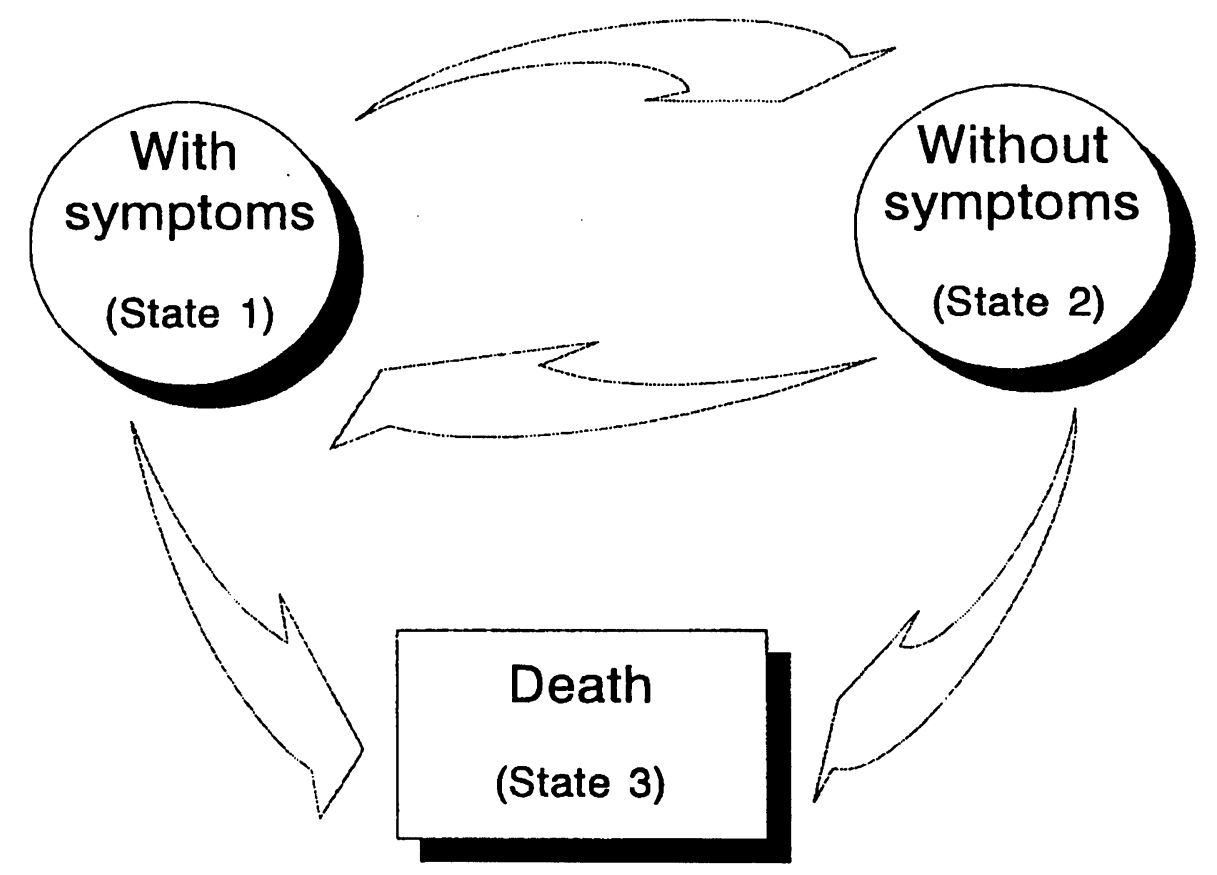

Figure 1. A three-state Markov process for analysing breast cancer

symptoms (state 2), on the date of the recurrence the patient is again considered with symptoms (state 1) and so on. Finally the patients who die are considered to be in state 3 at that date. Using these definitions, the observation is continuous (i.e., information on exact transition times between transient states is available).

Probabilities and Transition Intensities in a Markov Process

Let $E=\{1, \ldots, m\}$ be a state space consisting of $m$ disease states in which patients make independent transitions. Let $X(t)$ be the state occupied by an individual at time $t$ for $0 \leq t$ and $\{X(t): t \geq 0\}$ a collection of random variables that define a stochastic process. In this context, if the process is Markov, we denote $p_{i j}(s, t)$ the additional probability that the process shall be in state $j$ at time $t$ given that it was at time $s$ in state $i$, for $0 \leq s \leq t$.

Homogeneous processes are the simplest form of Markov models. In this case, assuming a stationary process, the transition probabilities satisfy

$p_{i j}(s, s+u)=p_{i j}(0, u)=p_{i j}(u)$ for $u, s \geq 0$, with

$\mathrm{p}(\mathrm{U})=\left(\mathrm{p}_{\mathrm{ij}}(\mathrm{u})\right)$ the $m x m$ transition probability matrix.

The transition intensities for the process, $q_{i j}$, are defined by

$$
\begin{array}{ll}
p_{i j}(h)=q_{i j} h+o(h) & i \neq j \quad i, j \in E \\
1-p_{i i}(h)=q_{i} h+o(h) & i \in E
\end{array}
$$

where $O(h)$ is an infinitesimal that satisfies $\frac{O(h)}{h} \rightarrow 0$ when $h \rightarrow 0$ and $Q=\left(q_{i j}\right)$ is the $m \times m$ transition intensity matrix satisfying $q_{i}=-q_{i i}=\sum_{j \neq l} q_{i j}$ for $i, j \in E$.

The relationship between $P(u)$ and $Q$ is

$$
P(u)=\exp (Q u)=\sum_{S=0}^{\infty} Q^{s} \frac{U^{s}}{S !}
$$

Let $T_{i}$ be the random variable "time elapsed in the state $i \in E$ ". It is known that this variable is distributed according to an exponential law with parameter $q_{i}$ and average $E\left[T_{i}\right]=\frac{1}{q_{i}}$.

Likelihood Function

Suppose that for each subject $\mathrm{c}$ in a cohort $(c=1$, 
..., N), $\mathrm{m}_{\mathrm{c}}$ transitions between states has been observed at tion times

$$
W_{c O}<W_{c l}<\ldots<W_{c m_{c}}
$$

If $m_{c}$ transitions has been observed for each subject ( $c=1$, $\ldots, N)$, then there is a set of $N\left(m_{c}+1\right)$ times in which there was a jump from a state to another. These times can be ordered to form a partition of the time given by $N\left(m_{c}+1\right)$ cut points in which at least a transition between states has been observed. Let $\rho$ be such a partition with

$\wp=\left\{s_{1}, \ldots, s_{N\left(m_{c}+1\right)}\right\}$. To simplify the notation we denote $r$ $=N\left(m_{c}+1\right)-1$, so $\rho=\left\{s_{1}, \ldots, s_{r+1}\right\}$

There are $r+1$ cut points that form $r$ intervals with length $u_{k}=S_{k+1}-S_{k}, k=1, \ldots, r$. If $n_{i j k}$ denotes the number of patients who were in the state $i$ in the time $S_{k}$ and are in the state $j$ at the instant $S_{k+1}$, the likelihood function is

$$
L=\prod_{k=l}^{r}\left(\prod_{i=l}^{m} \prod_{j=l}^{m} p_{i j}\left(U_{k}\right)^{n_{j k}}\right)
$$

Two Methods to Estimate Intensity Matrix

This section shows how matrix $Q$ can be estimated when homogeneous Markov processes in continuous time are used. Assume that transition intensities depend on a beta vector of unknown parameters with components $\beta_{1}, \ldots, \beta_{b}$. That is, $q_{i j}=q_{i j}(\beta), i, j \in E$, but to simplify the notation write $p_{i j}(U)$ and $q_{i j}$ instead of $p_{i j}(U, \beta)$ and $q_{i j}(\beta)$.

\section{Kalbfleisch and Lawless Method}

By using $\varnothing$ partition, the likelihood function in (4) can be seen as an extension of the methodology of Kalbfleisch and Lawless ${ }^{8}$ when observations are continuous and transitions between states are observed at different times. In order to obtain the Maximum Likelihood Estimate (MLE) for $\beta$, the first and second partial derivatives of $\ln (L)$ are required. The Kalfleisch and Lawless method uses a quasi-Newton algorithm for this purpose in which it is necessary to compute complex calculations for every interval of the partition. ( See Appendix. )

\section{Approximate Method}

To estimate matrix $\mathrm{Q}$, an algorithm is proposed that consists of modeling transition probabilities with a simple function that depends on the transition intensities. From expressions (1) and (2), in a homogeneous Markov process the transition probabilities can be approximated by $p_{i j}(h)=q_{i j} h$ and $p_{i i}(h)=1-q_{i} h$ when $h \rightarrow 0$. If there is a sufficiently fine $\wp$ partition composed of very small intervals, $U_{k}$, whose lengths tend to be zero, the approxima-

$$
\begin{aligned}
& p_{i j}\left(u_{k}\right)=q_{i j} u_{k} \quad i, j \in E \\
& p_{i i}\left(u_{k}\right)=1-q_{i} u_{k} \quad k \in E
\end{aligned}
$$

can be used for all $k=1, \ldots, r$. Usually, $q_{i j}=\exp \left(\beta_{i j}\right)$ can be taken in order to avoid problems in the range of the estimates upon implementing the algorithm in a computer. The likelihood function given in (4) simplifies to

$$
\left.L=\prod_{k=1}^{r}\left(\prod_{i=l}^{m}\left(1-u_{k} \sum_{v \neq i}^{m} \exp \left(\beta_{i v}\right)\right)^{n_{j k}} \prod_{j \neq i}^{m}\left(u_{k} \exp \left(\beta_{i j}\right)\right)\right)^{j, k}\right)
$$

using this approximation.

The MLE for $\beta$ can be obtained using traditional partial derivatives of $\ln (L)$. In this case, these partial derivatives are calculated easily. The algorithm for fitting the model is iterative and it will asymptotically approach the best fitting values of the parameters. The convergence criterion, $\epsilon$, is the maximum relative change in all the parameters. The estimated transition probability matrix is $\hat{p}(u)=\exp (\hat{Q} u)$ where $\hat{Q}=\left(\hat{a}_{i j}\right)=\left(\exp \left(\hat{\beta}_{i j}\right)\right)$.

\section{Selection of an Initial Estimate}

As mentioned above, the algorithms for fitting the model are iterative, and will asymptotically approach the best solution. However, a serious problem in any algorithm is the selection of an appropriate initial value $\beta^{(0)}$. The calculations used to obtain an estimate of $\beta$ are complex, particularly in the Kalbfleisch and Lawless method, and the time invested in each iteration is high. A poor choice of the initial value will increase enormously the time necessary to obtain a final estimate and even could cause the algorithm does not to converge.

An initial estimate of the transition intensities based on the observed data can be obtained as follows:

From expression (1), in each interval $U_{k}$ it is obtained an approximation of the form

$$
q_{i j}\left(u_{k}\right)=\frac{p_{i j}\left(u_{k}\right)}{u_{k}} \quad i \neq j, i, j \in E
$$

If the value $p_{i j}\left(u_{k}\right)$ is estimated $\frac{n_{i j k}}{n_{i . k}}$, an initial estimate will be obtained for the transition intensity in each one of the intervals given by

$$
q_{i j}\left(u_{k}\right)=\frac{n_{i j k}}{n_{i, k} v_{k}} \quad i \neq j, i, j \in E
$$

where $k=1, \ldots, r$. Because the transition intensities are independent of the time, consider the means 


$$
q_{i j}^{(0)}=\frac{\sum_{k=1}^{r} q_{i j}\left(u_{k}\right)}{r} \quad i \neq j
$$

and the initial vector $\beta^{(0)}=\left(\log \left(q_{i j}^{(0)}\right)\right)_{i, j^{\prime}} \quad i \neq j$.

Figure 2 shows this method based on the breast cancer data mentioned above, where the dashed line represents the initial values, $q_{12}^{(0)}, q_{13}^{(0)}, q_{21}^{(0)}, q_{23}^{(0)}$, given respectively by $0.37,0.24,0.10,0.02$. The circles represent the values given by $(6)$, where $U_{k}$ units are years.

Two Methods for Breast Cancer Analysis

To analyze the breast cancer data, consider the transition intensity matrix

$$
Q=\left[\begin{array}{ccc}
-\left(a_{12}+a_{13}\right) & a_{12} & a_{13} \\
q_{21} & -\left(a_{21}+q_{23}\right) & q_{23} \\
0 & 0 & 0
\end{array}\right]
$$

The transition 2-3 corresponds to death from other causes which can not be controlled. Thus, the entry $q_{23}$ is included in the intensity matrix.

After building the $\delta$ partition, 35 different intervals were found between 0.002 and 0.260 years. The mean was 0.071 years. The $25^{\text {th }}, 50^{\text {th }}$, and $75^{\text {th }}$, percentiles were $0.026,0.049$ and 0.075 , respectively. These results show a sufficiently fine partition where most intervals (75\%) extended less than 0.075 years.

Table 1 shows a comparative analysis of the Kalbfleisch-Lawless vs the approximate method. The estimates obtained are very similar. The criterion of convergence was $\epsilon=0.00001$ and the implementation in a computer was accomplished through functions written for $S$ PLUS. ${ }^{16}$

Figure 3 shows the difference between estimated transition probability matrices from each method for each interval of length $u$. These differences have been calculated using Error $=\left\|\hat{P}_{1}(u)-\hat{P}_{\| 1}(u)\right\|_{2}$, where $\hat{P}_{I}(u)$ and $\hat{P}_{\| \prime}(u)$ are the estimated transition probability matrices from Kalbfleisch-Lawless and Approximate method respectively.

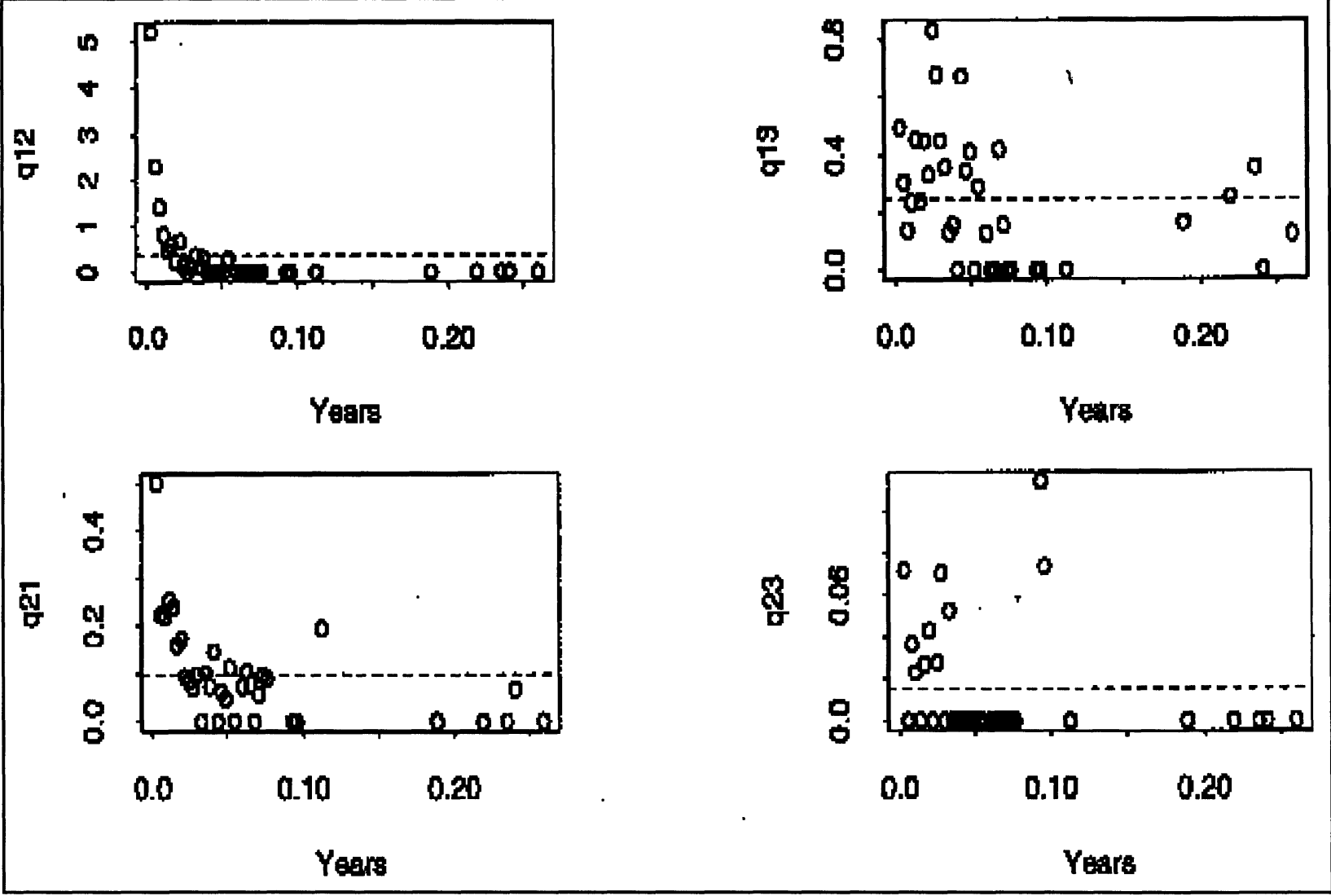

Figure 2. Initial estimation for transition intensities. Dashed lines is the mean of all transition intensities, used as initial value in iterative methods 


\section{RICARDO OCAÑA-RIOLA}

Table 1. Homogeneous Markov Models for Breast Cancer Data.

\begin{tabular}{lllll} 
& \multicolumn{2}{c}{ Kalbfleisch-Lawless } & & \multicolumn{2}{c}{ Approximate } \\
\cline { 2 - 5 } Transition intensities & Estimate & Standard error & Estimate & Standard error \\
\hline $\mathrm{q} \_\{12\}$ & 1.1653 & 0.0049 & 1.0738 & 0.0044 \\
$\mathrm{q} \_\{13\}$ & 0.2929 & 0.0024 & 0.2729 & 0.0023 \\
$\mathrm{q} \_\{21\}$ & 0.1235 & 0.0008 & 0.1175 & 0.0008 \\
$\mathrm{q} \_\{23\}$ & 0.0150 & 0.0003 & 0.0153 & 0.0003 \\
\hline
\end{tabular}

If in the breast cancer study, the approximate algorithm is used instead of the method of Kalbfleisch and Lawless, the maximum error is 0.036 . The estimated intensity matrix is similar by using either algorithm. However the approximate method is less costly computationally.

Mean sojourn times in each state $i=1,2$ were obtained from $1 / \hat{q}_{i}$, that is, 0.69 years ( 8.2 months) and 7.22 years, respectively, for the mean sojourn time with and without symptoms.

Figures 4 shows the estimated transition probabilities between states, obtained from $\hat{P}(u)=\exp (\hat{Q} u)$. The 5 -years probability of death from with symptoms and without symptoms state was 0.30 and 0.15 respectively.
The 5 -years survival probability was 0.55 . Note that the 5 years Kaplan-Meier survival rate was $58 \%$ for these patients.

\section{Conclusion}

Multistate Markov models offer some advantages on traditional survival models for studying disease history events. Using this sort of model, it is possible to estimate the proportions of patients who shall be in each disease state in the future. Therefore, highly relevant information for health planning services can be obtained.

In this article, the partition of time used was for

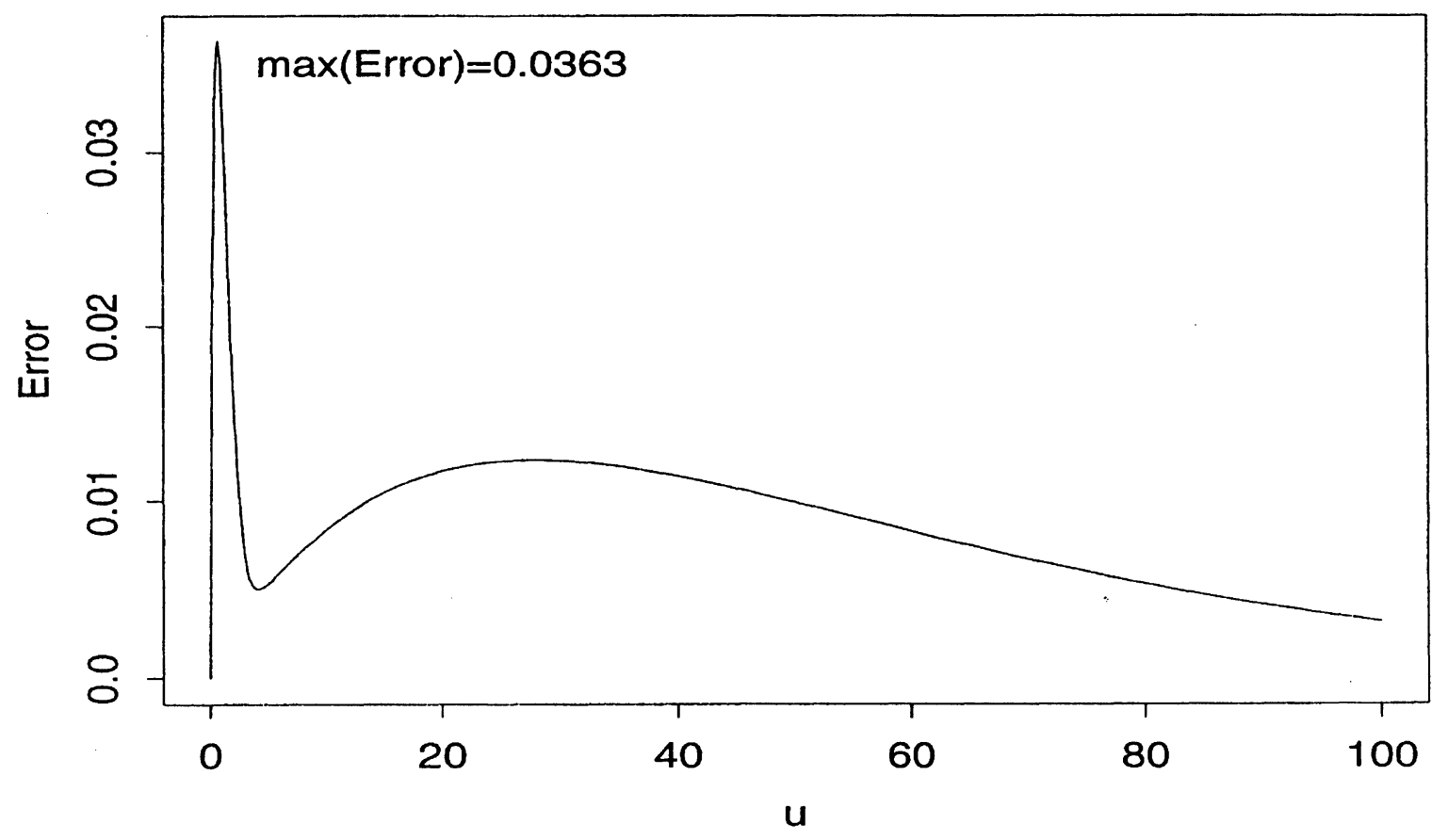

Figure 3. Differences among the transiton probability matrices obtained from Kalbfleisch-Lawless algorithm and Approximation method. These differences have been calculated using Error $=\left\|\hat{P}_{I}(u)-\hat{P}_{\| \prime}(u)\right\|_{2}, 0<u<100$ 


\section{WITH SYMPTOMS}

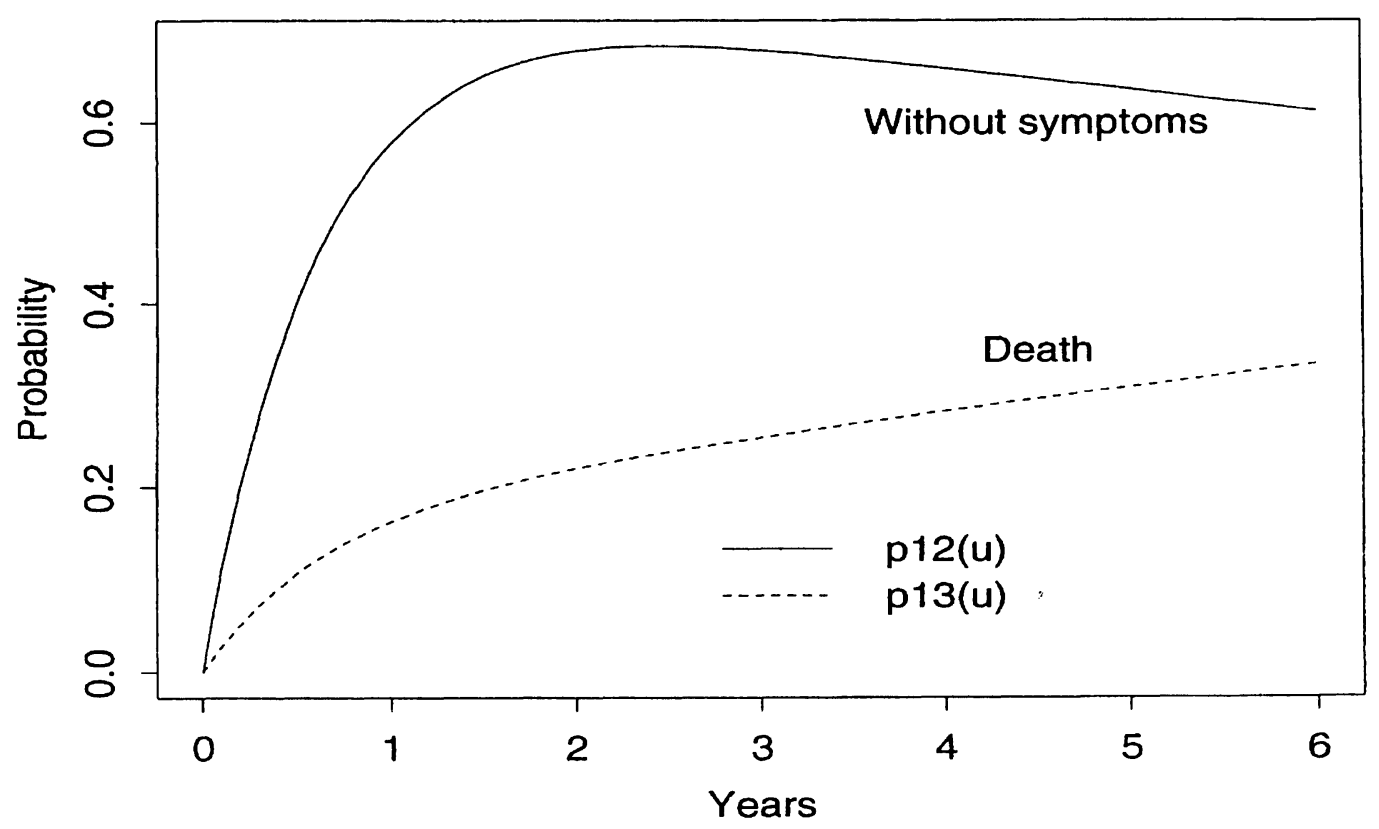

Figure 4. Estimated transition probabilities from with symptoms state to without symptoms state and death states

continuous observations. In this situation, the methodology proposed by Kalbfleisch and Lawless has been extended, and an approximate method was proposed in order to estimate intensity matrix. If the intervals in which patients are observed are sufficiently small, the KalbfleischLawless algorithm can be simplified using the approximate method, obtaining similar MLEs.

In survival studies, the use of models that incorporate covariates permits further analysis of patients' survival. When multistate models are used, it is also possible to study the effect of covariates on different transitions between states in patients disease history. Some authors have worked on the introduction of covariates in multistate processes and particularly in homogeneous Markov processes. ${ }^{8,17}$ However they mention the increased complexity of analysis in this sort of model, where an additional problem is the shortage of standard software.

In spite of these problems, the introduction of covariates in stochastic processes are required to explain the effect of these factors on disease history events. In this case, a homogeneous Markov model with covariates would be an interesting option. An advantage of the approximate method proposed in this paper is the ease with which covariates can be incorporated in the likelihood function, therefore the study of prognostic factors is not difficult. Interesting results for breast cancer survival and breast cancer recurrence using the approximate method with TNM variables have been found. ${ }^{18}$

Computing approximate MLEs replaces the intensity transition matrix with a first order Taylor series. In this article, an empirical comparison showed that, in some cases, it can be a good approximation. Similar conclusions in other applications, so the question of when the Taylor expansion is liable to produce accurate approximations and on developing a diagnosis for examining the accuracy of the approximation is of interest. The results obtained, therefore, could be an interesting finding in applying Markov processes to Health Sciences and Epidemiology.

\section{References}

1. Kaplan, E. L., \& Meier, P. (1958). Non parametric estimation from incomplete observations. $J$. Am. Stat. Assoc, 53, 457-481.

2. Cox, D. R. (1972). Regression models and lifetime (with discussion). J. Roy. Stat. Soc. B. Met., 34, 187-220.

3. Prentice, R. L., Kalbfleisch, J. D., Peterson Jr., A. V., Flournoy, N. S., Farewell, V. T., \& Breslow, N. (1978). The analysis of failure times in the presence of competing risks. Biometrics, 34, 541-554. 
4. Chiang, C. L. (1968). An introduction to stochastic processes and their applications. New York: John Wiley \& Sons.

5. Aalen, O. O. (1978). Non-parametric estimation of partial transition probabilities in multiple decrement models. Ann. Stat., 6, 534-545.

6. Andersen, P. K. (1988). Multistate models in survival analysis: A study of nephropathy and mortality in diabetes. Stat. Med., 7, 661-670.

7. Andersen, P. K, \& Borgan, O. (1985). Counting process models for life history date: A review (with discussion). Scand. J. Stat., 12, 97-158.

8. Kalbfleisch, J. D., \& Lawless, J. F. (1985). The analysis of panel data under a Markov assumption. J. Am. Stat. Assoc., 80, 863-871.

9. Kay, R. A. (1986). Markov model for analyzing cancer markers and disease states in survival studies. Biometrics, 42, 855-865.

10. De Gruttola, V., \& Lagakos, S. W. (1989). Analysis of doubly-censored survival date, with application to AIDS. Biometrics, 1989, 45, 1-11.

11. Frydman, H. A. (1992). Nonparametric estimation procedure for a periodically observed threestate Markov process, with application to AIDS. J. Roy. Stat. Soc. B Met., 54, 853-866.
12. Mariotto, A. B., Mariotti, S., Pezzotti, P., Rezza, G., \& Verdecchia, A. (1992). Estimation of the Acquired Immunodeficiency Syndrome incubation period in intravenous drug users: a comparison with male homosexual. Am. J. Epidemiol., 135, 428-437.

13. Islam, M. A. (1994). Multistate survival models for transitions and reverse transitions: an application to contraceptive use date. J. Roy. Stat. Soc. A Sta., 157, 441-455.

14. Martínez, C. (1994). El cáncer en Granada: incidencia y mortalidad 1988-1990. Granada: EASP.

15. WHO. (1980). WHO handbook for reporting results of cancer treatment, WHO offset publication No. 48., Geneva.

16. S-Plus. (1998). S-PLUS 4 programmer's guide. Seattle: MathSoft, Inc.

17. Pastorello, S. (1993). La mobilità nel mercato del lavoro: un analisi econometrica con osservacioni in tempo discreto. Statistica, 53, 185-206.

18. Ocaña-Riola, R., Sánchez-Cantalejo, E., \& Martínez-García, C. (2000). Homogeneous Markov processes for breast cancer analysis. Unpublished Technical Report.

Appendix

Summary of the Kalbfleisch and Lawless Method

The Newton-Raphson method is usually applied in order to obtain MLE for $\beta$. In this case, first and second partial derivatives of $\ln (L)$ with respect to each unknown parameter $\beta_{h}(h=1, \ldots, b)$ are required, that is

$$
\begin{aligned}
& S_{h}(\beta)=\frac{\partial \ln (L)}{\partial \beta_{h}}=\sum_{k=1}^{r} \sum_{i=1}^{m} \sum_{j=1}^{m} n_{i j k} \frac{\frac{\partial p_{i j}\left(u_{k}\right)}{\partial \beta_{h}}}{p_{i j}\left(u_{k}\right)} \\
& J_{h r}(\beta)=\frac{\partial^{2} \ln (L)}{\partial \beta_{h} \partial \beta_{r}}=\sum_{k=1}^{r} \sum_{i=1}^{m} \sum_{j=1}^{m} n_{i j k}\left(\frac{\frac{\partial^{2} p_{i j}\left(u_{k}\right)}{\partial \beta_{h} \partial \beta_{r}}}{p_{i j}\left(u_{k}\right)}-\frac{\frac{\partial p_{i j}\left(u_{k}\right)}{\partial \beta_{h}} \frac{\partial p_{i j}\left(u_{k}\right)}{\partial \beta_{r}}}{p_{i j}\left(u_{k}\right)^{2}}\right)
\end{aligned}
$$

We obtain then a vector $S(\beta)=\left(S_{h}(\beta)\right)_{b \times l}$ of dimension $b$ and a $b \times b$ squared matrix $J(\beta)=\left(J_{h r}(\beta)\right)_{b \times b}$. However this method can be simplified using a quasi-Newton method that employs, instead of $J_{h r}(\beta)$, the information matrix $M(\beta)=E[-J(\beta)]$ with elements

$$
M_{h r}(\beta)=\sum_{k=1}^{r} \sum_{i=l}^{m} \sum_{j=l}^{m} \frac{\partial p_{i j}\left(u_{k}\right)}{\partial \beta_{h}} \frac{\partial p_{i j}\left(u_{k}\right)}{\partial \beta_{r}} \frac{n_{i, k}}{p_{i j}\left(u_{k}\right)}
$$


where $n_{i . k}=\sum_{j \in E} n_{i j k}$.

If $\beta^{(0)}$ is an initial estimate of $\beta$ we can built the sequence

$$
\beta^{(n+1)}=\beta^{(n)}+M\left(\beta^{(n)}\right)^{-1} S\left(\beta^{(n)}\right)
$$

which converges to the MLE for $\beta$, assuming that $M\left(\beta^{(n)}\right)$ is nonsingular in each iteration. 


\section{BRIEF REPORTS \\ Two-Sided Equivalence Testing Of The Difference Between Two Means}

\author{
R. Clifford Blair \\ Department of Epidemiology and Biostatistics \\ College of Public Health, \& \\ Jaeb Center For Health Research \\ University of South Florida
}

\author{
Stephen R.Cole \\ Department of Epidemiology \\ Bloomberg School of Public Health \\ The Johns Hopkins University
}

Studies designed to examine the equivalence of treatments are increasingly common in social and biomedical research. Herein, we outline the rationale and some nuances underlying equivalence testing of the difference between two means. Specifically, we note the odd relation between tests of hypothesis and confidence intervals in the equivalence setting.

Keywords: Equivalence, Statistical inference, Hypothesis testing, Confidence intervals

\section{Introduction}

Studies designed explicitly to examine the equivalence of two (or more)treatments are increasingly common in social and biomedical research. In such studies the null hypothesis maintains that the difference between treatments is at least of some specified magnitude, while the alternative specifies a lesser difference. Some consequences of stating hypotheses in this fashion are not obvious. For example, intention-to-treat analyses do not carry the same robust interpretation when there is noncompliance (Robins, 1988),random measurement error may bias toward rejecting the null (Jones, et al.,1996), and significantly larger sample sizes may be required (Makuch \& Johnson, 1986). In order to understand these and other consequences of equivalence testing one must first have an understanding of the basic tenets underlying the methodology.

The purpose of this report is to briefly outline the rationale and some of the nuances underlying equivalence testing of the difference between two means. For simplicity the context involves the difference between means but the explanations afforded apply with equal force to tests of the difference between two adjusted means as might be obtained from a two group ANCOVA analysis. Topics to

R.Clifford Blair is Professor and Interim Chair, Department of Epidemiology and Biostatistics, College of Public Health MDC-56, University of South Florida, 13201 Bruce B.Downs Blvd., Tampa FL, 33612-3805. E-mail: cblair@hsc.usf.edu. His areas of expertise are in computerintensive statistical methods, multiple end point analysis, and control of family-wise error. Stephen R.Cole is Assistant Research Professor, Department of Epidemiology, The Johns Hopkins School of Hygiene and Public Health. He specializes in longitudinal data analysis, computer-intensive applications, and testing issues in medicine. be covered include, establishing equivalence by means of hypothesis tests, establishing equivalence by means of one and two-sided confidence intervals, power considerations, and efficacy tests.

Establishing 2-Sided Equivalence by a Pair of Nested Hypothesis Tests

Two-sided equivalence tests (ET) of two means begin with the establishment of an equivalence interval defined by the constants $\mathrm{c}$ and $-\mathrm{c}$. Equivalence is declared if it can be established that the value $\mu_{1}-\mu_{2}$ lies within the interval (-c,c).In order to make this determination, two onesided tests of significance must be carried out as depicted in Figure 1.In this figure $-\mathrm{c}$ and $\mathrm{c}$ are set to -1 and 1 units, respectively. The curves depict the sampling distribution of $\bar{X}_{1}-\bar{X}_{2}$ under each of the null hypotheses to be tested. In order to establish equivalence the null hypothesis $\mu_{1}-\mu_{2} \geq 1$ must be rejected in favor of the alternative $\mu_{1}-\mu_{2}<1$ in order to establish the fact that $\mu_{1}-\mu_{2}$ is below the upper bound of the equivalence interval. Similarly, a second test is necessary to show that $\mu_{1}-\mu_{2}$ is greater than the lower bound of the equivalence interval.

Notice that both tests must attain significance in order to declare equivalence. Notice also that both null hypotheses cannot be true. Therefore, the Type I error rate will be determined by the critical region of only one of the two curves. If $\mu_{1}-\mu_{2}=1$, the probability of a type I error $(\alpha)$ is the shaded critical region of the right hand curve.

Also of interest is the fact that the nominal level of the test establishes an upper bound for Type I errors rather than an explicit level. This derives from two factors: (1) If the null value exceeds c (e.g., 2 units) or is less than -c, the Type I error rate will necessarily be decreased. This is common to standard (i.e., efficacy) one-sided tests and will not be discussed here. (2) In the event that the standard error 


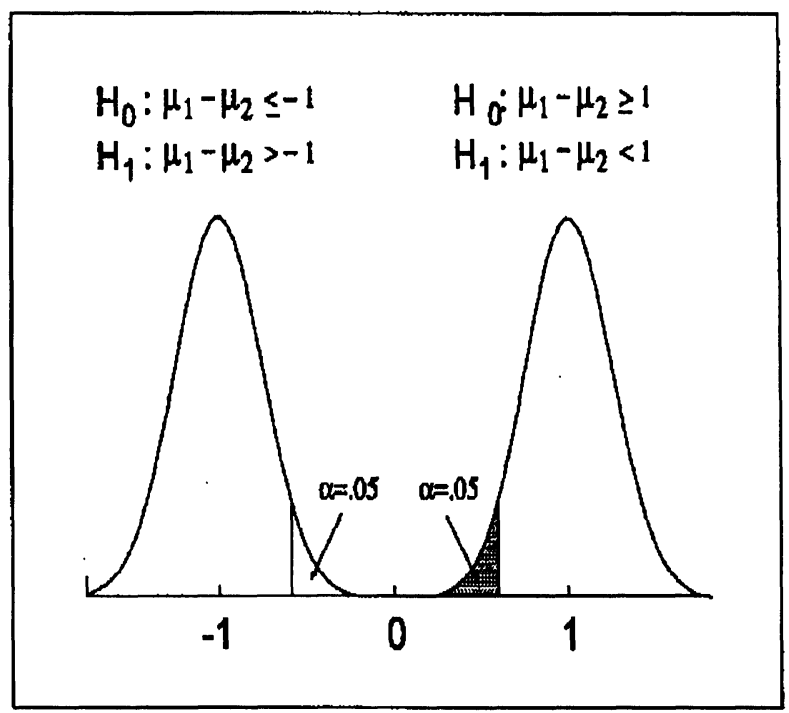

Figure 1: Hypothesis tests to establish quivalence of two means.

of the test statistic (SE) is too large and/or the length of the equivalence interval is too small, the two critical regions will overlap to a significant degree thereby producing a conservative test. This situation is depicted in Figure 2 where the Type I error rate is represented by the gray shaded area in the critical region of the right hand curve. In the extreme, the two critical regions may completely overlap so that the Type I error rate will be zero.

Establishing Equivalence by Means of One and Two-Sided Confidence Intervals

As with standard efficacy tests (Cox \& Hinkley, 1974), there is a relationship between tests of hypotheses and confidence intervals used to establish equivalence. The relationship for equivalence is somewhat different from that for efficacy, however.

As depicted in Figure 3, the distance between the hypothesized null value and the beginning of the critical region is (approximately) 1.65 standard errors (SE). Because the upper end of a one-sided 95 percent confidence inter$\mathrm{val}$ is given by $\mathrm{U}=\mathrm{TS}+1.65 \mathrm{SE}$ where TS is the test statistic $\left(\bar{X}_{1}-\bar{X}_{2}\right.$ in the present case), it follows that any TS in the critical region of the right hand curve will produce a value of $U$ that is less than $c$ (or 1 in this sample).This situation is depicted in panel $\mathrm{A}$ of the figure. On the other hand, a value of $U$ that is greater than $c$ implies that TS is not in the critical region as shown in panel $\mathrm{B}$. Thus, a value of $\mathrm{U}$ less than $\mathrm{c}$ implies rejection of $\mathrm{H}_{0}$, while a value greater than $\mathrm{c}$ implies a failure to reject. The same logic applies to the lower end of a one-sided 95 percent confidence interval and a test of hypothesis carried out on the lower curve. Thus, noting that neither of two one-sided 95 percent confidence intervals overlap $\mathrm{c}$ or $-\mathrm{c}$ is equivalent

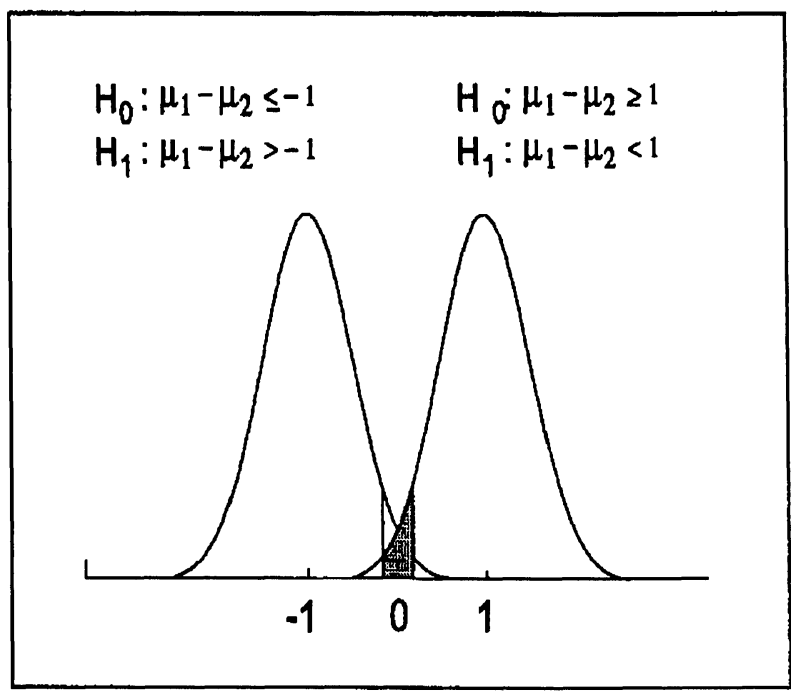

Figure 2: Conservative test of significance.

to noting that both hypothesis tests are significant.

It is obvious that a more direct assessment can be carried out by inspection of a two-sided 90 percent confidence interval where the lower and upper limits are constructed by subtracting and adding $1.65 \mathrm{SE}$ to TS. In this case, significance is established by noting that the 90 percent confidence interval is completely contained in the interval $(-c, c)$. In general, one would use a two-sided $100(1-2 \alpha)$ percent confidence interval to establish significance at the $\alpha$ level. Although convenient, reporting use of this methodology can lead to confusion on the part of readers not familiar with equivalence methods.

A statement of the form "A two-sided significance test was carried out at the .05 level by means of a 90 percent confidence interval " is almost certain to cause confusion. An equivalent statement concerning two one sided 95 percent confidence intervals seems slightly more palatable.

\section{Power Considerations}

For the present situation, power is defined as the probability of attaining significance when $\mu_{1}-\mu_{2}$ is contained in the interval (-c, c). Equivalently, power may be defined as the probability that a properly constructed confidence interval will be completely contained in the interval (-c, c) when $\mu_{1}-\mu_{2}$ is in the interval (-c, c). Power calculations are usually carried out under the assumption that $\mu_{1}-\mu_{2}$ $=0$, although other values may be chosen when the research situation warrants.

The shaded area of the middle distribution in Figure 4 depicts power of .95 for a two-sided equivalence test. Notice that the probability of failing to obtain a significant result in this situation is the unshaded portion in the tails 
Figure 3: Relationship of a confidence interval to a test of hypothesis.

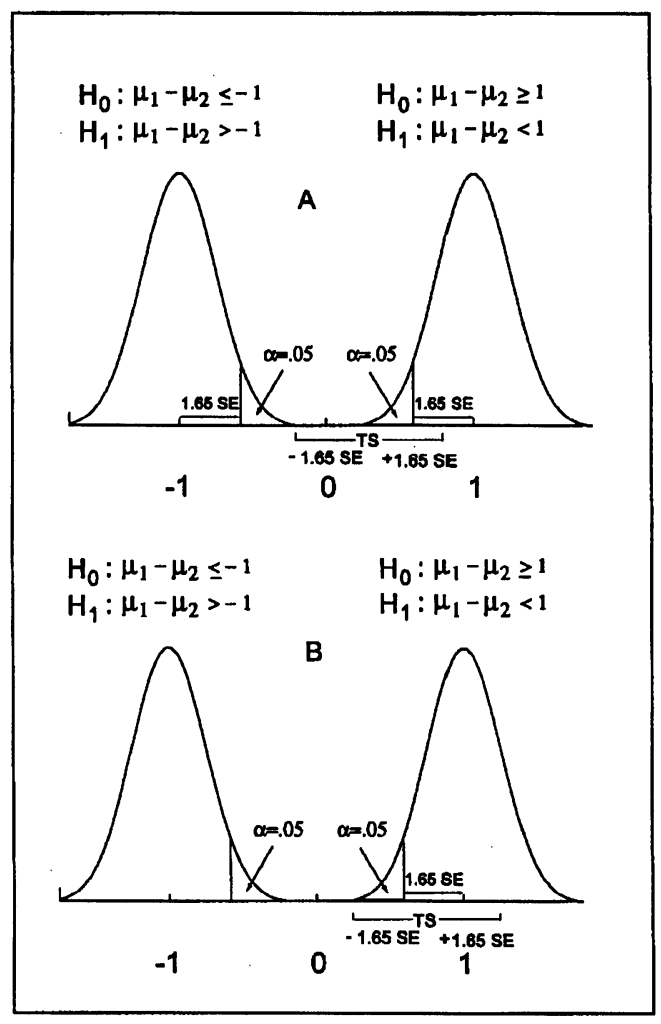

Figure 4: Power of a two-sided equivalence test.

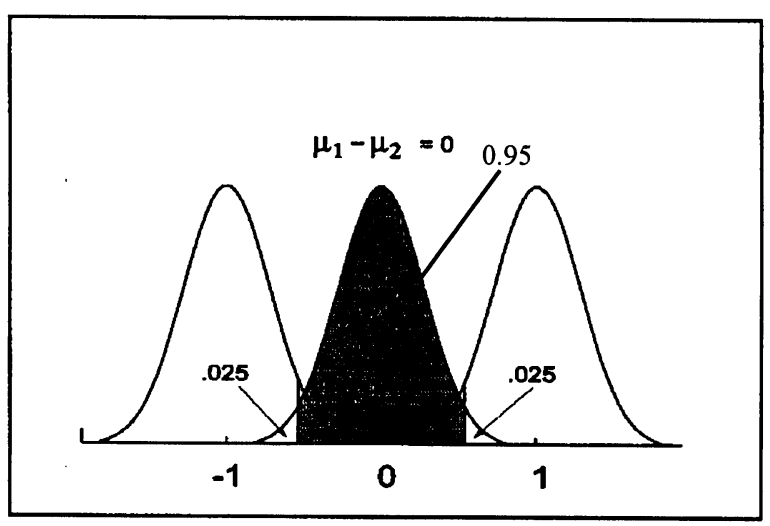

of the middle distribution. Because power is .95 , the unshaded area is .05 with .025 allocated to each tail. Power for equivalence tests is often lower than that for efficacy tests of the same sample size due to the typically smaller effect sizes associated with equivalence tests relative to efficacy tests.

\section{Efficacy Tests}

Inherent in every equivalence test is an efficacy test of the null hypothesis $\mu_{1}-\mu_{2}=0$. Look again at the middle
Figure 5: Using 95 percent CI to establish significance.

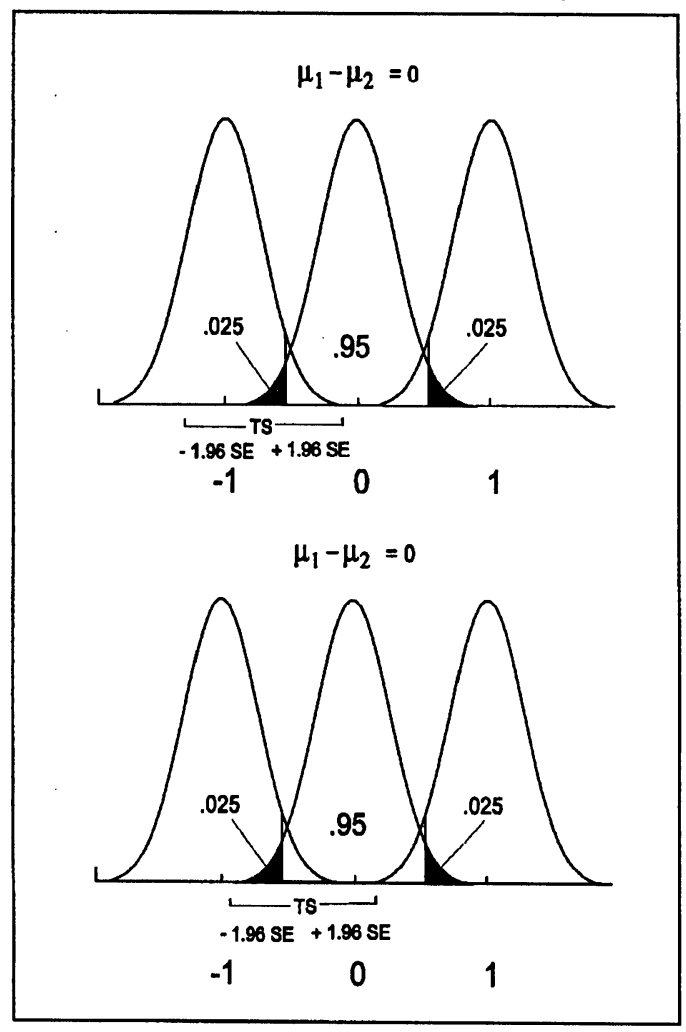

distribution in Figure 4. This is the sampling distribution of the TS when $\mu_{1}-\mu_{2}=0$ which in turn is the usual null distribution when testing the hypothesis $\mu_{1}-\mu_{2}=0$. The probability of rejecting the efficacy test when there is no difference is one minus the power of the equivalence test. Thus, when establishing the power of the equivalence test one is also establishing the Type I error rate of the efficacy test. This leads to the following result: If significance is attained with the equivalence test, one can conclude that the treatments are equivalent with probability of error being $\alpha$ (usually .05). When non-significance is attained with the equivalence test one can state that there is a significant difference with probability of error being one minus the power of the equivalence test. By setting power at .95 this error rate becomes the traditional .05 . Note that non-significance of the equivalence does not mean non-equivalence ( $\mu_{1}-\mu_{2}$ may well be in the equivalence interval), but does mean that there is some, albeit possibly practically irrelevant, difference in the treatments.

One last point should be noted. If the significanc level for the equivalence test is established at .025 and power at .95 the following results. (1) Significance can be determined by means of a two-sided 95 percent confidence interval. (2) If this interval is completely contained in $(-c, c)$, 
equivalence is established with probability of error being.025. (3) If this interval is not completely contained in $(-c, c)$, then a significant difference between $\mu_{1}$ and $\mu_{2}$ is established with probability of error being .05. (4) These determinations can also be made by noting whether or not zero is in the 95 percent confidence interval. (See Figure 5.) (5) The 95 percent confidence interval can be used to estimate $\mu_{1}-\mu_{2}$ in the usual manner.

\section{References}

Cox, D. R., \& Hinkley, D. V. (1974). Theoretical statistics. London: Chapman and Hall.

Jones, B., Jarvis, P., Lewis, J.A., \& Ebbutt, A. F. (1996). Trials to assess equivalence: The importance of rigorous methods. British Medical Journal, 313, 36-39.

Makuch, R. W., \& Johnson, R. F. (1986). Some issues in the design and interprettion of 'negative'clinical studies. Archives of Internal Medicine, 146, 989.

Robins, J. M. (1998). Correction for non-compliance in equivalence trials. Statistics in Medicine, 17, 269-302. 


\section{The Trouble With Trivials $(\mathrm{p}>.05)$}

\author{
Shlomo S. Sawilowsky \\ Educational Evaluation and Research \\ College of Education \\ Wayne State University
}

\author{
Jina S. Yoon \\ Educational Psychology \\ College of Education \\ Wayne State University
}

Trivials are effect sizes associated with statistically non-significant results. Trivials are like Tribbles in the Star Trek television show. They are cute and loveable. They proliferate without limit. They probably growl at Bayesians. But they are troublesome. This brief report discusses the trouble with trivials.

Keywords: Effect size, Meta analysis

Introduction

Among various reforms suggested to the American Educational Research Association's editorial policies directed at "editors, program chairs, and reviewers" (p. 28), Thompson (1996) recommended the reporting of effect sizes "regardless of whether statistical tests are or are not reported" (p. 29), "even [for] non-statistically significant effects" (1999, p. 67). Similar advice was given by Carver (1993), Hulburt (1994), Rosnow and Rosenthal (1989), and Wilkinson (1999).

Heuristic support in the form of a thought experiment designed to illustrate the concern with this suggested reform was given by Robinson and Levin (1997). They concluded that a better editorial practice is to "First convince us that a finding is not due to chance, and only then, assess how impressive it is" (p. 23).

Purpose of This Study

This study presents Monte Carlo evidence, which is more convincing than a thought experiment, to demonstrate the perils of reporting and interpreting effect sizes arising from nonstatistically significant research studies.

Shlomo S. Sawilowsky, Wayne Statue University Distinguished Faculty Fellow, is Professor and Chair, Educational Evaluation and Research, 351 College of Education, Wayne State University, Detroit, MI, 48202, e-mail: shlomo@edstat.coe.wayne.edu. His interests are in nonparametric, robust, exact, permuation, and other computerintensive methods, especially via Fortran. Jina Yoon is Assistant Professor, Educational Psychology, College of Education, Wayne State University. Her research interests are social emotional functioning of children and adolescents, school environments, and teacher-student relationships.
Methodology

A Fortran 95 program was written to randomly draw variates from a deMoivreian (i. e., normal) distribution and then randomly assigned to two groups $\left(n_{1}=n_{2}=10\right)$, with the first group designated the treatment and the second the control. A two-sided two independent samples $t$ test was conducted with nominal $\alpha=0.05 .10,000$ repetitions were conducted.

The effect were considered (a) under the truth of the null hypothesis, and (b) for shift in location parameter, which was simulated by adding a constant "c", representing 0.52 (a moderate effect size according to Cohen, 1988). This shift was selected to produce a power of about .2 for the $t$ test for the given sample size and $\alpha$ level.

Small sample size and power level were chosen to mimic applied research. A balanced layout and a theoretically normally distributed data set were chosen to demonstrate what happens under the best of circumstances with regard to layout and data distribution assumptions. Nominal $\alpha$ was selected at 0.05 due to Cohen (1994).

\section{Results}

The results are compiled in Table 1. The upper panel represents the various outcomes due to random numbers, where the effect size is modeled as zero. The entries were obtained by averaging the absolute value of $d$, given by the formula $d=\left(\bar{x}_{t}-\bar{x}_{c}\right) / s_{\text {pooled }}$, where $s_{\text {pooled }}$ refers to the pooled estimate of $\sigma$. (The absolute value was taken because the order of $\bar{x}_{f}$ and $\bar{x}_{c}$ is arbitrary). The upper panel demonstrates the trouble with reporting and interpreting effect sizes when the results of the experiment are statistically trivial. A fail to reject decision was reached in $95 \%$ of the repetitions of the experiment. Reporting an average effect size of 0.17 , which is approximately what Cohen (1988) judged to be a small effect size, is misleading because these 
Table 1. Effect Sizes for $\mathrm{n}_{1}=\mathrm{n}_{2}=10$, Gaussian Distribution, Nominal $\alpha=0.05$.

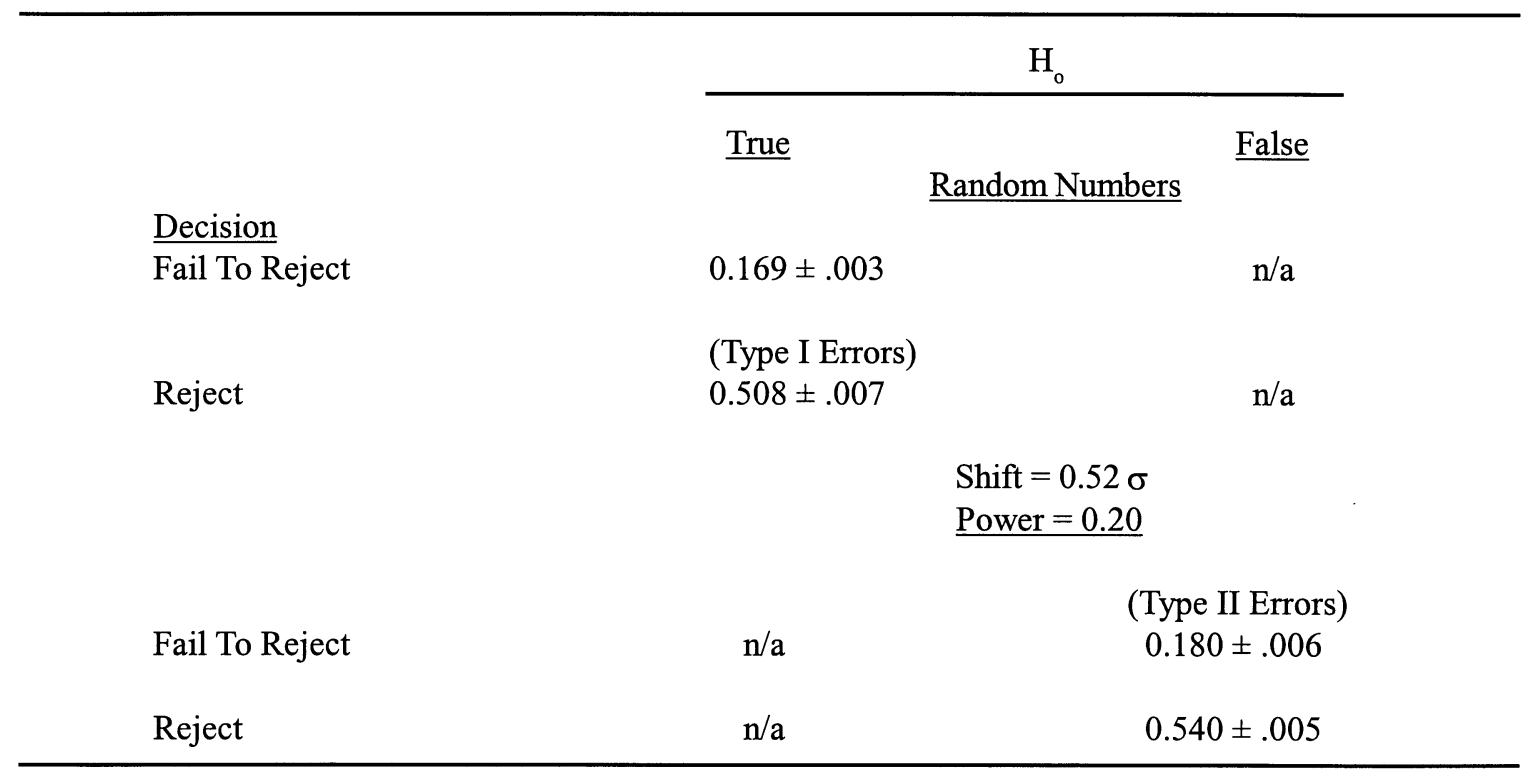

effect sizes are specious. There can be no effect size because none was modeled in the data generation.

(The remaining results aren't relevant to the main pronouncement of this paper, but are presented to complete the illustration. The adverse effects of making a Type I error is demonstrated, because an average effect size of 0.51 was obtained, a medium effect size, Cohen, 1988, when in fact the true effect size is zero.

In the second case, depicted by the lower panel, one-tailed power is represented by averaging the effect sizes. As predicted by Cohen's (1988) power tables, when the false null hypothesis is rejected, the average effect size reported and interpreted is a moderate 0.54 . This is a meaningful effect size to report and interpret.

However, when the $t$ test failed to reject the false null hypothesis, the resulting calculations indicate the effect size under consideration was only 0.18 . Similar results were obtained for the $t$ test when data were drawn from nonnormally distributed data, indicating that the $t$ test is (a) robust with respect to Type II errors, but more importantly, (b) is less powerful than competitors, such as the Wilcoxon Rank-Sum test, which would have rejected many more of these false null hypotheses.)

\section{Conclusion}

It was shown that effect sizes should not be reported or interpreted in the absence of statistical significance. As Shaver (1993) noted, even "an effect size of 1 or larger may reflect a trivial result" (p. 303, emphasis added). This is the trouble with trivials.

\section{References}

Carver, R. P. (1993). The case against statical significance testing, revisited. Journal of Experimental Education, 61, 287-291.

Cohen, J. J. (1988). Statistical power analysis for the behavioral sciences. $\left(2^{\text {nd }}\right.$ ed.) Hillsdale, NJ: Lawrence Earlbaum Associates.

Cohen, J. J. (1994). The Earth is round ( $<$.05). American Psychologist, 49, 997-1003.

Hulburt, R. T. (1994). Comprehending behavioral statistics. Pacific Grove, CA: Brooks/Cole.

Robinson, D. H., \& Levin, J. R. (1997). Reflections on statistical and substantive significance, with a slice of replication. Educational Researcher, 26, 21-26.

Rosnow, R. L., \& Rosenthal, R. (1989). Statistical procedures and the justification of knowledge in psychological science. American Psychologist, 44, 1276-1284.

Shaver, J. P. (1993). What statistical significance testing is, and what it is not. Journal of Experimental Education, 61, 293-316.

Thompson, B. (1996). AERA Editorial Policies regarding statistical significance testing: Three suggested reforms. Educational Researcher, 25, 26-30.

Thompson, B. (1999). Five methodology errors in educational research: A pantheon of statistical significance and other faux pas. In B. Thompson (Ed.), Advances in social science methodology, 5, 23-86.

Wilkinson, L., \& The Task Force on Statistical Inference (1999). Statistical methods in psychology journals: Guidelines and explanations. American Psychologist, 54, 594-604. 


\section{Using The $t$ Test With Uncommon Sample Sizes}

\author{
Shlomo S. Sawilowsky \\ Educational Evaluation \& Research \\ College of Education \\ Wayne State University
}

\author{
Barry S. Markman \\ Educational Psychology \\ College of Education \\ Wayne State University
}

Monte Carlo techniques were used to determine the effect of using common critical values as an approximation for uncommon sample sizes. Results indicate there can be a significant loss in statistical power. Therefore, even though many instructors now rely on computer statistics packages, the recommendation is made to provide more specificity (i.e., values between 30 and 60) in tables of critical values published in textbooks.

Keywords: $\mathrm{t}$ test, Critical values

\section{Introduction}

There is a practical problem that persists when using tabled critical values in introductory behavioral and social science statistics and research textbooks. We refer specifically to courses where statistical packages for the computer are not used. Critical $t$ values are only given for common degrees of freedom (df) and alpha levels. Consider calculating a two independent means t-test on response data for Condition A and Condition B in a layout with $\mathrm{n}=19$ per group. The df, $n_{1}+n_{2}-2$, is 36 . A survey of many popular textbooks showed most only had tabled values of $\mathrm{t}$ for df of $1-30,40,60,120$, and infinity. (A few textbooks also included $45 \mathrm{df}$.)

Which tabled value should be used for $36 \mathrm{df}$ in a classroom demonstration of the use of the $t$ test without a computer package? One possibility is to interpolate between the tabled values for 30 d.f. and 40 d.f. Instructors commonly eschew this approach because of the possibility that students will interpolate differently, obtain a different critical value, and subsequently cause confusion in the classroom. Another prevalent classroom solution is to use the critical $t$ values either for $30 \mathrm{df}$ or for $40 \mathrm{df}$, which are given in all textbooks reviewed. With a sample size of $\mathrm{N}=38(\mathrm{df}=36)$, the $\mathrm{t}$ statistic associated with $30 \mathrm{df}$ will be conservative, while the $t$ statistic associated with $40 \mathrm{df}$ will be liberal. An inspection of the $t$ table indicates only minor

Shlomo S. Sawilowsky, Wayne Statue University Distinguished Faculty Fellow, is Professor and Chair, Educational Evaluation and Research, 351 College of Education, Wayne State University, Detroit, MI, 48202, e-mail: shlomo@edstat.coe.wayne.edu. His interests are in nonparametric, robust, exact, permuation, and other computerintensive methods, especially via Fortran. Barry S. Markman is Professor of Educational Psychology at Wayne State University. His areas of interest are assessing ADHD using continuous performance tasks, test anxiety, anxiety and cancer, and learning theory. differences at $30 \mathrm{df}$ and $40 \mathrm{df}$, differences mainly in the $1 / 100$ ths place. How does the imprecision of using the approximate critical value affect the power properties of the t-test?

\section{Methodology and Results}

A Monte Carlo study was conducted to answer this question. A Fortran program was written for an IBM compatible computer accessing the PC version of the IMSL (1987) subroutine library. Random variates were drawn from a normal population and t-tests using the correct and approximate critical values were calculated for various alpha levels. A fail to reject vs. reject decision was recorded. This was repeated for 10,000 repetitions.

A treatment effect of shift in location parameter was modeled by adding a constant to each of the observations of one group. The constant was selected to obtain a power level of .500 for the correct $t$ value when nominal alpha was .100 . That same constant was used to obtain the power at the .050 and .010 alpha levels as well. The results are compiled in Table 1.

Table 1. Power Rates When Using the Higher $t\left(t_{h}\right), t$, And The Lower $\mathrm{t}\left(\mathrm{t}_{1}\right)$; Alpha $=.100, .050$, And $.010 ; \mathrm{n}_{1}=\mathrm{n}_{2}=$ 19; 10,000 Repetitions.

\begin{tabular}{cccc}
\hline$\alpha$ & $\mathrm{t}_{\mathrm{h}}$ & $\mathrm{t}$ & $\mathrm{t}_{\mathrm{1}}$ \\
\hline .100 & .494 & .500 & .501 \\
.050 & .368 & .374 & .376 \\
.010 & .160 & .172 & .172 \\
\hline
\end{tabular}

The results in Table 1 indicate that, at the cost of being slightly liberal, $t_{1}$ does not provide any appreciable power over the correct $t$. The power results for $t$ demonstrated a noticeable loss of power as high as $6.25 \%$. However, the temptation to use the conservative $t_{h}$, with its slight loss of power, sends the wrong message to students, in consideration of the abundant literature in education and 
psychology that suggests that treatment effects are often extremely small. In such situations the cavalier approach to the loss of any power to detect a false null hypothesis is exacerbated. (Moreover, we note that there remained staunch supporters of the superiority of the $t$ test vs the Wilcoxon test during the parametric vs nonparametric wars of the last quarter of the $20^{\text {th }}$ century, despite the fact that countless Monte Carlo studies demonstrated the power advantage of the $t$ test under normality was only a meager $1 \%-3 \%$ !)

\section{Conclusion}

A simple solution is proposed for the problem of uncommon df for sample sizes commonly used classroom instruction. It is recommend that textbook authors include the missing critical for 31 through $39 \mathrm{df}$ and 41 through $59 \mathrm{df}$.

Moreover, there also frequently arises the need for uncommon alpha levels. For example, consider conducting a "one-and-a-half-tailed" $t$ test (Ramsey, 1990).
Alpha is set to .04 in one tail to maximize power in the anticipated direction, and alpha is also set at .01 in the other tail. This will provide more power than the usual two-tailed test with .025 in each tail, while providing some protection against outcomes occurring in the wrong direction. Because .04 is an uncommon alpha level, Ramsey (1990) suggested using the $Z$ table as approximation critical values. This suggestion is clearly undesirable. Nevertheless, this may be a circumstance that cannot be adequately handled with tabled critical values found in the back of statistics textbooks.

\section{References}

IMSL (1987). IMSL Stat/Library user's manual: Fortran subroutines for statistical analysis. Version 1.0. Houston: Author.

Ramsey, P. H. (1990). 'One-and-a-half-tailed' tests of significance. Psychological Reports, 66, 653-654. 


\section{STATISTICAL SOFTWARE APPLICATIONS \& REVIEW Modeling Strategies In Logistic Regression With SAS, SPSS, Systat, BMDP, Minitab, And STATA}

\author{
Chao-Ying Joanne Peng \\ School of Education \\ Indiana University
}

\author{
Tak-Shing Harry So \\ School of Education \\ Indiana University
}

This paper addresses modeling strategies in logistic regression within the context of a real-world data set. Six commercially available statistical packages were evaluated in how they addressed modeling issues and in the accuracy of their regression results. Recommendations are offered for data analysts in terms of each package's strengths and weaknesses.

Keywords: Logistic regression, Analysis issues, Statistical packages, Binary outcome, Categorical variables, Statistical computing

\section{Introduction}

Among the variety of statistical methods that are employed to analyze social science data, regression methods are widely used in examining the relationship between an outcome variable and one or more predictor variables. One class of regression methods, logistic regression, is well suited for studying categorical or qualitative outcome variables. This technique is increasingly applied in social science research, especially in higher education (Austin, Yaffee, \& Hinkle, 1992; Cabrera, 1994). Logistic regression textbooks by Hosmer and Lemeshow (2000), Kleinbaum (1994), McCullagh and Nelder (1989), and Menard (1995) have been published within the last thirteen years. Other textbooks of multivariate statistics (e.g., Afifi \& Clark, 1990; Ryan, 1997; Tabachnick \& Fidell, 1996) have begun to include chapters on logistic regression in their recent editions. Because logistic regression does not assume that data are drawn from a multivariate normal distribution with equal variances and covariances for all variables (Efron, 1975; Lei \& Koehly, 2000; Press \& Wilson, 1978), it is less restrictive than linear discriminant function analysis. Thus, social science researchers have recognized logistic regression as a viable method for handling categorical outcome variables.

Despite the simplicity of logistic regression and the ease with which researchers are able to implement this technique using statistical software, most researchers are unaware how a number of modeling issues are dealt with by statistical software. This paper addresses these issues within

Chao-Ying Joanne Peng, Counseling \& Educational Psychology, School of Education, \#4050, 201 N. Rose Ave., Indiana University, Bloomington, IN, 47405. Email: peng@indiana.edu. Tak-Shing Harry So is at Indiana University. The authors acknowledge Gary M. Ingersoll, Lisa Kurtz, Dan J. Mueller, Edward St. John, Dale Weigel, Jim $\mathrm{Zhu}$, and Larry Hoezee for helpful comments. the context of a real-world data set. Six statistical packages were compared and contrasted in how they addressed these issues and in the accuracy of logistic regression results. We conclude this paper by offering evaluations of the six packages for logistic regression.

\section{Statistical Packages}

Six logistic regression procedures/commands implemented in SAS, SPSS, SYSTAT, BMDP, MINITAB, and $S T A T A$ were reviewed in order to understand how these popular and accessible packages handled logistic regression models:

I. the LOGISTIC procedure in SAS Release 8,

II. the LOGISTIC REGRESSION command in SPSS Release 10,

III. the LOGIT command in SYSTAT Release 9,

IV. the LR command in BMDP Release 7.1,

V. the BLOGISTIC command in MINITAB Release 13 , and

VI. the LOGISTIC command in STATA Release 6.

For the purpose of discussion, statistical packages refer to SAS, SPSS, SYSTAT, BMDP, MINITAB, and STATA software. Procedure refers to a procedure or main command in a statistical package that performs logistic regression, such as the LOGISTIC procedure in $S A S$, the LOGISTIC REGRESSION command in SPSS, etc. (For clarity, variable names are written with an underscore, e.g., labor force paid $=\underline{\text { lfp. }}$.)

Two types of logistic regression models (direct and stepwise) were fit to these data. Direct modeling permits researchers to specify predictors that represent main effects and interactions according to a theory-based proposition. Stepwise modeling yields best models according to statistical software's internal criteria and restrictions. This modeling approach is largely atheoretical; its use is popular among researchers, yet controversial among methodologists. 
Data

The "married women labor force participation" data (hereafter abbreviated as MWLFP) were provided by Mroz (1987). The data set contains profiles of 752 married white women who were recruited in 1975 for the Panel Study of Income Dynamics conducted at the University of Michigan. We were interested in explaining women's decision to enter the paid labor force in 1975 with their demographic information. The outcome variable (lifp) was coded 1 for women who worked for pay in 1975 and 0 otherwise. The predictors were: the age of women (age), number of children under the age of $5(\underline{\mathrm{k}})$, number of children between ages 6 and 18 (k618), the household's total income minus the wife's labor income (inc), the wife's estimated wage rate ( $\underline{\mathrm{wg}})$ plus two dichotomous variables indicating, respectively, whether the wife $(\underline{\mathrm{wc}})$ and the husband $(\underline{\underline{h c}})$ spent at least one year in college. Table 1 presents descriptive information of the eight variables.

\section{Direct Modeling}

A direct model (Model 1) was fit to the MWLFP data to explain the predicted odds of women entering the paid labor force (i.e., $\underline{\mathrm{f} p}=1$ ) in 1975. This model included four main effects- $\underline{\mathrm{k} 5}, \underline{\mathrm{k} 618}, \underline{\mathrm{hc}}, \underline{\mathrm{wc}}$ - plus one categorical variable (newage) and its interaction with wc.

Model 1:

predicted logit $(\underline{\mathrm{lfp}}=1)=\alpha+\beta_{1} \times \underline{\mathrm{k} 5}+\beta_{2} \times \underline{\mathrm{k} 618}+$ $\beta_{3} \times$ newage $1+\beta_{4} \times$ newage $2+\beta_{5} \times$ newage $3+\beta_{6} \times$ newage $4+$ $\beta_{7} \times$ newage 5 $+\beta_{8} \times \underline{\text { hc }}+\beta_{9} \times \underline{w c}+\beta_{10} \times(\underline{w c} *$ newage $)+\beta_{11} \times$ $\left(\underline{w c}^{*}\right.$ newage2 $)+\beta_{12} \times\left(\underline{w c}^{*}\right.$ newage $)+\beta_{13} \times\left(\underline{w c}^{*}\right.$ newage $)$ $+\beta_{14} \times\left(\right.$ wc$^{*}$ newage5 $)$.

The variable newage was transformed from the continuous variable age according to a 5 -year increment (i.e., 30 to 34,35 to $39, \ldots$, and 55 to 60 ) with the last category (women older than 54 years old) designated as the reference group.

After specifying Model 1 into the six statistical packages, we obtained very similar estimates for parameters and standard errors. All predictors reached the significance level of 0.05 , except for $\underline{\mathrm{k} 618}, \underline{\mathrm{hc}}$, and the interactions of wc with newage1 through newage5 (Table 2). The result implied that the odds for married women to enter the paid labor force in 1975 were related to the number of young children (5 years old or under), their age groups, and whether women had some college education.

Tests of individual parameter estimates are performed either by the likelihood ratio test, the Wald statistic, or the Score test. According to Jennings (1986), Long (1997), and Tabachnick and Fidell (1996), the likelihood ratio test is more powerful than the Wald test while the Score test is a normal approximation to the likelihood ratio test. $B M D P$ is the only package that computes the likelihood ratio test. The other five perform the Wald test [Table 3 (III) Results]. For categorical predictors, SAS, SPSS, and MINITAB automatically perform an overall test of design variables transformed from the same categorical predictor. In SYSTAT, this test is requested by the CONSTRAINT subcommand. This subcommand may also be applied to test two or more slope parameters simultaneously against zero. An equivalent option (the TEST statement) is available in SAS LOGISTIC. Both CONSTRAINT and TEST work in stepwise modeling only if the multiple predictors, to be tested simultaneously, are already selected into the model.

\section{Stepwise Modeling}

As stated earlier, the application of stepwise modeling is controversial among methodologists since the inclusion or removal of predictors is based entirely on statistical criteria. The substantive importance of predictors is often overlooked in the process. Small differences in the criteria can have a great impact on those marginally significant predictors. Furthermore, any model determined by the stepwise algorithm is sample-bound and atheoretical. The most serious of all problems is the positive bias introduced into parameter estimates, as with the stepwise discriminant function analysis (Kromrey, Foster-Johnson, \& Yi, 1997). Despite these criticisms, stepwise logistic modeling is a flourishing practice among higher education researchers (Peng, So, Stage, \& St. John, 2002). As Tabachnick and Fidell (1996) explain, it can assist researchers in generating and screening hypotheses.

Forward stepwise, or simply stepwise, modeling is one of four model selection methods available in five statistical packages we examined [Table 3 (II) Model Specification]. MINITAB was excluded because it does not provide a selection method. The stepwise method begins with only a constant in the model. At each subsequent step, the most important + predicator is added to the model. A predictor's importance is determined by its criterion statistic. Only when the largest criterion statistic is tested to be significant according to a preset entry p-level, will its corresponding predictor be selected into the model. Otherwise, the selection process stops. As a predictor is added to the model, all predictors already in the model are simultaneously reassessed to determine if any of them meets the criterion for removal, again according to a preset removal p-level.

To implement stepwise modeling, we considered all effects already contained in Model 1, plus additional main effects and interactions: inc, wg, wg2 (the squared value of $\underline{w g}), \underline{w c}^{*} w g$, and $\underline{w c}^{*} w g 2$. These interactions were included for the illustration of stepwise modeling only. Following suggestions from Hosmer and Lemeshow (2000), we adopted 0.15 and 0.20 as entry and removal p-levels, 
respectively. Two models ( 2 and 3 ) were identified by five packages. Model 2-the model identified by $S A S$ and $B M D P$ - contained ten predictors while SPSS, SYSTAT, and STATA selected Model 3 with eleven predictors (Table 2). Nine predictors in both models were identical: $\underline{\mathrm{k}}$, newage1$\underline{\text { newage } 5}$, inc, wg, and wg2. Two predictors, wc* wg and $\mathrm{wc}^{*} \mathrm{wg} 2$, appeared only in Model 3 , whereas $\underline{\mathrm{hc}}$ appeared only in Model 2. The difference between these two models was caused by a modeling restriction imposed by $S A S$ and $B M D P$. This restriction requires that all main-effects and lower-order interaction(s) be included in the model before a higher-order interaction is entered into the model. $S A S$ and $B M D P$ enforce this definition for hierarchical modeling during the model selection process while MINITAB and STATA do so only in direct modeling.

Which model, 2 or 3 , is a better model for the data? To answer this question fully, one needs to examine multiple descriptive as well as inferential statistics. They are described below:

Descriptive statistics. The Akaike Information Criterion (AIC) and the Schwarz Criterion (SC) provided by SAS were used to compare different models derived from the same sample. A smaller value indicates a better fit. The AIC and SC values for Model 2 (787.446 and 838.296), Model 3 (719.743 and 775.216), and Model 1 (966.078 and 1035.238) indicated that either Model 2 or 3 outperformed Model 1. Furthermore, Model 3 was a better model than Model 2. Other measures, such as $R^{2}$, Somers' $D_{x y}$, Gamma, Tau-a, and c statistics, also supported the same conclusions. Because Model 3 did not satisfy the hierarchical modeling restriction, we constructed one additional model-Model 4-for comparison with Model 3. Model 4 contained all predictors from Model 3 plus wc; it yielded similar values as Model 3 on all descriptive indices (Table 2).

Inferential statistics. Because Model 3 was nested in Model 4, we used the likelihood ratio test to test if the additional predictor (i.e., $\underline{\mathrm{wc}}$ ) had a zero coefficient (i.e., $\mathrm{H}_{0}: \beta_{\mathrm{wc}}=0$ ). The likelihood ratio test is based on the difference $(\mathrm{G})$ in the log-likelihood (LL) of both models. Under the null hypothesis that coefficients of additional predictors equal zero, the $\mathrm{G}$ statistic follows a chi-square distribution with degree(s) of freedom equal to the number of additional predictors. To test $\mathrm{H}_{0}: \beta_{\mathrm{wc}}=0$, the $\mathrm{G}$ statistic equals $-2[-347.8715-(-347.4725)]=0.789$. Because the $G$ statistic did not exceed the $\chi^{2}$ critical value of 3.841 with 1 degree of freedom and alpha of 0.05 , the null hypothesis was not rejected. We concluded that wc in Model 4 did not significantly improve the prediction. Hence, Model 3 was considered as good as Model 4 and better than either Model 1 or 2 .

\section{Evaluations of Six Logistic Regression Procedures}

An ideal statistical package for logistic regression should be user-friendly and comprehensive in its options and output. Each package we examined possesses certain features of this ideal package. Table 3 summarizes features and options available in all six procedures. An evaluation of each is given below:

I. SAS LOGISTIC is the most versatile procedure. Several selection methods are provided. Its ability to fit a broad class of binary response models, plus its provision to correct for over-sampling, over-dispersion, and bias introduced into predicted probabilities, sets it apart from the other five.

II. With dazzling graphic interfaces, SPSS LOGISTIC REGRESSION and SYSTAT LOGIT are userfriendly. They provide several selection methods, yet their goodness-of-fit statistics and diagnostic statistics are calculated from individual observations. Hence, they should not be interpreted as chi-square values.

III. $B M D P$ LR performs logistic regression on covariate patterns. It is a stepwise procedure that provides the greatest flexibility in selecting the "best" set of predictors, under the hierarchical modeling restriction. Unfortunately, it does not compute diagnostic statistics recommended by Hosmer and Lemeshow (2000), such as change in Pearson chi-square, change in deviance, or change in parameter estimates.

I. MINITAB BLOGISTIC is the simplest to use. It adopts the hierarchical modeling restriction in direct modeling. However, the absence of predictor selection methods may make it less appealing to some researchers.

II. STATA LOGISTIC provides the most detailed information on parameter estimates, yet its goodness-of-fit indices are limited. Its command language is easy to learn. It generates high quality graphics with a single command. Model selections are carried out in two procedures: SW for stepwise selection and LOGISTIC for logistic regression modeling. Multicollinearity among predictors is examined automatically during stepwise modeling.

In sum, we recommend MINITAB and STATA for beginners. If either SPSS or SYSTAT is the only package available, researchers must be aware that both compute the goodness-of-fit and diagnostic statistics from individual observations. Consequently, these statistics are inappropriate for statistical tests. $S A S$ and $B M D P$ are more suitable for experienced researchers. Their options are versatile and 
results are comprehensive and accurate. Last but not the least, researchers should always check default settings of a statistical package when performing logistic regression. Variations in results may be due to different defaults.

With the wide availability of sophisticated statistical software installed on high-speed computers, the anticipated use of logistic regression appears to be increasing. Researchers in social sciences are encouraged to apply this versatile technique fully to their data and evaluate competing models with supplementary statistics provided by statistical software.

\section{References}

Afifi, A. A., \& Clark, V. (1990). Computer-aided multivariate analysis (2nd ed.). New York, NY: Van Nostrand Reinhold.

Austin, J. T., Yaffee, R. A., \& Hinkle, D. E. (1992). Logistic regression for research in higher education. In J. C. Smart (ed.), Higher education: Handbook of theory and research, Vol. VIII, 379-410.

BMDP (1992). BMDP statistical software manual (Vol. 2). Los Angeles, CA: BMDP Statistical Software, Inc.

Cabrera, A. F. (1994). Logistic regression analysis in higher education: An applied perspective. In J. C. Smart (ed.), Higher Education: Handbook of theory and research, Vol. X, 225-256.

Efron, B. (1975). The efficiency of logistic regression compared to normal discriminant analysis. Journal of the American Statistical Association, 70, 892-898.

Homer, D. W., Jr., \& Lemeshow, S. (2000). Applied logistic regression $\left(2^{\text {nd }}\right.$ ed.). New York, NY: John Wiley \& Sons, Inc.

Jennings, D. E. (1986). Judging inference adequacy in logistic regression. Journal of the American Statistical Association, 81, 471-476.

Kleinbaum, D. G. (1994). Logistic regression: A selflearning text. New York, NY: Springer-Verlag.

Kromrey, J. D., Foster-Johnson, L., \& Yi, Q. (1997, March). Statistical bias resulting from the use of variables selection algorithms in discriminant function analysis: What do stepwise-built models represent? Paper presented at the Annual Meeting of American Educational Research Association, Chicago, IL.
Lei, P.-W., \& Koehly, L. M. (April, 2000). Linear discriminant analysis versus logistic regression: A comparison of classification errors. Paper presented at the Annual Meeting of American Educational Research Association, New Orleans, LO.

Long, J. S. (1997). Regression models for categorical and limited dependent variables. Thousand Oaks, CA: Sage.

McCullagh, P., \& Nelder, J. A. (1989). Generalized linear models (2nd ed.). London: Chapman \& Hall.

Menard, S. (1995). Applied logistic regression analysis. Sage University paper series on quantitative applications in the social sciences, 07-106. Thousand Oaks, CA: Sage.

Minitab (2000). MINITAB user's guide 2: Data analysis and quality tools, Release 13. State College, PA: Minitab Inc.

Mroz, T. A. (1987). The sensitivity of an empirical model of married women's hours of work to economic and statistical assumptions. Econometrica, 55, 765-799.

Peng, C. Y., So, T. S., Stage, F. K., \& St. John, E. P. (2002). The use and interpretation of logistic regression in higher education journals: Research in Higher Education, 43(3), 259-293.

Press, S. J., \& Wilson, S. (1978). Choosing between logistic regression and discriminant analysis. Journal of the American Statistical Association, 73, 699-705.

Ryan, T. P. (1997). Modern regression methods. New York, NY: John Wiley \& Son, Inc.

SAS (1999). SAS/STAT user's guide, version 8, volume 2. Cary, NC: SAS Institute Inc.

SPSS (1999a). SPSS regression models 10. Chicago, IL: SPSS Inc.

SPSS (1999b). SYSTAT 9.0 Statistics I. Chicago, IL: SPSS Inc.

Stata (1999). STATA reference manual release 6(Vols. 1-4). College Station: TX: Stata Press.

Tabachnick, B. G., \& Fidell, L. S. (1996). Using

multivariate statistics (3rd ed.). New York, NY: Harper Collins. 
Table 1

Descriptive Statistics for the Married Women Labor Force Participation Data

\begin{tabular}{|c|c|c|c|c|c|}
\hline Variable Name & Mean & $\begin{array}{l}\text { Standard } \\
\text { Deviation }\end{array}$ & Minimum & Maximum & Description \\
\hline Full Sample & 0.567 & 0.496 & 0 & 1 & $\begin{array}{l}1 \text { if wife is in the paid labor } \\
\text { force; else }=0\end{array}$ \\
\hline $\begin{array}{r}\text { k5 } \\
\text { Full Sample } \\
\text { Working Women } \\
\text { Non-working Women }\end{array}$ & $\begin{array}{l}0.238 \\
0.141 \\
0.366\end{array}$ & $\begin{array}{l}0.524 \\
0.392 \\
1.327\end{array}$ & $\begin{array}{l}0 \\
0 \\
0\end{array}$ & $\begin{array}{l}3 \\
2 \\
3\end{array}$ & $\begin{array}{l}\text { Number of children ages } 5 \text { or } \\
\text { younger }\end{array}$ \\
\hline $\begin{array}{r}\text { k618 } \begin{array}{r}\text { Full Sample } \\
\text { Working Women }\end{array} \\
\text { Non-working Women }\end{array}$ & $\begin{array}{l}1.352 \\
1.349 \\
1.357\end{array}$ & $\begin{array}{l}1.321 \\
1.317 \\
1.327\end{array}$ & $\begin{array}{l}0 \\
0 \\
0\end{array}$ & $\begin{array}{l}8 \\
8 \\
7\end{array}$ & $\begin{array}{l}\text { Number of children ages } 6 \text { to } \\
18\end{array}$ \\
\hline $\begin{array}{r}\text { Age } \\
\text { Full Sample } \\
\text { Working Women } \\
\text { Non-working Women }\end{array}$ & $\begin{array}{l}42.547 \\
41.988 \\
43.283\end{array}$ & $\begin{array}{l}8.073 \\
7.722 \\
8.468\end{array}$ & $\begin{array}{l}30 \\
30 \\
30\end{array}$ & $\begin{array}{l}60 \\
60 \\
60\end{array}$ & Wife's age in years \\
\hline $\begin{array}{r}\text { Wc } \\
\begin{array}{r}\text { Full Sample } \\
\text { Working Women }\end{array} \\
\text { Non-working Women }\end{array}$ & $\begin{array}{l}0.281 \\
0.335 \\
0.209\end{array}$ & $\begin{array}{l}0.450 \\
0.473 \\
0.407\end{array}$ & $\begin{array}{l}0 \\
0 \\
0\end{array}$ & $\begin{array}{l}1 \\
1 \\
1\end{array}$ & $\begin{array}{l}1 \text { if wife attended college; else } \\
0\end{array}$ \\
\hline $\begin{array}{r}\text { Hc } \\
\text { Full Sample } \\
\text { Working Women } \\
\text { Non-working Women }\end{array}$ & $\begin{array}{l}0.392 \\
0.415 \\
0.363\end{array}$ & $\begin{array}{l}0.489 \\
0.493 \\
0.482\end{array}$ & $\begin{array}{l}0 \\
0 \\
0\end{array}$ & $\begin{array}{l}1 \\
1 \\
1\end{array}$ & $\begin{array}{l}1 \text { if husband attended college; } \\
\text { else } 0\end{array}$ \\
\hline $\begin{array}{rr}\text { Wg } & \text { Full Sample } \\
\text { Working Women } \\
\text { Non-working Women }\end{array}$ & $\begin{array}{l}3.56 \\
4.17 \\
2.76\end{array}$ & $\begin{array}{l}2.64 \\
3.31 \\
0.81\end{array}$ & $\begin{array}{l}0.13 \\
0.13 \\
0.99\end{array}$ & $\begin{array}{r}25.00 \\
25.00 \\
5.80\end{array}$ & Wife's estimated wage rate \\
\hline Inc $\begin{array}{r}\text { Full Sample } \\
\text { Working Women } \\
\text { Non-working Women }\end{array}$ & $\begin{array}{l}20.156 \\
18.981 \\
21.698\end{array}$ & $\begin{array}{l}11.619 \\
10.564 \\
12.728\end{array}$ & $\begin{array}{l}1.120 \\
1.120 \\
1.500\end{array}$ & $\begin{array}{l}96 \\
91 \\
96\end{array}$ & $\begin{array}{l}\text { Family income excluding } \\
\text { wife's wages (in } \$ 1,000 \text { ) }\end{array}$ \\
\hline
\end{tabular}

Note. Full Sample: $\underline{\mathrm{N}}=752$. Working Women Sample: $\underline{\underline{n}}=427$. Non-working Women Sample: $\underline{\underline{n}}=325$ 
Table 2

Summary of Models $1,2,3$, and 4

\begin{tabular}{|c|c|c|c|c|}
\hline & Model 1 & Model $2^{\mathrm{a}}$ & Model $3^{b}$ & Model 4 \\
\hline CONSTANT & $\begin{array}{c}-0.5935 \\
(0.3325)_{c}\end{array}$ & $\begin{array}{c}3.4100 \\
(0.7113)^{\mathrm{c}}\end{array}$ & $\begin{array}{c}7.0724 \\
(1.0463)^{c}\end{array}$ & $\begin{array}{c}6.5700 \\
(1.1705)^{c}\end{array}$ \\
\hline$\underline{\mathrm{k} 5}$ & $\begin{array}{r}-1.3710^{* *} \\
(0.1955)^{c}\end{array}$ & $\begin{array}{c}-1.5196^{* *} \\
(0.2300)^{c}\end{array}$ & $\begin{array}{r}-1.3861^{* *} \\
(0.2290)^{c}\end{array}$ & $\begin{array}{c}-1.4038^{* *} \\
(0.2319)^{c}\end{array}$ \\
\hline$\underline{\mathrm{k} 618}$ & $\begin{array}{c}-0.1097 \\
(0.0703)^{c}\end{array}$ & -- & - & - \\
\hline \multicolumn{5}{|l|}{ age } \\
\hline newage 1 (age $<=34)$ & $\begin{array}{l}1.8425^{* *} \\
(0.4271)^{c}\end{array}$ & $\begin{array}{l}1.7787^{* *} \\
(0.4096)^{c}\end{array}$ & $\begin{array}{l}1.8844^{* *} \\
(0.4348)^{c}\end{array}$ & $\begin{array}{l}1.8582^{* *} \\
(0.4348)^{c}\end{array}$ \\
\hline newage2 $(34<$ age $<=39)$ & $\begin{array}{l}1.6956^{* *} \\
(0.4331)^{c}\end{array}$ & $\begin{array}{l}1.3882^{* *} \\
(0.3947)^{c}\end{array}$ & $\begin{array}{l}1.5615^{* *} \\
(0.4210)^{c}\end{array}$ & $\begin{array}{l}1.5391^{* *} \\
(0.4205)^{c}\end{array}$ \\
\hline newage $3(39<$ age $<=44)$ & $\begin{array}{l}1.1662^{* *} \\
(0.4175)^{c}\end{array}$ & $\begin{array}{l}1.0839 * * \\
(0.3888)^{c}\end{array}$ & $\begin{array}{l}1.0523^{* *} \\
(0.4132)^{\mathrm{c}}\end{array}$ & $\begin{array}{c}1.0289^{*} \\
(0.4128)^{c}\end{array}$ \\
\hline newage4 $(44<$ age $<=49)$ & $\begin{array}{c}0.9405^{*} \\
(0.3854)^{\mathrm{c}}\end{array}$ & $\begin{array}{l}1.0950^{* * *} \\
(0.3761)^{c}\end{array}$ & $\begin{array}{l}1.1647^{* *} \\
(0.4030)^{c}\end{array}$ & $\begin{array}{l}1.1401^{* *} \\
(0.4026)^{c}\end{array}$ \\
\hline newage $5(49<$ age $<=54)$ & $\begin{array}{c}0.4613 \\
(0.3999)^{c}\end{array}$ & $\begin{array}{c}0.5016 \\
(0.3937)^{c}\end{array}$ & $\begin{array}{c}0.3819 \\
(0.4216)^{c}\end{array}$ & $\begin{array}{c}0.3669 \\
(0.4207)^{c}\end{array}$ \\
\hline$\underline{\text { he }}$ & $\begin{array}{c}-0.1205 \\
(0.1938)^{c}\end{array}$ & $\begin{array}{c}0.3230 \\
(0.2083)^{c}\end{array}$ & -- & - \\
\hline inc & - & $\begin{array}{l}-0.0372^{* *} \\
(0.00967)^{c}\end{array}$ & $\begin{array}{l}-0.0325^{* *} \\
(0.00931)^{c}\end{array}$ & $\begin{array}{c}-0.0359^{* *} \\
(0.01)^{c}\end{array}$ \\
\hline$\underline{\text { wc }}$ & $\begin{array}{c}1.3834^{*} \\
(0.6541)^{c}\end{array}$ & -- & - & $\begin{array}{c}2.1547 \\
(2.5187)^{c}\end{array}$ \\
\hline$\underline{\text { wg }}$ & - & $\begin{array}{c}-2.9908^{* *} \\
(0.4517)^{c}\end{array}$ & $\begin{array}{c}-6.6937^{* *} \\
(0.8214)^{c}\end{array}$ & $\begin{array}{l}-6.2739^{* *} \\
(0.9306)^{c}\end{array}$ \\
\hline wg2 & - & $\begin{array}{l}0.5473^{* *} \\
(0.0747)^{c}\end{array}$ & $\begin{array}{l}1.3500^{* *} \\
(0.1614)^{\mathrm{c}}\end{array}$ & $\begin{array}{l}1.2723^{* *} \\
(0.1800)^{c}\end{array}$ \\
\hline$\underline{w} c^{*} w g$ & -- & -- & $\begin{array}{l}2.6923^{* *} \\
(0.3925)^{c}\end{array}$ & $\begin{array}{c}1.4177 \\
(1.5160)^{\mathrm{c}}\end{array}$ \\
\hline$\underline{w c}{ }^{*} \underline{w g} 2$ & -- & - & $\begin{array}{c}-0.8025^{* *} \\
(0.1150)^{c}\end{array}$ & $\begin{array}{r}-0.6186^{* *} \\
(0.2373)^{c}\end{array}$ \\
\hline \multicolumn{5}{|l|}{$w^{*}$ age } \\
\hline wc $^{*}$ newage 1 & $\begin{array}{c}-0.5837 \\
(0.7544)^{c}\end{array}$ & - & - & -- \\
\hline wc ${ }^{*}$ newage2 & $\begin{array}{c}-0.7515 \\
(0.7788)^{c}\end{array}$ & -- & -- & - \\
\hline wc* ${ }^{*}$ newage 3 & $\begin{array}{c}-0.9163 \\
(0.7637)^{c}\end{array}$ & -- & -- & -- \\
\hline wc* newage4 & -0.0448 & - & - & -- \\
\hline
\end{tabular}




\begin{tabular}{|c|c|c|c|c|}
\hline wc ${ }^{*}$ newage 5 & $\begin{array}{c}(0.7769)^{c} \\
-0.4655 \\
(0.7934)^{c}\end{array}$ & -- & -- & -- \\
\hline Likelihood Ratio Test & $\begin{array}{l}92.538^{* *} \\
(\underline{\mathrm{df}}=14)\end{array}$ & $\begin{array}{c}263.1693^{* *} \\
(\underline{d f}=10)\end{array}$ & $\begin{array}{c}332.8725^{* *} \\
(\underline{\mathrm{d} f}=11)\end{array}$ & $\begin{array}{c}333.671^{* *} \\
(\underline{\mathrm{df}}=12)\end{array}$ \\
\hline Score Test & $\begin{array}{l}87.547^{* *} \\
(\underline{\mathrm{df}}=14)\end{array}$ & $\begin{array}{c}136.975^{* *} \\
(\underline{\mathrm{df}}=10)\end{array}$ & $\begin{array}{c}137.0647^{* *} \\
(\underline{\mathrm{df}}=11)\end{array}$ & $\begin{array}{c}147.8995^{* *} \\
(\underline{\mathrm{df}}=12)\end{array}$ \\
\hline Wald Test & $\begin{array}{l}76.838^{* *} \\
(\underline{\mathrm{df}}=14)\end{array}$ & $\begin{array}{c}108.2304^{* *} \\
(\underline{\mathrm{df}}=10)\end{array}$ & $\begin{array}{c}120.1213^{* *} \\
(\underline{\underline{d f}}=11)\end{array}$ & $\begin{array}{c}118.6195^{* *} \\
(\underline{\mathrm{df}}=12)\end{array}$ \\
\hline Akaike's Criterion (AIC) & 966.078 & 787.446 & 719.743 & 720.945 \\
\hline Schwarz Criterion (SC) & 1035.238 & 838.296 & 775.216 & 781.041 \\
\hline Somers' $\underline{D}_{x y}$ & 0.397 & 0.638 & 0.688 & 0.687 \\
\hline Gamma & 0.405 & 0.639 & 0.689 & 0.688 \\
\hline Kendall's Tau-a & 0.195 & 0.314 & 0.338 & 0.338 \\
\hline c statistic & 0.698 & 0.819 & 0.844 & 0.844 \\
\hline
\end{tabular}

Note. Results were reported by SAS LOGISTIC unless noted otherwise.

${ }^{\mathrm{a}}$ Based on a stepwise model suggested by SAS and BMDP.

based on a stepwise model suggested by SPSS, SYSTAT, and STATA.

'The number in parentheses is the standard error for the parameter.

${ }^{*} \mathrm{p}<0.05 .{ }^{* *} \mathrm{p}<0.01$ 


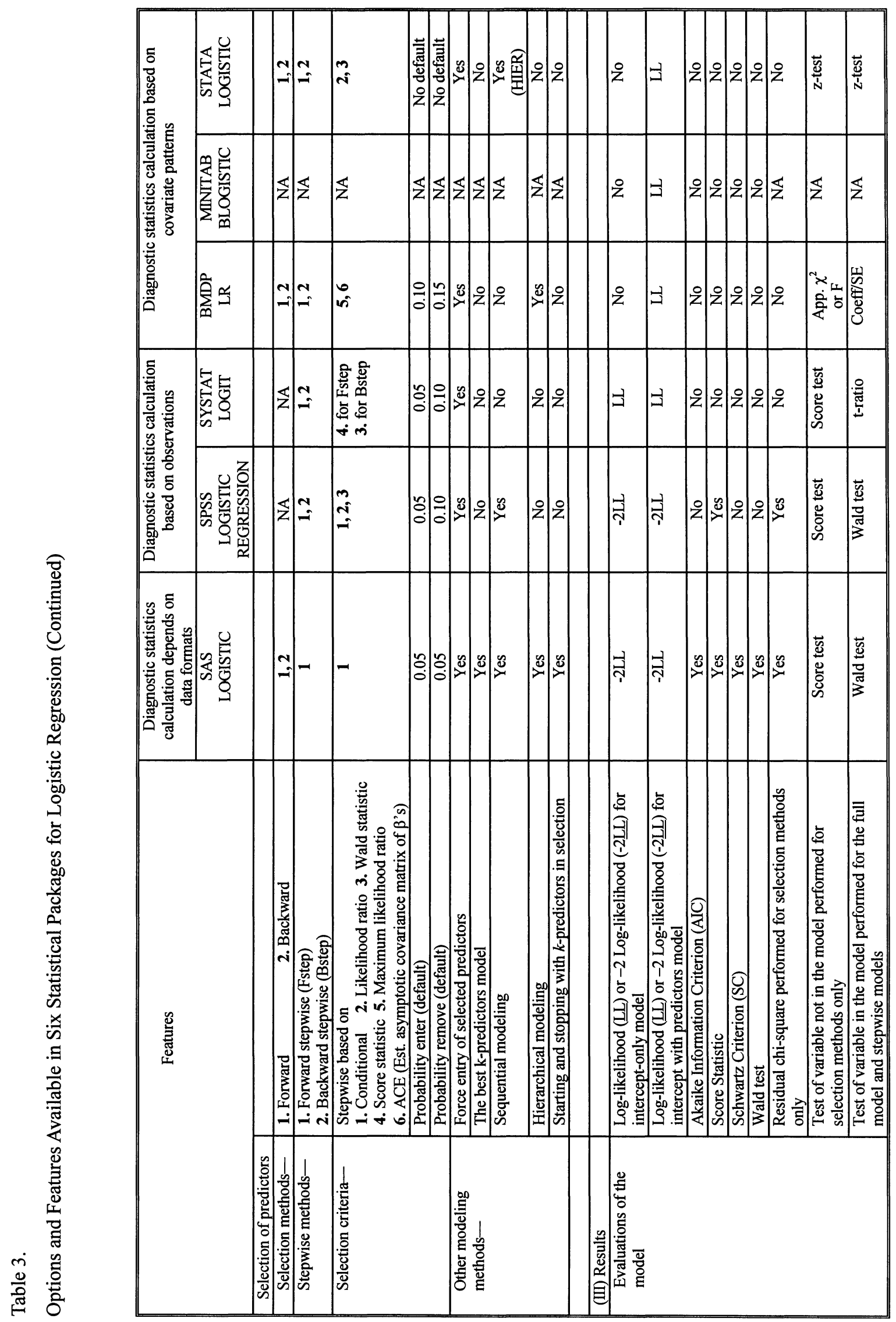


PENG \& SO

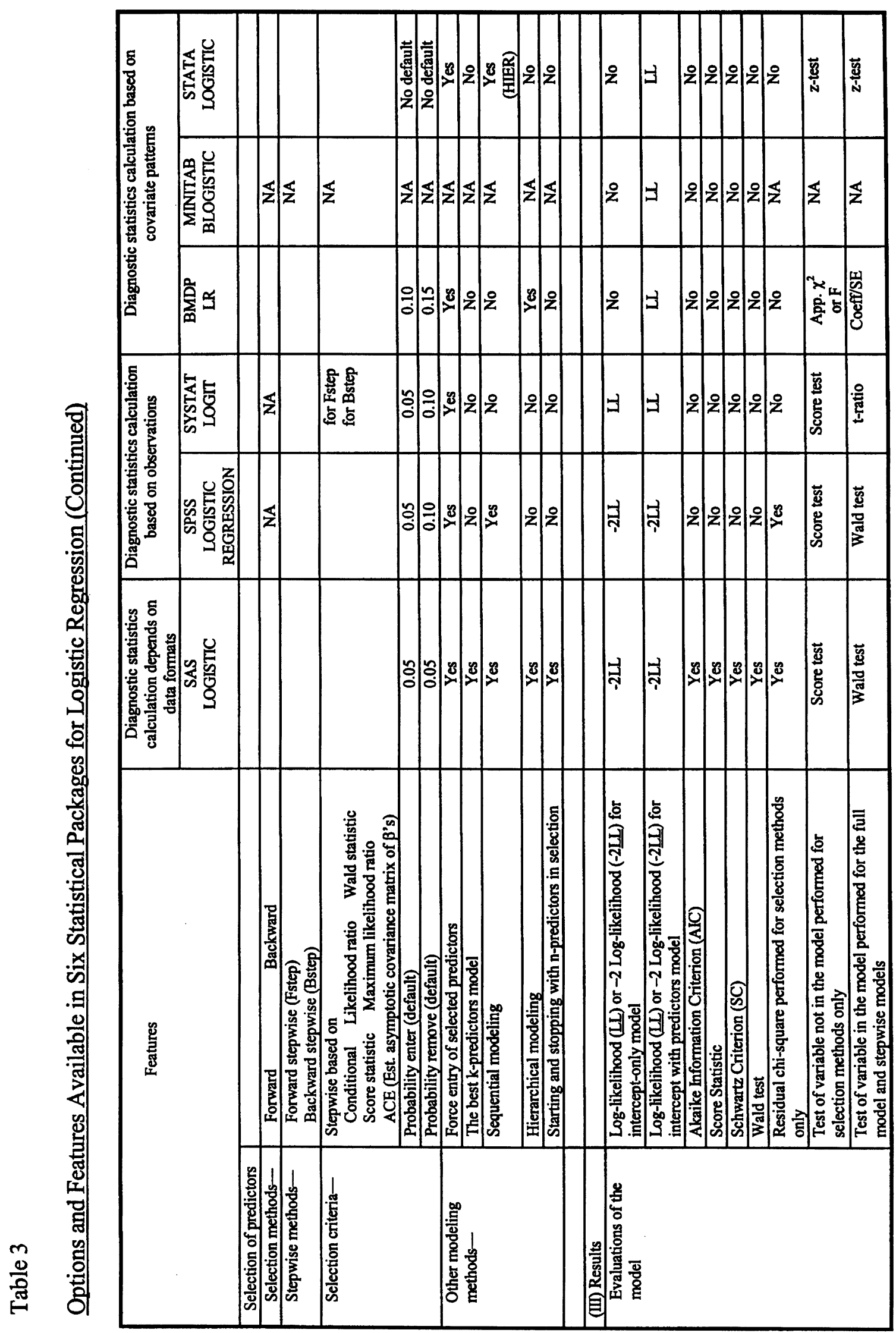




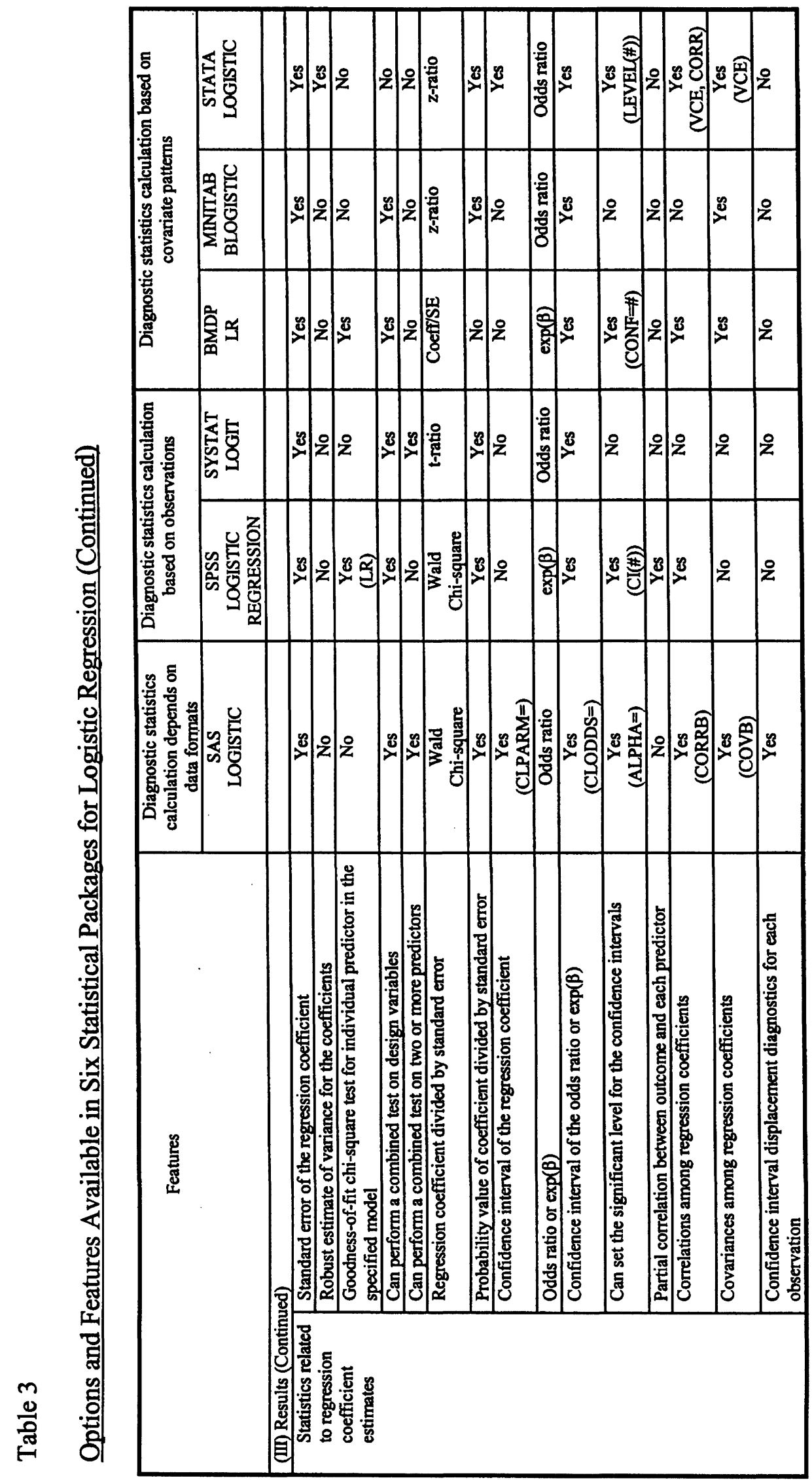




\section{Quantifying Bimodality Part I: An Easily Implemented Method Using SPSS}

\author{
B. W. Frankland \\ Department of Psychology \\ Dalhousie University
}

\author{
Bruno D. Zumbo \\ Measurement, Evaluation, \& Research Methodology \\ University of British Columbia
}

Scientists in a variety of fields are faced with the question of whether or not a particular sample of data are best described as unimodal or bimodal. We provide a simple and convenient method for assessing bimodality. The use of the non-linear algorithms in SPSS for modeling complex mixture distributions is demonstrated on a unimodal normal distribution (with 2 free parameters) and on bimodal mixture of two normal distributions (with 5 free parameters).

Keywords: Mixture modeling, Bimodality

\section{Introduction}

Research in the social, natural and health sciences (e.g., epidemiology, health care, education, psychology, sociology) are often faced with the question of whether or not a particular sample is best described as unimodal or bimodal (e.g., Do \& McLachlan, 1984; Hoffmann \& Miller, 1998; Knoll, Garver, Ramberg, Kingsbury, Croissant \& McDermott, B., 1998; Johnson \& Yantis, 1995; Reischies, Schaub \& Schlattmann, 1996; Roeder, 1994; Sussman, 1999; Volbrecht, Nerger \& Harlow, 1997). Issues of bimodality surface in fields as diverse as astrophysics (Roeder, 1990) and medicine (Ottong \& Garver, 1997). Fundamentally, this is the question of whether the set of data was extracted from one population or two populations (note that one can ask this question for data that is between subjects or within a single subject). The generic terms unimodal and bimodal are too vague for analysis, so to make the question more specific (and therefore testable), one can ask, do the data represent a single unimodal normal population? or do the data better represent a bimodal mixture of two normal distributions?

The current work is focused on the development of a fairly simple, but general, procedure for testing such alternatives. It should be noted that, although the techniques developed herein are focused on putative mixtures of normal distributions, they can be applied, in principle, to the

Bradley Frankland is Assistant Professor, Psychology Department, Life Sciences Centre, Dalhousie University, Hailifax, Nova Scotia, Canada, B3H 4J1. E-mail him at franklan@is.dal.ca.His areas of expertise include computational modeling, statistical methods, and cognition. Bruno D. Zumbo. is Professor, Measurement, Evaluation, and Research Methodology, and Associate Member of the Department of Statistics, University of British Columbia. His areas of statistical research include performance characteristics of standard procedures (both parametric and nonparametric) under non-standard conditions, multivariate analysis, and statistical theories of measurement. comparison of any set of theoretical distributions. The choice of the normal distribution as the starting point was not arbitrary: If the data is unimodal, then it is likely that the normal distribution is at least a good approximation ${ }^{1}$ and if the data is bimodal representing two populations, then it is likely that the normal distribution is a good approximation for each population. Regardless of the choice of distribution, the main point is to present a methodology that permits any researcher to quickly and easily discover the most appropriate parent population for a given data set.

In broad strokes, mixture modeling concerns modeling a statistical distribution by a mixture (or weighted sum) of other distributions. This modeling strategy comes under a variety of names in various disciplines: unsupervised concept learning (in artificial intelligence), intrinsic classification (in philosophy), or, classification, clustering, and numerical taxonomy.

It is admitted, a priori, that the solution offered herein is not an analytical solution to the question of bimodality. The point was to develop an accessible, flexible and, most importantly, accurate method that could be used to test any number of hypotheses. To achieve accessibility, the commercially available statistical package SPSS was used: It could be added that any statistical package should be capable of a comparable level of analysis. Flexibility demanded that the procedure place few restrictions on the nature of the hypothetical parent population. Too often, methods for exploring multimodal population distributions require constraints that are outside the interests of the research at hand.

For example, it is often assumed that the parent population must be normal distributions or mixtures of such (e.g., Yellott, 1971); such an assumption may not be reasonable in all cases. Although the current work is focused on mixtures of normal distributions, the techniques can be applied to any distribution. At the other end of the spectrum, to avoid any assumptions of nature of the parent distributions, some researchers (e.g., Yantis, Meyer \& Smith; 1991) assumed that it possible to obtain, by empirical 
means, pure unimodal distributions (separate experiments are run to obtain data that is "purely" unimodal) from which the mixture distribution can be constructed. Even if/when possible, the requirement is unwieldy for many experimental designs, particularly for quasi-experimental designs. Flexibility has the added benefit of permitting the analysis to be tailored to the experimental design, rather than vice versa $^{2}$.

Although an attempt has been made to make these routines accessible, the desire for accuracy precluded the simpler solutions such as analyses based on increased kurtosis (e.g., Hoffman \& Miller, 1998), increased variance (Eriksen \& Eriksen, 1972) or a shift in the mean (e.g., Eriksen \& Yeh, 1985). Such solutions tend to be inadequate because they cannot distinguish a mixture from a non-normal, but unimodal and symmetric, distribution (e.g., the $t$ distribution or the Cauchy). To complicate the issue, a mixture distribution may appear to be unimodal and symmetric (see Yantis et al. for more extensive discussion of alternative approaches). Interestingly, an advantage of the SPSS routines is that they provide a wealth of statistics pertaining to the fit of the solution.

Method

A set of empirically determined data is compared to two hypothetical population distributions: the first population is a unimodal normal distribution and the second population is a bimodal mixture of two normal distributions. Several steps are required to fit each population to the empirical data. First, the empirical data must be binned to create a histogram. Second, this binned data must be compared, using the non-linear regression algorithm of $S P S S$, to each hypothetical population. Finally, the fits for the two populations must be compared. For reasons of space and clarity, the development of a rigorous likelihood-ratio test of the best choice will be presented in a forthcoming paper. In the usual case, a researcher will have a single, or limited number of data sets. Hence, procedures are demonstrated within that context.

The crucial, though often ignored, step involves binning the data to create a probability histogram that accurately represents the data distribution. Critically, the most appropriate bin size (aka: bin widths) must be chosen, because an inappropriate choice will actually result in the loss of information. When working with a single or limited number of data sets, this can most easily be done "by eye/ hand". Various statistical or spreadsheet packages will also provide one with binned data but it must be remembered that these packages are generally tailored more to the goal of a pleasing presentation (e.g., limiting the number of bins to 20) than to accurate representation. A commonly accepted guideline is that no bin should have less than 5 counts ${ }^{3}$. In addition, when choosing bin sizes by hand, one can vary the bin widths, using narrow bins in the centre of the distribution and wide bins at the edges of the distribution which might result in a more accurate representation while maintaining a reasonable minimum bin count. Regardless of how one achieves it, for subsequent analyses, the SPSS data file is expected to contain the following variables:

\begin{tabular}{|c|c|}
\hline binnum & $\begin{array}{l}\text { bin number (not actually used, but } \\
\text { useful for humans) }\end{array}$ \\
\hline bincnt & count per bin \\
\hline$x l$ & $\begin{array}{l}\text { bin lower limit in the original } \\
\text { scores }\end{array}$ \\
\hline$x c$ & bin centre in the original scores \\
\hline$x u$ & $\begin{array}{l}\text { bin upper limit in the original } \\
\text { scores }\end{array}$ \\
\hline totalcnt & $\begin{array}{l}\text { total counts (total number of data } \\
\text { points) }\end{array}$ \\
\hline
\end{tabular}

It must be noted that before fitting, the count within each bin will be converted to a probability because fitting is based upon the numerical integration of the normal distribution. Hence, one needs the total number of counts (number of data points) as well as the counts per bin. The variable total counts (totalcnt) will be the same for all bins, and could be entered using a compute statement. One could simply enter probabilities per bin in place of count per bin and total count. Pragmatically, however, since one must maintain many significant digits, it is often easier to enter two integers (bincnt and totalcnt) than one long real number (e.g., prob) - and one is less likely commit a data entry error with simple integers.

To fit the data to the population, the fitting algorithm converts the theoretical population distribution into a histogram with bin sizes that are matched to the bin sizes of the data (i.e., the real data determines the bins sizes for the theoretical population). Then, the procedure adjusts the population parameters so that the counts per bin in the binned theoretical histogram matches (as best as possible) the counts per bin in the binned data histogram. Fitting is accomplished by the non-linear regression routines provided by $S P S S$.

Generally, a non-linear regression algorithm has four basic components: (1) the data to be fitted, (2) the function to be fitted, (3) the free parameters of the function to be fitted, and (4) the error or loss function of the function to be fitted. In this work, the data to be fitted is the proportion per bin in the data histogram $\left(\mathrm{Y}_{\mathrm{i}}\right.$, where $\mathrm{i}$ is the bin number). The function to be fitted is the proportion of the hypothetical population distribution that should fall within each bin, i (recall that bin sizes are determined from the data histogram). These proportions were determined from the theoretical unimodal normal distribution or from the theoretical bimodal mixture of two normal distributions. In the case of the unimodal normal distribution, the free parameters to be fitted are, the mean $(\mu)$ and standard 
deviation $\left(F\right.$, or variance, $\left.F^{2}\right)$ :

$$
N(\mu, \sigma)=\frac{1}{\sqrt{2 \pi \sigma^{2}}} e^{\frac{-\left(Y_{1}-\mu\right)^{2}}{2 \sigma^{2}}}
$$

In the case of the bimodal mixture of two normal distributions, there is a mean $\left(:_{1},:_{2}\right)$ and a standard deviation $\left(\mathrm{F}_{1}, \mathrm{~F}_{2}\right)$ for each normal distribution, as well as, the mixture proportion (8; note many authors use $\mathrm{B}$ to denote this parameter, which can be confused with the constant $B=3.14$, while others use $\alpha$, which can be confused with Type 1 Error Rate):

$$
\begin{aligned}
B\left(\mu_{1}, \sigma_{1}, \mu_{2}, \sigma_{2}, \pi\right) & =\lambda * \frac{1}{\sqrt{2 \pi \sigma_{1}^{2}}} e^{\frac{-\left(Y_{1}-\mu_{1}\right)^{2}}{2 \sigma_{1}^{2}}}+(1-\lambda) * \frac{1}{\sqrt{2 \pi \sigma_{2}^{2}}} e^{\frac{-\left(Y_{1}-\mu_{2}\right)^{2}}{2 \sigma_{2}^{2}}} \\
& =\lambda * N_{1}\left(\mu_{1}, \sigma_{1}\right)+(1-\lambda)^{*} N_{2}\left(\mu_{2}, \sigma_{2}\right)
\end{aligned}
$$

The loss function assesses the deviation between the data and the population. Although the cnlr routine allows one to define the error (or loss) function, the default of leastsquares error was used, which is the same as that which is used in ordinary least-squares regression (OLS).

The fitting algorithm returns the best-fit parameters, given the data and the error function. In SPSS, there are two possible non-linear regression routines. The $n l r$ (non-linear regression) procedure uses the LevenbergMarguart algorithm for fitting the data to the function, while the cnlr (constrained non-linear regression) uses a sequential quadratic search algorithm that permits constraints to be placed on the parameters. The basic Marquart algorithm is arguably the most general fitting routine (Bevington \& Robinson, 1992 p. 164), but the ability of cnlr to place bounds on the values provides some additional stability (this is more important within Monte Carlo simulations), so the results presented here cite only the $c n l r$ approach.

Application to a Single Set of Data

The fitting of two different data sets - one unimodal and one bimodal - is demonstrated. Both data sets consisted of data generated by SPSS. Hereafter, the data from the unimodal normal distribution will be labeled as unimodal data, while data generated from a mixture of two normal distributions will be labeled as bimodal data. Similarly, the function defining the unimodal normal distribution will be labeled as the unimodal function while the function defining the mixture or two normal distributions will be labeled as the bimodal function.

\section{Unimodal Data}

A unimodal data set of 500 data points were obtained from a unimodal function (a normal distribution) defined as $\mathrm{N}(:, \mathrm{F})=\mathrm{N}(0,1)$ using the SPSS command NOR$M A L$, which generates standard Normal pseudo-random variates. The resulting distribution is shown in Figure 1, and with empirical mean 0.001 and standard deviation 0.967 (median: 0.003 ). The process of binning produced a range of 79 bins for 500 data points with 12 bins per standard deviation, but in fact, only 64 bins contained nonzero counts (see Figure 1): With 500 data points, one can

\author{
Listing 1 \\ Fitting Algorithm for the Bimodal Function \\ compute $\quad$ prop $=$ observed $/$ total. \\ model program \\ mean $=0.0 \mathrm{sd}=1.0 \mathrm{c}=0.0$. \\ compute \\ $\mathrm{xa}=\operatorname{abs}(\mathrm{xl}-\mathrm{xc})$. \\ compute \\ $\mathrm{xb}=\mathrm{abs}(\mathrm{xu}-\mathrm{xc})$. \\ compute \\ $\mathrm{h} 1=(.398942 / \mathrm{sd}) * \exp \left(-\left(((\mathrm{xl}-\mathrm{mean}) * * 2) /\left(2 * \mathrm{sd} \mathrm{d}^{* *} 2\right)\right)\right)$. \\ compute \\ $\mathrm{h} 2=(.398942 / \mathrm{sd}) * \exp (-(((\mathrm{xc}-\mathrm{mean}) * * 2) /(2 * \mathrm{sd} * * 2)))$. \\ compute \\ $\mathrm{h} 3=(.398942 / \mathrm{sd}) * \exp \left(-\left(((\mathrm{xu}-\mathrm{mean}) * * 2) /\left(2^{*} \mathrm{sd}^{* *} 2\right)\right)\right)$. \\ compute \\ cnlr \\ preduni $=\left(.5 *(\mathrm{~h} 1+\mathrm{h} 2)^{*} \mathrm{xa}+.5^{*}(\mathrm{~h} 2+\mathrm{h} 3)^{*} \mathrm{xb}\right)+\mathrm{c}$. \\ prop \\ /bounds sd gt 0.0001 \\ $/$ pred $=$ preduni \\ $/$ save $=$ preduni residuni.
}


expect to obtain a few high z-scores, and consequently, bins in the tails that contain no counts. The algorithm only considers bins with non-zero counts: More will be said on this point at the end of this paper. The binned mean, 0.001 , and standard deviation, 0.967 (median: 0.001 ) were not different, providing a rudimentary check on the binning process. Such a check is more important when there are fewer data points.

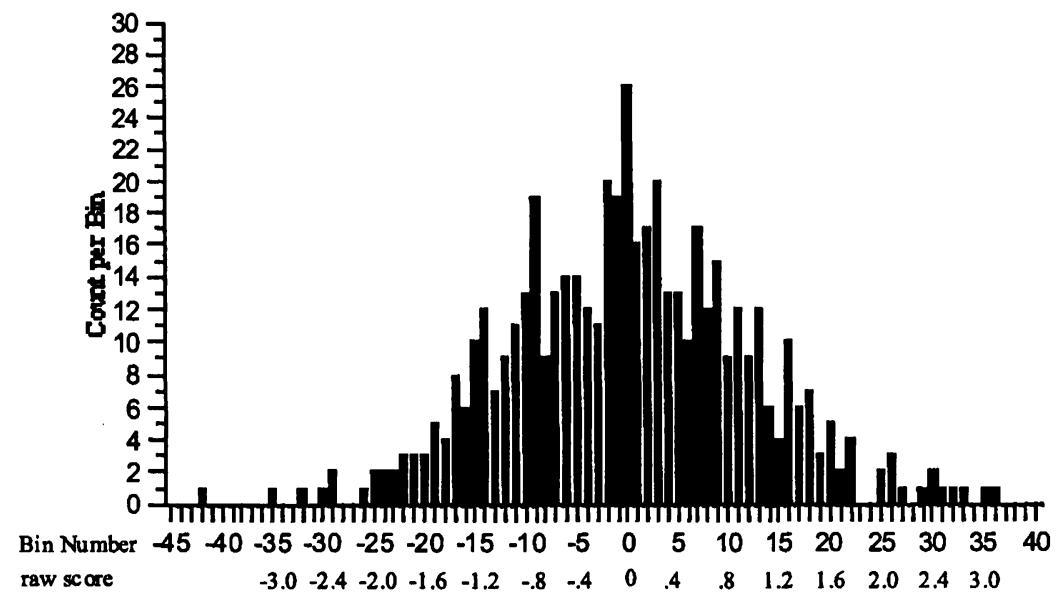

Figure 1 The unimodal distribution.

The first compute command simple converts the observed counts per bin into a proportion within each bin (percentages could also be used and would provide more significant digits in the output on some statistics, but percentages would require adjustments to the formula for the normal distribution).

All the compute statements between the model program and cnlr commands are setting up the fitting algorithm: All of these could be collapsed into one line, but it would not be as readable. Essentially, as shown in Figure 2 , the area defined by each bin (i.e., the integration) under the normal curve is computed: This area is the probability or proportion of scores that should fall into that bin. There are numerous ways to obtain this area, but in this case, it is computed using the trapezoidal rule for numerical integration: In this case, the proportion is computed from the sum of two trapezoid rule integrations, one for the area from the bin lower limit to the bin centre and one for the bin centre to the bin upper limit (hence, the $h 1, h 2$, and $h 3$ of the compute statements refer to the heights of the curve at the lower limit, centre and upper limit of the bin). The use of two trapezoids should simply provide greater resolution, particularly if a bin should happen to cover a large range (in units of standard deviations). It should be noted that, in the end, what really matters is the prediction per bin (the variable preduni) - how one generates the prediction is flexible. In fact, the use of a single trapezoid did not alter the results dramatically.

A constant term, $\mathrm{c}$, is included in the model to insure that the residuals sum to zero: It has minimal effect on the parameters returned or the fit. More is said on the rationale for its inclusion in a forthcoming paper.

It is the cnlr command that actually runs the analysis, using the previous information, subject to the constraints that follow (/bounds, /pred, and /save). Basically, cnlr tries to match the actual data (the variable prop) to the predictions of the model (the variable preduni) by adjusting the values of mean and sd (and c). It is the subcommand /pred that creates and names the predicted variable. The adjustment is performed iteratively - small changes are made to all variables, the fit is computed, and if the fit does not improve "substantially" with the new values, the processes stops.

Because the process is iterative, the program needs a starting value for all of the parameters that are to be adjusted. The model program command identifies the parameters to be adjusted and set their initial (starting) values. It is important to have good starting values: Poor starting values may result in no solution, or worse, in a "second best" solution that can masquerade as the correct solution. In a similar fashion, the subcommand /bounds allows one to set limits on the values of the fitted parameters. This can prevent the algorithm from drifting into a local solution, producing ridiculous values. In this case, since variances can never be less than zero, zero is used as a lower bound.

Finally, the subcommand /save preduni residuni saves and names the predicted scores and the residual scores onto the original data file. It is the residuals that are the key to the algorithm. The algorithm works by trying to minimize the residual. In fact, the algorithm minimizes: 


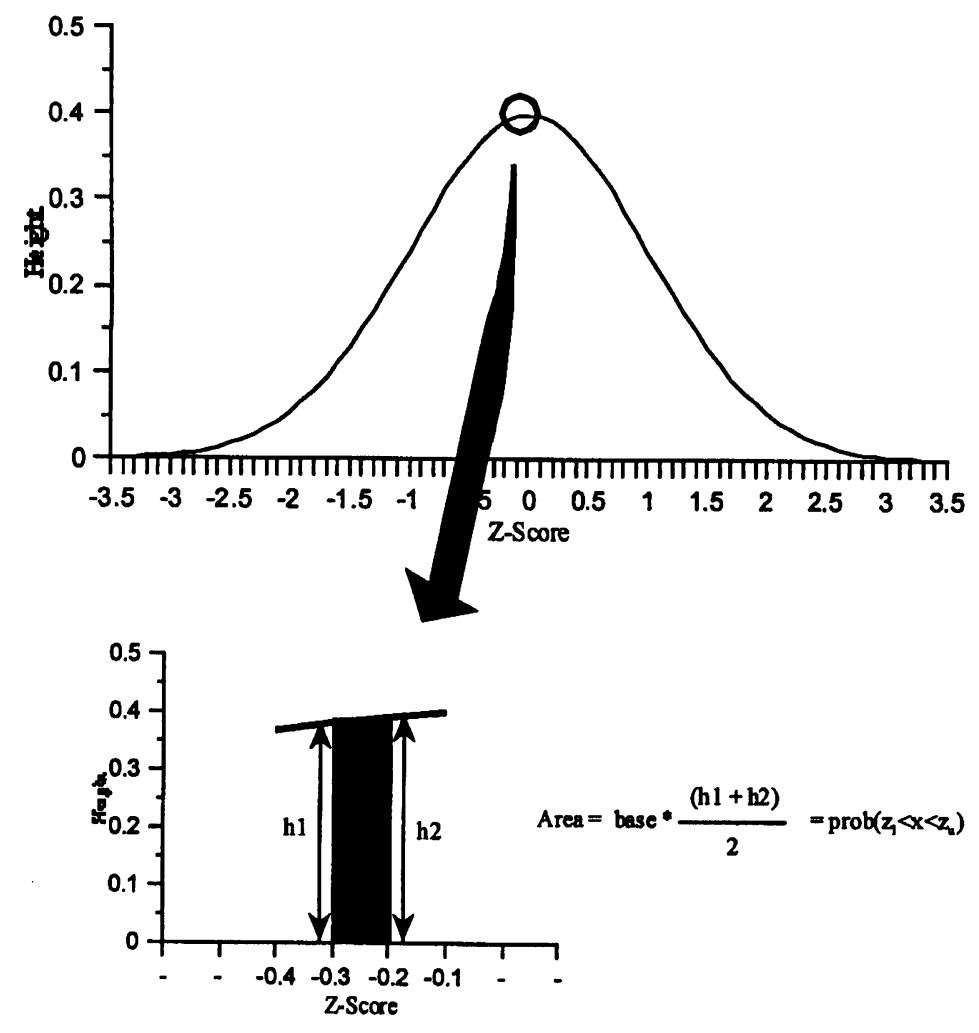

Figure 2 The trapezoid rule for obtaining the area under the curve (numerical integration). The accuracy of the integration depends on the magnitude of the base and the shape of the curve.

error $=\Sigma\left(Y_{\text {data }}-Y_{\text {predicted }}\right)^{2}=\Sigma\left(Y_{i}-\hat{Y}\right)^{2}=\Sigma$ residual $^{2}$ which is essentially the same error term as is used in any OLS regression. The only real difference (in this context) between the more common linear regression (the regression command in SPSS), and non-linear regression (cnlr) is that non-linear regression uses an iterative (trial and error) process.

In the case of the current unimodal data set, the algorithm returned a mean of $-0.008 \pm 0.044$, a standard deviation of $0.956 \pm 0.037$ and a constant of $0.000 \pm 0.001$ (cited errors are the standard errors of fit, analogous to the standard error on a slope in linear regression) These values, within the defined confidence intervals, are the same as the original measures.

In linear regression, one maximizes the fit between the predicted and actual score by minimizing the sum of the squared residuals. The measure of fit can be defined by numerous statistics, with $\mathrm{s}_{\text {error }}, \mathrm{R}_{\mathrm{Yi} \text { i. }}^{2}$ and $\mathrm{P}_{\mathrm{df}}^{2}$ being the most common. Similar measures can be used here and, in fact, the $c n l r$ and $n l r$ algorithms actually provide $\mathrm{SS}_{\text {error }}$ (hence, $\mathrm{s}_{\text {error }}^{2}$ ) and $\mathrm{R}_{\mathrm{Y}, \mathrm{i}}^{2}$ directly.

$\mathrm{SS}_{\text {error }}$ can be used on its own as an estimate of the degree of fit (Equation 3). Ideally, $\mathrm{SS}_{\text {error }}$ should be small, but beyond that, one cannot say much about the predicted value of $\mathrm{SS}_{\text {error }}$ : It depends on the underlying distribution, and scale of the data.

Another measure of fit is $\mathrm{R}_{\mathrm{Yi}}^{2}$, which is the correlation between the predicted proportion, or count, per bin $\left(\grave{i}_{\mathrm{i}}\right)$ and actual proportion, or count, per bin $\left(\mathrm{Y}_{\mathrm{i}}\right)$. It is completely analogous to $\mathrm{R}_{\mathrm{Y}, \mathrm{i}}^{2}$ in the more common linear regression, and it is essentially:

$$
\begin{gathered}
R_{Y . Y}^{2}=1-\frac{S S_{\text {error }}}{S S_{\text {total }}} \\
\text { where } \quad S S_{\text {error }}=\Sigma\left(Y_{i}-\hat{Y}\right)^{2} \\
S S_{\text {total }}=\Sigma\left(Y_{i}-\bar{Y}\right)^{2} \\
\bar{Y}_{i}=\text { the mean proportion }
\end{gathered}
$$


Because $R^{2}$ is just a correlation, it can be tested for significance, using the traditional Type I error rate of $\alpha=0.05$. However, little faith should be placed in "significance" since, in principle, one would not be testing a model that did not have some hope of fitting the data: It is the magnitude of $R^{2}$ that matters, particularly, the relative magnitude when comparing models. As shown in Figure 3, what is actually tested by $R^{2}$ is the nature of the relationship between the predicted bin proportion (or count) and the actual bin proportion (or count). For $R^{2}$ to apply, this relationship should be linear. Given that $R^{2}$ is affected by outliers, one should carefully check the tails of the distribution (theoretical and/or actual) for such points. In addition, non-linearities in the plot imply that the model is not a good match to the data, regardless of the magnitude of the $R^{2}$. In this case, the plot seems reasonable. Note that the standard residuals plots will provide the same information.

A third traditional test of the fit of a sample to a theoretical function is that of the $\chi_{\mathrm{df}}^{2}$ distribution, which uses actual counts (not proportions):

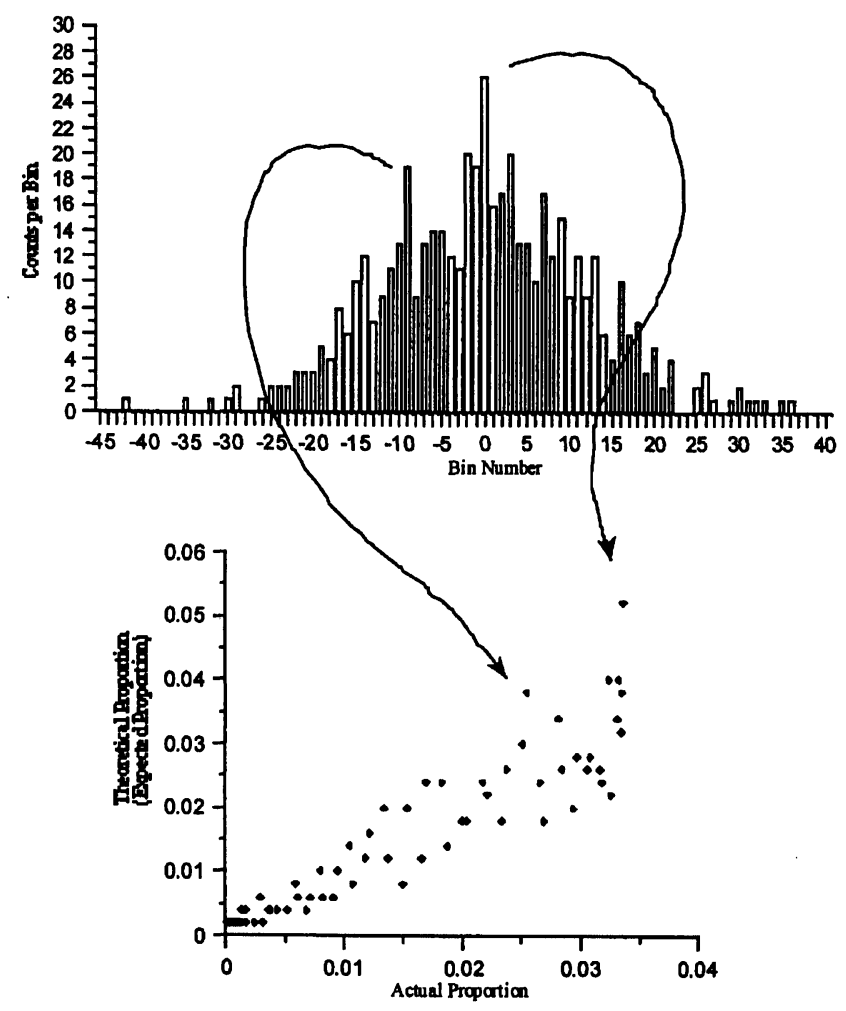

Figure 3 The test provided by $R^{2}$ compares theoretical bin counts (proportions) to actual bin counts (proportions). Note that the relationship is relatively linear, but that the variance is not perfectly constant.

$\chi_{d f}^{2}=\frac{\Sigma\left(C_{\text {obs }}-C_{\text {pred }}\right)^{2}}{C_{\text {pred }}}$

where $\quad C_{o b s}=$ the observed counts in each bin

$C_{\text {pred }}=$ the predicted counts in each bin

$d f=\#$ bins -2 since 2 parameters are estimated 
Essentially, if the sample is a good fit to the function, then the value of the $\chi_{\mathrm{df}}^{2}$ should be small. Traditionally, one uses a type 1 error rate of $\alpha=0.05$ that assumes that if the $\chi_{\mathrm{df}}^{2}$ is one of those values that falls into the upper $5 \%$ of the distribution, then the theoretically-derived distribution is not a good match to the data.

For the current data, $\mathrm{SS}_{\text {error }}=0.0015$ and $R_{Y Y Y}^{2}=$ 0.848 with an associated $F(1,62)=346.429(p<.000)$. Both $\mathrm{SS}_{\text {error }}$ and $R_{\mathrm{YYY}}^{2}$ are directly available within the output of the $c n l r$ (or $n l r$ ). The value of $\chi^{2}$ must be computed separately using the actual bin score and the saved predicted values. Here, $\chi_{62}^{2}=76.118(p>.100)$ meaning that the model does not deviate significantly from the data.

There is one crucial point that has been mentioned previously, is that in this fitting, bins with zero counts (see Figure 1) were not included in the analysis. The results might be different if the zero-count bins (particularly zerocount bins flanked by non-zero-count bins) were included. This is an issue that will be returned to in a forthcoming paper.

\section{Bimodal Data}

A bimodal data set consisting of 500 data points was generated from a bimodal function (a mixture of two normal distributions) defined as $B\left(\mu_{1}, \sigma_{1}{ }^{2}, \mu_{2}, \sigma_{2}{ }^{2}, \lambda\right)=$ $B(-1.0,1.0,1.0,1.0,0.5)$. Note that the population means were placed symmetrically around 0.0 , that the variances were equal and that the mixture proportion was 0.50 . Hence, by design, the mean of the distribution should be zero (a quick, but useful, check). The resulting distribution is shown in Figure 4, had a mean of 0.063 and standard deviation of 1.44 before binning (median: 0.091) and a mean of 0.064 and a standard deviation of 1.44 after binning (median: 0.063 ). The process of binning produced a range of 75 bins but only 58 contained non-zero counts (see Figure 4).

This data was then fit to the bimodal function (the mixture of two normal distributions). In this case, there are five parameters that are free to vary: two means $\left(\mu_{1}, \mu_{2}\right)$, two variances $\left(\sigma_{1}^{2}, \sigma_{2}^{2}\right)$, and the mixture proportion $(\lambda)$ as well as a constant. The algorithm is more complicated, but it is essentially the same as before. Note that the fitted statistics should reproduce the original population, with sampling error, as well as, drift due to binning. The fitting algorithm is shown in Listing 2.

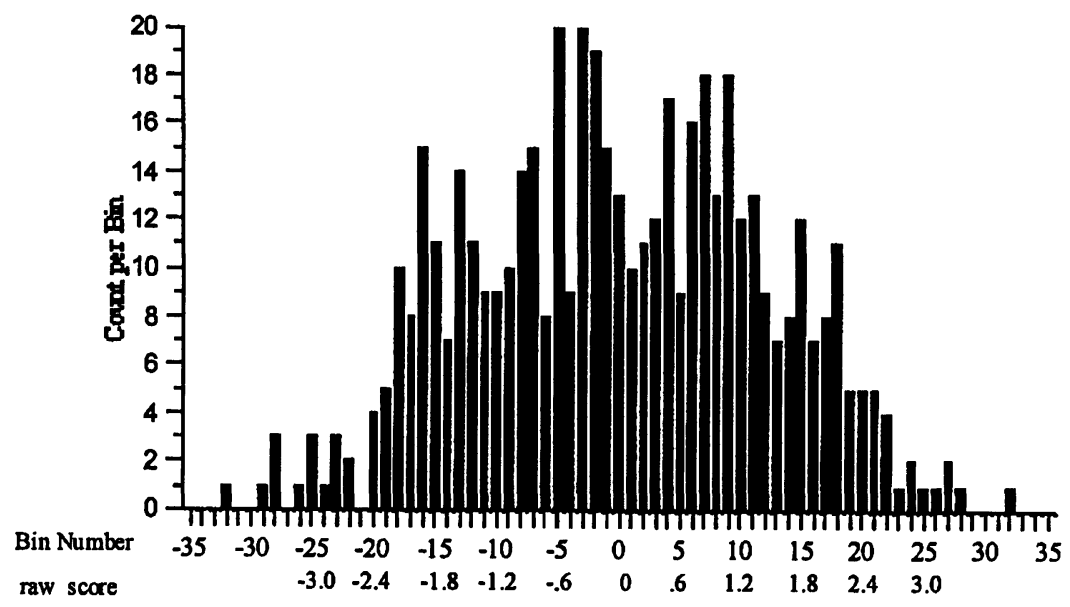

Figure 4 The bimodal distribution.

Listing 2

Fitting Algorithm for the Bimodal Function

compute $\quad$ prop $=$ observed/total $* 100$.

model program mean $1=-2.0$ mean $2=1.0 \mathrm{sd} 1=2.0 \mathrm{sd} 2=1.0$ ratio $=0.5 \mathrm{c}=0$.

compute $\quad \mathrm{xa}=\mathrm{abs}(\mathrm{xl}-\mathrm{xc})$.

compute $\quad x b=a b s(x u-x c)$.

compute $\quad \mathrm{h} 1=(.398942 / \mathrm{sd} 1) * \exp ((((\mathrm{xl}-\mathrm{mean} 1) * * 2) /(2 * \mathrm{sd} 1 * * 2)))$ ).

compute $\quad \mathrm{h} 2=(.398942 / \mathrm{sd} 1) * \exp \left(\left(\left((\mathrm{xc}-\mathrm{mean} 1)^{* *} 2\right) /(2 * \mathrm{sd} 1 * * 2)\right)\right)$.

compute $\quad \mathrm{h} 3=(.398942 / \mathrm{sd} 1) * \exp \left(\left(\left((\mathrm{xu}-\mathrm{mean} 1)^{* *} 2\right) /(2 * \mathrm{sd} 1 * * 2)\right)\right)$ ).

compute $\quad \mathrm{h} 4=(.398942 / \mathrm{sd} 2) * \exp \left(\left(\left((\mathrm{xl}-\text { mean } 2)^{* *} 2\right) /(2 * \mathrm{sd} 2 * * 2)\right)\right)$. 


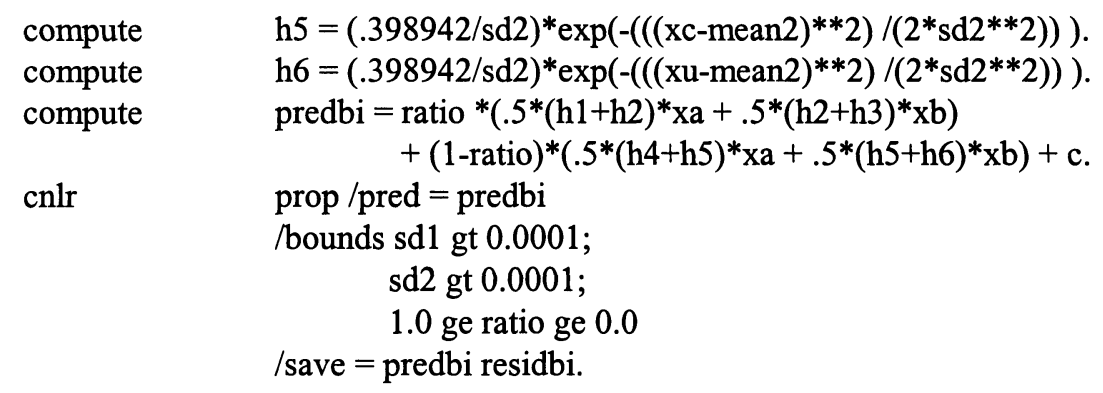

As before, the first compute command simply converts the observed counts per bin into a proportion. The compute commands between the model program and cnlr commands create the model. Again, in principle this could be done in one line. The model program command defines the parameters to be fitted and sets their initial values. The cnlr command defines the dependent variable, sets some bounds on the parameters and sets the routine to save the predicted and residual. The difference between this and the previous unimodal case is one of increasing complexity: There are no substantive changes.

In the case of the current bimodal data set, the values returned by the algorithm were $-1.140 \pm 0.828$ for first mean, $1.047 \pm 0.411$ for the first standard deviation, $0.967 \pm 0.746$ for the second mean, $1.049 \pm 0.366$ for the second standard deviation, $0.473 \pm 0.406$ for the mixing proportion, and $0.000 \pm 0.001$ for the constant (cited errors are the standard errors of fit analogous to the standard error on a slope in linear regression). The algorithm returns the original population parameters (within the defined confidence limits).

For the bimodal data, $\mathrm{SS}_{\text {error }}=0.0018$ and $\mathrm{R}_{\mathrm{Y} \cdot \hat{\mathrm{Y}}}^{2}$ $=0.751$ with an associated significance of $F(1,53)=168.928$ $(p<.000) \cdot \chi_{53}^{2}=46.277(p>.950)$, which implies that the model does not differ significantly from the data. Again, it must be emphasized that bins with zero counts (see Figure 4) were not included in the computations and, as such, result might be different if they were.

\section{Cross Fits}

In practice, one would not know whether the unimodal or bimodal function provided the proper fit to the data. To address this question, it is necessary to fit the data with both functions so that the best choice may be obvious. In an ideal world, the unimodal data will fit a unimodal function better than a bimodal function, and the bimodal data will fit the bimodal function better than the unimodal function. Hence, the previous unimodal data was fitted to the bimodal function and the previous bimodal data was fitted to the unimodal function.

The fitting of the unimodal data to the bimodal function produced $-0.016 \pm 0.091$ for first mean, $0.911 \pm$ 0.149 for the first standard deviation, $4.912 \pm 22.797$ for the second mean, $2.771 \pm 54.287$ for the second standard deviation, and $0.927 \pm 0.362$ for the mixing proportion. The constant was near zero, $0.001 \pm 0.003$. The bimodal fit suggests that the data can be modeled by the proper parent distribution centered at 0 and a wider distribution centered five standard deviations above the mean. The wider distribution provides only $7 \%$ of the data. Effectively, the bimodal function finds a unimodal distribution.

The fitting of the bimodal data to the unimodal function produced a mean of $-0.009 \pm 0.097$ and a standard deviation of $1.620 \pm 0.083$, with a constant near zero, $0.001 \pm 0.001$. These values were within expectation given that $\mu_{\text {bimodal }}=\lambda * \mu_{1}+(1-\lambda) * \mu_{2}=0.00$ and $\sigma_{\text {bimodal }}^{2}=\lambda$ $* \sigma_{1}^{2}+(1-\lambda) * \sigma_{2}^{2}+\lambda *(1-\lambda) *\left(\mu_{1}-\mu_{2}\right)^{2}=2.00$ Hence, fitting the bimodal data with a unimodal function finds a single distribution centered at zero, which is

Table 1

Fitting the unimodal and bimodal data with the unimodal and bimodal functions.

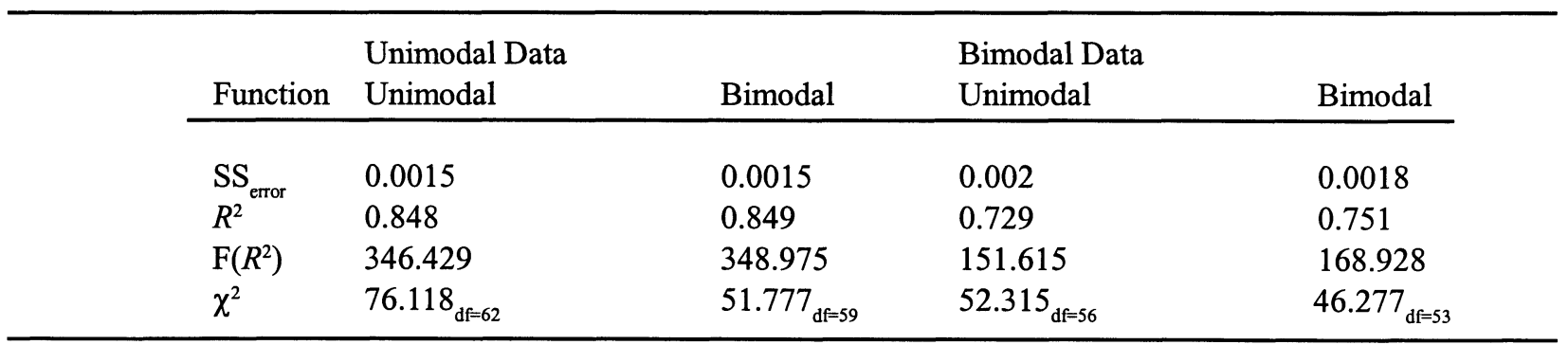


reasonable given that the data were symmetric about zero.

In many cases, the actual parameters produced are less important than the fits of the data to the alternative functions. Table 1 provides $\mathrm{SS}_{\text {error }}, R_{Y . Y}^{2}$ and $\chi_{\mathrm{df}}^{2}$ for the unimodal data fitted to both functions and for the bimodal data fitted to both functions. In the case of the unimodal data, both functions seem to fit equally well. As such, there would be no grounds for arguing that the function is bimodal (parsimony). In the case of the bimodal data, the bimodal function provides the better fit.

\section{Conclusion}

This work has been a demonstration of the application of commonly available statistical software, in this case SPSS, to solving the problem of assessing putative mixture distributions: particularly, decisions concerning a unimodal normal distribution or a bimodal mixture of two normal distributions. Routines were developed to enable anyone to determine the best-fit statistics for fitting data to a unimodal normal distribution or a bimodal mixture of two normal distribution.

The method presented has several advantages. The most important advantage is that the method is not limited to normal distributions or mixtures of two normal distributions. There is unlimited flexibility in the choice of fitting functions. In fact, the fitting function does not have be continuous (however, non-linear regression is best done with continuous functions). As noted previously, one only needs to be able to create a predicted bin count that can be compared to the actual bin count. The predicted bin count can be based on any function. The likelihood test to be presented later also has this advantage.

The second advantage is that one can modify the algorithm to obtain greater accuracy. Non-linear regression using a least-squares error term assumes that the error is a constant for all values of the independent variable. If bins have variable counts (or proportions), the error per bin is not constant. This could be a factor in the fit if the range of counts is large. SPSS non-linear regression allows one to specify the error term. As such, one could switch to weighted least-squares (non-linear) regression if greater accuracy is demanded. This change would also help to alleviate the problem of bins with low counts. It has not been presented in this work because it adds an additional layer of complication thereby obscuring the main points.

The third advantage is related to the first two. In particular situations, some parameters can be fixed. For example, the two variances may set to be equal, or either variance could be set to a constant (e.g., 1.0) or the mixture proportion may be fixed (e.g., 0.5). This would effectively improve the fit for the remaining parameters (assuming that the alterations were appropriate to the theoretical distribution) because it would increase the $\mathrm{df}$ per parameter. Such an approach might be more useful when there are only a few data points (or bins). In a similar fashion, the regression model has the flexibility to include a constant, or to not include a constant. If there is no constant, the model assumes that the regression line goes through the point $(X, \hat{Y})=(0,0)$. If the model does not include a constant when it should, then the resulting fit will be biased (i.e., the sum of the residuals will not be zero). In this work, it is arguable that the model does not need a constant because the $(0,0)$ point can be assumed (i.e., the predicted value should be zero, when the bin is empty). The caveat is that this is not strictly true when dealing with binned data. That is, bins with counts of zero may correspond to a non-zero theoretical probability. In fact, given a normal parent population, no bin should ever have a zero theoretical probability. Hence, an actual bin count of zero will correspond to some non-zero theoretical bin count (this will be small, but non-zero). As such the inclusion of a constant is prudent. In general, we expect that only $\chi^{2}$ will be dramatically affected by the removal of the constant $\left(\chi^{2}\right.$ can be very large when there is no constant, implying that the model would be biased without the constant).

In summary, the routine works; however, it must be cautioned that this algorithm is only considered an interim solution to the problem - one of many (cf., Eriksen \& Eriksen, 1972; Eriksen \& Yeh, 1985; Hartigan, 1974; Jones \& McLachlen, 1990; Müller \& Sawitzki, 1991; Roeder, 1990, 1994; Yantis, Meyer and Smith, 1991; Yellott, 1971). Hopefully, a proper fully parametric method for assessing bimodality will be developed, one that extracts all the information contained within each individual data point rather than working through the intermediary of a histogram. As has been shown, if not done carefully, the process of binning can alter the data. On the other hand, even if a proper parametric method is developed, it will necessarily be tied to particular parent distributions. As such, the algorithms developed herein will continue to serve some purpose with other parent distributions. We have ongoing research studying the methods presented herein in simulations. Forthcoming papers will discuss matters of fit indices and bin size.

\section{References}

Bevington, P. R., \& Robinson, D. K. (1992). Data Reduction and Error Analysis for the Physical Sciences. Toronto: McGraw Hill, Inc.

Do, K., \& McLachlan, G. L. (1984). Estimation of mixing proportions: A case study. Applied Statistics, 33 (2), 134-140.

Eriksen, C. W., \& Yeh, Y. Y. (1985). Allocation of attention in the visual field. Journal of Experimental Psychology: Human Perception and Performance, 11 (5), 583-597. 
Eriksen, C. W., \& Eriksen B. A. (1972). Visual backward masking as measured by voice reaction time. Perception and Psychophysics, 12 (1), 5-8.

Hartigan, J. A. (1974). Asymptotic distributions for clustering criteria. The Annals of Statistics, 6 (1), 117131.

Hayes, W. L. (1994). Statistics. NY: Holt, Rinehart and Winston.

Hoffman, J. P., \& Miller, A. S. (1998). Denominational influences on socially divisive issues: Polarization or continuity? Journal for the Scientific Study of Religion, 37 (3), 528-546.

Howell, D. (1995). Statistical Methods for Psychology. NY: Duxbury Press.

Johnson, D. N., \& Yantis, S. (1995). Allocating visual attention: Tests of a two-process model. Journal of Experimental Psychology: Human Perception \& Performance, 21 (6), 376-1390.

Jones, P. N., \& McLachlan, G. J. (1990). Algorithm AS 254: Maximum likelihood estimation from grouped and truncated data with finite mixture models. Applied Statistics, 39 (2), 273-312.

Knoll, J. L. IV, Garver, D. L., Ramberg, J. E., Kingsbury, S. J., Croissant, D., \& McDermott, B. (1998). Heterogeneity of the psychoses: Is there a neurodegenerative psychosis? Schizophrenia Bulletin, 24 (3), 365-379.

Müller, D. W., \& Sawitzki, G. (1991). Excess mass estimates and tests for multimodality. Journal of the American Statistical Association, 86, 738-746.

Ottong, S. E., \& Garver, D. L. (1997). A bimodal distribution of plasma HVA/MHPG in the psychoses. Psychiatry Research, 69, 97-103.

Reischies, F. M.., Schaub, R. T., \& Schlattmann, 3 P. (1996). Normal ageing, impaired cognitive functioning and senile dementia: A mixture distribution analysis. Psychological Medicine, 26 (4), 785-790.

Roeder, K. (1990). Density estimation with confidence set exemplified by superclusters and voids in the galaxies. Journal of the American Statistical Association, $85,617-624$.
Roeder, K. (1994). A graphical technique for determining the number of components in a mixture of normals. Journal of the American Statistical Association, $89,487-495$.

Sussman, H. M. (1999), A neural mapping hypothesis to explain why velar stops have an allophonic split. Brain and Language, 70 (2), 294-304.

Volbrecht, V. J., Nerger, J. L., \& Harlow, C. E. (1997). The bimodality of unique green revisited. Vision Research, 37 (4), 407-416.

Yantis, S., Meyer, D. E., \& Smith, J. E. K. (1991). Analysis of multimodal mixture distributions: New tests for stochastic models of cognition and action. Psychological Bulletin, 110, 350-374.

Yellott, J. I. (1971) Correction for fast guessing and the speed-accuracy tradeoff in choice reaction time. Journal of Mathematical Psychology, 8 (2), 159-199.

\section{End Notes}

The normal distribution can be considered a reasonable approximation by virtue of the central limit theorem. In addition, the limiting cases of the binomial, $\chi^{2}$, Poisson, $t$ and their derivatives are approximately normal.

2 Those who think this is not a serious concern should contemplate the standard use of ANOVA: Many psychological variables, when used as independent factors, have been forcefully cast as categorical in order to fit the analysis (e.g., clinical categories such as depression, drug levels); it is now difficult to present them in their true continuous form.

The determination of "some reasonable number" depends on the author: Ten for Bevington and Robinson (1992, p. 109), five to ten for Hayes (1994, p. 862), and five for Howell (1995, p. 115, 141). Values from Hayes and from Howell assume a $\chi^{2}$ distribution within each cell, while those of Bevington and Robinson assume a Poisson distribution within each cell. 


\section{Asymptotic And Exact Tests In $2 \times \mathrm{C}$ Ordered Categorical Contingency Tables With StatXact 2.0 - 4.0 \\ Margaret Posch \\ Office of the Dean \\ College of Education \\ Wayne State University}

The purpose of this study was to compare the statistical power of a variety of exact tests in the $2 \times \mathrm{C}$ ordered categorical contingency table using StatXact software. The Wilcoxon Rank Sum, Expected Normal Scores, Savage Scores (or its Log Rank equivalent), and Permutation tests were studied. Results indicated that the procedures were nearly the same in terms of comparative statistical power.

Keywords: Ordered-categorical contingency tables, 2 x C, Statistical power, Nonparametric tests

\section{Introduction}

The purpose of this study was to compare the statistical power of a variety of exact tests in the $2 \times \mathrm{C}$ ordered categorical contingency table using StatXact software. The Wilcoxon Rank Sum, Expected Normal Scores, Savage Scores (or its Log Rank equivalent), and Permutation tests were studied. Results indicated that the procedures were nearly the same in terms of comparative statistical power.

The development and wide-spread availability of personal computers with increased power in the early 1980 s to the present have provided user-friendly statistical packages which make it possible for the applied researcher to easily carry out computation-intensive statistical procedures with high-speed and accuracy. Randomization and permutation tests, examples of computer-intensive procedures, yield exact $p$ values instead of asymptotic $p$ values. Mehta and Patel (1995) underscored the importance of using exact $p$ values with an example, where the asymptotic Pearson Chi Squared $\left(\chi^{2}\right)$ test for row and column interaction in a $3 \times 9$ contingency table produced an observed test statistic of $\chi^{2}=22.29$. The $p$ value associated with this obtained value for $v(16)$ is 0.1342 . However, the $p$ value associated with the exact distribution of $\chi^{2}$ for the tail area to the right of 22.29 is 0.0013 , which indicates there was a significant row and column interaction in the contingency table. This clearly demonstrates the power superiority of using the exact $p$ value.

There are other reasons to support the preference of randomization and permutation tests over asymptotically-derived procedures in applied small samples research.

Margaret Posch, is Assistant Professor-Research, Office of the Dean, College of Education at Wayne State University. Her expertise is in research methods and statistical analysis in school transitional programs. She has served two terms as President of the Detroit Chapter of the American Statistical Association. Contact her at m.posch@wayne.edu.
Consider again the asymptotic $\chi^{2}$ test. It is well known that for sparse contingency tables, "the usual chi-squared asymptotic distribution... is not likely to yield accurate p-values" (Mehta \& Patel, 1995, p. 577). Moreover, the nature of small samples research lends itself to one or more (if not most) cells with expected values less than five. The statistical literature is replete with warnings about conducting the asymptotic $\chi^{2}$ test under these conditions. Some authors protest if even a single cell has an expected frequency of less than five, though others permit up to twenty percent of the cells with low expectancies.

The analysis of continency data, which results in the commonly applied asymptotic $\chi^{2}$ test, is frequent in behavioral and educational research. Of particular interest in this article is the ordinal categorical layout, which is comprised of two categorical groups with ordinal level outcomes. For example, in a study on the research experiences of doctoral students and publication rates after graduation, Troup-Leasure, Eichelberger, and Zigmond (1992) analyzed a $2 \times 3$ layout of apprenticeship (yes or no) and rate of publication per year (zero, less than one, one or more). Grissom (1994) examined a $2 \times 3$ layout of two types of marital therapies for which three levels of outcome (divorce, no change, improve) occurred. Frequently, in applied ordinal categorical studies such as these, the asymptotic $\chi^{2}$ or asymptotic Wilcoxon test is calculated, or the results are analyzed solely with descriptive statistics such as percentages or correlations.

\section{The Current Study}

Given the propensity of the $2 \mathrm{x} c$ ordinal categorical layout in applied research, the purpose of this study was to compare the statistical properties of computer-intensive nonparametric tests. The competing tests were the asymptotic $\chi^{2}$, which was included for comparison purposes, and the computer-intensive exact versions of the following four tests: Wilcoxon Rank Sum(WRS), Expected Normal Scores (ENS), Savage Scores (SS) or its Log Rank 
equivalent, and Permutation (P).

\section{Methodology}

Considerable small samples research has been conducted on the properties of various statistics using pseudo-random number generators to model real data sets. However, Micceri (1989) pointed out the "need for careful data scrutiny prior to analysis" (p.161), and therefore, real data sets were obtained for the current study using the sampling plan discussed below.

\section{Sampling Plan}

Twenty-nine education and psychology journals were canvassed, primarily from the four year period of 1992 - 1996. A list of journals, the number of articles in which 2 $\mathrm{x} c$ data sets were found, and the number of data sets, are compiled in Table 1 . The competing tests (asymptotic $\chi^{2}$, and the exact WRS, ENS, SS, and P) were then applied to the data sets obtained from these studies.

The software used was StatXact 2.0-4.0 (Gajjar, Mehta, Patel, \& Senchaudhuri, 1992) for exact nonpara- metric inference. (Note that version 5.0 has subsequently been released.) Results below permit a direct comparison of these statistics on real data commonly found in social and behavioral science research. The hypotheses being tested in all cases is the test for interaction. In some situations, this is a test of independence with regard to the outcome variable. In other cases, the data sets are simply cross tabulations of demographic variables.

\section{Results}

The survey yielded 149 ordinal categorical data sets of a 2 $\mathrm{x} c$ design collected from 73 articles published in refereed journals. Four distinct formats were encountered in the data: 1) the simple $2 \times c$ design; 2) a $4 \times c$ design, which consisted of a table with two distinct sets of two categories. For example, Table 2 shows four categories of psychological problems according to grade levels (used ordinally). For this data set, two $2 \times c$ tables were arbitrarily constructed to include Emotional and Conduct as the categories of the first table and ADD and LD as the categories in the second $2 \times c .3$ ) Nested designs were handled

Table 1. Education and Psychology Journals from 1992 - 1996 Canvassed.

\begin{tabular}{|c|c|}
\hline Psychology (\# of Articles, Data Sets) & Education (\# of Articles, Data Sets) \\
\hline American Journal of Community & American Educational Research Journal $(2,8)$ \\
\hline Psychology $(1,1)$ & Education $(1,2)$ \\
\hline American Journal of Family & Educational Researcher $(1,1)$ \\
\hline Therapy $(0,0)$ & Harvard Educational Review $(1,1)$ \\
\hline American Journal of Psychology $(0,0)$ & Journal for the Education of the Gifted $(1,2)$ \\
\hline Basic and Applied Social & Journal for Research in Mathematics Education $(3,3)$ \\
\hline Psychology $(0,0)$ & Journal of Negro Education $(1,8)$ \\
\hline Developmental Psychology $(0,0)$ & Journal of Special Education $(1,1)$ \\
\hline Educational Psychologist $(0,0)$ & Journal of Teacher Education $(0,0)$ \\
\hline Journal of Applied Psychology $(1,4)$ & Teacher Education and Special Education $(0,0)$ \\
\hline $\begin{array}{l}\text { Journal of Clinical Child } \\
\text { Psychology }(1,2)\end{array}$ & \\
\hline Journal of Consulting and Clinical & \\
\hline Psychology $(1,5)$ & \\
\hline Journal of Psychology: Interdisciplinary & \\
\hline and Applied $(3,4)$ & \\
\hline Perceptual and Motor Skills $(14,19)$ & \\
\hline Psychological Bulletin $(0,0)$ & \\
\hline Psychological Reports $(14,29)$ & \\
\hline Psychological Science $(2,5)$ & \\
\hline Psychology and Aging $(4,7)$ & \\
\hline Psychology in the Schools $(2,2)$ & \\
\hline Professional Psychology: Research & \\
\hline and Practice $(2,4)$ & \\
\hline Reading Psychology $(2,2)$ & \\
\hline School Psychology International $(1,5)$ & \\
\hline
\end{tabular}


as multiple data sets. For example, Cherian (1992) reported parental education on an ordinal scale of low, middle and high for boys and girls from families with varying life status of parent(s) (Table 3). This example yielded two other data sets, by gender and by life status, both of which engaged the same ordinal levels of parent education, or $c .4$ ) Other nested designs encouraged the summing of subdivisions, as noted in Table 4 . The table portrayed several subcategories of men and women who were placed in ordinal levels of young, middle-aged or senior age groups. The subdivisions of each gender category were summed to develop a single data set.

Sample sizes for the selected studies ranged from ten to 19,256 , and contained from three to eight ordinal levels $(c)$ of outcome data. Ninety-seven $(65.1 \%)$ of the studies had three ordinal levels, $32(21.5 \%)$ had 4 levels, $15(10.1 \%)$ had 5 levels, one $(.7 \%)$ had six levels, one (.7\%) had seven levels, and three (2\%) studies had eight

Table 2. Teacher's Perceptions Of Psychological Problems In Students (Morrow, 1995).

\begin{tabular}{lllll}
\hline Category & $\underline{\text { K-3 }}$ & $\underline{4-6}$ & $\underline{7-8}$ & $\underline{9-12}$ \\
Emotional & 187 & 110 & 23 & 56 \\
Conduct & 190 & 116 & 76 & 55 \\
ADD & 190 & 118 & 23 & 54 \\
LD & 184 & 113 & 23 & 54 \\
\hline
\end{tabular}

Table 3. Frequencies For Low-, Middle-, And High-Scoring Subjects On Parental Education From Families With One Or Both Parents Dead And With Both Parents Alive (Cherian, 1992).

\begin{tabular}{llll}
\hline One/Both Parents Dead & $\underline{\text { Low }}$ & $\underline{\text { Middle }}$ & $\underline{\text { High }}$ \\
Boys & 9 & 89 & 13 \\
Girls & 39 & 147 & 11 \\
Both Parents Alive & & & \\
Boys & 21 & 121 & 55 \\
Girls & 58 & 279 & 178 \\
\hline
\end{tabular}

Table 4. Number Of Note Pairs Matched By Age Group, Sex, And Occupational Level (Black, 1993).

\begin{tabular}{llll}
\hline Group & $\begin{array}{l}\text { Young } \\
(18-29)\end{array}$ & $\begin{array}{l}\text { Middle-aged } \\
(30-64)\end{array}$ & $\begin{array}{l}\text { Senior } \\
(65+)\end{array}$ \\
Men & & & \\
Blue Collar & 4 & 10 & 3 \\
White Collar & 3 & 18 & 4 \\
Professional & 0 & 7 & 3 \\
Student & 3 & 0 & 0 \\
Homemaker & 0 & 0 & 0 \\
Women & & & \\
Blue Collar & 0 & 0 & 0 \\
White Collar & 3 & 3 & 1 \\
Professional & 0 & 2 & 1 \\
Student & 2 & 0 & 0 \\
Homemaker & 1 & 5 & 4 \\
\hline
\end{tabular}


ordinal levels.

The $p$ values compiled in Table 5 for each nonparametric exact test, in addition to the asymptotic $p$ values for the $\chi^{2}$ test, include an asterisk if the value was significant at $\alpha=0.0500$. StatXact Turbo software carried out the decimal to four places; thus, $p$ values of 0.05 with values higher than zero in the third or fourth decimal place were not reported as significant.

Error messages sometimes occurred with larger data sets: "The problem (was) too large for the test op- tion". When this occurred, the test was rerun with the "Monte Carlo (MO) option". $P$ values for the Monte Carlo option result from employing 20,000 iterations (99\% confidence level), and are indicated in parentheses in Table 5. StatXact reported Monte Carlo results either as a confidence interval, or, for example as with data set 41 , as 0.0053 \pm 0.0013 . (Note that these lower and upper bounds of the interval are not included in Table 5.)

Table 5. $P$ Values For Tests Computed With StatXact.

\begin{tabular}{|c|c|c|c|c|c|c|c|c|c|c|c|}
\hline ID & WRS & SS & ENS & $\mathbf{P}$ & $\chi^{2}$ & ID & WRS & SS & ENS & $\mathrm{P}$ & $\chi^{2}$ \\
\hline 001 & .4225 & .4901 & .4572 & .4415 & .9336 & 039 & $.0000 *$ & $.0000^{*}$ & $.0000^{*}$ & $.0000^{*}$ & $.0000^{*}$ \\
\hline 002 & .3467 & .2980 & .3167 & .3467 & .8424 & 040 & .5000 & .4675 & .4838 & .6169 & .6005 \\
\hline 003 & .4073 & .4526 & .4073 & .4397 & .9208 & 041 & $(.0053)^{*}$ & $(.0091)^{*}$ & $(.0067)^{*}$ & $.0056 *$ & .1342 \\
\hline 004 & .5000 & .4675 & .4838 & .6169 & .6005 & 042 & $(.0163)^{*}$ & $(.0231)^{*}$ & $(.0171)^{*}$ & $.0161^{*}$ & .2012 \\
\hline 005 & $.0261^{*}$ & .0613 & $.0260^{*}$ & $.0392 *$ & .1146 & 043 & .1801 & .4194 & .1775 & .3230 & .0807 \\
\hline 006 & .2812 & .3907 & .3158 & .2511 & $.0056^{*}$ & 044 & $.0263 *$ & $.0235^{*}$ & $.0263^{*}$ & $.0330 *$ & .1311 \\
\hline 007 & $.0122 *$ & $.0122 *$ & $.0122 *$ & $.0203^{*}$ & $.0207^{*}$ & 045 & .1903 & .0985 & .2053 & .2570 & .0941 \\
\hline 008 & $(0.0)^{*}$ & $(0.0)^{*}$ & $(0.0)^{*}$ & $(0.0)^{*}$ & $.0000^{*}$ & 046 & $.0117^{*}$ & $.0014^{*}$ & $.0328^{*}$ & .0574 & $.0002 *$ \\
\hline 009 & $.0042 *$ & $.0012 *$ & $.0026^{*}$ & $.0026^{*}$ & $.0044^{*}$ & 047 & $.0000^{*}$ & $0^{*}$ & $.0000^{*}$ & $.0000^{*}$ & $.0000^{*}$ \\
\hline 010 & .4658 & .4768 & .4844 & .5071 & .9747 & 048 & $.0000^{*}$ & .00 & .00 & & $.0000^{*}$ \\
\hline 011 & .4570 & .4390 & .4522 & .4535 & .9975 & 049 & $.0289^{*}$ & 9* & $.0289^{*}$ & & .1025 \\
\hline 012 & .5831 & .2441 & .5831 & .5831 & $.0123 *$ & 050 & $(0.0)^{*}$ & $(0.0)^{*}$ & $(0.0)^{*}$ & & $.0002 *$ \\
\hline 013 & $.0024^{*}$ & $.0015^{*}$ & $.0024^{*}$ & $.0024 *$ & $.0085^{*}$ & 051 & .4254 & & .4773 & .46 & $.0161^{*}$ \\
\hline 014 & $.0201 *$ & .0805 & $.0201^{*}$ & $.0302^{*}$ & $.0360 *$ & 052 & $.0029 *$ & .02 & $(.0041)^{*}$ & .00 & $.0238 *$ \\
\hline 015 & $.0001 *$ & $.0001^{*}$ & $.0000^{*}$ & $.0001 *$ & $.0002^{*}$ & 053 & $.0000^{*}$ & $(.00$ & $.0000 *$ & .00 & $.0000^{*}$ \\
\hline 016 & $.0000^{*}$ & $.0001 *$ & $.0000^{*}$ & $.0000^{*}$ & $.0001 *$ & 054 & $.0014^{*}$ & $(.0001)^{*}$ & $.0020^{*}$ & $8^{*}$ & $.0023^{*}$ \\
\hline 017 & $.0016^{*}$ & $.0018^{*}$ & $.0019^{*}$ & $.0021^{*}$ & $.0130^{*}$ & 055 & .0938 & .0812 & .0914 & .1210 & .3271 \\
\hline 018 & $.0160 *$ & .0511 & $.0195^{*}$ & $.0216^{*}$ & .0953 & 056 & $.0000^{*}$ & $.0000^{*}$ & $.0000^{*}$ & $.0000 *$ & $.0001 *$ \\
\hline 019 & $.0004 *$ & $.0014^{*}$ & $.0004^{*}$ & $.0004 *$ & $.0017^{*}$ & 057 & .5000 & .4619 & .5000 & .5568 & .9004 \\
\hline 020 & .2755 & .4146 & .3834 & .4857 & .0756 & 058 & .2007 & .12 & .20 & .2052 & .4380 \\
\hline 021 & .2968 & .2184 & .2662 & .2902 & .6945 & 059 & $.0000^{*}$ & $.0000^{*}$ & $.0000^{*}$ & $.0000^{*}$ & $.0000^{*}$ \\
\hline 022 & .1475 & .2185 & .1667 & .1864 & .5167 & 060 & .1580 & .23 & .15 & .15 & .1049 \\
\hline 023 & .4488 & .2234 & .4909 & .4916 & .1238 & 061 & .3574 & .4266 & .3510 & .3510 & .2900 \\
\hline 024 & $.0001 *$ & $.0012^{*}$ & $.0002^{*}$ & $.0005^{*}$ & $.0003^{*}$ & 062 & .2486 & .1256 & .2070 & .2175 & .5384 \\
\hline 025 & $.0000^{*}$ & $.0000^{*}$ & $.0000^{*}$ & $.0000^{*}$ & $.0000^{*}$ & 063 & $.0400 *$ & $.0257^{*}$ & .0559 & $.0492 *$ & .1791 \\
\hline 026 & $.0135^{*}$ & .3396 & .0501 & .0825 & $.0000 *$ & 064 & .3997 & .4201 & .3997 & .4228 & .9574 \\
\hline 027 & $.0000^{*}$ & $.0005^{*}$ & $.0000^{*}$ & $.0000^{*}$ & $.0000^{*}$ & 065 & $(.4912)$ & $(.4985)$ & $(.4918)$ & .5017 & 9999 \\
\hline 028 & $.0324 *$ & $.0178^{*}$ & $.0324 *$ & $.0330^{*}$ & .0889 & 066 & .4592 & .4771 & .4585 & .4648 & .9228 \\
\hline 029 & .3467 & .4884 & .3467 & .3738 & .3865 & 067 & $(0.0)^{*}$ & $(0.0)^{*}$ & $(0.0)^{*}$ & $.0000^{*}$ & $.0000^{*}$ \\
\hline 030 & .0525 & $.0332 *$ & .0525 & .0535 & .1581 & 068 & $.0000^{*}$ & $.0000^{*}$ & $.0000^{*}$ & $.0000 *$ & $.0363 *$ \\
\hline 031 & .4414 & .4803 & .4414 & .4649 & .9315 & 069 & .3836 & .1582 & .4673 & .4675 & $.0363 *$ \\
\hline 032 & $.0000^{*}$ & $.0000^{*}$ & $.0000^{*}$ & $.0000^{*}$ & $.0000 *$ & 070 & .3836 & .1582 & .4673 & .4675 & $.0363 *$ \\
\hline 033 & .4959 & .4901 & .4971 & .5169 & .9996 & 071 & .3836 & .1582 & .4673 & .4675 & $.0363 *$ \\
\hline 034 & .5769 & .3288 & .4462 & .5769 & .4117 & 072 & .3836 & .1582 & .4673 & .4675 & $.0363 *$ \\
\hline 035 & .2464 & .2715 & .2587 & .2791 & .8731 & 073 & .1562 & .1876 & .1597 & .1703 & .7475 \\
\hline 036 & .3798 & .3543 & .3744 & .4082 & .9813 & 074 & .1575 & .1779 & .1514 & .1718 & .7512 \\
\hline 037 & .4361 & .4448 & .4407 & .4727 & .9968 & 075 & .0810 & .0726 & .0806 & .0858 & .3301 \\
\hline
\end{tabular}


Table 5. $P$ Values For Tests Computed With StatXact.

\begin{tabular}{|c|c|c|c|c|c|c|c|c|c|c|c|}
\hline ID & WRS & SS & ENS & $\mathrm{P}$ & $\chi^{2}$ & ID & WRS & SS & ENS & $\mathrm{P}$ & $\chi^{2}$ \\
\hline 077 & $.0269^{*}$ & $.0333^{*}$ & $.0276^{*}$ & $.0332 *$ & .1470 & 114 & .1272 & .0892 & .1584 & .1626 & .3264 \\
\hline 078 & .0913 & .1182 & .0913 & .1291 & .2751 & 115 & .2536 & .2814 & .2751 & .2982 & .7118 \\
\hline 079 & .3556 & .3333 & .3556 & .3556 & .5988 & 116 & .5257 & .5041 & .5046 & .5257 & 1.0000 \\
\hline 080 & $.0007^{*}$ & $.0043^{*}$ & $.0010^{*}$ & $.0011^{*}$ & $.0070^{*}$ & 117 & $.0000^{*}$ & $.0000 *$ & $.0000^{*}$ & $.0000^{*}$ & $.0000 *$ \\
\hline 081 & $.0000^{*}$ & $.0000^{*}$ & $.0000^{*}$ & $.0000^{*}$ & $.0000 *$ & 118 & $.0051^{*}$ & $.0047^{*}$ & $.0051^{*}$ & $.0059^{*}$ & $.0215^{*}$ \\
\hline 082 & $.0454^{*}$ & $.0265^{*}$ & .0570 & .0715 & .0828 & 119 & $.0023^{*}$ & $.0123^{*}$ & $.0030^{*}$ & $.0077^{*}$ & $.0039 *$ \\
\hline 083 & .4433 & .4234 & .4569 & .4904 & .9181 & 120 & $.0000^{*}$ & $.0000^{*}$ & $.0000^{*}$ & $.0000^{*}$ & $.0000 *$ \\
\hline 084 & $.0219^{*}$ & $.0037^{*}$ & $.0265^{*}$ & $.0431 *$ & $.0014 *$ & 121 & $.0034^{*}$ & $.0034^{*}$ & $.0034^{*}$ & $.0034^{*}$ & $.0006^{*}$ \\
\hline 085 & $.0475^{*}$ & .0622 & .0562 & .0583 & .3469 & 122 & $.0010^{*}$ & $.0013^{*}$ & $.0010^{*}$ & $.0010^{*}$ & $.0051^{*}$ \\
\hline 086 & $.0000^{*}$ & $.0000^{*}$ & $.0000^{*}$ & $.0000^{*}$ & $.0000^{*}$ & 123 & .0526 & .0868 & .0518 & .0530 & .1404 \\
\hline 087 & .0748 & .1062 & .0748 & .0777 & .2591 & 124 & .4368 & .4680 & .4369 & .4680 & .9644 \\
\hline 088 & $.0136^{*}$ & $.0152^{*}$ & $.0136^{*}$ & $.0164^{*}$ & .0824 & 125 & $.0000^{*}$ & $.0000^{*}$ & $.0000^{*}$ & $.0000^{*}$ & $.0000 *$ \\
\hline 089 & .2759 & .1823 & .2759 & .2799 & .4065 & 126 & .5581 & .4730 & .5581 & .5581 & .8956 \\
\hline 090 & $.0136^{*}$ & $.0152^{*}$ & $.0136^{*}$ & $.0164 *$ & .0824 & 127 & .2151 & .2697 & .2151 & .2195 & .3871 \\
\hline 091 & .5569 & .5223 & .5223 & .5569 & 1.0000 & 128 & $.0000^{*}$ & $.0000 *$ & $.0000^{*}$ & $.0000^{*}$ & $.0000^{*}$ \\
\hline 092 & .5569 & .5223 & .5223 & .5569 & 1.0000 & 129 & $.0269^{*}$ & $.0138 *$ & $.0251 *$ & $.0260^{*}$ & .0779 \\
\hline 093 & .1829 & .1939 & .1829 & .2010 & .6344 & 130 & .4672 & .2885 & .4697 & .4695 & .5258 \\
\hline 094 & .3696 & .3762 & .3696 & .3911 & .9396 & 131 & .3995 & .4954 & .3941 & .4255 & .5917 \\
\hline 095 & $(0.0)^{*}$ & $(0.0)^{*}$ & $(0.0)^{*}$ & $(0.0)^{*}$ & $.0000^{*}$ & 132 & .3685 & .3681 & .3667 & .4036 & .9015 \\
\hline 096 & $(0.0)^{*}$ & $(0.0)^{*}$ & $(0.0)^{*}$ & $(0.0)^{*}$ & $.0002^{*}$ & 133 & .0742 & $.0131 *$ & .0845 & .0690 & $.0308^{*}$ \\
\hline 097 & $(0.0)^{*}$ & $(0.0)^{*}$ & $(0.0)^{*}$ & $.0000 *$ & $.0000^{*}$ & 134 & $.0000^{*}$ & $.0000 *$ & $.0000 *$ & $.0000^{*}$ & $.0000 *$ \\
\hline 098 & $(0.0)^{*}$ & $(0.0)^{*}$ & $(0.0)^{*}$ & $(0.0)^{*}$ & $.0001^{*}$ & 135 & .3160 & .3571 & .3160 & .3430 & .8505 \\
\hline 099 & $(0.0)^{*}$ & $(0.0)^{*}$ & $(0.0)^{*}$ & $(0.0)^{*}$ & $.0000^{*}$ & 136 & .1665 & .2038 & .1419 & .1419 & $.0000^{*}$ \\
\hline 100 & $(0.0)^{*}$ & $(0.0)^{*}$ & $(0.0)^{*}$ & $(0.0)^{*}$ & $.0001 *$ & 137 & .4699 & .4397 & .4706 & .4949 & .9 .810 \\
\hline 101 & $.0000 *$ & $(0.0)^{*}$ & $.0000^{*}$ & $.0000^{*}$ & $.0000^{*}$ & 138 & .2250 & .3601 & .2786 & .2913 & .4336 \\
\hline 102 & $(0.0)^{*}$ & $(0.0)^{*}$ & $(0.0)^{*}$ & $(0.0)^{*}$ & $.0000^{*}$ & 139 & .2150 & .0809 & .1794 & .1794 & .2050 \\
\hline 103 & .4691 & .4926 & .4691 & .4838 & .9073 & 140 & .3748 & .4329 & .3748 & .4286 & .9040 \\
\hline 104 & .3824 & .2651 & .3417 & .3167 & .7407 & 141 & .3605 & .2346 & .3444 & .3527 & .5897 \\
\hline 105 & .4852 & .4943 & .4878 & .4949 & 9999 & 142 & $.0383^{*}$ & $.0136^{*}$ & $.0208^{*}$ & $.0382^{*}$ & .0689 \\
\hline 106 & .2804 & .2551 & .2754 & .2776 & .7979 & 143 & .4740 & .3986 & .4302 & .5000 & .8977 \\
\hline 107 & .2868 & .2580 & .2812 & .2832 & .7988 & 144 & .0767 & $.0367^{*}$ & .0534 & .0785 & .1733 \\
\hline 108 & .3600 & .1486 & .3265 & 3159 & .0681 & 145 & $.0000^{*}$ & $.0000^{*}$ & $.0000^{*}$ & $.0000^{*}$ & $.0002 *$ \\
\hline 109 & .2849 & .1507 & .2637 & 2577 & .2359 & 146 & $.0000^{*}$ & $.0000^{*}$ & $.0000^{*}$ & $.0000^{*}$ & $.0000^{*}$ \\
\hline 110 & .2980 & .1602 & .2760 & .2705 & .2455 & 147 & .3776 & .4209 & .3776 & .4160 & .9146 \\
\hline 111 & .3280 & .3801 & .3326 & .3564 & .8453 & 148 & $.0016^{*}$ & $.0061 *$ & $.0016^{*}$ & $.0028 *$ & $.0092 *$ \\
\hline 112 & .3993 & .4312 & .3993 & .4459. & .9365 & 149 & $.0257^{*}$ & $.0302^{*}$ & $.0257^{*}$ & $.0275^{*}$ & $.0178^{*}$ \\
\hline 113 & .3565 & .3815 & .3565 & .3712 & .7920 & & & & & & \\
\hline
\end{tabular}

The prevailing question is whether one exact test is more powerful than another. Table 6 carries the question of power one step further, displaying the frequency and the percent of significant results for all levels of ordinal outcomes. The results indicates comparable results for all procedures. categorical contingency tables, for both theoretical reasons and in terms of power to detect row and column interactions. On the basis of this study, the exact versions of the Wilcoxon, Expected Normals, Savage, and Permutation tests appear to be comparable in terms of statistical power.

Conclusion

Clearly, the $\chi^{2}$ should be avoided in analyzing ordered 
Table 6. Frequency (\%) of Significant Results of Exact Tests for Each Level of $c, \alpha=0.0500$.

\begin{tabular}{lllllll}
\hline \multicolumn{5}{c}{ Number Of Ordinal Levels, (N Of Data Sets) } \\
\cline { 3 - 7 } Test & $3(\mathrm{~N}=97)$ & $4(\mathrm{~N}=32)$ & $5(\mathrm{~N}=15)$ & $6(\mathrm{~N}=1)$ & $7(\mathrm{~N}=1)$ & $8(\mathrm{~N}=3)$ \\
WRS & $41(.25)$ & $8(.29)$ & $7(.27)$ & $0(0)$ & $0(0)$ & $0(0)$ \\
SS & $40(.25)$ & $7(.27)$ & $5(.19)$ & $0(0)$ & $0(0)$ & $0(0)$ \\
ENS & $39(.25)$ & $6(.22)$ & $6(.23)$ & $0(0)$ & $0(0)$ & $0(0)$ \\
P & $39(.25)$ & $6(.22)$ & $8(.31)$ & $1(1.00)$ & $0(0)$ & $0(0)$ \\
\hline
\end{tabular}

References

Basu, A. P. (1968). On a generalized savage statistic with applications to life testing. Annals of Mathematical Statistics, 39, 1591-1604.

Bhattacharyya, G. K., \& Johnson, R. A. (1977). Statistical concepts and methods. NY: Wiley.

Black, S. T. (1993). Comparing genuine and simulated suicide notes: A new perspective. Journal of Consulting and Clinical Psychology, 61, 699-702.

Blair, R. C. (1980). A comparison of the power of the two independent means $t$ test to that of the Wilcoxon's rank-sum test for samples of various populations. Unpublished doctoral dissertation, University of South Florida, Tampa, FL.

Bradley, J. V. (1968). Distribution-free statistical tests. Englewood Cliffs, NJ: Prentice-Hall.

Chernoff, H., \& Savage, I. R. (1958). Asymptotic normality and efficiency of certain nonparametric test statistics. Ann. Math. Statist, 29, 972-994.

Cherian, V. I. (1992). Relation of parental education and life status to academic achievement by Xhosa children. Psychological Reports, 70, 947-956.

Fisher, R. A., \& Yates, F. (1938). Statistical tables for biological, agricultural and medical research. Edinburgh: Oliver and Boyd.

Gajjar, Y., Mehta, C. R., Patel, N. \& Senchaudhuri, P. (1992). StatXact-Turbo statistical software for exact nonparametric inference user manual. MA: Cytel Software.

Grissom, R. J. (1994). Statistical analysis of ordinal categorical status after therapies. Journal of Consulting and Clinical Psychology, 62, 281-284.

Haber, A. (1990). Comments on The test of homogeneity for $2 \times 2$ contingency tables: A review of and some personal opinions on the controversy by G. Camilli. Psychological Bulletin, 108, 146-149.

Hajek, J. (1969). A course in nonparametric statistics. San Francisco, CA: Holden-Day.
Harter, H. L. (1969). Order statistics and their use in testing and estimation. Vol. 2. U. S. Government Printing Office, Washington, DC.

Hodges, J. L., Jr., \& Lehmann, E. L. (1960). Comparison of the Normal Scores and Wilcoxon tests. Proceedings of the Fourth Berkeley Symposium on Mathematical Statistics and Probability, Vol. 1, 307-317. Berkeley: University of California Press.

Kerlinger, F. N. (1973). Foundations of behavioral research (2nd ed.) New York: Holt, Rinehart, \& Winston.

Lehmann, E. L. (1975). Nonparametrics: Statistical methods based on ranks. San Francisco: Holden-Day.

Mann, H. B., \& Whitney, D. R. (1947). On a test of whether one of two random variables is stochastically larger than the other. Annals of Mathematical Statistics, $18,50-60$.

Marascuilo, L. \& Serlin, R. (1988). Statistical methods for the social and behavioral sciences. NY: W. H. Freeman.

May, R. B., \& Hunter, M. A. (1990). Some advantages of permutation tests. In B. Zumbo (Chair), Alternatives to classical statistical procedures. Symposium conducted at the annual meeting of the Canadian Psychological Association, Ottawa. (Abstract in Canadian Psychology, 31, 238).

Mehta, C. R. \& Patel, N. (1992). StatXact Turbo 2.0: Statistical software for exact nonparametric inference user manual. MA: Cytel Software.

Mehta, C. R. \& Patel, N. (1995). StatXact 3 for Windows: Statistical software for exact nonparametric inference user manual. MA: Cytel Software.

Micceri, T. (1989). The unicorn, the normal curve, and other improbable creatures. Psychological Bulletin, 105, 156-166.

Morrow, V. L. (1995). Teachers evaluate psychological problems and personal counseling needs of students. Education, 116, 130-136.

Norween, E. W. (1989). Computer intensive methods for testing hypotheses: An introduction. NY: Wiley. 
Owen, D.B. (1962). Handbook of statistical tables. Reading, MA: Addison-Wesley.

Randles, R. H., \& Wolfe, D. A. (1979). Introduction to the theory of nonparametric tests. NY: John Wiley.

Savage, R. (1956). Contributions to the theory of rank order statistics: The two-sample case. Annals of Mathematical Statistics, 27, 590-615.

Solorzano, D. G. (1992). An exploratory analysis of the effects of race, class, and gender on student and parent mobility aspirations. Journal of Negro Education, 61, 30-44.

Troup-Leasure, K. , Eichelberger, R. T., \& Zigmond, N. (1992). Research experiences of doctoral students and publication after graduation. Teacher Education and Special Education, 15, 183-193.

Van der Waerden, B. L. (1952). Order Tests for the two-sample problem and their power. Indagationes Math, 14, 453-458.

Van der Waerden, B. L. (1953). Errata. 80.

Wilcoxon, F. (1945). Individual comparisons by ranking methods. Biometrics, 1, 80-82.

\section{Appendix}

Studies Where Data Sets Were Located (Ordered According To ID in Table 5)

'Babad, E. (1995). Can accurate knowledge reduce wishful thinking in voters' predictions of election outcomes? The Journal of Psychology, 129, 285-300.

${ }^{2,3}$ Weiserbs, B., \& Gottlieb, J. (1995). The perception of risk over time as a factor influencing attitudes toward children with physical disabilities. The Journal of Psychology, 129, 689-699.

${ }^{4}$ Gallagher-Thompson, D., \& Steffen, A. M. (1994). Comparative effects of cognitive-behavioral and brief psychodynamic psychotherapies for depressed family caregivers. Journal of Consulting and Clinical Psychology, 62, 543-549.

${ }^{5}$ Speer, D. C. (1994). Can treatment research inform decision makers? Nonexperimental method issues and examples among older outpatients. Journal of Consulting and Clinical Psychology, 62, 560-568.

${ }^{6}$ Dancer, L. S., Anderson, A. J., \& Derlin, R. L. (1994). Use of log-linear models for assessing differential item functioning in a measure of psychological functioning. Journal of Consulting and Clinical Psychology, 62, 710-717.

${ }^{7}$ Gauthier, J., Côté, G., \& French, D. (1994). The role of home practice in the thermal biofeedback treatment of migraine headache. Journal of Consulting and Clinical Psychology, 62, 180-184.

${ }^{8}$ Storfer, M. D. (1995). Problems in left-right discrimination in a high-IQ population. Perceptual and Motor Skills, 81, 491-497.
${ }^{9}$ Zaslavsky, O. \& Peled, I. (1996). Inhibiting factors in generating examples by mathematics teachers and student teachers: The case of binary operation. Journal for Research in Mathematics Education, 27, 67-78.

${ }^{10,11}$ Morrow, V. L. (1995). Teachers evaluate psychological problems and personal counseling needs of students. Education, 116, 130-136.

${ }^{12,13}$ Halvari, H. \& Gjesme, T. (1995). Trait and state anxiety before and after competitive performance. Perceptual and Motor Skills, 81, 1059-1074.

${ }^{14}$ Forns-Santacana, M. \& Martorell-Balanzó, B. (1996). Relationships of personality factors with clinical dimensions and school achievement. Perceptual and Motor Skills, 82, 243-253.

${ }^{15}$ Toro, P. A. \& McDonnell, D. M. (1992). Beliefs, attitudes, and knowledge about homelessness: A survey of the general public. American Journal of Community Psychology, 20, 53-78.

${ }^{16}$ Cherian, V. I. (1994). Self-reports of corporal punishment of Xhosa children from broken and intact families and their academic achievement. Psychological Reports, 74, 867-874.

${ }^{17}$ Cherian, V. I. (1994). Relationship between parental aspiration and academic achievement of Xhosa children from broken and intact families. Psychological Reports, 74, 835-840.

${ }^{18} \mathrm{Hamm}, \mathrm{N} . \mathrm{H}$. (1994). Outcomes of the Minnesota smoking prevention program. Psychological Reports, $75,880-882$.

${ }^{19}$ Ines, T. M. \& Sacco, W. P. (1992). Factors related to correspondence between teacher ratings of elementary student depression and student self-ratings. Journal of Consulting and Clinical Psychology, 60, 140-142.

${ }^{20-31}$ Norris, F. H. (1992). Epidemiology of trauma: Frequency and impact of different potentially traumatic events on different demographic groups. Journal of Consulting and Clinical Psychology, 60, 409-418.

${ }^{32}$ Eysenck, H. J. (1992). The effects of psychotherapy: An evaluation. Journal of Consulting and Clinical Psychology, 60, 659-663.

${ }^{33}$ Sanders, M. R., Shepherd, R. W., Cleghorn, G., $\&$ Woolford, H. (1994). The treatment of recurrent abdominal pain in children: A controlled comparison of cognitive-behavioral family intervention and standard pediatric care. Journal of Consulting and Clinical Psychology, 62, 306-314.

${ }^{34}$ Black, St. T. (1993). Comparing genuine and simulated suicide notes: A new perspective. Journal of Consulting and Clinical Psychology, 61, 699-702.

${ }^{35-37}$ Jacobson, N. S., Fruzzetti, A. E., Dobson, K., Whisman, M., \& Hops, H. (1993). Couple therapy as a treatment for depression: II. The effects of relationship quality and therapy on depressive relapse. Journal of Consulting and Clinical Psychology 61, 516-519. 
${ }^{38,39}$ Mossman, D. (1994). Assessing predictions of violence: Being accurate about accuracy. Journal of Consulting and Clinical Psychology, 62, 783-792.

${ }^{40}$ Uehara, E. S., Smukler, M., \& Newman, F. L. (1994). Linking resource use to consumer level of need: Field test of the Level of Need-care Assessment (LONCA) method. Journal of Consulting and Clinical Psychology, 62, 695-709.

${ }^{41-43}$ Kingma, J. (1994). Age and gender distributions of pedestrian accidents across the life-span. Perceptual and Motor Skills, 79, 1680-1682.

${ }^{44,45}$ Finkel, D., McGue, M. \& Pedersen, N. (1995). Genetic influences on memory performance in adulthood: Comparison of Minnesota and Swedish twin data. Psychology and Aging, 10, 437-446.

46,47Bell, P. F., McKenna, J. P., \& Digman, Jr., R. H. (1995). Should psychologists obtain prescribing privileges? A survey of family physicians. Professional Psychology: Research and Practice, 26, 371-376.

${ }^{48}$ Simonson, S. D. (1995). A historical view of content area reading instruction. Reading Psychology: An International Quarterly, 16, 99-147.

${ }^{49} \mathrm{Kragler}$, S. (1995). The transition from oral to silent reading. Reading Psychology: An International Quarterly, 16, 395-408.

${ }^{50-54}$ Zimba, R. F. (1995). Secondary school students' risks that may promote HIV infection and the spread of AIDS. School Psychology International, 16, 67-78.

${ }^{55-60}$ Fukunishi, I. (1995). Psychological acceptance and alexithymia in spinal cord injury patients. Psychological Reports, 76, 475-481.

${ }^{61}$ Isoaho, R., Keistinen, T., Laippala, P. \& Kivelä, S. (1995). Chronic obstructive pulmonary disease and symptoms related to depression in elderly persons. Psychological Reports, 76, 287-297.

${ }^{62,63}$ Anstey, K., Stankov, L., \& Lord, S. (1993). Primary aging, secondary aging, and intelligence. Psychology and Aging, 8, 562-570.

${ }^{64}$ Schulz, R., Musa, D., Staszewski, J., \& Siegler, R. (1994). The relationship between age and major league baseball performance: Implications for development. Psychology and Aging, 9, 274-286.

${ }^{65,66}$ Spiro, III, A., Schnurr, P. P., \& Aldwin, C. M. (1994). Combat-related posttraumatic stress disorder symptoms in Older Men. Psychology and Aging, 9, 17-26.

${ }^{67}$ Goldberg, C. J. \& Botvin, G. J. (1993). Assertiveness in Hispanic adolescents: Relationship to alcohol use and abuse. Psychological Reports, 73, 227-238.

${ }^{68}$ Trankina, M. L. (1993). Gender differences in attitudes toward science. Psychological Reports, 73, 123130.

${ }^{69-72}$ Cherian, V. I. (1993). Gender, socioeconomic status, and mathematics achievement by Xhosa children. Psychological Reports, 73, 771-778.
${ }^{73,74}$ Erdman, R. A. M., Kooijman, M. Passchier, J., \& Stronks, D. L. (1993). The Dutch version of the Nottingham Health Profile: Investigations of psychometric aspects. Psychological Reports, 72, 1027-1035.

${ }^{75-77}$ Nagy, S. \& Nagy, M. C. (1992). Longitudinal examination of teachers' burnout in a school district. Psychological Reports, 71, 523-531.

${ }^{78,79}$ Beer, J. \& Beer, J. (1992). Depression, selfesteem, suicide ideation, and GPAs of high school students at risk. Psychological Reports, 71, 899-902.

${ }^{80,81}$ Cherian, V. I. (1992). Relation of parental education and life status to academic achievement by Xhosa children. Psychological Reports, 71, 947-956.

${ }^{82-84}$ Matthew, M. D., Franz, R. S., \& Weaver, C. N. (1992). Perceived financial status of male and female college professors. Psychological Reports, 70, 199-202.

${ }^{85}$ Kalliopuka, M. (1992). Social desirability related to children's age, sex, and willingness to help. Psychological Reports, 70, 479-482.

${ }^{86}$ May, C. P., Hasher, L. \& Stoltzfus, E. R. (1993). Optimal time of day and the magnitude of age differences in memory. Psychological Science, 4, 326-330.

${ }^{87-90}$ Costa, M. M. \& Gatz, M. (1992). Determination of authorship credit in published dissertations. Psychological Science, 3, 354 - 357.

${ }^{91-94}$ Blanchard, F. A., Crandall, C. S., Brigham, J. C., \& Vaughn, L. A. (1994). Condemning and condoning racism: A social context approach to interracial settings. Journal of Applied Psychology, 79, 993-997.

${ }^{95-102}$ Solorzano, D. G. (1992). An exploratory analysis of the effects of race, class, and gender on student and parent mobility aspirations. Journal of Negro Education, 61, 30-44.

${ }^{103}$ Kowner, R. \& Ogawa, T. (1993). The contrast effect of physical attractiveness in Japan. The Journal of Psychology, 127, 51-64.

${ }^{104,105}$ Adelman, H. S., Barker, L. A., \& Nelson, P. (1993). A study of a school-based clinic: Who uses it and who doesn't? Journal of Clinical Child Psychology, 22, 52-59.

${ }^{106-111}$ Gottfredson, D. C., Gottfredson, G. D., \& Hybl, L. G. (1993). Managing adolescent behavior: A multiyear, multischool study. American Educational Research Journal, 30, 179-215.

${ }^{112,113}$ Newman, R. S. \& Schwager, M. T. (1995). Students' help seeking during problem solving: Effects of grade, goal, and prior achievement. American Educational Research Journal, 32, 352-376.

${ }^{114}$ Powell, F. C. \& Wanzenried, J. W. (1993). Perceptions of Bush, Clinton, and Perot in relation to frequency of presidential debate viewing. Perceptual and Motor Skills, 77, 35-41.

${ }^{115}$ Hicks, R. A. \& Bautista, J. (1993). Snoring and nightmares. Perceptual and Motor Skills, 77, 433-434. 
${ }^{116} \mathrm{Kunz}$, J. (1993). Ice cream preference: Gender differences in taste and quality. Perceptual and Motor Skills, 77, 1097-1098.

${ }^{117}$ Hicks, R. A., Conti, P. A., \& Nellis, T. (1992). Arousability and stress-related physical symptoms: A validation study of Coren's Arousal Predisposition Scale. Perceptual and Motor Skills, 74, 659-662.

${ }^{118-122}$ Troup-Leasure, K. Eichelberger, R. T., \& Zigmond, N. (1992). Research experiences of doctoral students and publication after graduation. Teacher Education and Special Education, 15, 183-193.

${ }^{123} \mathrm{Lidz}, \mathrm{C}$. S. (1992). The extent of incorporation of dynamic assessment into cognitive assessment courses: A national survey of school psychology trainers. The Journal of Special Education, 26, 325-331.

${ }^{124}$ English, L. D. (1993). Children's strategies for solving two- and three-dimensional combinatorial problems. Journal for Research in Mathematics Education, 24, 255-273.

${ }^{125}$ Ferrini-Mundy, J. \& Gaudard, M. (1992). Secondary school calculus: Preparation or pitfall in the study of college calculus? Journal for Research in Mathematics Education, 23, 56-71.

${ }_{126,127}$ Ford, D. Y. (1993). Support for the achievement ideology and determinants of underachievement as perceived by gifted, above-average, and average Black students. Journal for the Education of the Gifted, 16, 280298.

${ }^{128}$ Orfield, G. (1992). Money, equity, and college access. Harvard Educational Review, 52, 337-372.

${ }^{129}$ Pickett, W. \& Burrill, D. F. (1994). The use of quantitative evidence in research: A comparative study of two literatures. Educational Researcher, 23, 18-21.

${ }^{130,131}$ Wrobel, N. H. (1993). Effect of patient age and gender on clinical decisions. Professional Psychology: Research and Practice, 24, 206-212.
${ }^{132}$ Harris, J. (1992). Teacher-completed child behavior checklist ratings as a function of classroom-based interventions: A pilot study. Psychology in the Schools, 29, 42-52.

${ }^{133}$ Santos de Barona, M. (1992). Infant and preschool evaluation services: Implications for school districts and personnel. Psychology in the Schools, 29, 237-247.

${ }^{134}$ Carrillo-de-la-Peňa \& Luengo, M. A. (1994). Time estimation and juvenile delinquency. Perceptual and Motor Skills, 79, 1559-1565.

${ }^{135}$ Eddowes, E. A., Aldridge, J., \& Culpepper, S. (1994). Primary teachers' classroom practices and their perceptions of children's attention problems. Perceptual and Motor Skills, 79, 787-790.

${ }^{136}$ Bortoli, L. \& Robazza, C. (1994). The Motor Activity Anxiety test. Perceptual and Motor Skills, 79, 299305.

${ }^{137,138}$ Gaulin, C. A. \& Campbell, T. F. (1994). Procedure for assessing verbal working memory in normal school-age children: Some preliminary data. Perceptual and Motor Skills, 79, 55-64.

${ }^{139-144}$ Speer, D. C. (1992). Clinically significant change: Jacobson and Truax (1991) revisited. Journal of Consulting and Clinical Psychology, 60, 402-408.

${ }_{145,146}$ Tanaka, S. (1994). Effects of a moderately difficult task on preschool children's concentration and their subsequent choices of task. Perceptual and Motor Skills, $78,699-700$.

${ }^{147}$ Nkaya,H. N., Huteau, M., \& Bonnet, J. (1994).

Retest effect on cognitive performance on the Raven-38 Matrices in France and in the Congo. Perceptual and Motor Skills, 78, 503-510.

${ }^{148,149} \mathrm{Grissom}, \mathrm{R} . \mathrm{J}$. (1994). Statistical analysis of ordinal categorical status after therapies. Journal of Consulting and Clinical Psychology, 62, 281-284. 


\section{Graduate Student Research Alternatives To $\mathrm{S}_{\mathrm{w}}$ In The Bracketed Interval Of The Trimmed Mean}

\author{
Jennifer Bunner \\ Wunderman Analytics, Detroit
}

\author{
Shlomo Sawilowsky \\ Educational Evaluation and Research \\ College of Education \\ Wayne State University
}

The aim of this Monte Carlo study is to examine alternatives to estimated variability in building bracketed intervals about the trimmed mean.

Keywords: Trimmed mean, Bracketed interval, Winsorized sample standard deviation, Robust tests

Introduction

The prevalence of nonnormally distributed data in applied studies has been documented (e.g., Micceri, 1989; Pearson $\&$ Please, 1975, Tan, 1982). Summary statistics, such as measures of central tendency, and parametric hypothesis tests, such as Student's t, are affected by nonnormal data, as many studies have also documented (e.g., Bradley, 1968, 1978; Blair, 1981; Blair \& Higgins, 1980a, 1980b, 1985).

Nonnormality arises for a variety of reasons. In some cases, the underlying distribution of the variable is exponential (e.g., growth or decay), multimodal lumpy (e.g., Micceri, 1989), mass at zero with gap (e.g., Sawilowsky \& Hillman, 1992), or some other non-Gaussian shape. In other cases, an essentially normal model can be adopted if perturbations, commonly called outliers, can be assumed to have contaminated the model. The latter case motivated the development of robust statistics.

Consider, for example, measures of central tendency for a single sample. The arithmetic mean, $\bar{x}$, is the most commonly used measure of the average. It is a sample statistic that is used as a point estimate of the population parameter $\mu$. However, it has a finite sample breakdown point of only $1 / n$. This implies that even a single observation can vastly distort the obtained value of $\bar{x}$, and hence, it is not a robust measure.

In contradistinction, the median is much more robust. Its finite sample breakdown point is approximately

Jennifer Bunner is a doctoral student in Educational Evaluation and Research, Wayne State University. She is a marketing analyst with Wunderman Analytics, 550 Towne Center Drive, Suite 300, Dearborn, MI, 48126, e-mail: jeni_bunner@det.wunderman.com. Shlomo S. Sawilowsky, Wayne Statue University Distinguished Faculty Fellow, is Professor and Chair, Educational Evaluation and Research, College of Education, Wayne State University. His interests are in nonparametric, robust, exact, permutation, and other computer-intensive methods, especially via Fortran.
$1 / 2$. Thus, almost half of the values could be untoward perturbations, and yet the value of the median remains unaffected. Despite this robustness property, the median never emerged as a popular measure of central tendency. Three possible reasons can be offered as an explanation for this unpopularity: (1) the sampling distribution of the median is intractable (requiring reliance on asymptotic variances or some other approach), making the construction of hypothesis tests difficult, (2) the sample median is usually not a very good estimate of the population median, and (3) the value of the median is actually determined based on only one number for $\mathrm{N}=$ odd (e.g., the point on the scale below which half of the observations fall), or within the upper and lower real limits of a single value for $\mathrm{N}=$ even, essentially ignoring the information contained in all of the other scores.

A well known alternative to dealing with nonnormally distributed data, where an essentially Gaussian structure can be assumed to exist underlying the data, is the trimmed mean $\left(\bar{x}_{t}\right)$. The trimmed mean is a compromise between the mean (i.e., trim $=$ zero) and the median (i.e., trim approximately equal to but less than $50 \%$ ).

The $2 \times 10 \%$ trimmed mean means that $10 \%$ of the observations are deleted from both sides of the data set. As an illustration, the $2 \times 10 \%$ trim is calculated on the data below by (1) ordering the data from low to high, (2) deleting the $.1 \times 10=1$ observation on the left and the $.1 \times$ $10=1$ observation on the right, and (3) computing the mean on the remaining 8 scores. This is illustrated in Table 1 .

A question that naturally arises in working with $\bar{x}_{t}$ is how to form a bracketed interval around it. In other words, how well does $\bar{x}_{\mathrm{t}}$ estimate the population mean $\mu$ ? For example, consider a $95 \%$ bracketed interval. From a frequentist's perspective, the purpose is to determine if one can be $95 \%$ sure that $\mu$ is contained within the interval built around the sample trimmed mean. A Bayesian's perspective would view this differently, and determine if many such intervals were formed, would $95 \%$ of those intervals contain the population mean. 
Table 1. Computing Trimmed Means.

\begin{tabular}{lllllllllll}
\hline Original & 85 & 92 & 87 & 93 & 99 & 86 & 88 & 90 & 73 & 91 \\
Ordered & 73 & 85 & 86 & 87 & 88 & 90 & 91 & 92 & 93 & 99 \\
Trimmed & & 85 & 86 & 87 & 88 & 90 & 91 & 92 & 93 & \\
\hline
\end{tabular}

$$
\bar{X}_{t}=\frac{85+86+87+88+90+91+92+93}{8}=89
$$

Many modern textbooks (e.g, Wilcox, 1996) address this question and give a formula similar to the following:

$$
\text { C... }\left.\right|_{1-\alpha}\left(\mu_{t}\right)=\bar{X}_{t} \pm\left(t_{1-\alpha} \times \frac{1}{1-2 \gamma} \times \frac{s_{w}}{\sqrt{n}}\right)
$$

where $\gamma$ is the amount of trimming and $s_{w}$ is the sample winsorized standard deviation.

Assume $\alpha=0.05$ and the amount to trim $g=.1$. The right side of (1) contains four expressions. The first term, $\bar{x}_{t}$, is the sample trimmed mean. With regard to the second expression, $t_{1-\alpha}$, Student's $t$ is two tailed, and degrees of freedom after trimming is $v=n-2 g-1$, where $\mathrm{g}$ is the percent to trim on one side. In the example above, $\mathrm{n}=$ $10, \mathrm{~g}=.1 \times 10=1$, and thus, $v=10-(2 \times 1)-1=7$. Therefore, $\mathrm{t}_{1-\alpha}=\mathrm{t}_{.975}=2.365$.

The third expression is a multiplier that is used to adjust the standard error (which is the fourth term) based on the amount of trimming. If there has been no trimming, this term reduces to 1 , leaving the full expression of the standard error. As the amount of trimming increases, the denominator decreases, and this multiplier increases.

The final expression, the standard error, is in fact the focus of the current paper. The $s_{w}$ term is a robust estimate of the population variance, which is unbiased after being divided by the square root of the sample size. The sample winsorzed standard deviation is obtained by "winsorizing the data", which is accomplished by recoding extreme values closer to the median.

For the current data, a $2 \times 10 \%$ winsorization is accomplished by recoding the two most extreme values back (i.e, the 73 is recoded to an 85 , and the 99 is recoded to a 93). Winsorization is a method of treating outliers without taking the harsh measure of deleting extreme values, but rather, recoding outliers to values that are toward the ends of the distribution but are more likely to be valid than perturbations. The value of $\mathrm{S}_{\mathrm{w}}$ for the example data is calculated as follows in Table 2. (See bottom of page.)

The standard deviation of the winsorized values is 3.2. For comparison, the standard deviation of the original scores is 6.8 .

An examination of the three right-most expressions that constitute the bracketed interval of the trimmed mean indicates that Formula (1), although widely circulated, certainly has no rigorous mathematical basis of support. There does not appear to be any justification for using the cdf of the $t$ distribution, unless an underlying Gaussian data structure is strictly assumed. Moreover, modifications to $v$ (e.g., Satterithwaite) are just as likely to ensure the sampling distribution of $\bar{x}_{t}$ is Student's $t$ as is the use of the multiplier in the third term. However, for the purposes of this paper, attention is turned to the use of $s_{w}$.

Wilcox (1996) and other textbooks that rely on some form of Formula (1) cite Tukey and McLaughlin (1963), which is the primary source for support of $s_{w}$. This paper is highly recommended to graduate students because it reads more like a fireside chat than a technical statistical paper. In this paper, Tukey and McLaughlin search for a robust measure of dispersion for the numerator of the fourth expression in Formula (1), recognizing that use of the sample standard deviation, which has the nonrobust arithmetic mean as its statistical engine, would be self-defeating in the presence of outliers.

The primary condition they sought to satisfy is that the average value of the denominator squared and the variance of the numerator are matched, or "in constant proportion over as broad a spectrum" (p. 337) of distributions as possible. Examination of the sample standard deviation of the trimmed mean based on this primary condition was shown to be unsatisfactory. Inspection of the results indicated that more consideration needed to be given to outliers than simply deleting them; hence, the winsorization approach was adopted.

However, there was no theoretical dependency requiring Tukey and McLaughlin's selection of the winsorized procedure as a robust measure of dispersion.

Table 2. Computing Winsorized Means.

\begin{tabular}{lllllllllll}
\hline Original & 85 & 92 & 87 & 93 & 99 & 86 & 88 & 90 & 73 & 91 \\
Ordered & 73 & 85 & 86 & 87 & 88 & 90 & 91 & 92 & 93 & 99 \\
Winsorized & 85 & 85 & 86 & 87 & 88 & 90 & 91 & 92 & 93 & 93 \\
\hline
\end{tabular}


Indeed, Lax (1985) identified over 150 different robust measures of dispersion, and the list is certainly longer than that. How might some other robust measure of variability perform in creating a bracketed interval for the trimmed mean?

\section{Purpose of the Study}

Given that the choice of $S_{w}$ was based on trial and error, and no theoretical underpinning, the purpose of this study is to examine the properties of bracketed intervals formed by using some alternative measures of dispersion.

\section{Methodology}

\section{Measures of Dispersion}

Three common measures of variability are considered: Mean Deviation $\left(\mathrm{S}_{\mathrm{md}}\right)$; Median Deviation $\left(\mathrm{S}_{\mathrm{mdd}}\right)$; and $\mathrm{MAD}$, the median absolute deviation $\left(\mathrm{S}_{\mathrm{mad}}\right.$ ). (Note that only the $\mathrm{S}_{\text {mad }}$ is considered a robust measure, as the other two procedures eventually incorporate an arithmetic mean.) We also present results for a new measure of dispersion described below that is noted as $\mathrm{S}_{\mathrm{bs}}$. All four measures are compared with $\mathrm{S}_{\mathrm{w}}$.

$\mathrm{S}_{\mathrm{md}}$

The mean deviation is defined as

$$
S_{m d}=\frac{\sum|X-\mu|}{N-1}
$$

The mean is subtracted from each score, the absolute value is taken, the results are summed, and then divided by N. For example, consider the original scores above, where the mean is 88.4 and median is 89 . The results are taken from Table $3 . \sum|X-\mu|=46$, and thus,
$\mathrm{S}_{\mathrm{md}}=5.11$.

$\mathrm{S}_{\mathrm{mdd}}$

This statistic is similar to the mean deviation, except it is based on the absolute value of the average of the median subtracted from each score, instead of the mean. The formula is

$$
S_{\text {mdd }}=\frac{\sum \mid X-\text { Median } \mid}{N-1}
$$

Because $\sum \mid X-$ Median $\mid=46, S_{\text {mdd }}=5.11$.

Note that coincidentally, this value is the same for $\mathrm{S}_{\mathrm{md}}$. Also, because the final value is obtained via the arithmetic mean for both $\mathrm{S}_{\mathrm{md}}$ and $\mathrm{S}_{\text {mdd }}$, the resulting statistics suffer from the lack of robustness ascribed to the arithmetic mean.

$\mathrm{S}_{\mathrm{mad}}$

$S_{\text {mad }}$ is similar to $S_{\text {mdd }}$, but with the important difference that instead of taking the mean of the absolute value of the deviations from the median, the median of the absolute value of the deviations from the median is taken, and thus, $\mathrm{S}_{\mathrm{mad}}$ is a robust statistic. The median of the values in the $3^{\text {rd }}$ column in Table 1 is 3 . Thus, $\mathrm{S}_{\mathrm{mad}}=3$.

$\mathrm{S}_{\mathrm{bs}}$

The idea behind the Bunner-Sawilowsky approach is to take into consideration the resulting histogram due to winsorizing, and attempt to smooth the end points. For example, if in a larger data set the winsorization method requires the recoding of the highest and lowest 10 values, then the endpoints of the distribution will have a mass at both recoding points, as noted in the Figure 1.

\begin{tabular}{|c|c|c|c|}
\hline Original Scores & $\underline{X-\mu}$ & X-Median & 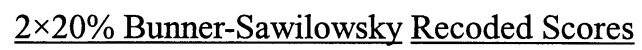 \\
\hline 73 & 15.4 & 16 & 86 \\
\hline 85 & 3.4 & 4 & 87 \\
\hline 86 & 2.4 & 3 & 86 \\
\hline 87 & 1.4 & 2 & 87 \\
\hline 88 & .4 & 1 & 88 \\
\hline 90 & 1.6 & 1 & 90 \\
\hline 91 & 2.6 & 2 & 91 \\
\hline 92 & 3.6 & 3 & 92 \\
\hline 93 & 4.6 & 4 & 91 \\
\hline 99 & 10.6 & 10 & 92 \\
\hline
\end{tabular}

Table 3. Computing Recorded Scores. 


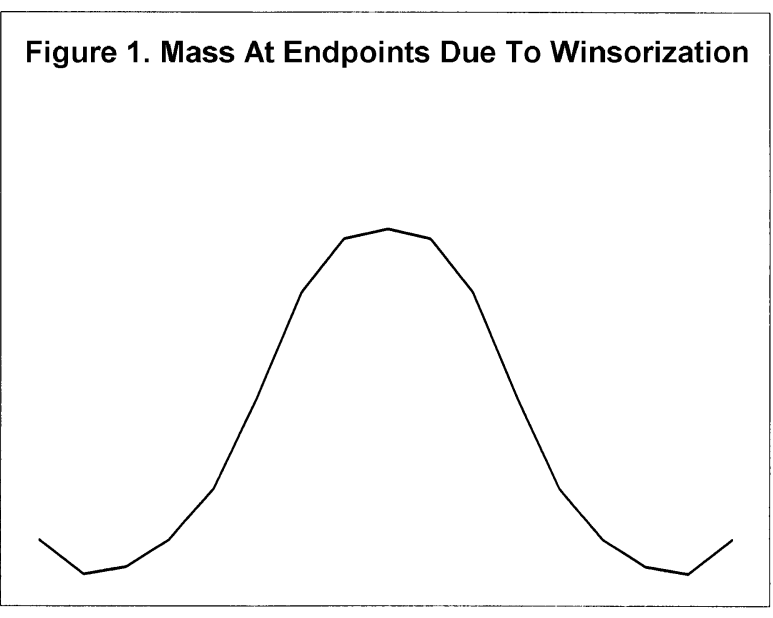

Suppose the winsorization was $2 \times 10 \%$, meaning for the original data set both the lowest and highest score would be recoded back one score when $\mathrm{N}=10$. In this case, the $\mathrm{S}_{\mathrm{bs}}$ procedure is identical to the winsorization. However, suppose that a $2 \times 20 \%$ recoding was desired, where two scores were to be recoded on each end of the distribution. In the original data set, the winsorized procedure would recode the 73 and the 85 to $86 \mathrm{~s}$, yielding a mass of three $86 \mathrm{~s}$; and the 99 and 93 would be recoded to $92 \mathrm{~s}$, yielding a mass of three $92 \mathrm{~s}$.

The Bunner-Sawilowsky approach smooths this mass by recoding the 73 to 86 , and the 85 to the next value score, which is an 87. Similarly, the 99 is recoded to a 92 , but the 93 is recoded to a 91 . The standard deviation of the recoded scores is 2.45 .

To summarize, the values for the example in descending order are $\mathrm{S}=6.8, \mathrm{~S}_{\mathrm{md}}=5.1, \mathrm{~S}_{\mathrm{mdd}}=5.1, \mathrm{~S}_{\mathrm{w}}=3.2$, $\mathrm{S}_{\text {mad }}=3$, and $\mathrm{S}_{\mathrm{bs}}=2.45$.

\section{Bracketed Intervals}

Two criteria were evaluated with regard to the performance of the various measures of dispersion being substituted into Formula (1) above. The first was the Type I error, where $\alpha=0.05$. The second was the width of the resulting interval, which is simply the range (upper - lower).

\section{Methodology}

The study proceeded as follows: A Minitab Version 13.1 macro was written to randomly select variates from a standard unit Gaussian (i.e., de Moivreian) distribution $\mathrm{N}(0,1)$, with samples of sizes $n=30$. Next, four models of outliers were used. They were:

- $\quad$ one wild score on the left of the distribution (1WL)

- two wild scores on the left of the distribution (2WL)

- three wild scores on the left of the distribution
(3WL)

- $\quad$ three wild scores on the left and one wild score on the right of the distribution (3WL-1WR)

The wild scores were created by taking the lowest score and subtracting 3.5; and where there were two wild scores to the left, also taking the second lowest score and subtracting 3.0; and where there were three wild scores to the left, also taking the third lowest score and subtracting 2.5; and where there were three wild scores to the left and one wild score to the right, also taking the highest score and adding 1.5. Because the $\mu$ and $\sigma$ of the population are 0 and 1 , respectively, this procedure takes the lowest score out an additional 3.5 standard deviations further from the mean, the second score is moved 3 standard deviations further from the mean, and so forth. The various measures of dispersion were computed, the resulting bracketed interval of the trimmed mean was calculated, the interval was checked to see if the population parameter was found within it, and the width of the interval was determined. Each experiment was repeated 1,000 times.

\section{Results}

The results are compiled in Table 4 below. Note that the common alternatives for measures of dispersion, the Mean Deviation, Median Deviation, and MAD resulted in Type I errors that were greatly inflated, typically from 0.05 to about 0.248 , almost five times nominal alpha. Even the use of the robust MAD statistic performed poorly. Although the width of the resulting intervals are typically about $45 \%$ narrower than bracketed intervals formed with the winsorized standard deviation, these procedures will no longer be considered due to their lack of ability in preserving Type I errors to nominal alpha.

The dispersion measure based on the BunnerSawilowsky approach resulted in robust Type I errors according to Bradley's (1968) liberal criteria, where $.5 \alpha \leq$ Type I error $\leq 1.5 \alpha$, or $0.025-.075$. These results were not within Bradley's conservative criteria, however, which is $.9 \alpha \leq$ Type I error $\leq 1.1 \alpha$, or $0.045-0.055$. The advantage of the Bunner-Sawilowsky approach, however, is that the resulting bracketed intervals are approximately $5.13 \%$ more narrow than the intervals formed by using the winsorized standard deviation.

\section{Conclusion}

The initial motivation for trying to improve on the bracketed interval of the trimmed mean was the consideration of no theoretical connection of the winsorized standard deviation to the trimmed mean. Furthermore, winsorization is a process that by definition creates a mass at the recoding points, which is at the extreme points of the distribution. 
Table 4. Width and Type I Error For Bracketed Interval of The Trimmed Mean For Various Alternatives of $\mathrm{S}_{\mathrm{w}}$, Gaussian Distribution With Perturbations; 1,000 repetitions, $\alpha=0.05$.

\begin{tabular}{|c|c|c|c|c|}
\hline \multicolumn{3}{|c|}{$1 \mathrm{WL}$} & \multicolumn{2}{|c|}{$2 \mathrm{WL}$} \\
\hline$\underline{\text { Statistic }}$ & Type I Error & Width & Type I Error & Width \\
\hline $\mathrm{S}_{\mathrm{w}}$ & .052 & .766 & .046 & .760 \\
\hline $\mathrm{S}_{\mathrm{hs}}$ & .064 & .727 & .061 & .724 \\
\hline $\mathrm{S}_{\mathrm{mad}}^{\mathrm{bS}}$ & $.222 *$ & .471 & $.226^{*}$ & .474 \\
\hline $\mathrm{S}_{\mathrm{md}}$ & $.267 *$ & .421 & $.263^{*}$ & .420 \\
\hline \multirow[t]{2}{*}{$\mathrm{S}_{\mathrm{mdd}}^{\mathrm{md}}$} & $.267^{*}$ & .415 & $.267^{*}$ & .415 \\
\hline & \multicolumn{2}{|l|}{ 3WL } & \multicolumn{2}{|c|}{$\underline{3 W L}-1 \mathrm{WR}$} \\
\hline $\mathrm{S}_{\mathrm{w}}$ & .050 & .768 & .054 & .765 \\
\hline $\mathrm{S}_{\mathrm{bs}}$ & .063 & .732 & .064 & .730 \\
\hline $\mathrm{S}_{\mathrm{mad}}$ & $.225^{*}$ & .471 & $.206^{*}$ & .474 \\
\hline $\mathrm{S}_{\mathrm{md}}$ & $.265^{*}$ & .423 & $.239 *$ & .439 \\
\hline $\mathrm{S}_{\mathrm{mdd}}$ & $.269 *$ & .416 & $.244^{*}$ & .434 \\
\hline
\end{tabular}

Note: $\mathrm{nWL}=$ Number of wild observations in the left tail. $\mathrm{nWR}=$ Number of wild observations in the right tail. $\mathrm{S}_{\mathrm{w}}=$ winsor. $\mathrm{S}_{\mathrm{bs}}=$ Bunner-Sawilowsky. $\mathrm{S}_{\mathrm{mad}}=$ Median Absolute Deviation. $\mathrm{S}_{\mathrm{md}}=$ Mean Deviation. $\mathrm{S}_{\mathrm{mdd}}=$ Median Deviation. * = Exceeds Bradley's (1968) liberal definition of robustness with respect to Type I error.

The new recoding scheme $\left(\mathrm{S}_{\mathrm{bs}}\right)$ examined in this paper ameliorated the mass at the recoded end points by smoothing out the tails of the distribution. The scheme investigated is equivalent to the usual winsorization when the number of values to be recoded is one. However, when additional points are identified as outliers, they are recoded to the next values closer to the median. If the four lowest values are noted as $\mathrm{x}_{1}, \mathrm{x}_{2}, \mathrm{x}_{3}$, and $\mathrm{x}_{4}$, and two values are to be recoded, then the usual winsorization procedure would recode both $x_{1}$ and $x_{2}$ to $x_{3}$. However, the $S_{b s}$ procedure would recode the value of $x_{1}$ to $x_{3}$ and $x_{2}$ to $x_{4}$. This, in effect, helps to reduce the mass at the recoding points.

Morever, the example data yielded the smallest estimate of variance for the $S_{b s}$ as compared with all other competitors investigated. This indicates its resistence to the presence of outliers. This property directly translated into producing bracketed intervals with widths smaller than that achieved by using the winsorized standard deviation in the bracketed interval of the trimmed mean formula.

An inspection of the results indicated that the $\mathrm{S}_{\mathrm{bs}}$ produced intervals that were more than $5 \%$ narrower than the usual winsorization. However, further study of this recoding scheme, and similar alternatives, is necessary because the Type I error rates were slightly inflated (e.g., $\approx$.06).

\section{References}

Blair, R. C. (1981). A reaction to 'Consequences Of Failure To Meet Assumptions Underlying The Fixed Effects Analysis of Variance and Covariance'. Review of Educational Research, 51, 499-507.

Blair, R. C., \& Higgins, J. J. (1980a). A comparison of the power of the $t$ test and Wilcoxon statistics when samples are draw from a certain mixed normal distribution. Evaluation Review, 4, 645-656.

Blair, R. C., \& Higgins, J. J. (1980b). A comparison of the power of the Wilcoxon's rank-sum statistic to that of Student's t statistic under various nonnormal distributions. Journal of Educational Statistics, 5, 309-355.

Blair, R. C., \& Higgins, J. J. (1985). A comparison of the power of the paired samples $t$ test to that of Wilcoxon's signed-ranks test under population shapes. $P s y$ chological Bulletin, 97, 119-128.

Bradley, J. V. (1968). Distribution-free statistical tests. Englewood Cliffs, NJ: Prentice Hall.

Bradley, J. V. (1978). Robustness? British Journal of Mathematical and Statistical Psychology, 31, 144-152. 
Lax, D. A. (1985). Robust estimators of scale: Finite sample performance in long-tailed symmetric distributions. Journal of the American Statistical Association, 80, 736-741.

Micceri, T. (1989). The unicorn, the normal curve, and other improbable creatures. Psychological Bulletin, 105, 156-166.

Minitab. (2000). MINITAB v. 13.1. State College, PA: Minitab, Inc.

Pearson, E. S., \& Please, N. W. (1975). Relation between the shape of population distribution and the robustness of four simple test statistics. Biometrika, 62, 223241.
Sawilowsky, S. S., \& Hillman, S. B. (1992). Power of the independent samples $t$ test under a prevalent psychometric distribution. Journal of Consulting and Clinical Psychology, 60, 240-243.

Tan, W. Y. (1982). Sampling distributions and robustness of $t, F$, and variance-ratio in two samples and ANOVA models with respect to departure from normality. Communications in Statistics, 11, 2485-2511.

Tukey, J. W., \& McLaughlin, D. H. (1963). Less vulnerable confidence and significance procedures for location based on a single sample: Trimming/Winsorization 1. Sankhya, 25, 331-352.

Wilcox, R. R. (1996). Statistics for the social sciences. San Diego, CA: Academic Press. 


\title{
JMASM Algrorithms and Code \\ JMASM1: RANGEN 2.0 (Fortran 90/95)
}

\author{
Gail F. Fahoome \\ Educational Evaluation \& Research \\ College of Education \\ Wayne State University
}

Rangen 2.0 is Fortran 90 module of subroutines used to generate uniform and nonuniform pseudo-random deviates. It includes uni1, an uniform pseudo-random number generator, and non-uniform generators based on uni1. The subroutines in Rangen 2.0 were written using Essential Lahey Fortran 90, a proper subset of Fortran 90. It includes both source code for the subroutines and a short description of each subroutine, its purpose, and the arguments including data type and usage.

Keywords: Psuedo-random number generators, Fortran subroutines

Introduction

Rangen is a collection of subroutines used to generate uniform and nonuniform pseudo-random deviates. Rangen 1.0, the original version, was written in Fortran 77 (IBM RT PC FORTRAN 77, Version 1.01) by R. Clifford Blair and published by IBM in 1987. It was developed for the IBM AIX Unix platform. Shlomo S. Sawilowsky produced Rangen 1.1, a minor translation of Rangen 1.0, for the PC environment, and added additional subroutines which are not included here. Rangen 2.0 is a translation of selected Rangen 1.1 subroutines from Fortran 77 to Fortran 90 using Essential Lahey Fortran 90 (ELF) (1998, Version 4.0). $E L F$ is a proper subset of Fortran 90 . The selected subroutines have been incorporated into a module.

The original version of Rangen included two uniform pseudo-random number generators, called Uni1 and Uni2. Blair noted that both had been extensively tested and "appear to be excellent generators" (1987, p. I-1). There were some differences between the two. For example, Uni1 is faster than Uni2, and thus may produce slightly less random deviates. However, "In most cases either generator will prove to be quite satisfactory" (Blair, p. I-1). Rangen 2.0 includes Uni1 and the nonuniform pseudo-random number generators based on Uni1. The names of the subroutines have retained the designation ' 1 ' so that Uni2 and the nonuniform pseudo-random number generators based on Uni2 can be added at a later date.

The material is presented in two sections. The first section contains the source code for the module Rangen 2.0. The second section contains a short description of each

Gail F. Fahoome is Lecturer, Educational Evaluation and Research, College of Education, Wayne State University. Contact her at 18968 Hamburg, Detroit, MI 48205 for all communication regarding this paper. E-mail her at ac7252@wayne.edu. Her areas of expertise are Monte Carlo methods, nonparametric statistics, and program evaluation. subroutine, its purpose, and the arguments including data type and usage. An example of a program segment is included for each subroutine, including the type declarations, initialization of variables, and the subroutine call. The output of the example is given so that the user may check output for accuracy.

The references for the algorithms used in the subroutines written by Blair are included in the description section of Rangen 2.0. Subroutine Normb1, and Ppnd2, a subroutine called by Normb1, are adaptations of code written by Beasley and Springer (1985). The citation and original permission to reproduce published in Rangen 1.0 are contained in the description of Normb1.

\section{Source Code for Rangen 2.0}

\section{module rangen}

implicit none

public:: cay 1 , chisq1, erl1, exp1, f1, lap1, Inor1, normb1, t1, uni1, ppnd2

contains

!

subroutine cay 1 (dseed, inp, $\mathrm{x}$ )

integer: : $i$

integer, intent(in): : inp

real, intent(out): : x(inp)

real: : $u(2), v 1, v 2$

real (kind $=8$ ), intent(in out): : dseed

do $\mathbf{i}=1$, inp

10 call uni 1 (dseed, $2, u$ )

$\mathrm{v} 1=2 * u(1)-1$

$\mathrm{v} 2=2 * \mathrm{u}(2)-1$

if $(\mathrm{v} 1 * * 2+\mathrm{v} 2 * * 2$.gt. 1$)$ go to 10

$x(i)=v 1 / v 2$

end do

return

end subroutine cay 1

!

subroutine chisq1(dseed, inp, idf, $y, x$ ) 
183

integer, intent(in):: inp, idf

integer: : $i$, idf 1

real, intent(in out):: $x$ (inp), y(inp)

real (kind $=8)$, intent(in out):: dseed

if (idf $==1$ ) go to 30

if $(\bmod (\mathrm{idf}, 2) /=0)$ go to 40

idf $1=$ idf $/ 2$

call erl1(dseed, inp, idf1, 2.0, y, x)

return

30 call normb1(dseed, inp, $x$ )

do $i=1$, inp

$x(i)=x(i) * * 2$

end do

return

40 idf1 $=($ idf- 1$) / 2$

call erl1(dseed, inp, idf1, 2.0, y, x)

call normb1(dseed, inp, y)

do $i=1$, inp

$x(i)=x(i)+y(i) * * 2$

end do

return

end subroutine chisq 1

!

subroutine erl1(dseed, inp, ia, $b, y, x$ )

integer, intent(in):: inp

integer, intent(in): : ia

integer: : $i, j$

real, intent(in): : $b$

real, intent(in out): : x(inp)

real, intent(in out):: $y$ (inp)

real:: py

real (kind $=8$ ), intent(in out): : dseed

if (ia > 50) go to 30

do $i=1$, inp

call uni1(dseed, ia, y)

py $=y(1)$

do $\mathrm{j}=2$, ia

py $=p y^{*} y(j)$

end do

$x(i)=-b * \log (p y)$

end do

return

30 do $i=1$, inp

call uni1(dseed, ia, y)

py $=y(1)$

do $\mathrm{j}=2,50$

py $=p y^{*} y(j)$

end do

$x(i)=-b * \log (p y)$

py $=0.0$

do $\mathrm{j}=51$, ia

py $=p y+\log (y(j))$

end do

$x(i)=-b * p y+x(i)$
GAIL FAHOOME

end do

return

end subroutine erl1

!

subroutine exp1(dseed, inp, sm, $x$ )

integer, intent(in)::inp

integer: : $i$

real, intent(out):: $x$ (inp)

real, intent(in): : sm

real (kind $=8)$, intent(in out): : dseed

call uni1(dseed, inp, $x$ )

do $i=1$, inp

$x(i)=(-s m) *(\log (x(i)))$

end do

return

end subroutine exp1

!

subroutine f1(dseed, inp, idfn, idfd, y1, y2, x)

integer, intent(in):: inp, idfn, idfd

integer: : $i$

real, intent(out):: $x$ (inp)

real, intent(in out):: y1(inp), y2(inp)

real (kind $=8$ ), intent(in out): : dseed

call chisq1(dseed, inp, idfn, y1, y2)

call chisq1(dseed, inp, idfd, $y 1, x$ )

do $i=1$, inp

$x(i)=(y 2(i) /$ real $($ idfn $)) /(x(i) /$ real (idfd $))$

end do

return

end subroutine f1

!

subroutine lap1(dseed, inp, $y, x$ )

integer, intent(in):: inp

integer: : $i$

real, intent(out):: $x$ (inp)

real, intent(in out):: $y$ (inp)

real (kind $=8$ ), intent(in out):: dseed

call uni1(dseed, inp, y)

call exp1(dseed, inp, 1.0, x)

do $i=1$, inp

if $(y(i)>=.5) x(i)=-x(i)$

end do

return

end subroutine lap1

!

subroutine Inor1(dseed, inp, am, sd, $x$ )

integer, intent(in):: inp

integer:: $i$

real, intent(in): : am, sd

real, intent(out): : x(inp)

real (kind $=8$ ), intent(in out):: dseed

call normb1(dseed, inp, $x$ )

do $i=1$, inp

$x(i)=\exp \left(s d^{*} x(i)+a m\right)$ 
end do

return

end subroutine Inor1

!

subroutine normb1(dseed1, inp1, x1)

integer, intent(in):: inp1

integer: $i$, ifault

real, intent(out): : x1(inp1)

real(kind $=8$ ), intent(in out): : dseed 1

real:: xtemp

call uni1(dseed1, inp1, x1)

do $\mathbf{i}=1$, inp 1

call ppnd $2(x 1(i), x t e m p$, ifault)

$x 1(i)=x$ temp

end do

return

end subroutine normb1

!

subroutine $t 1$ (dseed, inp, idf, $y, x$ )

integer, intent(in): : inp, idf

integer: : i

real, intent(out): : $x$ (inp)

real, intent(in out): : $y$ (inp)

real (kind $=8$ ), intent(in out): : dseed

call chisq1(dseed, inp, idf, $y, x$ )

call normb1(dseed, inp, $y$ )

do $i=1$, inp

$x(i)=y(i) / \operatorname{sqrt}((x(i) /$ real(idf $)))$

end do

return

end subroutine $\mathrm{t} 1$

!

subroutine uni 1 (dseed, inp, $x$ )

real(kind $=8$ ), intent(in out): : dseed

integer, intent(in): : inp

integer: : i

real, intent(out): : $x$ (inp)

do $i=1$, inp

dseed $=$ modulo $\left(16807.8^{*}\right.$ dseed, 2147483647._8)

$x(i)=$ dseed $/ 2147483648 . \_8$

end do

return

end subroutine uni 1

!

subroutine ppnd 2 ( $p$, ppndt, ifault)

real:: zero, split, half, one, a0, a1, a2, a3, b1, b2, b3, b4, \&

$c 0, c 1, c 2, c 3, d 1, d 2, q, r$

real,intent(in):: $p$

real, intent(out):: ppndt

integer, intent(out): : ifault

zero $=0.0 \mathrm{e} 0$

half $=0.5 \mathrm{e} 0$ one $=1.0 \mathrm{e} 0$

split $=0.42 \mathrm{e} 0$

$\mathrm{a} 0=2.50662823884 \mathrm{e} 0$

$\mathrm{a} 1=-18.61500062529 \mathrm{e} 0$

$\mathrm{a} 2=41.39119773534 \mathrm{e} 0$

$a 3=-25.44106049637 e 0$

b1 $=-8.47351093090 \mathrm{e} 0$

$b 2=23.08336743743 \mathrm{e} 0$

b3 $=-21.06224101826 \mathrm{e} 0$

b4 $=3.13082909833 \mathrm{e} 0$

!

$\mathrm{CO}=-2.78718931138 \mathrm{e} 0$

$\mathrm{c} 1=-2.29796479134 \mathrm{e} 0$

$\mathrm{c} 2=4.85014127135 \mathrm{e} 0$

$c 3=2.32121276858 \mathrm{e} 0$

$\mathrm{d} 1=3.54388924762 \mathrm{e} 0$

$\mathrm{d} 2=1.63706781897 \mathrm{e} 0$

ifault $=0$

$q=p$ - half

if (abs(q) > split) go to 1

$r=q^{*} q$

ppndt $=q *(((a 3 * r+a 2) * r+a 1) * r+a 0) /$

$((((b 4 * r+b 3) * r+b 2) * r+b 1) * r+o n e)$

return

$1 r=p$

if $(q>$ zero) $r=$ one $-p$

if $(r<=$ zero) go to 2

$r=\operatorname{sqrt}(-\log (r))$

ppndt $=(((c 3 * r+c 2) * r+c 1) * r+c 0) /$

$((\mathrm{d} 2 * r+\mathrm{d} 1) * r+o n e)$

if $(\mathrm{q}<$ zero $)$ ppndt $=$ _ppndt

return

2 ifault $=1$

ppndt $=$ zero

return

end subroutine ppnd 2

!

end module rangen 


\section{Description of Rangen 2.0 Subroutines}

CAY1

Reference: Rubinstein, R. Y. (1981).

Cay1 generates deviates from a Cauchy distribution.

Arguments: $\quad$ dseed $\quad$ - Input/output. Dseed must be an integer of type real (kind=8) in the exclusive range 1. 8 to 2147483647 ._8. Cay1 returns a new dseed.

inp - Input: The number of deviates to be returned.

$\mathrm{x} \quad-\quad$ Output: A vector of length inp containing the deviates.

Call: $\quad$ call cay $1(\mathrm{dseed}$, inp, $\mathrm{x}$ )

Example: $\quad$ Cayl is used to generate 100 deviates.

real:: $x(100)$

real $($ kind $=8)$ : : dseed

dseed $=12346$

call cay 1 (dseed, $100, \mathrm{x}$ )

Output:

dseed $=944541922$

$x(1)=-37.1592$

$x(100)=5.59855$

CHISQ1

Reference: Hastings, N. A., \& Peacock, J. B. (1974).

Chisq1 generates deviates from a chi-squared distribution with user-provided degrees of freedom.

Arguments: dseed - Input/output. Dseed must be an integer of type real (kind=8) in the exclusive range 1._8 to 2147483647._8. Chisq1 returns a new dseed.

inp - Input: The number of deviates (integer) to be returned.

idf - Input: The value (integer) of degrees of freedom.

y $\quad-\quad$ Work vector (real) of length inp.

$\mathrm{x} \quad-\quad$ Output: A real vector of length inp containing the deviates.

Call: $\quad$ call chisq1(dseed, inp, idf, $y, x)$

Example: $\quad$ Chisq1 is used to generate 100 deviates.

integer:: idf

integer, parameter:: inp $=100$

real:: $x$ (inp), $y$ (inp)

real (kind $=8$ ): : dseed

dseed $=12346$

idf $=3$

call chisq1(dseed, inp, idf, $y, x$ )

Output:

dseed $=1533170485$

$x(1)=4.69289$

$x(100)=1.86385$ 
ERL1

Reference: Hastings, N. A., \& Peacock, J. B. (1974).

Erl1 generates deviates from a Erlang distribution with user-provided degrees of freedom.

Arguments: $\quad$ dseed - Input/output. Dseed must be an integer of type real $(\mathrm{kind}=8)$ in the exclusive range 1. 8 to 2147483647 . 8 . Erl1 returns a new dseed.

$\begin{array}{lll}\text { inp } & \text { - Input: The number of deviates (integer) to be returned. } \\ \text { ia } & \text { - } & \text { Input: Shape parameter (integer) which must be greater than or equal to one. } \\ \text { b } & \text { - Input: Scale parameter (real) which must be greater than zero. } \\ \text { y } & \text { - } & \text { Work vector of length inp. } \\ \mathrm{x} & \text { - } & \text { Output: A real vector of length inp containing the deviates. }\end{array}$

Call: $\quad$ call erl1(dseed, inp, ia, $b, y, x)$

Example: $\quad$ Erl1 is used to generate 100 deviates.

integer:: ia

integer, parameter: : inp $=100$

real:: $x$ (inp), $y($ inp $), b$

real (kind $=8):$ : dseed

dseed $=12346$

ia $=2$

$b=3.5$

call erl1(dseed, inp, ia, b, y, x)

Output:

dseed $=1533170485$

$x(1)=8.30200$

$x(100)=1.27826$

EXP1

Reference: Morgan, B. J. T. (1984).

Exp1 generates deviates from an exponential distribution with user-provided mean and standard deviation.

Arguments: $\quad$ dseed $\quad$ - Input/output: Dseed must be an integer of type real (kind=8) in the exclusive range 1._8 to 2147483647._8. Exp1 returns a new dseed.

inp - Input: The number of deviates (integer) to be returned.

sm - Input: Mean and standard deviation (real) of the sampled population.

$x \quad$ - Output: A real vector of length inp containing the deviates.

Call: $\quad$ call exp1(dseed, inp, sm, $x$ )

Example: $\quad$ Exp1 is used to generate 100 deviates.

integer, parameter: : in $p=100$

real:: $x$ (inp), sm

real (kind $=8$ ): : dseed

dseed $=12346$

$\mathrm{sm}=1.0$

call exp1(dseed, inp, sm, $x$ )

Output:

dseed $=991974008$

$x(1)=2.33692$

$x(100)=0.772355$ 
$\mathrm{F} 1$ generates deviates from a $\mathrm{F}$ distribution with user-provided degrees of freedom.

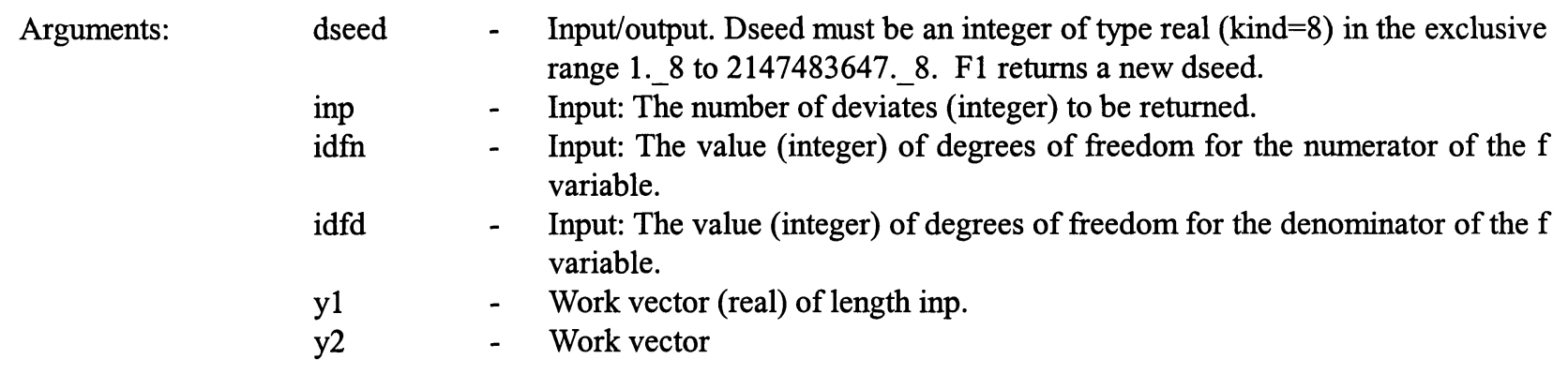

Call: $\quad$ call f1(dseed, inp, idfn, idfd, y1, y2, x)

Example: $\quad F 1$ is used to generate 100 deviates.

integer: : idfn, idfd

integer, parameter: : inp $=100$

real:: $x$ (inp), y1(inp), y2(inp)

real $($ kind $=8)$ : : dseed

dseed $=12346$

idfn $=3$

idfd $=5$

call f1(dseed, inp, idfn, idfd, y1, y2, x)

Output:

dseed $=580303867$

$x(1)=1.39239$

$x(100)=1.30237$

LAP1

Reference: Morgan, B. J. T. (1984).

Lap1 generates deviates from a Laplace (double exponential) distribution, using an exponential distribution with mean and standard deviation 1.0.

Arguments: $\quad$ dseed $\quad-$ Input/output: Dseed must be an integer of type real (kind=8) in the exclusive range 1._8 to 2147483647._8. Lap1 returns a new dseed.

inp - Input: The number of deviates (integer) to be returned.

y - - A work vector (real) of length inp.

$\mathrm{x} \quad$ - Output: A real vector of length inp containing the deviates.

Call: $\quad$ call lap1(dseed, inp, $y, x$ )

Example: Lap1 is used to generate 100 deviates.

integer, parameter: : inp $=100$

real: : $x$ (inp), $y$ (inp)

real $($ kind $=8)$ : : dseed

dseed $=12346$

call lap1(dseed, inp, $y, x)$

Output:

dseed $=1533170485$

$x(1)=0.588999$

$x(100)=0.336959$ 
LNOR1

Reference: Hastings, N. A., \& Peacock, J. B. (1974).

Lnor1 generates deviates from a lognormal distribution with user-provided parameters.

\begin{tabular}{|c|c|c|}
\hline Arguments: & $\begin{array}{l}\text { inp } \\
\text { am } \\
\text { sd } \\
x\end{array}$ & $\begin{array}{l}\text { Input/output: Dseed must be an integer of type real }(\mathrm{kind}=8) \text { in the exclusive } \\
\text { range } 1.8 \text { to } 2147483647.8 . \text { Lnor } 1 \text { returns a new dseed. } \\
\text { - } \quad \text { Input: The number of deviates (integer) to be returned. } \\
\text { - } \quad \text { Input: The mean of y (real) for } y=\ln (\mathrm{x}) \text {. } \\
\text { - } \quad \text { Input: The standard deviation }(\text { real) of y for } y=\ln (\mathrm{x}) \text {. } \\
\text { - } \quad \text { Output: A real vector of length inp containing the deviates. }\end{array}$ \\
\hline
\end{tabular}

Call: $\quad$ call Inor1(dseed, inp, am, sd, $x$ )

Example: $\quad$ Lnor1 is used to generate 100 deviates.

integer, parameter: : inp $=100$

real:: $x$ (inp), am, sd

real (kind $=8):$ : dseed

dseed $=12346$

$\mathrm{am}=0.0$

$\mathrm{sd}=\operatorname{sqrt}(2.0)$

call Inor1(dseed, inp, am, sd, x)

Output:

dseed $=991974008$

$x(1)=0.158828$

$x(100)=0.873557$

NORMB1

References: Beasley, J. D., \& Springer, S. G. (1985); Marsaglia, G., MacLaren, M. D., \& Bray, T. A. (1964).

Normb1 generates deviates from the standard normal distribution.

Arguments: $\quad$ dseed - Input/output: Dseed must be an integer of type real $(\mathrm{kind}=8)$ in the exclusive range 1._8 to 2147483647 ._8. Normb1 returns a new dseed.

inp - Input: The number of deviates (integer) to be returned.

$x \quad-\quad$ Output: A real vector of length inp containing the deviates.

Call: $\quad$ call normb1(dseed, inp, $x$ )

Example: $\quad$ Normb1 is used to generate 100 deviates.

integer, parameter: : inp $=100$

real:: $x$ (inp)

real (kind $=8)$ : : dseed

dseed $=12346$

call normb1(dseed, inp, $x$ )

Output:

dseed $=991974008$

$x(1)=-1.30103$

$x(100)=-0.095588$

Note: The subroutines Normb1 and Ppnd2 (called by Normb1) are adaptations of Beasley, J. D., \& Springer, S. G. (1985), "The Percentage Points of the Normal Distribution". Reproduced with permission from Applied Statistics Algorithms by Griffiths and Hill; Chichester, England: Ellis Horwood Limited. (See Blair, 1987.) 
$\mathrm{T} 1$ generates deviates from an exponential distribution with user-provided degrees of freedom.

Arguments: $\quad$ dseed - Input/output: Dseed must be an integer of type real (kind=8) in the exclusive range 1._8 to 2147483647 ._8. T1 returns a new dseed.

inp - Input: The number of deviates (integer) to be returned.

idf - Input: The degrees of freedom (integer) for the $t$ variable.

y - - Work vector (real) of length inp.

$\mathrm{x} \quad$ - Output: A real vector of length inp containing the deviates.

Call: $\quad$ call t1(dseed, inp, idf, $y, x$ )

Example: $\mathrm{T} 1$ is used to generate 100 deviates.

integer:: idf

integer, parameter: : in $\mathrm{p}=100$

real: : $x$ (inp), $y$ (inp)

real $($ kind $=8)$ : : dseed

dseed $=12346$

idf $=3$

call t1(dseed, inp, idf, $y, x$ )

Output:

dseed $=489858532$

$x(1)=-0.800539$

$x(100)=-0.945289$

UNI1

References: Learmonth, G. P., \& Lewis, P. A. W. (1973); Morgan, B. J. T. (1984).

Uni1 generates deviates from the uniform distribution.

Arguments: $\quad$ dseed $\quad$ Input/output: Dseed must be an integer of type real (kind=8) in the exclusive range 1._8 to 2147483647._8. Unil returns a new dseed.

inp - Input: The number of deviates (integer) to be returned.

$\mathrm{x} \quad-\quad$ Output: A real vector of length inp containing the deviates.

Call: $\quad$ call uni1(dseed, inp, $x$ )

Example: Uni1 is used to generate 100 deviates.

integer, parameter:: inp $=100$

real:: $x$ (inp)

real $($ kind $=8):$ : dseed

dseed $=12346$

call uni1(dseed, inp, $x$ )

Output:

dseed $=991974008$

$x(1)=0.0966244$

$x(100)=0.461924$ 


\section{References}

Beasley, J. D., \& Springer, S. G. (1985). The percentage points of the normal distribution. In P. Griffiths and I. D. Hill (Eds.), Applied statistics algorithms. Chichester, England: Ellis Horwood.

Blair, R. C. (1987). Rangen 1.0. Boca Raton, FL: IBM.

Hastings, N. A., \& Peacock, J. B. (1974). Statistical distributions. New York: Wiley \& Sons.

Learmonth, G. P., \& Lewis, P. A. W. (1973). Naval postgraduate school random number generator package LLRANDOM NPS55LW3061A. Monterey, California: Naval Postgraduate School.
Marsaglia, G., MacLaren, M. D., \& Bray, T. A. (1964). Communications of the Association for Computing Machinery, 7, 4-10.

Morgan, B. J. T. (1984). Elements of simulation. London: Chapman and Hall.

Rubinstein, R. Y. (1981). Simulation and the Monte Carlo method. New York: Wiley \& Sons. 


\title{
Generation of Combinations Using Excel
}

\author{
Constantine Stamatopoulos \\ Fisheries Department \\ Food and Agriculture Organization of the United Nations (FAO)
}

Theoretical development of combinations via enumeration methods are considered. An Excel marcro is provided.

Keywords: Combinations, Enumeration, Excel, Visual Basic

Introduction

The presented algorithm is applicable in situations where exact mathematical solutions are either not applicable or impractical, and simple enumeration approaches are used as alternatives for problem solving. By an enumeration approach, I mean a process by means of which all possible cases associated to a problem are first fully listed and then tested against a set of criteria for determining acceptable or optimal solutions.

Automatic enumeration of problem cases can be achieved in a wide variety of ways. Generally, such algorithms ought to respond to the following two operational requirements:

- The population of cases should not be resident in the computer memory. A current case that has been formulated and tested should also be the basis for generating the next one;

- All possible cases must be exhausted.

Combinations constitute a special category of enumeration approaches with a wide range of applications. In this article two examples are presented. The first concerns samples taken from a given population and tested against pre-set criteria. The second examines optimal geographical distribution of focal points that provide/receive services to/from other given points.

The computerized algorithm for generating combinations is written in Visual Basic and listed in Annex II. In its present form it records all combinations of $\mathbf{N}$ by $\mathbf{M}$ elements on a text file that can be used externally by a main program. It can also be easily modified for incorporation into the main program and used as a sub-routine.

Constantine Stamatopoulos is a graduate of mathematics from Athens University. Since 1971 he has been working for the Food and Agriculture Organization of the United Nations (FAO) in the sector of fisheries statistical development. Email: Constantine.Stamatopoulos@fao.org.
Methodology

Assume a finite set of $\mathbf{N}$ elements: $\mathbf{E}=\left\{\mathbf{e}_{1}, \mathbf{e}_{2}, \ldots, \mathbf{e}_{\mathrm{N}}\right\}$.

Any combination of $M$ elements $C=\left\{\mathbf{e}_{\mathbf{s}_{1}}, \mathbf{e}_{\mathrm{s}_{2}}, \ldots, \mathbf{e}_{\mathrm{s}_{\mathrm{M}}}\right\}$ with $\mathbf{M}$ between 1 and $\mathbf{N}$ is fully described by the array of subscripts: $\mathbf{s}_{\mathbf{1}}, \mathbf{s}_{\mathbf{2}}, \ldots, \mathbf{s}_{\mathbf{M}}$. Conventionally, also assume that the array of subscripts is written in a manner so as to have: $\mathbf{s}_{\mathbf{1}}>\mathbf{s}_{\mathbf{2}}>$,..., $>\mathbf{s}_{\mathbf{M}}$. In order to generate all combinations of $\mathbf{N}$ by $\mathbf{M}$, proceed as follows:

Step 1

Determine the first combination by the array of $\mathbf{M}$ subscripts: $s_{1}=N, s_{2}=N-1, \ldots, s_{M}=N-M+1$. Use it as the current combination.

Step 2

2.1 Check if current combination is the last one.

If the first subscript $\mathbf{s}_{\mathbf{1}}=\mathbf{M}$ this means that the current combination is the last one and the process is ended here.

2.2 Check for the first occurrence of $\mathbf{s}_{\mathbf{i}}=\mathbf{M}-\mathbf{i}+\mathbf{1}$

If this does not occur then apply Step 3 else apply Step 4.

Step 3

Generate a new combination: $\mathbf{s}_{\mathbf{1}}, \mathbf{s}_{\mathbf{2}}, \ldots, \mathbf{s}_{\mathbf{M}}-\mathbf{1}$, that is by subtracting 1 from the last subscript. Use it as current combination and repeat Step 2.

Step 4

Recall that $\mathbf{i}$ is the first occurrence for which: $\mathbf{s}_{\mathbf{i}}=\mathbf{M}-\mathbf{i}+\mathbf{1}$. Generate a new combination: $s_{1}=$ same, $s_{2}=$ same $, \ldots, s_{i-1}=s_{i-1}-1, s_{i}=s_{i-1}-2$, $\mathbf{s}_{\mathbf{i}+\mathbf{1}}=\mathbf{s}_{\mathbf{i}-\mathbf{1}}-\mathbf{3}$, etc. Use it as current combination and repeat Step 2. 
Table 1 - Example: Generation of combinations with $\mathrm{N}=6, \mathrm{M}=4$

\begin{tabular}{|c|c|c|c|c|c|}
\hline No. & $\mathbf{s}_{1}$ & $\mathbf{s}_{\mathbf{2}}$ & $\mathbf{s}_{3}$ & $\mathbf{s}_{4}$ & Remarks \\
\hline 1 & 6 & 5 & 4 & 3 & Generate first combination as per Step 1. Check conditions 2.1 and 2.2. Applv Step 3. \\
\hline 2 & 6 & 5 & 4 & 2 & Check conditions 2.1 and 2.2. Apply Step 3. \\
\hline 3 & 6 & 5 & 4 & 1 & Condition 2.2 holds for last subscript. Apply step 4. \\
\hline 4 & 6 & 5 & 3 & 2 & Check conditions 2.1 and 2.2. Apply Step 3. \\
\hline 5 & 6 & 5 & 3 & 1 & Condition 2.2 holds for last subscript. Apply step 4. \\
\hline 6 & 6 & 5 & 2 & 1 & Condition 2.2 holds for $3^{\text {rd }}$ subscript. Apply step 4. \\
\hline 7 & 6 & 4 & 3 & 2 & Check conditions 2.1 and 2.2. Apply Step 3. \\
\hline 8 & 6 & 4 & 3 & 1 & Condition 2.2 holds for last subscript. Apply step 4. \\
\hline 9 & 6 & 4 & 2 & 1 & Condition 2.2 holds for 3rd subscript. Apply step 4. \\
\hline 10 & 6 & 3 & 2 & 1 & Condition 2.2 holds for 2nd subscript. Apply step 4. \\
\hline 11 & 5 & 4 & 3 & 2 & Check conditions 2.1 and 2.2. Apply Step 3. \\
\hline 12 & 5 & 4 & 3 & 1 & Condition 2.2 holds for last subscript. Apply step 4. \\
\hline 13 & 5 & 4 & 2 & 1 & Condition 2.2 holds for $3^{\text {rd }}$ subscript. Apply step 4. \\
\hline 14 & 5 & 3 & 2 & 1 & Condition 2.2 holds for $2^{\text {nd }}$ subscript. Apply step 4 . \\
\hline 15 & 4 & 3 & 2 & 1 & End of process since $s_{1}=4$ \\
\hline
\end{tabular}

\section{Results}

Application 1

Given the set of numbers $1,2, \ldots, 10$ find if there exist combinations of three numbers with sums between and including 13 and 17, and product between and including 120 and 130.

Solution

The presented algorithm was used to generate all 10 by 3 combinations. Each combination was then tested to satisfy the given criteria. Of the resulting 120 combinations the following four have passed the test successfully:

\begin{tabular}{lll}
\hline Combination & Sum & Product \\
\hline 1043 & 17 & 120 \\
853 & 16 & 120 \\
763 & 16 & 126 \\
654 & 15 & 120 \\
\hline
\end{tabular}

Application 2

In this application there are twenty locations or points $\mathbf{P}_{\mathbf{i}}, \mathbf{i}=1,2, \ldots, 20$ with co-ordinates $\left(\mathbf{X}_{\mathbf{i}}, \mathbf{Y}_{\mathbf{i}}\right)$ as illustrated in Figure 1. The problem is to identify four servicing locations among these points such that their distances from the points being serviced constitute a global minimum.

\section{Solution}

The presented algorithm was used to generate all $\mathrm{N}=20$ by $M=4$ combinations. Each point in a combination was then connected to its closest points (including itself) and the sum of resulting distances was computed. Optimal combination was found to be the one consisting of points P3, P6, P12 and P16.

\section{Appendix I}

This Annex contains a listing of the computerized algorithm for generating $\mathbf{N}$ by $\mathbf{M}$ combinations. The steps for generating and running the program as an Excel macro are given below:

1. Type the program using a text editor and save it.

2. Select all program lines and copy them into the computer memory (i.e. CTL+c).

3. Create an Excel worksheet named COMB.XLS.

4. From the Tools menu select "Record new macro".

5. Specify a key combination to trigger the macro, i.e. CTL+a.

6. The Excel program will enter into macro recording mode. Using Tools select "Stop recording".

7. From the Tools menu select "Macros" and then "Edit macro"

8. Paste the program lines into the macrol subroutine (i.e. CTL $+v$ ).

9. Exit from macro edit mode.

10. Save the worksheet.

Each time COMB.XLS is called the "macros enabled" button must be used. To run the macro the following steps are required:

11. Enter variable $\mathbf{N}$ in cell B3, variable $\mathbf{M}$ in B4 and finish with ENTER.

12. Press CTL+a (or the key combination specified during the recording of the macro). 


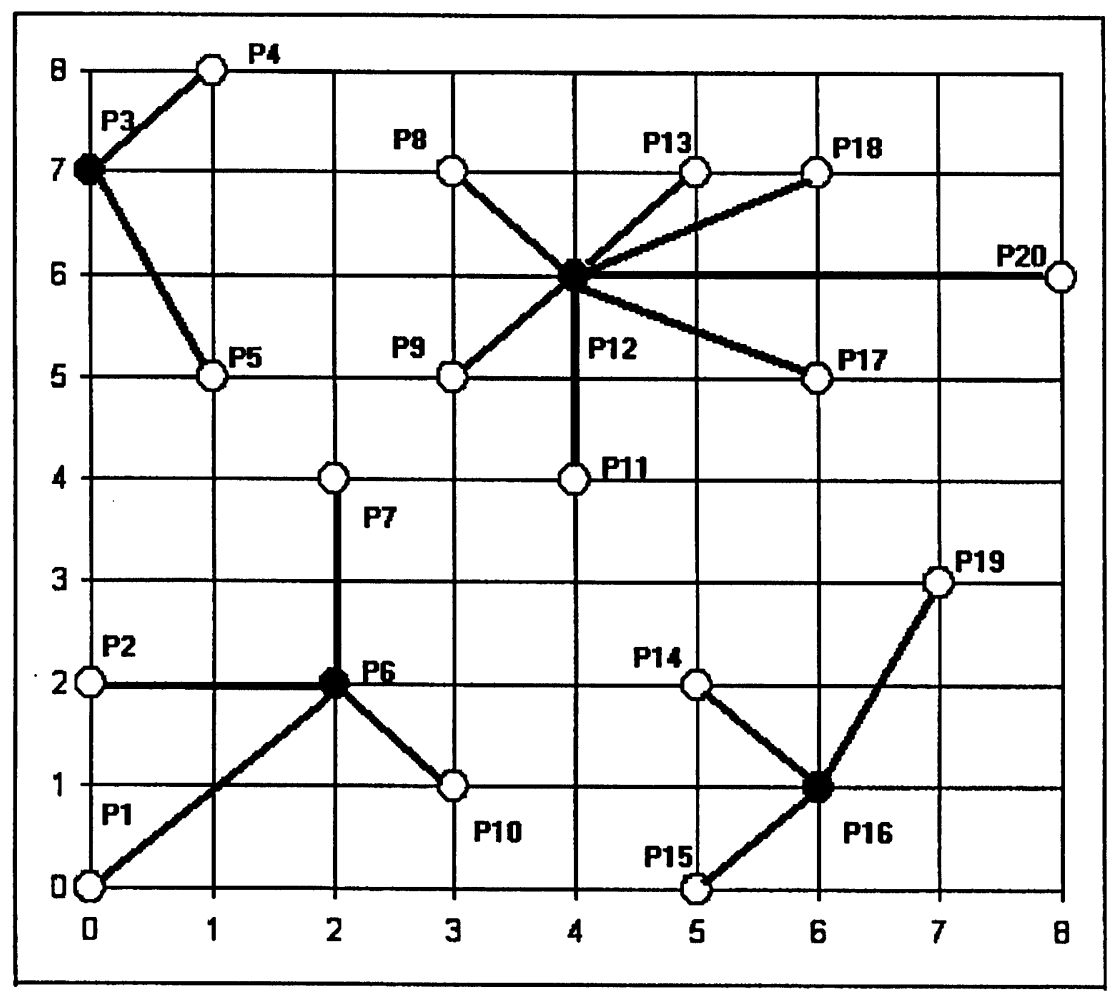

Figure 1: Given points and optimal "servicing" locations

The program will generate all $\mathbf{N}$ by $\mathbf{M}$ combinations on file C:ICOMBS.TXT. To change the name of the output file the third statement (displayed in bold in Annex II), must be changed accordingly.

Optionally, users may wish to include any text describing data entry and running instructions provided that the text does not use cells B3 and B4 reserved for the variables $\mathbf{N}$ and $\mathbf{M}$.

\section{Appendix II}

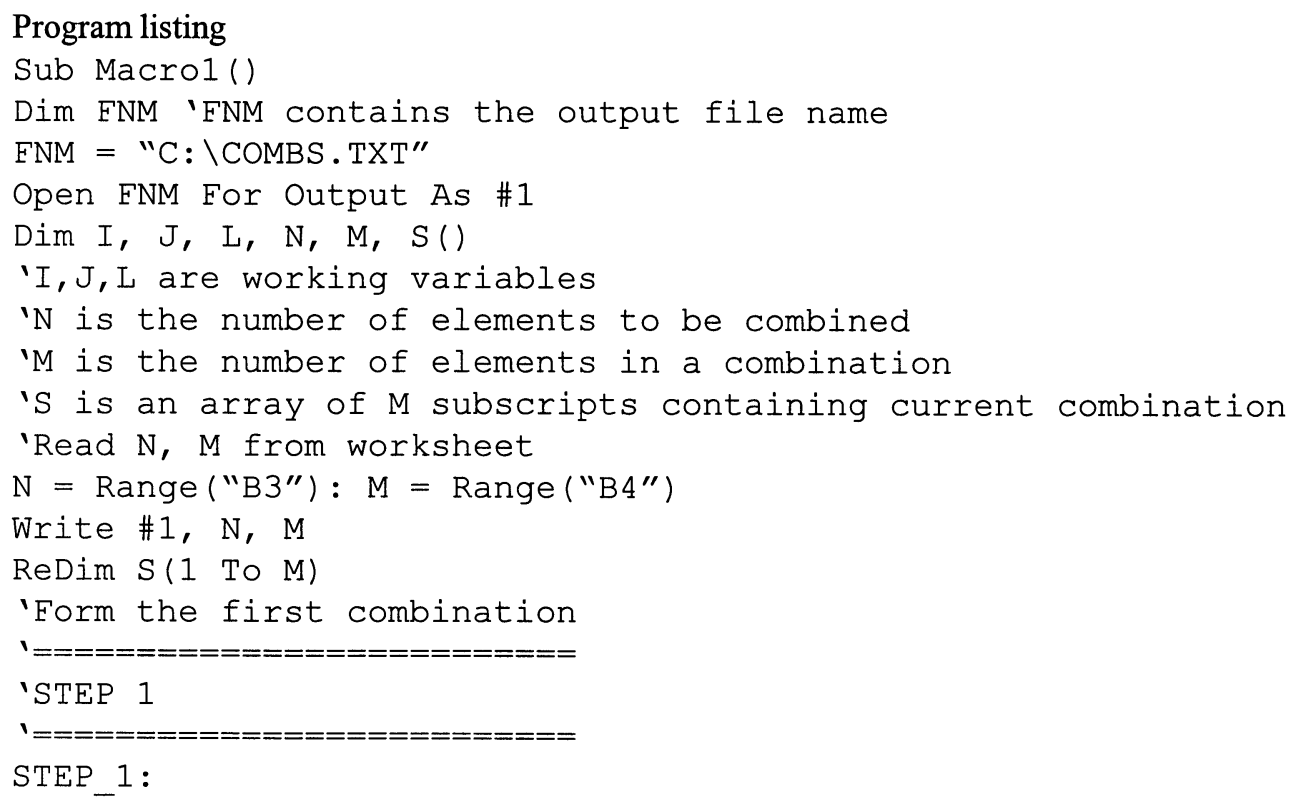




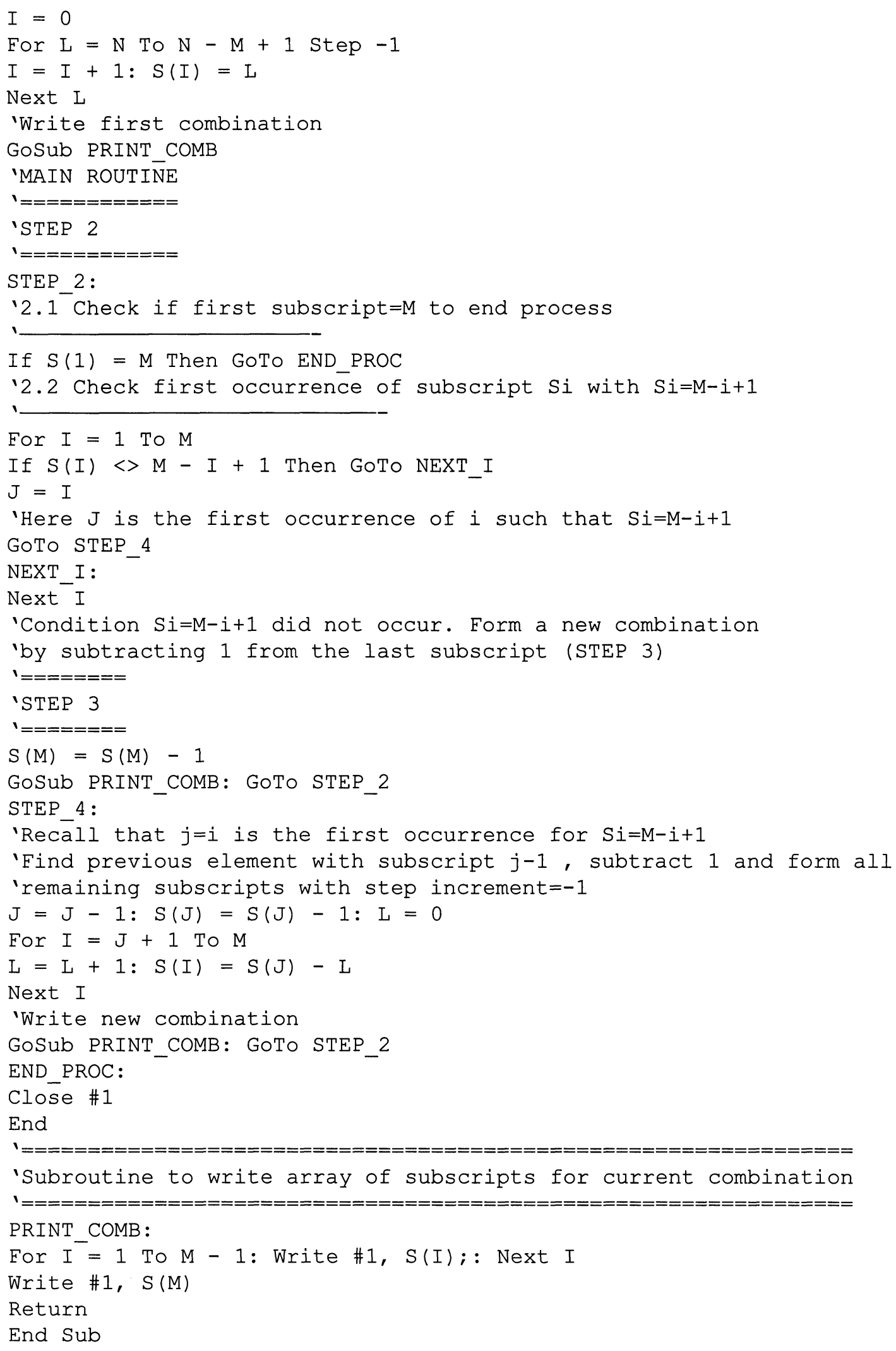




\title{
JMASM3: A Method for Simulating Systems of Correlated Binary Data
}

\author{
Todd C. Headrick \\ Southern Illinois University at Carbondale
}

An efficient algorithm is derived for generating systems of correlated binary data. The procedure allows for the specification of all pairwise correlations within each system. Intercorrelations between systems can be specified qualitatively. The procedure requires the simultaneous solution of a system of equations for obtaining the threshold probabilities to generate each system of binary data. A numerical example is provided to demonstrate that the procedure generates correlated binary variables that yield correlations in close agreement with the specified population correlations.

Keywords: Correlated binary variables, Simulation, Uniform random deviates

Introduction

The availability of the desktop computer has made simulation and Monte Carlo techniques widely applicable in statistical research. For example, Monte Carlo methods may be used to compare the small sample properties of a test statistic with its competitors or whether these properties are consistent with the statistic's asymptotic approximation (Headrick \& Rotou, 2001). Markov chain Monte Carlo methods (e.g., the Gibbs or slice sampler, Robert \& Casella, 1999) are also commonly used to generate posterior distributions to carry out Bayesian analyses. Further, these techniques are now applicable to many areas of research interest. Some examples include: bootstrap tilting (Hesterberg, 2001); conditional logistic regression (Mehta, Patel, \& Senchaudhuri, 2000); and likelihood inference with missing data (Gilks, Richardson, \& Spiegelhalter, 1998).

There may be occasions when it is desirable to investigate the properties of statistics that involve correlated binary data. Most procedures that generate correlated binary variables are based on an underlying joint distribution that is multivariate normal (e.g., Bahadur, 1961; Emirch \& Piedmonte, 1991; Leisch, 2001). This approach presents a problem because correlated binary variables with normal covariance structures may not provide a realistic simulation (Oman \& Zucker, 2001). Further, the popular Bahadur (1961) method requires the specification of higher order correlations. These higher order correlations are nuisance parameters that make the Bahadur (1961) procedure arduous (Parzen, Bellamy, Lipsitz, \& Fitzmaruice, 2001).

Thus, the purpose of this study is to derive a simple procedure that generates correlated binary variables. The proposed method simulates systems of multivariate binary data using a priori specified marginal probabilities and only requires a uniform random number generator.

Todd C. Headrick is Assistant Professor of statistics, Mail Code 4618, 222-J Wham Building, Southern Illinois University-Carbondale, IL, 62901. His areas of research interest are statistical computing, nonparametric statistics, and optimization. Email him at headrick@siu.edu.
Notation and a theoretical derivation of the proposed method are first provided. A numerical example is subsequently worked to demonstrate the procedure. Mathematica (Wolfram, Version 4.0,1999) notebooks are available from the author that simultaneously solves systems of equations that provide the probability threshold points for implementing the procedure. Fortran 77 source code is also provided in the Appendix to demonstrate the algorithm.

\section{Methodology}

Let $\pi_{i}$ be a pre-specified constant such that $\pi_{i} \in(0,1)$ and $U_{i}$ be a $K \times 1$ vector of uniform random deviates on the interval $(0,1)$ where $i=1, \ldots, T$. Let $Y_{1}$ represent a $K \times 1$ vector of a sequence of independent Bernoulli trials defined by

$$
Y_{1}=\left\{\begin{array}{ll}
1 & U_{1}<\pi_{1} \\
0 & U_{1}>\pi_{1}
\end{array} .\right.
$$

It follows that

$E\left[Y_{1}\right]=\mu_{Y_{1}}=\pi_{1}$, and

$\operatorname{Var}\left[Y_{1}\right]=\mu_{Y_{1}}\left(1-\mu_{Y_{1}}\right)=\pi_{1}\left(1-\pi_{1}\right)$.

For subscripts $i=2, \ldots, T$, let $Y_{i}$ represent the $i$ th $K \times 1$ vector of binary variables resulting from a sequence of independent trials defined by

$$
Y_{i}=\left\{\begin{array}{cc}
Y_{1} & U_{i}<\pi_{i} \\
Y_{1}+1 & U_{i}>\pi_{i} \text { and } Y_{1}=0 \\
1-Y_{1} & U_{i}>\pi_{i} \text { and } Y_{1}=1
\end{array}\right.
$$

Thus, we have

$$
\begin{aligned}
& E\left[Y_{i}\right]=\mu_{Y_{i}}=\pi_{1} \pi_{i}+\left(1-\pi_{1}\right)\left(1-\pi_{i}\right), \\
& E\left[Y_{1} Y_{i}\right]=\mu_{Y_{1}} \pi_{i}=\pi_{1} \pi_{i}, \text { and } \\
& \operatorname{Var}\left[Y_{i}\right]=\mu_{Y_{i}}\left(1-\mu_{Y_{i}}\right), \forall_{i>1} .
\end{aligned}
$$

Given the definitions of $Y_{1}$ and $Y_{i}$ in (1) and (4), let their measure of correlation, denoted as $\rho_{Y_{1} Y_{i}}$, be defined as 


$$
\begin{aligned}
\rho_{Y_{1} Y_{i}} & =\frac{\operatorname{Cov}\left[Y_{1}, Y_{i}\right]}{\sqrt{\operatorname{Var}\left[Y_{1}\right]} \sqrt{\operatorname{Var}\left[Y_{i}\right]}} \\
& =\frac{E\left[Y_{1} Y_{i}\right]-E\left[Y_{i}\right] E\left[Y_{i}\right]}{\left\{\left(E\left[Y_{1}^{2}\right]-\left(E\left[Y_{1}\right]\right)^{2}\right) \times\left(E\left[Y_{i}^{2}\right]-\left(E\left[Y_{i}\right]\right)^{2}\right)\right\}^{1 / 2}} \\
& =\frac{E\left[Y_{1} Y_{i}\right]-E\left[Y_{1}\right] E\left[Y_{i}\right]}{\left\{\left(E\left[Y_{1}\right]\left(1-E\left[Y_{i}\right]\right)\right) \times\left(E\left[Y_{i}\right]\left(1-E\left[Y_{i}\right]\right)\right)\right\}^{1 / 2}},
\end{aligned}
$$

where $E\left[Y_{1}^{2}\right]=E\left[Y_{1}\right]$ and $E\left[Y_{i}^{2}\right]=E\left[Y_{i}\right]$ because $Y_{1}$ and $Y_{i}$ are binary realizations of either 1 or 0 . Substituting equations (2), (3), (5), (6), and (7) into (8) yields:

$$
\rho_{Y_{1} Y_{i}}=\frac{\pi_{1} \pi_{i}-\mu_{Y_{1}} \mu_{Y_{i}}}{\sqrt{\mu_{Y_{1}}\left(1-\mu_{Y_{1}}\right)} \sqrt{\mu_{Y_{i}}\left(1-\mu_{Y_{i}}\right)}}
$$

Given $T$ vectors of $Y_{i}$, define $X_{i j}$ as the $j$-th $K \times 1$ vector of binary variables generated from $Y_{i}$ as follows:

$$
X_{i j}=\left\{\begin{array}{cc}
Y_{i} & U_{i j}<\pi_{i j} \\
Y_{i}+1 & U_{i j}>\pi_{i j} \text { and } Y_{i}=0 \\
1-Y_{i} & U_{i j}>\pi_{i j} \text { and } Y_{i}=1
\end{array}\right.
$$

where the second index $(j)$ runs faster than the first. As such, there are $N T$ vectors of $X_{i j}$. Note that the $N T$ vectors of $X_{i j}$ are independent of the $T$ vectors of $U_{i}$ and the constants of $\pi_{i j}$ are not necessarily equal $\pi_{i}$.

Taking expectations with respect to $X_{i j}$ and the product term of $X_{1 j} X_{1 k}$ gives

$$
\begin{aligned}
& E\left[X_{1 j}\right]=\mu_{X_{1 j}}=\pi_{1} \pi_{1 j}+\left(1-\pi_{1}\right)\left(1-\pi_{1 j}\right), \\
& E\left[X_{1 k}\right]=\mu_{X_{1 k}}=\pi_{1} \pi_{1 k}+\left(1-\pi_{1}\right)\left(1-\pi_{1 k}\right), \\
& E\left[X_{1 j} X_{1 k}\right]=\pi_{1} \pi_{1 j} \pi_{1 k}+\left(1-\pi_{1}\right)\left(1-\pi_{1 j}\right)\left(1-\pi_{1 k}\right),
\end{aligned}
$$

and hence

$$
\begin{aligned}
& \operatorname{Var}\left[X_{1 j}\right]=\mu_{X_{1 j}}\left(1-\mu_{X_{1 j}}\right), \\
& \operatorname{Var}\left[X_{1 k}\right]=\mu_{X_{1 k}}\left(1-\mu_{X_{1 k}}\right) .
\end{aligned}
$$

Taking expectations with respect to $X_{i j}$ and the product term $X_{i j} X_{i k}$ for $i=2, \ldots, T$ yields

$$
\begin{aligned}
& E\left[X_{i j}\right]=\mu_{X_{i j}}=\pi_{i j}\left(\pi_{1} \pi_{i}+\left(1-\pi_{1}\right)\left(1-\pi_{i}\right)\right)+\left(1-\pi_{i j}\right)\left(1-\left(\pi_{1} \pi_{i}+\left(1-\pi_{1}\right)\left(1-\pi_{i}\right)\right)\right) \\
& E\left[X_{i k}\right]=\mu_{X_{i k}}=\pi_{i k}\left(\pi_{1} \pi_{i}+\left(1-\pi_{1}\right)\left(1-\pi_{i}\right)\right)+\left(1-\pi_{i k}\right)\left(1-\left(\pi_{1} \pi_{i}+\left(1-\pi_{1}\right)\left(1-\pi_{i}\right)\right)\right) \\
& E\left[X_{i j} X_{i k}\right]=\pi_{i j} \pi_{i k}\left(\pi_{1} \pi_{i}+\left(1-\pi_{1}\right)\left(1-\pi_{i}\right)\right)+\left(1-\pi_{i j}\right)\left(1-\pi_{i k}\right)\left(1-\left(\pi_{1} \pi_{i}+\left(1-\pi_{1}\right)\left(1-\pi_{i}\right)\right)\right) \\
& \operatorname{Var}\left[X_{i j}\right]=\mu_{X_{i j}}\left(1-\mu_{X_{i j}}\right) \text {, and } \\
& \operatorname{Var}\left[X_{i k}\right]=\mu_{X_{i k}}\left(1-\mu_{X_{i k}}\right) .
\end{aligned}
$$

The correlations $\rho_{X_{1 j} X_{1 k}}$ and $\rho_{X_{i j} X_{i k}}$ can be determined by substituting the expressions in (11) through (15) and (16) through (20) into analogous definitions of (8) expressed in terms of the $X_{i j}$.

Given specified constants of $\pi_{i}$, an efficient number of correlated $X_{i j}$ vectors for each integer of $i=1$, the selection of $N=3$ yields a system of three equations (for three pairwise correlations $\left.\rho_{X_{11} X_{12}}, \rho_{X_{11} X_{13}}, \rho_{X_{12} X_{13}}\right)$ solvable in terms of three unknowns (probability threshold points of $\pi_{11}, \pi_{12}, \pi_{13}$ ). This system is constructed by first substituting the right-hand sides of (11) and (12) into (14) and (15). Subsequently substituting (11) through (15) into three equations of the form in (8) yields

$$
\begin{aligned}
& \rho_{X_{11} X_{12}}= \\
& \frac{\left[\pi_{1} \pi_{11} \pi_{12}+\left(1-\pi_{1}\right)\left(1-\pi_{11}\right)\left(1-\pi_{12}\right)\right]-\left[\pi_{1} \pi_{11}+\left(1-\pi_{1}\right)\left(1-\pi_{11}\right)\right]\left[\pi_{1} \pi_{12}+\left(1-\pi_{1}\right)\left(1-\pi_{12}\right)\right]}{\sqrt{\left[\pi_{1} \pi_{11}+\left(1-\pi_{1}\right)\left(1-\pi_{11}\right)\right]\left(1-\left[\pi_{1} \pi_{11}+\left(1-\pi_{1}\right)\left(1-\pi_{11}\right)\right]\right)} \sqrt{\left[\pi_{1} \pi_{12}+\left(1-\pi_{1}\right)\left(1-\pi_{12}\right)\right]\left(1-\left[\pi_{1} \pi_{12}+\left(1-\pi_{1}\right)\left(1-\pi_{12}\right)\right]\right)}} . \\
& \rho_{X_{11} X_{13}}= \\
& \frac{\left[\pi_{1} \pi_{11} \pi_{13}+\left(1-\pi_{1}\right)\left(1-\pi_{11}\right)\left(1-\pi_{13}\right)\right]-\left[\pi_{1} \pi_{11}+\left(1-\pi_{1}\right)\left(1-\pi_{11}\right)\right]\left[\pi_{1} \pi_{13}+\left(1-\pi_{1}\right)\left(1-\pi_{13}\right)\right]}{\sqrt{\left[\pi_{1} \pi_{11}+\left(1-\pi_{1}\right)\left(1-\pi_{11}\right)\right]\left(1-\left[\pi_{1} \pi_{11}+\left(1-\pi_{1}\right)\left(1-\pi_{11}\right)\right]\right)} \sqrt{\left[\pi_{1} \pi_{13}+\left(1-\pi_{1}\right)\left(1-\pi_{13}\right)\right]\left(1-\left[\pi_{1} \pi_{13}+\left(1-\pi_{1}\right)\left(1-\pi_{13}\right)\right]\right)}} . \\
& \rho_{X_{12} X_{13}}=\quad\left[\pi_{1} \pi_{12} \pi_{13}+\left(1-\pi_{1}\right)\left(1-\pi_{12}\right)\left(1-\pi_{13}\right)\right]-\left[\pi_{1} \pi_{12}+\left(1-\pi_{1}\right)\left(1-\pi_{12}\right)\right]\left[\pi_{1} \pi_{13}+\left(1-\pi_{1}\right)\left(1-\pi_{13}\right)\right] \\
& \frac{\sqrt{\left[\pi_{1} \pi_{12}+\left(1-\pi_{1}\right)\left(1-\pi_{12}\right)\right]\left(1-\left[\pi_{1} \pi_{12}+\left(1-\pi_{1}\right)\left(1-\pi_{12}\right)\right]\right.} \sqrt{\left[\pi_{1} \pi_{13}+\left(1-\pi_{1}\right)\left(1-\pi_{13}\right)\right]\left(1-\left[\pi_{1} \pi_{13}+\left(1-\pi_{1}\right)\left(1-\pi_{13}\right)\right]\right)}}{} .
\end{aligned}
$$


Specifying $\pi_{1}$ and selecting positive values for $\rho_{X_{11} X_{12}}, \rho_{X_{11} X_{13}}$, and $\rho_{X_{12} X_{13}}$ in (21), (22), and (23) and then simultaneously solving these equations gives the probability threshold points of $\pi_{11}, \pi_{12}, \pi_{13}$ to generate $X_{11}, X_{12}$, and $X_{13}$ with the desired intercorrelations for (10). Systems of equations analogous to (21), (22), and (23) can be created in the same manner for all $i=2, \ldots, T$ from equations (16) through (20). As such, $3 T$ vectors of $X_{i j}$ can be generated with three positive pairwise correlations within each system.

Without loss of generality, feasible solutions (i.e., $\left.0 \leq \pi_{i j} \leq 1\right)$ are obtainable provided that the following conditions hold with respect to the left-hand sides of (21), (22), and (23)

$$
\begin{aligned}
& \frac{\left(\rho_{X_{i 1} X_{i 2}}\right)\left(\rho_{X_{i 1} X_{i 3}}\right)}{\left(\rho_{X_{i 2} X_{i 3}}\right)} \leq 1, \\
& \frac{\left(\rho_{X_{i 1} X_{i 2}}\right)\left(\rho_{X_{i 2} X_{i 3}}\right)}{\left(\rho_{X_{i 1} X_{i 3}}\right)} \leq 1 \text {, and } \\
& \frac{\left(\rho_{X_{11} X_{i 3}}\right)\left(\rho_{X_{i 2} X_{i 3}}\right)}{\left(\rho_{X_{i 1} X_{i 2}}\right)} \leq 1 .
\end{aligned}
$$

These conditions must also hold with respect to the other $T-1$ systems of equations.

Because all $Y_{i}$ for $i=2, \ldots, T$ are a function of $Y_{1}$ from (4), intercorrelations between $X_{i j}$ belonging to different systems may also exist and can be analytically determined as follows:

$$
\rho_{X_{i j} X_{j j}}=\left(\rho_{X_{i j} Y_{i}}\right)\left(\rho_{Y_{i} Y_{j}}\right)\left(\rho_{Y_{j} X_{j j}}\right) \text {. }
$$

Further, and by inspection of (5) and (9), correlations in equation (25) may be negative, zero, or positive. Specifically, correlations are respectively negative, zero, or positive when $\pi_{i}<0.5, \pi_{i}=0.5$, or $\pi_{i}>0.5$ in equation (9) for all $i=2, \ldots, T$. Note that the correlations $\rho_{X_{i j} Y_{i}}$ and $\rho_{Y_{j} X_{j}}$ in (2.5) are derived analogously as (21),.., (23) from (8).

\section{Numerical Example}

Suppose it is desired to generate two systems of correlated binary data with correlations $\rho_{X_{11} X_{12}}=0.40$, $\rho_{X_{11} X_{13}}=0.50, \rho_{X_{12} X_{13}}=0.60$; and $\rho_{X_{21} X_{22}}=0.75$, $\rho_{X_{21} X_{23}}=0.80, \rho_{X_{22} X_{23}}=0.90$ and where the variables between the systems are inversely related. The following steps are taken:

1. Ensure that three pairwise intercorrelations within the systems satisfy (24a), (24b), and (24c).

2. Specify values for $\pi_{1}$ and $\pi_{2}$. Let $\pi_{1}=0.90$ and let $\pi_{2}=0.10$ to obtain the inverse relationships between the two systems.

3. Simultaneously solve the two systems of the form in
(21), (22) and (23) given the specified correlations and values of $\pi_{1}$ and $\pi_{2}$. The numerical equation solver FindRoot (Mathematica, 1999) yields solutions of $\pi_{11}=0.881247, \pi_{12}=0.924095, \pi_{13}=0.972456$, $\pi_{21}=0.939341, \pi_{22}=0.974722$, and $\pi_{23}=0.993961$.

4. Use equation (25) to determine the correlations between the variables of the two systems. The correlations are: $\rho_{X_{11} X_{21}}=-0.294484, \rho_{X_{11} X_{22}}=-0.331295, \rho_{X_{11} X_{23}}=-$ $0.353381, \rho_{X_{12} X_{21}}=-0.353381, \rho_{X_{12} X_{22}}=-0.397554$, $\rho_{X_{12} X_{23}}=-0.424057, \rho_{X_{13} X_{21}}=-0.441726, \rho_{X_{13} X_{22}}=-$ 0.496942 , and $\rho_{X_{13} X_{23}}=-0.530071$.

5. Given the parameters and solutions from steps 2 and 3 , generate the correlated binary data. Fortran 77 source code is presented in the Appendix to implement this example. Presented in Table 1 are population correlations and computed correlations for both within and between the two systems described in steps 1 and 4 . Single samples of binary data with $\mathrm{K}=5000$ were generated for each of the six variables as described in step 5. Inspection of Table 1 indicates that the proposed method generated correlated binary data that yielded computed correlations that were in close agreement with the population correlations.

\section{Conclusion}

As previously mentioned the systems of equations of the form in (21), (22), and (23) solve for the probability threshold points $\left(\pi_{i j}\right)$ for only positive correlations. However, it may be desirable to generate binary data with negative correlations. This can be accomplished by writing additional if-else structure statements in the Fortran 77 source code that simultaneously reversing the 1 's and 0 's within each selected vector. Such reversals will allow for the construction of negative correlations within the system(s).

More specifically, suppose that it was desired that

$\rho_{X_{11} X_{12}}=-0.40$, and $\rho_{X_{11} X_{13}}=-0.50$ in the numerical example. This can be accomplished by simultaneously reversing the 1 's and 0 's in the vectors $X_{12}$, and $X_{13}$. It should be noted that these changes would also create positive correlations between the systems of: $\rho_{X_{12} X_{21}}=0.353381$, $\rho_{X_{12} X_{22}}=0.397554, \rho_{X_{12} X_{23}}=0.424057, \rho_{X_{13} X_{21}}=0.441726$, $\rho_{X_{13} X_{22}}=0.496942$, and $\rho_{X_{13} X_{23}}=0.530071$.

It should also be pointed out that as the sample sizes become smaller the probability increases of obtaining vectors of either all 1's or all 0's (i.e., standard deviations of zero). Thus, one way to circumvent this problem in a larger simulation would be to initialize the correlation computed in the subroutine (e.g., PCOR in the Appendix) to zero when either standard deviation is zero and 
Table 1. Two systems of correlated binary data generated using the Fortran source code provided in the Appendix. A single sample of size $K=5000$ was drawn for each of the six variables.

\begin{tabular}{|c|c|c|c|}
\hline \multicolumn{2}{|c|}{ System 1} & \multicolumn{2}{|c|}{ System 2} \\
\hline \multicolumn{2}{|c|}{ Variables: $X_{11}, X_{12}, X_{13}$} & \multicolumn{2}{|c|}{ Variables: $X_{21}, X_{22}, X_{23}$} \\
\hline $\begin{array}{l}\text { Pop. Correlations } \\
\text { Within System } 1\end{array}$ & Computed Correlations & $\begin{array}{l}\text { Pop. Correlations } \\
\text { Within System } 2\end{array}$ & Computed Correlations \\
\hline$\rho_{X_{11} X_{12}}=0.40$ & 0.400780 & $\rho_{X_{21} X_{22}}=0.75$ & 0.745030 \\
\hline$\rho_{X_{11} X_{13}}=0.50$ & 0.503307 & $\rho_{X_{21} X_{23}}=0.80$ & 0.790314 \\
\hline$\rho_{X_{12} X_{13}}=0: 60$ & 0.591606 & $\rho_{X_{22} X_{23}}=0.90$ & 0.893551 \\
\hline \multicolumn{2}{|c|}{$\begin{array}{c}\text { Population Correlations } \\
\text { Between System } 1 \text { and System } 2\end{array}$} & \multicolumn{2}{|c|}{ Computed Correlations } \\
\hline \multicolumn{2}{|c|}{$\rho_{X_{11} X_{21}}=-0.294484$} & \multicolumn{2}{|c|}{-0.290305} \\
\hline \multicolumn{2}{|c|}{$\rho_{X_{11} X_{22}}=-0.331295$} & \multicolumn{2}{|c|}{-0.330829} \\
\hline \multicolumn{2}{|c|}{$\rho_{X_{11} X_{23}}=-0.353381$} & \multicolumn{2}{|c|}{-0.351456} \\
\hline \multicolumn{2}{|c|}{$\rho_{X_{12} X_{21}}=-0.353381$} & \multicolumn{2}{|c|}{-0.347825} \\
\hline \multicolumn{2}{|c|}{$\rho_{X_{12} X_{22}}=-0.397554$} & \multicolumn{2}{|c|}{-0.390941} \\
\hline \multicolumn{2}{|c|}{$\rho_{X_{12} X_{23}}=-0.424057$} & \multicolumn{2}{|c|}{-0.407341} \\
\hline \multicolumn{2}{|c|}{$\rho_{X_{13} X_{21}}=-0.441726$} & \multicolumn{2}{|c|}{-0.449708} \\
\hline \multicolumn{2}{|c|}{$\rho_{X_{13} X_{22}}=-0.496942$} & \multicolumn{2}{|c|}{-0.504316} \\
\hline \multicolumn{2}{|c|}{$\rho_{X_{13} X_{23}}=-0.530071$} & \multicolumn{2}{|c|}{-0.526768} \\
\hline
\end{tabular}

keep count of the zero correlations throughout the simulation. Thus, the total real number of replications could be computed by subtracting the number of times a zero correlation occurs due to standard deviations of zero (e.g., 50 occurrences) from the initialized total number of replications (e.g. 10,000). That is, the total real number of replications would be 9950 .

\section{References}

Bahadur, R. R. (1961). A representation of the joint distribution of responses to $n$ dichotomous items. In H. Solomon (Ed.), Studies in item analysis and prediction. Stanford, CA: Stanford University Press, 158-168.

Blair, R. C. (1987). RANGEN, Boca Raton, FL:

IBM.Emrich, L. J., \& Piedmonte, M. R. (1991). A method for generating high-dimensional multivariate binary variates. American Statistician, 45, 302-304.

Gilks, W. R., Richardson, S., \& Spiegelhalter, D. J. (1998). Markov chain Monte Carlo in practice. Boca Raton, FL: Chapman \& Hall/CRC.

Headrick, T. C. \& Rotou, O. (2001). An investigation of the rank transformation in multiple regression. Computational Statistics \& Data Analysis, 38, 203-215.
Hesterberg, T. (2001). Bootstrap tilting. The University of Florida Statistics Symposium on Monte Carlo in the New Millenium. Gainesville, FL.

Mehta, C. R., Patel, N. R., \& Senchaudhuri, P. (2000). Efficient Monte Carlo methods for conditional logistic regression. Journal of the American Statistical Association, 95, 99-108.

Leisch, F. (2001) The bindata package: Version 2. Vienna: Author.

Oman, S. D., \& Zucker, D. M. (2001). Modeling and generating correlated binary variables. Biometrika, 88 , 287-290.

Parzen, M., Bellamy, S., Lipsitz, S. R., \& Fitzmaurice, G. (2001). A random effects model for clustered data. (Unpublished), Graduate School of Business, University of Chicago.

Robert, C. P., \& Casella, G. (1999). Monte Carlo statistical methods. New York: Springer.

Wolfram, S. (1999). The Mathematica book (4th). Wolfram Media/Cambridge University Press. 


\section{APPENDIX}

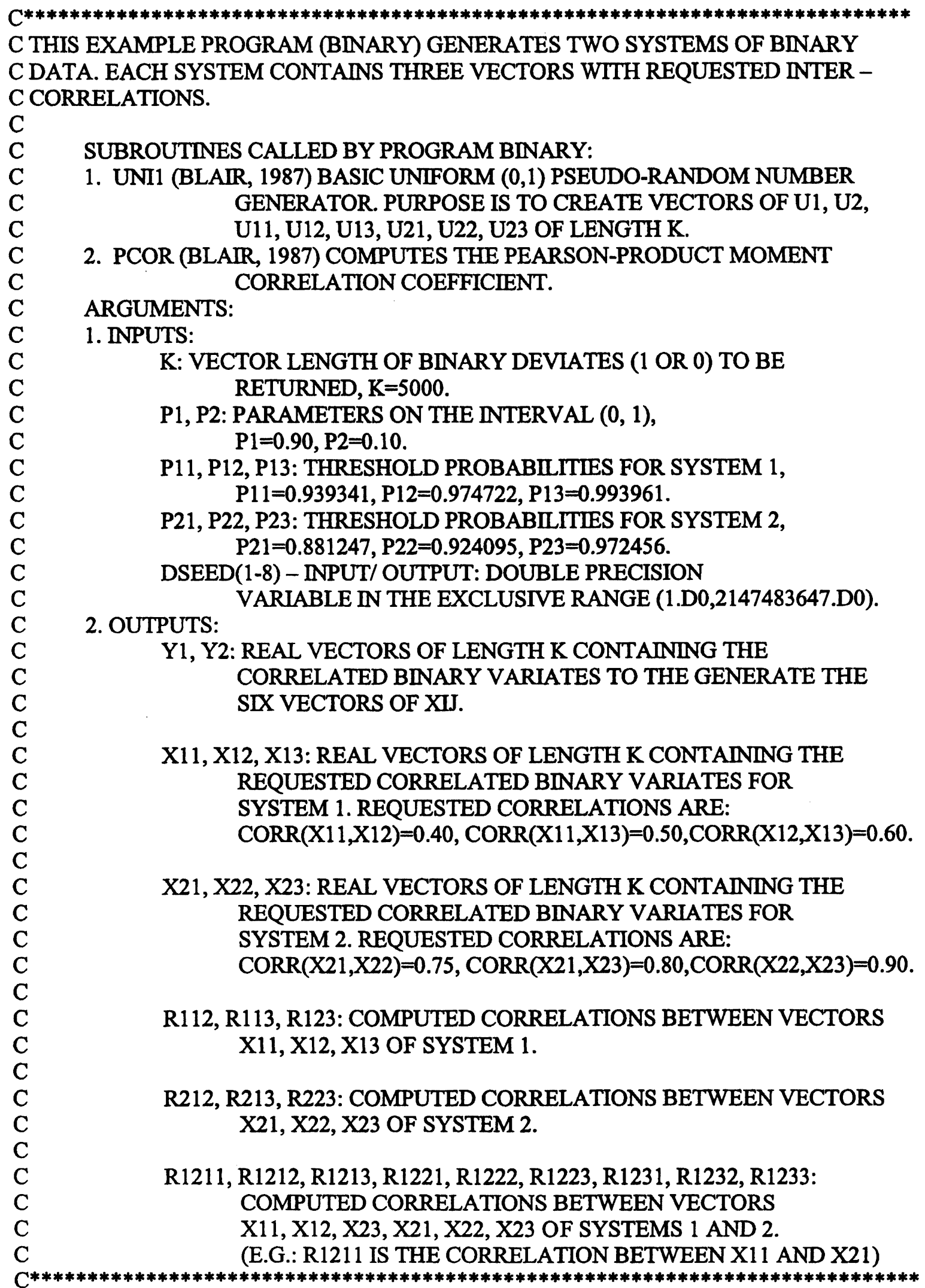


PROGRAM BINARY

REAL Y1(5000), Y2(5000), X11(5000), X12(5000), X13(5000), X21(5000), X22(5000), X23(5000), U1(5000), U2(5000), U11(5000), U12(5000), U13(5000), U21(5000), U22(5000), U23(5000), P1, P2, P11, P12, P13, P21, P22, P23, R112, R113, R123, R212, R213, R223, R1211, R1212, R1213, R1221, R1222, R1223, R1231, R1232, R1233

INTEGER I, K

DOUBLE PRECISION DSEED1, DSEED2, DSEED3, DSEED4, DSEED5, DSEED6, DSEED7, DSEED8

READ $\left({ }^{*},{ }^{*}\right) \mathrm{K}, \mathrm{P} 1, \mathrm{P} 2, \mathrm{P} 11, \mathrm{P} 12, \mathrm{P} 13, \mathrm{P} 21, \mathrm{P} 22, \mathrm{P} 23$

$\mathrm{C} * * * * * * * * * * * * * * * * * * * * * * * * * * * * * * * * * * * * * * * * * * * * * * * * * * * * * * * * * * * * * * * * * * * * * * * * * * * * *$

C INITIALIZE DSEED(1-8) AND CALL UNI1 TO GENERATE ARRAYS OF U1, U2, U11, C U12, U13, U21, U22, U23

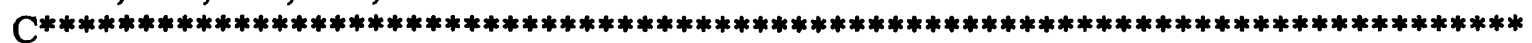

CALL UNI1 (DSEED1, K, U1)

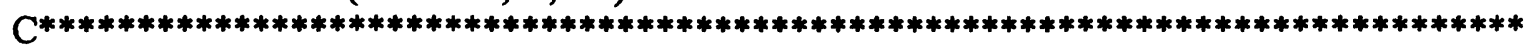

C CREATE CALI UNI1 STATEMENTS FOR U2,..,U23

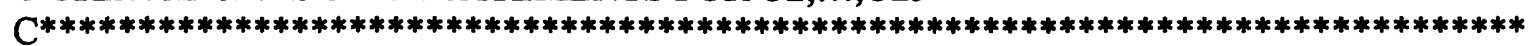

$\mathrm{DO} 10 \mathrm{I}=1, \mathrm{~K}$

IF ( U1(I) .LE. P1 ) THEN

$\mathrm{Y} 1(\mathrm{I})=1.0$

ELSE IF ( U1(1) .GE. P1) THEN

$\mathrm{Y} 1(\mathrm{I})=0.0$

ENDIF

IF ( U2(I) .LE. P2 ) THEN

$\mathrm{Y} 2(\mathrm{I})=\mathrm{Y} 1(\mathrm{I})$

ELSE IF (U2(I) .GE. P2 .AND. Y1(I) .EQ. 0) THEN

$\mathrm{Y} 2(\mathrm{I})=\mathrm{Y} 1(\mathrm{I})+1$

ELSE IF ( U2(I) .GE. P2 .AND. Y1(I) .EQ. 1) THEN

$\mathrm{Y} 2(\mathrm{I})=\mathrm{Y} 2(\mathrm{I})-1$

ENDIF

IF( U11(I) .LE. P11 ) THEN

$\mathrm{X} 11(\mathrm{I})=\mathrm{Y} 1(\mathrm{I})$

ELSE IF ( U11(I) .GE. P11 .AND. Y1(I) .EQ. 0) THEN

$\mathrm{X} 11(\mathrm{I})=\mathrm{Y} 1(\mathrm{I})+1$

ELSE IF ( U11(1) .GE. P11 .AND. Y1(I) .EQ. 1) THEN

$\mathrm{X} 11(\mathrm{I})=\mathrm{Y} 1(\mathrm{I})-1$

ENDIF

$\mathrm{C} * * * * * * * * * * * * * * * * * * * * * * * * * * * * * * * * * * * * * * * * * * * * * * * * * * * * * * * * * * * * * * * * * * * * * * * * * * * * *$

C CREATE IF-THEN STRUCTURES TO GENERATE VECTORS X12 AND X13 FROM Y1.

$\mathrm{C} * * * * * * * * * * * * * * * * * * * * * * * * * * * * * * * * * * * * * * * * * * * * * * * * * * * * * * * * * * * * * * * * * * * * * * * * * * * * *$

IF( U21(I) .LE. P21) THEN

$\mathrm{X} 21(\mathrm{I})=\mathrm{Y} 2(\mathrm{I})$

ELSE IF ( U21(I) .GE. P21 .AND. Y2(I) .EQ. 0) THEN

$\mathrm{X} 21(\mathrm{I})=\mathrm{Y} 2(\mathrm{I})+1$

ELSE IF ( U21(I) .GE. P21 .AND. Y2(I) .EQ. 1) THEN

$\mathrm{X} 21(\mathrm{I})=\mathrm{Y} 2(\mathrm{I})-1$

ENDIF

$\mathrm{C} * * * * * * * * * * * * * * * * * * * * * * * * * * * * * * * * * * * * * * * * * * * * * * * * * * * * * * * * * * * * * * * * * * * * * * * * * * * * *$

C CREATE IF-THEN STRUCTURES TO GENERATE VECTORS X22 AND X23 FROM Y2.

$\mathrm{C} * * * * * * * * * * * * * * * * * * * * * * * * * * * * * * * * * * * * * * * * * * * * * * * * * * * * * * * * * * * * * * * * * * * * * * * * * * * * *$

10 CONTINUE 
$\mathrm{C} * * * * * * * * * * * * * * * * * * * * * * * * * * * * * * * * * * * * * * * * * * * * * * * * * * * * * * * * * * * * * * * * * * * * * * * * * * * * *$ C CALL PCOR TO COMPUTE THE CORRELATIONS

$\mathrm{C} * * * * * * * * * * * * * * * * * * * * * * * * * * * * * * * * * * * * * * * * * * * * * * * * * * * * * * * * * * * * * * * * * * * * * * * * * * * * *$

CALL PCOR (X11, X12, K, R112)

CALL PCOR (X11, X13, K, R113)

CALL PCOR (X12, X13, K, R123)

CALL PCOR (X21, X22, K, R212)

CALL PCOR (X21, X23, K, R213)

CALL PCOR (X22, X23, K, R223)

CALL PCOR (X11, X21, K, R1211)

CALL PCOR (X11, X22, K, R1212)

CALL PCOR (X11, X23, K, R1213)

CALL PCOR (X12, X21, K, R1221)

CALL PCOR (X12, X22, K, R1222)

CALL PCOR (X12, X23, K, R1223)

CALL PCOR (X13, X21, K, R1231)

CALL PCOR (X13, X22, K, R1232)

CALL PCOR (X13, X23, K, R1233)

STOP

END 


\section{Announcing StatXact 5!}

StatXact 5, with over 100 procedures and a 1500 page manual that is really a textbook on exact methods, provides the world's most comprehensive collection of exact procedures for significance tests and confidence intervals. Among its new features, StatXact 5 now gives you a host of new procedures for the commonly-encountered two-binomial situation. Based on recent research (Agresti and Min, Biometrics 2000; Chan and Zang, Biometrics 1999), these procedures will give you more powerful exact $p$-values, and shorter exact confidence intervals.

\section{New In StatXact 5}

Unconditional exact tests for 2 binomials:

$\square$ Superiority

Non-inferiority

1 Equivalence

More powerful exact unconditional tests and shorter exact confidence intervals for differences and ratios of proportions

Unconditional exact McNemar's test

Exact interaction tests in stratified $2 \times \mathrm{C}$ tables

n comparison of $\mathrm{C}$ ordered binomials

a comparison two ordered multinomials

Exact test of trend for correlated binary data

Exact tests and confidence intervals for stratified Poisson data

While some standard software programs have a few exact tests, none has anywhere near the coverage of StatXact 5. StatXact 5, with over 100 tests and procedures, gives you exact $p$-values and confidence intervals for one-, two- and k-sample problems, $R \times C$ contingency tables, stratified $2 \times 2$ and $2 \times C$ contingency tables, goodness-of-fit tests, measures of association, binomial data, multinomial data, and censored survival data. Plus, StatXact 5 gives you exact power and sample size capabilities.

CYTEL Software Corporation $\bullet 675$ Massachusetts Ave., Cambridge, MA 02139 USA

Tel (617) 661-2011 • Toll Free (US) 866-298-3511 • Fax (617) 661-4405

http://www.cytel.com·E-mail: sales@cytel.com

INTERNATIONAL DISTRIBUTORS: Ask Int' (UK) e-mail: cyteluk@astu.com • Tel: +44(0) $1227795240 \bullet$ Faxi+44(0) 1227795 201; ID2 (Belgium) 


\section{Depth and flexibility for predicting numerical outcomes}

As a clear leader in statistical software, SPSS has what you need for analysis - and the complete analytical process.

SPSS is a modular, tightly integrated, full-featured product line. It's available for Windows and Macintosh desktops. Alternatively, it's available for many high-performance server platforms. The SPSS product line covers the full analytical process. SPSS' offering includes products for database access, data cleaning and management, as well as a broad range of analytical capabilities, and high-quality tabular and graphical output. You can even publish your SPSS results to the Web. This enables people who don't have SPSS installed on their machines to interact with results using their Web browsers. SPSS products are available through a variety of pricing and licensing programs, including student, graduate student and campus-wide licenses.

Take a look at some highlights in SPSS' line-up for predicting numerical outcomes and learn about just one aspect of SPSS' many offerings for the analytical process.

Sincerely,

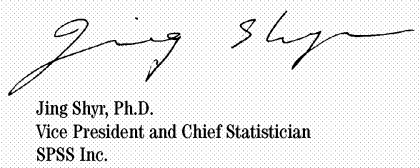

sPsS Inc.

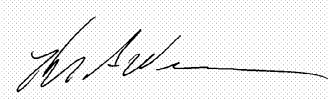

Kyle A. Weeks, Ph.D.

Senior Product Manager

SPSS Inc.

\section{Linear Mixed Models procedure}

Do you have data that display correlation and non-constant variability, such as data that

represent students nested within classrooms or consumers nested within families? You can model not only means but also variances and covariances in your data using the powerful Linear Mixed Models procedure. Its flexibility means you can formulate a wide variety of models such as multilevel models with fixed-effects covariances, hierarchical-linear models, randomeffects models, random-coefficient models and linear-growth models. In addition, you can work with repeated measure designs, including incomplete repeated measurements in which the number of observations varies across subjects.

General Linear Models (GLM) procedure multivariate

Do you need a flexible procedure that works simultaneously with related multiple dependent variables? SPSS' GLM multivariate procedure does just that - providing flexible design and contrast options to estimate means and variances and to test and predict means. Mix and match categorical and continuous data to build models. Because GLM multivariate doesn't limit you to one data type, you have options giving you a wealth of model-building possibilities. Also, you can easily visualize relationships using profile plots (interaction plots) resulting from estimated predicted mean values.

General Linear Models (GLM) procedure repeated measures

Do you need to measure the same people over time, for example, to measure how overall employee satisfaction increases or descreases? Using SPSS' GLM repeated measures procedure you can analyze variances when you make the same measurement a fixed number of times on individual subjects or cases. Get the flexibility to mix and match categorical and continuous-level predictors - including interactions. As with the GLM multivariate procedure, you can see relationships in your data using profile plots.

\section{Nonlinear Regression (NLR) and Constrained} Nonlinear Regression (CNLR) procedures Are you working with models that have nonlinear relationships, such as predicting coupon redemption as a function of time and number of coupons distributed? Estimate nonlinear equations using one of two SPSS procedures: NLR for unconstrained problems and CNLR for both constrained and unconstrained problems. CNLR empowers you to write your own algorithms. CNLR also gives you the flexibility to:

- Use linear and nonlinear constraints on any combination of parameters

- Estimate parameters by minimizing any smooth loss function (objective function)

- Compute bootstrap estimates of parameter standard errors and correlations

\section{Everything you need for predicting} numerical outcomes

SPSS' procedures for predicting numerical outcomes aren't limited to the ones we just described. The following procedures help give

SPSS 11.0 what you need for prediction:

- Linear Regression

- Weighted Least Squares Regression

- Two-Stage Least Squares

- Survival Analysis procedures

- Cox Regression with time-dependent covariates

- Kaplan-Meier

- Life Tables

Want to know what other statistics - including stats for identifying groups and time-series analysis and software SPSS offers for the complete analytical process? Visit www.spss.com/statisticalmethods to download a white paper, "Complete end-to-end analysis with SPSS 11.0." Do you like what you see? You can buy SPSS 11.0 online at www.spss.com/store or call (800) 543-9247.

SPSS BI helps people solve business problems using statistics and data mining. This predictive technology enables our customers in the commercial and public sectors to make better decisions and improve results. SPSS B1: software and services are used successfully in a wide range of applications. including customer attraction and retention, cross-selling, survey research. fraud detection. Web site performance, forecasting and scientific research SPSS BIs market-leading products include SPSS, Clementine, Answer Tree, Decisiontime and SigmaPlot?

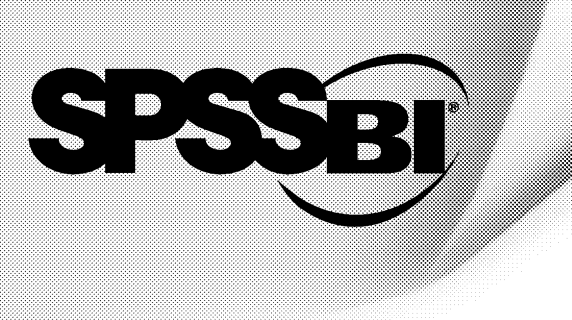

Call today

for an SPSS product catalog

(800) 543-9247 
"Perfection is achieved, not when there is nothing more to add, but when there is nothing left to take away."

- Antoine de Saint Exupery

F is a carefully crafted subset of the most recent version of Fortran, the world's most powerful numeric language.

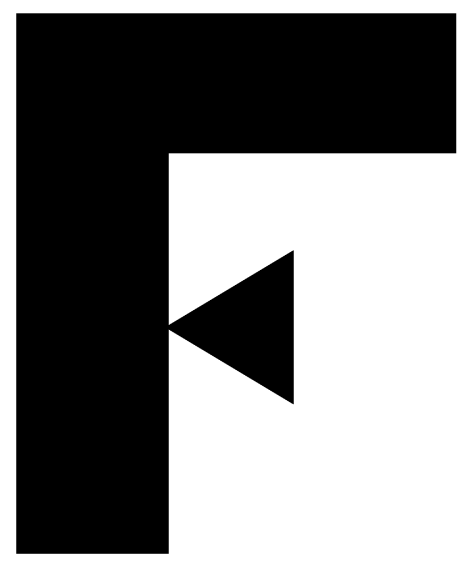

Using F has some very significant advantages:

- Programs written in F will compile with any Fortran compiler

- $\mathrm{F}$ is easier to use than other popular programming languages

- F compilers are free and available for Linux, Windows, and Solaris

- Several books on F are available

- F programs may be linked with C, Fortran 95, or older Fortran 77 programs

F retains the modern features of Fortran-modules and data abstraction, for example-but discards older error-prone facilities of Fortran.

It is a safe and portable programming language.

F encourages Module-Oriented Programming.

It is ideal for teaching a programming language in science, engineering, mathematics, and finance.

It is ideal for new numerically intensive programs.

The Fortran Company

11155 E. Mountain Gate Place, Tucson, AZ 85749 USA $+1-520-256-1455+1-520-760-1397$ (fax) http://www.fortran.com info@fortran.com 




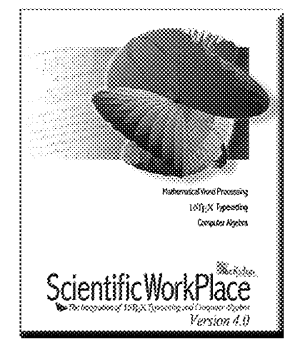

Math Word Processing

LATEX Typesetting Computer Algebra
The Gold Standard for Mathematical Publishing and the Easiest-to-Use Computer Algebra System

\section{Now in a new version!}

Scientific WorkPlace makes writing, publishing, and doing mathematics easier than you ever imagined possible.

- Enter text and mathematics naturally in the same paragraph

- Produce documents with or without $\mathrm{LAT}_{\mathrm{E}} \mathrm{X}$ typesetting

- Produce portable LATEX output

- Perform mathematical computations with both the $M u P A D^{\circledast}$ and Maple $^{\circledast}$ computer algebra engines

- Export documents as HTML, with mathematics exported as graphics or as MathML

- Use hyperlinking to create an entire web of Scientific WorkPlace documents

- And more

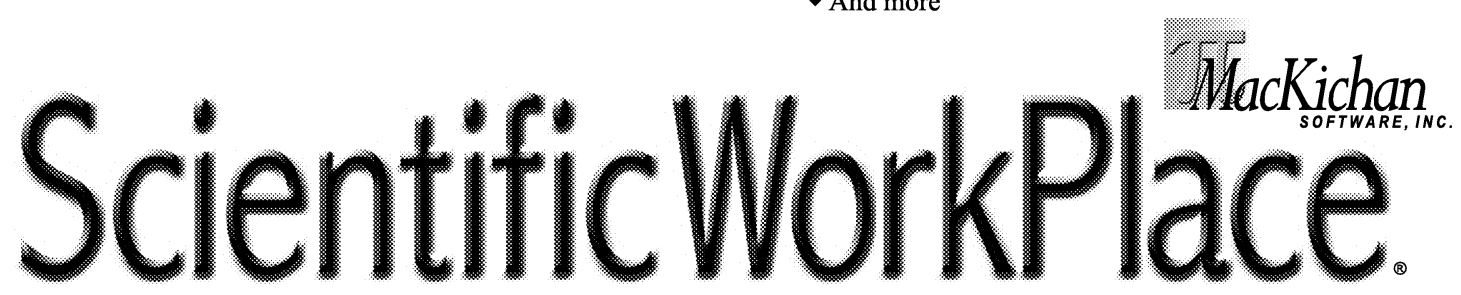

Email: info@mackichan.com • Toll Free: 877-724-9673 • Fax: 206-780-2857 Visit our website for free evaluation copies of all our software. www.mackichan.com/jmsm 

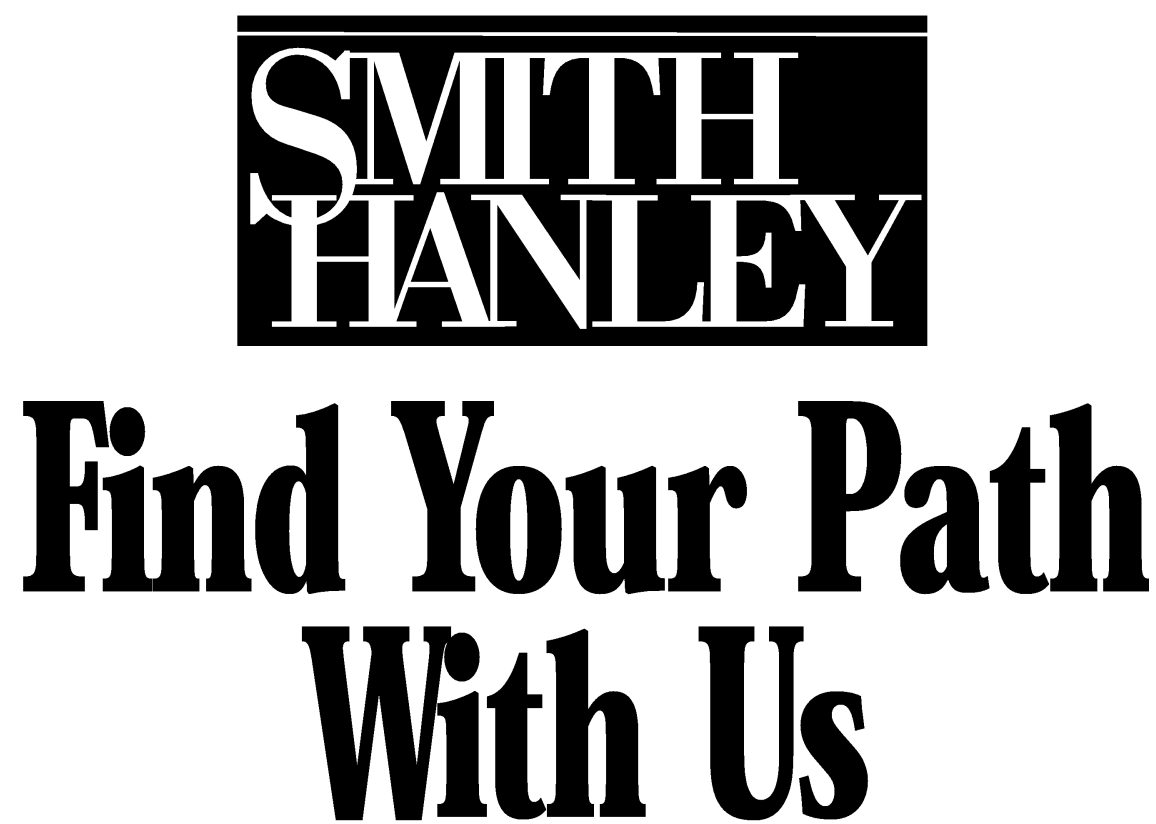

\section{HIGHLY SPECIALIZED PERMANENT \& CONTRACT OPPORTUNITIES AVAILABLE}

- BIOSTATISTICS

- SAS® PROGRAMMING

- DATA MANAGEMENT

- MARKETING SCIENCE

- RESEARCH

- MANUFACTURING

Permanent Placement

contach Tracey cmoser

80019:99.5627

Fax: 212:818:9067

perm@smithhanley.com

www.smithianley.com
Contract Staffing

Contrack Keitin Snelly

800.689 .9921

Faxx4.07.805.3020

contract@smith anley.com

ww.smith hanley-consulting.com

\section{Smith Hanley}

Vew York. Chicago Houston Southport Orlando 


\section{GGUM2000}

\section{Item Response Theory Models for Unfolding}

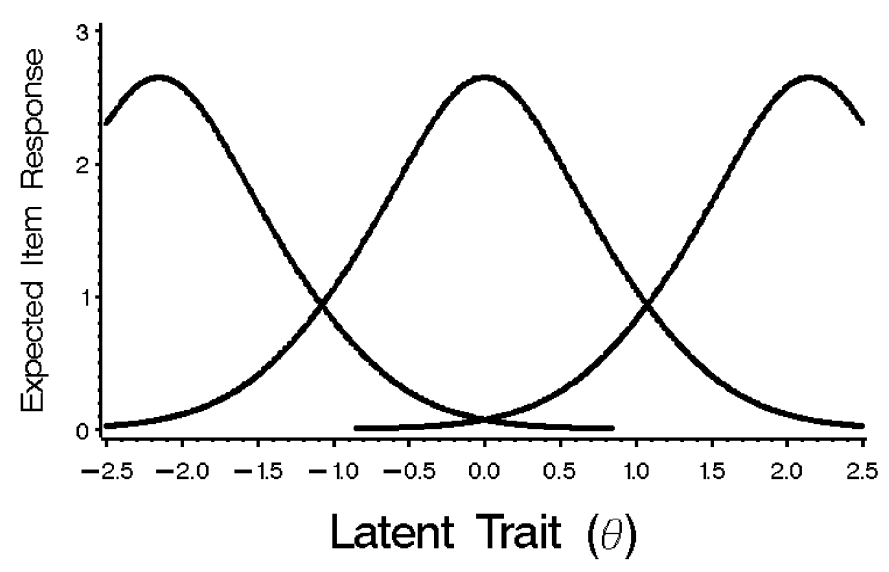

The GGUM2000 software system estimates parameters in a family of item response theory (IRT) models that unfold polytomous responses to questionnaire items. These models assume that persons and items can be jointly represented as locations on a latent unidimensional continuum. A single-peaked, nonmonotonic response function is the key feature that distinguishes unfolding IRT models from traditional, "cumulative" IRT models. This response function suggests that a higher item score is more likely to the extent that an individual is located close to a given item on the underlying continuum. Such single-peaked functions are appropriate in many situations including attitude measurement with Likert or Thurstone scales, and preference measurement with stimulus rating scales. This family of models can also be used to determine the locations of respondents in particular developmental processes that occur in stages.

The GGUM2000 system estimates item parameters using marginal maximum likelihood, and person parameters are estimated using an expected a posteriori (EAP) technique. The program allows for up to 100 items with 2-10 response categories per item, and up to 2000 respondents. The software is accompanied by a detailed user's manual. GGUM2000 is free and can be downloaded from:

\section{http://www.education.umd.edu/EDMS/tutorials}

Start putting the power of unfolding IRT models to work in your attitude and preference measurement endeavors. Download your free copy of GGUM2000 today! 


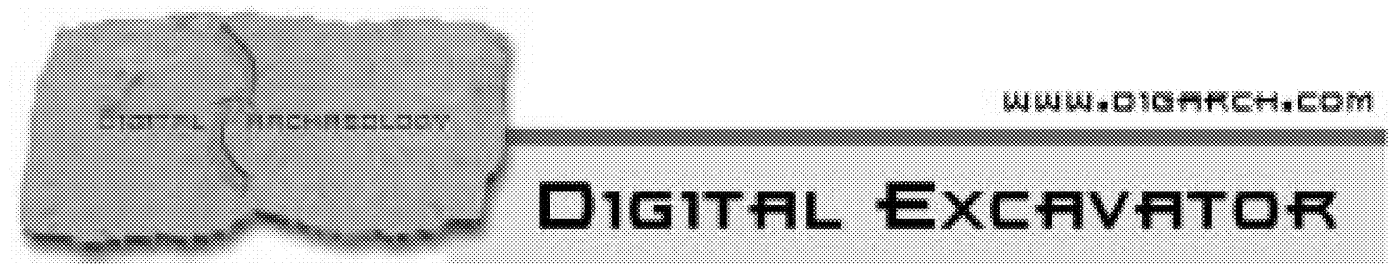

Are you involved in Data Modeling or Data Mining?

\section{Are you spending a large percentage of your time dealing with data issues?}

If so, you will be happy to know that we have developed a tool that specifically addresses the data prep tasks associated with data modeling and data mining. The tool is called the Digital Excavator from Digital Archaeology (www.digarch.com). Data modelers are well aware of the time-consuming and sometimes frustrating nature of data set-up. In many cases data preparation can represent $60 \%$ $80 \%$ of the data mining project length. With Digital Archaeology's Digital Excavator, data preparation tasks are streamlined, results are more accurate, and the modeler has more time to focus on finding the appropriate mathematical solution--rather than wasting time with painful data issues. Digital Archaeology's software is intuitive, visual, self-documenting, and deploys what a number of analysts and customers have termed the "most elegant" user interface for data analysis and exploration ever conceived. It's the only tool specifically designed for the data prep tasks of data modeling.

\section{Visit our website and see for yourself! $\quad$ >>> www.digarch.com}

Functions have been created which perform the following:

- Frequency Distributions

- Categorical Variable Profile

- Continuous Variable Profile

- Histograms

- De-duping

- $\quad$ Find and Replace Missing Values

- $\quad$ Find and Split Out Outliers

- Binning

- Correlation Matrix

- Cross-Tabs

- $\quad$ Panel Variables (Occupancy Map)

- Lag functions

- Decimal Scaling

- $\quad$ Rank and Sample Variables

- $\quad$ Recency, Frequency, Monetary Analysis

- N-Tile Distributions

- Gains Charts

- Many others
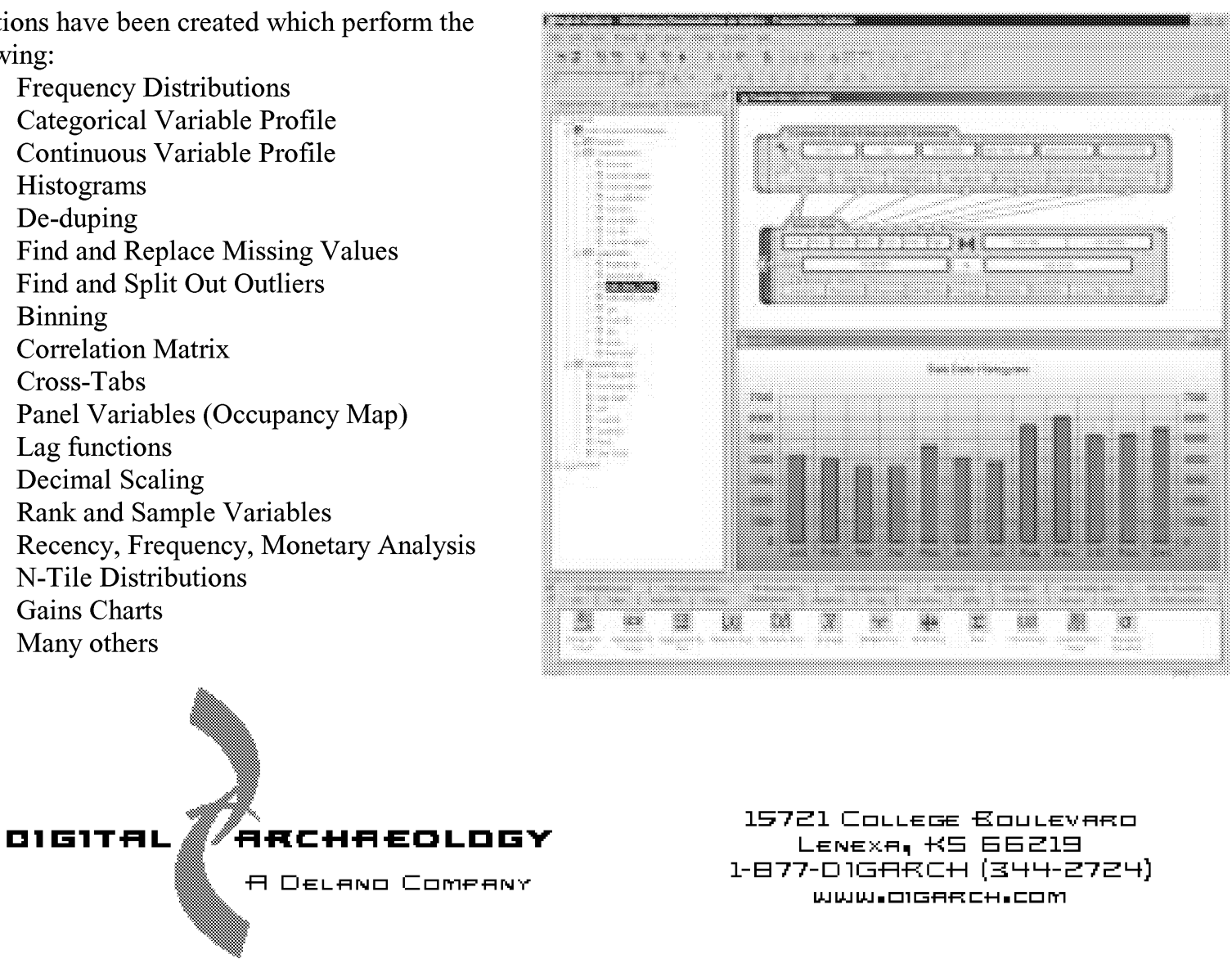


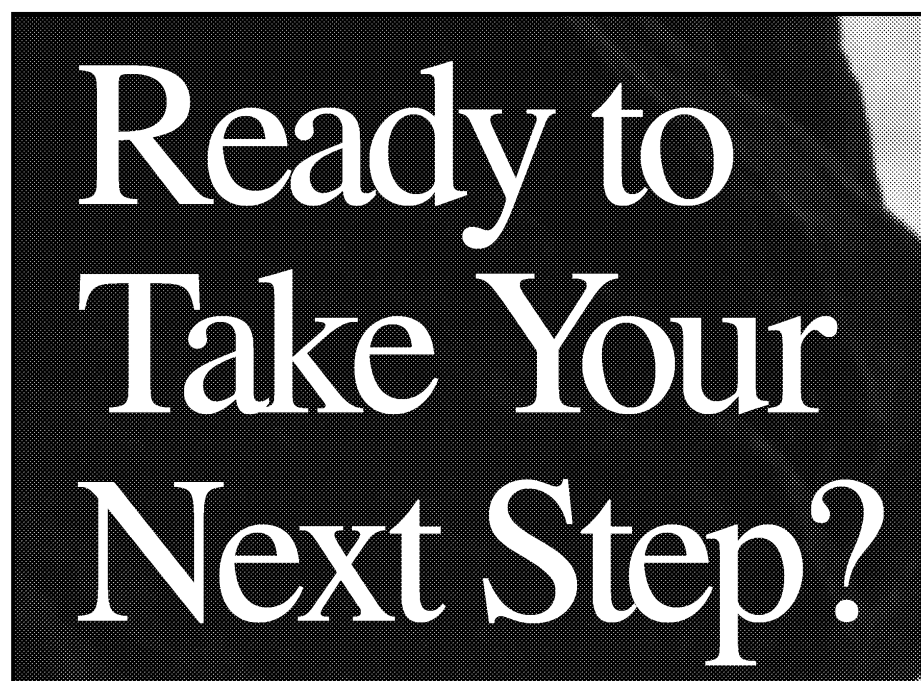

As your career climbs and each step requires careful planning, consider The Cambridge Group...

...Your Success is Our Business.

Business Statistics

-Quantitative Analysis

-Marketing Sciences/Research

-Econometrics

-Quality Assurance

stat@cambridgegroup.com

Consultant

\section{\& Contract Staffing}

-Biostatisticians

-SAS ${ }^{\circledR} /$ Statistical Programmers

- Clinical Data Managers

- Clinical Systems

-CRA's \& Clinical Monitors

- Medical Writers

-Project Managers

-Bioinformatics

contract@cambridgegroup.com

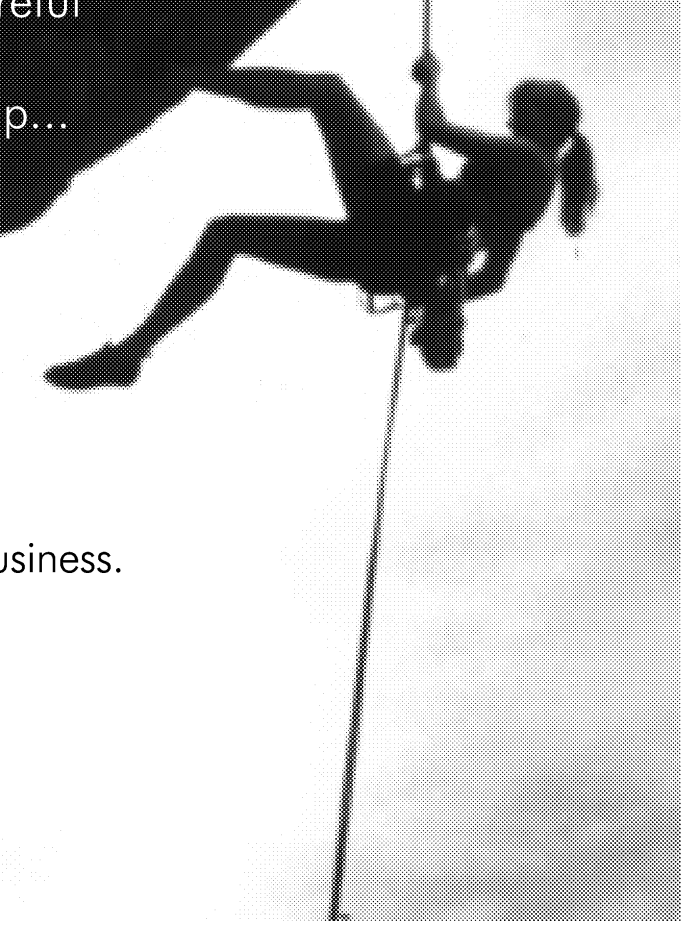

Clinical Computing

\& Data Management

-SAS Programming/Application

- Clinical Data Management

-Systems Design \& Analysis

QA@cambridgegroup.com

\section{Biostatistics}

- Clinical

-Preclinical/Nonclinical

-Health Outcomes

-PK/PD

biostat@cambridgegroup.com

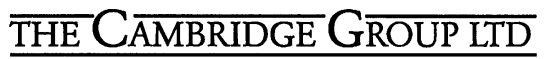

(800) 525-3396 fax (203) 226-3856 www.cambridgegroup.com 


\title{
U S C C E N S U S B U R E A U
}

\author{
TIME SERIES \\ RESEARCH POSITIONS
}

Applications and nominations are invited for one senior and three junior or intermediate research positions in time series analysis at the U.S. Census Bureau in the Washington D.C. area. The senior position of Principal Researcher involves research, consulting, and directing the work of less experienced researchers. It requires a Ph.D. in Statistics or a closely related field and a substantial record or original research in times series analysis including publications. The 2002 salary range is $\$ 78,265-\$ 119,682$ depending on qualifications and experience. The other positions require at least a Master's degree in Statistics or a closely related field, course work in time series analysis, and significant statistical computing experience. The 2002 salary range is $\$ 45,851-\$ 72,400$ depending on qualifications and experience.

The Census Bureau's methods and software for time series modeling and seasonal adjustment are used world-wide in government, industry, and academia. The available positions are on the staffs responsible for the further development, testing, training, and support of such methods and software for the Census Bureau's many economic surveys. Research topics of current interest include: state space modeling with sampling error components; non-Gaussian modeling for signal extraction; statistical graphics and other diagnostics for seasonal adjustment; automatic selection methods for ARIMA models, regressors and outlier treatments; and issues involved with the seasonal adjustment of short time series. The researchers also provide consultation, training, and documentation of methods and software developed and so must have good communication and personal skills.

For further information or to start the application process, please contact David F. Findley, Statistical Research Division, Census Bureau, Washington, DC 20233-9100 or david.f.findley@census.gov, or call 301-457-4983. U.S. citizenship is required.

The Census Bureau is an Equal Opportunity Employer

\section{ARE YOU INTERESTED IN AN ORGANIZATION DEVOTED TO EDUCATIONAL AND BEHAVIORAL STATISTICS?}

\author{
Become a member of the Special Interest Group-Educational Statisticians of the \\ American Educational Research Association ISIG-ES of AERAl!
}

The mission of SIG-ES is to increase interaction among educational researchers interested in the theory, applications, and teaching of statistics in the social sciences.

Each spring, as part of the overall AERA annual meeting, there are 7 sessions sponsored by SIG-ES devoted to educational statistics and statistics education. We also publish a twice-yearly electronic newsletter.

Past issues of the SIG-ES newsletter and other information regarding SIG-ES can be found at http://orme.uarkedu/edstatsig.htm.

To join SIG-ES you must be a member of AERA. You simply check the box next to SIG-Educational Statisticians on the list of SIGs the next time you renew your AERA membership. Dues are only $\$ 5.00$ per year.

If you are not an AERA member and would like to join both AERA and SIG-ES, membership information can be found at http://aera.net/members/index.htm

Contact Michael Seaman, President of SIG-ES, at Department of Educational Psychology, 26I Wardlaw College, University of South Carolina, Columbia, SC 29208. email: mseaman@sc.edu . 


\section{Numerical Recipes in Fortran from Cambridge University Press}

\section{Numerical Recipes in Fortran 77 \\ Volume 1 of Fortran Numerical Recipes \\ Second Edition \\ William H. Press, Saul A. Teukolsky, William T.Vetterling, and Brian P. Flannery}

"This reviewer knows of no other single source of so much material of this nature. Highly recommended."

$$
\text { - Choice }
$$

"... a valuable resource for those with a specific need for numerical software. The routines are prefaced with lucid, selfcontained explanations....highly recommended for those who require the use and understanding of numerical software."

-SIAM Review

1992992 pp. 0-521-43064-X Hardback $\$ 70.00$

\section{Highlights include:}

- A chapter on integral equations and inverse methods

4. Multigrid and other methods for solving partial differential equations

9. Improved random namber routines

- Wavelet transforms

- The statistical bootstrap method

- A chapter on "less numerical" algorithms including compression coding and arbitrary precision arithmetic:

\section{Numerical Recipes in Fortran 77 Example Book}

Second Edition

William T.Vetterling, Saul A. Teukolsky, William H. Press, and Brian P. Flannery

$1992 \quad 256 \mathrm{pp} . \quad 0-521-43721-0 \quad$ Paperback $\$ 35.00$

\section{Numerical Recipes in Fortran 90}

The Art of Parallel Scientific Computing

Volume 2 of Fortran Numerical Recipes

Second Edition

\section{William H. Press, Saul A. Teukolsky, William T.Vetterling, and Brian P. Flannery}

"This present volume will contribute decisively to a significant breakthrough, as it provides models not only of the numerical algorithms for which previous editions are already famed, but also of an excellent Fortran 90 style."

-From the Foreword by Michael Metcalf, one of Fortran 90 's original designers and author of FORTRAN 90 Explained

"This book is a classic and is essential reading for anyone concerned with the future of numerical calculation. It is beatifully produced, inexpensive for its content, and a must for any serious worker or student."

- Computing Reviews

Contains a detailed introduction to the Fortran 90 language and to the basic concepts of parallel programming, plus source code for all routines from the second edition of Numerical Recipes.

1996576 pp. 0-521-57439-0 Hardback $\$ 50.00$

\section{Numerical Recipes Multi-Language Code CDROM with LINUX or UNIX Single Screen License}

Source Code for Numerical Recipes in C, C++, Fortran 77, Fortran 90, Pascal, BASIC, Lisp and Modula 2 plus many extras

$2002 \quad 0.521-75036 \sim 9 \quad$ CD $R O M \quad \$ 150.00$

\section{Numerical Recipes Multi-Language Code CDROM with Windows, DOS, or Macintosh Single Screen License}

Source Code for Numerical Recipes in $\mathrm{C}, \mathrm{C}++$, Fortran 77 , Fortran 90, Pascal, BASIC, Lisp and Modula 2 plus many extras

$2002 \quad 0-521-75035-0 \quad$ CD-ROM $\$ 90.00$

Visit us.cambridge.org/numericalrecipes for more information on the complete line of Numerical Recipes products. 


\section{IMPORT XML-FORMATTED DATA INTO SAS EXPORT SAS DATA AS XML-FORMATTED DATA}

Now, an easy way to move CDISC and other XML-formatted data into or out of your SAS-based systems. You don't have to know perl, XSLT, Xpath, Java ${ }^{\circledR}$, or exotic languages. The remarkable Tekoa ${ }^{\text {sm }} \mathrm{XML}$ plug-in does it all for you.

Provided free of charge to any SAS user currently wrestling with XML. Developed by Zurich Biostatistics, the pioneer in SAS/XML integration.

\section{FREE. EASY. AND IT WORKS.}

Just e-mail Michael Palmer (mcpalmer@zbi.net) and receive the fully-functional, proven Tekoa XML plug-in by e-mail.

No charge. No obligation. No hassle. (We even support the tool. Imagine.)

\section{Zurich Biostatistics, Inc.}

45 Park Place South, PMB 178, Morristown, NJ 07960973 727-0025 wmw.zbi.net

\section{$X M L$ is easy if you know how. And we do.}

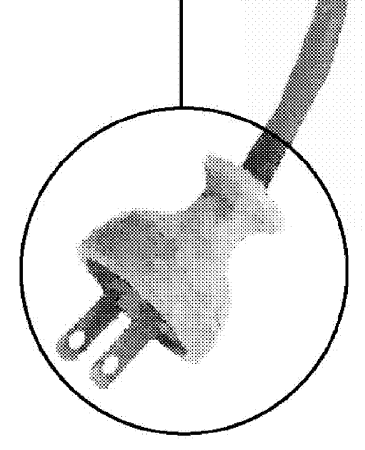

Tekoa XML Technology is a service mark of Zurich Biostatistics. In SAS is a registered trademark of SAS Institute Inc Java is a registered trademark of Sun Microsystems, Inc.

\section{Resampling Stats for Excel}

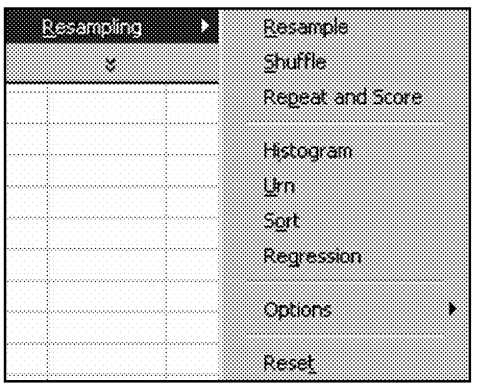

Select the data you want to resample, select "resample" or "shuffle," then specify an output range for the resampled data. Calculate a statistic of interest, select "Repeat \& Score," and the resampling operation will be repeated thousands (or tens of thousands) of times, and each time the value of your statistic of interest will be recorded. Does not use Excel's random number generator.

View complete user guide and download free 30-day trial at <www.resample.com>

\$249 commercial •\$149 personal/academic • \$89 student

612 N Jackson St., Arlington, VA 22201 Tel 703-522-2713 • Fax 703-522-5846 stats@resample.com

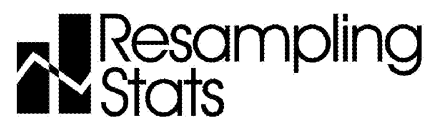




\section{ANNOUNCING LOGXACT 4}

\section{Only LogXact 4 can fit a logistic regression model to the data on this page - even LogXact 2 cannot do it.}

To solve this problem, you need a very powerful exact logistic regression algorithm.LogXact 4 implements a ground-breaking network Monte Carlo algorithm (published in JASA, April 2000), extending the scope of LogXact to problems previously beyond its capacity.

PLUS! LogXact 4 also provides exact Poisson regression (used extensively for cohort studies in epidemiology).

\section{Take the Cytel Challenge}

Data were gathered on 2,493 hospitalized patients, of whom 60 suffered from clostridium dificile, an acute form of diarrhea. 0 interest was the relationship between the occurrence of diarrhea and age, length of hospital stage, sex, use of the antibiotic Clindomycin, and the use of the antibiotic Cephalexin.

When you have data like these (low response rates,

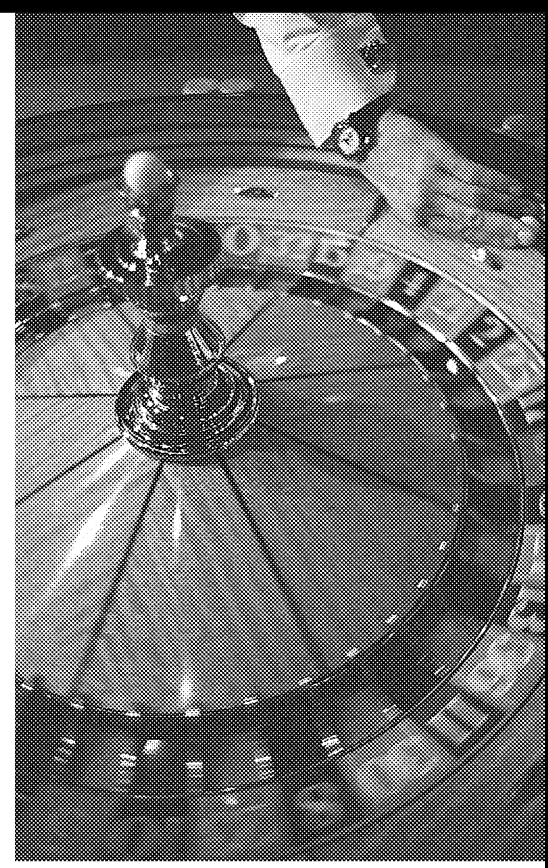

unbalanced covariates or small sample sizes), traditional logistic regression methods used by standard statistical packages often fail. But new LogXact 4 can fit models, test parameters and estimate coefficients even when the maximum likelihood method used by most statistical software can't. Plus, since LogXact gives you exact answers instead of approximations, it protects you from Type-l error.

60 cases of diarrhea among 2,493 hospitalized patients

\begin{tabular}{|c|c|c|c|c|}
\hline & Group 1 & Group 2 & $\cdot$ & Group 18 \\
\hline Cephalexin & 0 & 0 & - & 1 \\
\hline Clindomycin & 0 & 0 & • & 0 \\
\hline Sex & 1 & 1 & • & 0 \\
\hline Age & 0 & 0 & • & 1 \\
\hline LOS & 0 & 1 & • & 1 \\
\hline Diarrhea/Total $(60 / 2,493)$ & 0/174 & $1 / 113$ & - & $4 / 4$ \\
\hline
\end{tabular}

For the full data set, the solution and a free 30 day trial of LogXact 4, visit www.cytel.com

Call (617) 661-2011; fax (617) 661-4405; e-mail: sales@cytel.com

CYTEL Software Corporation $\bullet 675$ Massachusetts Ave., Cambridge, MA 02139 USA

Tel (617) 661-2011 • Toll Free (US) 866-298-3511 • Fax (617) 661-4405

http://www.cytel.com・E-mail: sales@cytel.com

INTERNATIONAL DISTRIBUTORS: Ask Int'I (UK) e-mail: cyteluk@asru.com • Tel: +44(0) $1227795240 \bullet$ Fax:+44(0) 1227795 201; ID2 (Belgium) 


\section{Instructions For Authors}

Upon acceptance of the manuscript, authors are charged US $\$ 60$ (US $\$ 30$ for graduate students), which must be paid prior to the creation of proofs. One copy of the issue in which the paper appears is sent to each author/co-author. Information regarding the cost of preprints and reprints are sent to the corresponding author only. Authors of invited papers are not charged for a copy of the issue in which their article appears.

The copyright is transmitted from the author(s) to JMASM for the entire usual period. After one year from the date of publication, the author(s) may republish the article in its entirety or in part if a footnote on the first page states, "Material in this article was initially published by the Journal of Modern Applied Statistical Methods", followed by the date, volume number, issue number, page numbers, mailing address, and e-mail address of JMASM.

To submit a manuscript for consideration, follow these instructions:

1. The most recent American Psychological Association style guidelines are preferred.

2a. For mailed submissions:

- $\quad$ four copies typed double spaced in 10 point font with 1" margins all around

- a computer file on a clearly labeled 31/2" "IBM Compatible" disk in WordPerfect (preferred), MS Word, or ordinary ASCII format. Note that at this time, submissions are not acceptable in Tex (or any of its derivatives), Adobe .pdf format, or non-PC formats (e.g., Macintosh).

2b. For e-mailed submissions:

- an external file attachment in either WordPerfect (preferred), MS Word, or ASCII format. Note that at this time, submissions are not acceptable in Tex (or any of its derivatives), Adobe .pdf format, or non-PC formats (e.g., Macintosh).

For all submissions:

- Title with name, address, and affiliation of all authors. Abstract containing 50 words maximum. Keywords. Provide 50 word maximum biographical sketch, including e-mail address, for all authors. Text maximum of 20 pages, including tables, figures, graphs, and references. Create tables in APA format without boxes or vertical lines. Place tables, figures, and graphs "in-line" at the appropriate place in the document, not at the end. Figures must be in .tif, .png, .jpg, and other formats readable by Adobe Illustrator or Photoshop. (Please inquire as to acceptable formats.) Article headings are Introduction, Methodology, Results, Conclusion, \& References. Subheadings are left-justified, and sub-subheadings are left-justified underscore. (Avoid underscore elsewhere.) Do not number sections, but number formulas, tables, and figures. Italics may be used. Use bold for matrices only. Avoid footnotes or endnotes. Do not number references.

- a statement that proper human subject protocols were followed, including informed consent

- a statement that the manuscript is not under consideration at another journal

3. There should be no material identifying authorship except on the title page.

4. Proofs will be sent by e-mail in Adobe .pdf format. Changes must be minimal and returned within 72 hours. A markup charge will be assessed for substantive changes.

5. The normal review period is about three months.

\section{Print and Online Subscriptions}

Print subscriptions including postage for professionals is US $\$ 60$ per year; graduate students is US $\$ 30$ per year; and libraries, universities, and corporations is US $\$ 195$ per year. Postage is an additional US $\$ 10$. Subscribers outside the US and Canada must pay a US $\$ 10$ surcharge for special processing. Online access will be available soon. Mail subscription requests with remittances to JMASM, P. O. Box 48023, Oak Park, MI 48237, USA. E-mail address for all correspondence is: jmasm@edstat.coe.wayne.edu.

\section{Notice To Advertisers}

Send requests for advertising information to jmasm@edstat.coe.wayne.edu.

\section{Call For Editorial Board Members and Ad Hoc Reviewers}

To serve on the Editorial Board or in an ad hoc reviewer capacity, please send a note indicating areas of expertise and your curriculum vitae as a WordPerfect or MS Word (PC format only) file attachment to jmasm@edstat.coe.wayne.edu. 


\section{Announcing StatXact 5!}

StatXact 5, with over 100 procedures and a 1500 page manual that is really a textbook on exact methods, provides the world's most comprehensive collection of exact procedures

for significance tests and confidence intervals. Among its new features, StatXact 5 now gives you a host of new procedures for the commonly-encountered two-binomial situation. Based on recent research (Agresti and Min, Biometrics 2000; Chan and

Zang, Biometrics 1999), these procedures will give you more powerful exact $\mathrm{p}$-values, and shorter exact confidence intervals.

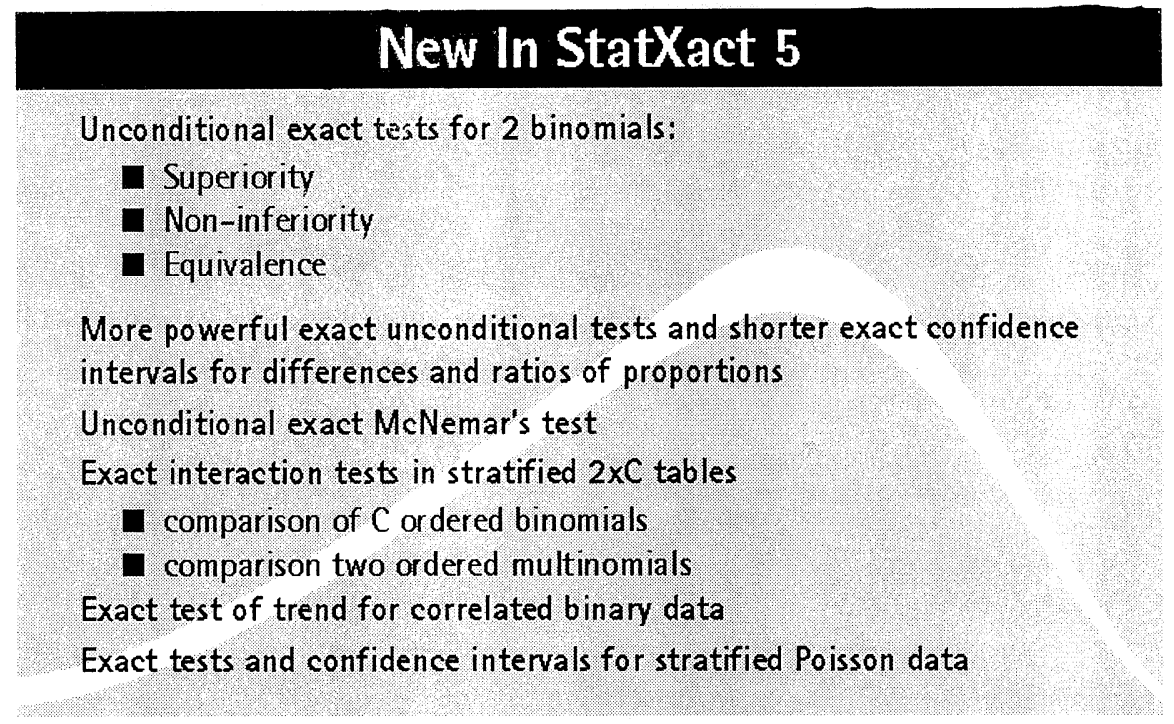

While some standard software programs have a few exact tests, none has amwhere near the coverage of StatXact 5. StatXact 5, with over 100 tests and procedures, gives you exact $p$-values and confidence intervals for one-, two- and k-sample problems, $\mathrm{R} \times \mathrm{C}$ contingency tables, stratified $2 \times 2$ and $2 \times \mathrm{C}$ contingency tables, goodness-of-fit tests, measures of association, binomial data, multinomial data, and censored survival data. Plus, StatXact 5 gives you exact power and sample size capabilities.

CYTEL Software Corporation - 675 Massachusetts Ave., Cambridge, MA 02139 USA

Tel (617) 661-2011 • Toll Free (US) 866-298-3511 • Fax (617) 661-4405 http://www.cytel.com • E-mail: sales@cytel.com 
"Perfection is achieved, not when there is nothing more to add, but when there is nothing left to take away."

- Antoine de Saint Exupery

F is a carefully crafted subset of the most recent version of Fortran, the world's most powerful numeric language.

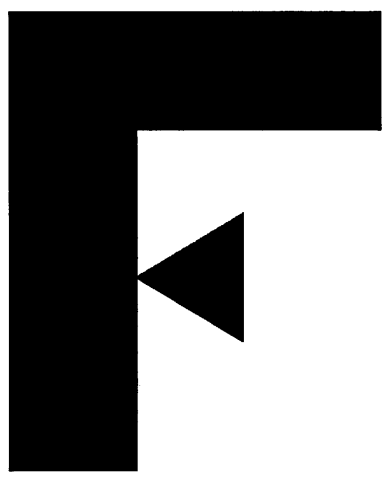

Using F has some very significant advantages:

- Programs written in F will compile with any Fortran compiler

- $F$ is easier to use than other popular programming languages

- F compilers are free and available for Linux, Windows, and Solaris

- Several books on F are available

- F programs may be linked with C, Fortran 95, or older Fortran 77 programs

F retains the modern features of Fortran-modules and data abstraction, for example- but discards older error-prone facilities of Fortran.

It is a safe and portable programming language.

\section{F encourages Module-Oriented Programming.}

It is ideal for teaching a programming language in science, engineering, mathematics, and finance.

It is ideal for new numerically intensive programs.

The Fortran Company

11155 E. Mountain Gate Place, Tucson, AZ 85749 USA +1-520-256-1455 +1-520-760-1397 (fax)

http://www.fortran.com info@fortran.com 